


\section{ELECTRIC MACHINES AND DRIVES}

Edited by Miroslav Chomat 


\section{Electric Machines and Drives}

http://dx.doi.org/10.5772/600

Edited by Miroslav Chomat

\section{Contributors}

Omar Taha Hegazy, Amr Amin, Joeri Van Mierlo, Mohamed Becherif, Asim Bensadeq, Eduardo Mendes, Assia Henni, Paul Lefley, Mohamed-Yacine Ayad, Jogendra Singh Thongam, Rachid Beguenane, Dan Valentin Nicolae, Kouzou Abdellah, Cristiane Cauduro Gastaldini, Rodrigo Zelir Azzolin, Rodrigo Padilha Vieira, Hilton Abílio Gründling, Miroslav Chomat, Jalal Nazarzadeh, Vahid Naeini, Mohamed Benhaddadi, Guy Olivier, Rachid Ibtiouen, Jessica Yelle, JeanFrançois Tremblay, Ademir Nied, José de Oliveira, Rafael De Farias Campos, Seleme Isaac Seleme, Jr., Luiz Carlos De Souza Marques, Waldiberto De Lima Pires, Hugo Gustavo Gomez Mello, Sebastiao Lauro Nau, Alexandre Postól Sobrinho

\section{(c) The Editor(s) and the Author(s) 2011}

The moral rights of the and the author(s) have been asserted.

All rights to the book as a whole are reserved by INTECH. The book as a whole (compilation) cannot be reproduced, distributed or used for commercial or non-commercial purposes without INTECH's written permission. Enquiries concerning the use of the book should be directed to INTECH rights and permissions department (permissions@intechopen.com).

Violations are liable to prosecution under the governing Copyright Law.

\section{(cc) BY}

Individual chapters of this publication are distributed under the terms of the Creative Commons Attribution 3.0 Unported License which permits commercial use, distribution and reproduction of the individual chapters, provided the original author(s) and source publication are appropriately acknowledged. If so indicated, certain images may not be included under the Creative Commons license. In such cases users will need to obtain permission from the license holder to reproduce the material. More details and guidelines concerning content reuse and adaptation can be foundat http://www.intechopen.com/copyright-policy.html.

\section{Notice}

Statements and opinions expressed in the chapters are these of the individual contributors and not necessarily those of the editors or publisher. No responsibility is accepted for the accuracy of information contained in the published chapters. The publisher assumes no responsibility for any damage or injury to persons or property arising out of the use of any materials, instructions, methods or ideas contained in the book.

First published in Croatia, 2011 by INTECH d.o.o.

eBook (PDF) Published by IN TECH d.o.o.

Place and year of publication of eBook (PDF): Rijeka, 2019.

IntechOpen is the global imprint of IN TECH d.o.o.

Printed in Croatia

Legal deposit, Croatia: National and University Library in Zagreb

Additional hard and PDF copies can be obtained from orders@intechopen.com

Electric Machines and Drives

Edited by Miroslav Chomat

p. cm.

ISBN 978-953-307-548-8

eBook (PDF) ISBN 978-953-51-5986-5 


\section{We are IntechOpen, the first native scientific \\ publisher of Open Access books}

$3,350+$

Open access books available

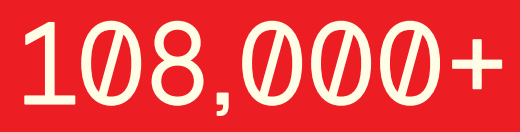

International authors and editors

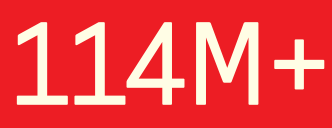

Downloads

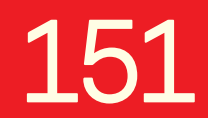

Countries delivered to

Our authors are among the

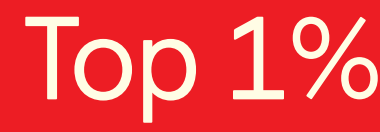

most cited scientists

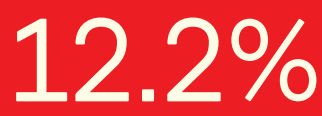

Contributors from top 500 universities

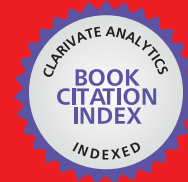

WEB OF SCIENCE ${ }^{\text {TM }}$

Selection of our books indexed in the Book Citation Index in Web of Science ${ }^{\mathrm{TM}}$ Core Collection (BKCI)

Interested in publishing with us?

Contact book.department@intechopen.com

Numbers displayed above are based on latest data collected.

For more information visit www.intechopen.com 



\section{Meet the editor}

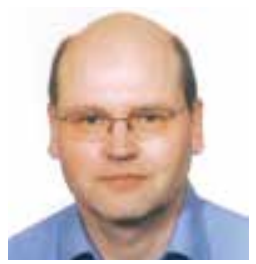

Miroslav Chomat attained his M.Sc. degree in Electrical Power Engineering from the Czech Technical University in Prague in 1988 and his Ph.D. degree in Electrical Engineering from the Institute of Electrical Engineering of the Academy of Sciences of the Czech Republic in 1994. He is currently a senior researcher and the head of the Department of Electrical Machines, Drives, and Power Electronics at the Institute of Thermomechanics AS CR, v.v.i. He is also an assistant professor at the Department of Electric Drives and Traction of the Czech Technical University in Prague. Between 1999 and 2000, he spent one year at the University of Wisconsin-Madison, USA working as a honorary research fellow. His research interests include numerical modeling and control of electrical machines, drives, power units and electronic converters. 



\section{Contents}

Preface XI

Chapter 1 Premium Efficiency Motors 1

M. Benhaddadi, G. Olivier, R. Ibtiouen, J. Yelle, and J-F Tremblay

Chapter 2 Electric Motor Performance Improvement Using Auxiliary Windings and Capacitance Injection 25

Nicolae D.V

Chapter 3 Magnetic Reluctance Method for Dynamical Modeling of Squirrel Cage Induction Machines 41

Jalal Nazarzadeh and Vahid Naeini

Chapter 4 Minimization of Losses in Converter-Fed Induction Motors-Optimal Flux Solution 61 Waldiberto de Lima Pires, Hugo Gustavo Gomez Mello, Sebastião Lauro Nau and Alexandre Postól Sobrinho

Chapter 5 Sensorless Vector Control of Induction Motor Drive - A Model Based Approach 77

Jogendra Singh Thongam and Rachid Beguenane

Chapter 6 Feedback Linearization of Speed-Sensorless Induction Motor Control with Torque Compensation 97

Cristiane Cauduro Gastaldini, Rodrigo Zelir Azzolin, Rodrigo Padilha Vieira and Hilton Abílio Gründling

Chapter 7 From Dynamic Modeling to Experimentation of Induction Motor Powered by Doubly-Fed Induction Generator by Passivity-Based Control 113 M. Becherif, A. Bensadeq, E. Mendes,

A. Henni, P. Lefley and M.Y Ayad

Chapter 8 A RMRAC Parameter Identification Algorithm Applied to Induction Machines 145 Rodrigo Z. Azzolin, Cristiane C. Gastaldini, Rodrigo P. Vieira and Hilton A. Gründling 
Chapter 9 Swarm Intelligence Based Controller for Electric Machines and Hybrid Electric Vehicles Applications 161 Omar Hegazy, Amr Amin, and Joeri Van Mierlo

\section{Chapter 10 Operation of Active Front-End Rectifier} in Electric Drive under Unbalanced Voltage Supply 195 Miroslav Chomat

Chapter 11 Space Vector PWM-DTC Strategy for Single-Phase Induction Motor Control 217

Ademir Nied, José de Oliveira, Rafael de Farias Campos,

Seleme Isaac Seleme Jr. and Luiz Carlos de Souza Marques

Chapter 12 The Space Vector Modulation PWM Control Methods Applied on Four Leg Inverters 233

Kouzou A, Mahmoudi M.O and Boucherit M.S 


\section{Preface}

This book focuses on a very important and diverse field of electric machines and drives. The history of the electric machine, which is the keystone of electromechanical energy conversion, dates back to the beginning of the nineteenth century. The names of famous scientists, such as Michael Faraday, Joseph Henry or Nikola Tesla, are associated with the invention of the rotating electric machine. Electric drives have quickly become an integral part of our everyday lives and we can hardly imagine our civilization without them. Electric drives play a vital part in industry, transportation as well as in modern households. If we counted the number of electric drives around every one of us today, we would certainly be surprised how big the number is.

Since the invention of the first electric machine, novel principles and designs have been appearing and the properties and parameters of electric machines have been steadily improving. The advent of power electronics and modern control circuitry at the end of the twentieth century caused a revolution in the field of electric drives. Nowadays, when modern technologies are available and advanced materials and techniques commonly utilized, formerly inconceivable results can be achieved in the field of modern electric drives.

The twelve chapters of the book written by renowned authors, both academics and practitioners, cover a large part of the field of electric machines and drives. Various types of electric machines, including three-phase and single-phase induction machines or doubly fed machines, are addressed. Most of the chapters focus on modern control methods of induction-machine drives, such as vector and direct torque control. Among others, the book addresses sensorless control techniques, modulation strategies, parameter identification, artificial intelligence, operation under harsh or failure conditions, and modelling of electric or magnetic quantities in electric machines. Several chapters give an insight into the problem of minimizing losses in electric machines and increasing the overall energy efficiency of electric drives, which is currently viewed as a priority.

I would like to express my gratitude to all the authors for their contributions, in which they shared their valuable experience and knowledge with the readers. It was their immense involvement that enabled the publication of this book. I would also like to thank the InTech staff for their great effort and support in preparation of the book. I hope it 
will benefit the field of electric machines and drives, provide the readers with a new point of view on this interesting branch of electrical engineering and possibly initiate many inventions and innovations in the future.

Miroslav Chomat Institute of Thermomechanics AS CR, v.v.i.

Czech Republic 


\title{
Premium Efficiency Motors
}

\author{
M. Benhaddadi ${ }^{1}$, G. Olivier ${ }^{1}$, R. Ibtiouen ${ }^{2}$, J. Yelle ${ }^{3}$, and J-F Tremblay ${ }^{3}$ \\ ${ }^{1}$ École Polytechnique de Montréal, dépt. de génie électrique, C.P. 6079 \\ Succursale Centre-ville, Montréal, Québec, H3C 3 A7 \\ 2École Nationale Polytechnique d'Alger, dépt. de génie électrique, Avenue Pasteur \\ BP 182, El Harrach, 16200 Alger \\ ${ }^{3}$ Cégep du Vieux Montréal, dépt. Technologie de génie électrique, 255 Ontario-Est \\ Montréal, Québec, Canada H2X 1X6 \\ 1,3 Canada \\ ${ }^{2}$ Algérie
}

\section{Introduction}

Despite its considerable potential for energy savings, energy efficiency is still far from realizing this potential. This is particularly true in the electrical sector (IEA, 2010). Why? There is no probably just one single answer to this question. A consequential response requires major multiform research and an analytical effort. No doubt that analysis of the interaction between energy efficiency policies and energy efficiency performance of economies accounts for a significant part of the effort.

In the future sustainable energy mix, a key role will be reserved for electricity, as GHG emissions reduction in this sector has to be drastically reduced. In this option, obvious conclusion is that large market penetration Premium motors needs a complex approach with a combination of financial incentives and mandatory legal actions, as industry doesn't invest according to least life cycle costs (DOE, 2010).

This present work illustrates the induced enormous energy saving potential, permitted by using high-efficiency motors. Furthermore, the most important barriers to larger highefficiency motors utilization are identified, and some incentives recommendations are given to overcome identified impediments.

In the present work, experimental comparison of the performance characteristics of $3 \mathrm{hp}$ Premium efficiency motors from three different manufacturers has been presented. The motors were tested according to Standard IEEE 112-B.

\section{Energy, climate change and electricity}

According to last report Intergovernmental Panel on Climate Change IPCC report (IPCC, 2007), the observed increase in global average temperatures since the mid-20th century is very likely due to the observed increase in anthropogenic greenhouse gas concentrations. Moreover, there is no doubt that discernible human influences now extend to other aspects of climate, including ocean warming, continental-average temperatures, temperature extremes and wind patterns. Stabilizing atmospheric carbon dioxide concentrations at twice 
the level of pre-industrial times is likely to require emissions reductions up to $90 \%$ below current levels by 2100 . Clearly, reductions of this magnitude can be achieved only by taking action globally and across all sectors of the economy. The electricity sector will undoubtedly need to assume a major share of the weight, according to its contribution to overall emissions estimated to be more $10000 \mathrm{Mt}$ (million tone) $\mathrm{CO}_{2 \text { eq }}$ per year.

As can be seen in fig.1, the electricity generation is dominantly produced from fossil fuels (coal, oil, and gas), and today's situation is the same as forty years ago (DOE, 2010). In the last XXI world energy congress, it is highlighted that electricity generation will still depend on fossil sources. In the meantime, according to (IEA, 2010), industry accounts for more $40 \%$ of the world 20000 TWh (terawatt hours, or so called billion kilowatt hours) electricity consumption, weighting more $4000 \mathrm{Mt} \mathrm{CO}_{2 \text { eq }}$ per year. Within the industrial sector, motor driven systems account for approximately $60 \%$ to $65 \%$ of the electricity consumed by North American (RNC 2004, DOE 2010) and European Union industries. Implementing high efficiency motor driven systems, or improving existing ones just by 1 to $2 \%$, could save up to 100-200 TWh of electricity per year. This would significantly reduce the need for new power plants. It would also reduce the production of greenhouse gases by more 100 million $\mathrm{CO}_{2 \text { eq }}$ per year and push down the total environmental cost of electricity generation.

The worldwide electric motors above $1 \mathrm{hp}$ can be estimated to be nowadays more 300 million units, with the annual sales of 34 million pieces. Typically, one-third of the electrical energy use in the commercial sector and two-thirds of the industrial sector feed the electrical motors (DOE, 2010). Moreover, the low voltage squirrel cage induction motor constitutes the industry workhorse. In particular industrial sector such as the Canadian petroleum and paper industry, the share of the energy used by electrical motors can reach 90\% (RNC 2004). Since induction motors are the largest electrical energy user, even small efficiency improvements will result in very large energy savings and contribute to reduce greenhouse gas emissions GHG. Furthermore, the declining resources combined environmental global warming concerns and with increasing energy prices make energy efficiency an imperative objective.

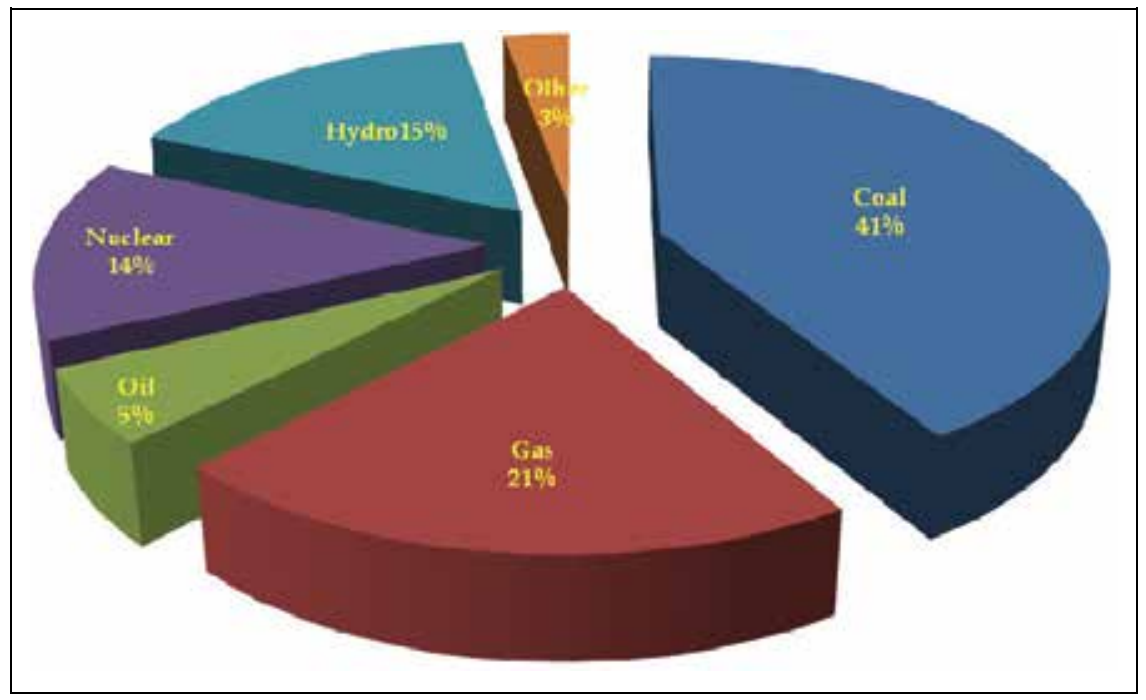

Fig. 1. Electricity generation by fuel 


\section{Motor losses segregation and efficiency}

The impact of a motor in terms of energy and economical costs depends on its performance during its lifetime. The motor performances are characterized by the efficiency with which it converts electrical energy into mechanical energy.

In Standard IEEE 112-B the losses are segregated and the efficiency is estimated by the following formula:

$$
\Delta \mathrm{P}_{\text {str }}=\mathrm{P}_{\text {in }}-\mathrm{P}_{\text {out }}-\left(\Delta \mathrm{P}_{\mathrm{el} 1}+\Delta \mathrm{P}_{\mathrm{el} 2}+\Delta \mathrm{P}_{\text {core }}+\Delta \mathrm{P}_{\text {mech }}\right)
$$

Where the electric input power, $P_{\text {in }}$, is measured with a power analyser and the output power, $\mathrm{P}_{\text {out, }}$ with a torque meter. The overall precision of efficiency assessment mainly depends on the torque estimation, and with the improved accuracy of recent power analysers and torque meters, this method can be considered accurate and reliable.

Motor efficiency is defined as a ratio motor mechanical output power and electrical input power. Hence in order to have a motor perform better, it is important to reduce its losses. The major motor losses are resistive losses in the stator and the rotor windings, and magnetic losses (hysteresis and eddy current losses) in the cores. Other losses include mechanical (bearing friction and ventilation), and stray load losses. High efficiency motor losses relative distribution is not so different at low efficiency one's; it's more dependent on the power. Their general distribution is illustrated in fig.2.

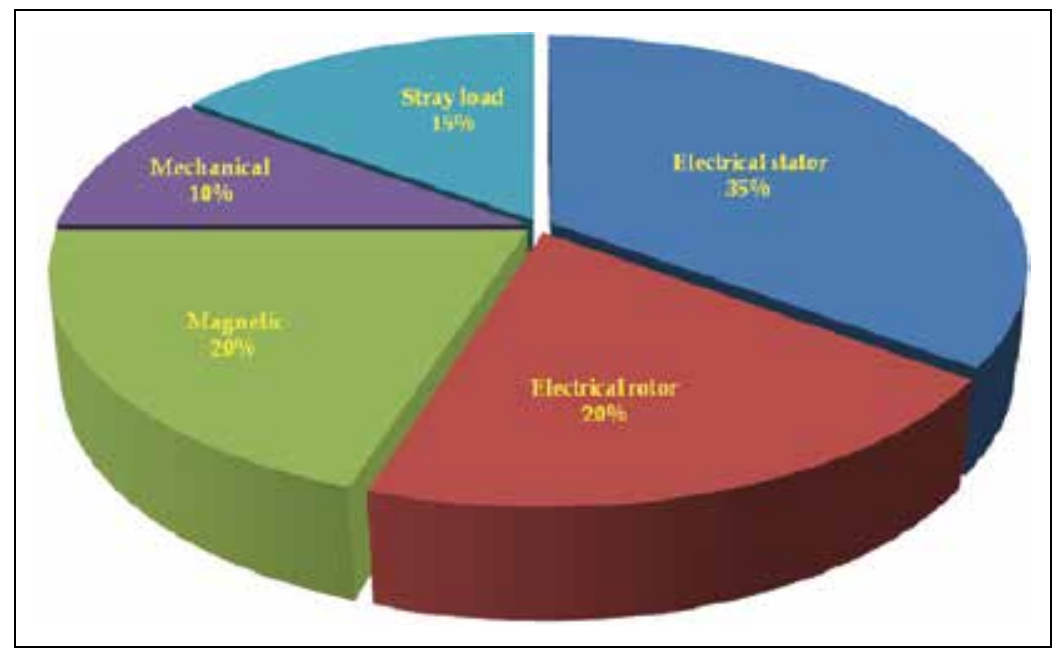

Fig. 2. Induction motor losses distribution

There are many ways to improve electric motor efficiency; the majority of them make the motor larger in diameter or overall sizes and, of course, more expensive.

- Winding stator $\left(\Delta \mathrm{P}_{\mathrm{el} 1}\right)$ and rotor $\left(\Delta \mathrm{P}_{\mathrm{el} 2}\right)$ losses are due to currents flowing through the stator windings and rotor bars. These losses can be reduced by decreasing the conductor current density in the stator windings, in the rotor bars and in the end rings. Using larger conductors lowers stator resistance, while the use of copper instead of aluminum reduces rotor losses (Parasiliti et al. 2002). Another way of decreasing stator losses is by reducing the number of turns. Unfortunately, this increases the starting current and maximum torque, as worsen the power factor. 
- Magnetic losses $\Delta \mathrm{P}_{\mathrm{m}}$ occurring in the stator and rotor laminations are caused by the hysteresis and eddy current phenomena. These losses can be decreased by using better grade magnetic steel, thinner laminations and by lowering the flux density (i.e larger magnetic cores). The better grade of laminations steel are still relatively very expensive. Cheaper manufacturing methods other than stamping are expected to become available in the near future.

- Mechanical losses $\Delta \mathrm{P}_{\text {mec }}$ are due to bearing friction and cooling fan air resistance. Improving the fan efficiency, the air flow and using low friction bearings result in a more efficient design. As these losses are relatively small, the efficiency gain is small too, but every improvement is welcome.

- Stray load losses $\Delta \mathrm{P}_{\text {st }}$ are due to leakage fluxes induced by load current, non-uniform current distribution, mechanical air-gap imperfection...These losses can be reduced by design optimisation and manufacturing method improvements.

As can be deducted, one of the most established methods of increasing motor efficiency is to use higher quality materials, inexorably increasing the motor cost, as most high performance materials are expensive materials. In a recurrent manner, the same problem of increased cost holds true for better construction techniques, such as smaller air gaps, copper rather than aluminum in the rotor construction, higher conductor slot fill, and segmented core stator construction. The resulting increase in motor cost is evaluated to be between $15 \%$ and $30 \%$.

\section{Testing standards}

In North America, the prevailing testing method is based on direct efficiency measurement method, as described in the Institute of Electrical and Electronics Engineers (IEEE) "Standard Test Procedure for Polyphase Induction Motors and Generators" IEEE 112-B and in its Canadian CSA 390 adaptation. The standard first introduced in 1984 and updated in 2004, requires the measurement of the mechanical power output and the electric input, and provide a value for the motor losses, where the additional stray load losses are extrapolated from their total by the following formula (1). So, the efficiency is extrapolated by:

$$
\eta=\frac{\text { Pout }}{\text { Pin }}=\frac{\text { Pout }}{\text { Pout }+\Delta \text { Pel } 1+\Delta \text { Pel } 2+\Delta P m+\Delta P m e c+\Delta P s t}
$$

In Europe, the prevailing testing method is based on an indirect efficiency measurement as defined in IEC 34-2 standard "Rotating electrical machines - Part 2: Methods for determining losses and efficiency of rotating electrical machinery from tests". The standard first introduced in 1972 and updated in 1997, attribute a fixed value, equal to o.5 \% of input power to the additional stray load losses.

These standards differ mainly by the method used to take into account the additional load losses (Aoulkadi \& Binder, 2008, Boglietti et al 2004, Nagorny et al. 2004, Elmeida et al. $2002 . .$.$) . Many papers have been published and some authors have illustrated, that IEC 34$ 2 has drawback with a noticeable influence on the testing of high efficiency motors, as the efficiency of this motor type is overestimated, particularly in the small motor size cases. Ultimately, standard IEC $34-2$ was found to be unrealistic with its $0.5 \% \mathrm{P}_{\text {in }}$ value for stray losses (Aoulkadi \& Binder, 2008, Renier et al. 1999, Boglietti et al 2004...). That is why, in 2007, IEC published a revised standard for efficiency classification no. 60034-2-1 which includes a test procedure largely comparable to IEEE 112-B or CSA C390. Newly 
harmonized standards for energy efficiency testing IEC 60034-2-1 can contribute to lowering barriers in global trade for energy efficient motor systems.

\section{Minimum energy performance standard MEPS and efficiency motor classification}

There are many different worldwide definitions for energy efficient motors, as until these last years, there was no consensus on what really represents an energy efficient motor. Technical barriers include non harmonized testing standards and efficiency classification. In reality, the key mandatory instrument is minimum energy performance standards (MEPS).

\subsection{MEPS in North America}

On October 1992, US Congress voted law, Energy Policy Act EPAct, which mandates strict energy efficiency standards for electrical appliances and equipment, including electric motors. Motor MEPS were for the first time introduced in 1992 when all partners were finally persuaded that voluntary measures are too slow, and no significant market transformation towards more efficient motors was possible otherwise.

EPAct requires that the general purpose electric motors meet the higher nominal efficiency requirements defined in the table of National manufacturer association NEMA Standard, and the implementation of the motor MEPS went into effect in 1997.

The Canadian Standard association developed a Canadian standard in 1993, and updated it in 1998. CAN/CSA C-390 set the requirement for minimum efficiency for new motors made or sold in Canada at the same value as the NEMA energy-efficient level.

The Energy Policy Act EPAct-92 motors covered are:

- General purpose

- Definite or special purpose in a general purpose application

- Continuous duty

- 2,4 \& 6 Pole

- $1-200 \mathrm{HP}$

- $230 / 460 /, 60 \mathrm{~Hz}$

The Canadian standard was furthermore extended to $575 \mathrm{~V}$ and IEC motors, and included $75 \%$ full load to reach maximum efficiency.

Some motors were not covered:

- Definite or special purpose in a non-general purpose application

- Slower speeds

- Inverter duty

- Multi-speed

- Totally enclosed air over TEAO, and totally enclosed not ventilated TENV

As a result of the mandatory standard that was endorsed as part of the EPAct-92, North America had a motor standard foundation that leads the new century world.

In 2002, NEMA and Consortium for energy efficiency CEE established a voluntary NEMA Premium level of efficiency, and the manufacturers began the next step in evolution with the implementation on voluntary basis MEPS NEMA Premium efficiency motors. NEMA premium efficiency standards (CEE 2007) have remained voluntary for a long period of 10 years. In spite of this, NEMA premium motors have been progressively gaining market share, as the overall benefits of Premium motors is incommensurable (more reliable, last 
longer, have longer warranties, run more quietly and cooler and produce less waste heat than their less-efficient counterparts). The trend is particularly well depicted in the work, illustrated in fig. 3.

The evolution of MEPS based on NEMA Premium is now moving from voluntary basis to legislated regulation, as the law implementation is awaited for December 2010. So, $1-200$ HP general purpose motors already covered by EPAct will change from NEMA MG-1, Table 12-11 Energy Efficient (Annex 1) to Table 12-12 NEMA Premium efficiencies levels (Annex 2), except for fire pump motors which remain at EPAct-92 level.

Moreover, the proposal expands the scope of enclosed 1-200 Hp motors, as several motor types not previously covered by EPAct-92 must meet EPAct efficiency levels. The added motors are:

- U-frame

- Design C

- Footless

- Close-coupled pump

- Vertical solid shaft normal thrust

- 8 poles

- All low voltages $\leq 600 \mathrm{~V}$ not previously covered, including IEC Metric frame motors from 90 frames and up.

- 201 to $500 \mathrm{HP}$ low voltage 2-8 pole general purpose motors, where Design B represent something like $3 / 4$ of total range.

It's appropriate to highlight that this additional coverage means that MEPS is extended to more $90 \%$ of the motors in the 1 to $200 \mathrm{Hp}$ range, despite the fact that some manufacturers expressed technical skepticism about meeting premium efficiency levels design $C$ and 900 rpm motors.

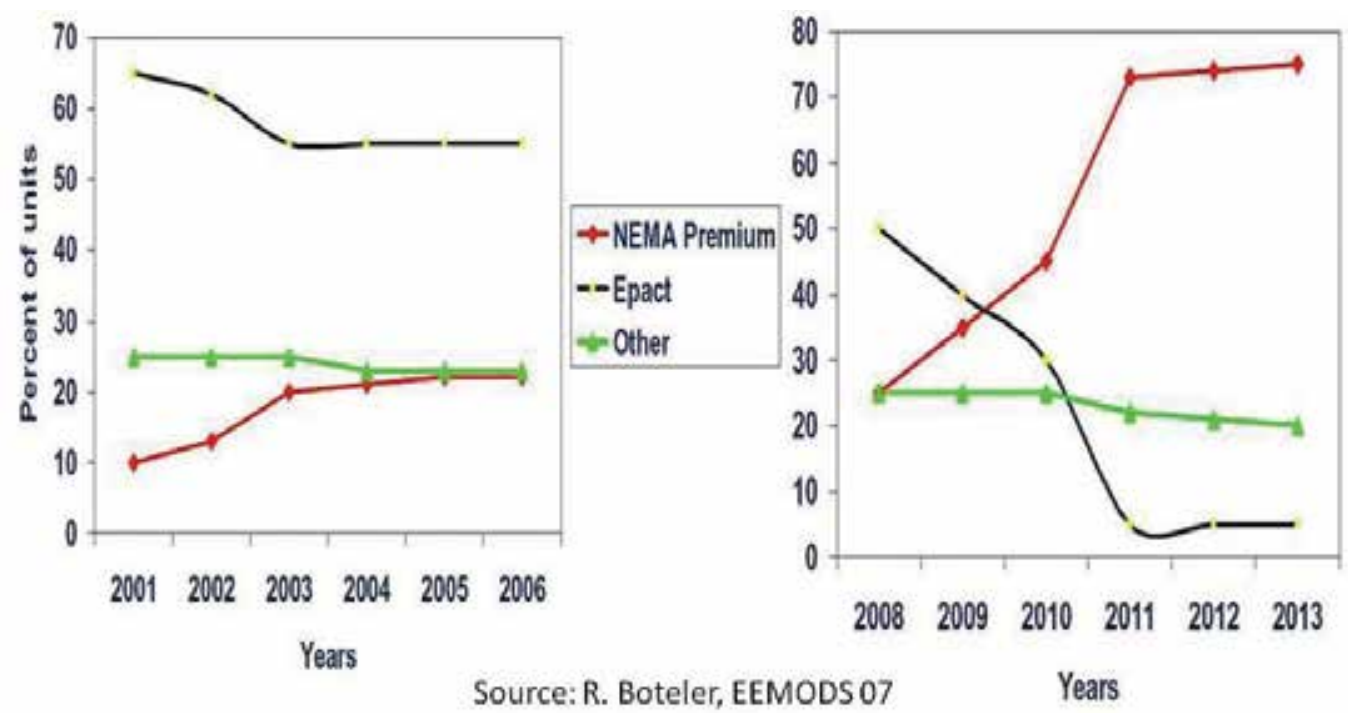

Fig. 3. Efficiency trend 2001-2006 and efficiency expected 2008-2013

On the other hand, EISA does not apply to motors exported outside North America, as well as EISA doesn't contain any requirement to replace electric motors in use. In addition, the 
law applies only to motors manufactured after December 19, 2010 and motors in inventory on that date can be sold or used without any additional constraints, but some customers may not probably want them.

Some Motor configurations are not covered by EISA law. These are:

- $\quad$ Design D with high slip

- Inverter duty with optimized windings for adjustable speed drives ASD use

- Customized OEM mounting

- Intermittent duty

- Integral with gearing or brake where motor cannot be used separately

- Submersible motors

- $\quad$ Single Phase motors

- DC motors

- $\quad$ Two-digit frames (48-56)

- Multi-speed

- Medium voltage motors

- Repaired motors

- TENV and TEAO enclosures

It's relevant to notice that regulation concerning single phase and three-phase small $(42 / 48$ and 56 frame) motors is ready, as Department of energy (DOE 2010) published in 2010 its final rule giving efficiency limits that must be meet by small general purpose electric motors, starting from 2015. Nevertheless, NEMA doesn't support regulation of this product, as many questions are still not solved while small induction motor is continually losing market part to switched reluctance and permanent magnet types.

\subsection{New IEC standard}

In 2007, IEC published a revised standard for efficiency testing no. 60034-2-1 which includes a test procedure largely comparable to IEEE 112-B or CSA C390, and in September 2008 a new standard no. 60034-30 for efficiency classification of electric motors.

New standard for efficiency classification is applicable to single speed, three-phase induction motors with the following parameters:

- $\quad$ Power from 0.75 to $375 \mathrm{~kW}$

- Voltage under $1000 \mathrm{~V}$

- 50 and $60 \mathrm{~Hz}$ frequency

- $\quad 2,4$ and 6 poles

- Duty S1 or S3

- All IP1x to $6 x$ and IC0x to $4 x$

- Networked

- All types of fixing, shaft extension, accessories

Some Motor configurations are not covered by IEC 60034-30. These are:

- Motors with reinforced isolation specifically designed for variable speed drives applications

- Motors which are fully integrated in a machine and cannot be tested separately

Newly 2008 harmonized standard for energy efficiency class 60034-30-2008 follow International Electrotechnical Commission (IEC) protocol and defines four induction motor efficiency classes: 
- $\quad$ Super Premium efficiency level IE4

- $\quad$ Premium efficiency level IE3

- High efficiency level IE2

- $\quad$ Standard efficiency level IE1

It's noticeable that, nowadays, Premium efficiency IE3 is the most efficient motor. Super Premium efficiency IE4 is a future new generation motor. It is awaited that in average, the losses reduction of IE4 should be $15 \%$ compared to IE3. So, IE4 is not a standard in fact, but just a level.

\subsection{MEPS in EU and BRIC countries}

In Europe, the European committee of manufacturers of electrical machines and power electronics CEMEP has classified $2 \& 4$ pole $1-90 \mathrm{~kW}$ motors into three levels:

- High (EFF1),

- Improved (EFF2), and

- $\quad$ Standard efficiency (EFF3).

The CEMEP classification has induced substantial EFF3 motors reduction and EFF2 market share promotion. Nevertheless, EFF1 market part is still modest. Meanwhile, European Union is considering prohibiting the sale of motors that don't meet EFF2 criteria in the near 2011 future.

The new EU MEPS system is based on the latest IEC standard, and it represents a significant step towards worldwide harmonization of efficiency regulations.

The challenges that EU countries facing are:

- From June 16, 2011, motors shall not be less efficient than IE1 (i.e. EFF2) and EFF3 motors will be banned

- From January 1, 2015, motors with rated output of 7.5 to $375 \mathrm{~kW}$ shall not be less efficient than IE2

- From January 1, 2017, motors with rated output of 7.5 to $375 \mathrm{~kW}$ shall not be less efficient than IE3

- From January 1, 2017, all motors with rated 0.75 to $375 \mathrm{~kW}$ shall not be less efficient than IE2 if equipped with adjustable speed drive (ASD).

BRIC countries (Brazil-Russia-India-China) motors and motor driven equipment are still relatively less efficient. For example, the efficiency of over $80 \%$ Chinese motors is $2-5 \%$ lower than international advanced ones. Chinese scientists consider that if efficiency of motor systems could be raised to the North American level, then 150 TWh of electricity would be saved each year. But China is making progress, by formulating a number of policies, laws and regulations on energy conservation. Beginning this 2010 year, the MEPS efficiency of newly added motors should reach international first-rate level Class 2, which is equivalent to IE1, or EFF2.

At the present time, 10 countries with a half of global electricity demand have motor MEPS endorsement. In the next two years, 15 new countries targeted for a next round until 2012 to reach around $80 \%$ of global electricity demand.

As earlier mentioned, the International Electrotechnical Commission (IEC), a worldwide organization for standardization comprising all national standards committees, defined in IEC 60034-30 (2009) four efficiency classes for single-speed cage-induction motors, and specified test procedures. It's important to notice that the so-called IE4 Super Premium Efficiency products are not commercially available yet, while lower efficiency motors in use 
now Eff3 disappears in the new classification. This new classification will be probably soon adopted worldwide in place of regional or local classification, as illustrated in table 1 . This new standard defines efficiency classes and their containing minimum values (conditions).

\begin{tabular}{|l|c|c|c|c|}
\hline Efficiency Class & IEC & USA/Canada & CEMEP & China \\
\hline $\begin{array}{l}\text { Super Premium } \\
\text { efficiency }\end{array}$ & IE4 & - & - & - \\
\hline Premium efficiency & IE3 & NEMA Premium & - & - \\
\hline High efficiency & IE2 & EPAct & EFF1 & Class 1 \\
\hline Standard efficiency & IE1 & - & EFF2 & Class 2 \\
\hline $\begin{array}{l}\text { Below standard } \\
\text { efficiency }\end{array}$ & - & - & EFF3 & Class 2 \\
\hline
\end{tabular}

Table 1. International motor efficiency classification

\section{Life cycle cost Premium motors}

An electric motor is somewhat cheap to buy, but expensive to run. For example, a $3 \mathrm{hp}$ Premium efficiency motor functioning 6000 hours per year consumes about $1000 \$$ of electricity at $\$ 0.07 / \mathrm{kWh}$. The purchase price for such a motor is about $500 \$$ and over the motor's 15-year life, the acquisition price represents only $3 \%$ of the lifetime costs, while the cost of electricity accounts for $97 \%$. Finally, a $2 \%$ increase in Premium motor efficiency over EFF1 translates in energy savings over that time nearly twice the cost difference. In addition, with a larger motor, the saving potential will be larger, and therefore payback periods would be shortened. For the $100 \mathrm{Hp}$ motor, the acquisition price represents only $1 \%$ of the lifetime costs, while the cost of electricity accounts for 99 \%!!! Fig. 4 depicts typical lifetime cycle cost motor in the conservative case (Benhaddadi \& Olivier, 2010a).

The average life cycle of the small power motors is of the order of 15 years, i.e. the equivalent of the average car range. The fundamental difference is in the fact that during this period, the cost of the electricity will represent $97 \%$ of the cost of useful life cycle of the electric motor, while for the car motor, it represents only $10 \%$. Moreover, the car's internal combustion motors can rarely overcome $50 \%$ efficiency, with an enormous negative impact to be paid in environmental pollution. We can deduct from this fact that the improvement of $1 \%$ of the electrical motor efficiency will have the same impact as the reduction of the $10 \%$ gas consumption car.

Moreover, the Canadian electricity costs are presently up to two times cheaper than elsewhere and high electricity prices reduce payback period. In addition, some Canadian utility companies and public agencies like Hydro Québec in Québec offer rebate programs to encourage customers to upgrade their standard motors to Premium efficiency (Benhaddadi \& Olivier, 2010b). For motors from 1 to $75 \mathrm{hp}$, this program allows $600 \$ / \mathrm{hp}$ to the customer and $150 \$ / h p$ to the distributor for each saved hp. Unfortunately, as a consequence of the lack of energy saving importance, the purchase of a new motor, as well as the rewinding of defective standard-efficiency motors, the choice of the motor is often driven by short term investment considerations, not on the cost of the electricity which can be saved.

The first law for energy efficient motors is the Energy Policy Act (EPAct) which mandates strict energy efficiency standards for electrical appliances and equipment. This law was first adopted in USA and became effective in Canada with the adoption of Standard CAN/CSA- 
C390-98. Today more than $75 \%$ of the motors sold in North America are Premium efficiency and EPAct machines. This clearly indicates the positive effect of the energy law. In light of the above, and taking into consideration the very slow market transformation with just voluntary and incentive measures, there's no doubt that Premium motors will monopolize the dominant part of the market in the near 2013 future. This is well illustrated in fig. 3. There is no doubt that the appropriate legislation is the best way of achieving that goal (Benhaddadi \& Olivier, 2009b). Only the latest energy efficient motor technologies should be manufactured and used. In general terms, North America is not on the leading edge for energy saving and conservation. Motor efficiency is an exception that should be at least maintained by EISA law implementation.

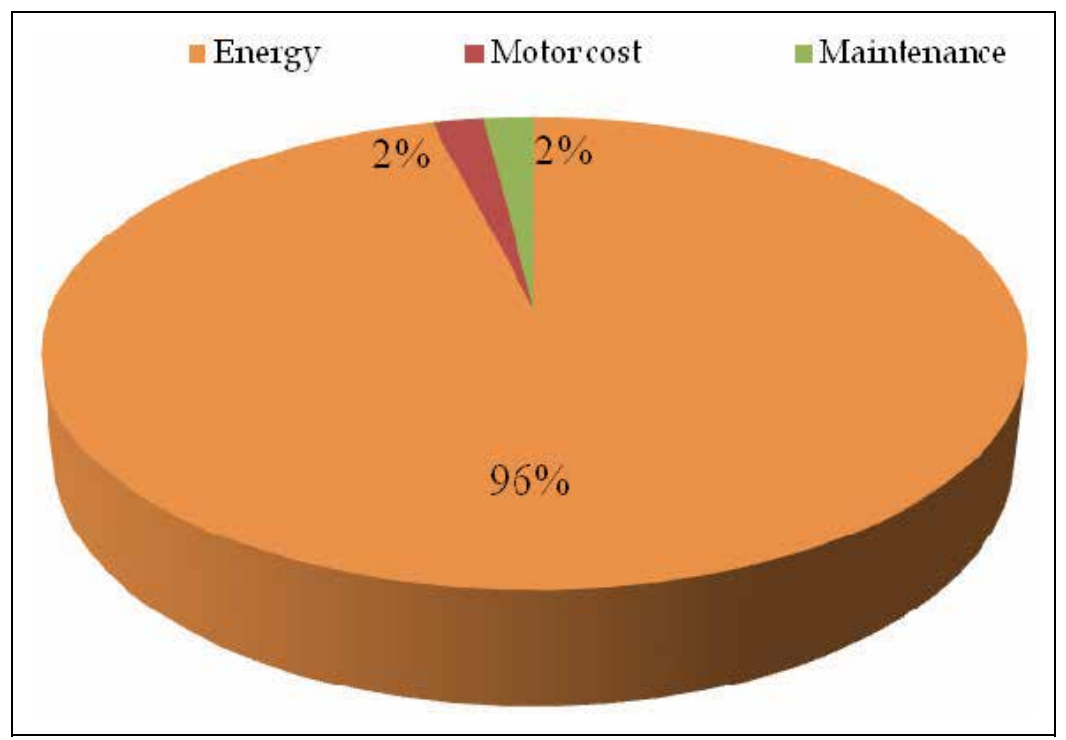

Fig. 4. Lifetime motor cost

\section{Barriers to high efficiency motors market penetration}

Despite the colossal energy saving potential and financial incentives programs, many companies are still reticent to invest in energy-efficiency motors. The reasons why the wellknown potential for energy saving energy is not exploited have been investigated, and the authors have identified several reasons why this potential is not yet fully exploited. The Grand paradox is that cost effective measures are not taken because of several illogical barriers. So, the most important barriers to high efficiency motors promotion are (Benhaddadi \& Olivier, 2008a):

- The energy costs are relatively so small that energy efficiency improvement isn't taken into consideration,

- Lower priority of energy savings importance, when other factors such as availability service, reliability, and first costs are of premium importance,

- Industry reluctance to change what is, a priori, a good functioning system,

- Doubt about success of energy efficiency programs, or the discount rates used to justify energy efficiency programs are too low, 
- Downtime replacement cost look like peanuts, but shutdown time to install new equipment is expensive and many companies don't accept this inconvenient,

- Reduced budget often makes reducing energy consumption as " poor parent ", inducing a lack of encouragement to make a decision,

- Implementing making-decision responsibility is often shared with many internal conflicting pressures and divergence and ultimate choice don't always belong to electrician engineers, who are energy savings conscious,

- Distributors regularly represent two or more motor manufacturers and they can advantage products from the manufacturer that offers the highest discount rather than high-efficiency ones,

- Usual predisposition to use stocked old motors rather than purchase high efficiency ones,

- It is not economically pragmatic to change a motor until it fails,

- Penchant to have the failed motor repaired rather than replaced by high efficiency ones,

- Degradation efficiency of repaired motor cannot be simply illustrated,

- Annual running hours are not sufficiently high to induce satisfactory payback.

\section{Incentive policies to overcome barriers}

Experience derived from many energy saving initiatives around the world showed that the most successful programs are based on a combination of technical and promotional information, educational tools and financial incentives. If technicians and engineers would be trained in system design integration and least lifecycle cost as a goal, no doubt that the problem of inefficient industrial equipment should be solved (Benhaddadi \& Olivier, 2008a). Consequently, to overcome the identified obstacles need a combination of the following measures:

- Premium priority: For the companies, the energy saving status has to arrive at legislative endorsement, like is the case for safety and quality insurance,

- Incentive programs: to reinforce energy savings promotion politics, much higher discount rates should be used to evaluate the cost-effectiveness of energy efficiency policies, programs or measures,

- Highlighted information and diffusion: this information must be of practical value, and sufficiently demonstrative with real pilot projects,

- Environmental concern: It's necessary to reinforce ecological policy criteria and support environmental friendly companies. This follows the principle that the saved energy is the most environmentally friendly one. A particularly promising concept is the emissions trading scheme, which could be enable companies to claim emissions credits for investments that reduce energy consumption,

- Legislation: to legislate against recalcitrant and to impose to market the gradual approach of the "carrot and the stick", where the carrot represents the incentives and the stick stands for refractory.

The authors strongly believed in the need to enhance policy measures aimed at reducing the demand for energy and the resultant environmental impact. We therefore welcome the increased interest in energy conservation in Canada. But, the accumulated experience clearly show that putting in place incentives and voluntary measures for the energy efficiency of electric motors is not sufficient, as it is prerequisite to implement mandatory measures for 
larger market penetration (fig. 5). The carbon savings from this measure have the potential to make a significant contribution to emissions target reduction.

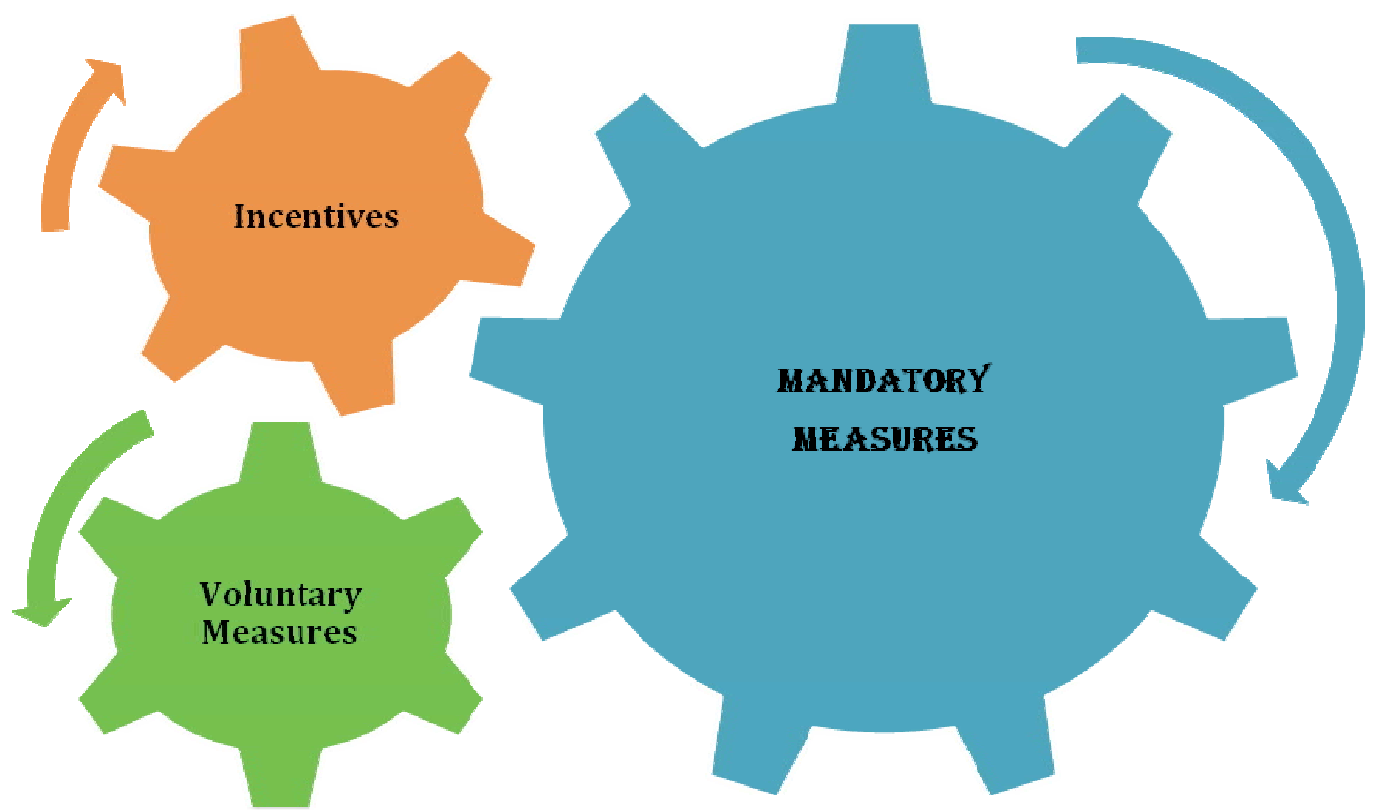

Fig. 5. Incentives, voluntary and mandatory measures impact

\section{Experimental setup and results}

Figure 6 depicts the experimental laboratory set-up. The motors are fed by three single phase autotransformers and direct torque control, DTC drives. The motors are mechanically loaded by dc machine connected through a precision torque-speed transducer. A motor/harmonic power meter is used for measuring real and reactive power, currents, voltages and power factor. The motors are mechanically loaded by dc machine connected through a high precision torque-speed transducer.

The measurements were taken in similar conditions and each motor was loaded until thermal equilibrium was reached, while each of the two benches can be used to determine the efficiency.

The two benches are sufficiently flexible and require minimum adjustments when different Premium motors are tested (Benhaddadi et al. 2010b). Several $3 \mathrm{hp}$ Premium motors from different 3 manufacturers were tested. Figures 7 and 8 show the stators and rotors of the three different motors.

The measurements were taken in similar conditions and each motor was loaded until thermal equilibrium was reached.

For each of the motors B \& C, Fig. 9 shows the variation of the efficiency versus the mechanical torque in the case of direct $60 \mathrm{~Hz}$ feeding, without drive. The results for motor A are not provided, as they are the same as for the motor B. One can deduct that when the motors are feed from the rated $230 \mathrm{~V}$ grid, the difference between the measured efficiency at rated load and the nameplate value is less a $0.2 \%$, But, most electric motors are designed to function at $50 \%$ to $100 \%$ of rated value. Fig. 9 also shows that maximum Premium motor 


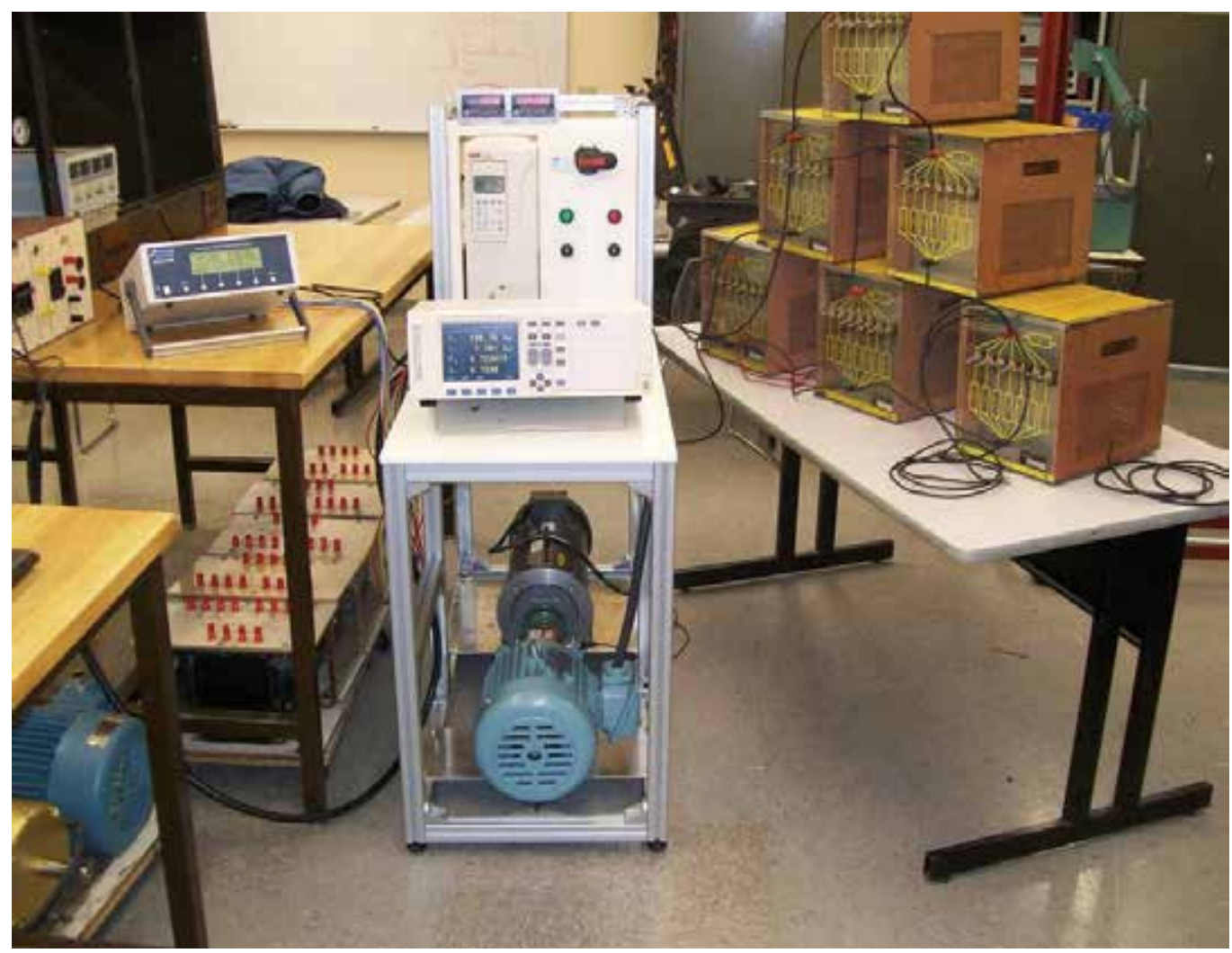

Fig. 6. Measurement setup with instrumentation

efficiency is near $75 \%$ of rated load, and tends to decrease substantially below about $50 \%$ load. Moreover, experienced overloaded motors don't significantly lose efficiency, as they are designed with a 1.15 factor service. It's also relevant to notice that the two motors give the same efficiency value just for the rated regime, as this efficiency difference is significant $(1.5 \%)$ when the motor is under loaded (Benhaddadi et al. 2010b).

Next step is to analyze the feeding voltage impact. The voltages choice are made taking into account practical considerations: $230 \mathrm{~V}$, and 200.

- The first one is the motor nameplate indication, which is in accordance with an industrial available $240 \mathrm{~V}$ feeding voltage,

- The second one is the real full-time laboratory available voltage. These two voltages were obtained with three one-phase transformers.

The experimental results obtained in the grid feeding case (without drive) and illustrated in fig. 10 show that feeding voltage has an important impact on efficiency value. With rated $230 \mathrm{~V}$ voltage and load values, efficiency is $89.3 \%$, i.e. $0.2 \%$ less than nameplate value. When the feeding voltage is decreased to $208 \mathrm{~V}$ and $200 \mathrm{~V}$ values, the efficiency decrease up to $2 \%$. So, additional losses occur when a $230 \mathrm{~V}$ motor is operated at or below 208 volts. The motor show lower full-load efficiency, slips more, and produces less torque.

In another hand, the ASD (adjustable speed drive) deployment to control motor can substantially reduce energy consumption. The ASD advantages and energy consumption reduction are nowadays well documented (Benhaddadi \& Olivier, 2007). But, at the present 


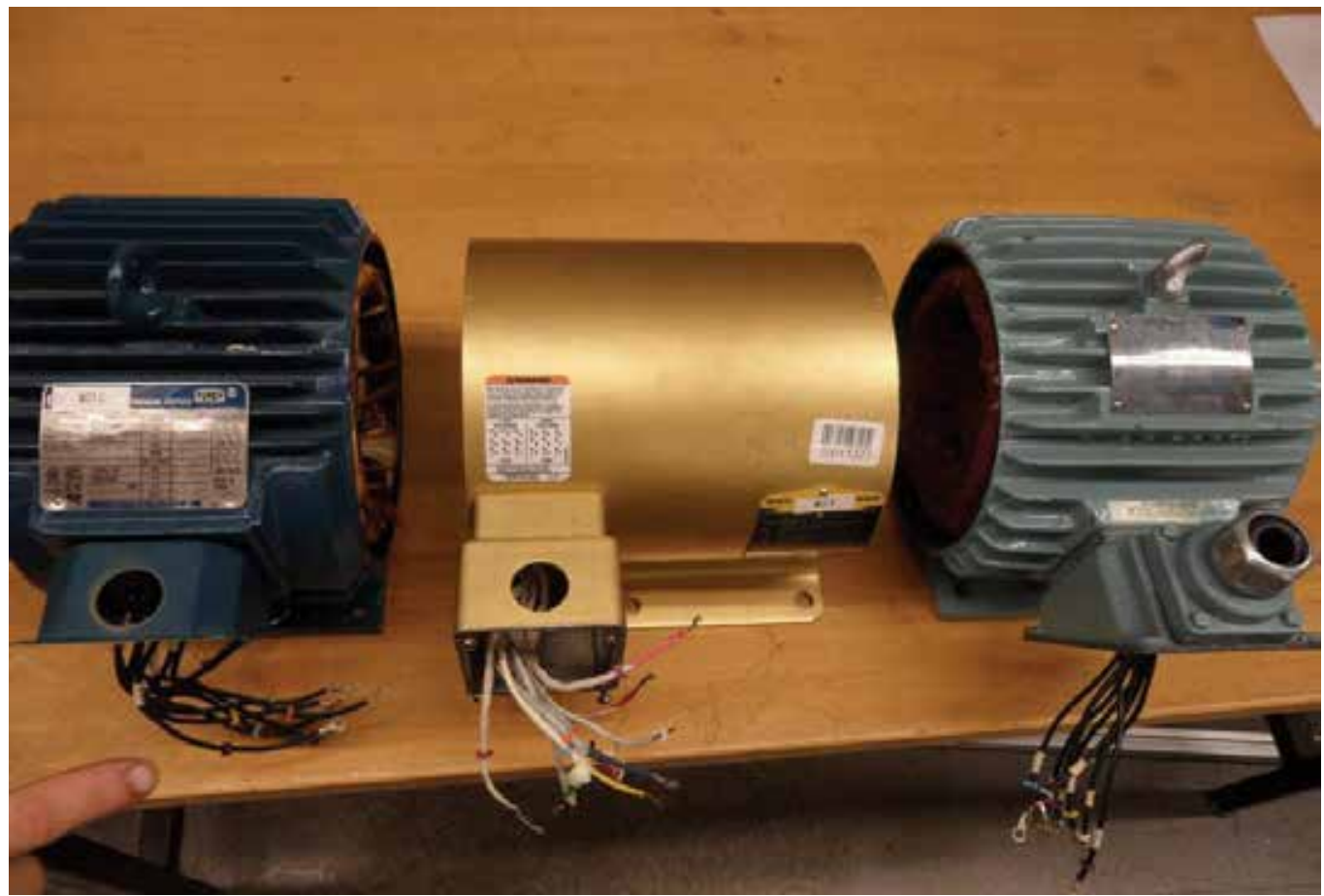

Fig. 7. Stator Premium motors

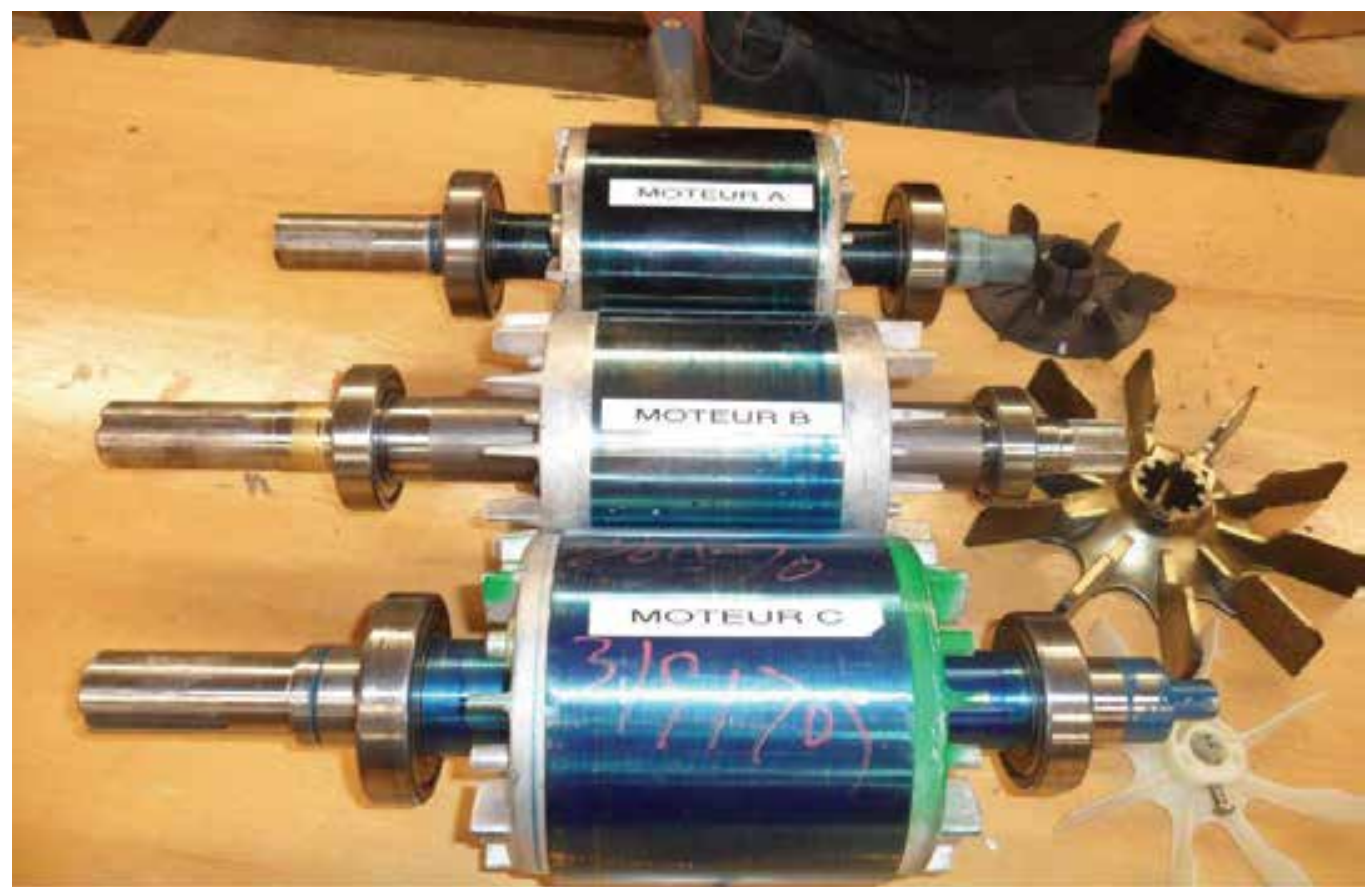

Fig. 8. Rotor Premium motors 
time, many companies use ASD to feed their motors, whenever they don't need speed regulation. In the energy saving point of view, we must be careful with this makingdecision, as ASD introduces supplementary losses in the motor and the drive. As can be seen in fig. 11 and 12, the drive introduces a noticeable reduction of the motor system efficiency. This decrease reaches approximately $4 \%$ in the rated regime, withdrawing totally energy savings induced by Premium efficiency motor use. Further investigations to correctly understand the extents of the losses introduced by the controller for all frequencies are under consideration.

The other problem is that if Premium motors are misapplied, they may not achieve predicted energy savings and may result in diminished performance efficiency. For centrifugal pumps, an increase in operating speed will increase the required power by the third power of the speed ratio. For example, by substituting Premium $1760 \mathrm{rpm}$ motor to EPAct $1740 \mathrm{rpm}$ one, a $20 \mathrm{rpm}$ increase in the speed induce $3.5 \%$ increase in the load, as $(1760 \div 1740)^{3}=(1.014)^{3}=1.035$. So, when replacing a standard efficiency motor, one must be careful, as a Premium motor with lower or equal full-load speed must be selected to avoid the energy increase that may negate the predicted energy savings resulting from a higher efficiency.

It's important to notice that to date, there is no agreement that allows the determination of ASD system efficiency at any given frequency. The ideal situation is to obtain a family of efficiency curves for diverse torques and frequencies, including overrated values, as experimentally illustrated in fig. 13.

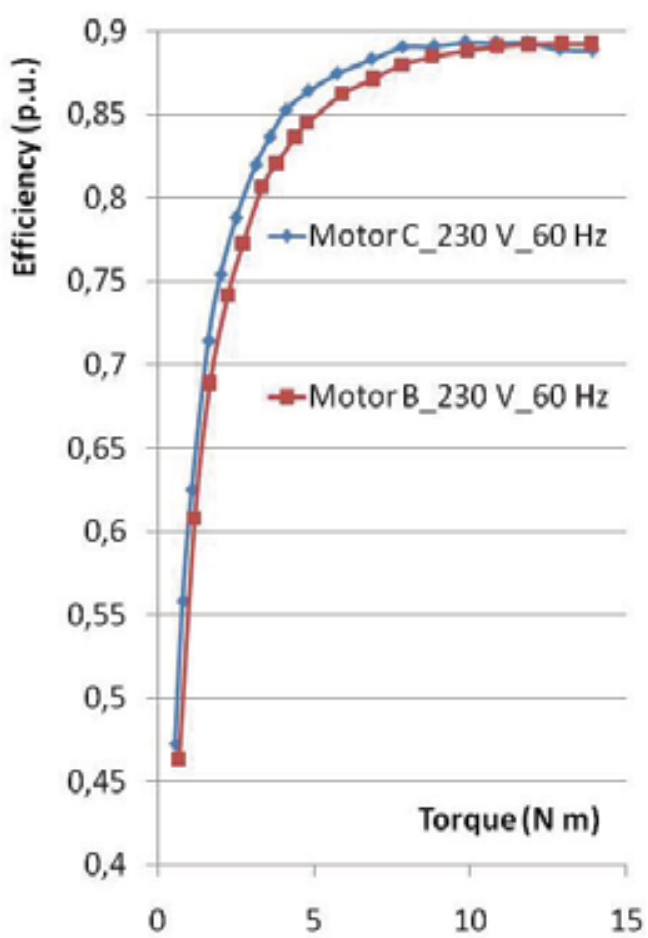

Fig. 9. Manufacturing technology impact

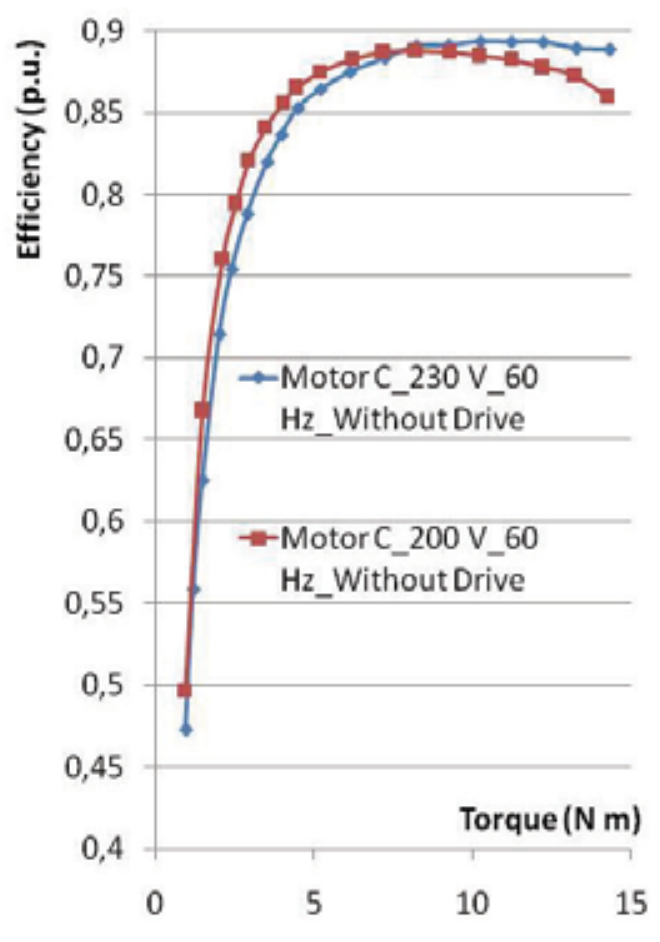

Fig. 10. Viltage feeding impact 


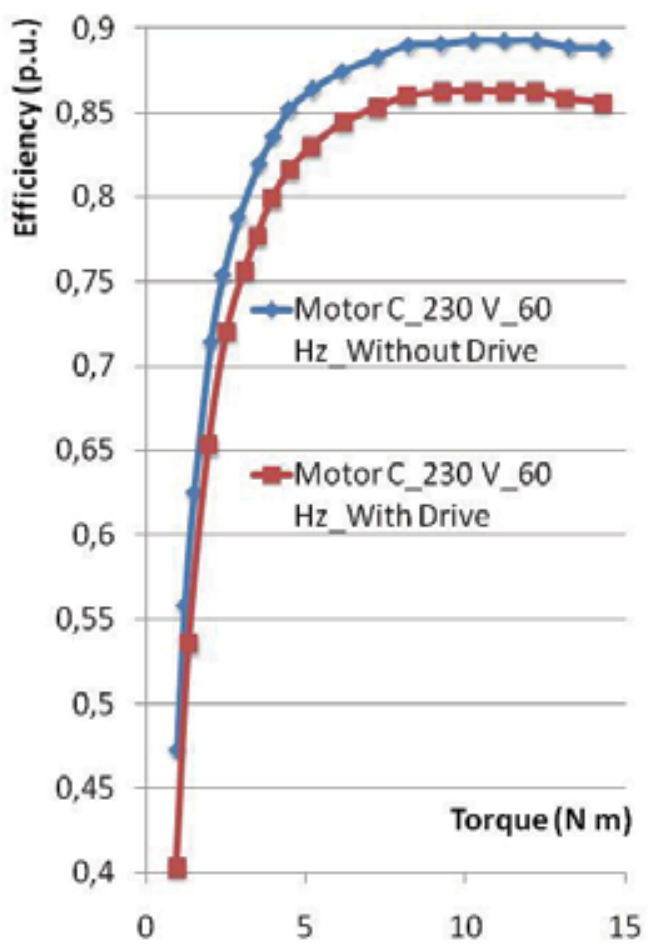

Fig. 11. Drive impact

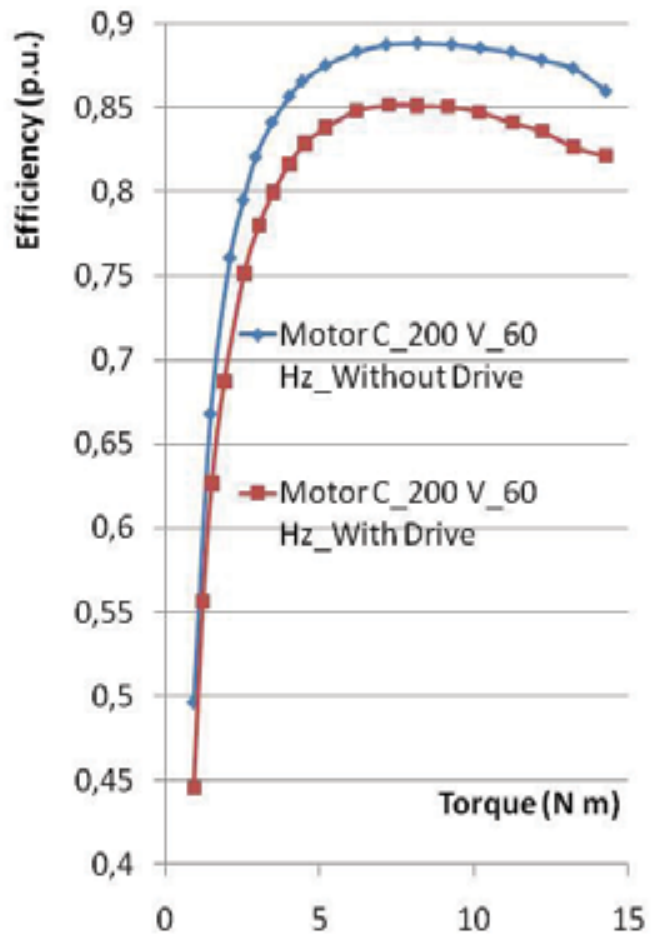

Fig. 12. Drive impact

But, to generalize results, there are two difficulties: the first one can be illustrated by the results presented in fig.14, where we can see that the same $3 \mathrm{Hp}$ motors issued from two different manufacturers can have the same efficiency for $60 \mathrm{~Hz}$ frequency feeding voltage, but different efficiency for another $30 \mathrm{~Hz}$ frequency (fig. 14). For each of these two motors B $\&$ C, Fig. 14 shows the variation of the efficiency versus the mechanical torque. In the $60 \mathrm{~Hz}$ case, the difference between the measured efficiency at rated load is negligible, while it reaches $2 \%$ in the $30 \mathrm{~Hz}$ feeding frequency.

The second difficulty is about $50-60 \mathrm{~Hz}$ feeding frequency dilemma. As earlier illustrated, 50 $\mathrm{Hz}$ frequency gives better efficiency beginning from $2 / 3$ load, while $60 \mathrm{~Hz}$ is better for low loads. It's noticeable that the same results were obtained for Motor B.

Considering that for the same frequency, two Premium motors issued from two different manufacturers can show significantly different efficiency in low frequencies, one must be careful in generalizing the conclusions of this research. Moreover, the same motor tested for different frequencies can yield to different losses repartitions. So, before claiming that the obtained results are, or are not in agreement with findings of other authors, several other Premium efficiency motors from different constructors should be tested. The mentioned work is under consideration.

\section{Conclusions}

In the future sustainable energy mix, a key role will be reserved for electricity, as GHG emissions reduction in this sector has to be drastically reduced. In this option, obvious 
conclusion is that large market penetration Premium motors needs a complex approach with a combination of financial incentives and mandatory legal actions, as industry doesn't invest according to least life cycle costs.

The US Energy Policy Act and the Canadian Energy Efficient Act, along with the implementation of NEMA Premium efficiency levels, have lead to North American leadership on motor efficiency implementation. In general terms, North America is not on the leading edge for energy saving and conservation. Motor efficiency is an exception that should be at least maintained. Next step is to get Tax incentives to promote early retirement of older inefficient pre-EPAct motors by replacing instead of repairing

Experimental comparison of the performance characteristics of $3 \mathrm{hp}$ Premium efficiency induction motors has been presented. The motors were tested according to Standard IEEE 112-B. In the rated frequency and voltage case, the experimental results are in good agreement with nameplate manufacturer's information. Particularly, a comparison of the rated operating point shows that, the discrepancy is approximately $0.2 \%$.

However, in low voltage/frequency applications, the use of a variable speed drive introduces extra losses and the overall efficiency can be noticeably reduced. The experimental results show that feeding voltage has an important impact on efficiency value, while efficiency at low frequencies depends on a certain level at manufacturer technology. From a global energy saving point of view, the ASD application to Premium efficiency motors should be promoted just when adjustable speed is needed.

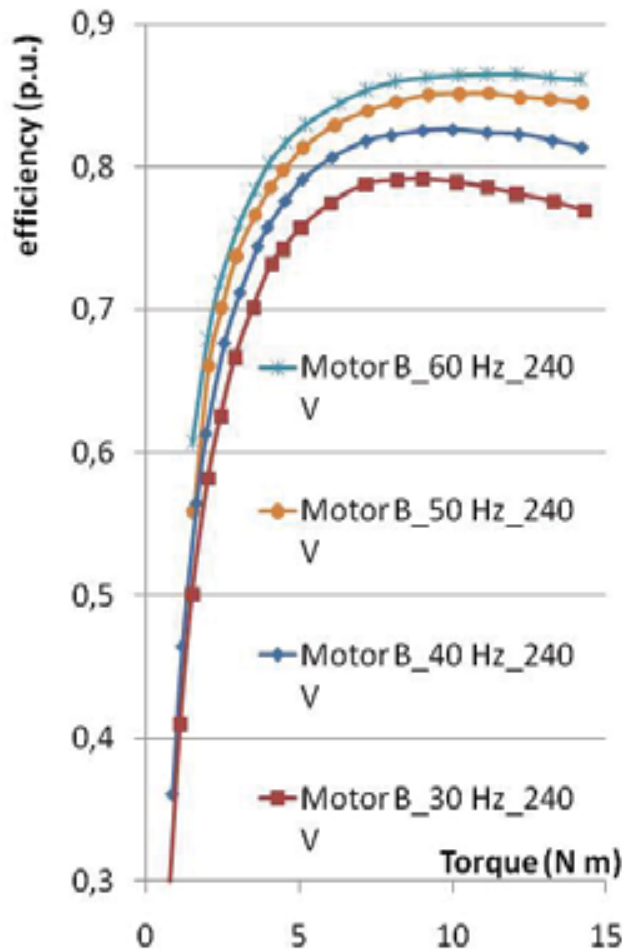

Fig. 13. Frequency impact

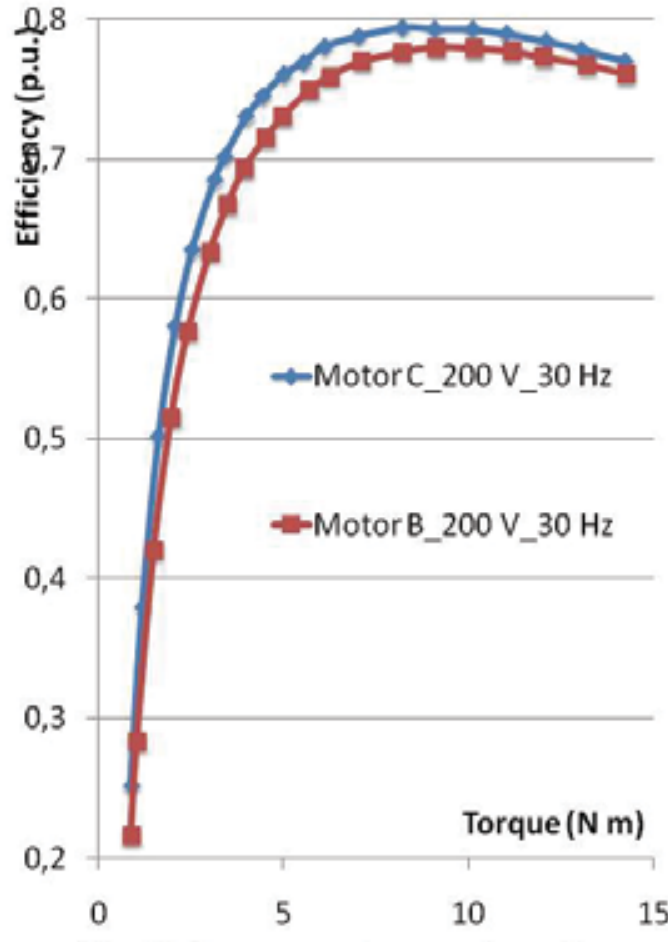

Fig. 12. Frequency \& manufacturing technology impact 


\begin{tabular}{|c|c|c|c|c|c|c|c|c|}
\hline \multirow{2}{*}{ HP } & \multicolumn{9}{|c|}{ ODP } & \multicolumn{4}{c|}{ TEFC } \\
\cline { 2 - 9 } & 2 Pole & 4 Pole & 6 Pole & 8 Pole & 2 Pole & 4 Pole & 6 Pole & 8 Pole \\
\hline 1.0 & 0.0 & 82.5 & 80.0 & 74.0 & 75.5 & 82.5 & 80 & 74 \\
\hline 1.5 & 82.5 & 84.0 & 84.0 & 75.5 & 82.5 & 84 & 85.5 & 77 \\
\hline 2.0 & 84.0 & 84.0 & 85.5 & 85.5 & 84 & 84 & 86.5 & 82.5 \\
\hline 3.0 & 84.0 & 86.5 & 86.5 & 86.5 & 85.5 & 87.5 & 87.5 & 84 \\
\hline 5.0 & 85.5 & 87.5 & 87.5 & 87.5 & 87.5 & 87.5 & 87.5 & 85.5 \\
\hline 7.5 & 87.5 & 88.5 & 88.5 & 88.5 & 88.5 & 89.5 & 89.5 & 85.5 \\
\hline 10.0 & 88.5 & 89.5 & 90.2 & 89.5 & 89.5 & 89.5 & 89.5 & 88.5 \\
\hline 15.0 & 89.5 & 91.0 & 90.2 & 89.5 & 90.2 & 91 & 90.2 & 88.5 \\
\hline 20.0 & 90.2 & 91.0 & 91.0 & 90.2 & 90.2 & 91 & 90.2 & 89.5 \\
\hline 25.0 & 91.0 & 91.7 & 91.7 & 90.2 & 91 & 92.4 & 91.7 & 89.5 \\
\hline 30.0 & 91.0 & 92.4 & 92.4 & 91.0 & 91 & 92.4 & 91.7 & 91 \\
\hline 40.0 & 91.7 & 93.0 & 93.0 & 91.0 & 91.7 & 93 & 93 & 91 \\
\hline 50.0 & 92.4 & 93.0 & 93.0 & 91.7 & 92.4 & 93 & 93 & 91.7 \\
\hline 60.0 & 93.0 & 93.6 & 93.6 & 92.4 & 93 & 93.6 & 93.6 & 91.7 \\
\hline 75.0 & 93.0 & 94.1 & 93.6 & 93.6 & 93 & 94.1 & 93.6 & 93 \\
\hline 100.0 & 93.0 & 94.1 & 94.1 & 93.6 & 93.6 & 94.5 & 94.1 & 93 \\
\hline 125.0 & 93.6 & 94.5 & 94.1 & 93.6 & 94.5 & 94.5 & 94.1 & 93.6 \\
\hline 150.0 & 93.6 & 95.0 & 94.5 & 93.6 & 94.5 & 95 & 95 & 93.6 \\
\hline 200.0 & 94.5 & 95.0 & 94.5 & 93.6 & 95 & 95 & 95 & 94.1 \\
\hline 250.0 & 94.5 & 95.4 & 95.4 & 94.5 & 95.4 & 95 & 95 & 94.5 \\
\hline 300.0 & 95.0 & 95.4 & 95.4 & 94.5 & 95.4 & 95.4 & 95 & 0 \\
\hline 350.0 & 95.0 & 95.4 & 95.4 & 94.5 & 95.4 & 95.4 & 95 & 0 \\
\hline 400.0 & 95.4 & 95.4 & 0.0 & 0.0 & 95.4 & 95.4 & 0 & 0 \\
\hline 450.0 & 95.8 & 95.8 & 0.0 & 0.0 & 95.4 & 95.4 & 0 & 0 \\
\hline 500.0 & 95.8 & 95.8 & 0.0 & 0.0 & 95.4 & 95.8 & 0 & 0 \\
\hline & & & & & & & & \\
\hline
\end{tabular}

Annex 1. NEMA MG-1 Table 12-11 Full-Load Efficiencies of Energy Efficient Motors (EPAct) 


\begin{tabular}{|c|c|c|c|c|c|c|}
\hline \multirow{2}{*}{$\mathrm{HP}$} & \multicolumn{3}{|c|}{ ODP } & \multicolumn{3}{|c|}{ TEFC } \\
\cline { 2 - 7 } & 2 Pole & 4 Pole & 6 Pole & 2 Pole & 4 Pole & 6 Pole \\
\hline 1 & 77 & 85.5 & 82.5 & 77 & 85.5 & 82.5 \\
\hline 1.5 & 84 & 86.5 & 86.5 & 84 & 86.5 & 87.5 \\
\hline 2 & 85.5 & 86.5 & 87.5 & 85.5 & 86.5 & 88.5 \\
\hline 3 & 85.5 & 89.5 & 88.5 & 86.5 & 89.5 & 89.5 \\
\hline 5 & 86.5 & 89.5 & 89.5 & 88.5 & 89.5 & 89.5 \\
\hline 7.5 & 88.5 & 91 & 90.2 & 89.5 & 91.7 & 91 \\
\hline 10 & 89.5 & 91.7 & 91.7 & 90.2 & 91.7 & 91 \\
\hline 15 & 90.2 & 93 & 91.7 & 91 & 92.4 & 91.7 \\
\hline 20 & 91 & 93 & 92.4 & 91 & 93 & 91.7 \\
\hline 25 & 91.7 & 93.6 & 93 & 91.7 & 93.6 & 93 \\
\hline 30 & 91.7 & 94.1 & 93.6 & 91.7 & 93.6 & 93 \\
\hline 40 & 92.4 & 94.1 & 94.1 & 92.4 & 94.1 & 94.1 \\
\hline 50 & 93 & 94.5 & 94.1 & 93 & 94.5 & 94.1 \\
\hline 60 & 93.6 & 95 & 94.5 & 93.6 & 95 & 94.5 \\
\hline 75 & 93.6 & 95 & 94.5 & 93.6 & 95.4 & 94.5 \\
\hline 100 & 93.6 & 95.4 & 95 & 94.1 & 95.4 & 95 \\
\hline 125 & 94.1 & 95.4 & 95 & 95 & 95.4 & 95 \\
\hline 150 & 94.1 & 95.8 & 95.4 & 95 & 95.8 & 95.8 \\
\hline 200 & 95 & 95.8 & 95.4 & 95.4 & 96.2 & 95.8 \\
\hline 250 & 95 & 95.8 & 95.4 & 95.8 & 96.2 & 95.8 \\
\hline 300 & 95.4 & 95.8 & 95.4 & 95.8 & 96.2 & 95.8 \\
\hline 350 & 95.4 & 95.8 & 95.4 & 95.8 & 96.2 & 95.8 \\
\hline 400 & 95.8 & 95.8 & 95.8 & 95.8 & 96.2 & 95.8 \\
\hline 450 & 95.8 & 96.2 & 96.2 & 95.8 & 96.2 & 95.8 \\
\hline 500 & 95.8 & 96.2 & 96.2 & 95.8 & 96.2 & 95.8 \\
\hline
\end{tabular}

Annex 2. NEMA MG-1 Table 12-12 Full-Load Efficiencies for $60 \mathrm{~Hz}$ NEMA Premium Efficient Electric Motors Rated 600 Volts or less 


\begin{tabular}{|c|c|c|c|c|}
\hline & Rating & \multicolumn{3}{|c|}{ Poles } \\
\cline { 3 - 5 } Rating (kW) & (hp) & $\mathbf{2}$ & $\mathbf{4}$ & $\mathbf{6}$ \\
\hline 0.75 & 1 & 72.1 & 72.1 & 70.0 \\
\hline 1.1 & 1.5 & 75.0 & 75.0 & 72.9 \\
\hline 1.5 & 2 & 77.2 & 77.2 & 75.2 \\
\hline 2.2 & 3 & 79.7 & 79.7 & 77.7 \\
\hline 3 & 4 & 81.5 & 81.5 & 79.7 \\
\hline 4 & 5.5 & 83.1 & 83.1 & 81.4 \\
\hline 5.5 & 7.5 & 84.7 & 84.7 & 83.1 \\
\hline 7.5 & 10 & 86.0 & 86.0 & 84.7 \\
\hline 11 & 15 & 87.6 & 87.6 & 86.4 \\
\hline 15 & 20 & 88.7 & 88.7 & 87.7 \\
\hline 18.5 & 25 & 89.3 & 89.3 & 88.6 \\
\hline 22 & 30 & 89.9 & 89.9 & 89.2 \\
\hline 30 & 40 & 90.7 & 90.7 & 90.2 \\
\hline 37 & 50 & 91.2 & 91.2 & 90.8 \\
\hline 45 & 60 & 91.7 & 91.7 & 91.4 \\
\hline 55 & 75 & 92.1 & 92.1 & 91.9 \\
\hline 75 & 75 & 92.1 & 92.1 & 91.9 \\
\hline 90 & 125 & 92.7 & 92.7 & 92.6 \\
\hline 110 & 150 & 93.0 & 93.0 & 92.9 \\
\hline 132 & 175 & 93.3 & 93.3 & 93.3 \\
\hline 160 & 215 & 93.5 & 93.5 & 93.5 \\
\hline 200 & 270 & 93.8 & 93.8 & 93.8 \\
\hline 260 & 350 & 94.0 & 94.0 & 94.0 \\
\hline 300 & 400 & 94.0 & 94.0 & 94.0 \\
\hline 335 & 450 & 94.0 & 94.0 & 94.0 \\
\hline 375 & 500 & 94.0 & 94.0 & 94.0 \\
\hline
\end{tabular}

Table 3. Standard Efficiency IE1 $50 \mathrm{~Hz}$

\begin{tabular}{|c|c|c|c|c|}
\hline Rating & Rating & \multicolumn{3}{|c|}{ Poles } \\
\cline { 3 - 5 }$(\mathbf{k W})$ & $(\mathbf{h p})$ & $\mathbf{2}$ & $\mathbf{4}$ & $\mathbf{6}$ \\
\hline 0.75 & 1 & 77.0 & 78.0 & 73.0 \\
\hline 1.1 & 1.5 & 78.5 & 79.0 & 75.0 \\
\hline 1.5 & 2 & 81.0 & 81.5 & 77.0 \\
\hline 2.2 & 3 & 81.5 & 83.0 & 78.5 \\
\hline 3 & 4 & 84.5 & 85.0 & 83.5 \\
\hline 4 & 5.5 & 86.0 & 87.0 & 85.0 \\
\hline 5.5 & 7.5 & 87.5 & 87.5 & 86.0 \\
\hline 7.5 & 10 & 87.5 & 88.5 & 89.0 \\
\hline 11 & 15 & 88.5 & 89.5 & 89.5 \\
\hline 15 & 20 & 89.5 & 90.5 & 90.2 \\
\hline 18.5 & 25 & 89.5 & 91.0 & 91.0 \\
\hline 22 & 30 & 90.2 & 91.7 & 91.7 \\
\hline 30 & 40 & 91.5 & 92.4 & 91.7 \\
\hline 37 & 50 & 91.7 & 93.0 & 91.7 \\
\hline 45 & 60 & 92.4 & 93.0 & 92.1 \\
\hline 55 & 75 & 93.0 & 93.2 & 93.0 \\
\hline 75 & 75 & 93.0 & 93.2 & 93.0 \\
\hline 90 & 125 & 93.0 & 93.5 & 94.1 \\
\hline 110 & 150 & 94.1 & 94.5 & 94.1 \\
\hline 150 & 200 & 94.1 & 94.5 & 94.1 \\
\hline 185 & 250 & 94.1 & 94.5 & 94.1 \\
\hline 200 & 270 & 94.1 & 94.5 & 94.1 \\
\hline 260 & 350 & 94.1 & 94.5 & 94.1 \\
\hline 300 & 400 & 94.1 & 94.5 & 94.1 \\
\hline 335 & 450 & 94.1 & 94.5 & 94.1 \\
\hline 375 & 500 & 94.1 & 94.5 & 94.1 \\
\hline & & & & \\
\hline
\end{tabular}

Table 4. Standard Efficiency IE1 60Hz

Annex 3. CEI efficiencies 


\begin{tabular}{|c|c|c|c|c|}
\hline & Rating & \multicolumn{3}{|c|}{ Poles } \\
\cline { 3 - 5 } Rating (kW) & (hp) & $\mathbf{2}$ & $\mathbf{4}$ & $\mathbf{6}$ \\
\hline 0.75 & 1 & 77.4 & 79.6 & 75.9 \\
\hline 1.1 & 1.5 & 79.6 & 81.4 & 78.1 \\
\hline 1.5 & 2 & 81.3 & 82.8 & 79.8 \\
\hline 2.2 & 3 & 83.2 & 84.3 & 81.8 \\
\hline 3 & 4 & 84.6 & 85.5 & 83.3 \\
\hline 4 & 5.5 & 85.8 & 86.6 & 84.6 \\
\hline 5.5 & 7.5 & 87.0 & 87.7 & 86.0 \\
\hline 7.5 & 10 & 88.1 & 88.7 & 87.2 \\
\hline 11 & 15 & 89.4 & 898.0 & 88.7 \\
\hline 15 & 20 & 90.3 & 90.6 & 89.7 \\
\hline 18.5 & 25 & 90.9 & 91.2 & 90.4 \\
\hline 22 & 30 & 91.3 & 91.6 & 90.9 \\
\hline 30 & 40 & 92.0 & 92.3 & 91.7 \\
\hline 37 & 50 & 92.5 & 92.7 & 92.2 \\
\hline 45 & 60 & 92.9 & 93.1 & 92.7 \\
\hline 55 & 75 & 93.2 & 93.5 & 93.1 \\
\hline 75 & 75 & 93.8 & 94.0 & 93.7 \\
\hline 90 & 125 & 94.1 & 94.2 & 94.0 \\
\hline 110 & 150 & 94.3 & 94.5 & 94.3 \\
\hline 132 & 175 & 94.6 & 94.7 & 94.6 \\
\hline 160 & 215 & 94.8 & 94.9 & 94.8 \\
\hline 200 & 270 & 95.0 & 95.1 & 95.0 \\
\hline 260 & 350 & 95.0 & 95.1 & 95.0 \\
\hline 300 & 400 & 95.0 & 95.1 & 95.0 \\
\hline 335 & 450 & 95.0 & 95.1 & 95.0 \\
\hline 375 & 500 & 95.0 & 95.1 & 95.0 \\
\hline
\end{tabular}

Table 5. High Efficiency IE2 50Hz

\begin{tabular}{|c|c|c|c|c|}
\hline Rating & Rating & \multicolumn{3}{|c|}{ Poles } \\
\cline { 3 - 5 }$(\mathbf{k W})$ & $\mathbf{h p})$ & $\mathbf{2}$ & $\mathbf{4}$ & $\mathbf{6}$ \\
\hline 0.75 & 1 & 75.5 & 82.5 & 80.0 \\
\hline 1.1 & 1.5 & 82.5 & 84.0 & 85.5 \\
\hline 1.5 & 2 & 84.0 & 84.0 & 86.5 \\
\hline 2.2 & 3 & 85.5 & 87.5 & 87.5 \\
\hline 3.7 & 5 & 87.5 & 87.5 & 87.5 \\
\hline 5.5 & 7.5 & 88.5 & 89.5 & 89.5 \\
\hline 7.5 & 10 & 89.5 & 89.5 & 89.5 \\
\hline 11 & 15 & 90.2 & 91.0 & 90.2 \\
\hline 15 & 20 & 90.2 & 91.0 & 90.2 \\
\hline 18.5 & 25 & 91.0 & 92.4 & 91.7 \\
\hline 22 & 30 & 91.0 & 92.4 & 91.7 \\
\hline 30 & 40 & 91.7 & 93.0 & 93.0 \\
\hline 37 & 50 & 92.4 & 93.0 & 93.0 \\
\hline 45 & 60 & 93.0 & 93.6 & 93.6 \\
\hline 55 & 75 & 93.0 & 94.1 & 93.6 \\
\hline 75 & 75 & 93.6 & 94.5 & 94.1 \\
\hline 90 & 125 & 94.5 & 94.5 & 94.1 \\
\hline 110 & 150 & 94.5 & 95.0 & 95.0 \\
\hline 150 & 200 & 95.0 & 95.0 & 95.0 \\
\hline 185 & 250 & 95.4 & 95.4 & 95.0 \\
\hline 200 & 270 & 95.4 & 95.4 & 95.0 \\
\hline 260 & 350 & 95.4 & 95.4 & 95.0 \\
\hline 300 & 400 & 95.4 & 95.4 & 95.0 \\
\hline 335 & 450 & 95.4 & 95.4 & 95.0 \\
\hline 375 & 500 & 95.4 & 95.4 & 95.0 \\
\hline & & & & \\
\hline
\end{tabular}

Table 6. High Efficiency IE2 60Hz (EPACT)

Annex 3. CEI efficiencies 


\begin{tabular}{|c|c|c|c|c|}
\hline \multirow[b]{2}{*}{ Rating (kW) } & \multirow{2}{*}{$\begin{array}{c}\text { Rating } \\
\text { (hp) }\end{array}$} & \multicolumn{3}{|c|}{ Poles } \\
\hline & & 2 & 4 & 6 \\
\hline 0.75 & 1 & 80.7 & 82.5 & 78.9 \\
\hline 1.1 & 1.5 & 82.7 & 84.1 & 81.0 \\
\hline 1.5 & 2 & 84.2 & 85.3 & 82.5 \\
\hline 2.2 & 3 & 85.9 & 86.7 & 84.3 \\
\hline 3 & 4 & 87.1 & 87.7 & 85.6 \\
\hline 4 & 5.5 & 88.7 & 88.6 & 86.8 \\
\hline 5.5 & 7.5 & 89.2 & 89.6 & 88.0 \\
\hline 7.5 & 10 & 90.1 & 90.4 & 89.1 \\
\hline 11 & 15 & 91.2 & 91.4 & 90.3 \\
\hline 15 & 20 & 91.9 & 92.1 & 91.2 \\
\hline 18.5 & 25 & 92.4 & 92.6 & 91.7 \\
\hline 22 & 30 & 92.7 & 93.0 & 92.2 \\
\hline 30 & 40 & 93.3 & 93.6 & 92.9 \\
\hline 37 & 50 & 93.7 & 93.9 & 93.3 \\
\hline 45 & 60 & 94.0 & 94.2 & 93.7 \\
\hline 55 & 75 & 94.3 & 94.6 & 94.1 \\
\hline 75 & 75 & 94.7 & 95.0 & 94.6 \\
\hline 90 & 125 & 95.0 & 95.2 & 94.9 \\
\hline 110 & 150 & 95.2 & 95.4 & 95.1 \\
\hline 132 & 175 & 95.4 & 95.6 & 95.4 \\
\hline 160 & 215 & 95.6 & 95.8 & 95.6 \\
\hline 200 & 270 & 95.8 & 96.0 & 95.8 \\
\hline 260 & 350 & 95.8 & 96.0 & 95.8 \\
\hline 300 & 400 & 95.8 & 96.0 & 95.8 \\
\hline 335 & 450 & 95.8 & 96.0 & 95.8 \\
\hline 375 & 500 & 95.8 & 96.0 & 95.8 \\
\hline
\end{tabular}

Table 7. Premium Efficiency IE3 $50 \mathrm{~Hz}$

\begin{tabular}{|c|c|c|c|c|}
\hline Rating & Rating & \multicolumn{3}{|c|}{ Poles } \\
\cline { 3 - 5 }$(\mathbf{k W})$ & $\mathbf{( h p )}$ & $\mathbf{2}$ & $\mathbf{4}$ & $\mathbf{6}$ \\
\hline 0,75 & 1 & 77,0 & 85,5 & 82,5 \\
\hline 1,1 & 1,5 & 84,0 & 86,5 & 87,5 \\
\hline 1,5 & 2 & 85,5 & 86,5 & 88,5 \\
\hline 2,2 & 3 & 86,5 & 89,5 & 89,5 \\
\hline 3,7 & 5 & 88,5 & 89,5 & 89,5 \\
\hline 5,5 & 7,5 & 89,5 & 91,7 & 91,0 \\
\hline 7,5 & 10 & 90,2 & 91,7 & 91,0 \\
\hline 11 & 15 & 91,0 & 92,4 & 91,7 \\
\hline 15 & 20 & 91,0 & 93,0 & 91,7 \\
\hline 18,5 & 25 & 91,7 & 93,6 & 93,0 \\
\hline 22 & 30 & 91,7 & 93,6 & 93,0 \\
\hline 30 & 40 & 92,4 & 94,1 & 94,1 \\
\hline 37 & 50 & 93,0 & 94,5 & 94,1 \\
\hline 45 & 60 & 93,6 & 95,0 & 94,5 \\
\hline 55 & 75 & 93,6 & 95,4 & 94,5 \\
\hline 75 & 75 & 94,1 & 95,4 & 95,0 \\
\hline 90 & 125 & 95,0 & 95,4 & 95,0 \\
\hline 110 & 150 & 95,0 & 95,8 & 95,8 \\
\hline 150 & 200 & 95,4 & 96,2 & 95,8 \\
\hline 185 & 250 & 95,8 & 96,2 & 95,8 \\
\hline 200 & 270 & 95,8 & 96,2 & 95,8 \\
\hline 260 & 350 & 95,8 & 96,2 & 95,8 \\
\hline 300 & 400 & 95,8 & 96,2 & 95,8 \\
\hline 335 & 450 & 95,8 & 96,2 & 95,8 \\
\hline 375 & 500 & 95,8 & 96,2 & 95,8 \\
\hline
\end{tabular}

Table 8. Premium Efficiency IE3 60Hz (NEMA Premium)

Annex 3. CEI efficiencies

\section{Annex 4: Acronyms}

- ASD Adjustable speed drives

- ACEEE American Council for and Energy Efficient Economy

- CEE Consortium for Energy Efficiency

- DOE Department of Energy

- EISA Energy Independence \& Security Act

- EPAct Energy Policy act

- EU European Union

- $\quad$ GHG Greenhouse gas emissions

- IPCC International Panel on climate change

- OEM Original equipment manufacturer

- MG 1 Motor Generator

- MEPS Minimum energy performance standard MEPS

- MDM Motor Decisions Matter (MDM

- NEMA National Electrical Manufacturers Association 


\section{References}

Aoulkadi, M., Binder, A.: "Evaluation of different measurement methods to determine stray load losses in induction machines,". IEEE Trans. On Industrial Electronics, vol. 2 No 1, 2008

Benhaddadi M., Olivier G.: (2010a) “La promotion de l'économie d'énergie électrique passe par son juste prix,". 79e Congrès de l'AFAS, Montréal, Canada, 2010

Benhaddadi M., Olivier G., and Yelle J.: (2010b) "Premium efficiency motors effectiveness,". IEEE International symposium on power electronics, electrical drives, automation and motion SPEEDAM 2010, Pisa, Italy, 2010

Benhaddadi M., Olivier G., Labrosse D., Tétrault P.: (2009a) “Premium efficiency motors and energy saving potential,". IEEE International electric machines and drives conference, IEEE_IEMDC, Miami, USA, 2009

Benhaddadi M., Olivier G.: (2009b) "L'économie d'énergie : une affaire de législation,". Communication présentée au 78e Congrès de l'ACFAS, Ottawa, Canada, 2009

Benhaddadi M., Olivier G.: (2008a) "Barriers and incentive policies to high-efficiency motors and drives market penetration,". IEEE International symposium on power electronics, electrical drives, automation and motion SPEEDAM 2008, Ischia, Italy,

Benhaddadi M., Olivier G.: (2008b) "Le génie électrique à la rescousse des économies d'énergie dans l'industrie,". Communication présentée au 76e Congrès de l'ACFAS, Québec, Canada, 2008

Benhaddadi M., Olivier G.: (2008c) “Dilemmes énergétiques,". Presses de l’Université du Québec, Québec, 2008, 216p.

Benhaddadi, M., Olivier G.: (2007) "Energy savings by means of generalization adjustable speed drive Utilization,". IEEE Canadian Conference on Electrical and Computer Engineering, Vancouver, 2007.

Benhaddadi M., Olivier G.: (2004) "Including Kyoto in electrical engineering curriculum,". IEEE Canadian Conference on Electrical and Computer Engineering, Niagara Falls, 2004

Boglietti, A. Cavagnino, A. Lazzari, M. Pastorelli, , M.: (2004) “International standards for the induction motor efficiency evaluation: a critical analysis o A. $\mathrm{f}$ the stray-load loss determination,". IEEE Trans. On Industry Appl., vol.40, No 5, 2004

Bonnett, A.H., Yung, C.: (2008) "Increased efficiency versus increased reliability: A comparison of pre-EPAct, EPAct, and premium-efficiency motors,". IEEE Industry application Magazine, vol.2, 2008

BP (2010) British Petroleum statistical review of world energy (2010), June 2010

CEE (2007) Energy-efficiency incentive programs: Premium-efficiency motors and adjustable speed drives in the US and Canada, (2007) prepared by consortium for energy efficiency, may 2007, www.cee.org

DOE (2010) Energy Information Administration, Official energy statistics from the US government,

DOE/EIA http://www.eia.doe.gov

Elmeida, A.I. Ferreira, F.FJ. Busch, J.F. Angers P. (2002) “Comparative analysis of IEEE-112 B and IEC 34-2 efficiency testing standards using stray load losses in low-voltage three phase cage induction motors," IEEE Industry applications, March-April 2002, pp. 608-614

Energy Policy Act EPACT and motor testing understanding, IEEE-112 Method B, 
Evans, B.D., Crissman, J., Gobert, G.: (2008) “Test results for energy savings,”. IEEE Industry application Magazine, vol.2, 2008

Finlay, W.R. Veerkamp B., Gehring D., and Hanna: P. (2009)“Improving motor efficiency levels globally,". IEEE Industry application Magazine, vol.15, 2009

IEA (2010) International Energy Agency, OECD/IEA http:/ / oecd.org./

IEA (2009) The experience with energy efficiency policies and programs in IEA countries: learning from the critics, (2009) International energy agency information paper, august 2009

IPCC (2007) Contribution of working group I to the fourth assessment report of the intergovernmental panel on climate change, summary for policymakers, http:/ / www.ipcc.ch

Nagorny, A., Wallace, A., Von Jouanne, A.: “Stray load loss efficiency connections,". IEEE Industry application Magazine, vol.10, issue 3, may-june 2004

NRC (2004) Energy-efficient motor systems assessment guide, Canadian industry program for energy conservation (2004), CIPEC, Natural Resources Canada, 2004

Parasiliti, F., Villani, M., Paris, C., Walti, O. Songini, G. Novello, A. Rossi T.: (2004) “Threephase induction motor efficiency improvements with die-cast cooper rotor cage and premium steel,". IEEE International symposium on power electronics, electrical drives, automation and motion SPPEDAM 2004, Capri, Italy, 2004

RNC (2004) Ressources Naturelles Canada: (2004) “Guide d'évaluation du rendement des systèmes moteurs éconergétiques," RNC, 2004

Renier, B. Hameyer, K., Belmans, R.: (1999) "Comparison standards for determining efficiency of three phase induction motors,". IEEE Trans. On Energy Conversion, vol.14, No 3, 1999

Rooks, J.A., Wallace: (2004) “Energy efficiency of VSDs," IEEE Industry applications Magazine, vol.10, issue 3, 2004

UE (2003) Commission Européenne, Direction générale énergie et transport: (2003) "Motor

challenge programme,". UE, Bruxelles, 2003 


\title{
Electric Motor Performance Improvement Using Auxiliary Windings and Capacitance Injection
}

\author{
Nicolae D.V \\ Tshwane University of Technology \\ South Africa
}

\section{Introduction}

Generally, some electric machines such as induction machines and synchronous reluctance motors require reactive power for operation. While the reactive power required by a synchronous machine can be taken from the power source or supplied by the machine itself by adjustment of the field current, the power factor of an induction machine is always lagging and set by external quantities (i.e., the load and terminal voltage). Poor power factor adversely affects the distribution system and a cost penalty is frequently levied for excessive VAr consumption.

Power factor is typically improved by installation of capacitor banks parallel to the motor. If the capacitor bank is fixed (i.e. that it can compensate power factor only for a fixed load), when the load is variable, then the compensation is lost. Some authors (El-Sharkawi et al, 1984, Fuchs and Hanna, 2002) introduced the capacitors using thyristor/triac controllers; by adjusting the firing angle, the capacitance introduced in parallel with the motor becomes variable and thus compensating the power factor for any load. Other works (Suciu et al, 2000.) consider the induction motor as an RL load and power factor is improved by inserting a variable capacitor (through a bridge converter) which is adjusted for unity according with the load. For the above methods, the capacitive injection is directly into the supply. Another method conceived for slip ring induction motor was to inject capacitive reactive power direct into the rotor circuit (Reinert and Parsley, 1995; Suciu, et al. 2002).

The injection of reactive power can be done through auxiliary windings magnetically coupled with the main windings (E. Muljadi et al. 1989; Tamrakan and Malik, 1999; Medarametla et al. 1992; Umans, and H. L. Hess, 1983; Jimoh and Nicolae, 2006, 2007). This compensating method has also been applied with good results not only for induction motors but also for a synchronous reluctance motor (Ogunjuyigbe et al. 2010).

\section{Method description}

\subsection{Physical solution}

The method described in this chapter makes use of two three-phase stator windings. One set, the main winding (star or delta), is connected directly to the source. The other set of windings - auxiliary, is only magnetically coupled to the main winding. All windings have the same shape and pitch, but may have different turn numbers and wire sizes; usually smaller in order to be accommodated in the slots together with the stator. The windings are 
arranged in slots such that there is no phase shift between the two windings. Figure 1 shows a possible arrangement of the windings for a four pole induction machine.

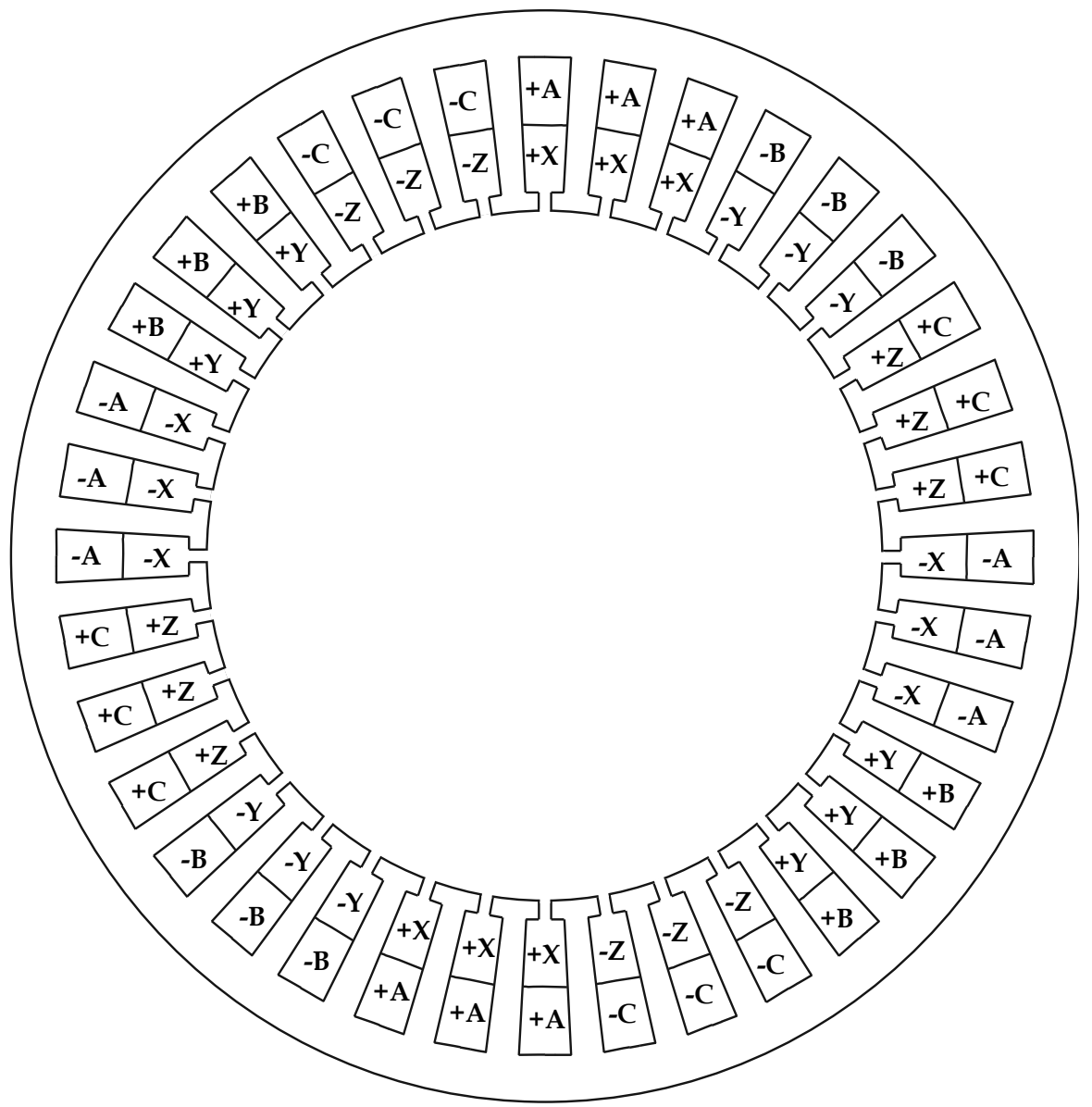

Fig. 1. High Power factor induction machines-windings arrangement

\subsection{Auxiliary windings connections}

As mention above, the main winding can have delta or star connection. Figure 2 shows the main winding connected in star and the auxiliary windings connected in generic (a), star (b) and delta (c) to the capacitor bank via a static switch.

Figure 3 shows a simpler way to inject capacitive reactive power. In this method, the auxiliary windings are in "single -phase connection" with the apparent advantage of using only one capacitor and static switch.

\subsection{Variable Capacitors}

In order to achieve a compensation for various loading of the machine, the compensating capacitor should be able to be varied. This capability is obtained through connecting a fixed capacitor via a static switch. The static switch can be achieved using thyristors or IGBTs in bidirectional configuration. 


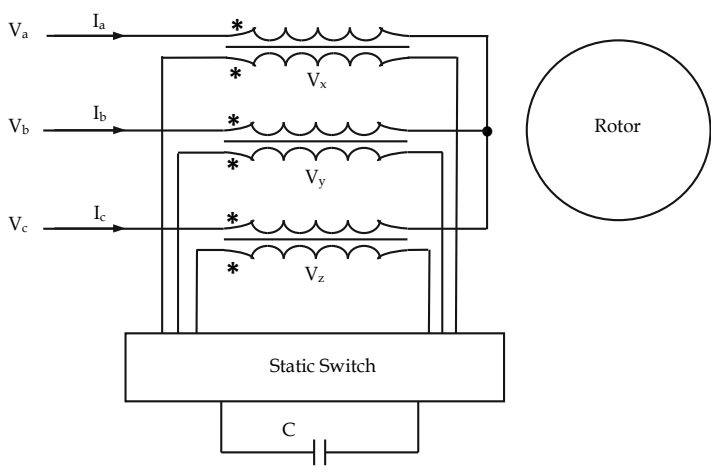

(a)

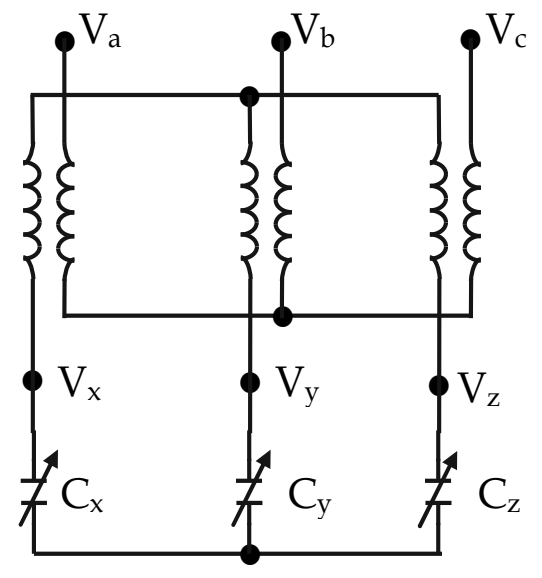

(b)

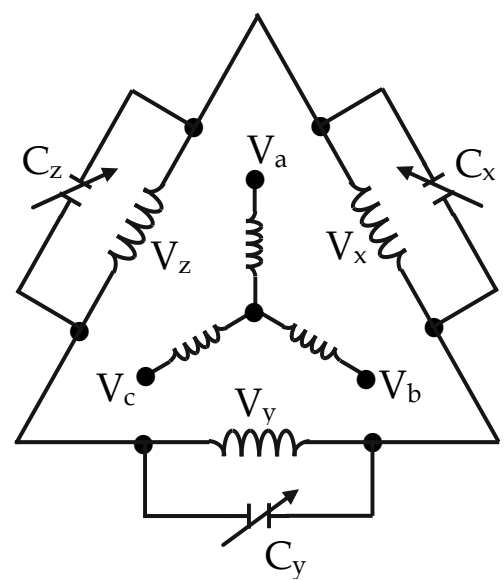

(c)

Fig. 2. Auxiliary windings: a) generic connection; b) star connection; c) delta connection

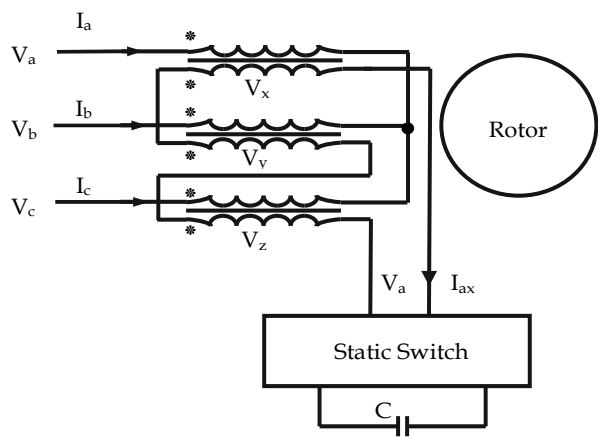

Fig. 3. Auxiliary windings: "single -phase connection" 


\subsubsection{Thyristor-based variable capacitor}

Figure 4 shows the use of thyristor to accomplish a variable capacitor. The inductor $\mathrm{L}_{\mathrm{r}}$ is introduced to reduce - limit the surge current; it is relatively small and does not affect the overall capacitive behaviour.

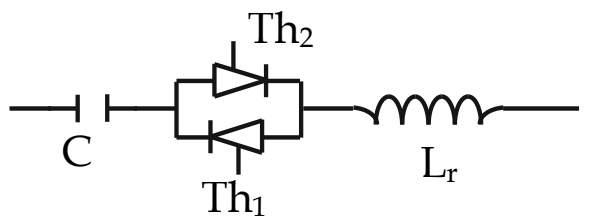

Fig. 4. Variable capacitor using bidirectional thyristor

The equivalent capacitance depends on the delay angle. Due to the phase angle control, the device introduces harmonic currents.

\subsubsection{IGBT-based variable capacitor}

The above drawback can be address using IGBTs in bidirectional configuration (Figure 5) and a switching frequency higher then operational frequency $(50 \mathrm{~Hz})$.

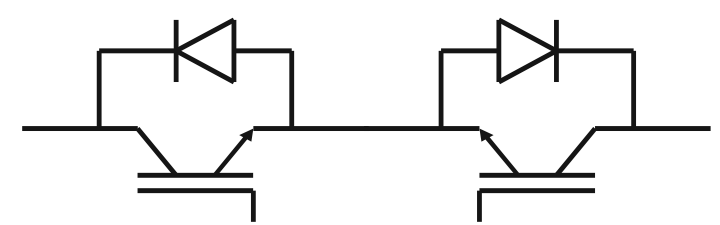

Fig. 5. IGBT in bidirectional topology

Figure 6 shows a configuration to achieve a variable capacitor using two bidirectional static switches. The main capacitor $C_{1}$ is introduced in the auxiliary winding circuit, via a bidirectional switch $S w_{1}$, for a period of time depending on the duty cycle $(\delta)$ of the switching frequency; in this time the bidirectional switch $\mathrm{Sw}_{2}$ is OFF. When $\mathrm{Sw}_{1}$ is OFF, the capacitor is discharged. The reactor $\mathrm{L}_{\mathrm{r}}$ limits the capacitive surge current without affecting the capacitive behaviour.

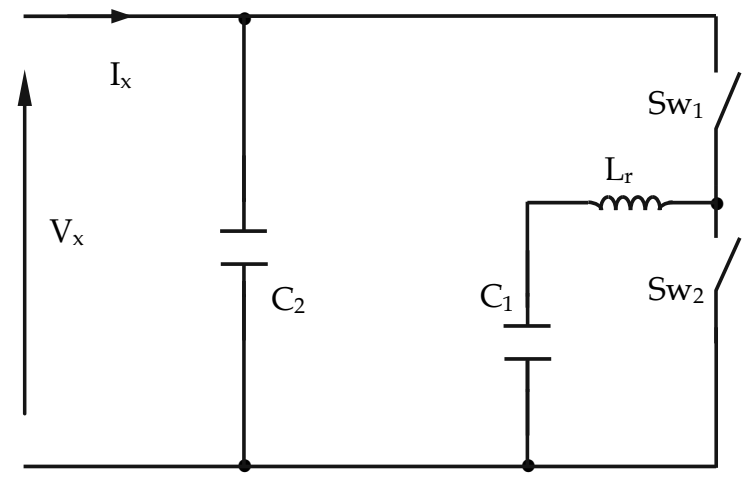

Fig. 6. Variable capacitor using two IGBTs in bidirectional topology 
The capacitor $C_{2}$, much smaller than $C_{1}$ is connected to mitigate the voltage spikes during switching off the main capacitor. Thus, the equivalent capacitor can be written as:

$$
\mathrm{C}_{\mathrm{eq}}=\delta \times \mathrm{C}_{1}+\mathrm{C}_{2}
$$

\subsubsection{Variable capacitor H-topology}

Figure 7 shows a single-phase $\mathbf{H}$ topology to achieve a variable capacitor. This configuration using $\mathrm{H}$-bridge bidirectional topology obtains a higher equivalent capacitance for the same fixed one as reference. In this configuration, the reactor $L_{r}$ has the same purpose of limiting the surge capacitive current, while $C_{2}$ also of small value mitigates the voltage spikes. The equivalent capacitance could be express as:

$$
\mathrm{C}_{\mathrm{eq}}=\mathrm{C}_{2}+\frac{\mathrm{C}_{1}}{(2 \delta-1)^{2}}
$$

It can be notice that the equivalent capacitance could increase significant when the duty cycle approaches $50 \%$. In practice, the switches are not ideal and there is no "infinite increase" of the equivalent capacitance.

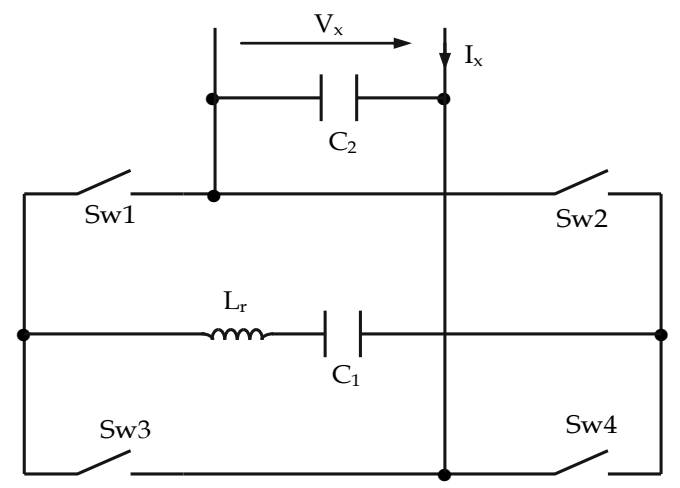

Fig. 7. Variable capacitor using H-bridge bidirectional topology

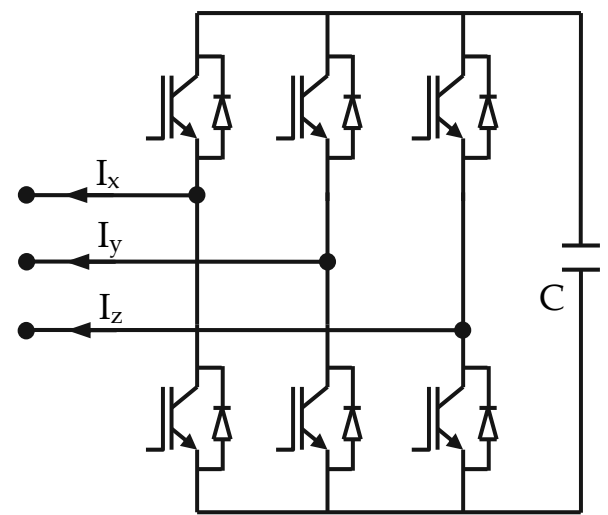

Fig. 8. Variable capacitor using three-phase H-bridge topology 
Another solution to achieve a variable capacitance, or rather to generate a capacitive current was proposed using a three-phase $\mathbf{H}$ topology as PWM inverter (E. Muljadi, et al 1989; Tamrakan and Malik, 1999) as presented in Figure 8. The converter injects capacitive reactive power into auxiliary windings and thus improving the power factor of the motor.

\section{Mathematical model}

The machine is treated as having two three-phase windings and the voltages equations system can be written as:

$$
\begin{gathered}
{\left[\mathrm{V}_{\mathrm{abcs}}\right]=\left[\mathrm{R}_{1}\right]\left[\mathrm{I}_{\mathrm{abc}}\right]+\frac{\mathrm{d}}{\mathrm{dt}}\left[\lambda_{\mathrm{abc}}\right]} \\
0=\left[\mathrm{R}_{2}\right]\left[\mathrm{I}_{\mathrm{xyz}}\right]+\frac{\mathrm{d}}{\mathrm{dt}}\left[\lambda_{\mathrm{xyz}}\right]+\mathrm{Vc}_{\mathrm{xyz}} \\
0=\left[\mathrm{R}_{\mathrm{r}}\right]\left[\mathrm{I}_{\mathrm{abcr}}\right]+\frac{\mathrm{d}}{\mathrm{dt}}\left[\lambda_{\mathrm{abcr}}\right]
\end{gathered}
$$

where

$$
\begin{gathered}
\mathrm{V}_{\mathrm{abc}}=\left[\begin{array}{lll}
\mathrm{V}_{\mathrm{a}} & \mathrm{V}_{\mathrm{b}} & \mathrm{V}_{\mathrm{c}}
\end{array}\right]^{\mathrm{T}} \\
\mathrm{I}_{\mathrm{abcs}}=\left[\begin{array}{lll}
\mathrm{I}_{\mathrm{a}} & \mathrm{I}_{\mathrm{b}} & \mathrm{I}_{\mathrm{c}}
\end{array}\right]^{\mathrm{T}} ; \lambda_{\mathrm{abc}}=\left[\begin{array}{lll}
\lambda_{\mathrm{a}} & \lambda_{\mathrm{b}} & \lambda_{\mathrm{c}}
\end{array}\right]^{\mathrm{T}} \\
{\left[\mathrm{R}_{1}\right]=\left[\begin{array}{ccc}
\mathrm{r}_{\mathrm{a}} & 0 & 0 \\
0 & \mathrm{r}_{\mathrm{b}} & 0 \\
0 & 0 & \mathrm{r}_{\mathrm{c}}
\end{array}\right] \quad\left[\mathrm{R}_{2}\right]=\left[\begin{array}{ccc}
\mathrm{r}_{\mathrm{x}} & 0 & 0 \\
0 & \mathrm{r}_{\mathrm{y}} & 0 \\
0 & 0 & \mathrm{r}_{\mathrm{z}}
\end{array}\right]}
\end{gathered}
$$

Note that indices " 1 " refer to the main winding and " 2 " to the auxiliary winding.

$$
\left[\begin{array}{c}
\lambda_{\text {abcs }} \\
\lambda_{\text {xyz }} \\
\lambda_{\text {abcr }}
\end{array}\right]=\left[\begin{array}{ccc}
\mathrm{L}_{\text {abc }} & \mathrm{L}_{\text {abcsxyz }} & \mathrm{L}_{\text {abcsr }} \\
\mathrm{L}_{\text {xyzabcs }} & \mathrm{L}_{\text {xyz }} & \mathrm{L}_{\text {xyzabcr }} \\
\mathrm{L}_{\text {abcrs }} & \mathrm{L}_{\text {abcrxyz }} & \mathrm{L}_{\text {abcr }}
\end{array}\right]\left[\begin{array}{c}
\mathrm{I}_{\text {abcs }} \\
\mathrm{I}_{\text {xyz }} \\
\mathrm{I}_{\text {abcr }}
\end{array}\right]
$$

The inductances in eq. (9) are time dependent, and this make the equation difficult and time consuming to solve. In order to obtain constant parameters, the voltage equations (3-5) are then transformed to the rotor reference frame. To achieve this, the equations are multiplied with an appropriate transformation matrix $K(\theta)$ to obtain:

$$
\begin{gathered}
{[K(\theta)]\left[\mathrm{V}_{\mathrm{abcs}}\right]=\left[\mathrm{R}_{1}\right][\mathrm{K}(\theta)]\left[\mathrm{I}_{\mathrm{abcs}}\right]+\frac{\mathrm{d}}{\mathrm{dt}}[\mathrm{K}(\theta)]\left[\lambda_{\mathrm{abcs}}\right]} \\
0=\left[\mathrm{R}_{2}\right][\mathrm{K}(\theta)]\left[\mathrm{I}_{\mathrm{xyzs}}\right]+\frac{\mathrm{d}}{\mathrm{dt}}[\mathrm{K}(\theta)]\left[\lambda_{\text {xyzs }}\right]+[\mathrm{K} \theta]\left[\mathrm{V}_{\text {cxyzs }}\right]
\end{gathered}
$$




$$
0=\left[\mathrm{R}_{\mathrm{r}}\right]\left[\mathrm{K}\left(\theta_{\mathrm{r}}\right)\right]\left[\mathrm{I}_{\mathrm{abcr}}\right]+\frac{\mathrm{d}}{\mathrm{dt}}\left[\mathrm{K}\left(\theta_{\mathrm{r}}\right)\right]\left[\lambda_{\mathrm{abcr}}\right]
$$

When these equations are expanded, after substantial matrix manipulations, it resolves to:

$$
\begin{gathered}
{\left[\mathrm{V}_{\mathrm{qdo1}}\right]=\left[\mathrm{R}_{\mathrm{s} 1}\right]\left[\mathrm{I}_{\mathrm{qdo1}}\right]+\frac{\mathrm{d}}{\mathrm{dt}}\left[\lambda_{\mathrm{qd} 01}\right]+\varpi\left[\lambda_{\mathrm{qdo1}}\right]} \\
0=\left[\mathrm{R}_{2}\right]\left[\mathrm{I}_{\mathrm{qdo} 2}\right]+\frac{\mathrm{d}}{\mathrm{dt}}\left[\lambda_{\mathrm{qd} 02}\right]+\varpi\left[\lambda_{\mathrm{qdo} 0}\right]+\left[\mathrm{V}_{\mathrm{qd} 02}\right] \\
0=\left[\mathrm{R}_{\mathrm{r}}\right]\left[\mathrm{I}_{\mathrm{qdor}}\right]+\frac{\mathrm{d}}{\mathrm{dt}}\left[\lambda_{\mathrm{qd} 0 \mathrm{r}}\right]+\varpi_{\mathrm{r}}\left[\lambda_{\mathrm{qdo1}}\right]
\end{gathered}
$$

where

$$
\varpi\left[\begin{array}{ccc}
0 & 1 & 0 \\
-1 & 0 & 0 \\
0 & 0 & 0
\end{array}\right] ; \varpi_{r}=\left(\omega-\omega_{r}\right)\left[\begin{array}{ccc}
0 & 1 & 0 \\
-1 & 0 & 0 \\
0 & 0 & 0
\end{array}\right]
$$

and

$$
\left[\mathrm{Vc}_{\mathrm{qd} 02}\right]=\frac{1}{\mathrm{C}} \int\left[\mathrm{I}_{\mathrm{qd} 02}\right] \mathrm{dt}+\varpi\left[\mathrm{Vc}_{\mathrm{qd} 02}\right]
$$

Neglecting the ' 0 ' sequence since we initially are assuming a balanced system, the expression for the stator and rotor flux linkages, resolves into the matrix:

$$
\left[\begin{array}{c}
\lambda_{\mathrm{q} 1} \\
\lambda_{\mathrm{d} 1} \\
\lambda_{\mathrm{q} 2} \\
\lambda_{\mathrm{d} 2} \\
\lambda_{\mathrm{qr}} \\
\lambda_{\mathrm{dr}}
\end{array}\right]=\left[\begin{array}{cccccc}
\mathrm{L}_{\mathrm{ls} 1}+\mathrm{L}_{\mathrm{m}} & 0 & \mathrm{~L}_{\mathrm{lm}}+\mathrm{L}_{\mathrm{m}} & 0 & \mathrm{~L}_{\mathrm{m}} & 0 \\
0 & \mathrm{~L}_{\mathrm{ls} 1}+\mathrm{L}_{\mathrm{m}} & 0 & \mathrm{~L}_{\mathrm{lm}}+\mathrm{L}_{\mathrm{m}} & 0 & \mathrm{~L}_{\mathrm{m}} \\
\mathrm{L}_{\mathrm{lm}}+\mathrm{L}_{\mathrm{m}} & 0 & \mathrm{~L}_{\mathrm{ls} 2}+\mathrm{L}_{\mathrm{m}} & 0 & \mathrm{~L}_{\mathrm{m}} & 0 \\
0 & \mathrm{~L}_{\mathrm{lm}}+\mathrm{L}_{\mathrm{m}} & 0 & \mathrm{~L}_{\mathrm{ls} 2}+\mathrm{L}_{\mathrm{m}} & 0 & \mathrm{~L}_{\mathrm{m}} \\
\mathrm{L}_{\mathrm{m}} & 0 & \mathrm{~L}_{\mathrm{m}} & 0 & \mathrm{~L}_{\mathrm{lsr}}+\mathrm{L}_{\mathrm{m}} & 0 \\
0 & \mathrm{~L}_{\mathrm{m}} & 0 & \mathrm{~L}_{\mathrm{m}} & 0 & \mathrm{~L}_{\mathrm{lsr}}+\mathrm{L}_{\mathrm{m}}
\end{array}\right]\left[\begin{array}{c}
\mathrm{I}_{\mathrm{q} 1} \\
\mathrm{I}_{\mathrm{d} 1} \\
\mathrm{I}_{\mathrm{q} 2} \\
\mathrm{I}_{\mathrm{d} 2} \\
\mathrm{I}_{\mathrm{qr}} \\
\mathrm{I}_{\mathrm{dr}}
\end{array}\right]
$$

\section{Equivalent model}

\subsection{Symmetrical loaded auxiliary windings}

When each phase of the auxiliary windings is loaded with equal capacitors (C), eventually star connected, the entire circuit is having a symmetrical behaviour and the equivalent circuit is very simple as shown in Figure 9.

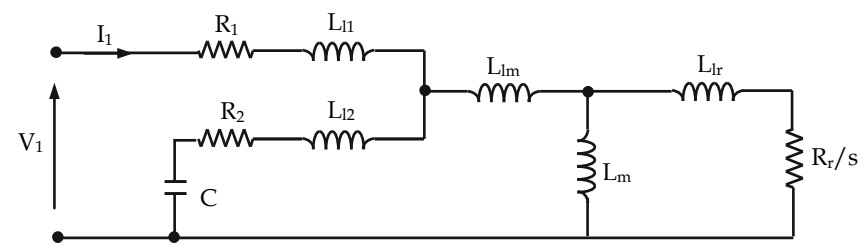

Fig. 9. Equivalent circuit for symmetrical loading auxiliary winding 
This equivalent circuit has two branches, each having separate resistance $\left(R_{1}-\right.$ main and $R_{2}$ auxiliary) and leakage reactance ( $\mathrm{L}_{11}$ - main and $\mathrm{L}_{12}$ - auxiliary) together with a common mutual leakage inductance $\mathrm{L}_{\mathrm{lm}}$, which occurs due to the fact that the two set of windings occupy the same slots and therefore mutually coupled by their leakage flux. The mutual inductance that occurs between main winding and rotor is represented by $\mathrm{Lm}_{\mathrm{m}}$.

Other parameters of the equivalent circuit are: main winding resistance $R_{1}$, auxiliary winding resistance $R_{2}$, main winding leakage reactance $L_{1}$, auxiliary winding leakage inductance $\mathrm{L}_{2}$; mutual leakage inductance $\mathrm{L}_{\mathrm{lm}}$, rotor leakage inductance $\mathrm{L}_{\mathrm{lr}}$; and the rotor resistance $R_{r}$ and " $s$ " is the slip.

In this analysis there is no need to refer the auxiliary winding quantities to the main winding, because the two sets of windings are wound for the same number of turns with a transformation ratio of one.

The above equivalent circuit helps us to determine the input impedance seen from the supply and the condition for unity power factor.

$$
Z_{\text {in }}=R_{1}+j X_{11}+\frac{\left(R_{2} R_{3}-X_{2} X_{3}\right)+j\left(X_{2} R_{3}+X_{3} R_{3}\right)}{\left(R_{2}+R_{3}\right)+j\left(X_{2}+X_{3}\right)}
$$

Where: $R_{1}$ and $j X_{11}$ are the components of the main winding (per-phase impedance), $X_{2}$ is the equivalent reactance of the auxiliary winding including the compensating capacitor, $R_{2}$ is the resistance (per-phase) of the auxiliary winding, $R_{3}$ and $X_{3}$ are the equivalent resistance and reactance of the paralleling the rotor branch with magnetizing branch:

$$
\begin{gathered}
\mathrm{X}_{2}=\frac{1}{\omega \mathrm{C}}-\mathrm{X}_{\mathrm{l} 2} \\
\mathrm{X}_{3}=\operatorname{Im}\left\{\mathrm{j} \mathrm{X}_{\mathrm{lm}}+\left[\left(\mathrm{R}_{\mathrm{r}} / \mathrm{s}\right)+\mathrm{j} \mathrm{X}_{\mathrm{lr}}\right] / /\left(\mathrm{j} \mathrm{X}_{\mathrm{m}}\right)\right\} \\
R_{3}=\operatorname{Re}\left\{\left[\left(\mathrm{R}_{\mathrm{r}} / \mathrm{s}\right)+\mathrm{j} \mathrm{X}_{\mathrm{lr}}\right] / /(\mathrm{jXm})\right\}
\end{gathered}
$$

The condition for unity power factor is:

$$
\operatorname{Im}\left\{Z_{\text {in }}\right\}=0
$$

Which, after some mathematical manipulation can be written as:

$$
\alpha X_{2}^{2}+\beta X_{2}+\gamma=0
$$

With:

$$
\begin{gathered}
a=X_{3}+X_{11} \\
\beta=-R_{2} R_{3}+\left(R_{1}+R_{2}\right) R_{3}+2 X_{3} X_{11} \\
\gamma=-X_{3}\left[R_{2} R_{3}-\left(R_{2}+R_{3}\right) R_{2}\right]+X_{11}\left[\left(R_{2}+R_{3}\right)^{2}+X_{3}^{2}\right]
\end{gathered}
$$


The equation (24) together with relations (25) to (27) produces two solutions, which means for a given slip (s) there are two values for the capacitor satisfying the condition for unity power factor. The practical/recommended value is the high $\mathrm{X}_{2}$ or small $\mathrm{C}$ connected to the auxiliary winding in order to have a small current through it.

\subsection{Asymmetrical loaded auxiliary windings.}

If the auxiliary windings are connected as "single-phase configuration", then the system has an asymmetrical behaviour. This connection is obtained by connecting in series the three auxiliary windings and thus we can write: $I_{x}=I_{y}=I_{z}=I_{X}$. Using this condition of current in the expansion that results to equations (3) - (18) it can be written: $\mathrm{I}_{\mathrm{d} 2}=\mathrm{I}_{\mathrm{q} 2}=0$, thus the resulting expression from this can be represented in a d-q-0 equivalent circuit of Figure 10 shown below.

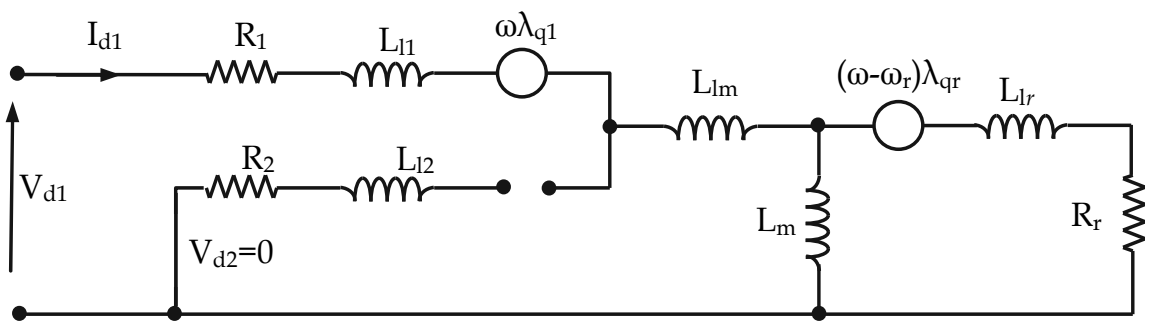

(a)

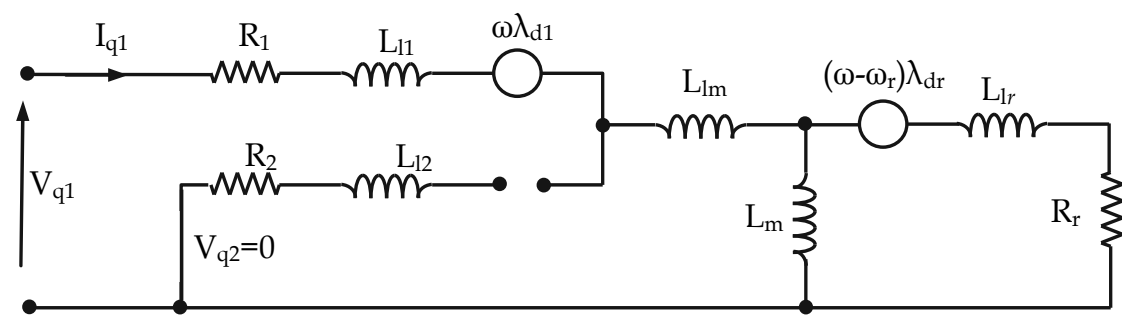

(b)

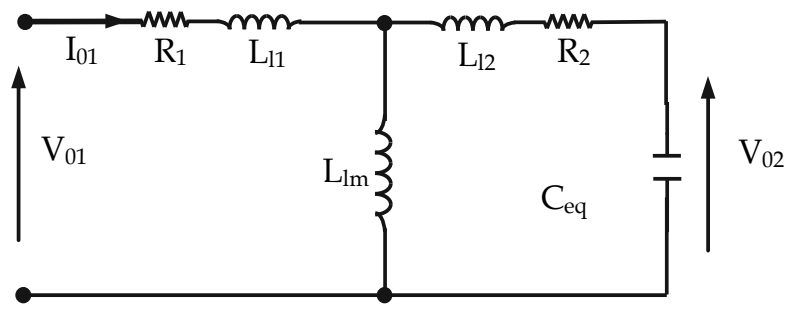

(c)

Fig. 10. The "d-q-0" equivalent circuit: a) "d" - equivalent circuit; b) "q" - equivalent circuit; c) " 0 " - equivalent circuit 
The particularity of connecting the auxiliary windings in a single phase winding creates an asymmetrical situation which brings about the relevance of the zero sequence.

The power factor of the machine could be defined by the argument of $Z_{a}$, which is $V_{a} / I_{a}$. And this is expressed as:

$$
\begin{gathered}
Z_{a}=\frac{V_{a}}{I_{a}}=\frac{V_{q 1}+V_{01}}{I_{q 1}+I_{01}} \\
V_{01}(C)=\left(-j \omega C+Z_{02}\right) \times I_{02} \times \frac{\left(Z_{01}+Z_{l m}\right)}{Z_{l m}}
\end{gathered}
$$

As can be observed from the equations (28) and (29), the power factor of the machine depends on $\mathrm{C}$ and thus it can be brought to unity by means of adjusting the equivalent capacitance.

It should be also noticed that the asymmetrical behaviour of the auxiliary windings connected as "single-phase configuration" has got a significant draw-back namely creating torque ripple. This disadvantage should not be overseen by the simplicity of the physical solution. Given this, further in this, we will consider only the symmetrical loading of the auxiliary winding.

\section{Concept validation}

For the validation of this concept of improving performances of the induction motor injecting capacitive reactive power via auxiliary windings, a standard $4 \mathrm{~kW}, 380 \mathrm{~V}, 50 \mathrm{~Hz}, 4$ pole, 36 slots with frame size DZ113A induction motor have been used. The stator was rewound with two full-pitches, single layer windings (see Figure 1).The main windings delta connected have same number of turns like in the original motor but the size of the wire has been reduced to accommodate the auxiliary windings. The auxiliary windings were connected in delta in order to reduce current through them. For this study, the auxiliary windings have the same number of turns as main windings. The rotor is unchanged. Table 1 shows the parameters of the modified motor under test obtained using the IEEE test procedure. The mutual leakage inductance $X_{\mathrm{lm}}$ is very small compared to the other reactance's (E. Muljadi et al, 1989).

\begin{tabular}{|l|c|}
\hline Description of data & Values \\
\hline Main Winding Rated Voltage & $380 \mathrm{~V}$ \\
\hline Auxiliary Winding Rated Voltage & $380 \mathrm{~V}$ \\
\hline Number of poles & 4 \\
\hline Magnetizing Reactance $\left(\mathrm{X}_{\mathrm{m}}\right)$ & $37.86 \Omega$ \\
\hline Main winding phase resistance $\left(\mathrm{R}_{1}\right)$ & $4.33 \Omega$ \\
\hline Auxiliary winding phase resistance $\left(\mathrm{R}_{2}\right)$ & $18.1 \Omega$ \\
\hline Main winding leakage reactance $\left(\mathrm{X}_{11}\right)$ & $6.97 \Omega$ \\
\hline Auxiliary winding leakage reactance $\left(\mathrm{X}_{\mathrm{l}}\right)$ & $6.97 \Omega$ \\
\hline Rotor resistance $\left(\mathrm{R}_{\mathrm{r}}\right)$ & $1.35 \Omega$ \\
\hline Rotor leakage reactance $\left(\mathrm{X}_{\mathrm{Ir}}\right)$ & $1.97 \Omega$ \\
\hline Full load main winding current & $8.6 \mathrm{~A}$ \\
\hline
\end{tabular}

Table 1. Specific parameters of the induction motor under test 
Firstly, the motor was tested without compensation; the results are presented in Table 2.

\begin{tabular}{|c|c|c|c|c|c|c|}
\hline $\mathrm{I}_{\mathrm{L}}(\mathrm{A})$ & $\mathrm{T}_{\mathrm{L}}(\mathrm{Nm})$ & $\mathrm{P}(\mathrm{W})$ & $\mathrm{S}(\mathrm{VA})$ & $\mathrm{PF}$ & Slip & RPM \\
\hline 5.43 & 1.83 & 678.6 & 3568.7 & 0.17 & 0.0026 & 1496 \\
\hline 5.56 & 4.73 & 1734 & 3655.1 & 0.43 & 0.0060 & 1491 \\
\hline 6.02 & 5.49 & 1992 & 3957.5 & 0.49 & 0.0086 & 1487 \\
\hline 6.88 & 7.53 & 2709 & 4522.9 & 0.61 & 0.0153 & 1477 \\
\hline 7.74 & 9.89 & 3540 & 5088.2 & 0.70 & 0.0213 & 1468 \\
\hline 8.61 & 12.1 & 4179 & 5653.6 & 0.74 & 0.056 & 1416 \\
\hline
\end{tabular}

Table 2. Experimental data for the motor under test

\subsection{Simulation results}

Based on Matlab platform, a simulation model has been built (Figure 11). The parameters from Table 1 have been used for Matlab model after delta-star transformation.

The rotor resistor was simulated using a variable resistor depending on slip. The model was run firstly to show the capability of adjusting the power factor via the static switch.

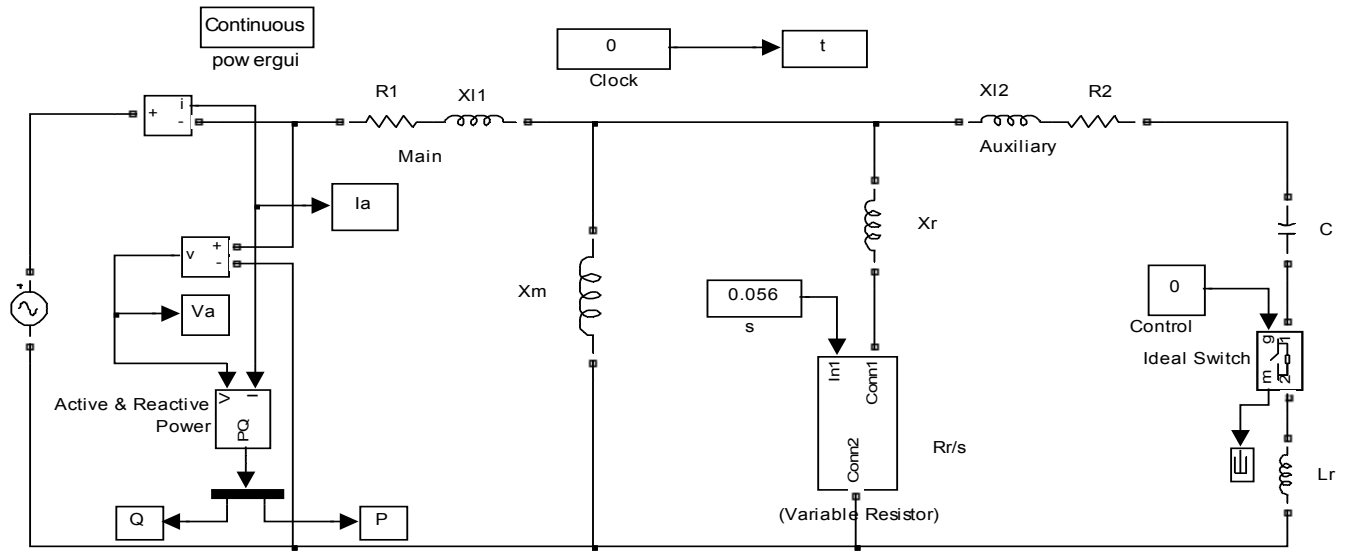

Fig. 11. Basic simulation model

\subsubsection{Thyristor-based variable capacitor}

One of the first solutions to introduce a variable capacitor was to use an "ac-ideal" switch based on two thyristors anti-parallel connected. The test was done using a $100 \mu \mathrm{F}$ and a delay angle of $45^{\circ}$ for a slip of 0.0026 (no load). This results in a reduction of reactive power drawn by the motor from $1193 \mathrm{VAr}$ to $46 \mathrm{VAr}$. The main drawback of this switching solution is the strong distorting currents introduced as could be seen in Figure 12.

As could be noticed, both currents main and auxiliary are very much distorted. Obviously, the power factor compensation is accompanied with degrading of all other performances of the motor. 


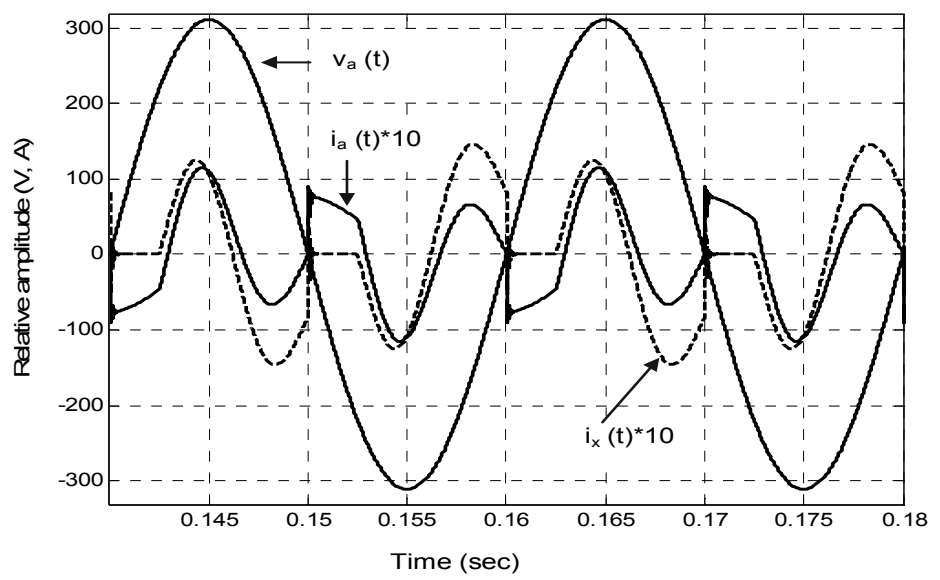

Fig. 12. Electric parameters $\left(v_{a}, i_{a}\right.$ and $\left.i_{x}\right)$ for compensated model using thyristor-based variable capacitor

\subsubsection{IGBT-based variable capacitor}

The other solution tested to obtaining variable capacitor was that of Figure 6 . The values for the two capacitors were chosen as: $\mathrm{C}_{1}=100 \mu \mathrm{F}$ and $\mathrm{C}_{2}=1 \mu \mathrm{F}$. The validation simulation was done for a slip of $s=0.0026$; the switching frequency was $1 \mathrm{kHz}$ and then the duty cycle manually adjusted. Figure 13 shows the main and auxiliary currents toward supply voltage for a duty cycle of $40 \%$ (Fig.13. a) and 80\% (Fig.13. a).
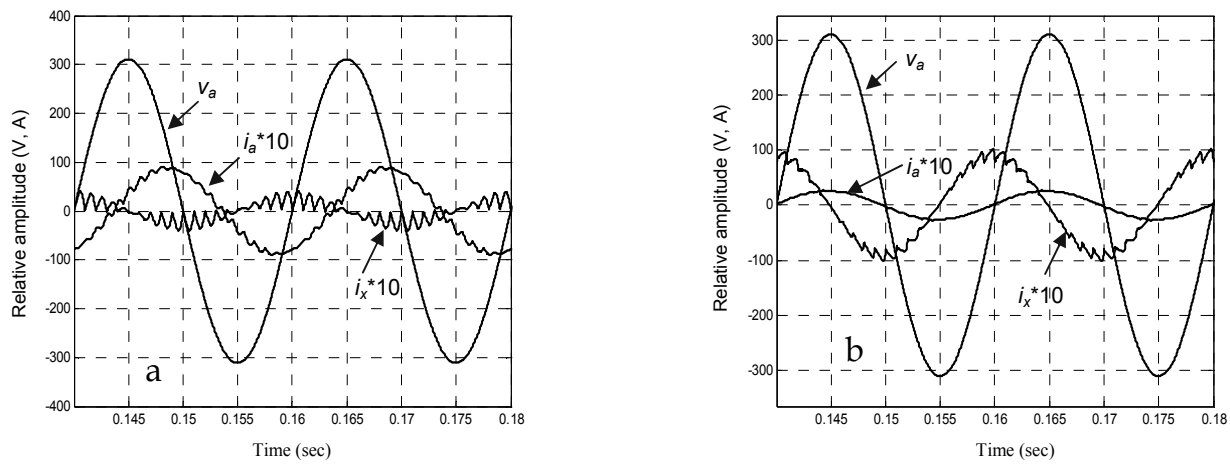

Fig. 13. Main and auxiliary currents for $\mathrm{s}=0.0026,1 \mathrm{kHz}$ and: a) $\delta=40 \%$; b) $\delta=80 \%$.

As can be noticed a duty cycle of $40 \%$ does adjust the shift between the main current and voltage marginally increasing the power factor from 0.15 to 0.32 lagging. When the duty cycle was increased to $80 \%$, then the main current arrived in phase with the voltage. It can also be noticed a relative high ripple especially for low duty cycle.

Figure 14 shows the same simulation conditions, but now the switching frequency was raised to $4 \mathrm{kHz}$. As can be noticed the ripple has been reduced significant.

The other methods of producing variable capacitive reactive power, meaning the H-bridge topologies require a more complex control system and are not addressed in this study. 

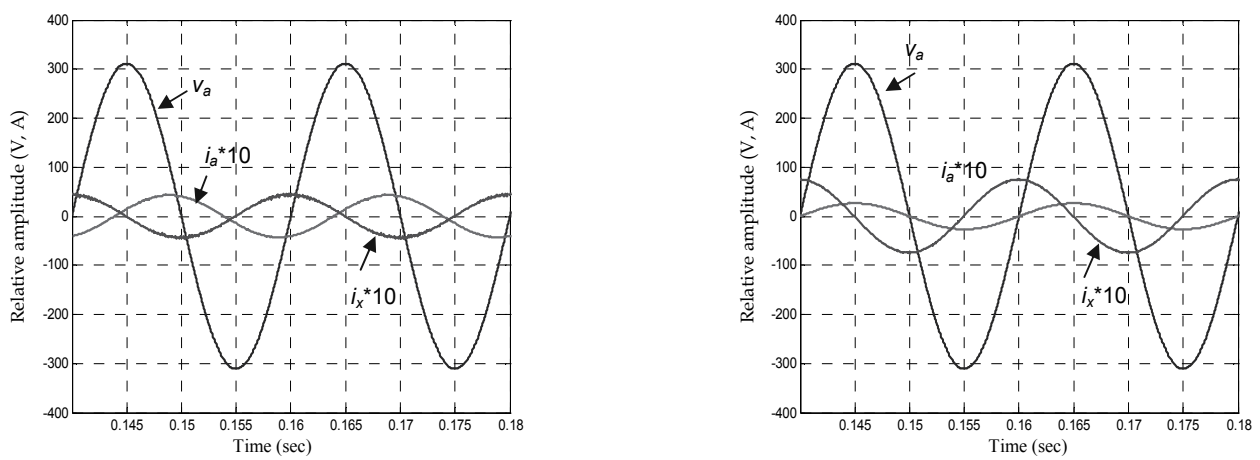

Fig. 14. Main and auxiliary currents for $\mathrm{s}=0.0026,4 \mathrm{kHz}$ and: a) $\delta=40 \%$; b) $\delta=80 \%$.

\subsubsection{Capacitance versus slip for unity power factor}

Now, the model was run for each value of the slip (speed) given in Table 2. The result of these simulations is the capacitance producing unity power factor (see Table 3).

\begin{tabular}{|c|c|c|c|c|c|c|}
\hline $\begin{array}{c}\mathrm{S} \\
(\mathrm{n})\end{array}$ & $\begin{array}{c}\mathrm{C}_{\mathrm{Y}} \\
(\mu \mathrm{F})\end{array}$ & $\begin{array}{c}\mathrm{I}_{\mathrm{a}} \\
(\mathrm{A})\end{array}$ & $\begin{array}{c}\mathrm{P}_{\mathrm{a}} \\
(\mathrm{W})\end{array}$ & $\begin{array}{c}\mathrm{Q}_{\mathrm{a}} \\
(\mathrm{VAr})\end{array}$ & $\begin{array}{c}\mathrm{S}_{\mathrm{a}} \\
(\mathrm{VA})\end{array}$ & $\mathrm{PF}$ \\
\hline 0.0026 & 0 & 5.49 & 184 & 1193 & 1207 & 0.152 \\
\cline { 2 - 7 }$(1496)$ & 81 & 1.65 & 362 & 9 & 363 & 0.999 \\
\hline 0.006 & 0 & 5.65 & 366 & 1187 & 1242 & 0.295 \\
\cline { 2 - 7 }$(1491)$ & 81 & 2.57 & 565 & 11 & 565 & 0.999 \\
\hline 0.0086 & 0 & 5.86 & 505 & 1186 & 1289 & 0.392 \\
\cline { 2 - 7 }$(1487)$ & 81.6 & 3.27 & 718 & 15 & 719 & 0.999 \\
\hline 0.0153 & 0 & 6.71 & 855 & 1200 & 1473 & 0.58 \\
\cline { 2 - 7 }$(1477)$ & 87 & 5.21 & 1143 & 1 & 1143 & 0.999 \\
\hline 0.0213 & 0 & 7.68 & 1160 & 1229 & 1689 & 0.686 \\
\cline { 2 - 7 }$(1468)$ & 90 & 6.82 & 1499 & 17 & 1499 & 0.999 \\
\hline 0.056 & 0 & 8.31 & 1330 & 1252 & 1826 & 0.728 \\
\cline { 2 - 7 }$(1491)$ & 90.6 & 7.66 & 1684 & 27 & 1686 & 0.999 \\
\hline
\end{tabular}

Table 3. Simulation results: capacitance versus slip for unity power factor

It is interesting to observe that the capacitance producing unity power factor does not vary so much as presented in other studies (E. Muljadi et al. 1989; Tamrakan and Malik, 1999) concerning similar method of injecting capacitive reactive power.

Moreover, it could be found a fix value of $75 \mu \mathrm{F}$ which maintain a high power factor irrespective of the load/slip. Table 4 shows the simulation results for the fixed capacitor and variable load/slip.

Figure 15 shows the variation of the power factor versus slip and Figure 16 shows the variation of the supply current versus slip for compensated with a fix $75 \mu \mathrm{F}$ capacitor per phase and uncompensated machine. 


\begin{tabular}{|c|c|c|c|c|c|c|}
\hline $\mathrm{s}$ & $\begin{array}{c}\mathrm{C}_{\mathrm{Y}} \\
(\mu \mathrm{F})\end{array}$ & $\begin{array}{c}\mathrm{I}_{\mathrm{a}} \\
(\mathrm{A})\end{array}$ & $\begin{array}{c}\mathrm{P}_{\mathrm{a}} \\
(\mathrm{W})\end{array}$ & $\begin{array}{c}\mathrm{Q}_{\mathrm{a}} \\
(\mathrm{VAr})\end{array}$ & $\begin{array}{c}\mathrm{S}_{\mathrm{a}} \\
(\mathrm{VA})\end{array}$ & $\mathrm{PF}$ \\
\hline 0.0026 & 75 & 1.57 & 330 & 101 & 345 & 0.956 \\
\hline 0.006 & 75 & 2.47 & 531 & 111 & 542 & 0.978 \\
\hline 0.0086 & 75 & 3.16 & 683 & 123 & 694 & 0.984 \\
\hline 0.0153 & 75 & 4.91 & 1066 & 170 & 1079 & 0.987 \\
\hline 0.0213 & 75 & 6.44 & 1396 & 230 & 1415 & 0.987 \\
\hline 0.056 & 75 & 7.29 & 1580 & 272 & 1603 & 0.986 \\
\hline
\end{tabular}

Table 3. Simulation results: capacitance versus slip for unity power factor

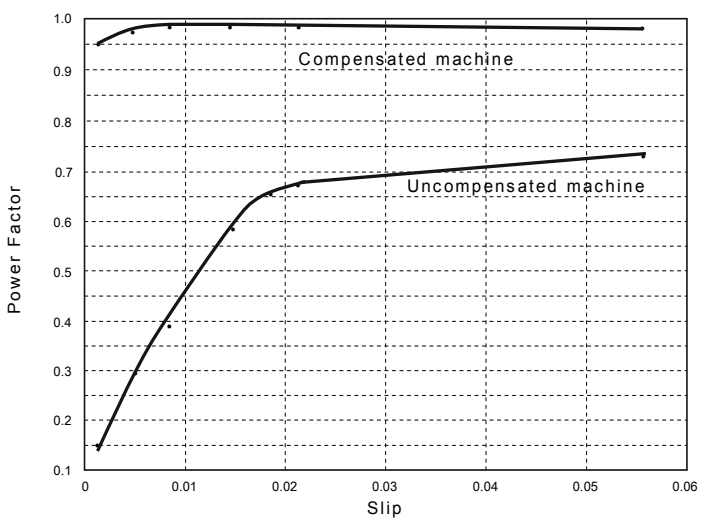

Fig. 15. Power Factor versus slip

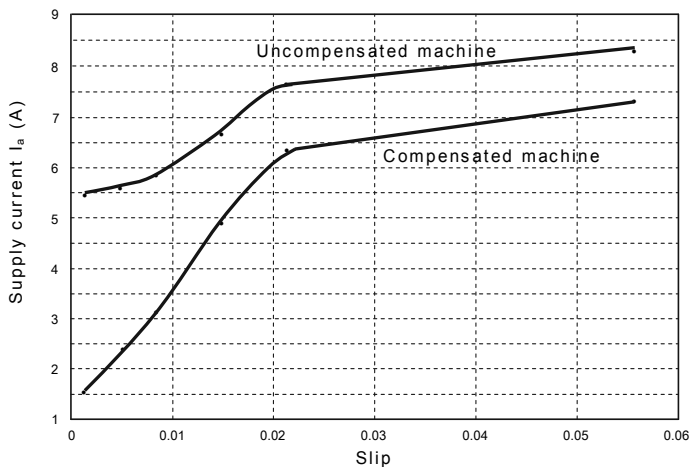

Fig. 16. Supply current versus slip

\subsection{Experimental validation}

After rewinding the machine under test with both sets of windings delta connected, three identical capacitors were connected to the auxiliary. Then the "compensated" motor was loaded gradually to get the same speeds as for the "uncompensated" motor.

The testing started with a capacitance of $25 \mu \mathrm{F}$ as found during the simulation: $\mathrm{C}_{\Delta}=\mathrm{C}_{Y} / 3$. This did not produce the results found via the simulation. Then a value of $28 \mu \mathrm{F}$ was found to give a relative even power factor of approximate 0.95 in the entire loading range (see Table 4 ). 
Figure 17 shows the phase voltage $\left(\mathrm{v}_{\mathrm{an}}\right)$ and current $\left(\mathrm{i}_{\mathrm{a}}\right)$ for the uncompensated (Fig. 17.a) and compensated (Fig. 17.b) with slip of 0.0026. It can be observed, the power factor increased from 0.17 to 0.937 which represent $450 \%$ improvement for no load. For full load the improvement in power factor is $29 \%$.

\begin{tabular}{|c|c|c|c|c|c|c|}
\hline $\mathrm{I}_{\mathrm{L}}(\mathrm{A})$ & $\mathrm{T}_{\mathrm{L}}(\mathrm{Nm})$ & $\mathrm{P}(\mathrm{W})$ & $\mathrm{S}(\mathrm{VA})$ & PF & Slip & RPM \\
\hline 1.71 & 2.81 & 1058 & 1129 & 0.937 & 0.0026 & 1496 \\
\hline 2.71 & 4.73 & 1694 & 1789 & 0.947 & 0.0060 & 1491 \\
\hline 3.49 & 5.83 & 2191 & 2304 & 0.951 & 0.0086 & 1487 \\
\hline 5.46 & 7.63 & 2709 & 3604 & 0.955 & 0.0153 & 1477 \\
\hline 6.92 & 9.29 & 3442 & 4567 & 0.952 & 0.0213 & 1468 \\
\hline 7.85 & 13.8 & 4937 & 5181 & 0.953 & 0.056 & 1416 \\
\hline
\end{tabular}

Table 2. Experimental data for the motor under test

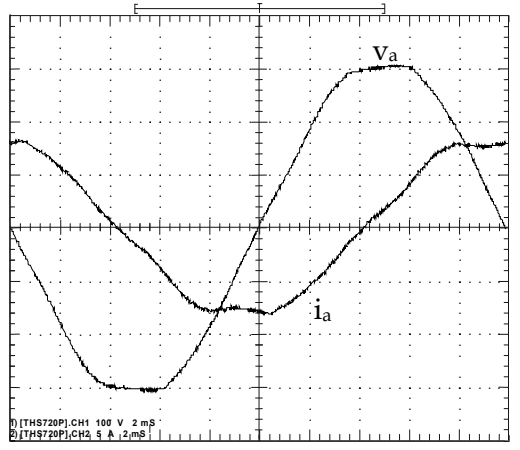

(a)

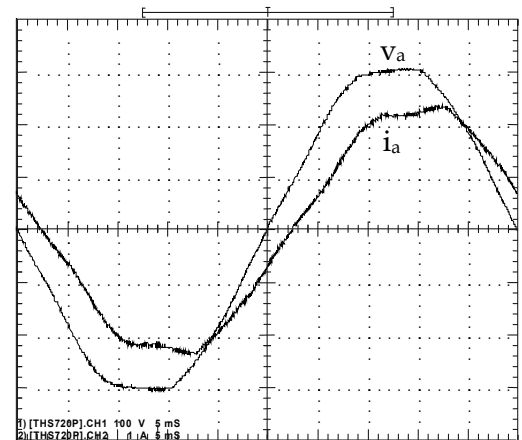

(b)

Fig. 17. Voltage and current for: a) uncompensated motor; b) compensated motor

\section{Comments}

This study proved that direct injecting capacitance reactance through auxiliary winding does improve the power factor of the induction motor; it also increases the ratio torque over current. What is also very interesting is this method achieves a "flat" variation of power factor with respect of load variation. This is a very important improvement given the fact that majority of induction motors do not work at constant full load where the classic design produces maximum performances.

However, this method introduces extra copper losses reducing the overall efficiency and increasing the operation temperature.

This study did not intended to elucidate the full effect of the method upon the torque especially the existence of the influence produced by the current flowing through auxiliary windings. The only aspect clearly noticed was the torque ripple introduced by the "singlephase" auxiliary winding connection.

Further more, the same method was applied to a synchronous reluctance motor (Ogunjuyigbe et al, 2010). The same type of stator winded similarly as above was used with a salient milled rotor obtained from the corresponding squirrel cage induction motor. The experimental results show an improvement of the power factor from 0.41-0.69 to 0.93-0.97 for the entire loading range. 
The economic benefits are related with the savings on demand especially for places where a large number of three-phase induction motors are used under variable loading.

\section{References}

M. A El-Sharkawi, S. S Venkata, T. J Williams, and N. G Butler, “An adaptive Power Factor Controller for Three-Phase Induction Generators", Paper 84 SM 672-2 presented at the IEEE/PES Summer Meeting, Seattle, Washington, July 15-20, 1984.

Fuchs, E.F. Hanna, W.J.; "Measured efficiency improvements of induction motors with thyristor/triac controllers", , IEEE Transaction on Energy Conversion, Volume 17, Issue 4, Dec. 2002 pp. 437 - 444

Suciu, C.; Dafinca, L.; Kansara, M.; Margineanu, I.; "Switched capacitor fuzzy control for power factor correction in inductive circuits", IEEE 31st Annual Power Electronics Specialists Conference, 2000. Vol. 2, pp. 773 - 777

C. Suciu, M. Kansara, P. Holmes and W. Szabo, "Performance Enhancement of an Induction Motor by Secondary Impedance Control, IEEE Trans. On Energy Conversion, Vol. 17, No. 2, June 2002

J. Reinert, M.J. Parsley, "Controlling the speed of $8 \times 1$ induction motor by resonating the rotor \&wit," in IEEE Transactions on Industry Applications, Vol. 31, No. 4, July/August 1995, pp. 887-891.

E. Muljadi, T.A. Lipo, D.W. Novotny, "Power Factor Enhancement of Induction Machines by Means of Solid State Excitation," IEEE Trans. on Power Electronics, Vol. 4, No. 4, pp. 409418, Oct. 1989.

I. M Tamrakan and O.P Malik, "Power Factor Correction of Induction motors Using PWM Inverter Fed Auxiliary Stator Winding", IEEE Transaction on Energy Conversion, Vol. 14, No.3, Sept, 1999, pp. 426-432

J. B. Medarametla, M. D. Cos, and Baghzouz, "Calculations and measurement of unity plus three-phase induction motor," IEEE Transactions on Energy Conversion, vol. 7, no. 4, pp. 732-738, 1992.

S. D. Umans, and H. L. Hess, "Modelling and analysis of a the Wanlass three-phase induction motor configuration," IEEE Transaction on Power Apparatus and Systems, vol. 102, no. 9, pp. 2912-2916, 1983.

R. Spée and A. Wanllace, "Comparative Evaluation Of Power-Factor Improvement Techniques For Squirrel cage Induction Motors", Industry Applications Society Annual Meeting, 1990.

A.A. Jimoh and D.V. Nicolae, "Performance Analysis of a Three-Phase Induction Motor with Capacitance Injection", OPTIM'06, 10 th International Conference on Optimization of Electrical and Electronic Equipments, Brasov, Romania, May 17-20, 2006

D.V. Nicolae and A.A. Jimoh, "Three-Phase Induction Motor with Power Electronic Controlled Single-Phase Auxiliary Stator Winding", PESC'07, The 38th IEEE Power Electronics Specialists Conference, Orlando, USA, June 17-21, 2007

A.S.O. Ogunjuyigbe, A.A. Jimoh, D.V. Nicolae and E.S. Obe, "Analysis of Synchronous Reluctance Machine with Magnetically Coupled Three Phase Windings and Reactive Power compensation", IET Electric Power Applications, 2010, Vol. 4, Iss. 4, pp 291-303 


\title{
Magnetic Reluctance Method for Dynamical Modeling of Squirrel Cage Induction Machines
}

\author{
Jalal Nazarzadeh and Vahid Naeini \\ Faculty of Engineering \\ Shahed University, Tehran
}

Iran

\section{Introduction}

Nowadays, induction machines play important role in electromechanical energy conversion in industry. These machines are often operated in critical conditions where can cause unexpected failures and outages. Generally, stator and bearing faults, broken rotor bar and end-rings, air-gap irregularities are some of the major faults in an induction machine (Al-Shahrani, 2005; Sprooten, 2007) which may be situated the induction machines in out of service (Siddique et al., 2005). Fourier analysis for stator currents (Bellini et al., 2001; Benbouzid, 2000; Jung et al., 2006), torque and rotor speed, acoustic noise and temperature analysis (Siddique et al., 2005) are some classical techniques which introduced for identification and diagnosis of induction machines faults. Additionally, other heuristic methods were proposed to monitor of the induction machines for fault detection. For instance, neural network modelling were applied to monitor an induction machine for fault detection (Su \& Chong, 2007). Also, space vector of rotor magnetic field (Mirafzal \& Demerdash, 2004) based on artificial intelligent approaches and pendulous oscillation of the rotor magnetic field were proposed. Recently, a new technique based on the analysis of three-phase stator current envelopes was presented (Mirafzalet \& Demerdash, 2008). In all monitoring and fault detection techniques, we need to tune up the monitoring systems based on response of induction machines for proper operations. However, experimental set up for testing any arbitrary fault conditions are not practical. Thus, an accuracy dynamic and steady state models of induction machines are very important for this propose.

Also, for dynamical modelling of induction machines, space harmonic distribution, core saturation and loss are often neglected in abc quantitative and two-axis methods (Krause et al., 1995). Thus, these approaches do not have an efficient accuracy for modelling of induction machines in asymmetrical and non-linear conditions. For considering distribution rotor bars, coupled magnetic circuit method (Muñoz \& Lipo, 1999), abc quantitative based on rotor bar currents (Alemi \& Nazarzadeh, 1996) can be utilized. Furthermore, winding function method may be used to include the stator winding distribution effect in the air gap flux (Luos et al., 1995). However, in all mentioned methods, the core saturation, stator and rotor teeth effects and distributions of the rotor and stator windings can not be investigated, simultaneously. Also, Finite Element Method (FEM) is a professional technique for analysis of any electromagnetic systems, which needs to magnetic and geometry details of the systems (Faiz 
et al., 2002). This method is very accurate and flexible, but due to complexity, the dynamical modelling of an induction machine is quite complicated. Contrary to FEM, the Magnetic Equivalent Circuit Method (MECM) can apply to analysis of the electro magnetics problems with lower complexity. Magnetic saturation, space harmonics in stator and rotor teeth, stator windings and distributed structure of a squirrel-cage rotor can be considered by MECM for modelling and analysis of any induction machines (Jeong et al., 2003; Ostovic, 1989). In this approach, the non-linear reluctances of flux paths use to configure magnetic equivalent circuit. This method has less complexity than FEM for dynamical modelling of induction machines. Therefore, developing an exact details model of induction machine for analysis of transient, sensitivity and fault diagnosis in the asymmetrical conditions are very essential.

The present chapter introduces methodology of MECM for modelling and analysis of asymmetrical non-linear systems in transient and steady state conditions. MECM is very suitable method for finding a generalized accurate dynamical model of squirrel cage induction machines with asymmetrical conditions. For evaluation of the method, several simulations in linear and non-linear conditions are made. Also, some simulations results for induction machines with broken bar faults and core saturation conditions are included to illustrate capability of the method in asymmetrical conditions.

\section{Electric and magnetic based model of squirrel cage induction machines}

For detailed modelling of any electromagnetic systems, we have to find a correlation between electric and magnetic variables of the system. Generally, a set of non-linear differential equations presents dynamical model of a electromagnetic system that by using numerical analysis, transient response of the electrical variables can be obtained. In addition, non-linear algebraic equations illustrate non-linear relations between electrical and magnetic variables. MECM provides an augmented model of the electromagnetic systems, in which we can achieve all variables of the systems in transient and steady state, simultaneously. Also, the main advantages of MECM for modelling of induction machines are; simple algorithm for including distribution winding, stator and rotor teeth effects and magnetic core saturation phenomena. Global non-linear model of squirrel cage induction machines can be offered in algebraical (magnetic) and differential (electric) equations which will be presented in the following sections.

\section{Magnetic equivalent circuit of induction machines}

Fig. 1 shows a part of rotor and stator structures for a typical squirrel cage induction machine that magnetic circuit elements are presented for rotor and stator teeth and yoke. Numbers of rotor and stator teeth are considered by $h$ and $l$, respectively. Also in this figure, magnetic mutual permeances of rotor and stator teeth in air gap are shown. Due to non-linear characteristics of flux and magnetic current in iron core, permeances of the rotor and stator in the magnetic cores are illustrated as non-linear elements. $G_{i, j}$ is linear permeance of flux path between $i^{\text {th }}$ stator and $j^{\text {th }}$ rotor teeth in the air gap.

\subsection{Magnetic node equations}

Due to the fact that magnetic permeances of each stator and rotor teeth make several magnetic loops in the air gap, we apply node magnetic potential equations to the each air gap nodes for 


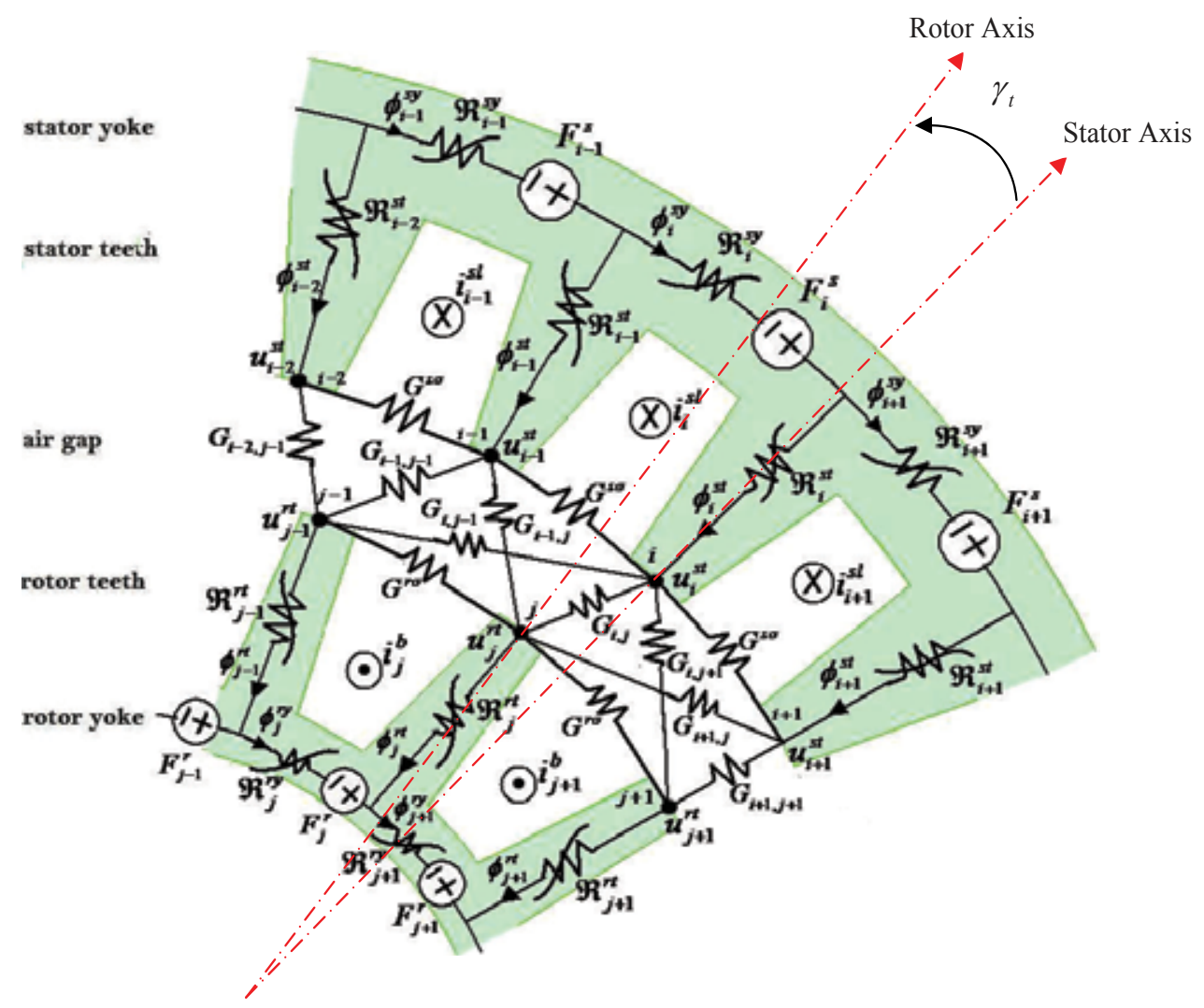

Fig. 1. Magnetic equivalent circuit of induction machine

simplicity in algebraic magnetic equations. For instance, sum of magnetic fluxes in $i^{t h}$ air gap node (stator tooth) have to be zero; thus we can write:

$$
\left(u_{i}^{s}-u_{i+1}^{s}\right) G^{s \sigma}+\left(u_{i}^{s}-u_{i-1}^{s}\right) G^{s \sigma}+\left(u_{i}^{s}-u_{1}^{r}\right) G_{i 1}+\cdots+\left(u_{i}^{s}-u_{l}^{r}\right) G_{i l}=\phi_{i}^{s t}
$$

where $u_{i}^{s}, u_{i}^{r}$ and $\phi_{i}^{s t}$ are magnetic potential of stator, rotor and flux of $i^{\text {th }}$ stator tooth and $G^{s \sigma}$ and $G_{i, j}$ are permeances of stator neighbour slots and mutual permance between $i^{\text {th }}$ stator with $j^{\text {th }}$ rotor teeth, respectively.

Similar Eq. (1), continuity principle in the teeth fluxes at $i^{\text {th }}$ rotor node yields as:

$$
\left(u_{i}^{r}-u_{i+1}^{r}\right) G^{r \sigma}+\left(u_{i}^{r}-u_{i-1}^{r}\right) G^{r \sigma}+\left(u_{i}^{r}-u_{1}^{s}\right) G_{i 1}+\cdots+\left(u_{i}^{r}-u_{h}^{s}\right) G_{i h}=-\phi_{i}^{r t}
$$

In Eq. (2), $\phi_{i}^{s t}$ and $G^{r \sigma}$ are flux of $i^{\text {th }}$ rotor tooth and permeance of rotor slot, respectively. Consequently, node potential equations for equivalent circuit of Fig. 1 along the air gap can be written as:

$$
\begin{gathered}
\mathbf{A}_{s s} \mathbf{U}_{s t}+\mathbf{A}_{s r} \mathbf{U}_{r t}=\Psi_{s t} \\
\mathbf{A}_{r s} \mathbf{U}_{s s}+\mathbf{A}_{r r} \mathbf{U}_{r t}=-\Psi_{r t}
\end{gathered}
$$


where $\mathbf{A}_{s s} \in \mathbb{R}^{h \times h} \hat{\mathbf{a}} \breve{A}$ ć, $\mathbf{A}_{r r} \in \mathbb{R}^{l \times l}$ and $\mathbf{A}_{s r} \in \mathbb{R}^{h \times l}$ are air gap permeance coefficients matrices that we can written as:

$$
\begin{aligned}
& \mathbf{A}_{s s}=\left[\begin{array}{ccccccc}
2 G^{s \sigma}+\sum_{j=1}^{l} G_{1, j} & -G^{s \sigma} & 0 & \cdots & 0 & 0 & -G^{s \sigma} \\
-G^{s \sigma} & 2 G^{s \sigma}+\sum_{j=1}^{l} G_{2, j} & -G^{s \sigma} & \cdots & 0 & 0 & 0 \\
\vdots & \vdots & \vdots & \ddots & \vdots & \vdots & \vdots \\
-G^{s \sigma} & 0 & 0 & \cdots & 0 & -G^{s \sigma} & 2 G^{s \sigma}+\sum_{j=1}^{l} G_{h, j}
\end{array}\right] \\
& \mathbf{A}_{r r}=\left[\begin{array}{ccccccc}
2 G^{r \sigma}+\sum_{i=1}^{h} G_{i, 1} & -G^{r \sigma} & 0 & \cdots & 0 & 0 & -G^{r \sigma} \\
-G^{r \sigma} & 2 G^{r \sigma}+\sum_{i=1}^{h} G_{i, 2} & -G^{r \sigma} & \cdots & 0 & 0 & 0 \\
\vdots & \vdots & \vdots & \ddots & \vdots & \vdots & \vdots \\
-G^{r \sigma} & 0 & 0 & \cdots & 0 & -G^{r \sigma} & 2 G^{r \sigma}+\sum_{i=1}^{h} G_{i, l}
\end{array}\right] \\
& \mathbf{A}_{s r}=\mathbf{A}_{r s}^{T}=\left[\begin{array}{cccc}
G_{1,1} & G_{1,2} & \cdots & G_{1, l} \\
G_{2,1} & G_{2,2} & \cdots & G_{2, l} \\
\vdots & \vdots & \ddots & \vdots \\
G_{h, 1} & G_{h, 2} & \cdots & G_{h, l}
\end{array}\right]
\end{aligned}
$$

Also, $\Psi_{s t} \in \mathbb{R}^{h \times 1}, \Psi_{r t} \in \mathbb{R}^{l \times 1}, \mathbf{U}_{s t} \in \mathbb{R}^{h \times 1}$ and $\mathbf{U}_{r t} \in \mathbb{R}^{l \times 1}$ are stator and rotor teeth fluxes vectors, stator and rotor magnetic scalar potentials vectors, respectively. These vectors can be presented as:

$$
\begin{aligned}
\Psi_{s t} & =\left[\begin{array}{llll}
\phi_{1}^{s t} & \phi_{2}^{s t} & \cdots & \phi_{h}^{s t}
\end{array}\right]^{T} \\
\Psi_{r t} & =\left[\begin{array}{llll}
\phi_{1}^{r t} & \phi_{2}^{r t} & \cdots & \phi_{l}^{r t}
\end{array}\right]^{T} \\
\mathbf{U}_{s t} & =\left[\begin{array}{llll}
u_{1}^{s t} & u_{2}^{s t} & \cdots & u_{h}^{s t}
\end{array}\right]^{T} \\
\mathbf{U}_{r t} & =\left[\begin{array}{llll}
u_{1}^{r t} & u_{2}^{r t} & \cdots & u_{l}^{r t}
\end{array}\right]^{T}
\end{aligned}
$$

In Eq. (5), $G^{s \sigma}$ is linear permeances between each successive stator slots with constant geometric permeability. This permeance can be obtained from (see Fig. 1):

$$
G^{s \sigma}=\mu_{0} \frac{A^{s \sigma}}{L^{s \sigma}}
$$

Similarly, for $G^{r \sigma}$ we can write:

$$
G^{r \sigma}=\mu_{0} \frac{A^{r \sigma}}{L^{r \sigma}}
$$

where $A^{s \sigma}, L^{s \sigma}, A^{r \sigma}, L^{r \sigma}$ and $\mu_{0}$ are cross section and length of stator and rotor slot opening and air permeability, respectively. Furthermore, mutual permeance between $i^{t h}$ and $j^{\text {th }}$ slots of the rotor and stator teeth depends to rotor mechanical angle $\left(\gamma_{i}(t)\right)$, momentarily. Fig.2-a 


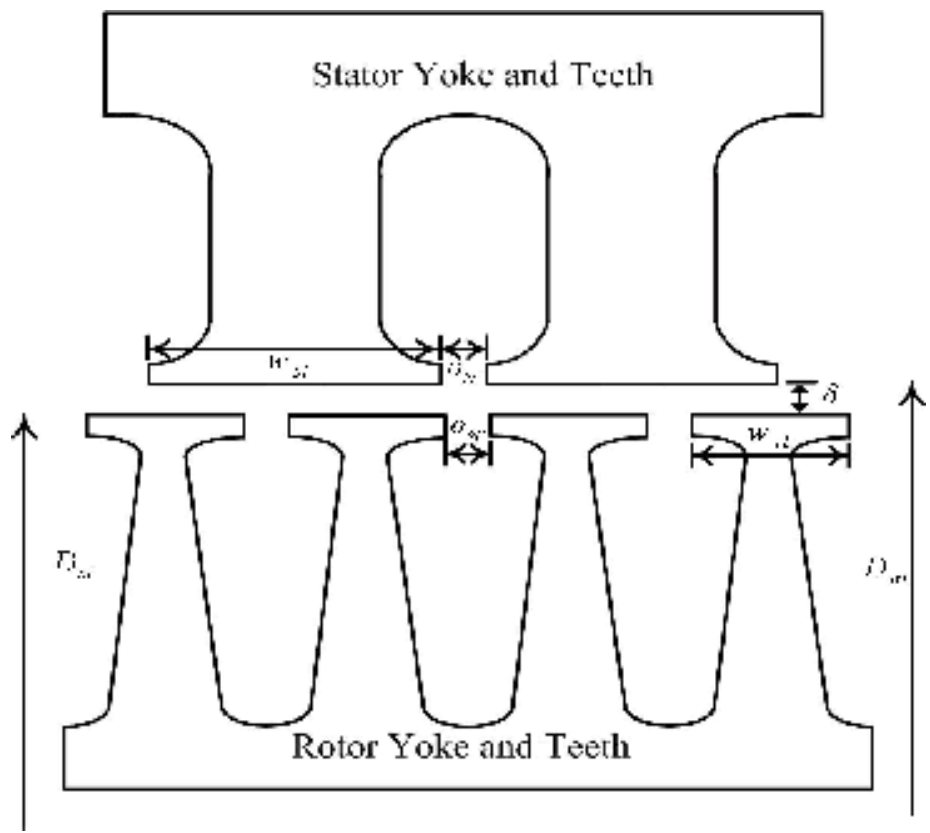

(a) Geometric of the stator and rotor teeth

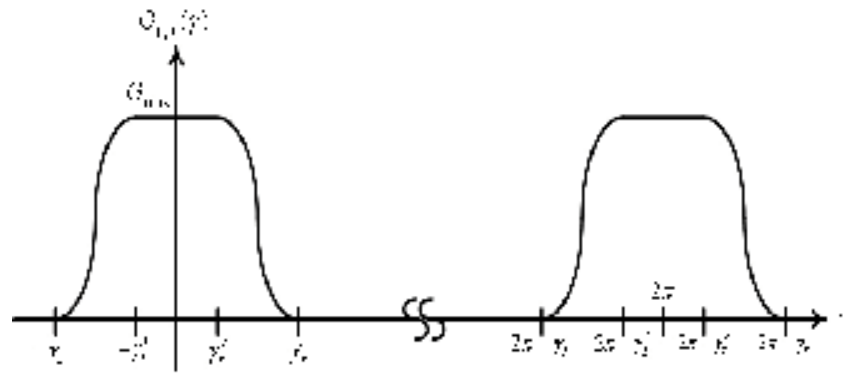

(b) Approximation function

Fig. 2. A typical air gap permeance between stator and rotor teeth

shows a typical geometry of the stator and rotor teeth. In this case, stator and rotor mutual permeance can be approximated by:

$$
G_{i, j}(\gamma)=\left\{\begin{array}{lc}
G_{\max } & 0<\gamma<\gamma_{t}^{\prime} \quad 2 \pi-\gamma_{t}^{\prime}<\gamma<2 \pi \\
G_{\max } \frac{1+\cos \pi \frac{\gamma-\gamma_{t}^{\prime}}{\gamma_{t}-\gamma_{t}^{\prime}}}{2} & \gamma_{t}^{\prime}<\gamma<\gamma_{t} \\
G_{\max } \frac{1+\cos \pi \frac{\gamma-2 \pi+\gamma_{t}^{\prime}}{\gamma_{t}-\gamma_{t}^{\prime}}}{2} & 2 \pi-\gamma_{t}<\gamma<2 \pi-\gamma^{\prime} \\
0 & \gamma_{t}<\gamma<2 \pi-\gamma_{t}
\end{array}\right.
$$

where $\gamma_{t}$ and $\gamma_{t}^{\prime}$ are two mechanical angles which are depended to the stator and rotor teeth geometry. For instance, in Fig. 2-a; $\gamma_{t}$ and $\gamma^{\prime} t$ can be obtained as: 


$$
\begin{gathered}
\gamma_{t}=\frac{w_{s t}+w_{r t}+o_{s s}+o_{s r}}{D_{a g}} \\
\gamma_{t}^{\prime}=\frac{\left|w_{s t}-w_{r t}\right|}{D_{a g}}
\end{gathered}
$$

where $w_{s t}, w_{r t}, o_{s s}$ and $o_{s r}$ are dimensions of stator and rotor which are shown in Fig. 2-a and $D_{a g}$ is :

$$
D_{a g}=\frac{D_{s i}+D_{r o}}{2}
$$

The maximum value of air gap permeance $G_{\max }$ can be written as

$$
G_{\max }=\mu_{0} \frac{l \times \min \left[w_{s t}, w_{r t}\right]}{\delta}
$$

where $l$ and $\delta$ are lengths of machine and air gap.

\subsection{Magnetic mesh equations}

Eqs. (3) and (4) present $l+h$ node equations which are shown relation between magnetic potentials of teeth nodes with stator and rotor teeth fluxes. Attention to Fig. 1, two neighbour stator teeth and yoke paths make a simple mesh in each stator slots, that sum of the magnetic potentials in these mesh have to be zero. For instance, magnetic mesh equation in $i^{\text {th }}$ and $(i+1)^{t h}$ stator teeth and yoke can be written as:

$$
u_{i}^{s t}-u_{i-1}^{s t}-\Re_{i-1}^{s t} \phi_{i-1}^{s t}+\Re_{i}^{s t} \phi_{i}^{s t}+\Re_{i}^{s y} \phi_{i}^{s y}=F_{i}^{S}
$$

Similarly, mesh equation for $i^{\text {th }}$ rotor tooth and yoke with $(i+1)^{\text {th }}$ rotor tooth can be obtained as:

$$
u_{i}^{r t}-u_{i-1}^{r t}+\Re_{i-1}^{r t} \phi_{i-1}^{r t}-\Re_{i}^{r t} \phi_{i}^{r t}+\Re_{i}^{r y} \phi_{i}^{r y}=F_{i}^{r}
$$

Thus, magnetic mesh equations for all rotor and stator meshes can be expressed as:

$$
\begin{aligned}
& \mathbf{A}_{u s t} \mathbf{U}_{s t}+\mathbf{A}_{\Psi_{s y}} \Psi_{s y}+\mathbf{A}_{\varphi s t} \Psi_{s t}=\mathbf{F}_{s} \\
& \mathbf{A}_{u r t} \mathbf{U}_{r t}+\mathbf{A}_{\phi r y} \Psi_{r y}+\mathbf{A}_{\phi r t} \Psi_{r t}=\mathbf{F}_{r}
\end{aligned}
$$

In Eqs. (21) and (22), $\mathbf{A}_{\phi s t} \in \mathbb{R}^{h \times h}$ and $\mathbf{A}_{\phi r t} \in \mathbb{R}^{l \times l}$ are diagonal coefficients matrices of the stator and rotor teeth reluctance that we can find as:

$$
\mathbf{A}_{\phi s t}=\left[\begin{array}{cccccc}
\Re\left(B_{1}^{s t}\right) & 0 & 0 & \cdots & 0 & -\Re\left(B_{h}^{s t}\right) \\
-\Re\left(B_{1}^{s t}\right) & \Re\left(B_{2}^{s t}\right) & 0 & \cdots & 0 & 0 \\
0 & -\Re\left(B_{2}^{s t}\right) & \Re\left(B_{3}^{s t}\right) & \cdots & 0 & 0 \\
\vdots & \vdots & \vdots & \ddots & \vdots & \vdots \\
0 & \cdots & 0 & \cdots & \Re\left(B_{h-1}^{s t}\right) & 0 \\
0 & \ldots & 0 & \cdots & -\Re\left(B_{h-1}^{s t}\right) & \Re\left(B_{h}^{s t}\right)
\end{array}\right]
$$




$$
\mathbf{A}_{\phi r t}=\left[\begin{array}{cccccc}
-\Re\left(B_{1}^{r t}\right) & 0 & 0 & \cdots & 0 & \Re\left(B_{l}^{r t}\right) \\
\Re\left(B_{1}^{r t}\right) & -\Re\left(B_{2}^{r t}\right) & 0 & \cdots & 0 & 0 \\
0 & \Re\left(B_{2}^{r t}\right) & -\Re\left(B_{3}^{r t}\right) & \cdots & 0 & 0 \\
\vdots & \vdots & \vdots & \ddots & \vdots & \vdots \\
0 & \cdots & 0 & \cdots & -\Re\left(B_{l-1}^{r t}\right) & 0 \\
0 & \ldots & 0 & \cdots & \Re\left(B_{l-1}^{r t}\right) & -\Re\left(B_{l}^{r t}\right)
\end{array}\right]
$$

also $\mathbf{A}_{\phi s y} \in \mathbb{R}^{h \times h}$ and $\mathbf{A}_{\phi r y} \in \mathbb{R}^{l \times l}$ are stator and rotor yoke reluctance coefficients matrices which can be written as

$$
\begin{aligned}
& \mathbf{A}_{\phi s y}=\operatorname{diag}\left(\Re\left(B_{1}^{s y}\right), \Re\left(B_{2}^{s y}\right), \cdots, \Re\left(B_{h}^{s y}\right)\right) \\
& \mathbf{A}_{\phi r y}=\operatorname{diag}\left(\Re\left(B_{1}^{r y}\right), \Re\left(B_{2}^{r y}\right), \cdots, \Re\left(B_{l}^{r y}\right)\right)
\end{aligned}
$$

which $\Psi_{s y} \in \mathbb{R}^{h \times 1}, \Psi_{r y} \in \mathbb{R}^{l \times 1}$ are stator and rotor yoke fluxes vectors and $\mathbf{F}_{S} \in \mathbb{R}^{h \times 1}$ and $\mathbf{F}_{r} \in \mathbb{R}^{l \times 1}$ are stator and rotor ampere-turn vectors, respectively. These vectors are considered as:

$$
\begin{aligned}
\Psi_{s y} & =\left[\begin{array}{llll}
\phi_{1}^{s y} & \phi_{2}^{s y} & \cdots & \phi_{h}^{s y}
\end{array}\right]^{T} \\
\Psi_{r y} & =\left[\begin{array}{llll}
\phi_{1}^{r y} & \phi_{2}^{r y} & \cdots & \phi_{l}^{r y}
\end{array}\right]^{T} \\
\mathbf{F}_{s} & =\left[\begin{array}{llll}
F_{1}^{s} & F_{2}^{s} & \cdots & F_{h}^{s}
\end{array}\right]^{T} \\
\mathbf{F}_{r} & =\left[\begin{array}{llll}
F_{1}^{r} & F_{2}^{r} & \cdots & F_{l}^{r}
\end{array}\right]^{T}
\end{aligned}
$$

Also, $\mathbf{A}_{u s t} \in \mathbb{R}^{h \times h}$ and $\mathbf{A}_{u r t} \in \mathbb{R}^{l \times l}$ are constant matrices which are given by:

$$
\mathbf{A}_{u s t}=\mathbf{A}_{u r t}=\left[\begin{array}{cccccc}
1 & 0 & 0 & \cdots & 0 & -1 \\
-1 & 1 & 0 & \cdots & 0 & 0 \\
0 & -1 & 1 & \cdots & 0 & 0 \\
\vdots & \vdots & \vdots & \ddots & \vdots & \vdots \\
0 & 0 & 0 & \cdots & 1 & 0 \\
0 & 0 & 0 & \cdots & -1 & 1
\end{array}\right]
$$

\subsection{Teeth and yoke flux relations}

For completing of the magnetic model of an induction machine, we need to find a relation between teeth and yoke fluxes in mesh equations (Eqs. (21) and (22)). Attention to Fig. 1, the relations between stator teeth and yoke fluxes can be obtained by applying magnetic flux continuity principle in the yoke nodes. Thus, we have:

$$
\phi_{i}^{s y}=\phi_{i}^{s t}+\phi_{i+1}^{s y}
$$


Similarly, relation between the rotor fluxes are:

$$
\phi_{j+1}^{r y}=\phi_{j}^{r t}+\phi_{j}^{r y}
$$

Eqs. (32) and (33) can be presented in matrix form as:

$$
\begin{aligned}
& \Psi_{s t}=\mathbf{A}_{s y t} \Psi_{s y} \\
& \Psi_{r t}=\mathbf{A}_{r y t} \Psi_{r y}
\end{aligned}
$$

where $\mathbf{A}_{s y t} \in \mathbb{R}^{h \times h}$ and $\mathbf{A}_{r y t} \in \mathbb{R}^{l \times l}$ can be written as

$$
\mathbf{A}_{r y t}=-\mathbf{A}_{s y t}=\left[\begin{array}{cccccc}
-1 & 1 & 0 & \cdots & 0 & 0 \\
0 & -1 & 1 & \cdots & 0 & 0 \\
0 & 0 & -1 & \cdots & 0 & 0 \\
\vdots & \vdots & \vdots & \ddots & \vdots & \vdots \\
0 & 0 & 0 & \cdots & -1 & 1 \\
1 & 0 & 0 & \cdots & 0 & -1
\end{array}\right]
$$

In a squirrel cage induction machine, a stator winding is not concentrated in single slot, but it is distributed along air gap for harmonics reduction, full utilization of core and reduction of mechanical stress to the winding. Thus, flux of stator coil equals the sum of fluxes of stator teeth in the coil pitch. If three phase flux vector of the stator windings denotes as $\Psi_{3 \phi}$, we can find a matrix relation between stator teeth and windings fluxes as:

$$
\Psi_{3 \phi}=\mathbf{M}_{c f} \Psi_{s t}
$$

where $\mathbf{M}_{c f} \in \mathbb{R}^{3 \times h}$ is a connected matrix which can be obtained based on the connection diagram of stator windings.

\section{Core saturation characteristic}

Generally, magnetic cores of any electrical machines have non-linear characteristic curve $(B-H)$, thus the elements of reluctance matrices in Eqs. (23) and (24) are depended to their fluxes. For inserting the non-linearity characteristic of the magnetic core to mesh equations, a non-linear permeability dependent to the core field density are used. For this purpose, a non-linear permeability is defined as:

$$
\mu(B)=\mu_{0} \mu_{r}(B)=\frac{\partial B}{\partial H}
$$

So, the reluctances of flux path in the stator and rotor cores can be written as:

$$
\Re\left(B_{i}^{k}\right)=\frac{L_{i}^{k}}{\mu\left(B_{i}^{k}\right) A_{i}^{k}} \quad \begin{gathered}
k=s y, s t, r y, r t \\
i=1,2, \ldots
\end{gathered}
$$

where $L_{i}^{k}$ and $A_{i}^{k}$ are $i^{t h}$ length and cross section of flux path in the stator and rotor cores, respectively. Tangent, exponential and piecewise linear functions may be used to approximate the saturation curve (Chen et al., 2005). In this chapter, the magnetic core permeability is 


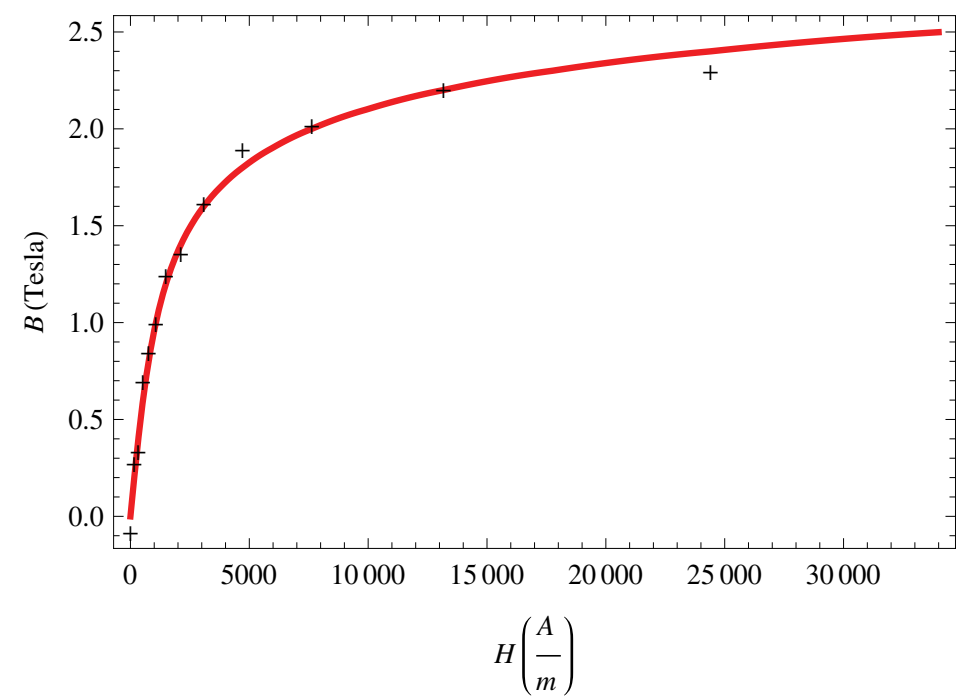

Fig. 3. Core saturation curve

considered as:

$$
\mu(B)=b \times e^{a \times B^{2}}
$$

where $a$ and $b$ are two constants which can be chosen for the best fitting of the saturation curve $(B-H)$ with this relation. By integrating Eq. (38) and combining with Eq. (40), the magnetic field intensity $H$ can be written as

$$
H(B)=\int \frac{1}{\mu(B)} d B=\frac{\sqrt{\pi} \operatorname{Erf}(\sqrt{a} \times B)}{2 b \sqrt{a}}
$$

where $\operatorname{Erf}$ is error function which is defined as: (Gautschi, 1964):

$$
\operatorname{Erf}(B)=\int e^{B^{2}} d B
$$

For instance, Fig. 3 shows a saturation curve $(B-H)$ for a silicon steel core that we can find two constants in Eq. (40) for the best fitting of the saturation curve. In this case, the two constants can be found as:

$$
\begin{aligned}
& a=-0.8 \\
& b=1000
\end{aligned}
$$

\section{Excitation vectors of reluctance network}

For determining relations between three phase currents with ampere-turn in the stator magnetic circuits, we give a contour between $(i-1)^{t h}$ and $i^{\text {th }}$ stator teeth, yoke and slot in Fig. 1. Also, we suppose that combining of three phase conductors are placed in the stator slots. Therefore, by applying Ampere's law to this contour, $(i)^{\text {th }}$ ampere-turn of the stator magnetic circuits $\left(F_{i}^{S}\right)$ in Eq. (21) can be expressed as:

$$
F_{i}^{S}=w_{i}^{a} i_{a}+w_{i}^{b} i_{b}+w_{i}^{c} i_{c}
$$




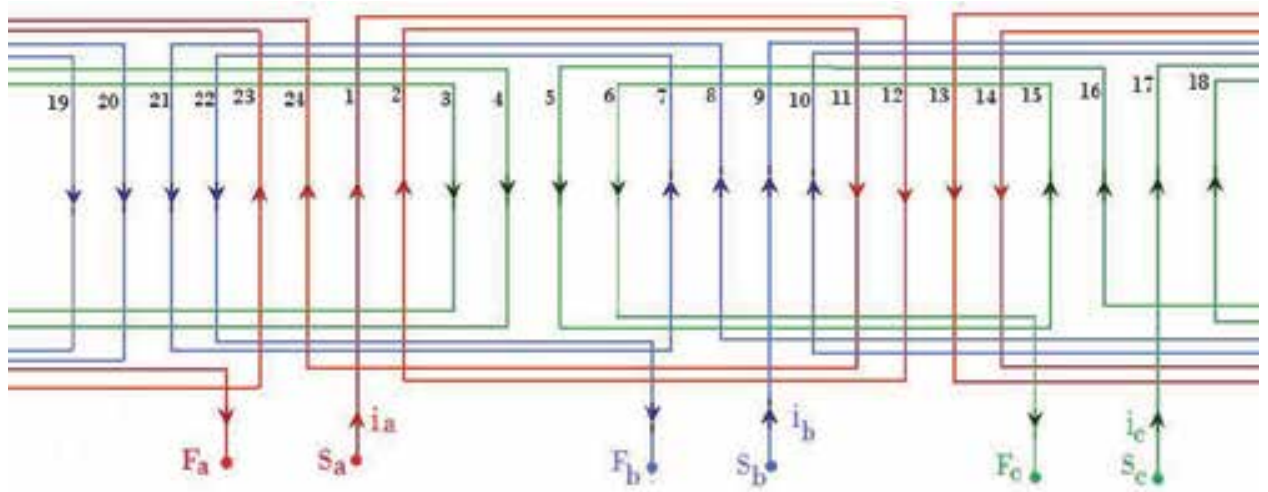

Fig. 4. Single layer distributed winding with 24 slot

where $w_{i}^{a}, w_{i}^{b}$ and $w_{i}^{c}$ are conductor numbers of phase $a, b$ and $c$ in the $i^{t h}$ stator slot, respectively. Values and signs of these parameters will depend to connections and directions of windings in the $i^{t h}$ slot. For instance, Fig. 4 shows a single layer three phase winding with two poles and 24 slots, which can generate a rotating magnetic field in the air gap. In this case, relation between ampere-turn of the stator magnetic circuits and stator currents can be determined as:

$$
\left[\begin{array}{c}
F_{1}^{s} \\
F_{2}^{s} \\
F_{3}^{s} \\
\vdots \\
F_{22}^{S} \\
F_{23}^{s} \\
F_{24}^{S}
\end{array}\right]=\left[\begin{array}{ccc}
-w^{a} & 0 & 0 \\
-w^{a} & 0 & 0 \\
0 & 0 & w^{c} \\
\vdots & \vdots & \vdots \\
0 & w^{b} & 0 \\
-w^{a} & 0 & 0 \\
-w^{a} & 0 & 0
\end{array}\right]\left[\begin{array}{c}
i_{a} \\
i_{b} \\
i_{c}
\end{array}\right]
$$

Thus, the stator ampere-turn vector $\left(\mathbf{F}_{\mathcal{S}}\right)$ in Eq. (21) may be presented as

$$
\mathbf{F}_{S}=\mathbf{W}_{c} \mathbf{i}_{S}
$$

where $\mathbf{W}_{c} \in \mathbb{R}^{h \times 3}$, is a connection matrix that shows number of conductors in all of the stator slots. Also, $\mathbf{i}_{s}$ is a vector of three phase currents that we can arrange as:

$$
\mathbf{i}_{s}=\left[\begin{array}{lll}
i_{a} & i_{b} & i_{c}
\end{array}\right]^{T}
$$

In single layer winding, each slot can carry only one phase current, thus rows of the connection matrix in Eq. (46) have only one non-zero element. These non-zero elements can be determined by attention to stator winding topology.

In other respect, currents of rotor bars in squirrel cage induction machines are equal to current of conductors in the rotor slots. Therefore, similar to above mentioned method; the ampere-turn in rotor slots $\left(\mathbf{F}_{r}\right)$ in Eq. (22) can be determined by:

$$
\mathbf{F}_{r}=\mathbf{i}_{b}
$$


where $\mathbf{i}_{b}$, the rotor bars currents vector is defined as

$$
\mathbf{i}_{b}=\left[\begin{array}{llll}
i_{1}^{b} & i_{2}^{b} & \cdots & i_{l}^{b}
\end{array}\right]^{T}
$$

Substituting Eqs. (34), (35), (46) and (48) into (21) and (22) and combining with Eqs. (3) and (4), the magnetic algebraic equations of squirrel cage induction machines in matrix form can be augmented as

$$
\left[\begin{array}{cccc}
\mathbf{A}_{\phi s y}+\mathbf{A}_{\phi s t} \mathbf{A}_{s y t} & 0 & \mathbf{A}_{u s t} & 0 \\
0 & \mathbf{A}_{\phi r y}+\mathbf{A}_{\phi r t} \mathbf{A}_{r y t} & 0 & \mathbf{A}_{u r t} \\
-\mathbf{A}_{s y t} & 0 & \mathbf{A}_{s s} & \mathbf{A}_{s r} \\
0 & \mathbf{A}_{r y t} & \mathbf{A}_{r s} & \mathbf{A}_{r r}
\end{array}\right]\left[\begin{array}{c}
\Psi_{s y} \\
\Psi_{r y} \\
\mathbf{U}_{s t} \\
\mathbf{U}_{r t}
\end{array}\right]=\left[\begin{array}{c}
\mathbf{W}_{c} \mathbf{i}_{s} \\
\mathbf{i}_{b} \\
0 \\
0
\end{array}\right]
$$

Due to core saturation characteristic is a non-linear curve, the matrix Eq. (50) is non-linear and some coefficient matrices depends to the core fluxes density. Thus, ordinary methods cannot be employed for solving Eq. (50). Furthermore, rotor and stator currents are depended to stator three phase source voltages and rotor speed in differential equation forms. Therefore, for detailed analysis of squirrel cage induction machine, it is necessary to solve an electric, mechanic and magnetic algebraic differential equations, simultaneously

\section{Electrical voltage equations of squirrel cage induction machines}

Generally, a set of differential equations in an electrical machine is used to describe rates of the electrical and mechanical variables. These equations establish the relationship between fluxes, currents and the three phase source voltage variables. In the next section, rotor and stator voltage relations are be derived.

\subsection{Stator voltage equations}

For an induction machine, we can write electrical differential equations in stator windings as:

$$
\mathbf{V}_{s}=\mathbf{R}_{s} \mathbf{i}_{s}+\frac{d}{d t} \Lambda_{s}
$$

where $\mathbf{V}_{s}$ and $\mathbf{R}_{s}$ are voltage and stator resistances matrix, respectively which are defined by

$$
\begin{gathered}
\mathbf{V}_{s}=\left[\begin{array}{lll}
v_{a} & v_{b} & v_{c}
\end{array}\right]^{T} \\
\mathbf{R}_{s}=\operatorname{diag}\left[\begin{array}{lll}
r_{a} & r_{b} & r_{c}
\end{array}\right]
\end{gathered}
$$

Moreover, $\Lambda_{S}$ is linkage flux vector and equals to the product of turn number of stator windings and the phase fluxes. Thus, we can write:

$$
\begin{gathered}
\Lambda_{s}=\left[\begin{array}{lll}
\lambda_{a} & \lambda_{b} & \lambda_{c}
\end{array}\right]^{T} \\
\Lambda_{s}=w_{c} \Psi_{3 \varphi}
\end{gathered}
$$

By substituting Eqs. (34) and (37) into (55), we obtain:

$$
\Lambda_{s}=w_{c} \mathbf{M}_{c f} \mathbf{A}_{s y t} \Psi_{s y}
$$




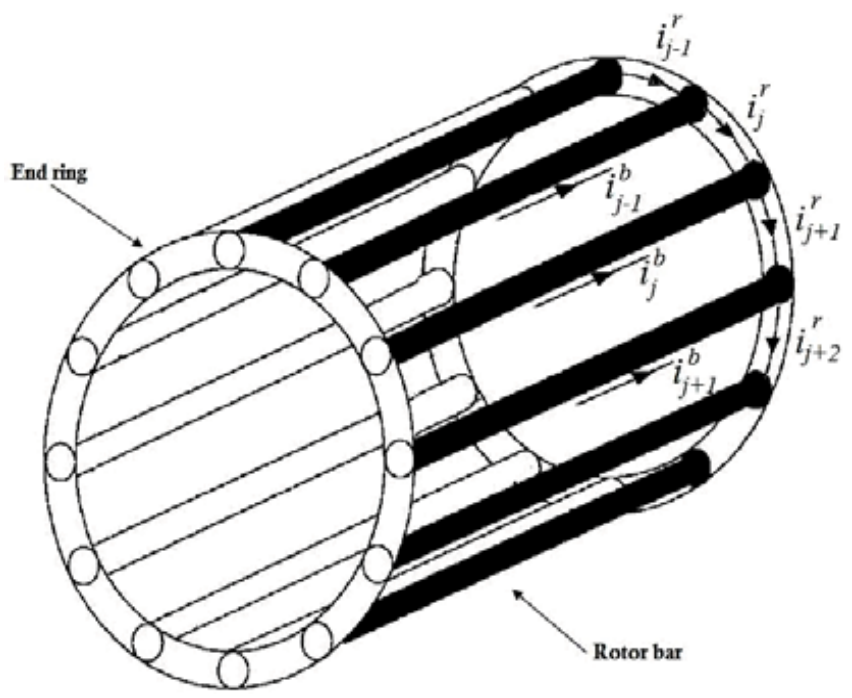

Fig. 5. Structure of squirrel cage rotor

\subsection{Rotor voltage equations}

Fig. 5 shows a structure of squirrel cage rotor in an induction machine. The rotor topology has $l+1$ mesh, thus we can write $l+1$ independent differential equations to describe electrical dynamic of rotor variables. Suppose, the rotor rings segments have a symmetrical structure, thus number of $l$ independent equations will be enough for modelling of the rotor dynamics. According to Fig. $1, j^{\text {th }}$ tooth of rotor is surrounded by $j^{\text {th }}$ and $(j+1)^{\text {th }}$ rotor bars. Thus, based on currents directions of bars and ring segments which are shown in the Fig. 4; the voltage equation in this loop can be given by:

$$
\frac{d}{d t} \phi_{j}^{r t}=-2 i_{j+1}^{r} r_{j+1}^{r}-i_{j}^{b} r_{j}^{b}+i_{j+1}^{b} r_{j+1}^{b}
$$

This relation can be rearranged in the matrix form and the electrical differential equations for the rotor cage can be obtained as

$$
\frac{d}{d t} \Psi_{r t}=-2 \mathbf{J}_{r} \mathbf{R}_{r} \mathbf{i}_{r}-\mathbf{A}_{r y t} \mathbf{R}_{b} \mathbf{i}_{b}
$$

where the vector $\mathbf{i}_{r} \in \mathbb{R}^{l \times 1}, \mathbf{R}_{b} \in \mathbb{R}^{l \times l}$ and $\mathbf{R}_{r} \in \mathbb{R}^{l \times l}$ denote ring segment currents, rotor bar and ring segment resistance matrices, receptively. These vectors are given by:

$$
\begin{aligned}
\mathbf{i}_{r} & =\left[\begin{array}{llll}
i_{1}^{r} & i_{2}^{r} & \cdots & i_{l}^{r}
\end{array}\right]^{T} \\
\mathbf{R}_{r} & =\operatorname{diag}\left[\begin{array}{llll}
r_{1}^{r} & r_{2}^{r} & \cdots & r_{l}^{r}
\end{array}\right] \\
\mathbf{R}_{b} & =\operatorname{diag}\left[\begin{array}{llll}
r_{1}^{b} & r_{2}^{b} & \cdots & r_{l}^{b}
\end{array}\right]
\end{aligned}
$$


Also, constant matrix $\mathbf{J}_{r} \in \mathbb{R}^{l \times l}$ is defined as

$$
\mathbf{J}_{r}=\left[\begin{array}{cccccc}
0 & 1 & 0 & \cdots & 0 & 0 \\
0 & 0 & 1 & \cdots & 0 & 0 \\
0 & 0 & 0 & \cdots & 0 & 0 \\
\vdots & \vdots & \vdots & \ddots & \vdots & \vdots \\
0 & 0 & 0 & \cdots & 0 & 1 \\
1 & 0 & 0 & \cdots & 0 & 0
\end{array}\right]
$$

Moreover, there are two current vectors in left hand side of Eq. (58) which have to find a relation between them. For this propose, Kirchhoff's Current Law $(K C L)$ can be used to determined a relation between rotor bars and ring segments currents. By applying $K C L$ to $j^{\text {th }}$ node (bar) in the rotor cage (Fig. 4), we can write:

$$
i_{j}^{b}=i_{j+1}^{r}-i_{j}^{r}
$$

Therefore, for $l$ nodes in the rotor cage; Eq. (63) can be expressed in matrix form as:

$$
\mathbf{i}_{b}=\mathbf{A}_{r y t} \mathbf{i}_{r}
$$

By substituting Eqs. (64) into (58), we can get:

$$
\frac{d}{d t} \Psi_{r t}=\left(-2 \mathbf{J}_{r} \mathbf{R}_{r}+\mathbf{A}_{r y t} \mathbf{R}_{b} \mathbf{A}_{r y t}\right) \mathbf{i}_{r}
$$

Up to this stage, two separate sets of non-linear equations are derived to established of algebraical (magnetic) and dynamicalal (electric) models of a squirrel cage induction machine. By combining of Eqs. (50), (51) and (65), the total algebria-differential equations of the system can be augmented as:

$$
\left[\begin{array}{cccccc}
w_{c} \mathbf{M}_{c f} \mathbf{A}_{s y t} p & 0 & 0 & 0 & \mathbf{R}_{s} & 0 \\
0 & \mathbf{A}_{r y t} p & 0 & 0 & 0 & 2 \mathbf{J}_{r} \mathbf{R}_{r}-\mathbf{A}_{r y t} \mathbf{R}_{b} \mathbf{A}_{r y t} \\
\mathbf{A}_{\phi s y}+\mathbf{A}_{\phi s t} \mathbf{A}_{s y t} & 0 & \mathbf{A}_{u s t} & 0 & -\mathbf{W}_{c} & 0 \\
0 & \mathbf{A}_{\phi r y}+\mathbf{A}_{\phi r t} \mathbf{A}_{r y t} & 0 & \mathbf{A}_{u r t} & 0 & \mathbf{A}_{r y t} \\
-\mathbf{A}_{s y t} & 0 & \mathbf{A}_{s s} & \mathbf{A}_{s r} & 0 & 0 \\
0 & -\mathbf{A}_{r y t} & \mathbf{A}_{r s} & \mathbf{A}_{r r} & 0 & 0
\end{array}\right]\left[\begin{array}{c}
\Psi_{s y} \\
\Psi_{r y} \\
\mathbf{U}_{s t} \\
\mathbf{U}_{r t} \\
\mathbf{i}_{s} \\
\mathbf{i}_{r}
\end{array}\right]=\left[\begin{array}{c}
\mathbf{V}_{s} \\
0 \\
0 \\
0 \\
0 \\
0
\end{array}\right]
$$

where $p$ denotes time derivative operator $\left(\frac{d}{d t}\right)$.

\subsection{Mechanical differential equations}

Some of the matrix coefficients in Eq.(66) are depended to mechanical instantaneous angle $\gamma$. In variable speed conditions, the mechanical variables of the system can be determined by solving differential equations of the rotor angel and speed. Generally, mechanical torque balance equation can be expressed as:

$$
J \frac{d \omega}{d t}=T_{e m}-T_{l}
$$




\begin{tabular}{lcc}
\hline \hline Quantity & Symbol & Value \\
\hline Power & $P$ & $1.1 \mathrm{kw}$ \\
Voltage & $V$ & $220 \mathrm{~V}$ \\
Frequency & $f$ & $50 \mathrm{~Hz}$ \\
Number of pole & $p$ & 2 \\
Stator resistance & $r_{s}$ & $5 \Omega$ \\
Rotor bar resistance & $r_{b}$ & $20 \mu \Omega$ \\
Rotor ring sector resistance & $r_{e}$ & $1.1 \mu \Omega$ \\
Inertia moment & $j$ & $0.02 \mathrm{kgm}^{2}$ \\
Number of turns per slot & & 68 \\
Number of rotor slots & & 18 \\
\hline \hline
\end{tabular}

Table 1. Parameters of squirrel cage induction machine

in which

$$
\frac{d \gamma}{d t}=\omega
$$

also, $\omega, J, T_{l}$ and $T_{e m}$ are the rotor angular speed, total inertia on the shaft, load torque and electromagnetic torque, respectively. The electromagnetic torque is depended to $\mathrm{mmf}$ along air gap and derivative of air gap permeances with respect to rotor angel $(\gamma)$ (Ostovic, 1989). For an induction machines, we can express as:

$$
T_{e m}=\sum_{i=1}^{h} \sum_{j=1}^{l}\left(u_{i}^{s t}-u_{j}^{r t}\right)^{2} \frac{d G_{i, j}(\gamma)}{d \gamma}
$$

By substuting Eqs.(7), (10) and (11) into (69), air gap electromagnetic torque can be obtained as:

$$
T_{e m}=\left(\mathbf{U}_{s t}^{T}-\mathbf{U}_{r t}^{T}\right) \frac{d}{d \gamma} \mathbf{A}_{\mathbf{s r}}\left(\mathbf{U}_{s t}-\mathbf{U}_{r t}\right)
$$

Therefore, the mechanical non-linear differential equations (67) to (70), with the magnetic non-linear differential-algebraic equations (66), describe the generalized non-linear dynamic model of squirrel cage induction machine. Because of non-linearity of the model, the advantage numerical solution methods must be used. In the next section, some simulation results are presented for demonstration of capability validation of the new model. After that, asymmetrical situations of squirrel cage induction machine such as broken rotor bar fault with saturation effects are analysed and evaluated.

\section{Simulations and experiment of results}

Table 1 shows the electrical parameters of the squirrel cage induction machine which is used to obtain numerical simulation and experimental results. Air gap, rotor and stator slots and other main dimensions of the induction machines are presented in Fig. (5) and windings connection diagram are assumed similar to Fig. (4). In the next section, some different dynamical conditions are implemented to obtain numerical results of the non-linear model of induction machines. 

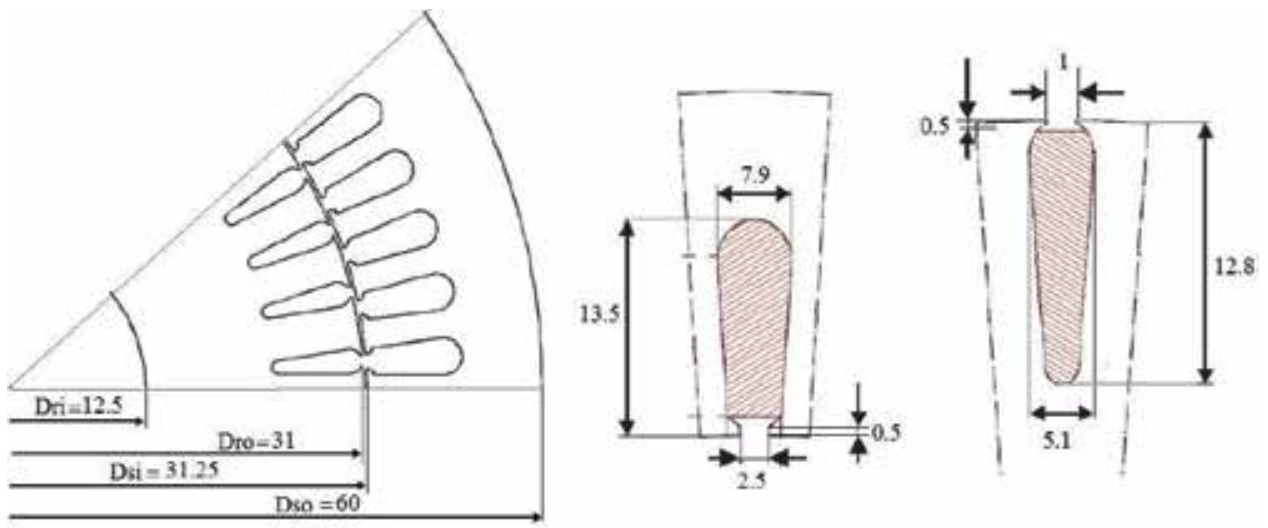

Fig. 6. Basic construction and main diameters and stator and rotor slot shapes

\subsection{Simulation in healthy condition}

For simulation study, the healthy conditions of a squirrel cage induction machine with saturable iron core are given. At first, free starting-up is considered and at $t=0.3 \mathrm{sec}$, a mechanical load $\left(T_{l}=10 \mathrm{Nm}\right)$ is applied to the rotor shaft. The results of the simulations are shown in Figs. 7 and 8. Speed-torque transient acceleration of the system is presented in Fig. 7-a, that rotor speed reaches to steady state value at about $t=0.2 \mathrm{sec}$. Also, transient electromagnetic torque are shown in Fig. 7-a where low order harmonics are appeared in the electromagnetic torque on the transient duration. This is a common behaviour in the induction machines, which can be obtained from classical method such as two axes theory. But the high order harmonics in transient and steady state are also appeared in the results. At $t=0.3 \mathrm{sec}$ load torque changes form zero to $T_{l}=10 \mathrm{~N} . \mathrm{m}$, electromagnetic torque increase by decreasing of the rotor speed. Fig. 7-b shows dynamic of three phase stator currents which decrease by increasing the rotor speed. Other dynamic performance for flux and rotor bar currents are shown in Fig. 8. The results show that, high order harmonics are observed in the rotor and stator teeth fluxes and currents. The rotor teeth and slots are moved opposite the stator teeth and slots, thus radial fluxes will have variable permeances in the air gap. This is caused a slot harmonics appears in the the all variables of the system. Fig 8-a illustrates that, slot harmonics deform the stator teeth flux in the transient and steady state conditions. Because of the machine is symmetric, all variables of machine such as the stator currents and stator teeth flux are balanced.

\subsection{Simulation in faulty condition}

In this simulation, analysis of the induction machine with a broken bar is done to determined the steady state performances of the machine. Fig. 9-a shows the steady state current of the stator winding with linear and non-linear core characteristics. The rotor speed and torque are $307.7 \mathrm{rad} / \mathrm{sec}$ and $10 \mathrm{~N} . \mathrm{m}$, respectively. Similar to the last conditions, slot harmonics are appeared on the stator currents in the both causes (linear and non-linear magnetic core). However, stator current has low order harmonics with the non-linear iron core. Stator teeth fluxes in two cases are approximately equal. Also, Fig. 9-c presents that the bar currents near to the broken bar are strongly changed. But attention to Fig 9-d, this situation is not appeared in induction machine with non-linear magnetic core. Therefore, disturbances of rotor broken bar in the saturated machines cannot effectively transfer to the stator currents. 


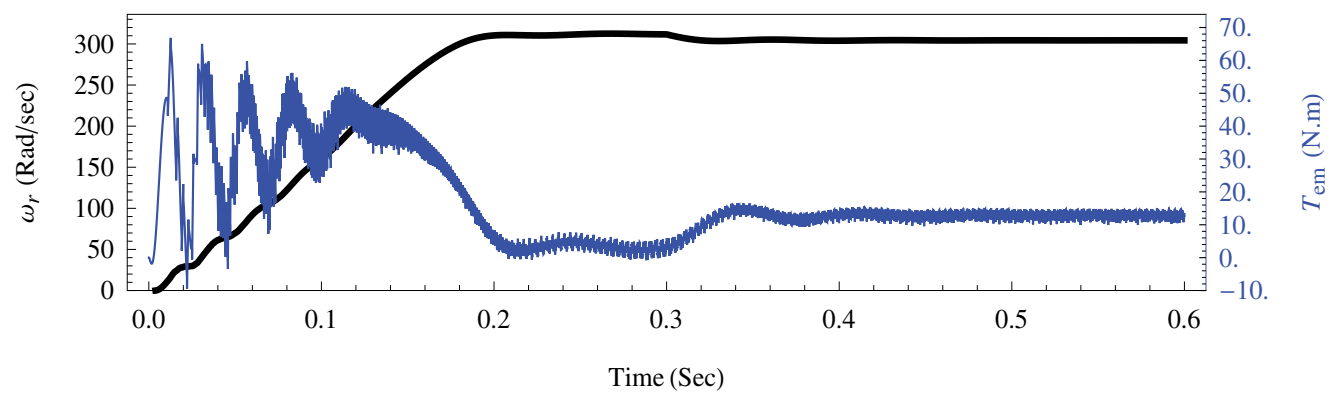

(a) Torque-speed dynamic

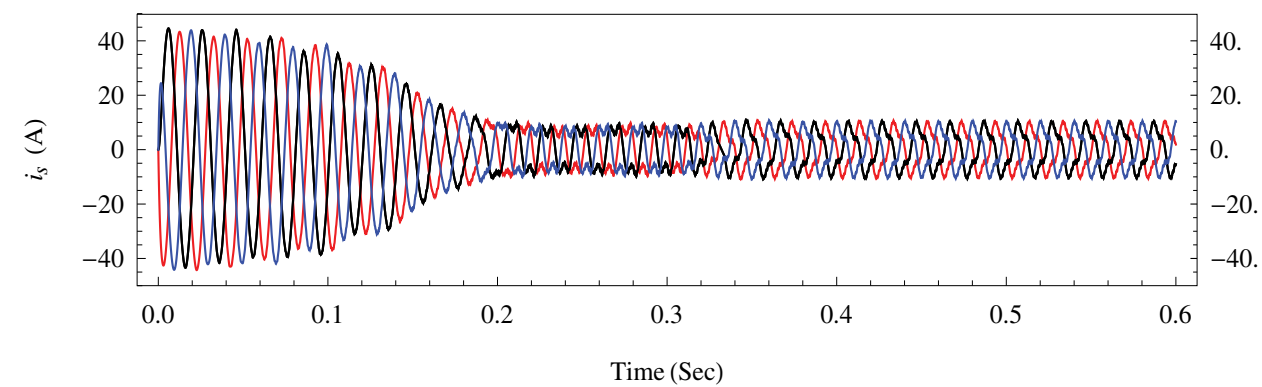

(b) Stator currents

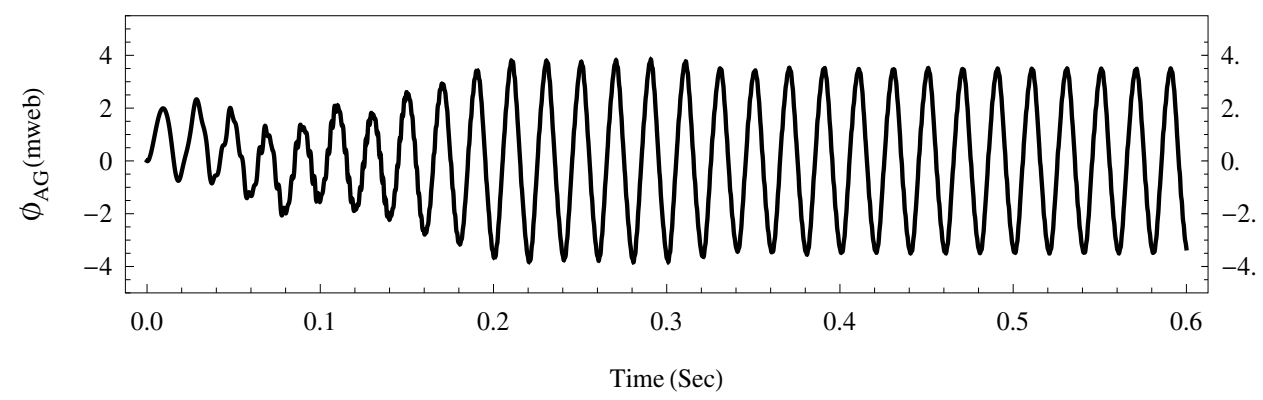

(c) Air gap flux

Fig. 7. Starting-up transient of induction machine

\section{Conclusion}

In this chapter, a generalized non-linear dynamical model of the squirrel cage induction machines was presented. In this modelling, magnetic saturation effects in iron core, space harmonic distributed of fluxes in the rotor and stator teeth, the stator windings and rotor bar distribution were considered. Some simulations results showed that, presented model has high accuracy and efficiency for asymmetrical analysis such as broken bar conditions. The effects of broken bar will not clearly appear when the machine core is saturated. 


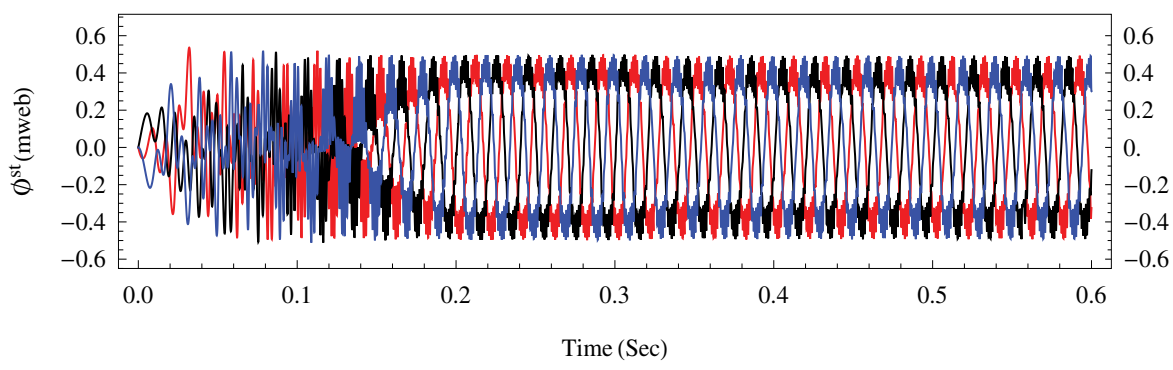

(a) Stator teeth Fluxes

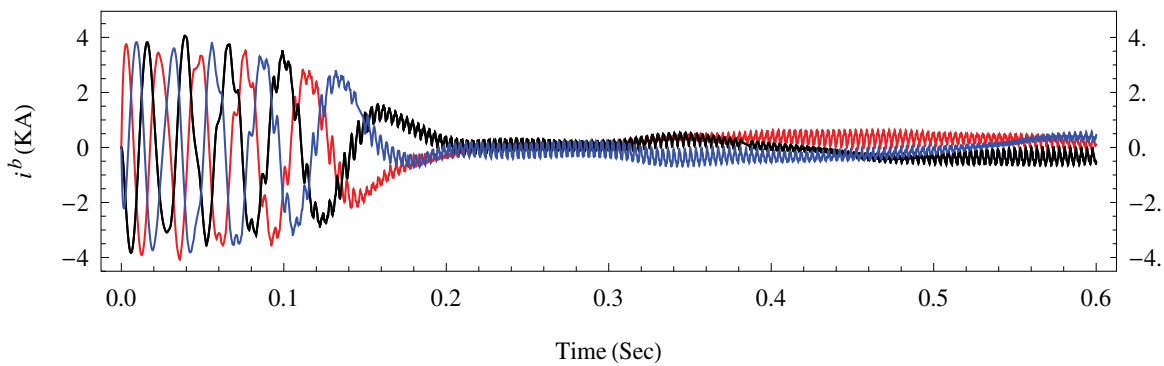

(b) Rotor bar currents

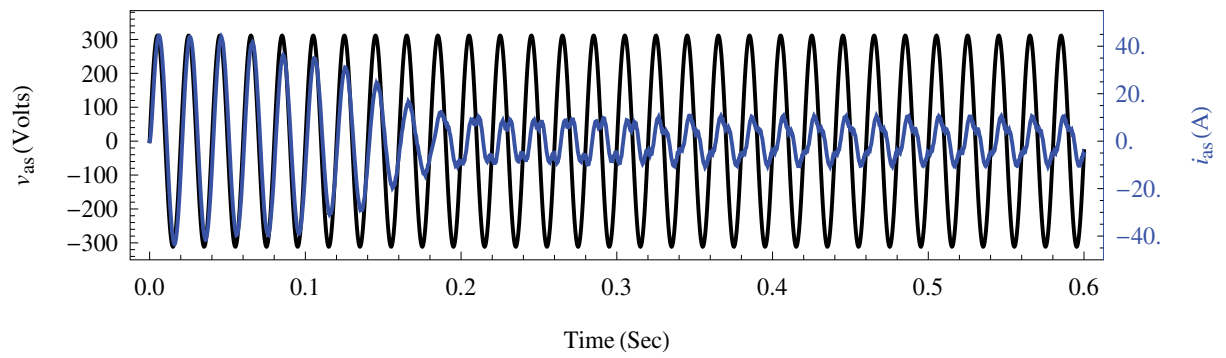

(c) Stator voltage and current

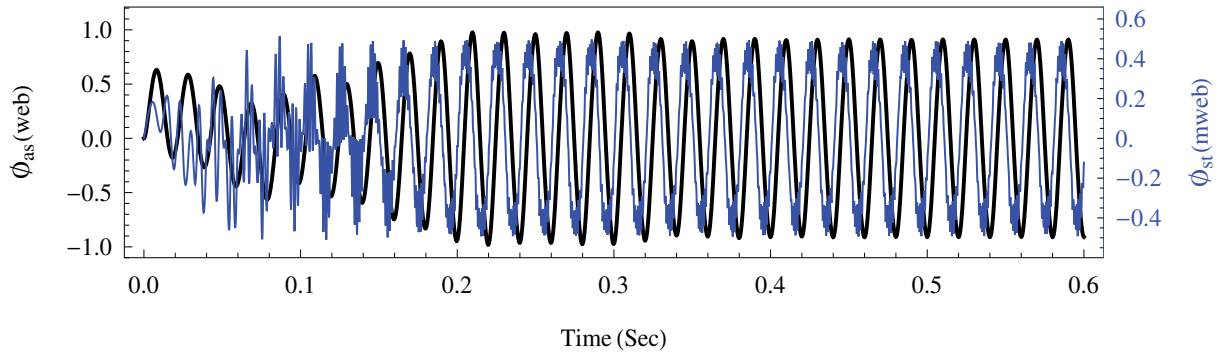

(d) Tooth and phase fluxes

Fig. 8. Starting-up transient of induction machine 


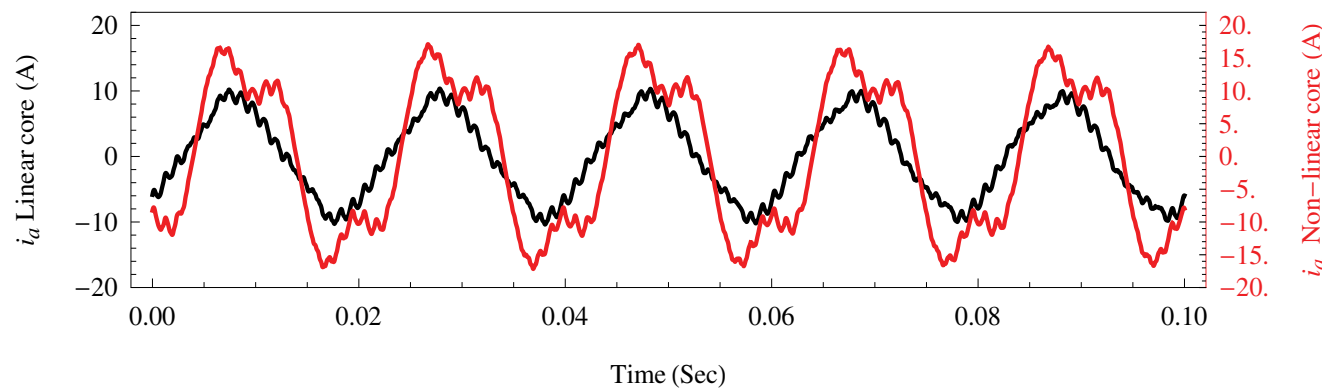

(a) Stator current

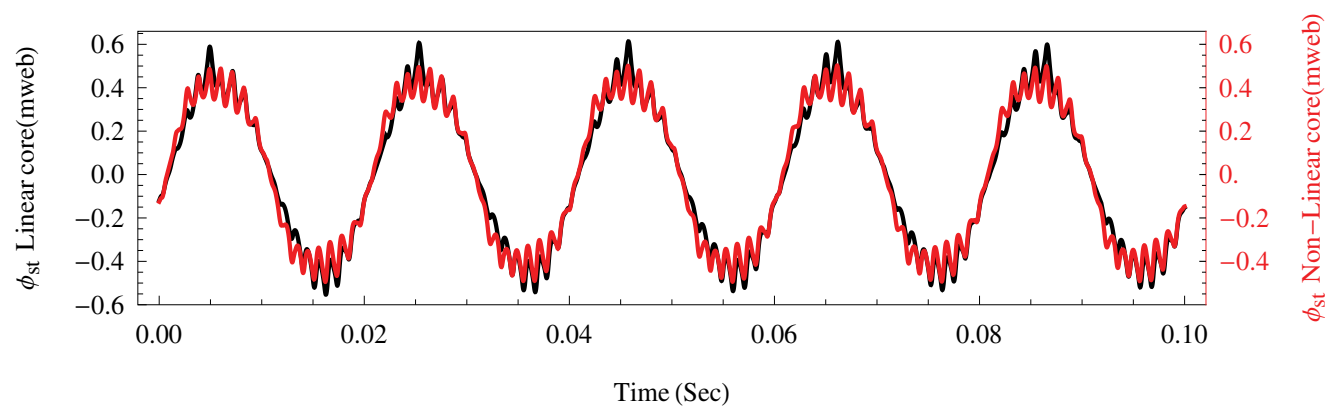

(b) Stator flux

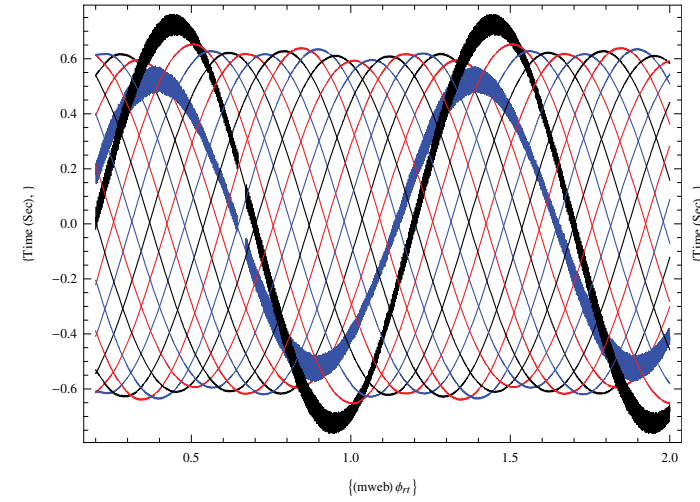

(c) Rotor fluxes with linear core

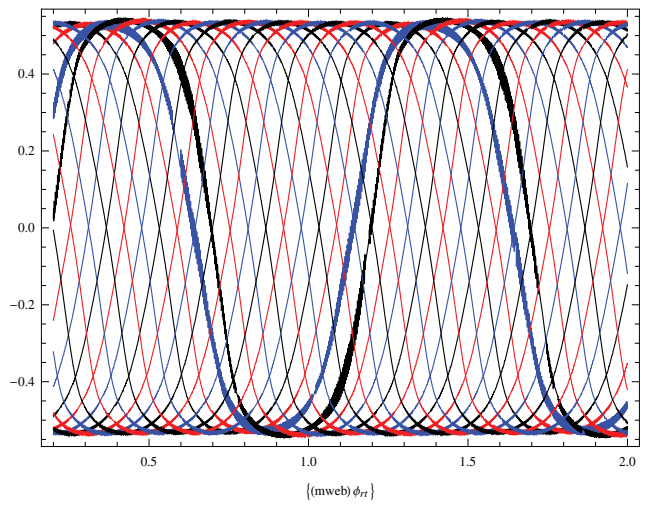

(d) Rotor fluxes with non-linear core

Fig. 9. Steady state fluxes and currents of faulty induction machine with a broken bar for linear and non-linear core

\section{References}

Alemi, P. \& Nazarzadeh, J. (2006). The Induction machines modeling based on bars current for rotor fault analysis, $21^{\text {th }}$ International Power System Conference, PSC 2006, 477-483, Niroo Research Institute, 13-15 Nov. 2006, Tehran, Iran 
Al-Shahrani, A. S. (2005). Influence of adjustable speed drive on induction motor fault detection using stator current monitoring. Ph.D. thesis, Oregon State University

Bellini, A.; Filippetti, F.; Franceschini, G.; Tassoni, C. \& Kliman G. B. (2001). Quantitative evaluation of induction motor broken bars by means of electrical signature analysis. IEEE Transactions on Industry Applications, Vol. 37, No. 5, (Sep./Oct. 2001), 1248-1255

Benbouzid, M. E. H. (2000). A review of induction motors signature analysis as a medium for faults detection.IEEE Transactions on Industry Electronics, Vol. 47, No. 5, (Oct. 2000), 984-993

Chen, S. D.; Lin, R. L \& Cheng, C. K.(2005). Magnetizing inrush model of transformers based on structure parameters.IEEE Transactions on Industry Electronics, Vol. 20, No. 3, (July 2005), 1947-1954

Faiz, J.; Sharifian, M. B. B.; Feyzi, M. R.; \& Shaarbafi, K. (2002). A complete lumped equivalent circuit of three-phase squirrel-cage induction motors using two-dimensional finite-elements technique. IEEE Transactions on Energy Conversion, Vol. 17, No. 3, (Spet. 2002), 363-367

Gautschi W. (1964). Error function and Fresnel integrals, Handbook of Mathematical Functions, NBS Appl. Math. Series, Vol. 55, U.S. Government Printing Office, Washington, D.C.

Jeong, J. H.; Lee E. W. \& Cho, H. K. (2003). Analysis of transient state of the squirrel cage induction motor by using magnetic equivalent circuit method, Sixth International Conference on Electrical Machines and Systems, Vol. 2, 720-723, 2003

Jung, J. H.; Lee, J. J. \& Kwon, B. H. (2006). Online diagnosis of induction motors using MCSA. IEEE Transactions on Industry Electronics, Vol. 53, No. 6, (Dec 2006), 1842-1852

Krause, P. C.; Wasynczuk, O. \& Sudhoff, S. D. (2002). Analysis of Electric Machinery and Drive Systems (2nd Edition), Wiley-IEEE Press, ISBN 047114326X, New York.

Luos, X.; Liao, Y.; Toliyaty, H.; El-Antably, A. \& Lipos, T. A. (1995). Multiple coupled circuit modeling of induction machines. IEEE Transactions on Industry Applications, Vol. 31, No.2, (Mar./Apr. 1995), 311-318

Mirafzal, B.\& Demerdash, N. A. O. (2004). Induction machine broken-bar fault diagnosis using the rotor magnetic field space-vector orientation. IEEE Transactions on Industry Applications, Vol. 40, No. 2, (Mar./Apr. 2004), 534-542

Mirafzal, B.\& Demerdash, N. A. O. (2008). Induction machine broken bar and stator short-circuit fault diagnostics based on three-phase stator current envelopes. IEEE Transactions on Industry Applications, Vol. 55, No. 3, (March 2008), 1310-1318

Muñoz, A. R. \& Lipo, T. A. (1999). Complex vector model of the squirrel-cage induction machine including instantaneous rotor bar currents. IEEE Transactions on Industry Applications, Vol. 35, No. 6, (Nov./Dec. 1999), 1332-1340

Ostovic, V. (1989). A novel method for evaluation of transient states in saturated electric machine. IEEE Transactions on Industry Applications, Vol. 25, No. 1, (Feb. 1989), 96-1000

Ostovic, V. (1989). Dynamics of Saturated Machines, Springer-Verlag, ISBN 0387970797, New York

Siddique, A.; Yadava, G. S. \& Singh, B. (2005). A review of stator fault monitoring techniques of induction motors. IEEE Transactions on Energy Conversion, Vol. 20, No. 1, (March 2005), 106-114

Sprooten, J. (2007). Finite element and electrical circuit modeling of faulty induction machines study of internal effects and fault detection techniques. Ph.D. thesis, Department of Bio, Electro and Mechanical Systems (BEAMS), University Libre de Bruxelles 
$\mathrm{Su}, \mathrm{H}$. \& Chong, K. T. (2007). Induction machine condition monitoring using neural network modelling. IEEE Transactions on Industry Applications, Vol. 54, No. 1, (Feb. 2007), 241-249 


\title{
Minimization of Losses in Converter- Fed Induction Motors - Optimal Flux Solution
}

\author{
Waldiberto de Lima Pires, Hugo Gustavo Gomez Mello, \\ Sebastião Lauro Nau and Alexandre Postól Sobrinho \\ WEG Equipamentos Eletricos S.A. - Motores \\ Research and Development of Product Department \\ Av. Pref. Waldemar Grubba, 3000 - malote 41 \\ Jaraguá do Sul, SC - 89256-900 \\ Brazil
}

\section{Introduction}

When a TEFC induction motor fed by static frequency converter drives a load which demands constant torque throughout the operation range, the low speeds are thermally critical, because the motor losses (heat sources) do not vary much as a function of the speed, but the ventilation efficiency decreases as the operation speed falls down, since the fan is installed on the very motor shaft. In such cases, when operating at low speeds, the temperature rise often exceeds the limits of the motor thermal class due to the lack of cooling. In order to prevent this problem the industry has traditionally adopted one of the following solutions: independent ventilation (a small auxiliary motor is used to exclusively drive the fan that provides the main motor cooling) or oversizing (the motor used in the application provides a higher torque than the rated load demand). However, neither one nor the other of these two options are attractive, as both, besides increasing the space required for the installation, still increase the motor price [1].

Frequency converters usually apply to the motor a constant voltage/frequency ratio throughout the operation range, so that no loss control is provided. But the study on the motor losses composition and its relation with voltage, frequency, magnetic flux and current, allied with the study on the influence of the ventilation on the temperature rise of the motor, has led to an optimal voltage/frequency ratio, which minimizes the total motor losses at each speed [2]. This way, by implementing the automatic control of the voltage/frequency ratio in the converter, the motor loss minimization can be automatically obtained throughout the frequency range, so that the motor temperature rise is kept within the thermal class limits even at low speeds with reduced ventilation.

\section{Determination of losses}

The fast growth of the number of industrial applications using static frequency converters recently observed in variable speed drives has encouraged the meticulous study of losses in magnetic materials under PWM supply by several researchers $[3,4,5,6,7,8]$. They have 
shown that such losses depend on a number of control parameters, such as the modulation index, the number of levels and pulses of the frequency converter and the duration of PWM signal pulses. On the other hand, Boglietti et alli have concluded that the flux waveforms resulting from PWM supply differ from those resulting from sinusoidal supply just except for a small ripple, which depends on the switching frequency, and that above approximately $5 \mathrm{kHz}$ the iron losses can be considered independent of this parameter [9]. Such studies represent the first step towards the understanding of the losses behavior in electric motors under PWM supply, that involves a higher degree of complexity and is not restricted exclusively to the magnetic materials issue, but includes also additional losses in the conductors and due to the cooling system and depends, besides the control parameters, on some machine design parameters, such as the flux density, the lamination geometry and the connection of windings $[3,10,11,12]$, as well as on other variables inherent to the manufacturing process [13].

For the purposes of this study, however, the analysis of the motor losses can be simplified, so that it is enough to separate the total motor losses in three key components:

$$
P=P f e+P j+P m e c
$$

where:

$P f e$ - Iron losses, which depend on the flux density (or magnetic induction), the frequency and the quality of the magnetic material.

$P j$ - Losses by Joule effect, which depend on the currents flowing through the stator windings and the rotor bars.

Pmec - Mechanical losses due to cooling system (fan coupled to the shaft) and friction, which depend on the speed.

The iron losses are classically considered as being composed of two portions: Hysteresis losses $(\mathrm{pH})$ and induced eddy current (Foucault) losses $(\mathrm{pF})$. For a lamination sample tested in Epstein Frame with sinusoidal supply, the hysteresis losses are directly proportional to the frequency $(f)$ and to the square of the magnetic induction $\left(B^{2}\right)$, while the eddy current losses are proportional to the square of both the frequency $\left(f^{2}\right)$ and the magnetic induction $\left(B^{2}\right)$, especially for induction values above one Tesla $(1 \mathrm{~T})$. However, in the induction motor the iron losses present a much more induction-dependent behavior than the quadratic ratio obtained with normalized samples of the magnetic material in Epstein Frame tests. In low-voltage threephase induction motors manufactured with fully processed steel laminations tested under different saturation levels, iron losses presented a dependance on the induction close to $B^{4}$ for inductions above 1,2 T (usual value for industrial motors), as presented in Fig. 1.

The fundamental theory of the electric machines shows that the torque provided by the induction motor is directly proportional to the product between the magnetic flux and the electric current $[14,15]$. Then in order to keep a constant torque, if the flux increases the current can decrease (and vice-versa). As the Joule losses are directly proportional to the square of the current, these losses can be considered as inversely proportional to the square of the magnetic flux. From the Faraday-Lenz law of induction, one can easily demonstrate that the magnetic flux in the motor is directly proportional to the ratio between the electromotive force $(E)$ and the frequency. Considering the steady-state model of equivalent circuit of the induction motor per-phase (Fig. 2), it can be noted that at the base frequency the voltage drop in the primary impedance has little significance, so that the flux can be considered as proportional to the $V 1 / f$ (voltage/frequency) ratio. 


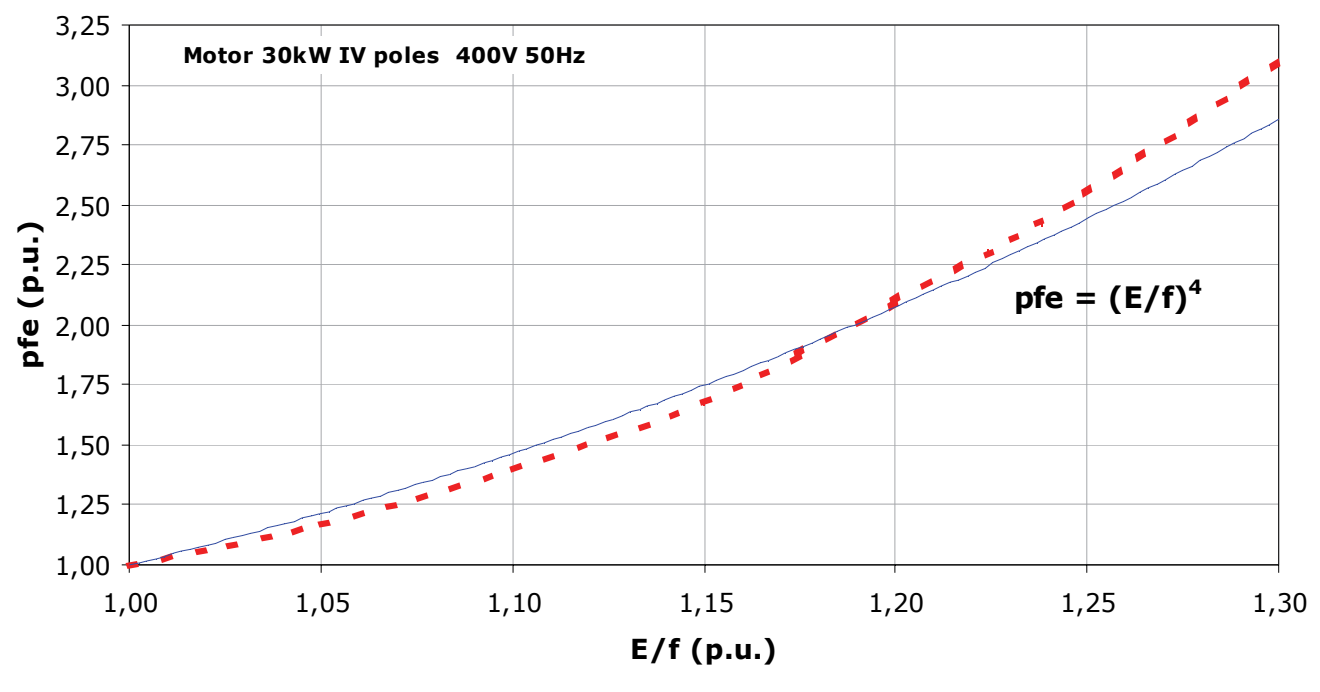

Fig. 1. Iron losses $x$ Magnetic induction for an industrial three-phase induction motor

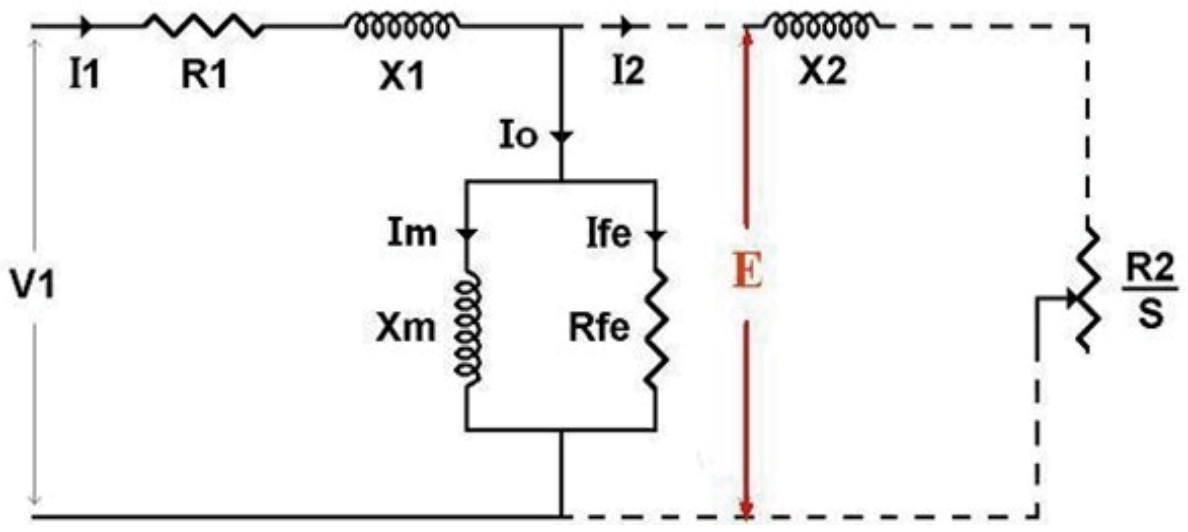

Fig. 2. Steady state equivalent circuit of the induction motor per-phase

For low operating frequencies, however, in which the input voltage is reduced, the voltage drop in the primary resistance becomes important and can be no longer despised. By neglecting the influence of the primary reactance, electromotive force $\mathrm{E}$ is given by

$$
E=V-R_{1} l_{1}=V-A V
$$

The voltage drop at the stator branch $(\Delta V)$ then depends directly on the stator current (I1). As Fig. 2 shows, the motor current can be decomposed into two components: one concerning magnetization and the other concerning torque production.

$$
t_{1}=\sqrt{t_{0}^{2}+t_{2}^{2}}
$$

Taking rated voltage as the base, $E / f$ ratio per unit can be written as: 


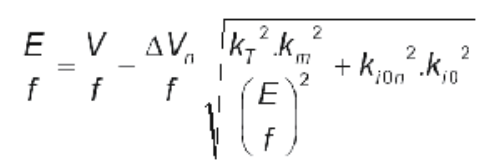

where:

$\Delta V n$ - Voltage drop per unit with rated frequency and load.

$f$ - Motor operating frequency per unit, considering rated frequency $f n$ as the base.

In (4), the square root results in a correction factor, which is function of the motor current and whose terms are explained in the following paragraphs.

It should be taken into account that, as the frequency (and consequently, the rotation) is reduced, the mechanical losses decrease in a nearly cubic proportion to it $\left(f^{3}\right)$. The mechanical losses do not affect the iron losses, but they act as an additional load to the motor, therefore they must be considered as a torque to be added to the rated torque available on the shaft. Its reduction implies current reduction and so reduction of Joule losses in the conductors $(P j)$.

Thus it is possible to rewrite the induction motor total losses per unit $p$, for operation with variable voltage and frequency, as follows:

$$
\rho=p_{i n}\left[\frac{k_{i}{ }^{2} \cdot k_{n i}{ }^{2}}{\left(\frac{E}{f}\right)^{2}}+k_{i 0 n}{ }^{2} \cdot k_{i 0}{ }^{2}\right]+p_{r i n}\left(\frac{E}{f}\right)^{4} f+p_{F a}\left(\frac{E}{f}\right)^{4} f^{2}
$$

where:

pin - Total Joule losses with the motor operating at rated conditions of load, voltage and frequency.

$\mathrm{pHn}$ - Total hysteresis losses with the motor operating at rated conditions of load, voltage and frequency.

$p F n$ - Total eddy current losses with the motor operating at rated conditions of load, voltage and frequency.

Motors manufactured with low loss magnetic core (fully processed silicon steel) operating at rated conditions typically present values of $80 \%, 12 \%$ and $8 \%$, for parameters $\mathrm{pin}, \mathrm{pH} n$ and $p F n$, respectively. The remaining parameters of (5) will be opportunely explained ahead.

The term of (5) in brackets refers to the motor Joule losses and depends on the total motor current. The second and third terms refer to the motor iron losses for hysteresis and eddy currents, respectively. The magnetic induction was conveniently replaced by the $E / f$ ratio. There is no explicit term for the motor total mechanical losses in the equation, because they are embedded in the first term, in accordance with what was mentioned before, by means of the factor $k m$ defined below:

$$
k_{m}=\left(\frac{1+p_{m n} f^{3}}{1+p_{m n}}\right)
$$

where $p m n$ is the mechanical losses at rated speed referred to the rated output power $P n$.

The aim of this study is to minimize the motor losses, in order to reduce its temperature rise, so that the need of both the torque reduction (oversizing) and the use of independent ventilation can be prevented. In (5), this is considered by means of the derating factor $k T$, 
which will be addressed later on this paper. It should be noted that the torque affects only the current-dependent losses, not influencing the iron losses. Therefore, $\mathrm{km}$ and $\mathrm{kT}$ are torque correction factors required to compensate for the effects of the speed variation, which influences the portion of losses related to the load current.

KiOn is the no-load current factor, defined by (7).

$$
k_{\text {ion }}=\frac{t_{3}}{t_{n}}
$$

where:

Io - No-load current under rated voltage and frequency.

In - Full-load current under rated voltage and frequency.

Due to the non-linearity of the magnetization curve of the laminations, the $E / f$ ratio increase causes the no-load current to increase according to (8). This peculiar behavior of the no-load current was observed experimentally (Fig. 3), and is taken into account in (5) by means of the factor ki0.

$$
\begin{aligned}
& k_{i 0}=\left(\frac{E}{f}\right)^{3,4} \text { para } \frac{E}{f} \geq 1 \\
& k_{i 0}=\left(\frac{E}{f}\right) \text { para } \frac{E}{f}<1
\end{aligned}
$$

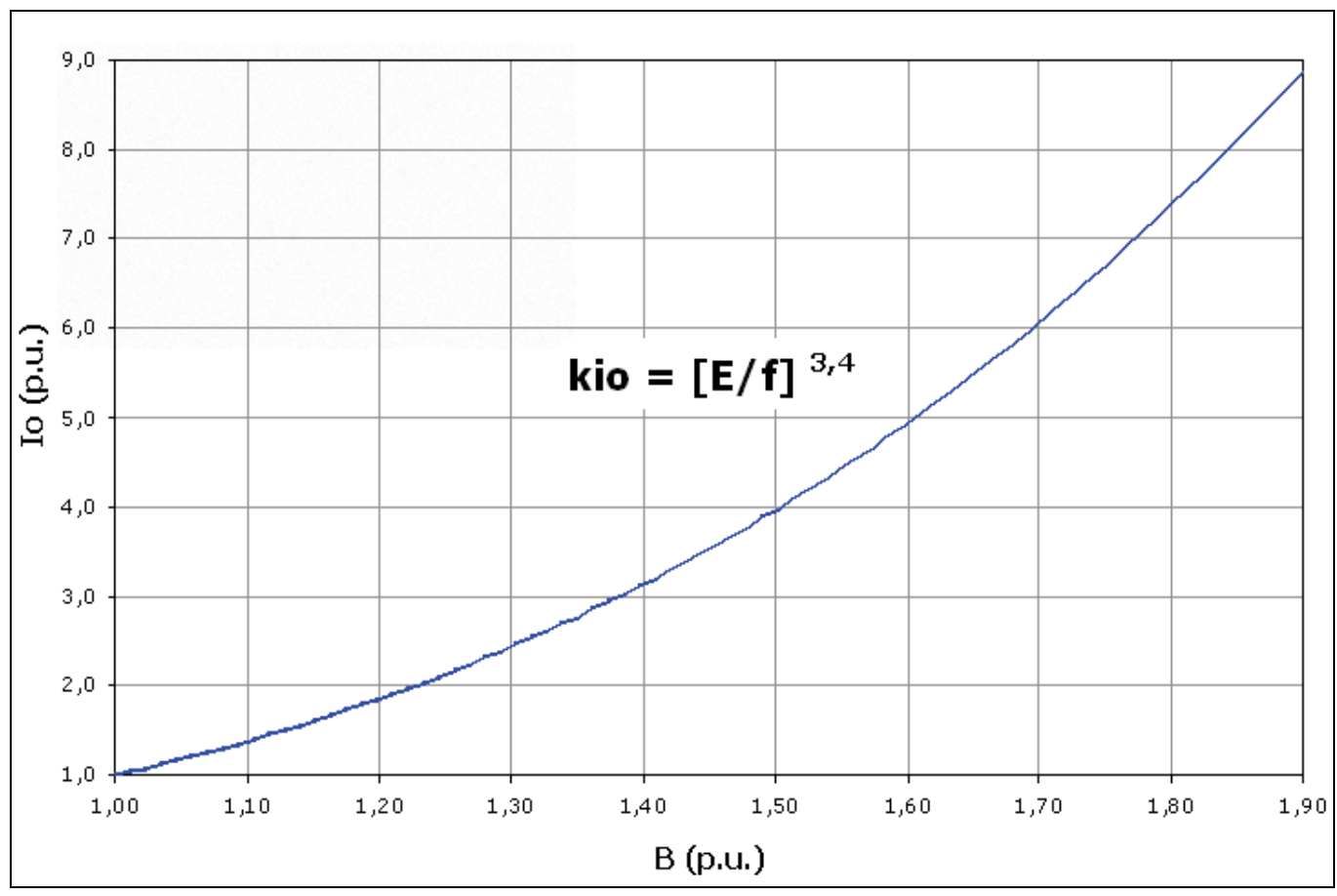

Fig. 3. Magnetizing current x E/f 


\section{Minimization of losses}

The analysis of (5) shows that the induction motor global losses depend on both the operation frequency and the induction (or magnetic flux). Then the values of $V / f$ that minimize the motor global losses change with the operation frequency, so that it is necessary to find the minimum losses at each frequency, with different values of $V / f$. Fig. 4 shows the total losses calculated as function of the frequency for various values of the $V / f$ ratio.

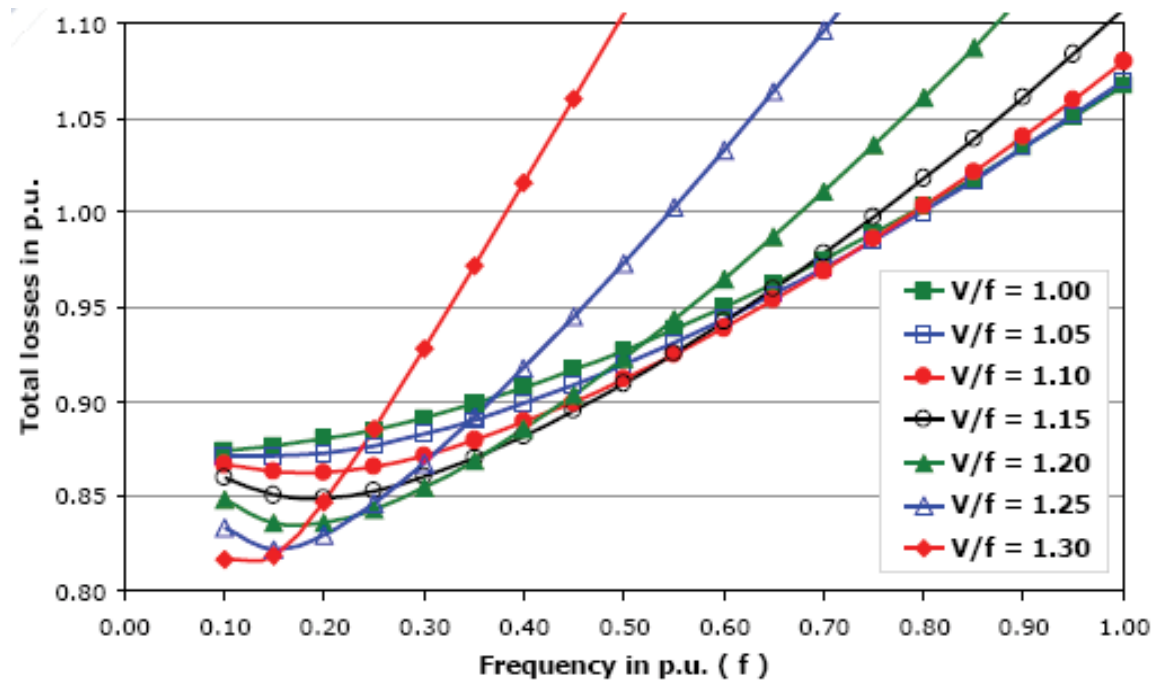

Fig. 4. Total losses $x$ frequency curve for several $\mathrm{V} / \mathrm{f}$ ratios at rated torque

Fig. 5 derives from the family of curves above and represents the $\mathrm{V} / \mathrm{f}$ ratios theoretically obtained, which minimize the total losses of the motor at each operation frequency.

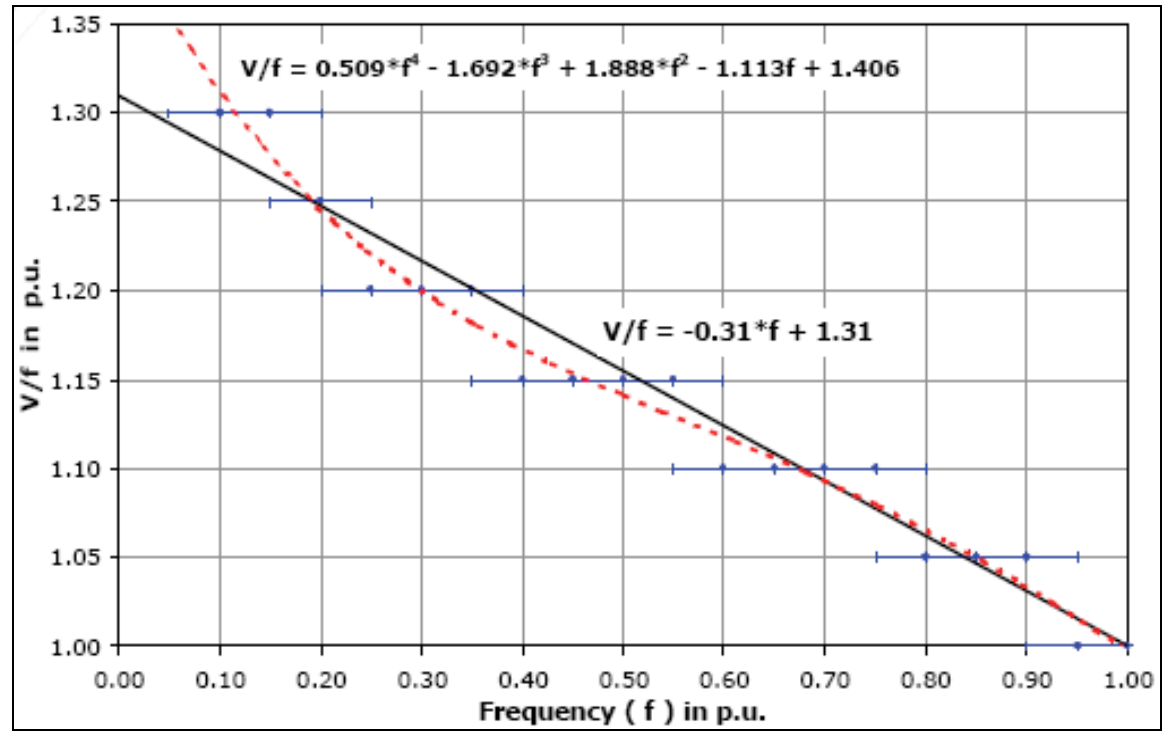

Fig. 5. V/f $x$ frequency curve for minimization of total losses 


\section{Influence of ventilation reduction}

Thermal calculations implemented for a number of motors of distinct frame sizes and power ratings considering speed variation, combined with experiments and tests performed with several motors at rated load, varying separately the fan speed from zero to base speed, led to the conclusion that TEFC three-phase motors of a wide output range present a similar thermal behavior. Fig. 6 represents the temperature rise per unit of low-voltage 4-pole cage induction motors manufactured with die cast iron frame as a function of the fan speed per unit.

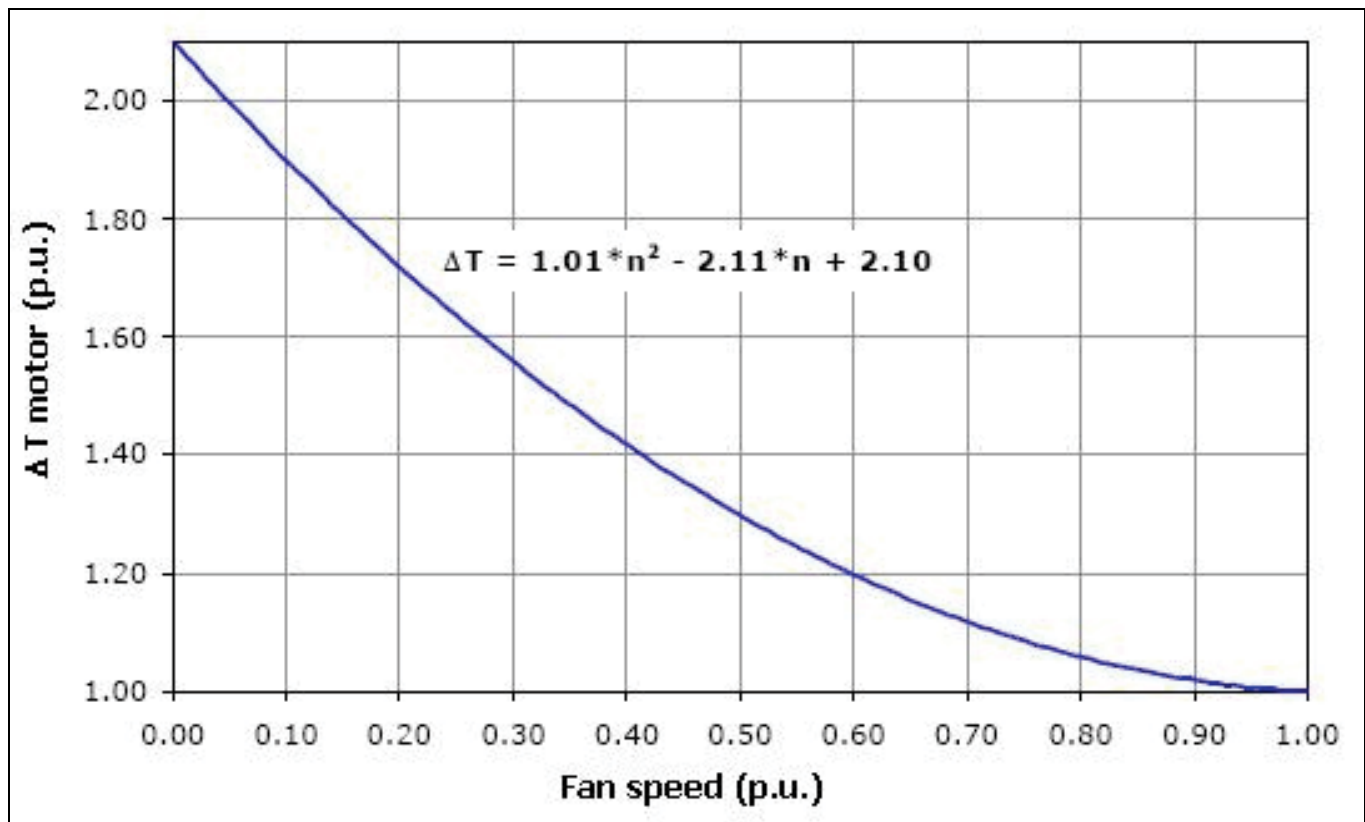

Fig. 6. Temperature rise $x$ fan speed at rated load

In this approach, for each desired value of frequency and for each value of $V / f$ ratio according to Fig. 5, the total losses $p$ are calculated according to (5). Fig. 6 determines the influence of the ventilation reduction on the motor temperature rise ( $\Delta$ Tmotor). So in order for the required motor temperature rise to be assured, it is necessary to calculate a new value of $p$, henceforth referred to as $p^{\prime}$, according to (9).

$$
p^{\prime}=\left(\begin{array}{c}
\Delta T \\
\Delta T_{n: x i s}
\end{array}\right) p_{n}
$$

where:

$p^{\prime}$ - total motor losses for the required temperature rise, considering the ventilation reduction.

$p n$ - total motor losses at rated conditions.

If, for instance, the maximum required temperature rise is the limit of the insulation system thermal class, then $\Delta T=\Delta$ Tclass. If, otherwise, the maximum required temperature rise is $\Delta T n$, then $\Delta T=1$. 
Once known the total losses $p^{\prime}$ that will cause the required temperature rise in the motor with reduced ventilation, then it is possible to calculate the convenient derating factor using (10):

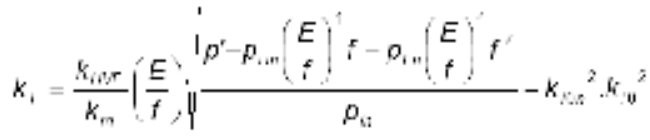

where $k H V F$ is the harmonic voltage factor as defined by NEMA [16]. It was placed in the equation originally conceived as (5) for the influence of the PWM supply voltage harmonics to be also considered on the motor temperature rise. For most of the modern static frequency converters $k H V F$ is 0.95 .

As a consequence of the cooling reduction, $k T$ is usually lower than 1 . However, if the minimized total losses are such that reduce the motor temperature rise even with poor ventilation, $k T$ can be higher than 1 . Similarly, if $k T$ is calculated for an insulation class temperature rise, it will be normally higher than 1 if the rated motor temperature rise is much below the insulation class temperature rise limit.

Fig. 7 presents an example of loss reduction achieved with the proposed method. A threephase, $30 \mathrm{~kW}$, 4-pole induction motor was tested at constant rated torque within the frequency range from 0.1 to 1.0 (p.u.). The results are presented for three different situations: calculation with constant flux, calculation with optimal flux and testing with optimal flux.

The loss reduction obtained with the proposed technique, as shown in Fig. 7, results in a better thermal performance of the motors operating with optimal flux. Comparing the motor temperature rises when operating at constant flux condition to those when operating at optimal flux condition, it is remarkable a behavior similar to that outlined in Fig. 8, as can be checked in the experimental results presented in section VI.

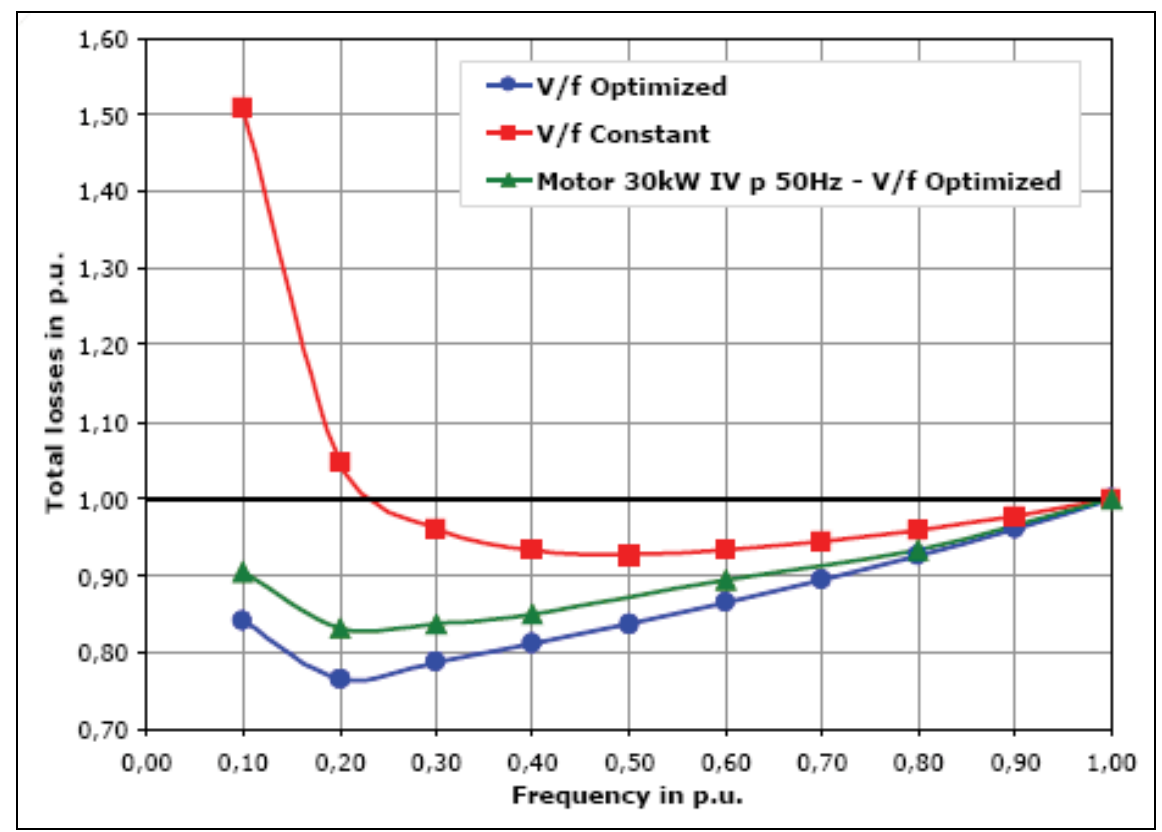

Fig. 7. Total losses (p.u.) $x$ frequency (p.u.) 


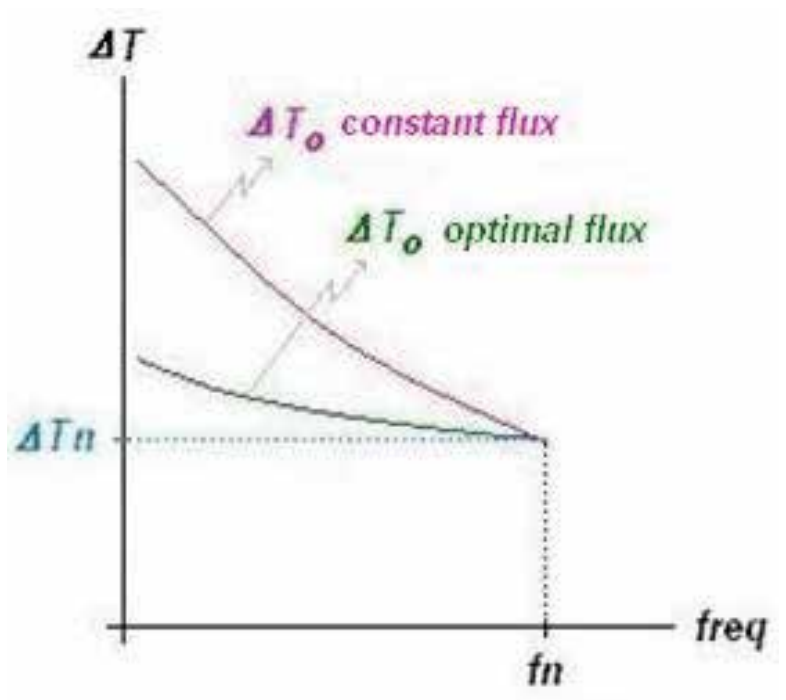

$\Delta \mathrm{T}_{\mathrm{n}}$ : temperature of thermal stabilization of the motor fed by sinusoidal supply (mains) $\Delta \mathbf{T}_{0}$ : temperature of thermal stabilization of the motor fed by converter supply fn: operation base frequency of the motor

Fig. 8. Temperature rise $\mathrm{x}$ operation frequency, sketch of the motor performance under different flux conditions

\section{Validation and implementation of the optimal flux curve}

To validate the proposed technique, temperature rise tests with speed/ventilation variation were accomplished for a wide range of industrial motor ratings. This way, it was possible to compare the thermal performances of converter-fed motors when under constant flux (rated losses) and optimal flux (minimum losses) conditions.

Before the implementation of the automatic function for optimal flux selection by the converter, drives with suitably modified softwares were used, so that specific flux values could be manually adjusted. This allowed the practical correction of the curve obtained by means of mathematical calculations and the finding of the actual optimal flux curve that was implemented in the converters used in the tests, into which were incorporated the automatic function for the optimal flux setting.

As the sensorless vector control enables the magnetic flux of the motor to be directly altered, this was the control type employed in all tests with converter. The switching frequency used in all tests with converter was $2.5 \mathrm{kHz}$.

\section{A. NEMA High Efficiency (NHE) Motors}

The motors to be tested were selected considering the worst horsepower/frame size ratios criteria. The following machines were used in the tests, all of them 4-pole (predominant polarity in low-voltage industrial applications) and all of them with class F insulation: $5 \mathrm{hp}$ (NEMA 184T); $20 \mathrm{hp}$ (NEMA 256T); $50 \mathrm{hp}$ (NEMA 326T) and $150 \mathrm{hp} \mathrm{(NEMA} \mathrm{444T).}$ Occasionally, for investigation of specific issues, tests were also realized with motors that are not related above. 
It should be noted that the loss minimization technique was conceived and developed specifically for low operation frequencies (below 0.5 p.u.). Thus, at base (rated) frequency, in this case $60 \mathrm{~Hz}$, the flux value for the minimum losses condition is the rated flux. The tests were conducted with full load being continuously applied until the motor thermal stabilization.

\section{B. NEMA Premium Efficiency (NPE) Motors}

Considering that NEMA Premium Efficiency motors tend to present lower temperature rises than NEMA High Efficiency motors, few NPE motors were tested just for the effectiveness of the proposed loss minimization technique to be corroborated with such motors. Despite that tests were conducted with 2- and 4-pole motors of very distinct horsepower rates, in order to extend the solution also for machines of other polarities. The following motors were tested: 5 hp (NEMA 184T) and $150 \mathrm{hp} \mathrm{(NEMA} \mathrm{444T).}$

The tests were conducted in a way similar to the followed for NHE motors: continuous application of full load until the thermal stabilization of the motor.

\section{EFF1 (IE3) Motors}

The several motors tested were chosen according to the criterion of the most critical frame size/horsepower ratios. They were all 4-pole machines, since this polarity is typical in lowvoltage applications involving speed variation. Motors with base frequencies of both 50 and $60 \mathrm{~Hz}$ were tested, for a wider verification of the proposed solution, as listed below:

- $\mathrm{f}_{\text {base }}=60 \mathrm{~Hz}: 3 \mathrm{hp}$ (IEC 90L), $12.5 \mathrm{hp} \mathrm{(IEC} \mathrm{132M),} 50 \mathrm{hp}$ (IEC 200L), $75 \mathrm{hp}$ (IEC 225SM) and 150 hp (IEC 280 SM).

- $\mathrm{f}_{\text {base }}=50 \mathrm{~Hz}: 2 \mathrm{hp}$ (IEC 90L), $10 \mathrm{hp}$ (IEC 132M), $40 \mathrm{hp}$ (IEC 200L), $75 \mathrm{hp}$ (IEC 225SM) and $150 \mathrm{hp}$ (IEC $280 \mathrm{SM}$ ).

Regarding the temperature rise tests, it should be noted that at 50 or $60 \mathrm{~Hz}$ the motors were always fed by the mains (AC power line), that is, sinusoidal supply was used, while all the other tests were accomplished with the motors fed by converter in optimal flux condition for loss minimization. Due to the fact that EFF1 (IE3) motors usually present lower efficiency levels than NHE and NPE motors, they tend to operate a little bit warmer than the latter. Because of that, unlike the tests performed with NHE and NPE motors, sometimes derating factors were applied in the tests with EFF1 (IE3) motors at low frequencies. However, in all cases the tests were performed following S1 duty as well.

\section{Experimental results}

The results contained in Tables I and II evidence the effectiveness of the proposed loss minimization technique for NEMA High Efficiency and NEMA Premium Efficiency motors: all the motors tested with optimal flux got the thermal stabilization at a lower temperature than with constant flux, at all frequencies analysed. Furthermore, Tables I and II show that, when operating at minimum loss condition, NHE and NPE motors can provide the rated torque continually throughout the operation range, even at low frequencies.

Tables I and II present some lacking results of low frequency (typically $5 \mathrm{~Hz}$ or below) tests. In these cases, the respective temperature rise test at full load could not be concluded, either because the motor was already running too hot, forcing the interruption of the test before thermal stabilization was reached, or because the dynamometer could no longer apply the 
required test load after a period, due to its functioning principle (braking/loading caused by the Foucault effect) and given the increased slip of the motor when it is heated. Temperature rise tests that could not be concluded properly with constant flux, but could be normally performed in the optimal flux condition, are themselves evidences of the thermal performance improvement of the motor, provided by the loss minimization technique.

\begin{tabular}{|c|c|c|c|c|c|c|}
\hline Source & $\begin{array}{l}\text { Operation } \\
\text { Frequency }\end{array}$ & Flux & $5 \mathrm{hp}$ & $20 \mathrm{hp}$ & $50 \mathrm{hp}$ & $150 \mathrm{hp}$ \\
\hline $\begin{array}{l}\text { Mains } \\
\text { supply }\end{array}$ & $\begin{array}{c}60 \mathrm{~Hz} \\
\text { sinusoidal }\end{array}$ & constant & 50.3 & 48.1 & 53.8 & 67.7 \\
\hline \multirow{7}{*}{ 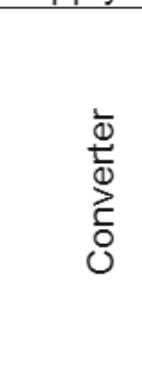 } & $60 \mathrm{~Hz}$ & \begin{tabular}{|c|} 
constant \\
optimal
\end{tabular} & 51.3 & 59.1 & 77.7 & 86.6 \\
\hline & \multirow{2}{*}{$15 \mathrm{~Hz}$} & constant & 72.8 & 79.8 & 122.3 & 100.3 \\
\hline & & optimal & 72.8 & 62.4 & 79.8 & 82.6 \\
\hline & \multirow{2}{*}{$10 \mathrm{~Hz}$} & constant & 101.0 & 104.7 & & 125.3 \\
\hline & & optimal & 77.8 & 67.7 & 88.1 & 83.2 \\
\hline & \multirow{2}{*}{$5 \mathrm{~Hz}$} & constant & & & & \\
\hline & & optimal & 82.8 & 80.6 & 115.8 & 97.4 \\
\hline
\end{tabular}

Table I. Temperature rise tests results (K) 4-pole nhe motors - s1 duty

\begin{tabular}{|c|c|c|c|c|c|}
\hline Source & $\begin{array}{l}\text { Operation } \\
\text { Frequency }\end{array}$ & Flux & $5 h p-4 p$ & $150 h p-4 p$ & $5 h p-2 p$ \\
\hline $\begin{array}{l}\text { Mains } \\
\text { supply }\end{array}$ & $\begin{array}{c}60 \mathrm{~Hz} \\
\text { sinusoidal }\end{array}$ & constant & 28.4 & 66.5 & 31.1 \\
\hline \multirow{11}{*}{ 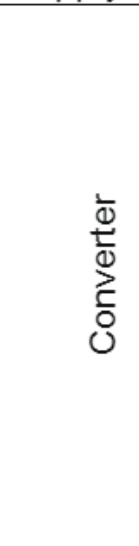 } & $60 \mathrm{~Hz}$ & $\begin{array}{c}\text { constant } \\
\text { optimal }\end{array}$ & 32.5 & * & * \\
\hline & \multirow{2}{*}{$30 \mathrm{~Hz}$} & constant & 39.9 & * & * \\
\hline & & optimal & 36.5 & * & $\star$ \\
\hline & \multirow{2}{*}{$10 \mathrm{~Hz}$} & constant & 65.5 & * & 40.2 \\
\hline & & optimal & 55.0 & * & 29.5 \\
\hline & \multirow{2}{*}{$5 \mathrm{~Hz}$} & constant & 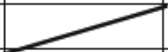 & * & r \\
\hline & & optimal & 67.9 & * & 82.1 \\
\hline & \multirow{2}{*}{$4 \mathrm{~Hz}$} & constant & & * & * \\
\hline & & optimal & 100.0 & * & * \\
\hline & \multirow{2}{*}{$3 \mathrm{~Hz}$} & constant & 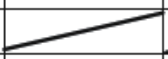 & 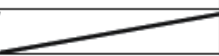 & * \\
\hline & & optimal & * & 87.4 & * \\
\hline
\end{tabular}

*Tests that have not been performed

Table II. Temperature rise tests results (K) npe motors - s1 duty

The analysis of the data presented in the tables above by means of graphs makes the interpretation of results easier. Fig. 9 evidences the advantages of using the optimal flux technique with a NHE, $20 \mathrm{hp}$, 4-pole motor.

Some results of temperature rise tests realized with EFF1 (IE3) motors under optimal flux condition are graphically presented in Figs. 10 to 13. 


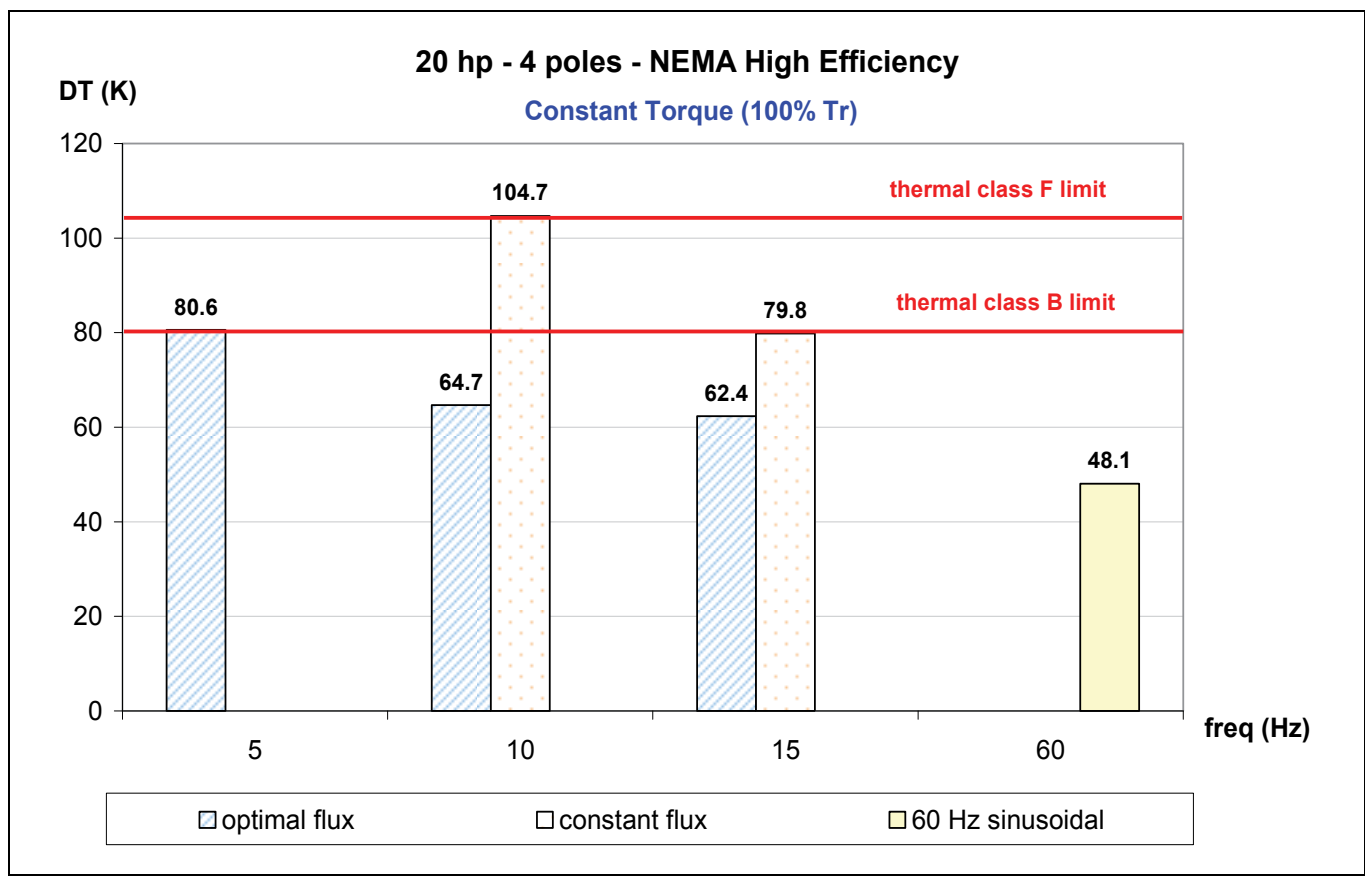

Fig. 9. Examples of results of temperature rise tests accomplished with NHE motor

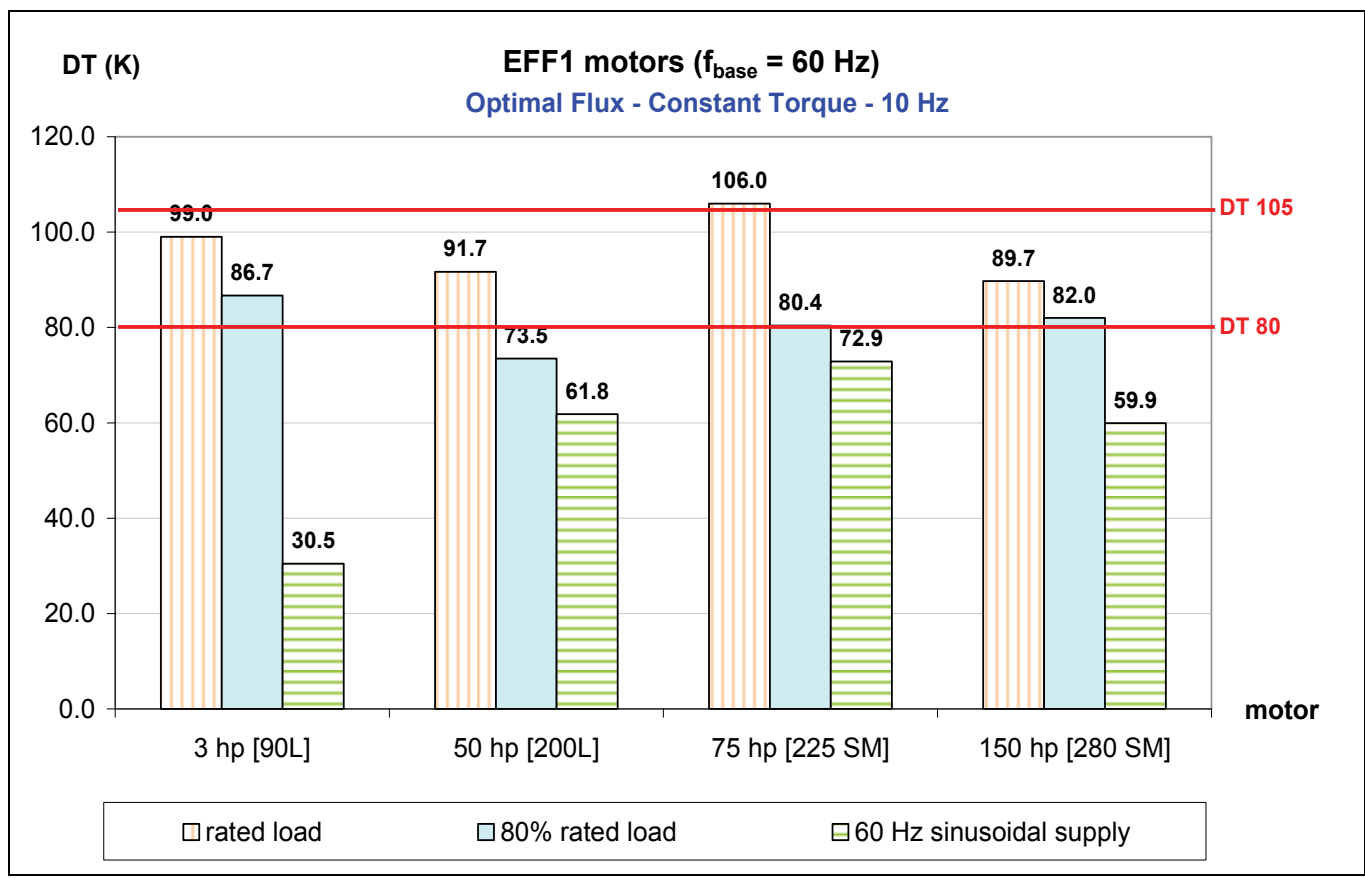

Fig. 10. Results of temperature rise tests conducted with EFF1 ( $\left.f_{\text {base }}=60 \mathrm{~Hz}\right)$ motors running at $10 \mathrm{~Hz}$ 


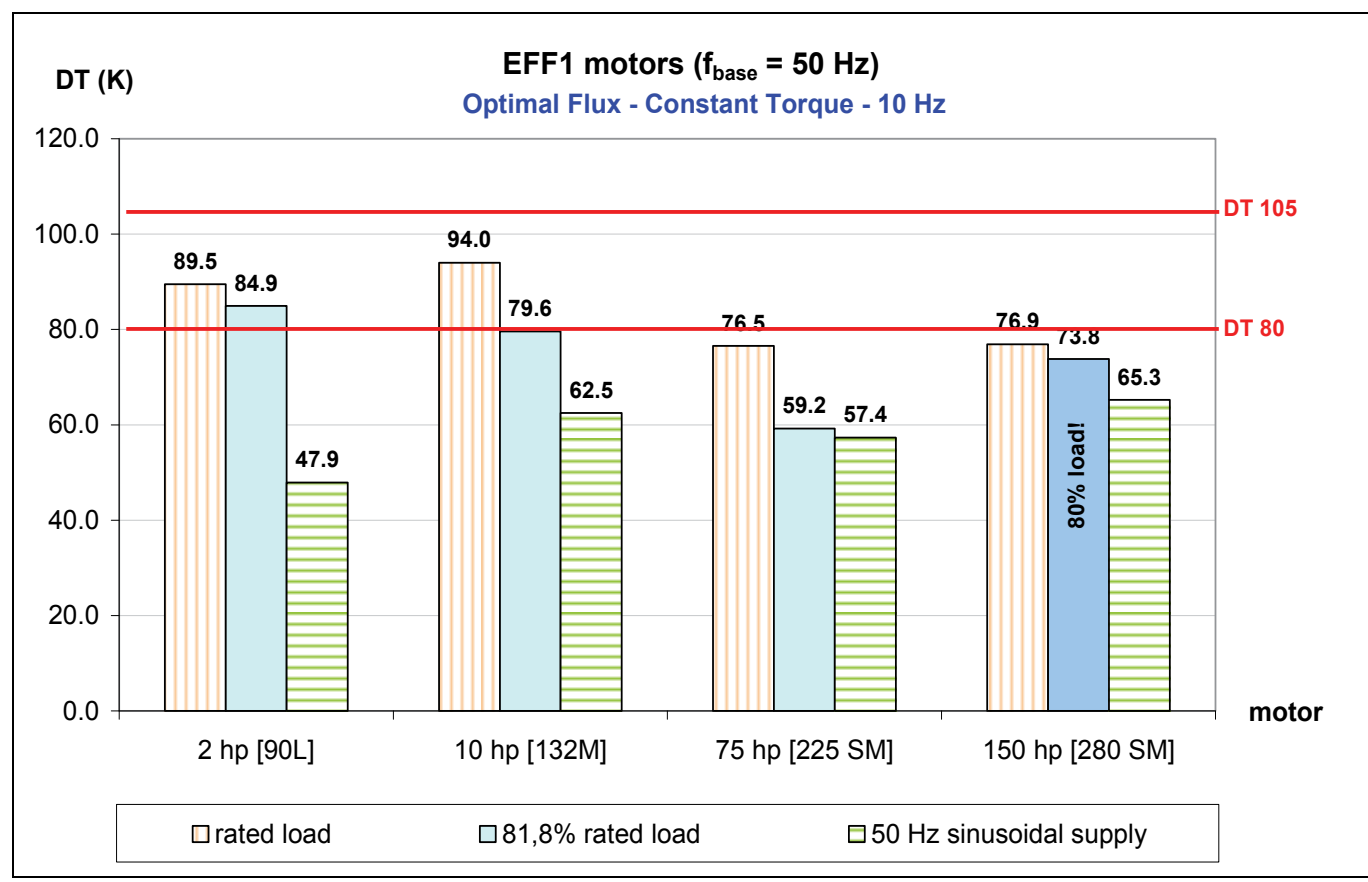

Fig. 11. Results of temperature rise tests conducted with EFF1 $\left(f_{\text {base }}=50 \mathrm{~Hz}\right)$ motors running at $10 \mathrm{~Hz}$

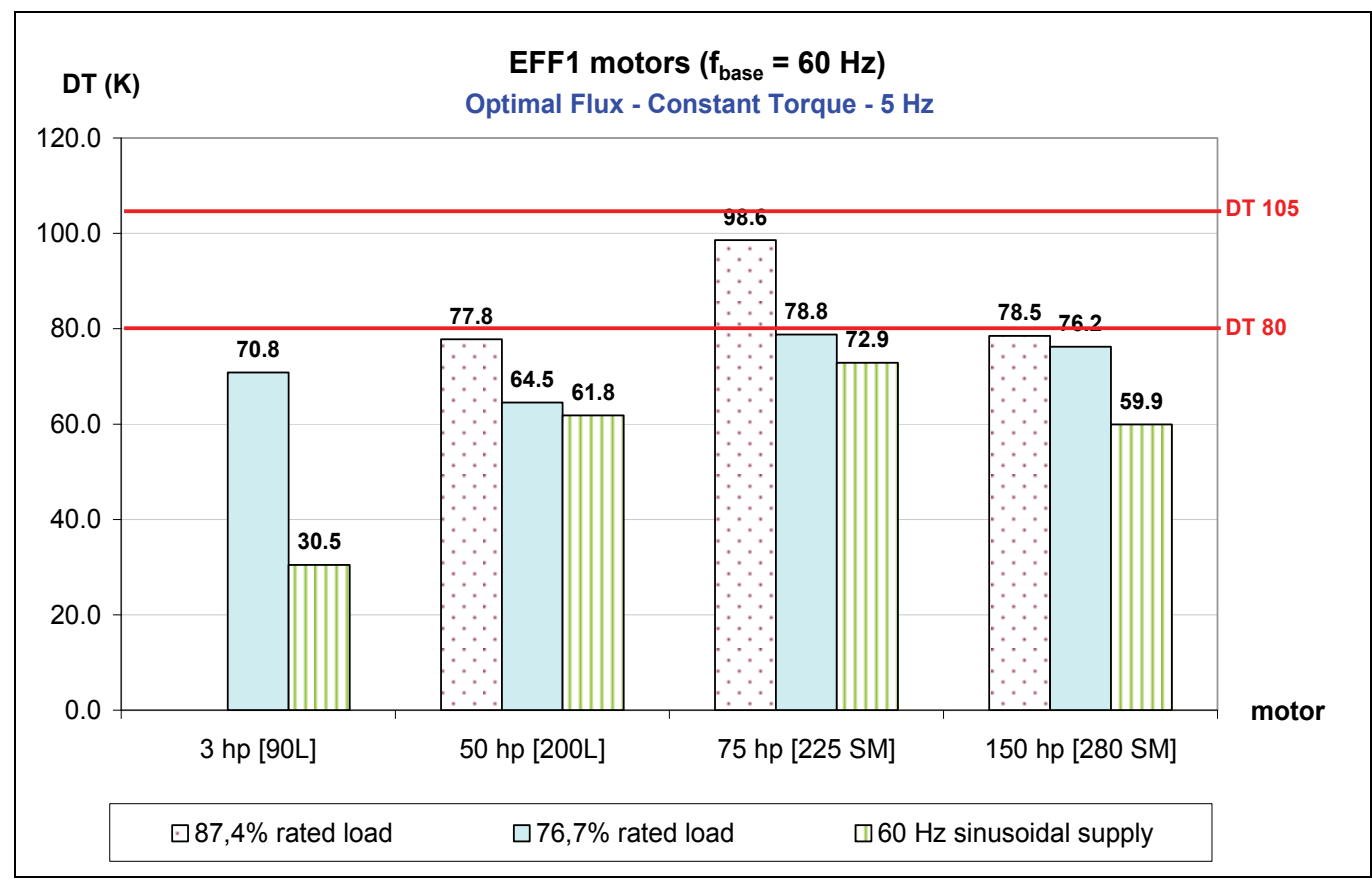

Fig. 12. Results of temperature rise tests conducted with EFF1 ( $\left.f_{\text {base }}=60 \mathrm{~Hz}\right)$ motors running at $5 \mathrm{~Hz}$ 


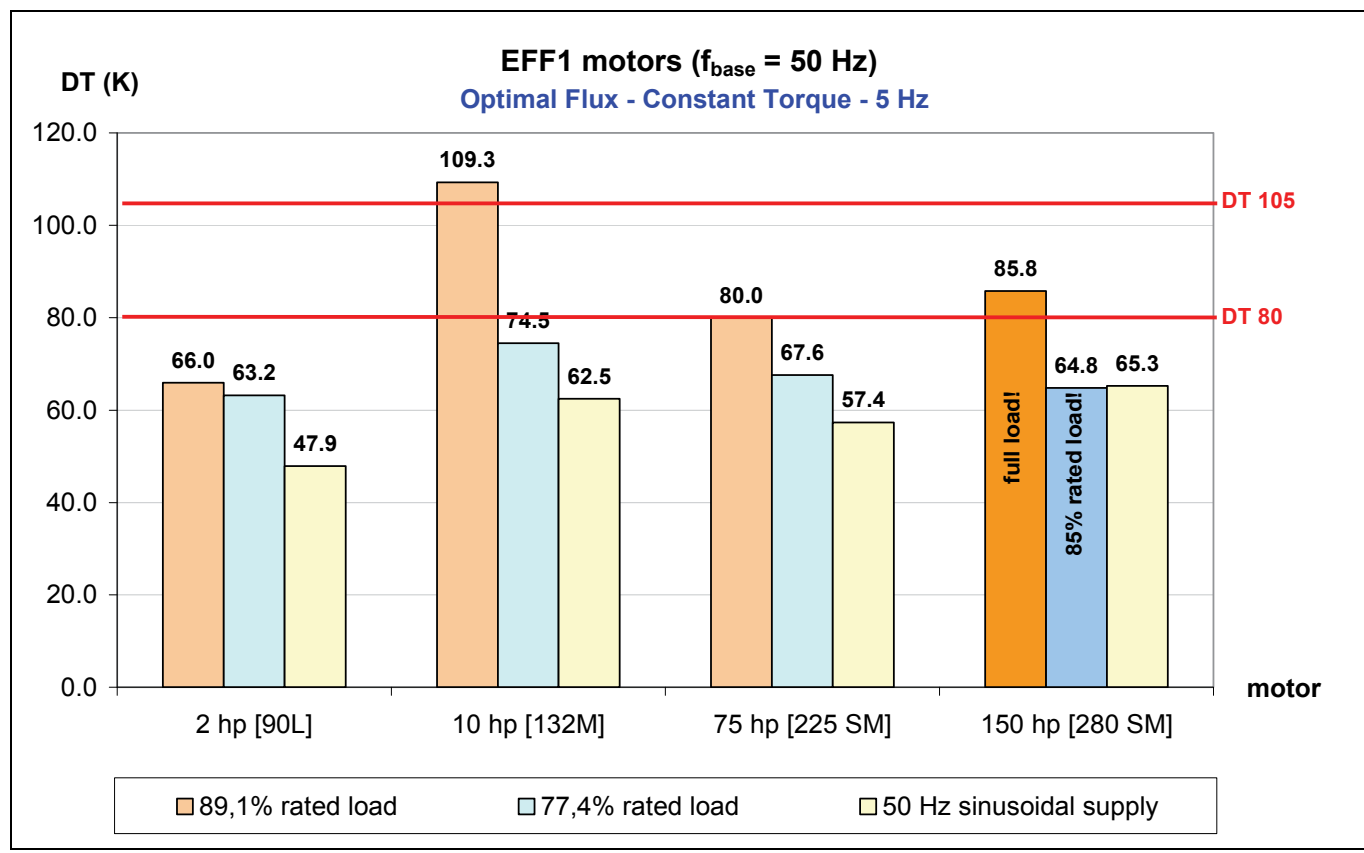

Fig. 13. Results of temperature rise tests conducted with EFF1 $\left(f_{\text {base }}=50 \mathrm{~Hz}\right)$ motors running at $5 \mathrm{~Hz}$

\section{Conclusions}

The study on the behavior of 3-phase induction low voltage motors' losses as a function of parameters such as load, operation frequency and magnetic induction (flux density), brought about the conception of an equation for magnetic flux that minimizes the total motor losses in each operation frequency. This is very interesting, given that variable speed drives usually involve operation throughout a speed range. Frequency reduction causes the motor iron losses to decrease, thus allowing the magnetic induction (or flux) to be increased at low operation speeds, enabling the motor to provide torque with lower current. The flux increase results from an increment in voltage/frequency ratio, accomplished in a growingly more accentuated manner the lower the operation frequency.

The mathematical equation that has been created was implemented in commercial static frequency converters and the study validation was carried out by means of the analysis of specific motor lines and the execution of tests with a number of frequency converters and induction motors of distinct sizes, using the optimal flux ratios theoretically determined. The tests led to the definition of the actual optimal flux curve as well as new derating curves valid for motors working under optimal flux condition, which evidence the advantages provided by the proposed technique (Fig. 14).

According to Fig. 14, it is possible to note that if temperature rise of thermal class $\mathrm{F}\left(105^{\circ} \mathrm{C}\right)$ is permitted on the motor windings, the loss minimization technique prevents the need of torque reduction for operation of the motor at low frequencies (until 0.1 p.u.). If temperature rise of thermal class $\mathrm{B}\left(80^{\circ} \mathrm{C}\right)$ is required, the loss minimization technique allows for the application of a smoother torque derating than the needed for the motor running with constant flux and rated losses. 


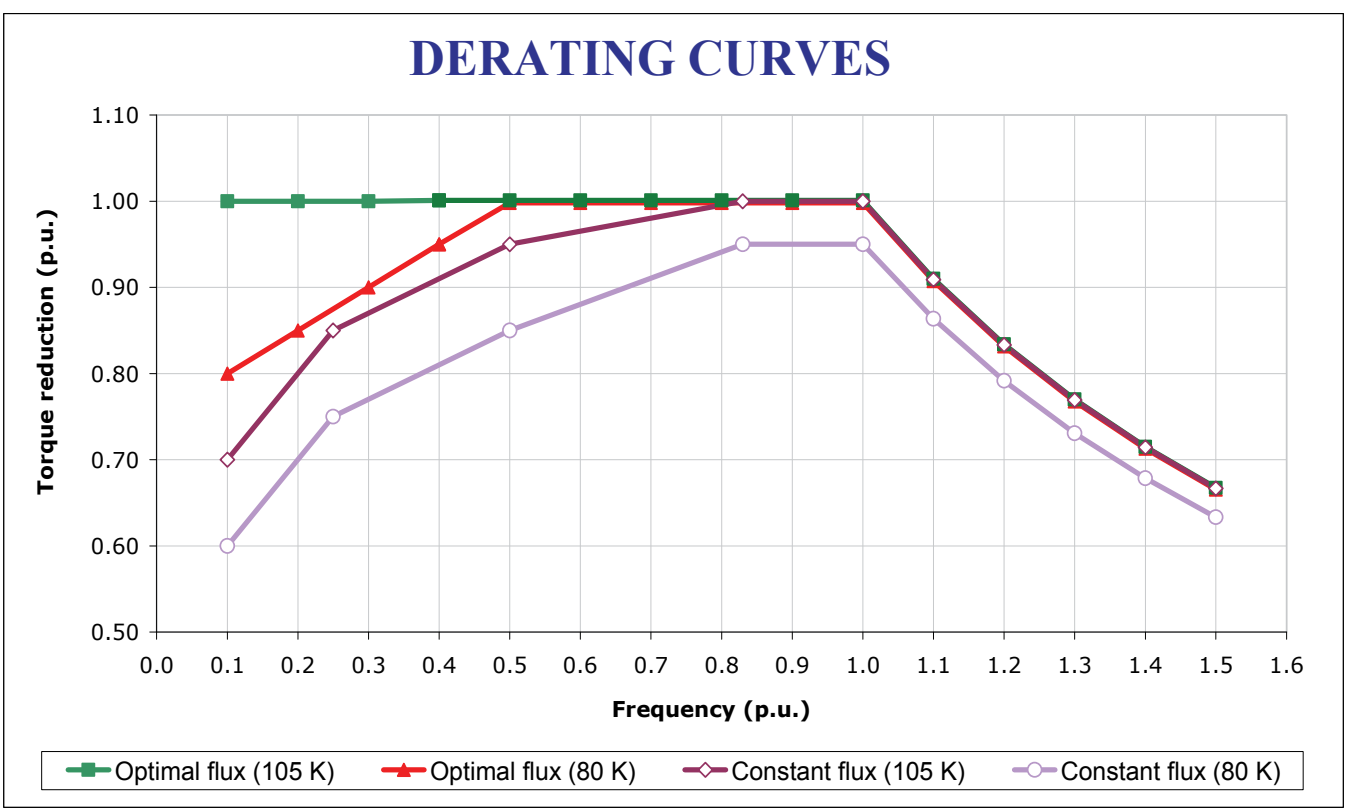

Fig. 14. The new derating (torque reduction) curves proposed evidence the advantages provided by the optimal flux solution

The implementation of the optimal flux curve into commercial frequency converters resulted in the development of a solution (converter + motor) to optimize/improve the variable speed drives with constant torque loads.

The next step of this development (ongoing) is improving the Optimal Flux Solution, so that it can be advantageously used with any type of load driven by converter-fed induction motors.

\section{References}

[1] PCT/BR2004/000074, "Static frequency converter with automatic function for optimizing magnetic flux and minimizing losses in electric induction motors".

[2] S. L. Nau and A. P. Sobrinho, "Optimal voltage/frequency curve for inverter-fed motor". Proceedings of the 3rd International Conference on Energy Efficiency in Motor Driven Systems (EEMODS), Treviso, Italy, 2002.

[3] R. Kaczmarek; M. Amar and F. Protat, "Iron loss under PWM voltage supply on Epstein frame and in induction motor core". IEEE Transactions on Magnetics, Vol. 32, No.1, January 1996.

[4] A. Boglietti; P. Ferraris; M. Lazzari and F. Profumo, "Iron losses in magnetic materials with six-step and PWM inverter supply". IEEE Transactions on Magnetics, Vol. 27, No. 6, November 1991.

[5] L. T. Mthombeni and P. Pillay, "Core losses in motor laminations exposed to highfrequency or nonsinusoidal excitation". IEEE Transactions on Industry Applications, Vol. 40, No. 5, September-October 2004. 
[6] M. S. Lancarotte; C. Goldemberg and A. A. Penteado Jr, "Estimation of FeSi core losses under PWM or DC bias ripple voltage excitations". IEEE Transactions on Energy Conversion, Vol. 20, No. 2, June 2005.

[7] A. J. Moses and N. Tutkun, "Investigation of power loss in wound toroidal cores under PWM excitation". IEEE Transactions on Magnetics, Vol. 33, No. 5, September 1997.

[8] A. Boglietti; M. Chiampi; M. Repetto; O. Bottauscio and D. Chiarabaglio, "Loss separation analysis in ferromagnetic sheets under PWM supply". IEEE Transactions on Magnetics, Vol. 34, No. 4, July 1998.

[9] A. Boglietti; P. Ferraris; M. Lazzari and M. Pastorelli, "About the possibility of defining a standard method for iron loss measurement in soft magnetic materials with inverter supply". IEEE Transactions on Industry Applications, Vo. 33, No. 5, September-October 1997.

[10] C. Cester; A. Kedous-Lebouc and B. Cornut, "Iron loss under practical working conditions of a PWM powered induction motor". IEEE Transactions on Magnetics, Vol. 33, No. 5, September 1997.

[11] A. Ruderman and R. Welch, "Electrical machine PWM loss evaluation basics". Proceedings of the $4^{\text {th }}$ International Conference on Energy Efficiency in Motor Driven Systems (EEMODS), Heidelberg, Germany, 2005.

[12] M. Sokola; V. Vuckovic and E. Levi, "Measurement of iron losses in PWM inverter fed induction machines". Proceedings of the 30th Universities Power Engineering Conference (UPEC), London, UK, 1995.

[13] A. C. Smith and K. Edey, "Influence of manufacturing processes on iron losses". Proceedings of the 7th International Conference on Electrical Machines and Drives Conference Publication No. 412. September 1995.

[14] K. Kostenko e L. Piotrovski, Máquinas Eléctricas - Volume II - Máquinas de Corrente Alternada (tradução de original russo), Ed. Lopes da Silva, Porto, Portugal, 1979.

[15] P. C. Krause, Analysis of Electric Machinery, McGraw Hill, New York, USA, 1996.

[16] NEMA Standard MG1-2003, Part 30 - Application considerations for constant speed motors used on a sinusoidal bus with harmonic content and general purpose motors used with adjustable-voltage or adjustable-frequency controls or both. 


\title{
Sensorless Vector Control of Induction Motor Drive - A Model Based Approach
}

\author{
Jogendra Singh Thongam ${ }^{1}$ and Rachid Beguenane ${ }^{2}$ \\ ${ }^{1}$ Department of Renewable Energy Systems, \\ STAS Inc., Chicoutimi, QC \\ ${ }^{2}$ Department of ECE, Royal Military College, \\ Kingston, ON \\ Canada
}

\section{Introduction}

Induction machines are more rugged, compact, cheap and reliable in comparison to other machines used in similar applications. Vector controlled induction motor drive outperforms the dc motor drive because of higher transient current capability, increased speed range and lower rotor inertia.

Sensors widely used in electric drives degrade the reliability of the system especially in hostile environments and require special attention to electrical noise. Moreover, it is difficult to mount sensors in certain applications in addition to extra expenses involved. Therefore, a lot of researches are underway to develop accurate speed estimation techniques. With sensorless vector control we have a decoupled control structure similar to that of a separately excited dc motor retaining the inherent ruggedness of the induction motor at the same time. Speed sensorless control technique first appeared in (Abbondante \& Brennen, 1975). The commonly used methods for speed estimation are Model Reference Adaptive System (MRAS) (Schauder, 1992; Tajima \& Hori, 1993; Peng \& Fukao, 1994; Choy et al., 1996), Neural Networks (Simoes \& Bose, 1995; Fodor et al., 1995; Ben-Brahim \& Kudor, 1995; Kim et al., 2001; Toqeer \& Bayindir, 2003; Haghgoeian et al., 2005), Extended Kalman Filter (EKF) (Kim et al.,1994, Comnac et al., 2001; Ma \& Gui, 2002; Du et al., 1995; Thongam \& Thoudam, 2004) and Nonlinear Observer (Bodson et al., 1995; Liu et al., 2001, Pappano et al., 1998).

The aim of this chapter is to provide with a brief overview of high performance sensorless induction motor drive. There exist two approaches to speed estimation for sensorless control of induction machine: non model based approach and model based approach. The non model based technique tracks a machine anisotropy: either saturation for flux estimation or rotor slotting for rotor position estimation, whereas the model based technique rely mostly on back emf voltage associated with fundamental component excitation of the machine. Model-based observers are considered very well adapted for state estimation and allow, in most cases, a stability proof and a methodology to tune observer gains. Among the observers the reduced order observers are more frequently implemented than the full order ones as they don't require heavy computations. Two sensorless vector control strategies using machine model-based estimation are presented in this chapter. 


\section{Speed estimation}

Rotor speed has been considered as a constant by many researchers in speed estimation problem (Schauder, 1992; Tajima \& Hori, 1993; Peng \& Fukao, 1994; Kim et al., 1994; Comnac et al., 2001; Ma \& Gui, 2002; Du et al., 1995; Minami et al., 1991; Veleyez-Reyes \& Verghese., 1992; Veleyez-Reyes et al., 1989). The idea is that the speed changes slowly compared to electrical variables. Adopting such an approach allowed speed estimation without requiring the knowledge of mechanical parameters of the drive system such as load torque, inertia etc. In (Schauder, 1992; Tajima \& Hori, 1993; Peng \& Fukao, 1994) speed was estimated using model reference adaptive system considering it as an unknown constant parameter. In (Kim et al., 1994; Comnac et al., 2001; Ma \& Gui, 2002; Du et al., 1995) the speed was considered as an unknown constant state of the machine and extended kalman filter (EKF) was used to estimate it. Recursive least square estimation method was used in (Minami et al., 1991; Veleyez-Reyes \& Verghese, 1992; Veleyez-Reyes et al., 1989) for speed estimation considering speed as an unknown constant parameter and found out the value of estimated speed that best fits the measured and calculated data in the dynamic equations of the motor.

In this section we present a sensorless vector control strategy using machine model-based speed estimation (Thongam \& Ouhrouche, 2007). The proposed method does not require taking derivative of the measured signals unlike that of (Peng \& Fukao, 1994; Minami et al., 1991; Veleyez-Reyes \& Verghese, 1992; Veleyez-Reyes et al., 1989). The method is also simpler to implement than implementing EKF. In this method the model of the motor used for estimation is derived by introducing a new variable which is a function of rotor flux and speed assuming that rotor speed varies slowly in comparison to electrical states and hence its derivative can be conveniently equated to zero in the machine model used for estimation. The sensorless method presented here in this chapter is based on observing this new variable. A reduced order observer is implemented for estimating the new variable using which the rotor speed is estimated.

\subsection{Induction machine model}

The induction motor model in stationary stator reference frame $\alpha-\beta$ may be written in vector matrix form as

$$
\begin{gathered}
\frac{d \boldsymbol{\psi}_{r}}{d t}=A_{11} \boldsymbol{\psi}_{r}+A_{12} \boldsymbol{i}_{s} \\
\frac{d \boldsymbol{i}_{s}}{d t}=\boldsymbol{A}_{21} \boldsymbol{\psi}_{r}+\boldsymbol{A}_{22} \boldsymbol{i}_{s}+\boldsymbol{A}_{23} \boldsymbol{v}_{s}
\end{gathered}
$$

where

$$
\boldsymbol{A}_{11}=-\left(R_{r} / L_{r}\right) \boldsymbol{I}+\omega \boldsymbol{J}, \boldsymbol{A}_{12}=\left(L_{m} R_{r} / L_{r}\right) \boldsymbol{I}
$$$$
\boldsymbol{A}_{21}=\frac{L_{m}}{\sigma L_{s} L_{r}}\left\{\left(R_{r} / L_{r}\right) \boldsymbol{I}-\omega \boldsymbol{J}\right\}
$$

$$
\boldsymbol{A}_{22}=-\left\{R_{S} /\left(\sigma L_{s}\right)+R_{r} L_{m}^{2} /\left(\sigma L_{s} L_{r}^{2}\right)\right\} \boldsymbol{I}, \quad \boldsymbol{A}_{23}=1 /\left(\sigma L_{s}\right) \boldsymbol{I},
$$$$
\boldsymbol{I}=\left[\begin{array}{ll}
1 & 0 \\
0 & 1
\end{array}\right], \quad \boldsymbol{J}=\left[\begin{array}{cc}
0 & -1 \\
1 & 0
\end{array}\right],
$$

$\psi_{r}=\left[\begin{array}{ll}\psi_{r \alpha} & \psi_{r \beta}\end{array}\right]^{T}$ is the rotor flux, $\boldsymbol{i}_{s}=\left[\begin{array}{ll}i_{s \alpha} & i_{s \beta}\end{array}\right]^{T}$ is the stator current, $\boldsymbol{v}_{s}=\left[\begin{array}{ll}v_{s \alpha} & v_{s \beta}\end{array}\right]^{T}$ is the stator voltage and $\sigma=1-L_{m}^{2} /\left(L_{s} L_{r}\right)$ is the leakage coefficient. 
Now, we introduce a new quantity into the motor model which when introduced will make the right hand side of conventional motor model given by equations (1) and (2) independent of the unknowns - the rotor flux and speed. Let's define the new quantity as

$$
\boldsymbol{Z}=-\boldsymbol{A}_{11} \boldsymbol{\psi}_{r}
$$

A new motor model is obtained after introducing the new quantity as given below:

$$
\begin{gathered}
\frac{d \psi_{r}}{d t}=A_{12} i_{S}+A_{14} \boldsymbol{Z} \\
\frac{d \boldsymbol{i}_{s}}{d t}=\boldsymbol{A}_{22} \boldsymbol{i}_{S}+\boldsymbol{A}_{23} \boldsymbol{v}_{s}+\boldsymbol{A}_{24} \boldsymbol{Z} \\
\frac{d \boldsymbol{Z}}{d t}=\boldsymbol{A}_{32} \boldsymbol{i}_{S}+\boldsymbol{A}_{34} \boldsymbol{Z}
\end{gathered}
$$

where $\boldsymbol{A}_{14}=-\boldsymbol{I}, \boldsymbol{A}_{24}=\left\{L_{m} /\left(\sigma L_{s} L_{r}\right)\right\} \boldsymbol{I}, \boldsymbol{A}_{32}=\left(L_{m} R_{r}^{2} / L_{r}^{2}\right) \boldsymbol{I}-\omega\left(L_{m} R_{r} / L_{r}\right) \boldsymbol{J}$ and $\boldsymbol{A}_{34}=\boldsymbol{A}_{11}$.

\subsection{Observer structure and speed estimation}

The proposed speed estimation algorithm is based on observing the newly defined quantity which is a function of rotor flux and speed. Equation (5) and (6) are used for constructing a Gopinath's reduced order observer (Gopinath, 1971) for estimating the newly defined quantity. The observer is as given below

$$
\frac{d \hat{\boldsymbol{Z}}}{d t}=\boldsymbol{A}_{32} \boldsymbol{i}_{S}+\boldsymbol{A}_{34} \hat{\boldsymbol{Z}}+\boldsymbol{G}\left(\frac{d \boldsymbol{i}_{S}}{d t}-\frac{d \hat{\boldsymbol{i}}_{S}}{d t}\right)
$$

where $\boldsymbol{G}=\left[\begin{array}{cc}g_{1} & -g_{2} \\ g_{2} & g_{1}\end{array}\right]$ is the observer gain. Using equation (5) for $\frac{d \hat{\boldsymbol{i}}_{s}}{d t}$ the observer equation becomes

$$
\frac{d \hat{\boldsymbol{Z}}}{d t}=\boldsymbol{A}_{32} \boldsymbol{i}_{s}+\boldsymbol{A}_{34} \hat{\boldsymbol{Z}}+\boldsymbol{G}\left(\frac{d \boldsymbol{i}_{s}}{d t}-\boldsymbol{A}_{22} \boldsymbol{i}_{s}-\boldsymbol{A}_{23} v_{s}-\boldsymbol{A}_{24} \hat{\boldsymbol{Z}}\right)
$$

The observer poles can be placed at the desired locations in the stable region of the complex plane by properly choosing the values of the elements of the $\mathbf{G}$ matrix. In order to avoid taking derivative of the stator current in the algorithm we introduce another new quantity

$$
\boldsymbol{D}=\hat{\boldsymbol{Z}}-\boldsymbol{G} \boldsymbol{i}_{S}
$$

Finally, the observer is of the following form:

$$
\frac{d}{d t} \boldsymbol{F}=\left(\boldsymbol{A}_{32}+\boldsymbol{A}_{34} \boldsymbol{G}-\boldsymbol{G} \boldsymbol{A}_{22}-\boldsymbol{G} \boldsymbol{A}_{24} \boldsymbol{G}\right) \boldsymbol{i}_{s}-\boldsymbol{G} \boldsymbol{A}_{23} \boldsymbol{v}_{s}+\left(\boldsymbol{A}_{34}-\boldsymbol{G} \boldsymbol{A}_{24}\right) \boldsymbol{D}
$$




$$
\hat{\boldsymbol{Z}}=\boldsymbol{D}+\boldsymbol{G} \boldsymbol{i}_{S}
$$

The block diagram of the $\mathbf{Z}$ observer is shown in Fig. 1 .

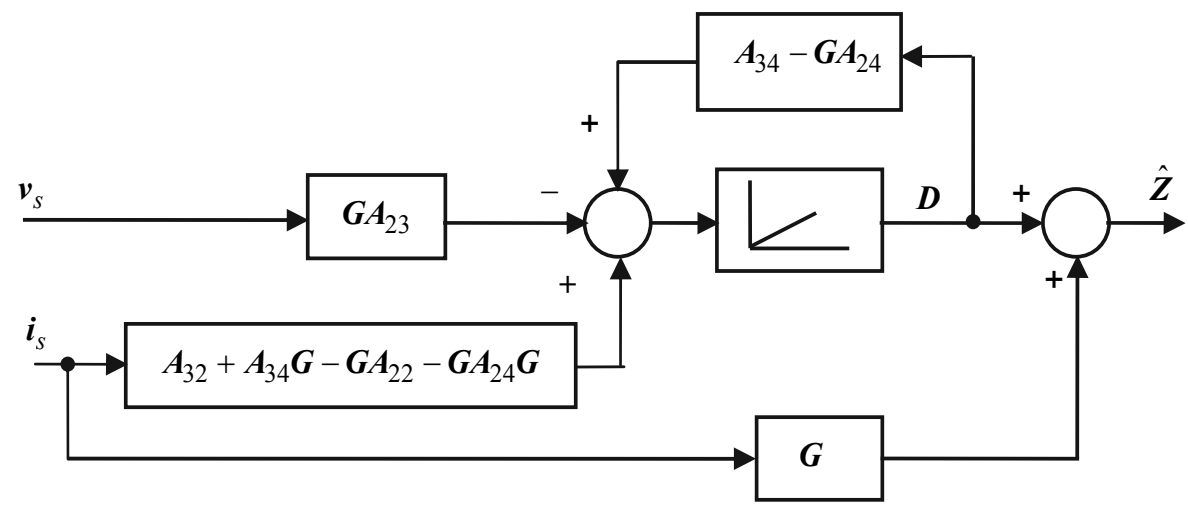

Fig. 1. Block diagram of $\boldsymbol{Z}$ observer

Assuming no parameter variation and no speed error, the equation for error dynamics is given by

$$
\frac{d}{d t} \tilde{\boldsymbol{Z}}=\frac{d}{d t}(\boldsymbol{Z}-\hat{\boldsymbol{Z}})=\left(\boldsymbol{A}_{34}-\boldsymbol{A}_{24} \boldsymbol{G}\right) \tilde{\boldsymbol{Z}}
$$

Eigenvalues of $\left(\boldsymbol{A}_{34}-\boldsymbol{A}_{24} \boldsymbol{G}\right)$ are the observer poles which are as given below:

$$
P_{o b s 1,2}=-\left(\frac{R_{r}}{L_{r}}+\frac{L_{m}}{\sigma L_{s} L_{r}} g_{1}\right) \pm j\left(\omega-\frac{L_{m}}{\sigma L_{s} L_{r}} g_{2}\right)
$$

The desired observer dynamics can be imposed by proper selection of observer gain G.

Next, let's see how the rotor speed is computed. It can be seen that the observed quantity is a function of rotor flux and speed. Performing matrix multiplication of $\psi_{r}^{T} \boldsymbol{J}$ with equation (3) we have

$$
Z_{\alpha} \psi_{r \beta}-Z_{\beta} \psi_{r \alpha}=\left(\psi_{r \alpha}^{2}+\psi_{r \beta}^{2}\right) \omega
$$

This is a simple equation which does not involve derivative or integration. To use it directly for speed computation we need to know the rotor flux; and as for $Z_{\alpha}$ and $Z_{\beta}$ we can use the estimated values. The required flux is obtained from the reference. Rearranging the above equation we have the equation used for rotor speed computation as given by

$$
\hat{\omega}=\frac{\hat{Z}_{\alpha} \psi_{r \beta}^{*}-\hat{Z}_{\beta} \psi_{r \alpha}^{*}}{{\psi_{r \alpha}^{*}}^{2}+\psi_{r \beta}^{* 2}}
$$

The coefficient matrices $A_{32}$ and $A_{34}$ in the observer equation are updated with the estimated values of rotor speed. 
It is to be noted here that the model of the motor used in implementing the observer algorithm has been developed assuming that the derivative of the rotor speed is zero. It is valid to make such an assumption since the dynamics of rotor speed is much slower than that of electrical states. Moreover, such an assumption allows estimation without requiring the knowledge of mechanical quantities of the drive such as load torque, inertia etc.

\subsection{Simulation results}

Simulation is carried out in order to validate the speed estimation algorithm presented. The block diagram of the sensorless indirect vector controlled induction motor drive incorporating the proposed speed estimator is shown in Fig. 2. The results of simulation are shown in Fig. 3 - Fig. 5.

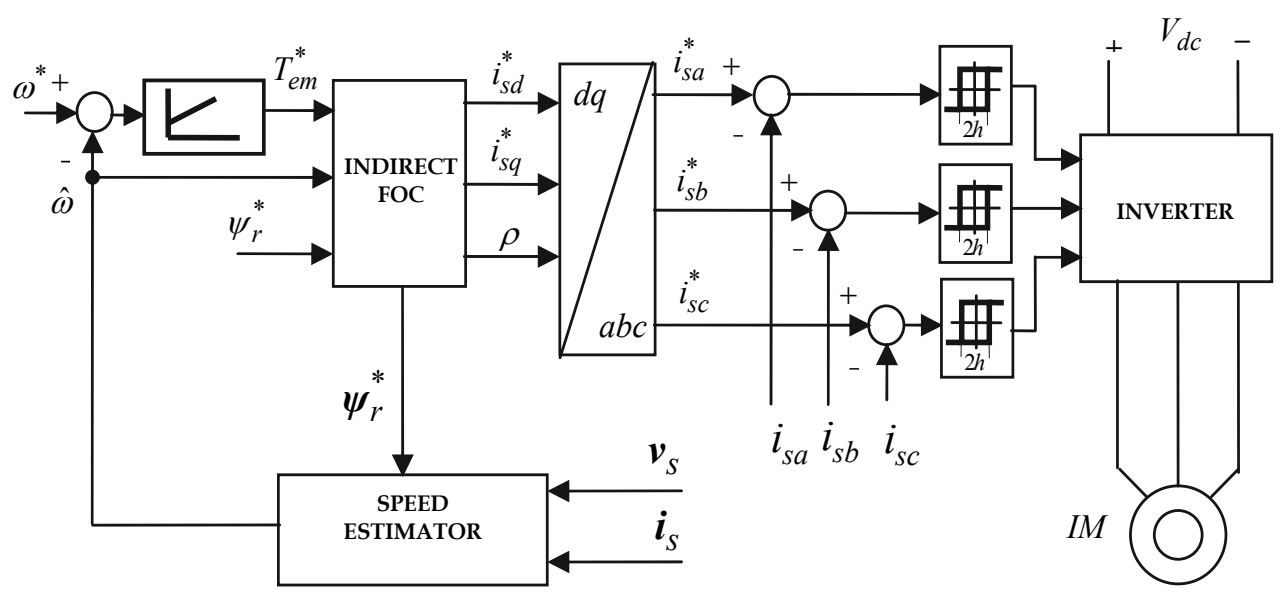

Fig. 2. Sensorless indirect VC induction motor drive

Initially, the drive is run at no load. It is accelerated from rest to $150 \mathrm{rad} / \mathrm{s}$ at $0.15 \mathrm{sec}$. and then, the speed is reversed at $2.5 \mathrm{sec}$. The speed is reversed again at $5.5 \mathrm{sec}$. The speed of the motor $(\omega)$, estimated speed $(\hat{\omega})$ and reference speed $\left(\omega^{*}\right)$ are shown in Fig. 3 (a). Fig. 3 (b) shows speed estimation error $(\omega-\hat{\omega})$. The newly defined quantity $(Z)$ and its estimated value $(\hat{Z})$ are shown in Fig. 3 (c) and its estimation error $(Z-\hat{Z})$ is shown in Fig. 3 (d).

The estimation algorithm and the drive response are then verified under loading and unloading conditions. The unloaded drive is started at $0.15 \mathrm{sec}$ and full load is applied at 1 $\mathrm{sec}$; then load is completely removed at $2 \mathrm{~s}$. Later, after speed reversal, full load is applied at $4 \mathrm{sec}$ and the load is completely removed at $5 \mathrm{sec}$. Fig. 4 shows the speed estimation result and response of the sensorless drive system.

Then, the sensorless induction motor drive is run under fully loaded condition at various operating speeds. The drive is started at full load at $0.15 \mathrm{~s}$ to $150 \mathrm{rad} / \mathrm{s}$ and the speed is reduced in steps in order to observe the response of the loaded drive at various speeds. Fig. 5 shows the estimation results and response of the loaded drive. 


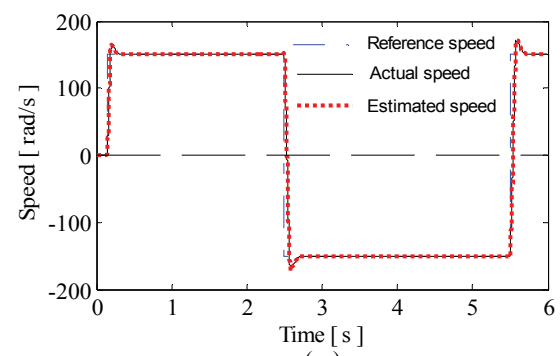

(a)

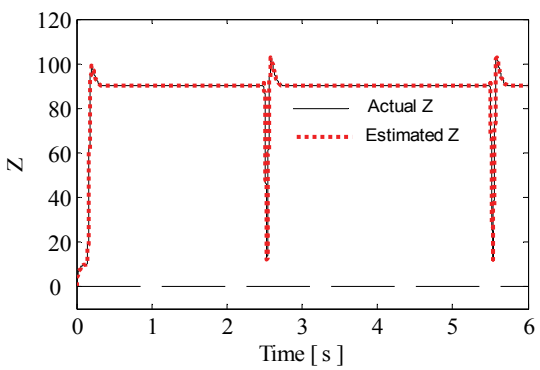

(c)

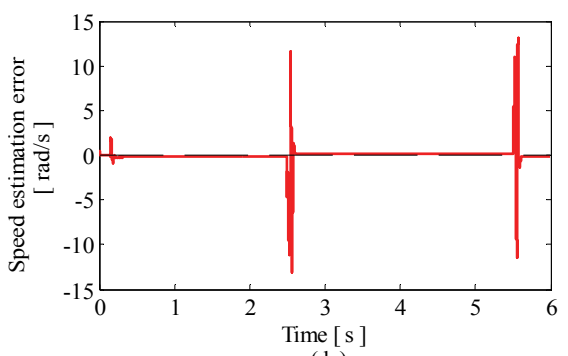

(b)

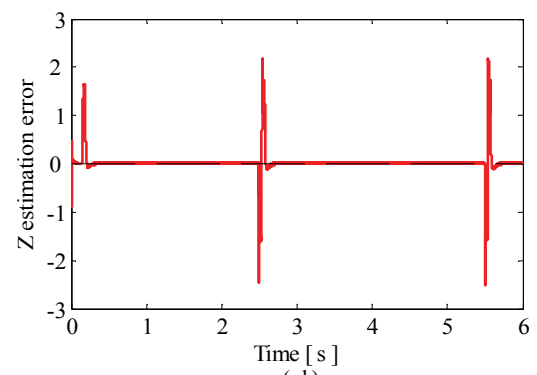

(d)

Fig. 3. Acceleration and speed reversal at no load; (a) reference, actual and estimated speeds; (b) speed estimation error; (c) actual $Z$ and estimated $Z$ and (d) $Z$ estimation error

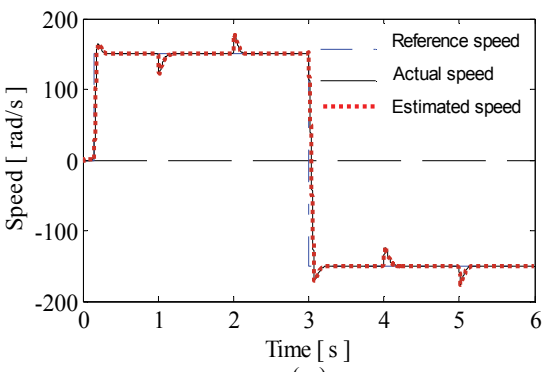

(a)

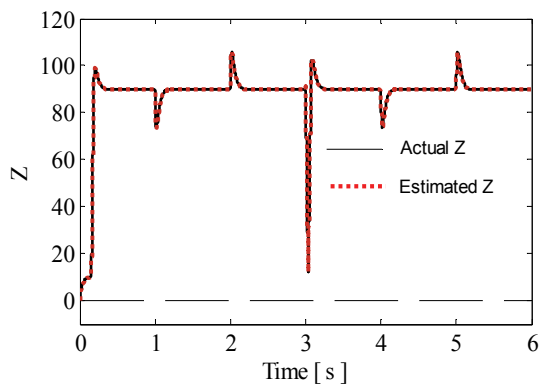

(c)

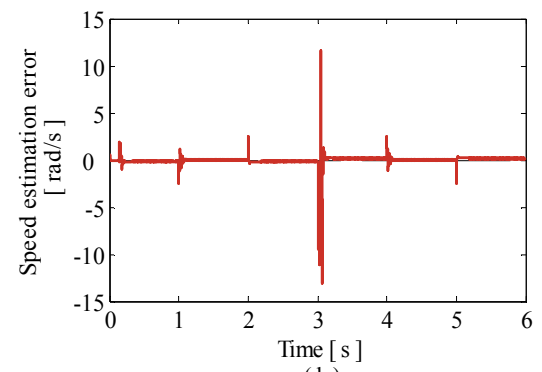

(b)

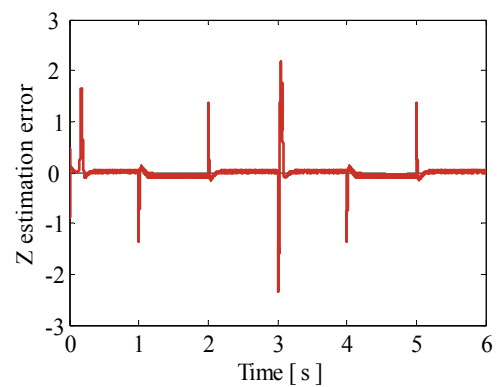

(d)

Fig. 4. Application and removal of load; (a) reference, actual and estimated speeds; (b) speed estimation error; (c) actual $Z$ and estimated $Z$ and (d) $Z$ estimation error 


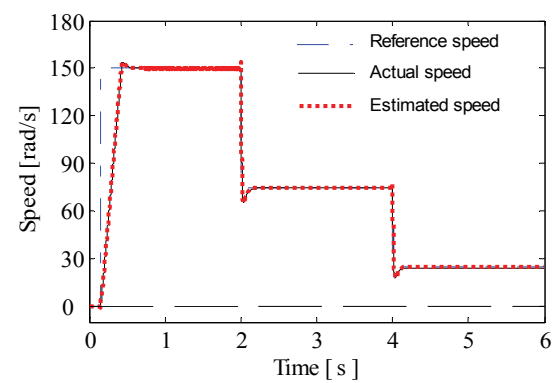

( a )

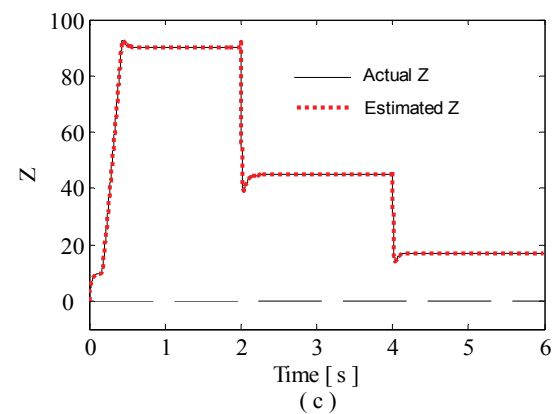

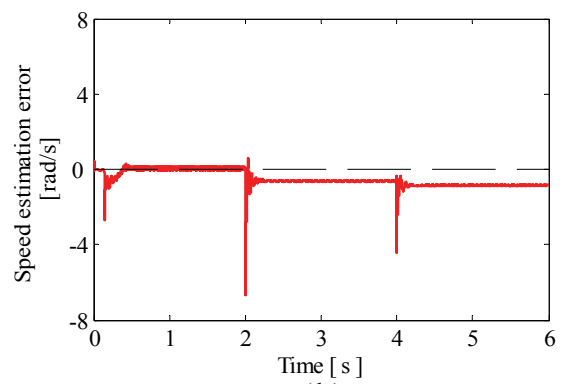

(b)

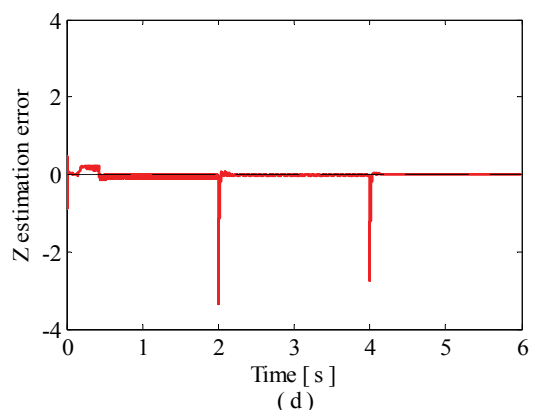

(d)

Fig. 5. Operation at full load at various speeds; (a) reference, actual and estimated speeds; (b) speed estimation error; (c) actual $Z$ and estimated $Z$ and (d) $Z$ estimation error

\subsection{Improvement in speed estimation}

It is observed that the estimation algorithm presented above gives good estimation accuracy under both dynamic and steady state conditions. However, it is found that the estimation accuracy decreases with decrease in speed. This is because of the fact that the estimation algorithm uses the command flux for speed estimation and not the actual rotor flux which however is little bit different from the command value. With the reduction in speed $Z$ decreases and therefore the flux error is more prominently visible in the estimated speed. The problem is overcome in this section by using a rotor flux observer based on the voltage model of the machine along with the observer of the newly defined quantity. This allows accurate speed estimation in various operating ranges. The speed is computed using (15) after replacing the command flux by the estimated one. Further, due to the obvious advantages of dc current regulators over ac current regulators as regards its robustness, and load and operating point independence (Rowan \& Kerkman, 1986) the control system uses dc current regulators. The rotor flux estimator and the control scheme are presented in the following subsections.

\subsubsection{Flux estimation}

The voltage model of induction motor is given by

$$
\boldsymbol{\psi}_{r}=\frac{L_{r}}{L_{m}}\left\{\int\left(\boldsymbol{v}_{s}-R_{s} \boldsymbol{i}_{s}\right) d t-\sigma L_{s} \boldsymbol{i}_{s}\right\}
$$

The rotor flux can be estimated using (16). However, the integration in (16) produces a problem of dc off-set and drift component in low speed region. Therefore, a first order low 
pass filter (LPF) is used instead of integration. The phase error in the low speed region produced due to LPF is approximately compensated by adding low pass filtered reference flux with the same time constant as above, and producing the estimated rotor flux (Ohtani et al., 1992). The estimator equation is given as

$$
\hat{\boldsymbol{\psi}}_{r}=\frac{L_{r}}{L_{m}}\left\{\left(\boldsymbol{v}_{s}-R_{s} \boldsymbol{i}_{S}\right) \frac{\tau}{1+\tau s}-\sigma L_{s} \boldsymbol{i}_{s} \frac{\tau s}{1+\tau s}\right\}+\boldsymbol{\Psi}_{r}^{*} \frac{1}{1+\tau s}
$$

where $\tau$ is the LPF time constant. The command rotor flux $\boldsymbol{\Psi}_{r}^{*}$ in (17) is obtained as follows:

$$
\boldsymbol{\Psi}_{r}^{*}=\left[\begin{array}{l}
\Psi_{r \alpha}^{*} \\
\Psi_{r \beta}^{*}
\end{array}\right]=\left[\begin{array}{l}
\Psi_{r}^{*} \cos \rho^{*} \\
\Psi_{r}^{*} \sin \rho^{*}
\end{array}\right]
$$

where $\Psi_{r}^{*}=L_{m} i_{s d}^{*}$ and $\rho^{*}$, the command rotor flux angle, is as given by

$$
\rho^{*}=\int \omega_{e}^{*} d t
$$

$\omega_{e}^{*}$, the command rotor flux speed, is computed as given below

$$
\omega_{e}^{*}=\omega_{s l}^{*}+\hat{\omega}
$$

The command slip speed $\omega_{s l}^{*}$ is given by

$$
\omega_{s l}^{*}=\frac{R_{r} i_{q s}^{*}}{L_{r} i_{d s}^{*}}
$$

\subsubsection{Speed estimation}

The equation (15) after modification is used for speed computation. In place of reference flux, estimated flux is used for speed computation as given below

$$
\hat{\omega}=\frac{\hat{Z}_{\alpha} \hat{\psi}_{r \beta}-\hat{Z}_{\beta} \hat{\psi}_{r \alpha}}{\hat{\psi}_{r \alpha}{ }^{2}+\hat{\psi}_{r \beta}{ }^{2}}
$$

\subsubsection{Simulation results}

Simulation is carried out in order to verify the accuracy of the estimation algorithm and to see the response of the sensorless drive system. The block diagram of sensorless vector controlled induction motor drive incorporating the flux and speed estimators is shown in Fig. 6.

First, the sensorless drive is run at no load at various speeds to verify the performance of the observer under no load condition. The drive is started at no-load and is run at various speeds by increasing it in steps to $10 \mathrm{rad} / \mathrm{s}, 50 \mathrm{rad} / \mathrm{s}, 100 \mathrm{rad} / \mathrm{s}$ and $150 \mathrm{rad} / \mathrm{s}$ at $0.3 \mathrm{sec}, 1.5$ sec, $3 \mathrm{sec}$ and $4.5 \mathrm{sec}$ respectively. The speed of the motor $(\omega)$, estimated speed $(\hat{\omega})$, reference speed $\left(\omega^{*}\right)$ and speed estimation error $(\omega-\hat{\omega})$ are shown in Fig. 7 (a). Fig. 7 (b) shows the actual $Z$, estimated $Z$ and $Z$ estimation error $(Z-\hat{Z})$. 


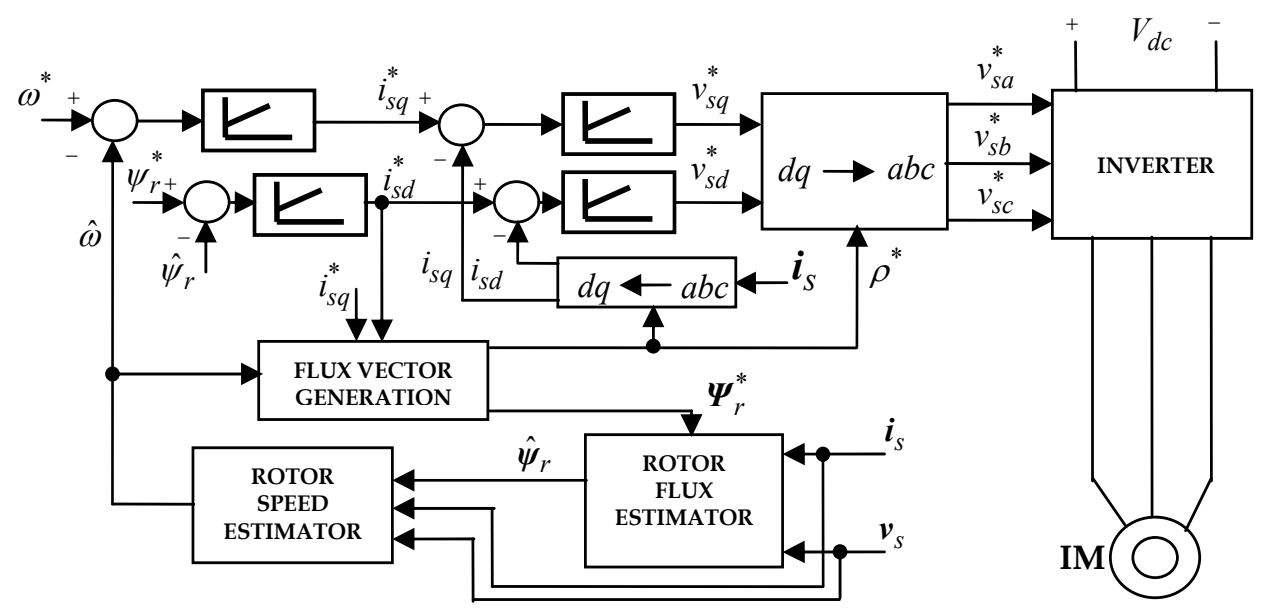

Fig. 6. Sensorless vector controlled induction motor drive

Then, the performance of the estimator and drive response are verified on loading and unloading. The drive is started at no-load at $0.25 \mathrm{~s}$ to a speed of $150 \mathrm{rad} / \mathrm{s}$ and full load is applied at $1 \mathrm{sec}$ and then the load is removed completely at $2 \mathrm{sec}$. Later, after speed reversal, full load is applied at $4 \mathrm{~s}$ and the load is completely removed at $5 \mathrm{sec}$. The response of the drive on application and removal of load is shown in Fig. 8.
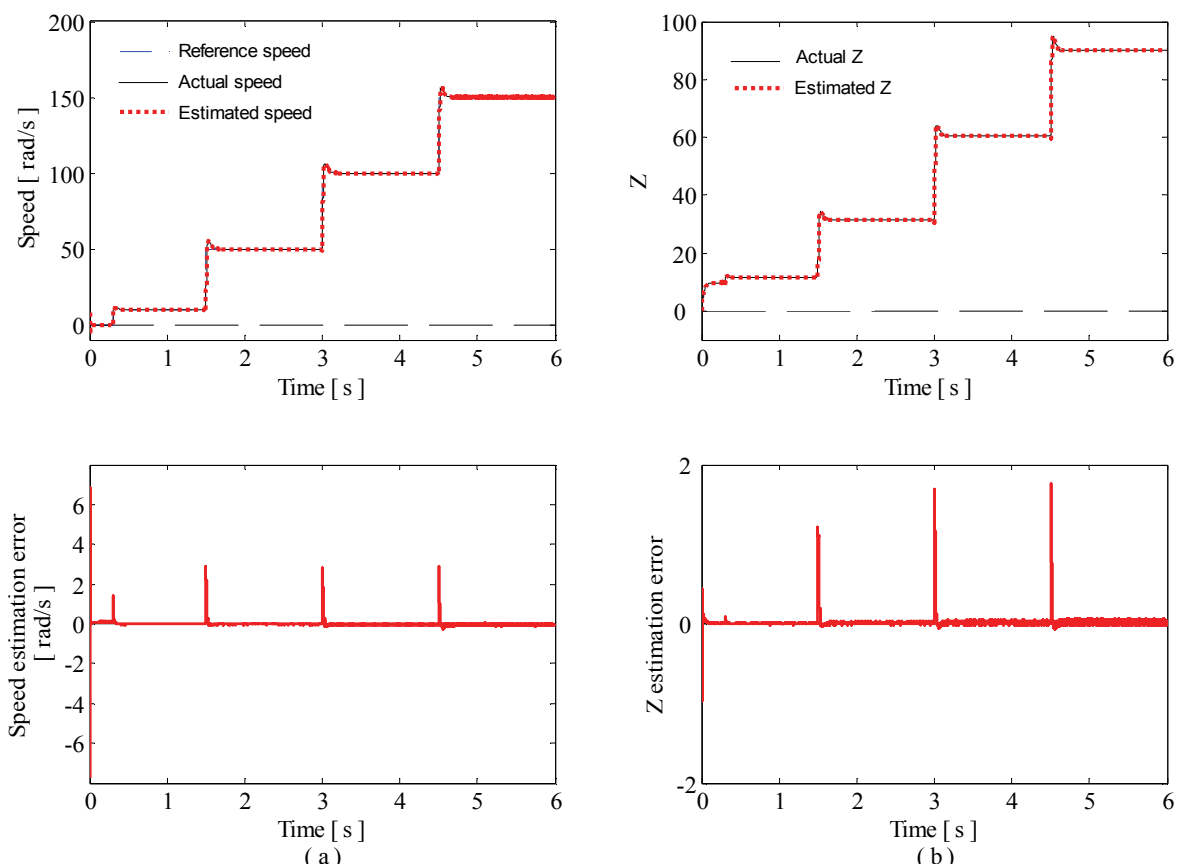

Fig. 7. No load operation at various speeds; (a) reference, actual, estimated speeds, and speed estimation error; (b) actual Z, estimated $Z$, and $Z$ estimation error 

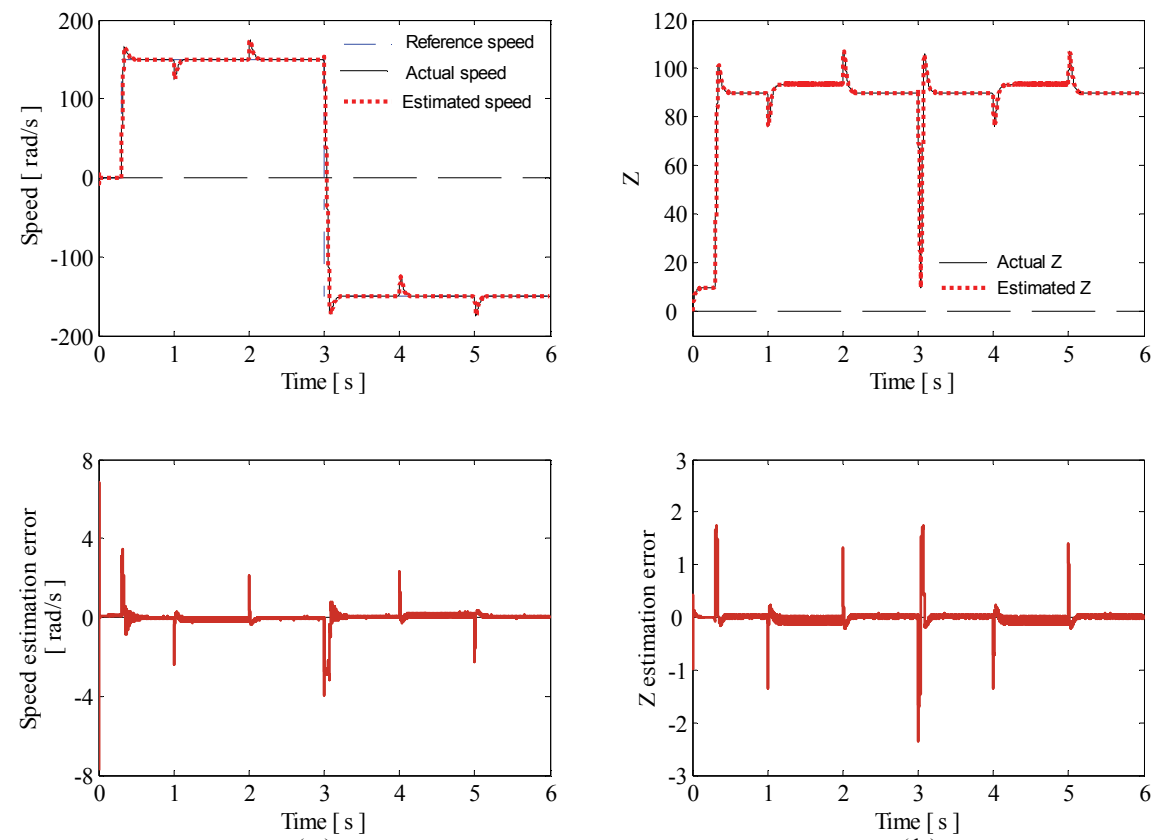

(a)

(b)

Fig. 8. Application and removal of load; (a) reference, actual, estimated speeds, and speed estimation error; (b) actual $Z$, estimated $Z$, and $Z$ estimation error.
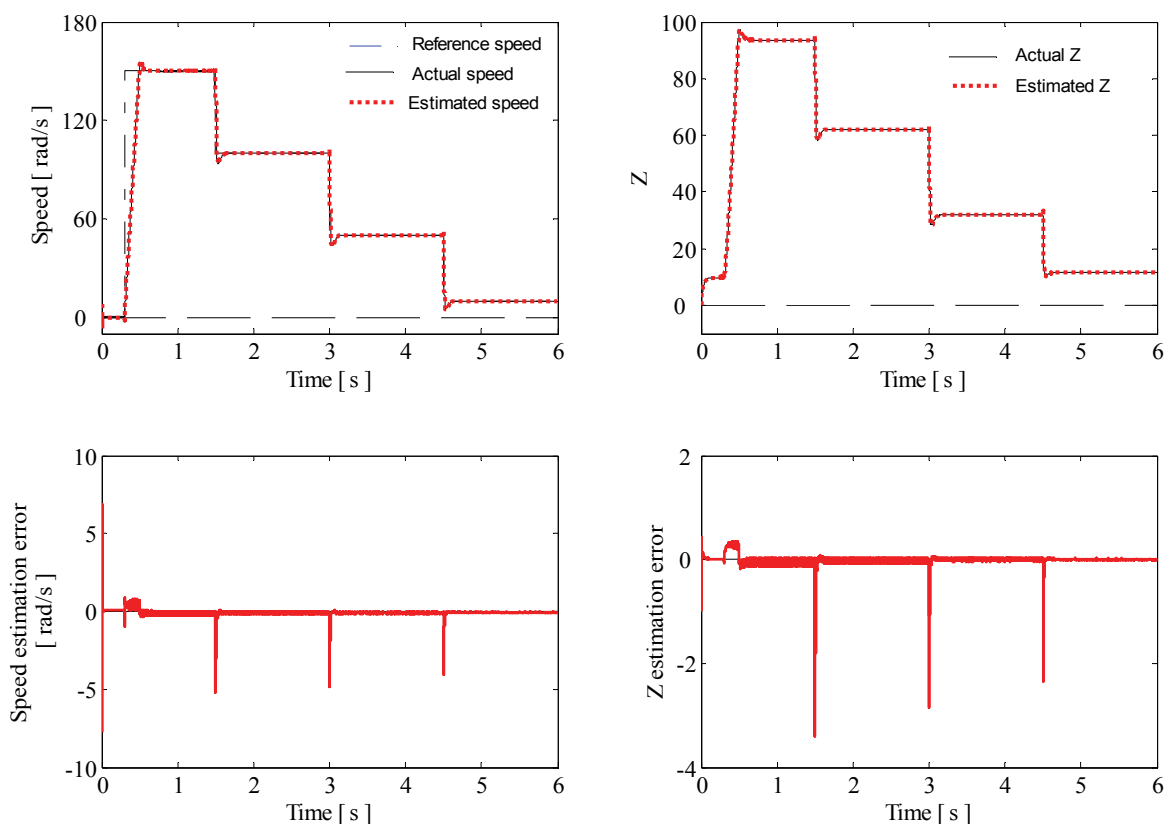

(a)

(b)

Fig. 9. Full load operation at various speeds; (a) reference, actual, estimated speeds, and speed estimation error; (b) actual $Z$, estimated $Z$, and $Z$ estimation error. 
The estimator performance and drive response are then verified under fully loaded condition of the drive at various operating speeds. The fully loaded machine is accelerated to $150 \mathrm{rad} / \mathrm{s}$ at $0.3 \mathrm{~s}$, and then the speed is reduced in steps to $100 \mathrm{rad} / \mathrm{s}, 50 \mathrm{rad} / \mathrm{s}$ and 10 $\mathrm{rad} / \mathrm{s}$ at $1.5 \mathrm{sec}, 3 \mathrm{sec}$ and $4.5 \mathrm{sec}$ respectively. Fig. 9 shows the estimation results and response of the drive during the operation.

Good speed estimation accuracy was obtained under both dynamic and steady state conditions under various operating conditions and response of the VC induction motor drive incorporating the estimation algorithms was found to be good. The speed estimation algorithm presented in this section depends upon the knowledge of the rotor flux, whereas, the rotor flux estimator is independent of rotor speed and requiring only the measurable stator terminal quantities, the stator voltage and current.

\section{Flux and speed estimation}

Induction machines do not allow rotor flux to be easily measured. The current model and the voltage model are the traditional solutions, and their benefits and drawbacks are well known. Various observers for flux estimation were analyzed in the work by Verghese and Sanders (Verghese \& Sanders, 1988) and Jansen and Lorenz (Jansen \& Lorenz, 1994). Over the years several other have been presented, many of which include speed estimation (Tajima \& Hori, 1993; Kim et al., 1994; Ohtani et al., 1992; Kubota et al., 1993; Sathiakumar, 2000; Yan et al., 2000).

Tajima \& Hori (Tajima \& Hori, 1993) proposed MRAS (Schauder, 1992) with novel pole allocation method for speed estimation while rotor flux estimation was done using Gopinath's observer. Extended Kalman Filter was used in (Kim et al., 1994) for estimating the rotor flux and speed using a full order model of the motor assuming that rotor speed is a constant. Ohtani et al (Ohtani et al., 1992) used the voltage model for flux estimation overcoming the problem associated with integrator and low pass filter while speed was obtained using a frequency controller. A speed adaptive flux observer was proposed in (Kubota et al., 1993) for estimating rotor flux and speed. Gopinath style reduced order observer was used in (Sathiakumar, 2000) for estimating the rotor flux while the speed was computed using an equation derived from the motor model. Yan et al (Yan et al., 2000) proposed a flux and speed estimator based on the sliding-mode control methodology.

In this section, we present a new flux estimation algorithm for speed sensorless rotor flux oriented controlled induction motor drive (Thongam \& Ouhrouche, 2006). The proposed method is based on observing the variable $Z$ introduced in Section 2 which when introduced makes the right hand side of the conventional motor model independent of rotor flux and speed. Rotor flux estimation is achieved using an equation obtained after introduction of the newly defined quantity into the Blaschke equation or commonly known as the current model; while, speed is computed using a simple equation obtained using the new quantity $Z$.

\subsection{Estimation of rotor flux and speed}

The speed computation equation (22) obtained in section 2.4.2 requires the knowledge of rotor flux and $\mathbf{Z}$. Here, we present a joint rotor flux and speed estimation algorithm. The block diagram of the proposed rotor flux and speed estimation algorithm is shown in Fig. 10. 


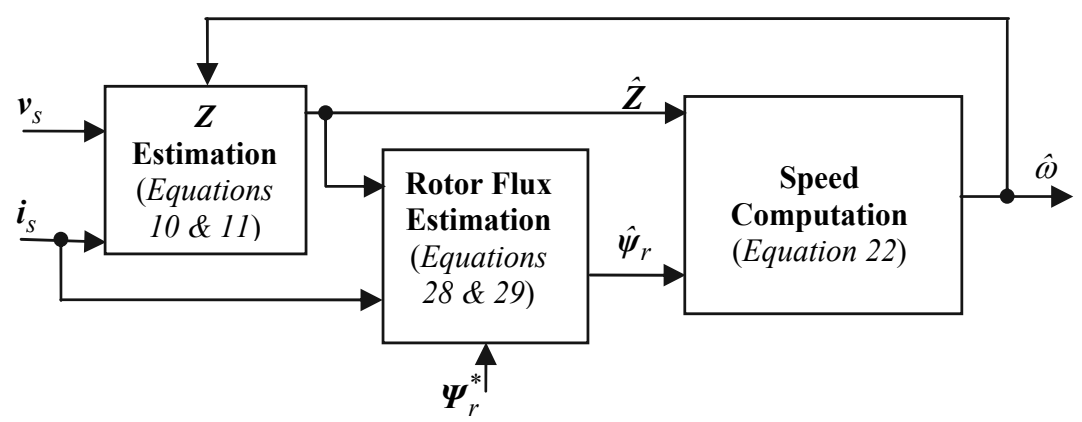

Fig. 10. Rotor flux and speed estimator

Rotor flux may be obtained directly using equation (4) which is obtained after introducing the newly defined quantity $\mathbf{Z}$ into the Blaschke equation as

$$
\hat{\boldsymbol{\psi}}_{r}=\int\left(\boldsymbol{A}_{12} i_{S}+\boldsymbol{A}_{14} \boldsymbol{Z}\right) d t
$$

However, rotor flux computation by pure integration suffers from dc offset and drift problems. To overcome the above problems a low pass filter is used instead of pure integrator and the phase error due to low pass filtering is approximately compensated by adding low pass filtered reference flux with the same time constant as used above (Ohtani et al., 1992). The equation of the proposed rotor flux estimator is given below

$$
\hat{\boldsymbol{\psi}}_{r}=\frac{\tau}{1+\tau S}\left(\boldsymbol{A}_{12} i_{S}+\boldsymbol{A}_{14} \boldsymbol{Z}\right)+\frac{1}{1+\tau S} \boldsymbol{\Psi}_{r}^{*}
$$

where $\tau$ is the LPF time constant. The command rotor flux $\boldsymbol{\Psi}_{r}^{*}$ is obtained as follows

$$
\boldsymbol{\Psi}_{r}^{*}=\left[\begin{array}{c}
\Psi_{r \alpha}^{*} \\
\Psi_{r \beta}^{*}
\end{array}\right]=\Psi_{r}^{*}\left[\begin{array}{c}
\cos \rho^{*} \\
\sin \rho^{*}
\end{array}\right]=L_{m} i_{s d}^{*}\left[\begin{array}{c}
\cos \rho^{*} \\
\sin \rho^{*}
\end{array}\right]
$$

The command rotor flux angle $\rho^{*}$ is obtained by integrating the command rotor flux speed as given by

$$
\rho^{*}=\int \omega_{e}^{*} d t=\int\left(\omega_{s l}^{*}+\hat{\omega}\right) d t
$$

The command slip speed $\omega_{s l}^{*}$ is given by

$$
\omega_{s l}^{*}=\frac{R_{r} i_{q s}^{*}}{L_{r} i_{d s}^{*}}
$$

We know that the equation of the back emf is given by:

$$
\boldsymbol{e}=\frac{L_{m}}{L_{r}} \frac{d \boldsymbol{\psi}_{r}}{d t}=\frac{L_{m}}{L_{r}}\left(\boldsymbol{A}_{12} \boldsymbol{i}_{s}+\boldsymbol{A}_{14} \boldsymbol{Z}\right)
$$




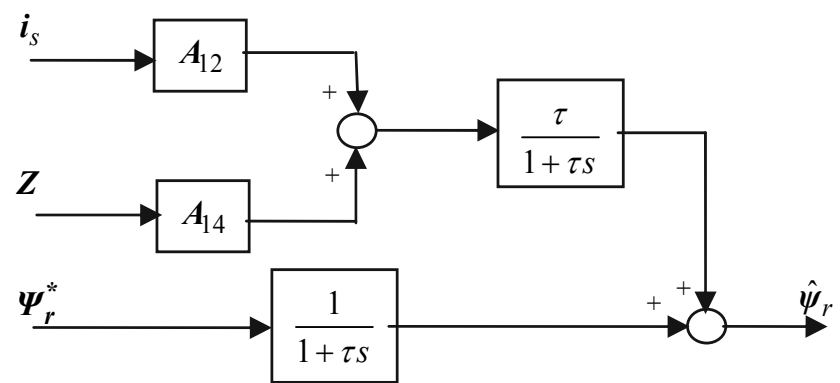

Fig. 11. Rotor Flux Estimator

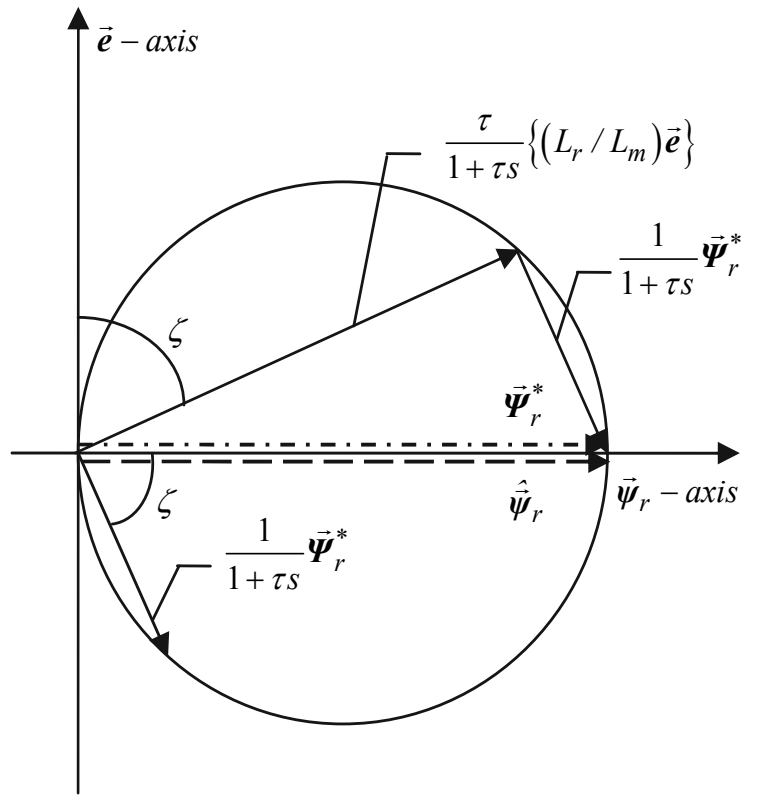

Fig. 12. Obtaining estimated rotor flux

Now, equation (24) may also be written as

$$
\hat{\boldsymbol{\psi}}_{r}=\frac{\tau}{1+\tau s}\left(\frac{L_{r}}{L_{m}} \boldsymbol{e}\right)+\frac{1}{1+\tau s} \boldsymbol{\Psi}_{r}^{*}
$$

Block diagram of the rotor flux estimator is shown in Fig. 11. Fig.12 explains how estimated flux is obtained using equation (29).

\subsection{Simulation results}

Simulation is carried out in order to validate the performance of the proposed flux and speed estimation algorithm. The proposed rotor flux and speed estimation algorithm is 
incorporated into a vector controlled induction motor drive. The block diagram of the sensorless vector controlled induction motor drive incorporating the proposed estimator is shown in Fig. 13. The sensorless drive system is run under various operating conditions.

First, acceleration and speed reversal at no load is performed. A speed command of 150 $\mathrm{rad} / \mathrm{s}$ at $0.5 \mathrm{~s}$ is given to the drive system which was initially at rest, and then the speed is reversed at $3 \mathrm{~s}$. The response of the drive is shown in Fig. 14. Fig. 14 (a) shows reference $\left(\omega^{*}\right)$, actual $(\omega)$, estimated $(\hat{\omega})$ speed, and speed estimation error $(\omega-\hat{\omega})$. The module of the actual $\left(\left|\boldsymbol{\Psi}_{r}\right|\right)$, estimated $\left(\left|\hat{\boldsymbol{\Psi}}_{r}\right|\right)$ rotor flux, and rotor flux estimation error $\left(\left|\boldsymbol{\Psi}_{r}\right|-\left|\hat{\boldsymbol{\Psi}}_{r}\right|\right)$ are shown in Fig. 14 (b). Fig. 14 (c) and (d) shows respectively the locus of the actual and estimated rotor fluxes.

The drive is then run at various speeds under no load condition. It is accelerated from rest to $10 \mathrm{rad} / \mathrm{s}$ at $0.5 \mathrm{~s}$, then accelerated further to $50 \mathrm{rad} / \mathrm{s}, 100 \mathrm{rad} / \mathrm{s}$ and $150 \mathrm{rad} / \mathrm{s}$ at $1.5 \mathrm{~s}, 3 \mathrm{~s}$ and $4.5 \mathrm{~s}$ respectively. Fig. 15 shows the estimation of rotor flux and speed, and the response of the sensorless drive system.

Then, the drive is subjected to a slow change in reference speed profile (trapezoidal), the results of which are shown in Fig. 16.

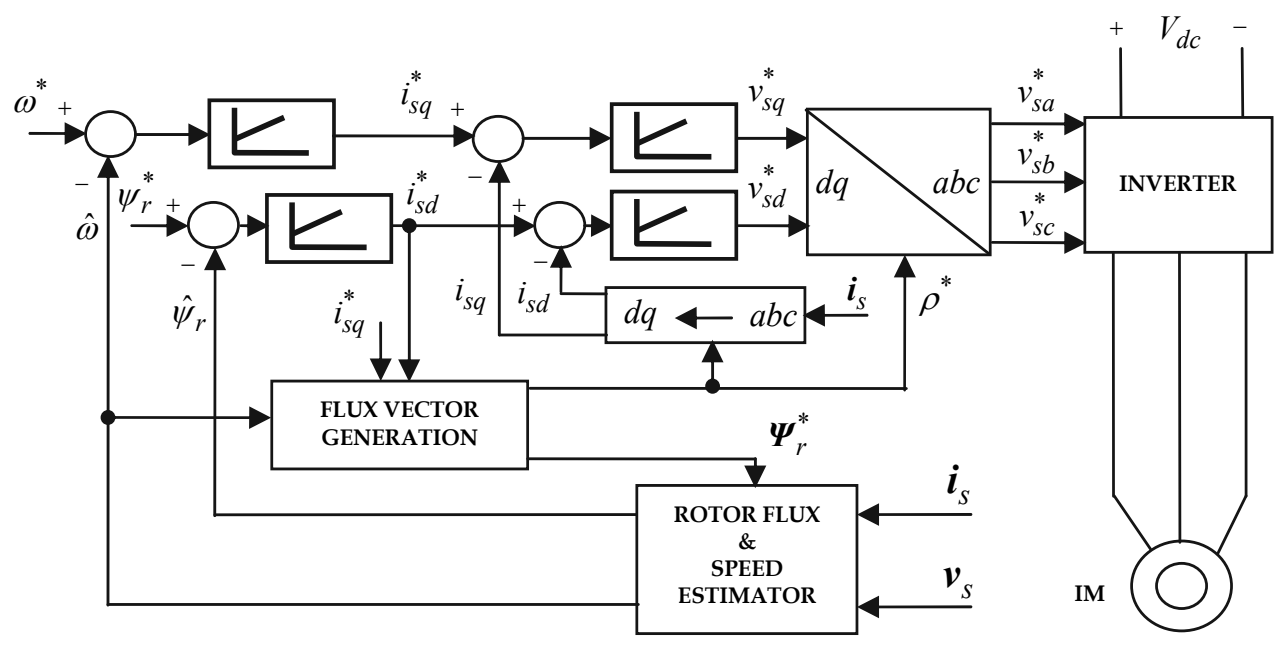

Fig. 13. Sensorless vector controlled induction motor drive

Further, the performance of the estimator is verified under loaded conditions at various operating speeds. The fully loaded drive is accelerated to $150 \mathrm{rad} / \mathrm{s}$ at $0.5 \mathrm{~s}$ and then decelerated in steps to $100 \mathrm{rad} / \mathrm{s}, 50 \mathrm{rad} / \mathrm{s}$ and $10 \mathrm{rad} / \mathrm{s}$ at $1.5 \mathrm{~s}, 3 \mathrm{~s}$ and $4.5 \mathrm{~s}$ respectively. Fig. 17 shows the estimation results and response of the loaded drive system.

Then, we test the performance of the estimator on loading and unloading. The drive at rest is accelerated at no-load to $150 \mathrm{rad} / \mathrm{s}$ at $0.5 \mathrm{~s}$ and full load is applied at $1 \mathrm{~s}$; we then remove the load completely at $2 \mathrm{~s}$. Later, after speed reversal, full load is applied at $4 \mathrm{~s}$, then, the load is removed completely at $5 \mathrm{~s}$. Fig. 18 shows the estimation results and the response of the sensorless drive. 

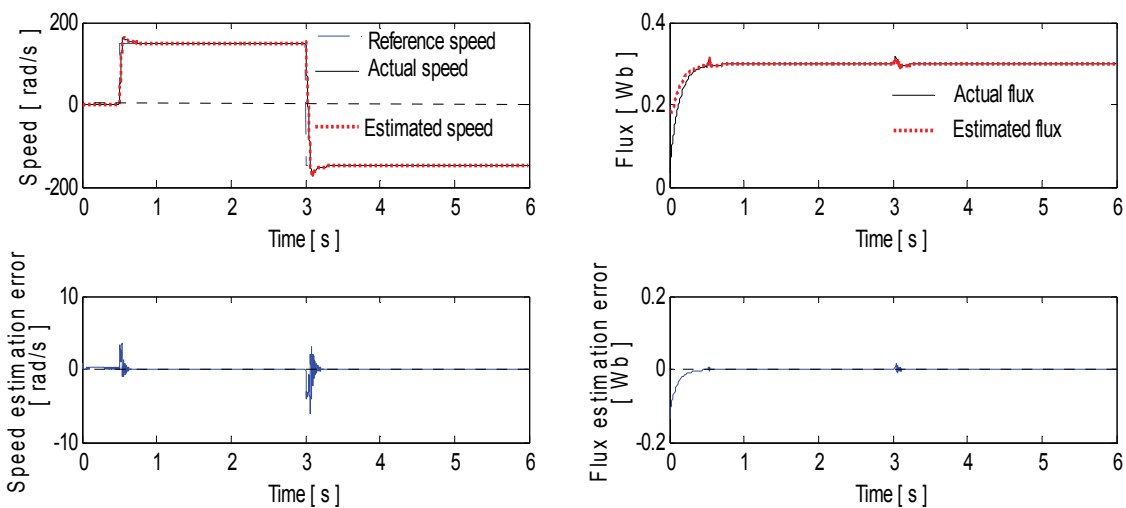

(a)

(b)

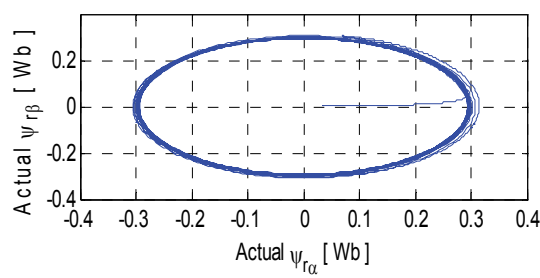

(c)

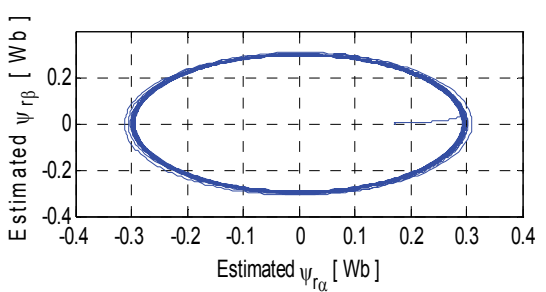

(d)

Fig. 14. Acceleration and speed reversal of the sensorless drive at no-load
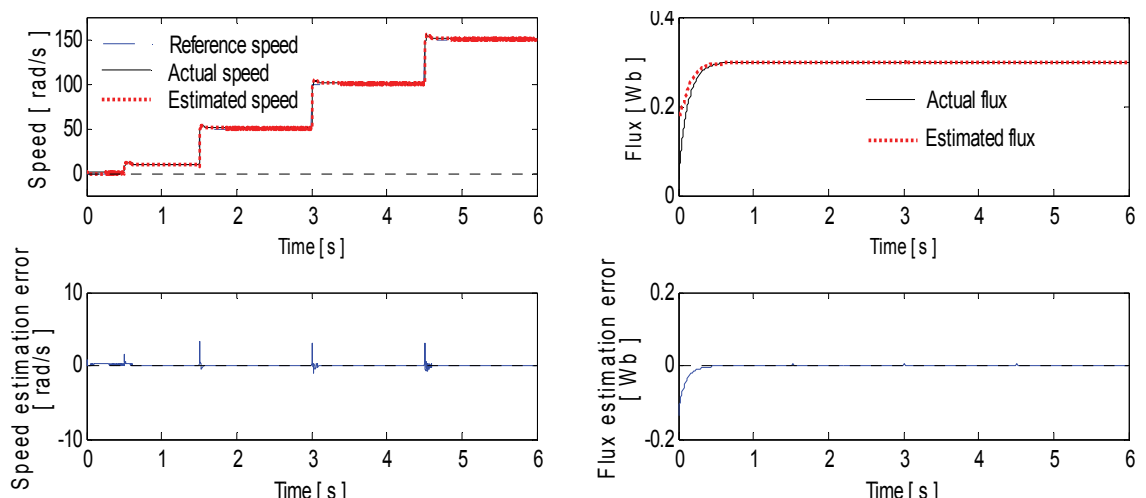

(a)

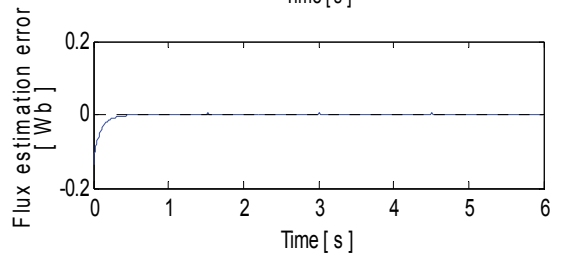

(b)

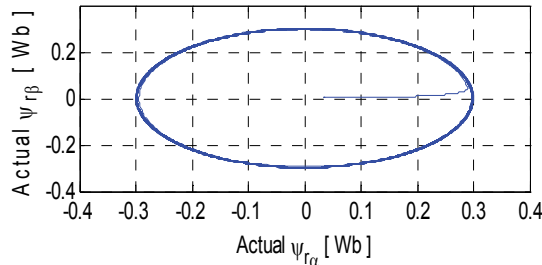

(c)

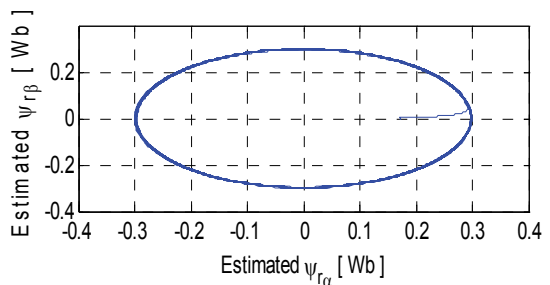

(d)

Fig. 15. No-load operation of the sensorless drive with step increase in speeds 

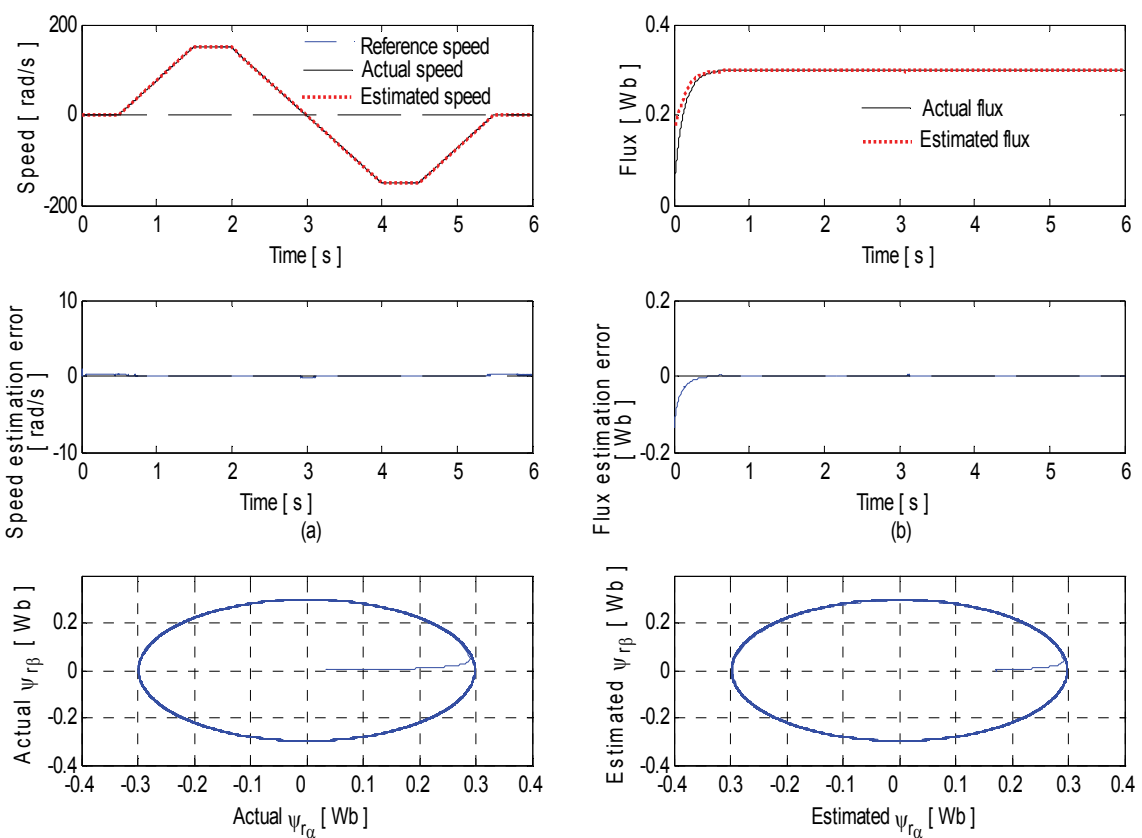

(c)

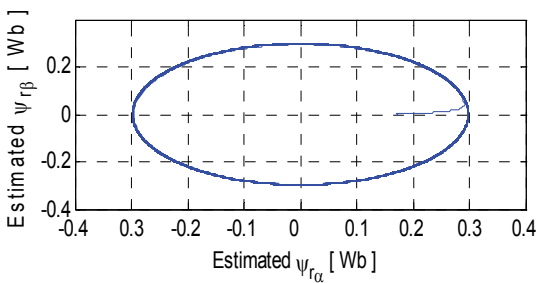

(d)

Fig. 16. No-load operation of the sensorless drive with trapezoidal reference speed
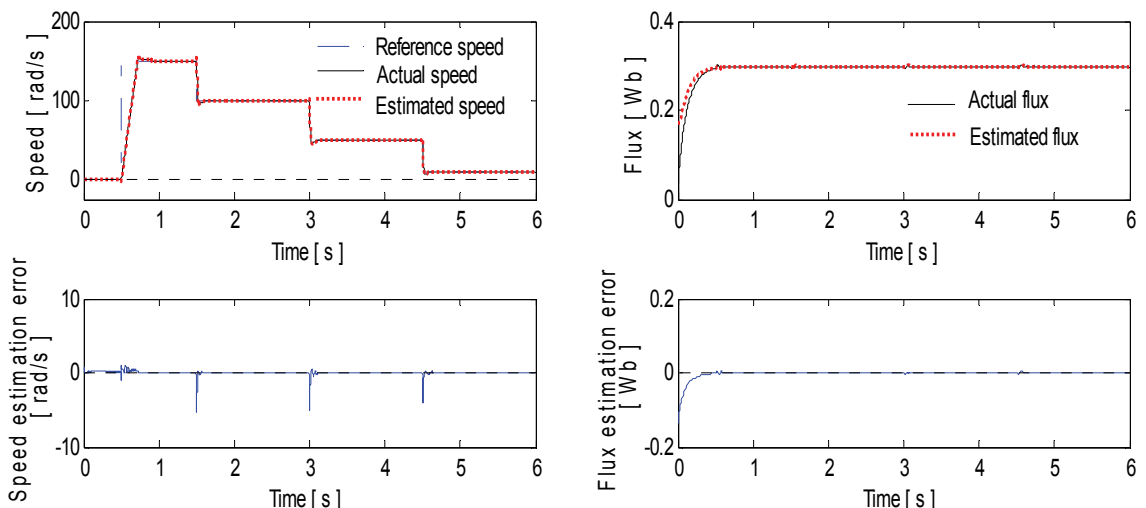

(a)

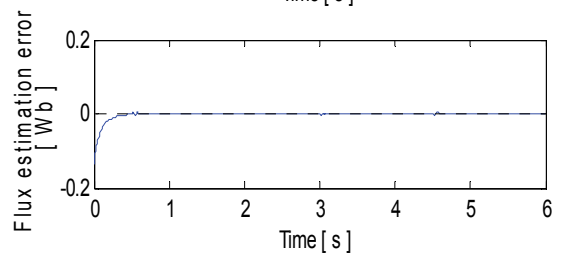

(b)

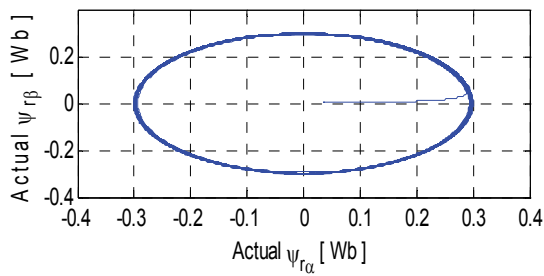

(c)

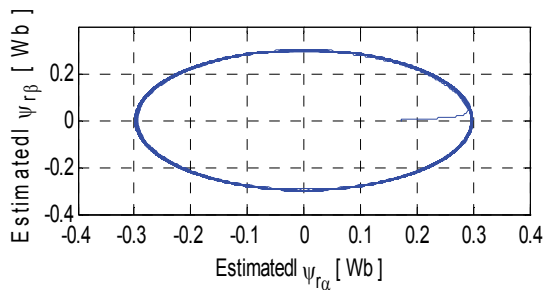

(d)

Fig. 17. Operation of the sensorless drive at full load at various speeds 

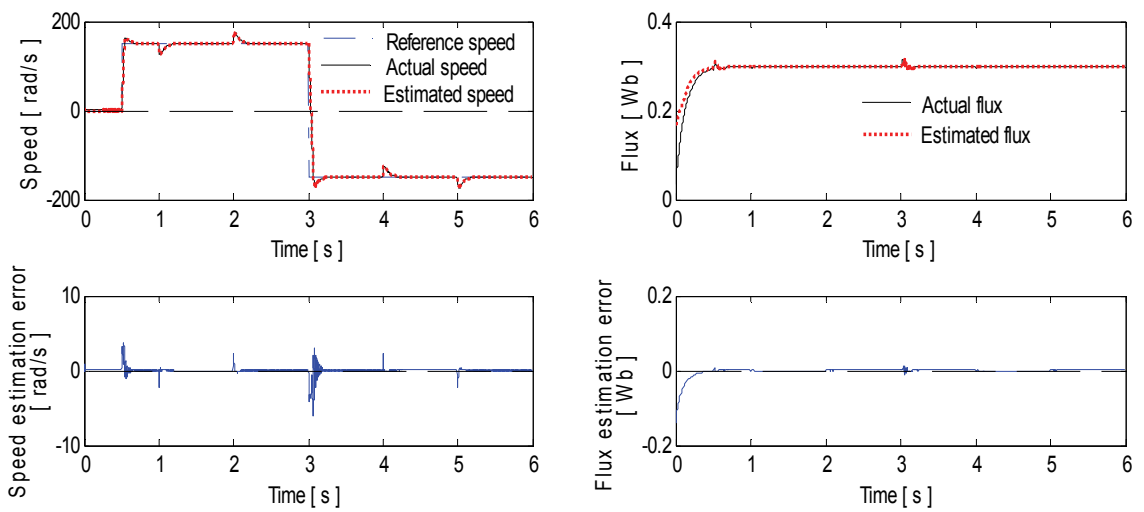

(a)

(b)

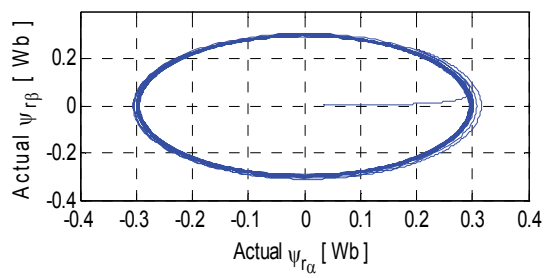

(c)

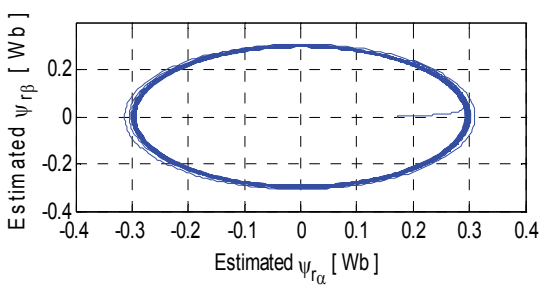

(d)

Fig. 18. Drive response on application and removal of load

\section{Conclusion and future works}

In this chapter we have presented some methods of sensorless vector control of induction motor drive using machine model-based estimation. Sensorless vector control is an active research area and the treatment of the whole model based sensorless vector control will demand a book by itself.

First, a speed estimation algorithm in vector controlled induction motor drive has been presented. The proposed method is based on observing a newly defined quantity which is a function of rotor flux and speed. The algorithm uses command flux for speed computation. The problem of decrease in estimation accuracy with the decrease in speed was overcome using a flux observer based on voltage model of the machine along with the observer of the newly defined quantity, and satisfactory results were obtained.

Then, a joint rotor flux and speed estimation algorithm has been presented. The proposed method is based on a modified Blaschke equation and on observing the newly defined quantity mentioned above. Good estimation accuracy was obtained and the response of the sensorless vector controlled drive was found to be satisfactory.

The mathematical model of the motor used for implementing the estimation algorithm was derived with the assumption that the rotor speed dynamics is much slower than that of electrical states. Therefore, increase in estimation accuracy of the proposed algorithms will be observed with the increase in the size of the machine used.

The machine model developed in this chapter may be used in future for machine parameter estimation. The newly defined quantity presented in this chapter contains rotor resistance information as well, in addition to that of rotor flux and speed. Therefore, future research efforts may be made towards developing rotor resistance estimation algorithm using the 
new machine model. Further, in the proposed algorithms rotor flux was necessary for speed estimation. Future research efforts may also be made towards developing a speed estimation algorithm for which the knowledge of rotor flux is not necessary.

\section{References}

Abbondante, A. \& Brennen, M. B. (1975). Variable speed induction motor drives use electronic slip calculator based on motor voltages and currents. IEEE Trans. Ind. Appl, Vol. 1A-11, No. 5, Sept/Oct, pp. 483-488.

Ben-Brahim, L. \& Kudor, T. (1995). Implementation of an induction motor speed estimator using neural networks. Proceedings of International Power Electronics Conference, IPEC 1995, Yokohama, April, pp. 52-58.

Bodson, M.; Chiasson, J. \& Novotnak, R. T. (1995). Nonlinear Speed Observer for High Performance Induction Motor Control. IEEE Trans. Ind. Elec, Vol. 42, No. 4, Aug. pp. 337-343.

Choy, I.; Kwon, S. H.; Lim J. \& Hong, S. W. (1996). Robust Speed Estimation for Tacholess Induction Motor Drives. IEEE Electronics Letters, Vol. 32, No. 19, pp. 1836-1838.

Comnac V.; Cernat M.; Cotorogea, M. \& Draghici, I. (2001). Sensorless Direct Torque and Stator Flux Control of Induction Machines Using an Extended Kalman Filter", Proceedings of IEEE Int. Conf. on Control Appl, Mexico, Sept. 5-7, pp. 674-679.

Du T.; Vas, P. \& Stronach, F. (1995). Design and Application of Extended Observers for Joint State and Parameter Estimation in High Performance AC Drives. IEE Proc. Elec. Power Appl., Vol. 142, No. 2, pp. 71-78.

Fodor, D. ; Ionescu, F. ; Floricau, D. ; Six, J.P. ; Delarue, P. ; Diana, D. \& Griva, G. (1995). Neural Networks Applied for Induction Motor Speed Sensorless Estimation. Proceedings of the IEEE International Symposium on Industrial Electronics, ISIE' 95, July 10-14, Athens, pp. 181-186.

Gopinath, B. (1971). On the Control of Linear Multiple Input-Output Systems. Bell System Technical Journal, Vol. 50, No. 3, March, pp. 1063-1081.

Haghgoeian, F.; Ouhrouche, M. \& Thongam, J. S. (2005). MRAS-based speed estimation for an induction motor sensorless drive using neural networks. WSEAS Transactions on Systems, Vol. 4, No. 12, December, pp. 2346-2352.

Jansen, P. L. \& Lorenz, R. D. (1994). A physically insightful approach to the design and accuracy assessment of flux observers for field oriented induction machine drives. IEEE Trans. Ind. App., Vol. 30, No. 1, Jan. /Feb., pp. 101-110.

Kim, S. H.; Park, T. S.; Yoo, J. Y. \& Park, G. T. (2001). Speed-sensorless vector control of an induction motor using neural network speed estimation. IEEE Trans. Ind. Elec, Vol. 48, No. 3, June, pp. 609-614.

Kim, Y. R.; Sul S. K. \& and Park, M. H. (1994). Speed sensorless vector control of induction motor using extended Kalman filter. IEEE Trans. Ind. Appl., Vol. 30, No. 5, Sept/Oct, pp. 1225-1233.

Kubota, H.; Matsuse K. \& Nakano, T. (1993). DSP-based speed adaptative flux observer of induction motor. IEEE Trans. Ind. Appl, Vol. 29, No. 2, March/April, pp. 344-348.

Liu, J. J.; Kung, I. C. \& Chao, H. C. (2001). Speed estimation of induction motor using a nonlinear identification technique. Proc. Natl. Sci. Counc. ROC (A), Vol. 25, No. 2, pp. 107-114. 
Ma, X. \& Gui, Y. (2002). Extended Kalman filter for speed sensor-less DTC based on DSP. Proc. of the $4^{\text {th }}$ World Cong. on Intelligent Control and Automation, Shanghai, China, June 10-14, pp. 119-122.

Minami, K.; Veley-Reyez, M.; Elten, D.; Verghese, G. C. \& Filbert, D. (1991). Multi-stage speed and parameter estimation for induction machines. Proceedings of the IEEE Power Electronics Specialists Conf., Boston, USA, pp. 596-604.

Ohtani, T.; Takada, N. \& and Tanaka, K. (1992). Vector control of induction motor without shaft encoder. IEEE Trans. Ind. Appl, Vol. 28, No. 1, Jan/Feb, pp. 157-164.

Pappano, V.; Lyshevski, S. E. \& Friedland, B. (1998). Identification of induction motor parameters. Proceedings of the 37th IEEE Conf. on Decision and Control, Tampa, Florida, USA, December 16-18, pp. 989-994.

Peng, F. Z. \& Fukao, T. (1994). Robust speed identification for speed sensorless vector control of induction motors. IEEE Trans. Ind. Appl, Vol. 30, No. 5, Sept/Oct., pp. 1234-1240.

Rowan, T. M. \& Kerkman, R. J. (1986). A new synchronous current regulator and an analysis of current-regulated PWM inverters. IEEE Trans. Ind. Appl, Vol. IA-22, No. 4, July/Aug., pp. 678-690.

Schauder, C. (1992). Adaptive speed identification for vector control of induction motors without rotational transducers. IEEE Trans. Ind. Appl, Vol. 28, No. 5, Sept./Oct., pp. 1054-1061.

Sathiakumar, S. (2000). Dynamic flux observer for induction motor speed control. Proceedings of Australian Universities Power Engineering Conf. AUPEC 2000, Brisbane, Australia, 24-27 Sept., pp. 108-113.

Simoes, M. G. \& Bose, B. K. (1995). Neural network based estimation of feedback signals for a vector controlled induction motor drive. IEEE Trans. Ind. Appl., Vol. 31, May/June, pp. 620-629.

Tajima, H. \& Hori, Y. (1993). Speed sensorless field-orientation control of the induction machine. IEEE Trans. Ind. Appl., Vol. 29, No. 1, pp. 175-180.

Thongam, J. S. \& Thoudam, V. P. S. (2004). Stator flux based speed estimation of induction motor drive using EKF. IETE Journal of Research, India, Vol. 50, No. 3. May-June, pp 191-197.

Thongam, J. S. \& Ouhrouche, M. (2006). Flux estimation for speed sensorless rotor flux oriented controlled induction motor drive. WSEAS Transactions on Systems, Vol. 5, No. 1, Jan., pp. 63-69.

Thongam, J. S. \& Ouhrouche, M. (2007). A novel speed estimation algorithm in indirect vector controlled induction motor drive. International Journal of Power and Energy Systems, Vol. 27, No. 3, 2007, pp. 294-298.

Toqeer, R. S. \& Bayindir, N. S. (2003). Speed estimation of an induction motor using Elman neural network. Neuro Computing, Volume 55, Issues 3-4, October, pp. 727- 730.

Velez-Reyes, M.; Minami, K. \& Verghese, G. C. (1989). Recursive speed and parameter estimation for induction machines", IEEE/IAS Ann. Meet. Conf. Rec., San Diego, pp. 607-611.

Veleyez-Reyes, M. \& Verghese, G. C. (1992). Decomposed algorithms for speed and parameter estimation in induction machines. IFAC Symposium on Nonlinear Control System Design, Bordeaux, France, pp. 77-82. 
Verghese, G. C. \& Sanders, S. R. (1988). Observers for flux estimation in induction machines. IEEE Trans. Ind. Elec, Vol. 35, No. 1, Feb., pp. 85-94.

Yan, Z.; Jin C. \& Utkin, V. I. (2000). Sensorless sliding-mode control of induction motors. IEEE Trans. Ind. Elec, Vol. 47, No. 6, Dec., pp. 1286-1297. 


\title{
Feedback Linearization of Speed-Sensorless Induction Motor Control with Torque Compensation
}

\author{
Cristiane Cauduro Gastaldini ${ }^{1}$, Rodrigo Zelir Azzolin ${ }^{2}$, \\ Rodrigo Padilha Vieira ${ }^{3}$ and Hilton Abílio Gründling ${ }^{4}$ \\ 1,2,3,4 Federal University of Santa Maria \\ ${ }^{2}$ Federal University of Rio Grande \\ ${ }^{3}$ Federal University of Pampa \\ Brazil
}

\section{Introduction}

This chapter addresses the problem of controlling a three-phase Induction Motor (IM) without mechanical sensor (i.e. speed, position or torque measurements). The elimination of the mechanical sensor is an important advent in the field of low and medium IM servomechanism; such as belt conveyors, cranes, electric vehicles, pumps, fans, etc. The absence of this sensor (speed, position or torque) reduces cost and size, and increases reliability of the overall system. Furthermore, these sensors are often difficult to install in certain applications and are susceptible to electromagnetic interference. In fact, sensorless servomechanism may substitute a measure value by an estimated one without deteriorating the drive dynamic performance especially under uncertain load torque.

Many approaches for IM sensorless servomechanism have been proposed in the literature is related to vector-controlled methodologies. One of the proposed nonlinear control methodologies is based on Feedback Linearization Control (FLC), as first introduced by (Marino et al., 1990). FLC provides rotor speed regulation, rotor flux amplitude decoupling and torque compensation. Although the strategy presented by (Marino et al., 1990) was not a sensorless control strategy, fundamental principles of FLC follow servomechanism design of sensorless control strategies, such as (Gastaldini \& Grundling, 2009; Marino et al., 2004; Montanari et al., 2007; 2006).

The purpose of this chapter is to present the development of two FLC control strategies in the presence of torque disturbance or load variation, especially under low rotor speed conditions. Both control strategies are easily implemented in fixed point DSP, such as TMS320F2812 used on real time experiments and can be easily reproduced in the industry. Furthermore, an analysis comparing the implementation and the limitation of both strategies is presented. In order to implement the control law, these algorithms made use of only two-phase IM stator currents measurement. The values of rotor speed and load torque states used in the control algorithm are estimated using a Model Reference Adaptive System (MRAS) (Peng \& Fukao, 1994) and a Kalman filter (Cardoso \& Gründling, 2009), respectively.

This chapter is organized as follows: Section 2 presents the fifth-order IM mathematical model. Section 3 introduces the feedback linearization modelling of IM control. A simplified 
FLC control strategy is described in Section 4. The proposed methods for speed and torque estimation, MRAS and Kalman filter algorithms, respectively, are developed in Sections 5 and 6. State variable filter is used to obtain derivative signals necessary for implementation of the control algorithm, and this is presented in section 7. Digital implementation in fixed point DSP TMS320F2812 and real time experimental results are given in Section 8. Finally, Section 9 presents the conclusions.

\section{Induction motor mathematical model}

A three-phase $\mathrm{N}$ pole pair induction motor is expressed in an equivalent two-phase model in an arbitrary rotating reference frame (q-d), according to (Krause, 1986) and (Leonhard, 1996) according to the fifth-order model, as

$$
\begin{gathered}
\frac{d}{d t} I_{q s}=-a_{12} I_{q s}-\omega_{s} I_{d s}+a_{13} a_{11} \lambda_{q r}-a_{13} N \omega \lambda_{d r}+a_{14} V_{q s} \\
\frac{d}{d t} I_{d s}=-a_{12} I_{d s}+\omega_{s} I_{q s}+a_{13} a_{11} \lambda_{d r}+a_{13} N \omega \lambda_{q r}+a_{14} V_{d s} \\
\frac{d}{d t} \lambda_{q r}=-a_{11} \lambda_{q r}-\left(\omega_{s}-N \omega\right) \lambda_{d r}+a_{11} L_{m} I_{q s} \\
\frac{d}{d t} \lambda_{d r}=-a_{11} \lambda_{d r}+\left(\omega_{s}-N \omega\right) \lambda_{q r}+a_{11} L_{m} I_{d s} \\
\frac{d}{d t} \omega=\mu \cdot\left(\lambda_{d r} I_{q s}-\lambda_{q r} I_{d s}\right)-\frac{B}{J} \omega-\frac{T_{L}}{J} \\
T_{e}=\mu \cdot J \cdot\left(\lambda_{d r} I_{q s}-\lambda_{q r} I_{d s}\right)
\end{gathered}
$$

In equations (1)-(6): $\mathbf{I}_{\mathbf{s}}=\left(I_{q s}, I_{d s}\right), \lambda_{\mathbf{r}}=\left(\lambda_{q r}, \lambda_{d r}\right)$ and $\mathbf{V}_{\mathbf{s}}=\left(V_{q s}, V_{d s}\right)$ denote stator current, rotor flux and stator voltage vectors, where subscripts $\mathrm{d}$ and $\mathrm{q}$ stand for vector components in (q-d) reference frame; $\omega$ is the rotor speed, the load torque $T_{L}$, electric torque $T_{e}$ and $\omega_{s}$ is the stationary speed, $\theta_{0}$ is the angular position of the $(\mathrm{q}-\mathrm{d})$ reference frame with respect to a fixed stator reference frame $(\alpha-\beta)$, where physical variables are defined. Transformed variables related to three-phase (RST) system are given by

$$
\mathbf{x}_{\alpha \beta}=\mathbf{K} \cdot \mathbf{x}_{\mathbf{R S T}}
$$

Let

$$
\mathbf{x}_{\mathbf{q d}}=\mathbf{e}^{j \theta_{0}} \mathbf{x}_{\alpha \beta}
$$

with $\mathbf{e}^{j \theta_{0}}=\left[\begin{array}{cc}\cos \theta_{0} & -\sin \theta_{0} \\ \sin \theta_{0} & \cos \theta_{0}\end{array}\right]$ and $\mathbf{K}=\sqrt{\frac{2}{3}}\left[\begin{array}{ccc}1 & -\frac{1}{2} & -\frac{1}{2} \\ 0 & -\frac{\sqrt{3}}{2} & -\frac{\sqrt{3}}{2}\end{array}\right]$.

$\mathbf{x}_{\mathbf{q d}}$ and $\mathbf{x}_{\alpha \beta}$ stand for two-dimensional voltage flux and stator current vector, respectively on $(\mathrm{q}-\mathrm{d})$ and $(\alpha-\beta)$ reference frame.

The relations between mechanical and electrical parameters in the above equations are $a_{0} \triangleq L_{s} L_{r}-L_{m}^{2}, a_{11} \triangleq \frac{R_{r}}{L_{r}}, a_{12} \triangleq\left(\frac{L_{s} L_{r}}{a_{0}} \frac{R_{s}}{L_{s}}+\frac{L_{m}^{2}}{a_{0}} a_{11}\right), a_{13} \triangleq \frac{L_{m}}{a_{0}}, a_{14} \triangleq \frac{L_{r}}{a_{0}}$ and $\mu \triangleq \frac{N L_{m}}{J L_{r}}$; 
where $R_{s}, R_{r}, L_{s}$ and $L_{r}$ are the stator/rotor resistances and inductances, $L_{m}$ is the magnetizing inductance, $J$ is the rotor inertia, $B$ is the viscous coefficient and $N$ is the number of pole pairs. In the control design, the viscous coefficient of (5) is considered to be approximately zero, i.e. $B \approx 0$.

\section{Feedback Linearization Control}

The feedback Linearization Control (FLC) general specifications are two outputs - rotor speed and rotor flux modulus, as

$$
\mathbf{y}_{\mathbf{1}}=\left[\begin{array}{ll}
\omega & \sqrt{\lambda_{q r}^{2}+\lambda_{d r}^{2}}
\end{array}\right]^{T} \triangleq\left[\begin{array}{ll}
\omega & \left|\lambda_{\mathbf{r}}\right|
\end{array}\right]^{T}
$$

which is controlled by two-dimensional stator voltage vector $\mathbf{V}_{\mathbf{s}}$, on the basis of measured variables vector $\mathbf{y}_{2}=\mathbf{I}_{\mathbf{s}}$. The development concept of this control strategy is completely described in (Marino et al., 1990) and it will be omitted here. Following the concept of indirect field orientation developed by Blaschke, (Krause, 1986) and (Leonhard, 1996), the purpose of FLC control is to align rotor flux vector with the d-axis reference frame, i.e.

$$
\lambda_{d r}=\left|\lambda_{\mathbf{r}}\right| \quad \lambda_{q r}=0
$$

The condition expressed in (10) guarantees the exact decoupling of flux dynamics of (1)-(4) from the speed dynamics. Once rotor flux is not directly measured, only asymptotic field orientation is possible, according to (Marino et al., 1990) and (Peresada \& Tonielli, 2000), then

$$
\lim _{t \rightarrow \infty} \lambda_{d r}=\left|\lambda_{\mathbf{r}}\right| \quad \lim _{t \rightarrow \infty} \lambda_{q r}=0
$$

It is defined $\mathbf{y}_{1}^{*}=\left[\begin{array}{ll}\omega_{r e f} & \lambda_{r}^{*}\end{array}\right]^{T}$, where $\omega_{r e f}$ and $\lambda_{r}^{*}$ are reference trajectories of rotor speed and rotor flux. The speed tracking, flux regulation control problem under speed sensorless conditions is formulated considering IM model (1)-(5) under the following conditions:

(a) Stator currents are measurable;

(b) Motor parameters are known and considered constant;

(c) Load torque is estimated and it is applied after motor flux excitation;

(d) Initial conditions of IM state variables are known;

(e) $\lambda_{r}^{*}$ is the flux constant reference value and estimated speed $\widehat{\omega}$ and reference speed $\omega_{r e f}$ are the smooth reference bounded speed signals.

FLC equations are developed considering the fifth-order IM model under the assumption that estimated speed tracks real speed, and therefore it is acceptable to replace measured speed with estimated speed (i.e. $\widehat{\omega}_{k} \approx \omega$ ). In addition, the torque value is estimated using a Kalman filter. Fig. 1 presents the block diagram of FLC Control.

\subsection{Flux controller}

From the decoupling properties of field oriented transformation (10), the control objective of the flux controller is to generate a flux vector aligned with the d-axis to guarantee induction motor magnetization.

Then, substituting (10) in (4)

$$
i_{d s}^{*}=\left(a_{11} \lambda_{r}^{*}+\frac{d}{d t}\left|\lambda_{\mathbf{r}}\right|\right) \frac{1}{a_{11} L_{m}}
$$



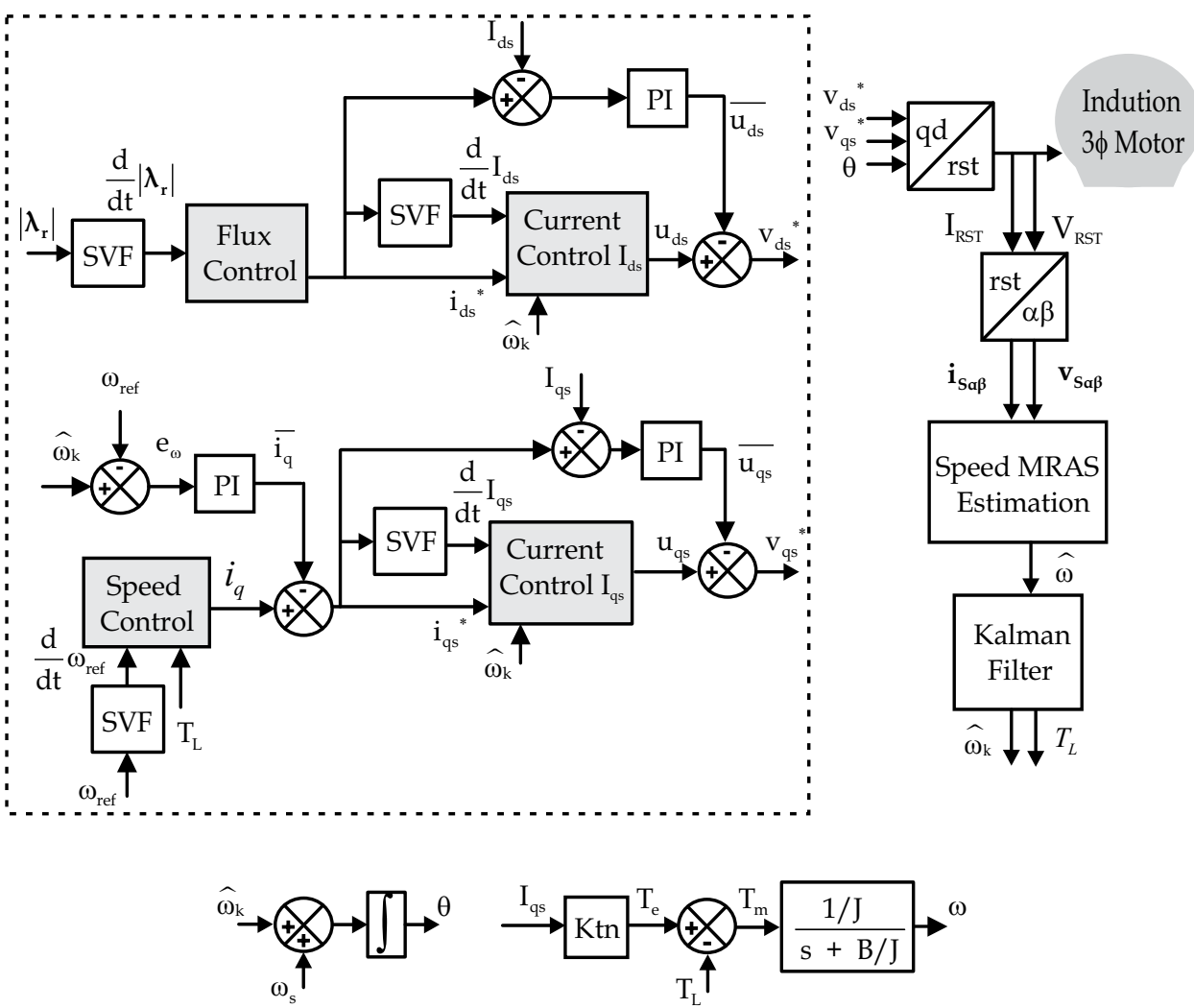

Fig. 1. Feedback Linearization Control proposed

The rotor flux $\left|\lambda_{\mathbf{r}}\right|$ is estimated by a model derived from the induction motor mathematical model, (3) and (4), that makes use of measured stator currents $\left(I_{q s}, I_{d s}\right)$ and estimated speed $\widehat{\omega}$ variables.

$$
\frac{d}{d t} \lambda_{\mathbf{r}}=-a_{11} \lambda_{\mathbf{r}}-j\left(\omega_{s}-N \omega\right) \lambda_{\mathbf{r}}+a_{11} L_{m} \mathbf{I}_{\mathbf{s}}
$$

where the stationary speed is $\omega_{s}=N \widehat{\omega}+\frac{a_{11} L_{m}}{\lambda^{*}} i_{q s}^{*}$. The digital implementation of the flux controller is made using Euler discretization and the derivative rotor flux signal is obtained by a state variable filter (SVF).

\subsection{Speed controller}

The speed control algorithm uses the same strategy adopted for the flux subsystem and it is computed from (5), as

$$
i_{q}=\frac{1}{\mu \lambda_{r}^{*}}\left(\frac{\widehat{T}_{L}}{J}+\frac{d}{d t} \omega_{r e f}\right)
$$

To compensate for speed error between estimated speed and reference speed, (i.e. $\left.e_{\omega}=\widehat{\omega}-\omega_{\text {ref }}\right)$, a proportional integral compensation is proposed, as follows 


$$
\overline{i_{q}}=\left(k_{p \_i q}+\frac{k_{i \_i q}}{s}\right) e_{\omega}
$$

These gains values $\left(k_{p \_i q}, k_{i \_i q}\right)$ are determined considering an induction motor mechanical model. The reference quadrature component stator speed current is derived from (14)-(15), as

$$
i_{q s}^{*}=i_{q}-\overline{i_{q}}
$$

In DSP implementation, the speed controller is discretized using the Euler method and the rotor speed derivative (14) is computed by a SVF.

\subsection{Currents controller}

From (1) and (2), the currents controller is obtained, as

$$
u_{q s}=\frac{1}{a_{14}}\left(a_{12} i_{q s}^{*}+\omega_{s} i_{d s}^{*}+a_{13} \lambda_{r}^{*} N\left(\omega_{r e f}+e_{\omega}\right)+\frac{d}{d t} I_{q s}\right)
$$

and

$$
u_{d s}=\frac{1}{a_{14}}\left(a_{12} i_{d s}^{*}+\omega_{s} i_{q s}^{*}+a_{11} a_{13} \lambda_{r}+\frac{d}{d t} I_{d s}\right)
$$

where proportional integral gains of the current error

$$
\bar{u}_{q s}=\left(k_{p v}+\frac{k_{i v}}{s}\right) \widetilde{i}_{q s}
$$

and

$$
\bar{u}_{d s}=\left(k_{p v}+\frac{k_{i v}}{s}\right) \widetilde{i}_{d s}
$$

in which $\widetilde{i}_{q s}=I_{q s}-i_{q s}^{*}$ and $\widetilde{i}_{d s}=I_{d s}-i_{d s}^{*}$.

These gains $\left(k_{p v}, k_{i v}\right)$ are determined considering a simplified induction motor electrical model, which is obtained by load and locked rotor test. Hence, current controllers are expressed as

$$
\begin{aligned}
& v_{q s}^{*}=u_{q s}-\bar{u}_{q s} \\
& v_{d s}^{*}=u_{d s}-\bar{u}_{d s}
\end{aligned}
$$

In DSP, currents controller are digitally implemented using discretized equation (17)-(22) based on the Euler method, and the stator current derivative is obtained by SVF using stator currents measures.

\section{Simplified feedback linearization control}

In order to reduce the number of computation requirements, a simplified feedback linearization control scheme is proposed. In this control scheme, one part of the current controller (6)-(7) is suppressed and only a proportional integral controller is used. This modification minimizes the influence of parameters variation in the control system.

Fig. 2 presents the block diagram of the Simplified FLC proposed.

The currents controller of simplified FLC are defined as 


$$
\begin{aligned}
& v_{q s}^{*}=\left(k_{p v}+\frac{k_{i v}}{s}\right) \widetilde{i}_{q s} \\
& v_{d s}^{*}=\left(k_{p v}+\frac{k_{i v}}{s}\right) \widetilde{i}_{d s}
\end{aligned}
$$

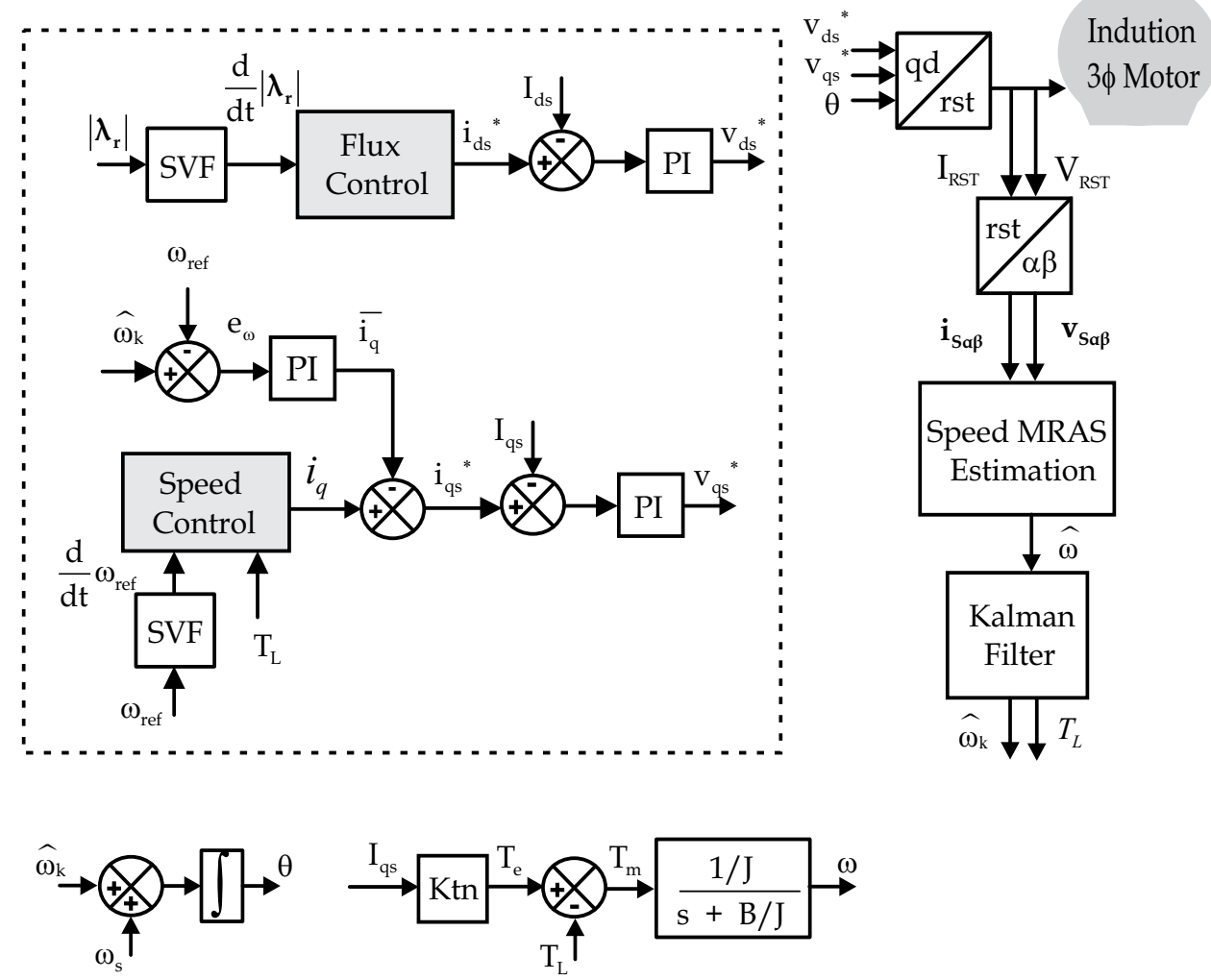

Fig. 2. Proposed Simplified Feedback Linearization Control

Flux and Speed Controller are computed exactly as in the previous scheme, as (12) and (14)-(16).

\section{Speed estimation - MRAS algorithm}

A squirrel-cage three-phase induction motor model expressed in a stationary frame can be modelled using complex stator and rotor voltage as in (Peng \& Fukao, 1994)

for squirrel-cage IM $\mathbf{v}_{\mathbf{r}}=0$

$$
\mathbf{v}_{\mathbf{s}}=R_{s} \mathbf{i}_{\mathbf{s}}+L_{s} \frac{d}{d t} \mathbf{i}_{\mathbf{s}}+L_{m} \frac{d}{d t} \mathbf{i}_{\mathbf{r}}
$$

$$
0=R_{r} \mathbf{i}_{\mathbf{r}}-j N \omega L_{r} \mathbf{i}_{\mathbf{r}}-j N \omega L_{m} \mathbf{i}_{\mathbf{s}}+L_{r} \frac{d}{d t} \mathbf{i}_{\mathbf{r}}+L_{m} \frac{d}{d t} \mathbf{i}_{\mathbf{s}}
$$


The voltage and the current space vectors are given as $\overline{\mathbf{x}}=x_{\alpha}+j x_{\beta}, \overline{\mathbf{x}} \in\left\{\mathbf{v}_{\mathbf{s}}, \mathbf{i}_{\mathbf{s}}, \mathbf{i}_{\mathbf{r}}\right\}$, relative to the transformed variables present in (7). The induction motor magnetizing current is expressed by

$$
\mathbf{i}_{\mathbf{m}}=\frac{L_{r}}{L_{m}} \mathbf{i}_{\mathbf{r}}+\mathbf{i}_{\mathbf{s}}
$$

Two independent observers are derived to estimate the components of the counter-electromotive vectors.

$$
\begin{gathered}
\widehat{\mathbf{e}}_{\mathbf{m}}=\frac{L_{m}^{2}}{L_{r}} \mathbf{i}_{\mathbf{m}}=\frac{L_{m}^{2}}{L_{r}}\left(\omega \mathbf{i}_{\mathbf{m}}-\frac{1}{T_{r}} \mathbf{i}_{\mathbf{m}}+\frac{1}{T_{r}} \mathbf{i}_{\mathbf{s}}\right) \\
\mathbf{e}_{\mathbf{m}}=\mathbf{v}_{\mathbf{s}}-R_{s} \mathbf{i}_{\mathbf{s}}-\sigma L_{s} \frac{d}{d t} \mathbf{i}_{\mathbf{s}}
\end{gathered}
$$

where $\sigma=1-\frac{L_{m}}{L_{s} L_{r}}$. The instantaneous reactive power maintains the magnetizing current, and its value is defined by cross product of the counter-electromotive and stator current vector

$$
\mathbf{q}_{\mathrm{m}}=\mathbf{i}_{\mathbf{s}} \otimes \mathbf{e}_{\mathrm{m}}
$$

Substituting (28) and (29) for $\mathbf{e}_{\mathbf{m}}$ in (30) and noting that $\mathbf{i}_{\mathbf{s}} \otimes \mathbf{i}_{\mathbf{s}}=0$, which gives

$$
\mathbf{q}_{\mathbf{m}}=\mathbf{i}_{\mathbf{s}} \otimes\left(\mathbf{v}_{\mathbf{s}}-\sigma L_{s} \frac{d}{d t} \mathbf{i}_{\mathbf{s}}\right)
$$

and

$$
\widehat{\mathbf{q}}_{\mathbf{m}}=\frac{L_{m}^{2}}{L_{r}}\left(\left(\mathbf{i}_{\mathbf{m}} \odot \mathbf{i}_{\mathbf{s}}\right) \omega+\frac{1}{T_{r}}\left(\mathbf{i}_{\mathbf{m}} \otimes \mathbf{i}_{\mathbf{s}}\right)\right)
$$

Then, $\mathbf{q}_{\mathbf{m}}$ is the reference model of reactive power and $\widehat{\mathbf{q}}_{\mathbf{m}}$ is the adjustable model. The estimated speed is produced by the proportional integral adaptation mechanism error of both models, and an MRAS system can be drawn as in Fig.3

This algorithm is customary for speed estimation and simple to implement in fixed point DSP, such as in (Gastaldini \& Grundling, 2009; Orlowska-Kowalska \& Dybkowski, 2010; Vieira et al., 2009).

The SVF blocks are state variable filters and are explained in greater detail in Section 7 . These filters compute derivative signals and are applied in voltage signals to avoid addition noise and phase delay among the vectors as was proposed by (Martins et al., 2006).

\section{Load torque estimation - Kalman filter}

The reduced mechanical IM system can be represented by the following equations

$$
\begin{gathered}
\frac{d}{d t}\left[\begin{array}{c}
\omega \\
T_{L}
\end{array}\right]=\left[\begin{array}{cc}
-\frac{B_{n}}{J} & -\frac{1}{J} \\
0 & 0
\end{array}\right]\left[\begin{array}{l}
\omega \\
T_{L}
\end{array}\right]+\left[\begin{array}{c}
\frac{1}{J} \\
0
\end{array}\right] T_{e} \\
y=\left[\begin{array}{ll}
1 & 0
\end{array}\right]\left[\begin{array}{c}
\omega \\
T_{L}
\end{array}\right]
\end{gathered}
$$

The Kalman Filter could be used to provide the value of torque load or disturbances - $T_{L}$. Since (15)-(16) is nonlinear, the Kalman filter linearizes the model at the actual operating 


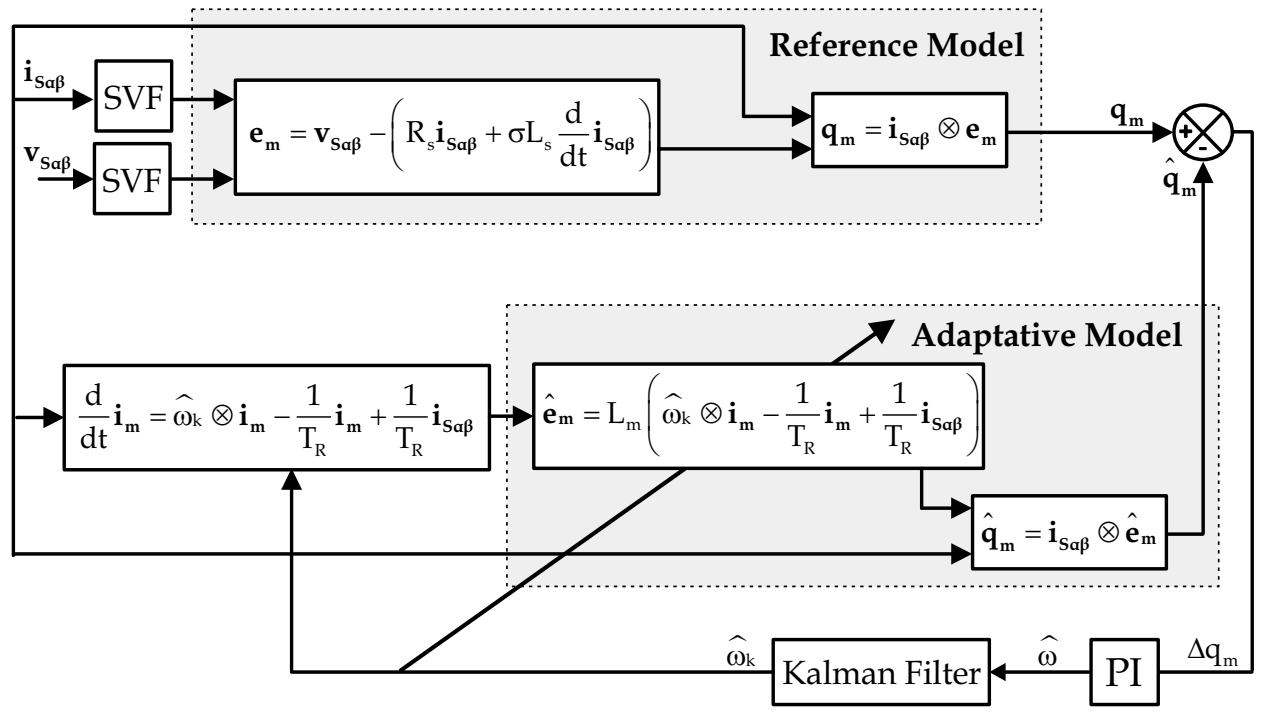

Fig. 3. Reactive Power MRAS Speed Estimation

point (Aström \& Wittenmark, 1997). In addition, this filter takes into account the signal noise, which could be generated as pulse width modulation drivers. Assuming the definitions $\mathbf{x}_{k}=\left[\begin{array}{ll}\widehat{\omega}_{k} & \widehat{T}_{L}\end{array}\right]^{T}, \mathbf{A}_{\mathbf{m}}=\left[\begin{array}{cc}-\frac{B_{n}}{J} & -\frac{1}{J} \\ 0 & 0\end{array}\right], \mathbf{B}_{\mathbf{m}}=\left[\begin{array}{c}\frac{1}{J} \\ 0\end{array}\right], \mathbf{C}_{\mathbf{m}}=\left[\begin{array}{ll}1 & 0\end{array}\right]$ and $y_{k}=\widehat{\omega}$. Then, the recursive equation for the discrete time Kalman Filter (De Campos et al., 2000) is described by

$$
\mathbf{K}(k)=\mathbf{P}(k) \mathbf{C}_{\mathbf{m}}^{T}\left(\mathbf{C}_{\mathbf{m}} \mathbf{P}(k) \mathbf{C}_{\mathbf{m}}^{T}+\mathbf{R}\right)^{-1}
$$

where $\mathbf{K}(k)$ is the Kalman gain. The covariance matrix $\mathbf{P}(k)$ is given by

$$
\mathbf{P}(k+1)=\left(\mathbf{I}-\mathbf{A}_{\mathbf{m}} t_{s}\right)\left(\mathbf{P}(k)-\mathbf{K}(k) \mathbf{C}_{\mathbf{m}} \mathbf{P}(k)\right)\left(\mathbf{I}-\mathbf{A}_{\mathbf{m}} t_{S}\right)^{T}+\left(\mathbf{B}_{\mathbf{m}} t_{S}\right) \mathbf{Q}\left(\mathbf{B}_{\mathbf{m}} t_{S}\right)^{T}
$$

Therefore, the estimated torque $\widehat{T}_{L}$ is one observed state of the Kalman filter

$$
\widehat{\mathbf{x}}_{\mathbf{k}}(k+1)=\left(\mathbf{I}-\mathbf{A}_{\mathbf{m}} t_{s}\right) \widehat{\mathbf{x}}_{\mathbf{k}}(k)+\mathbf{B}_{\mathbf{m}} t_{s} u(k)+\left(\mathbf{I}-\mathbf{A}_{\mathbf{m}} t_{s}\right) \mathbf{K}(k)\left(\widehat{\omega}-\mathbf{C}_{\mathbf{m}} \widehat{\mathbf{x}}_{\mathbf{k}}(k)\right)
$$

giving $\widehat{\omega} \approx \widehat{\omega}_{k}$ and $\widehat{\mathbf{x}}_{\mathbf{k}}(k)=\left[\begin{array}{cc}\widehat{\omega}_{k} & T_{L}\end{array}\right]^{T}$.

The matrices $\mathbf{R}$ and $\mathbf{Q}$ are defined according to noise elements of predicted state variables, taking into account the measurement noise covariance $\mathbf{R}$ and the plant noise covariance $\mathbf{Q}$.

\section{State variable filter}

The state variable filter (SVF) is used to mathematically evaluate differentiation signals. This filter is necessary in the implementation of FLC and MRAS algorithms. The transfer function of SVF is of second order as it is necessary to obtain the first order derivative.

$$
G_{s v f}=\frac{\omega_{s v f}}{\left(s+\omega_{s v f}\right)^{2}}
$$


where $\omega_{s v f}$ is the filter bandwidth defined at around 5 to 10 times the input frequency signal $u_{\text {svf. }}$.

The discretized transfer function, using the Euler method, can be performed in state-space as

$$
\mathbf{x}_{\mathbf{s v f}}(k+1)=\mathbf{A}_{\mathbf{s v f}} \mathbf{x}_{\mathbf{s v f}}(k)+\mathbf{B}_{\mathbf{s v f}} u_{s v f}(k)
$$

where $\mathbf{A}_{\mathbf{s v f}}=\left[\begin{array}{cc}1 & 1 \\ -\omega_{s v f}^{2} & 1-2 \omega_{s v f}\end{array}\right], \mathbf{B}_{\mathbf{s v f}}=\left[\begin{array}{c}0 \\ \omega_{\mathrm{svf}}^{2}\end{array}\right]$ and $\mathbf{x}_{\mathbf{s v f}}=\left[\begin{array}{l}x_{1} \\ x_{2}\end{array}\right]$.

The state variables $x_{1}$ and $x_{2}$ represent the input filtered signal and input derivative signal.

\section{Experimental results}

Sensorless control schemes were implemented in DSP based platform using TMS 320F2812. Experimental results were carried out on a motor with specifications: $1.5 \mathrm{cv}, 380 \mathrm{~V}, 2.56 \mathrm{~A}, 60$ $\mathrm{Hz}, R_{s}=3.24 \Omega, R_{r}=4.96 \Omega, L_{r}=404.8 m H, L_{s}=402.4 m H, L_{m}=388.5 m H, N=2$ and nominal speed of $188 \mathrm{rad} / \mathrm{s}$.

The experimental analyses are carried out with the following operational sequence:

1) The motor is excited (during $10 \mathrm{~s}$ to $12 \mathrm{~s}$ ) using a smooth flux reference trajectory.

2) Starting from zero initial value, the rotor speed reference grows linearly until it reaches the reference value. Thus, the reference rotor speed value is kept constant.

3) During stand-state, a step constant load torque is applied.

In order to generate load variation for torque disturbance analyses, the DC motor is connected to an induction motor driving-shaft. Then, the load shaft varies in accordance with DC motor field voltage and inserting a resistance on its armature. Fig. 4 and Fig. 5 depict performance of both control schemes: FLC control and simplified FLC control with rotor speed reference of $18 \mathrm{rad} / \mathrm{s}$. In these figures measure speed, estimated speed, stator (q-d) currents and estimated torque are illustrated.

Fig. 6 and Fig. 7 present experimental results with $36 \mathrm{rad} / \mathrm{s}$ rotor speed reference.

Fig. 8 and Fig. 9 show FLC control and Simplified FLC with $45 \mathrm{rad} / \mathrm{s}$ rotor speed reference.

The above figures present experimental results for low rotor speed range of FLC control and Simplified FLC control applying load torque. In accordance with the figures above, both control schemes present similar performances in steady state. It is verified that both schemes respond to compensated torque variations. With respect to Simplified FLC, it is necessary to carrefully select fixed gains in order to guarantee the alignment of the rotor flux on the $d$ axis.

\section{Conclusion}

Two different sensorless IM control schemes were proposed and developed based on nonlinear control - FLC Control and Simplified FLC Control. These control schemes are composed of a flux-speed controller, which is derived from a fifth-order IM model. In the implementation of feedback linearization control (FLC), the control algorithm presents a large number of computational requirements. In the simplified FLC scheme, a substitution of FLC currents controllers by two PI controllers is proposed to generate the stator drive voltage. In order to provide the rotor speed for both control schemes, a MRAS algorithm based on reactive power is applied.

To correctly evaluate whether this Simplified FLC does not affect control performance, a comparative experimental analysis of a FLC control and a simplified FLC control is presented. Experimental results in DSP TMS 320F2812 platform show the performance of both systems 


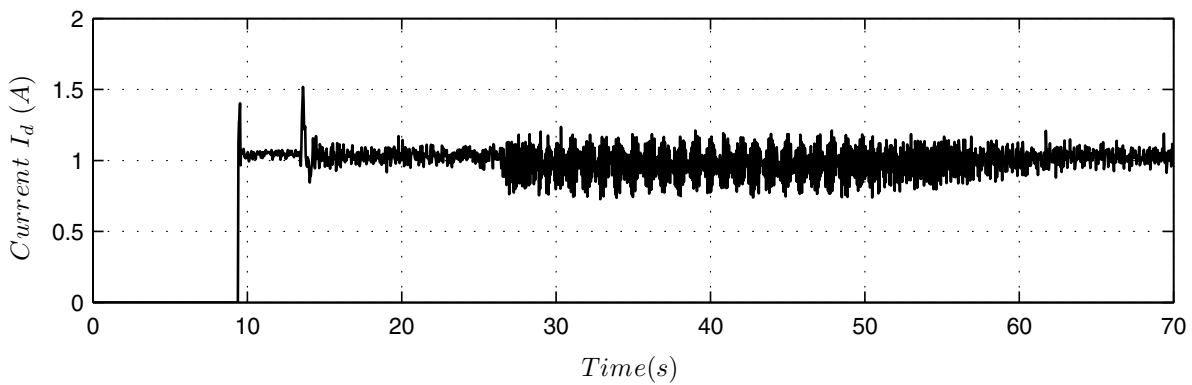

(a) IM Stator Current $I_{d s}$

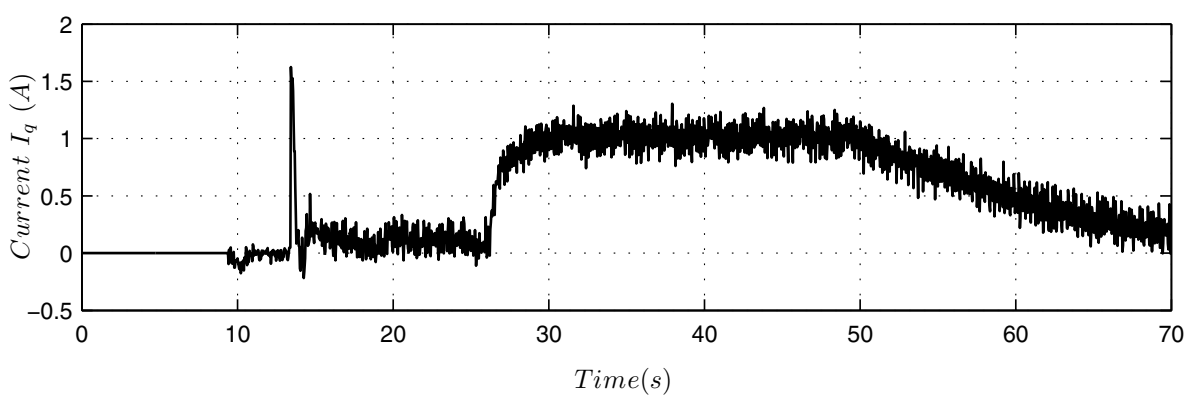

(b) IM Stator Current $I_{q s}$

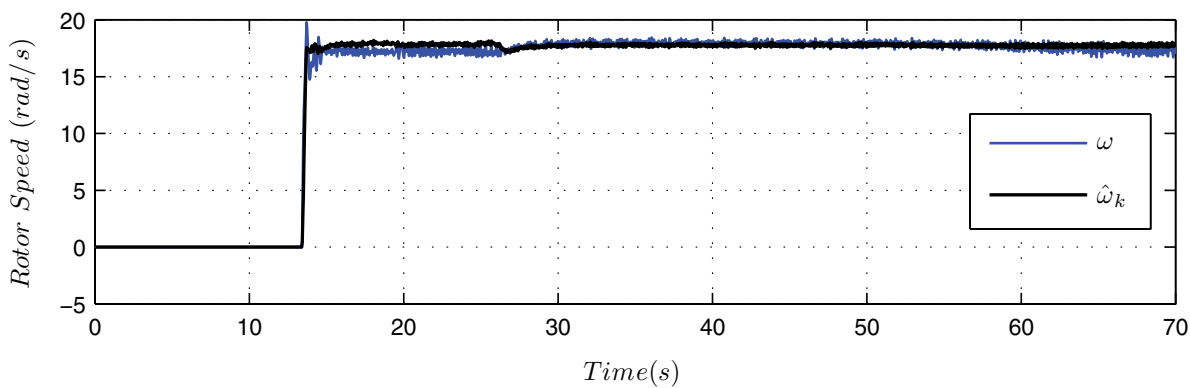

(c) Rotor Speed - Estimated and Encoder Measurement

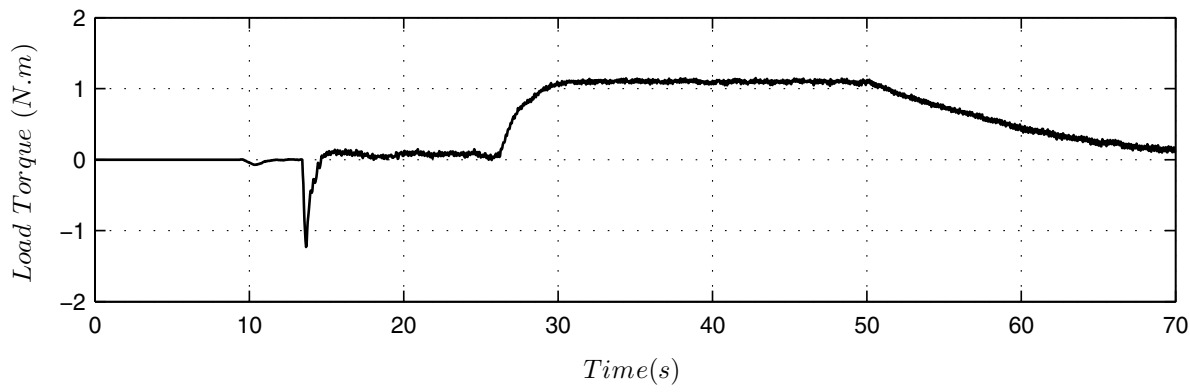

(d) Estimated Load Torque

Fig. 4. FLC control with $18 \mathrm{rad} / \mathrm{s}$ rotor speed reference 


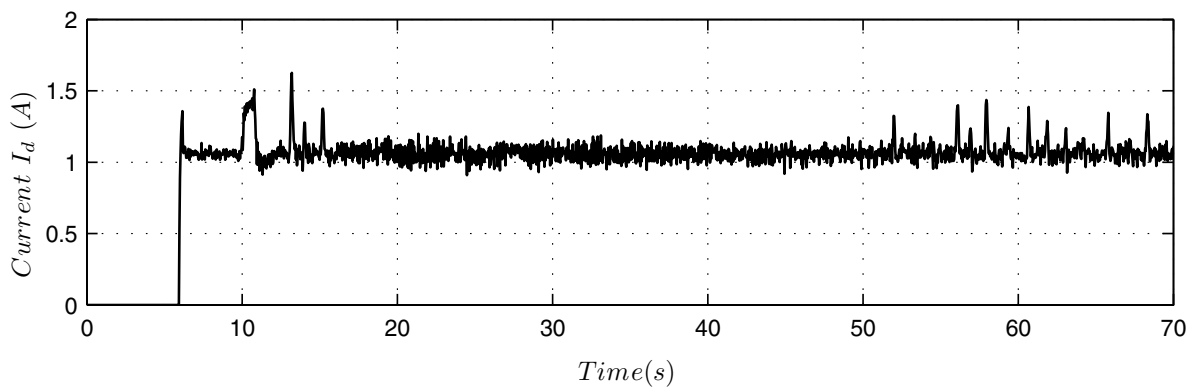

(a) IM Stator Current $I_{d s}$

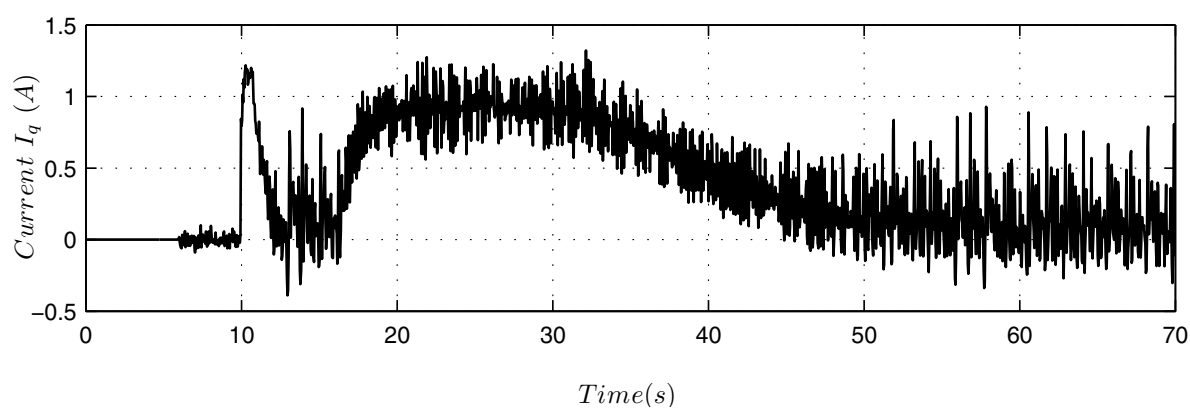

(b) IM Stator Current $I_{q s}$

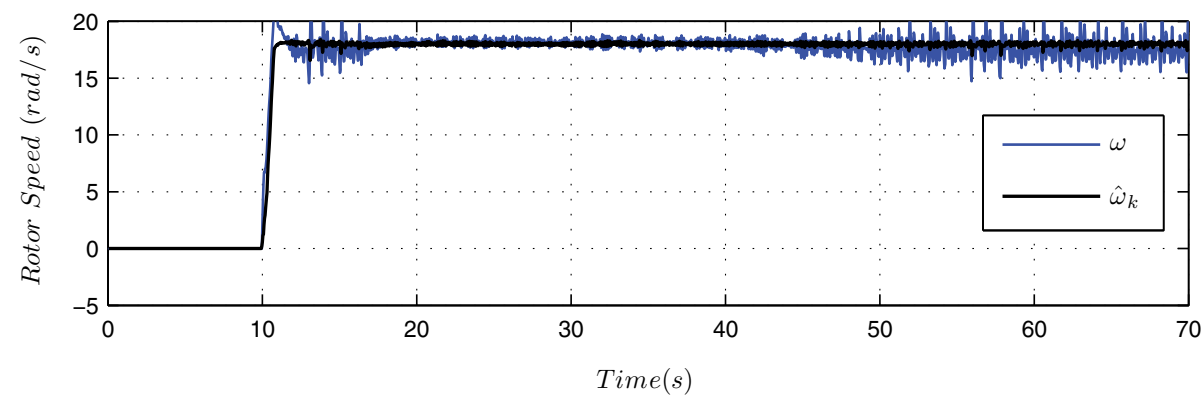

(c) Rotor Speed - Estimated and Encoder Measurement

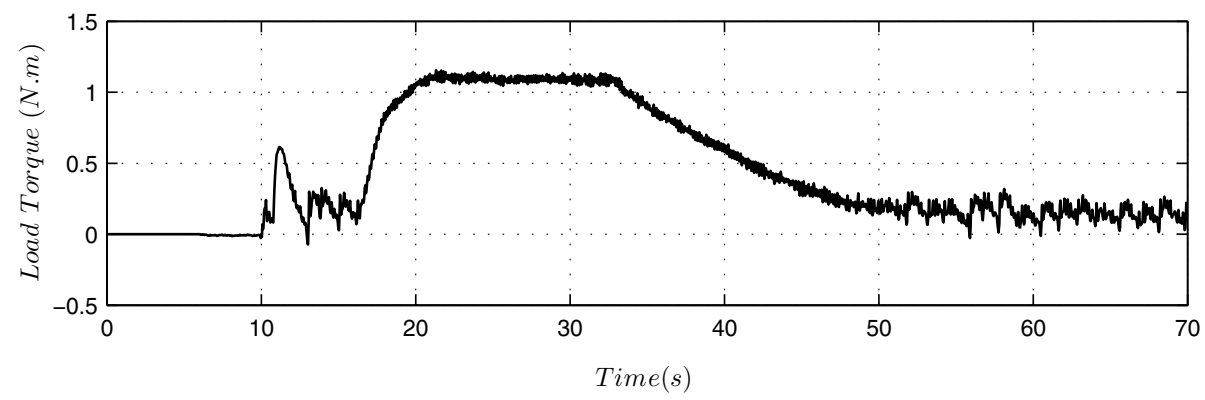

(d) Estimated Load Torque

Fig. 5. Simplified FLC control with $18 \mathrm{rad} / \mathrm{s}$ rotor speed reference 


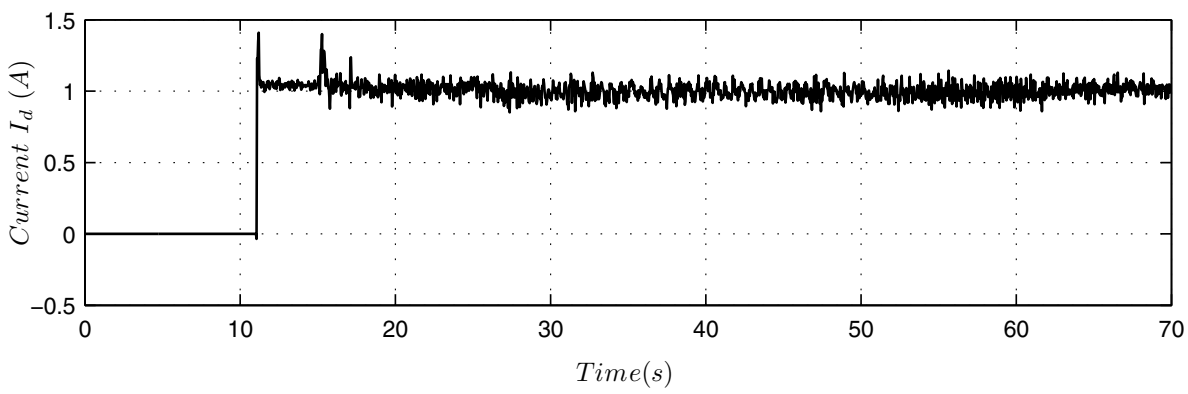

(a) IM Stator Current $I_{d s}$

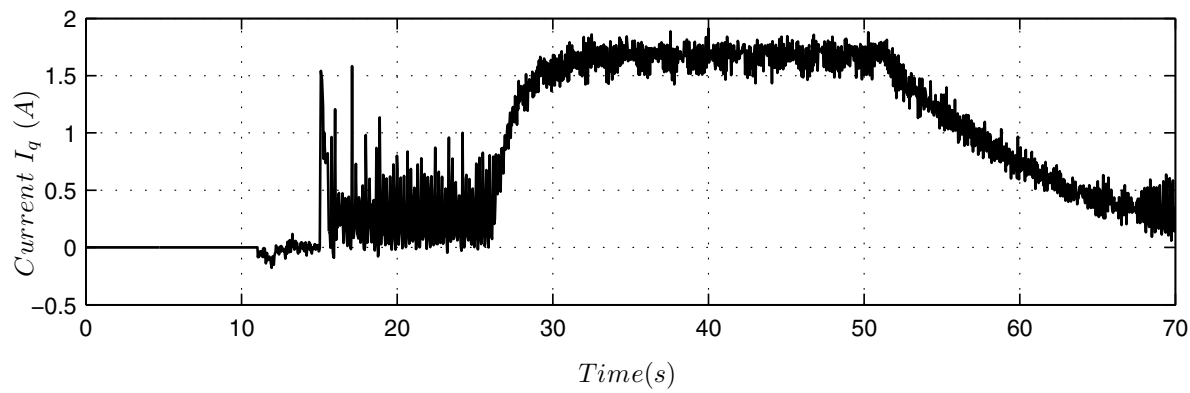

(b) IM Stator Current $I_{q s}$

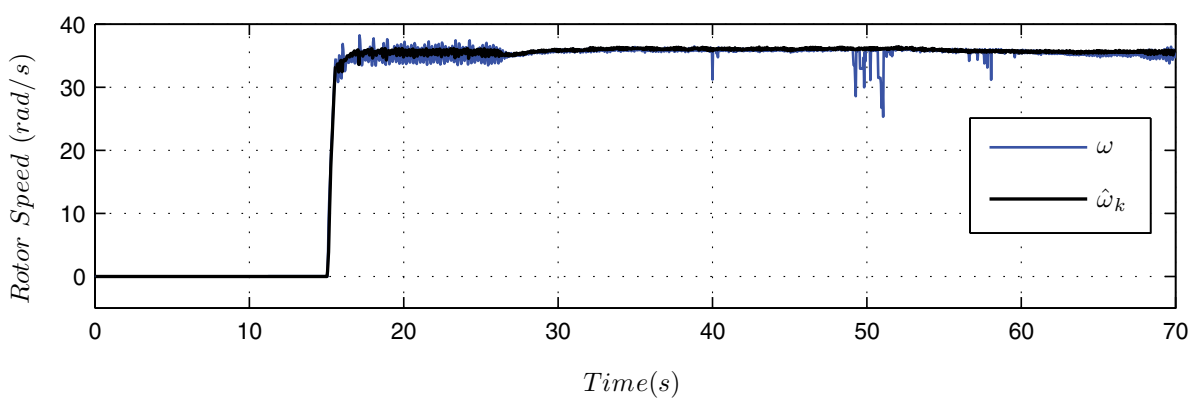

(c) Rotor Speed - Estimated and Encoder Measurement

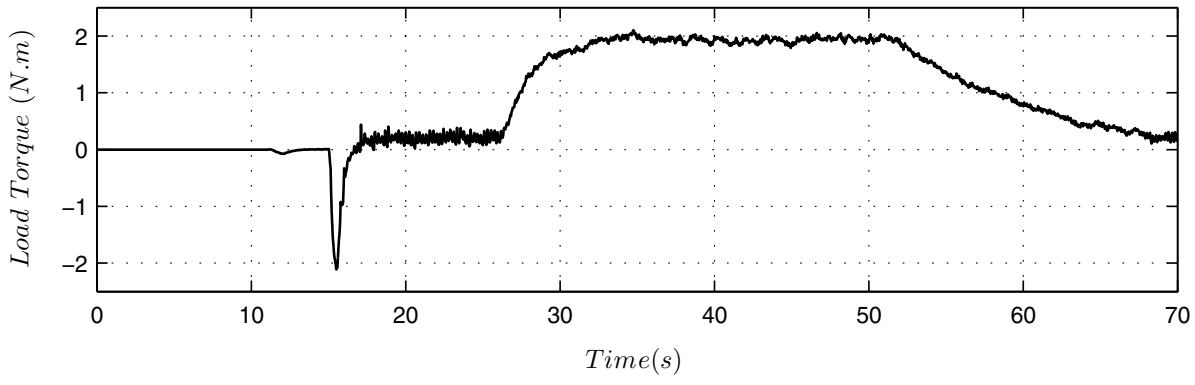

(d) Estimated Load Torque

Fig. 6. FLC control with $36 \mathrm{rad} / \mathrm{s}$ rotor speed reference 


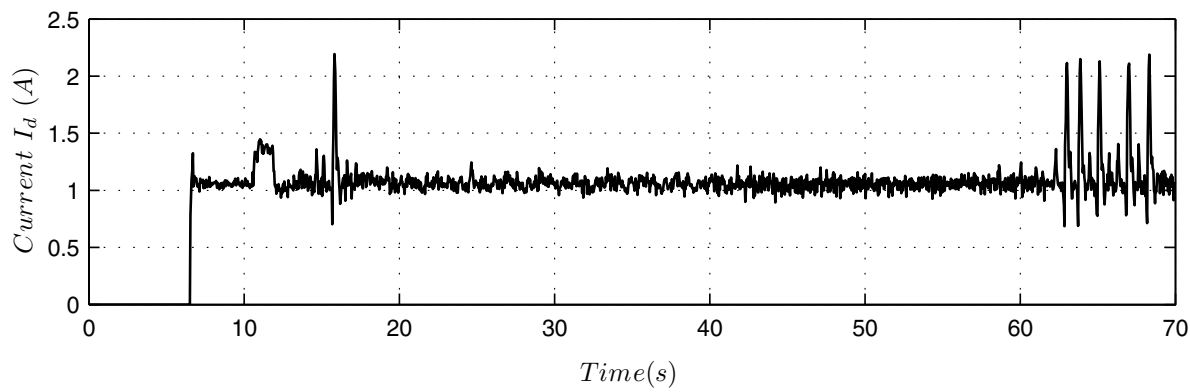

(a) IM Stator Current $I_{d s}$

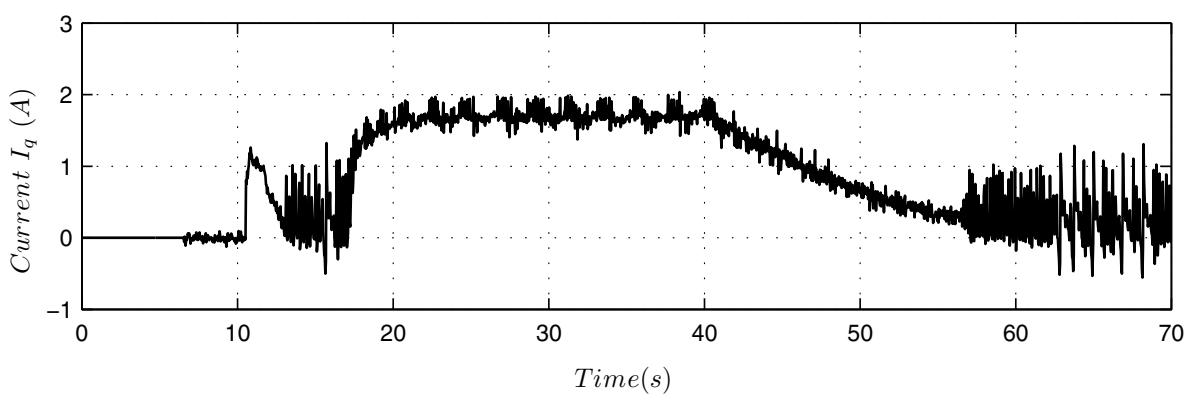

(b) IM Stator Current $I_{q s}$

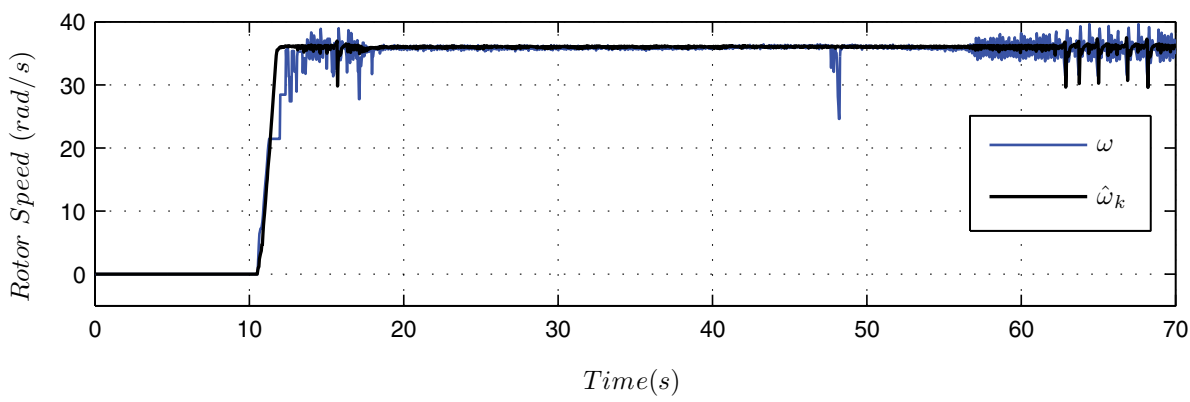

(c) Rotor Speed - Estimated and Encoder Measurement

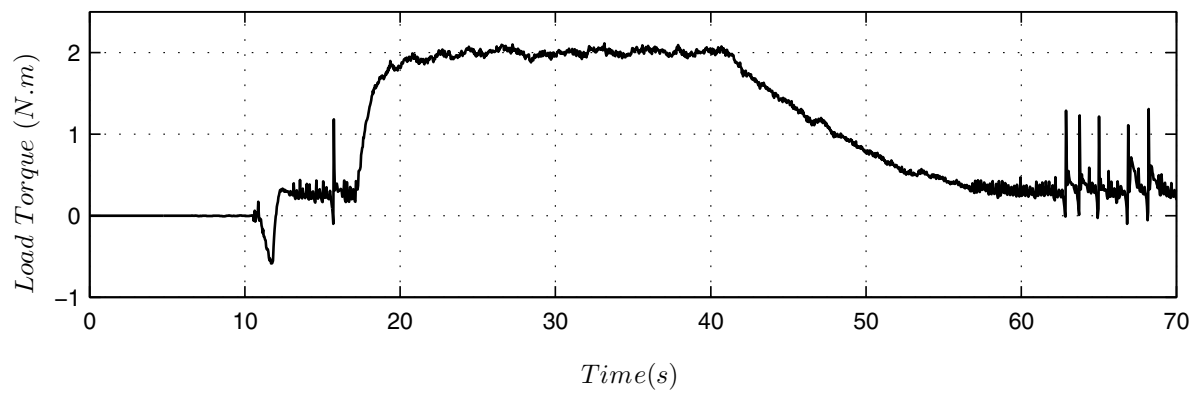

(d) Estimated Load Torque

Fig. 7. Simplified FLC control with $36 \mathrm{rad} / \mathrm{s}$ rotor speed reference 


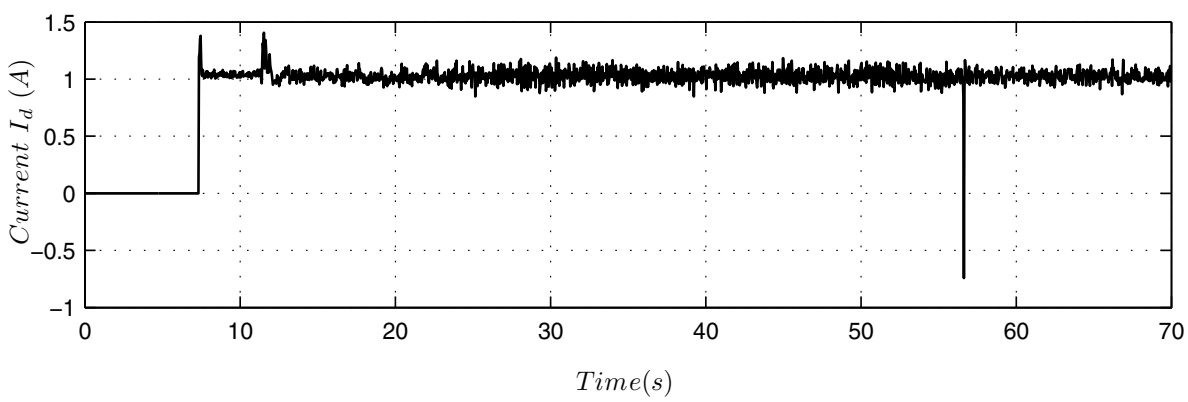

(a) IM Stator Current $I_{d s}$

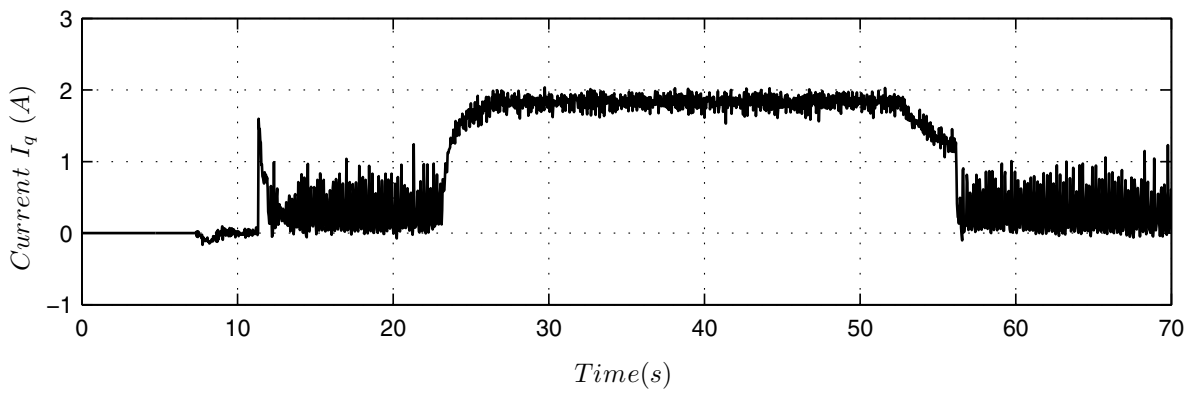

(b) IM Stator Current $I_{q s}$

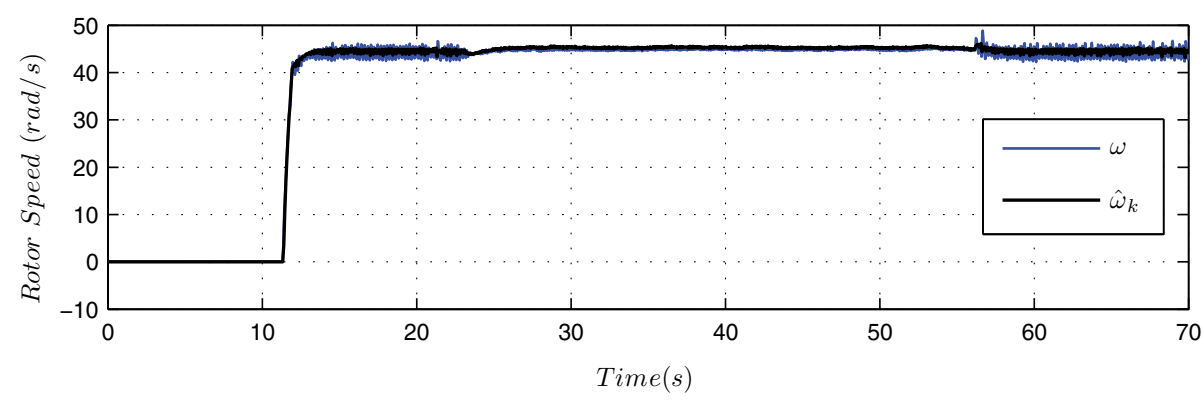

(c) Rotor Speed - Estimated and Encoder Measurement

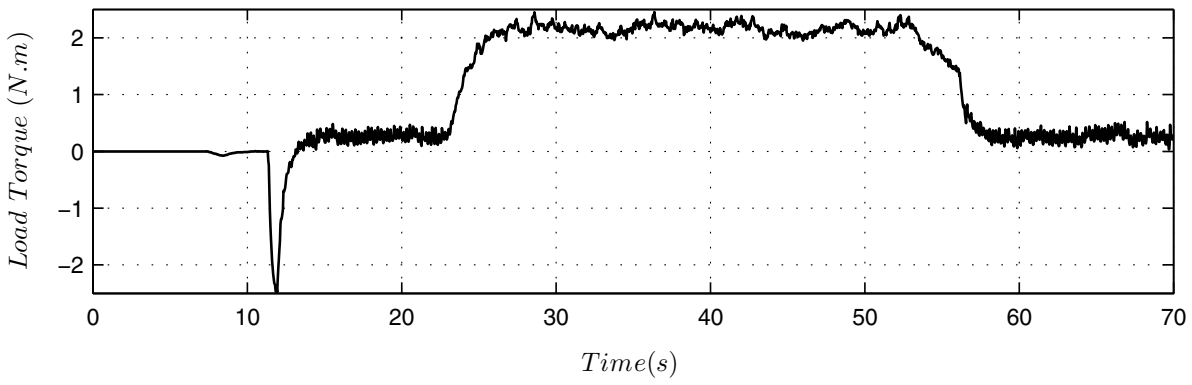

(d) Estimated Load Torque

Fig. 8. FLC control with $45 \mathrm{rad} / \mathrm{s}$ rotor speed reference 


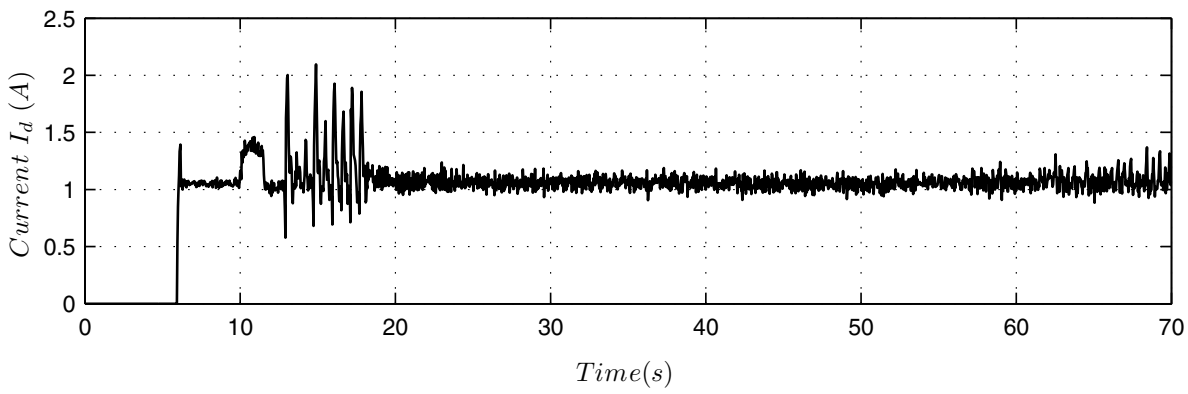

(a) IM Stator Current $I_{d s}$

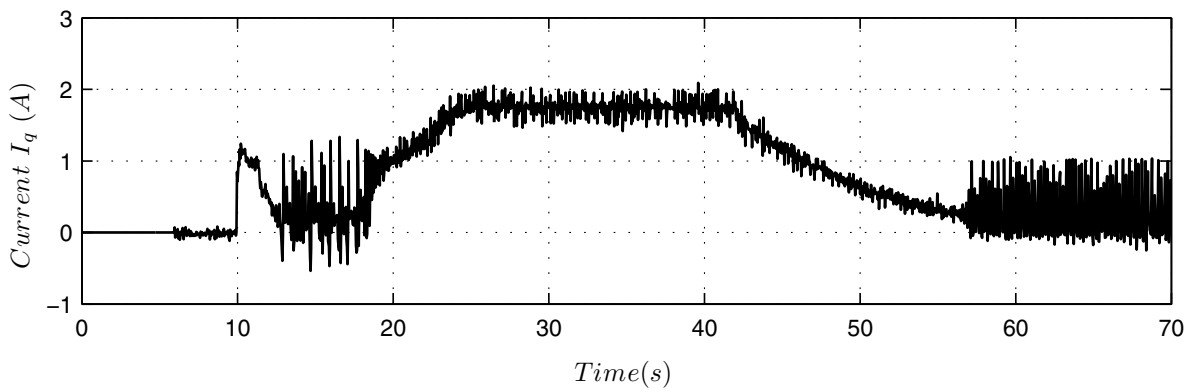

(b) IM Stator Current $I_{q s}$

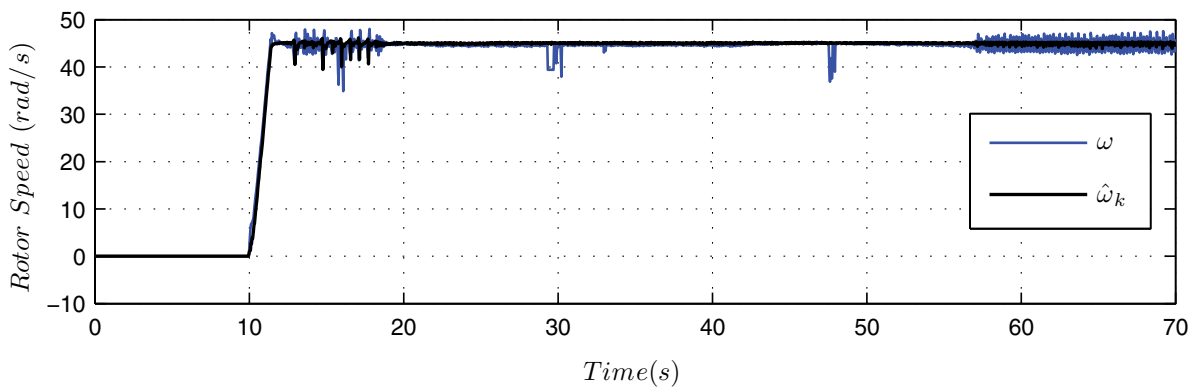

(c) Rotor Speed - Estimated and Encoder Measurement

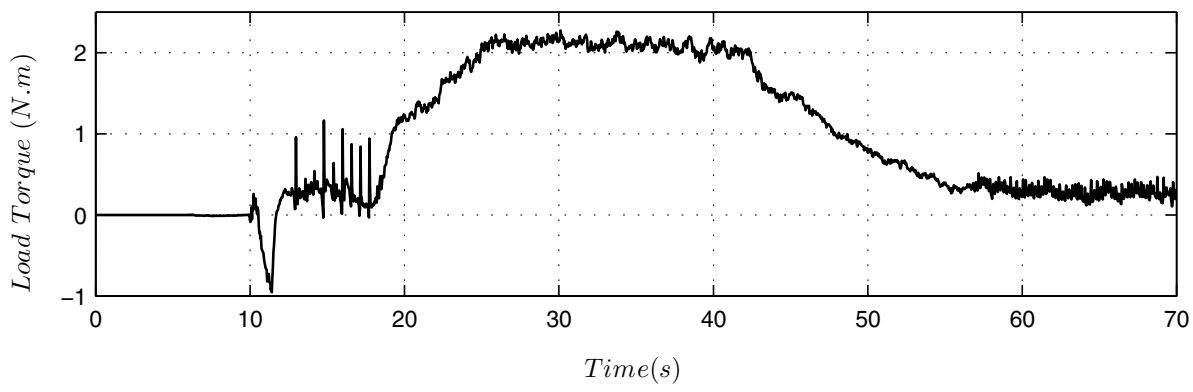

(d) Estimated Load Torque

Fig. 9. Simplified FLC control with $45 \mathrm{rad} / \mathrm{s}$ rotor speed reference 
in the $18 \mathrm{rad} / \mathrm{s}, 36 \mathrm{rad} / \mathrm{s}$ and $45 \mathrm{rad} / \mathrm{s}$ rotor speed range. Both control schemes present similar performance in steady-state. Hence, the proposed modification of the FLC control allows a simplification of the control algorithm without deterioration in control performance. However, it may necessary to carefully evaluate the gain selection in the simplified FLC control, to guarantee rotor flux alignment on the $\mathrm{d}$ axis, as well as, to guarantee speed-flux decoupling. Both control schemes indicate sensitivity with model parameter variation, and one way to overcome this would be the is development of an adaptive FLC control laws on FLC control.

\section{References}

Aström, K. \& Wittenmark, B. (1997). Computer-Controlled Systems: Theory and Design, Prentice-Hall.

Cardoso, R. \& Gründling, H. A. (2009). Grid synchronization and voltage analysis based on the kalman filter, in V. M. Moreno \& A. Pigazo (eds), Kalman Filter Recent Advances and Applications, InTech, Croatia, pp. 439-460.

De Campos, M., Caratti, E. \& Grundling, H. (2000). Design of a position servo with induction motor using self-tuning regulator and kalman filter, Conference Record of the 2000 IEEE Industry Applications Conference, 2000.

Gastaldini, C. \& Grundling, H. (2009). Speed-sensorless induction motor control with torque compensation, 13th European Conference on Power Electronics and Applications, EPE '09, pp. $1-8$.

Krause, P. C. (1986). Analysis of electric machinery, McGraw-Hill.

Leonhard, W. (1996). Control of Electrical Drives, Springer-Verlag.

Marino, R., Peresada, S. \& Valigi, P. (1990). Adaptive partial feedback linearization of induction motors, Proceedings of the 29th IEEE Conference on Decision and Control, 1990, pp. $3313-3318$ vol.6.

Marino, R., Tomei, P. \& Verrelli, C. M. (2004). A global tracking control for speed-sensorless induction motors, Automatica 40(6): 1071 - 1077.

Martins, O., Camara, H. \& Grundling, H. (2006). Comparison between mrls and mras applied to a speed sensorless induction motor drive, 37th IEEE Power Electronics Specialists Conference, PESC '06., pp. 1 -6.

Montanari, M., Peresada, S., Rossi, C. \& Tilli, A. (2007). Speed sensorless control of induction motors based on a reduced-order adaptive observer, IEEE Transactions on Control Systems Technology 15(6): 1049 -1064.

Montanari, M., Peresada, S. \& Tilli, A. (2006). A speed-sensorless indirect field-oriented control for induction motors based on high gain speed estimation, Automatica 42(10): $1637-1650$.

Orlowska-Kowalska, T. \& Dybkowski, M. (2010). Stator-current-based mras estimator for a wide range speed-sensorless induction-motor drive, IEEE Transactions on Industrial Electronics 57(4): $1296-1308$.

Peng, F.-Z. \& Fukao, T. (1994). Robust speed identification for speed-sensorless vector control of induction motors, IEEE Transactions on Industry Applications 30(5): 1234 -1240.

Peresada, S. \& Tonielli, A. (2000). High-performance robust speed-flux tracking controller for induction motor, International Journal of Adaptive Control and Signal Processing, 2000.

Vieira, R., Azzolin, R. \& Grundling, H. (2009). A sensorless single-phase induction motor drive with a mrac controller, 35th Annual Conference of IEEE Industrial Electronics,IECON '09., pp. $1003-1008$. 


\title{
From Dynamic Modeling to Experimentation of Induction Motor Powered by Doubly-Fed Induction Generator by Passivity-Based Control
}

\author{
M. Becherif ${ }^{1}$, A. Bensadeq ${ }^{2}$, E. Mendes ${ }^{3}$, A. Henni $^{4}$, \\ P. Lefley ${ }^{5}$ and M.Y Ayad ${ }^{6}$ \\ ${ }^{1}$ UTBM, FEMTO-ST/FCLab, UMR CNRS 6174, 90010 Belfort Cedex \\ ${ }^{2}$ AElectrical Power \& Power Electronics Group, Department of Engineering \\ ${ }^{3}$ Grenoble INP - LCIS/ESISAR, BP 5426902 Valence Cedex 9 \\ ${ }^{4}$ Alstom Power - Energy Business Management \\ ${ }^{5}$ Electrical Power \& Power Electronics Group, Department of Engineering \\ University of Leicester \\ ${ }^{6}$ IEEE Member \\ $1,3,4,6$ France \\ $2,5 \mathrm{UK}$
}

\section{Introduction}

DFIG wind turbines are nowadays more widely used especially in large wind farms. The main reason for their popularity when connected to the electrical network is their ability to supply power at constant voltage and frequency while the rotor speed varies, which makes it suitable for applications with variable speed, see for instance (10), (11). Additionally, when a bidirectional AC-AC converter is used in the rotor circuit, the speed range can be extended above its synchronous value recovering power in the regenerative operating mode of the machine. The DFIG concept also provides the possibility to control the overall system power factor. A DFIG wind turbine utilizes a wound rotor that is supplied from a frequency converter, providing speed control together with terminal voltage and power factor control for the overall system.

DFIGs have been traditionally used to convert mechanical power into electrical power operating near synchronous speed. Some advantages of DFIGs over synchronous or squirrel cage generators include the high overall efficiency of the system and the low power rating of the converter, which is only rated by the maximum rotor voltage and current. In a typical scenario the prime mover is running at constant speed, and the main concern is the static optimization of the power flow from the primary energy source to the grid. A good introduction to the operational characteristic of the grid connected DFIG can be found in (5). We consider in this paper the isolated operation of a DFIG driven by a prime mover, with its stator connected to a load-which is in this case an IM. Isolated generating units are economically attractive, hence increasingly popular, in the new era of the deregulated market. The possibility of a DFIG supplying an isolated load has been indicated in (6), (7) where some 
mention is made of the steady-state control problem. In (8) a system is presented in which the rotor is supplied from a battery via a PWM converter with experimental results from a $200 \mathrm{~W}$ prototype. A control system based on regulating the rms voltage of the DFIG is used which results in large voltage deviations and very slow recovery following load changes. See also $(9 ; 12)$ where feedback linearization and sliding mode principles are used for the design of the motor speed controller.

This paper presents a dynamic model of the DFIG-IM and proves that this system is Blondel-Park transformable. It is also shown that the zero dynamics is unstable for a certain operating regime. We implemented the passivity-based controller (PBC) that we proposed in (3) to a $200 \mathrm{~W}$ DFIG interconnected with an IM prototype available in IRII-UPC (Institute of Robotics and Industrial Informatics - University Polytechnic of Catalonia). The setup is controlled using a computer running RT-Linux. The whole system is decomposed in a mechanical subsystem which plays the role of the mechanical speed loop, controlled by a classical PI and an electrical subsystem controlled by the PBC where the model inversion was used to build a reference model.

The proposed PBC achieves the tracking control of the IM mechanical rotor speed and flux norm, the practical advantage of the PBC consists of using only the measurements of the two mechanical coordinates (Motor and Generator positions). The experiments have shown that the $\mathrm{PBC}$ is robust to variations in the machines' parameters.

In addition to the PBC applied to the electrical subsystem, we proposed a classical PI controller, where the rotor voltage control law is obtained via a control of the stator currents toward their desired values, those latter are obtained by the inversion of the model.

In the sequel, and for the control of the electrical subsystem a combination of the PBC + Proportional action for the control of the stator currents is applied. The last controller is a combination of PBC + PI action for the control of the stator currents.

The stability analysis is presented. The simulations and practical results show the effectiveness of the proposed solutions, and robustness tests on account of variations in the machines' parameters are also presented to highlight the performance of the different controllers.

The main disadvantage of the DFIG is the slip rings, which reduce the life time of the machine and increases the maintenance costs. To overcome this drawback an alternative machine arrangement is proposed, in section 6, which is the Brushless Doubly Fed twin Induction Generator (BDFTIG). The system is anticipated as an advanced solution to the conventional doubly fed induction generator (DFIG) to decrease the maintenance cost and develop the system reliability of the wind turbine system. The proposed BDFTIG employs two cascaded induction machines each consisting of two wound rotors, connected in cascade to eliminate the brushes and copper rings in the DFIG. The dynamic model of BDFTIG with two machines' rotors electromechanically coupled in the back-to-back configuration is developed and implemented using Matlab/Simulink.

\section{System configuration and mathematical model}

The configuration of the system considered in this paper is depicted in Fig.1. It consists of a wound rotor DFIG, a squirrel cage IM and an external mechanical device that can supply or extract mechanical power, e.g., a flywheel inertia. The stator windings of the IM are connected to the stator windings of the generator whose rotor voltage is regulated by a bidirectional converter. The electrical equivalent circuit is shown in Fig. 2. The main interest in this 
configuration is that it permits a bidirectional power flow between the motor, which may operate in regenerative mode, and the generator.

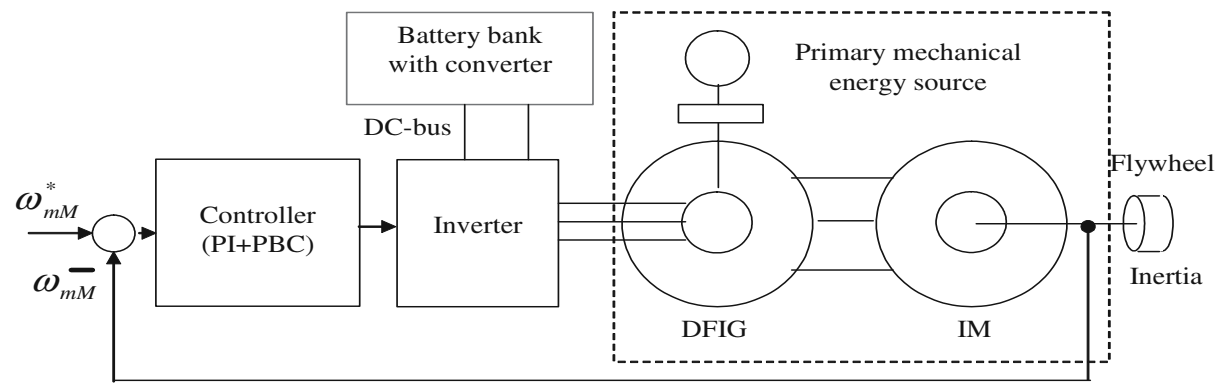

Fig. 1. System configuration with speed controller.

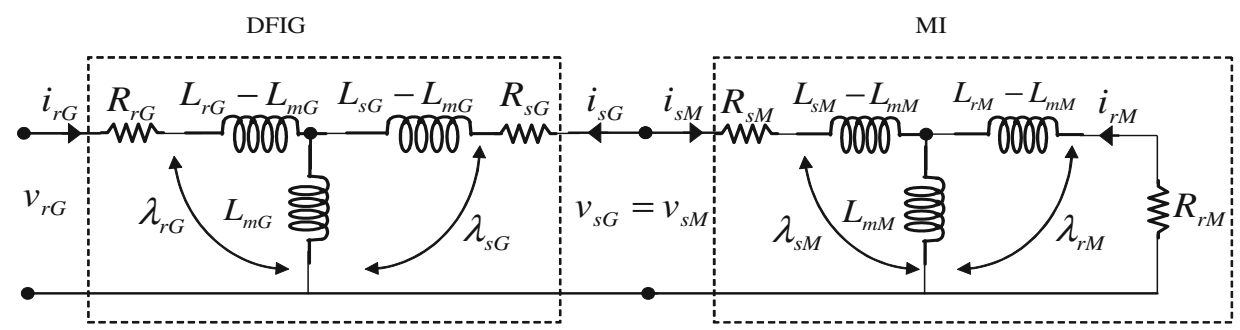

Fig. 2. Equivalent circuit of the DFIG with IM.

In Fig. 3, we show a power port viewpoint description of the system. The DFIG is a three-port system with conjugated power port variables ${ }^{1}$ prime mover torque and speed, $\left(\tau_{L G}, \omega_{G}\right)$, and rotor and stator voltages and currents, $\left(v_{r G}, i_{r G}\right),\left(v_{s G}, i_{s G}\right)$, respectively. The IM, on the other hand, is a two-port system with port variables motor load torque and speed, $\left(\tau_{L M}, \omega_{M}\right)$, and stator voltages and currents. The DFIG and the IM are coupled through the interconnection

$$
\begin{aligned}
v_{S G} & =v_{S M} \\
i_{S G} & =-i_{S M} .
\end{aligned}
$$

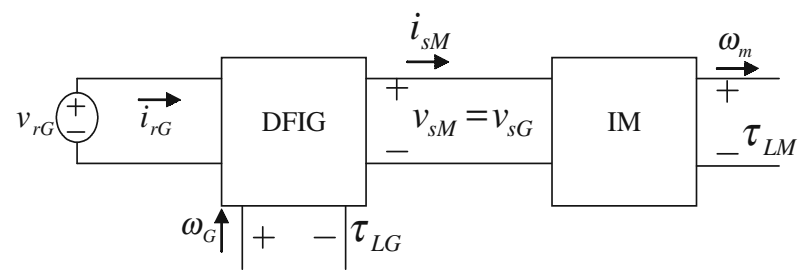

Fig. 3. Power port representation of the DFIG with IM.

To obtain the mathematical model of the overall system ideal symmetrical phases with uniform air-gap and sinusoidally distributed phase windings are assumed. The permeability

\footnotetext{
${ }^{1}$ The qualifier "conjugated power" is used to stress the fact that the product of the port variables has the units of power.
} 
of the fully laminated cores is assumed to be infinite, and saturation, iron losses, end winding and slot effects are neglected. Only linear magnetic materials are considered, and it is further assumed that all parameters are constant and known. Under these assumptions, the voltage balance equations for the machines are

$$
\begin{aligned}
\dot{\lambda}_{s G}+R_{s G} i_{s G} & =v_{s G} \\
\dot{\lambda}_{r G}+R_{r G} i_{r G} & =v_{r G} \\
\dot{\lambda}_{s M}+R_{s M} i_{s M} & =v_{s M} \\
\dot{\lambda}_{r M}+R_{r M} i_{r M} & =0
\end{aligned}
$$

where $\lambda_{s G}, \lambda_{r G}\left(\lambda_{s M}, \lambda_{r M}\right)$ are the stator and rotor fluxes of the DFIG (IM, resp.), $L_{s G}, L_{r G}, L_{m G}$ $\left(L_{s M}, L_{r M}, L_{m M}\right)$ are the stator, rotor, and mutual inductances of the DFIG (IM, resp.); $R_{s G}, R_{r G}$ $\left(R_{s M}, R_{r M}\right)$ are the stator and rotor resistances of the DFIG (IM, resp.).

The interconnection (1) induces an order reduction in the system. To eliminate the redundant coordinates, and preserving the structure needed for application of the $\mathrm{PBC}$, we define

$$
\lambda_{s G M}=\lambda_{s G}-\lambda_{s M}
$$

which upon replacement in the equations above, and with some simple manipulations, yields the equation

$$
\dot{\lambda}+R i=B v_{r G}
$$

where we have defined the vector signals

$$
\lambda=\left[\begin{array}{c}
\lambda_{r G} \\
\lambda_{s G M} \\
\lambda_{r M}
\end{array}\right], \quad i=\left[\begin{array}{c}
i_{r G} \\
i_{s G} \\
i_{r M}
\end{array}\right]
$$

and the resistance and input matrices

$$
R=\operatorname{diag}\{\underbrace{R_{r G}}_{R_{1}} I_{2}, \underbrace{\left(R_{s G}+R_{s M}\right)}_{R_{2}} I_{2}, \underbrace{R_{r M}}_{R_{3}} I_{2}\}, \quad B=\left[\begin{array}{ll}
I_{2} & 0
\end{array}\right]^{T} \in \mathbb{R}^{6 \times 2}
$$

To complete the model of the electrical subsystem, we recall that fluxes and currents are related through the inductance matrix by

$$
\lambda=L(\theta) i,
$$

where the latter takes in this case the form

$$
L(\theta)=\left[\begin{array}{ccc}
L_{r G} I_{2} & L_{m G} e^{-J n_{G} \theta_{G}} & 0 \\
L_{m G} e^{J n_{G} \theta_{G}} & \left(L_{S G}+L_{S M}\right) I_{2} & -L_{m M} e^{J n_{M} \theta_{M}} \\
0 & -L_{m M} e^{-J n_{M} \theta_{M}} & L_{r M} I_{2}
\end{array}\right]
$$

where $n_{G}, n_{M}$ denote the number of pole pairs, $\theta_{G}, \theta_{M}$ the mechanical rotor positions (with respect to the stator) and to simplify the notation we have introduced

$$
\theta=\left[\begin{array}{l}
\theta_{G} \\
\theta_{M}
\end{array}\right], \quad J=\left[\begin{array}{cc}
0 & -1 \\
1 & 0
\end{array}\right]=-J^{T}, \quad e^{J x}=\left[\begin{array}{cc}
\cos (x) & -\sin (x) \\
\sin (x) & \cos (x)
\end{array}\right]=\left(e^{-J x}\right)^{T} .
$$




$$
\begin{aligned}
L^{-1}(\theta) & =\frac{1}{\Delta}\left[\begin{array}{ccc}
{\left[L_{r M}\left(L_{s G}+L_{s M}\right)-L_{m M}^{2}\right] I_{2}} & -L_{m G} L_{r M} e^{-J n_{G} \theta_{G}} & -L_{m G} L_{m M} e^{-J\left(n_{G} \theta_{G}-n_{M} \theta_{M}\right)} \\
-L_{m G} L_{r M} e^{J n_{G} \theta_{G}} & L_{r G} L_{r M} I_{2} & L_{r G} L_{m M} e^{J n_{M} \theta_{M}} \\
-L_{m G} L_{m M} e^{J\left(n_{G} \theta_{G}-n_{M} \theta_{M}\right)} & L_{r G} L_{m M} e^{-J n_{M} \theta_{M}} & {\left[L_{r G}\left(L_{s G}+L_{s M}\right)-L_{m G}^{2}\right] I_{2}}
\end{array}\right] \\
& \triangleq \frac{1}{\Delta}\left[\begin{array}{ccc}
L_{11}^{\prime} & L_{12}^{\prime} & L_{13}^{\prime} \\
L_{12}^{\prime T} & L_{22}^{\prime} & L_{23}^{\prime} \\
L_{11}^{\prime T} & L_{23}^{\prime T} & L_{33}^{\prime}
\end{array}\right]
\end{aligned}
$$

where

$$
\Delta=L_{r G}\left[L_{r M}\left(L_{s G}+L_{s M}\right)-L_{m M}^{2}\right]-L_{r M} L_{m G}^{2}<0
$$

We recall that, due to physical considerations, $R>0, L(\theta)=L^{T}(\theta)>0$ and $L^{-1}(\theta)=$ $L^{-1^{T}}(\theta)>0$.

A state-space model of the (6-th order) electrical subsystem is finally obtained replacing (7) in (6) as

$$
\Sigma_{e}: \dot{\lambda}+R L(\theta)^{-1} \lambda=B v_{r G}
$$

The mechanical dynamics are obtained from Newton's second law and are given by

$$
\Sigma_{m}: J_{m} \ddot{\theta}+B_{m} \dot{\theta}=\tau-\tau_{L}
$$

where $J_{m}=\operatorname{diag}\left\{J_{G}, J_{M}\right\}>0$ is the mechanical inertia matrix, $B_{m}=\operatorname{diag}\left\{B_{G}, B_{M}\right\} \geq 0$ contains the damping coefficients, $\tau_{L}=\left[\tau_{L G}, \tau_{L M}\right]^{T}$ are the external torques, that we will assume constant in the sequel. The generated torques are calculated as usual from

$$
\tau=\left[\begin{array}{c}
\tau_{G} \\
\tau_{M}
\end{array}\right]=-\frac{1}{2} \frac{\partial}{\partial \theta}\left(\lambda^{T}[L(\theta)]^{-1} \lambda\right)
$$

From (7), we obtain the alternative expression

$$
\tau=\frac{1}{2} \frac{\partial}{\partial \theta}\left(i^{T} L(\theta) i\right) .
$$

The following equivalent representations of the torques, that are obtained from direct calculations using (7), (8) and (14), will be used in the sequel

$$
\begin{aligned}
\tau & =\left[\begin{array}{c}
-L_{m G} i_{r G}^{T} J e^{-J n_{G} \theta_{G}} i_{s G} \\
-L_{m M} i_{s G}^{T} J e^{J n_{M} \theta_{M}} i_{r M}
\end{array}\right] \\
& =\left[\begin{array}{c}
-\frac{n_{G}}{R_{s G}+R_{s M}} \dot{\lambda}_{s G M}^{T} J\left(\lambda_{s G M}-L_{m M} e^{J n_{M} \theta_{M}} i_{r M}\right) \\
\frac{n_{M}}{R_{r M}} \dot{\lambda}_{r M}^{T} J \lambda_{r M}
\end{array}\right]
\end{aligned}
$$

\subsection{Modeling of the DFIG-IM in the stator frame of the two machines}

It has been shown in (4) and (3) that the DFIG-IM is Blondel-Park transformable using the following rotating matrix:

$$
\operatorname{Rot}\left(\sigma, \theta_{G}, \theta_{M}\right)=\left[\begin{array}{ccc}
e^{(J \sigma)} & 0 & 0 \\
0 & e^{\left(J\left(\sigma+n_{G} \theta_{G}\right)\right)} & 0 \\
0 & 0 & e^{\left(J\left(\sigma+n_{G} \theta_{G}-n_{M} \theta_{M}\right)\right)}
\end{array}\right]
$$


where $\sigma$ is an arbitrary angle.

The model of the DFIG-IM in the stator frame of the two machines is given by (see (4) and (3) for in depth details):

$$
\left\{\begin{array}{c}
{\left[\begin{array}{c}
\dot{\hat{\lambda}}_{r G} \\
\dot{\lambda}_{s M G} \\
\hat{\hat{\lambda}}_{r M}
\end{array}\right]+\left[\begin{array}{ccc}
a I_{2}-n_{G} \dot{\theta}_{G} J & b I_{2} & 0 \\
a I_{2}-n_{G} \dot{\theta}_{G} J & e I_{2} & -c I_{2}+n_{M} \dot{\theta}_{M} J \\
0 & -d I_{2} & c I_{2}-n_{M} \dot{\theta}_{M} J
\end{array}\right]\left[\begin{array}{c}
\widehat{\lambda}_{r G} \\
\lambda_{s M G} \\
\hat{\lambda}_{r M}
\end{array}\right]=\left[\begin{array}{c}
I_{2} \\
I_{2} \\
0
\end{array}\right] \widehat{v}_{r G}} \\
{\left[\begin{array}{c}
J_{G} \dot{\omega}_{G} \\
J_{M} \dot{\omega}_{M}
\end{array}\right]+\left[\begin{array}{cc}
B_{G} & 0 \\
0 & B_{M}
\end{array}\right]\left[\begin{array}{c}
\omega_{G} \\
\omega_{M}
\end{array}\right]+\left[\begin{array}{c}
f \lambda_{s M G}^{T} J \widehat{\lambda}_{r G} \\
-f \lambda_{s M G}^{T} J \hat{\lambda}_{r M}
\end{array}\right]=\left[\begin{array}{c}
-\tau_{L G} \\
-\tau_{L M}
\end{array}\right]}
\end{array}\right.
$$

or

$$
\left\{\begin{array}{c}
{\left[\begin{array}{c}
\dot{\hat{\lambda}}_{r G} \\
\dot{\lambda}_{s M G} \\
\hat{\hat{\lambda}}_{r M}
\end{array}\right]+\left(R+L_{G} n_{G} \dot{\theta}_{G}+L_{M} n_{M} \dot{\theta}_{M}\right)\left[\begin{array}{c}
\hat{i}_{r G} \\
i_{S M} \\
\hat{i}_{r M}
\end{array}\right]=\left[\begin{array}{c}
I_{2} \\
I_{2} \\
0
\end{array}\right] \widehat{v}_{r G}} \\
{\left[\begin{array}{c}
J_{G} \dot{\omega}_{G} \\
J_{M} \dot{\omega}_{M}
\end{array}\right]+\left[\begin{array}{cc}
B_{G} & 0 \\
0 & B_{M}
\end{array}\right]\left[\begin{array}{c}
\omega_{G} \\
\omega_{M}
\end{array}\right]+\left[\begin{array}{c}
f \lambda_{s M G}^{T} J \widehat{\lambda}_{r G} \\
-f \lambda_{s M G}^{T} J \hat{\lambda}_{r M}
\end{array}\right]=\left[\begin{array}{c}
-\tau_{L G} \\
-\tau_{L M}
\end{array}\right]}
\end{array}\right.
$$

$\lambda_{S M G}$ corresponds to the total leakage flux of the two machines referred to the stators of the machines.

$L_{s M G}$ represent the total leakage inductance.

with

$$
\left\{\begin{array}{c}
R=\left[\begin{array}{ccc}
\widehat{R}_{r G} I_{2} & 0 & 0 \\
\widehat{R}_{r G} I_{2} & \left(R_{s G}+R_{s M}\right) I_{2} & -\widehat{R}_{r M} I_{2} \\
0 & 0 & \widehat{R}_{r M} I_{2}
\end{array}\right] \\
L_{G}=L_{M G}\left[\begin{array}{ccc}
-J & J & 0 \\
-J & J & 0 \\
0 & 0 & 0
\end{array}\right] \text { et } L_{M}=L_{M M}\left[\begin{array}{ccc}
0 & 0 & 0 \\
0 & J & J \\
0 & -J & -J
\end{array}\right]
\end{array}\right.
$$

with the positive parameters: $a=\widehat{R}_{r G} L_{M G^{\prime}}^{-1} b=\widehat{R}_{r G} L_{s M G^{\prime}}^{-1} c=\widehat{R}_{r M} L_{M M^{\prime}}^{-1} d=\widehat{R}_{r M} L_{s M G^{\prime}}^{-1}$ $e=\left(\widehat{R}_{r G}+R_{s G}+R_{s M}+\widehat{R}_{r M}\right) L_{s M G^{\prime}}^{-1} f=L_{s M G^{\prime}}^{-1}$ and the following transformations:

$$
\begin{array}{ll}
\widehat{\lambda}_{r G}=\frac{L_{m G}}{L_{r G}} e^{J n_{G} \theta_{G}} \lambda_{r G,} & \widehat{v}_{r G}=\frac{L_{m G}}{L_{r G}} e^{J n_{G} \theta_{G}} v_{r G} \\
\widehat{\lambda}_{r M}=\frac{L_{m M}}{L_{r M}} e^{J n_{M} \theta_{M}} \lambda_{r M}, & \widehat{i}_{r G}=\frac{L_{r G}}{L_{m G}} e^{J n_{G} \theta_{G}} i_{r G}
\end{array}
$$

\section{Properties of the model}

In this section, we derive some passivity and geometric properties of the model that will be instrumental to carry out our controller design.

\subsection{Passivity}

An explicit power port representation of the DFIG interconnected to the IM is presented in Fig.4 


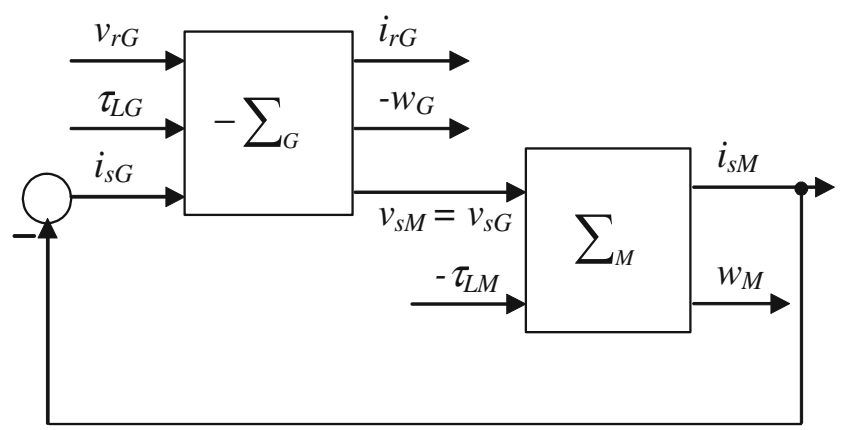

Fig. 4. Explicit power port representation of the DFIG with IM.

Claim 1. The interconnection of the DFIG with the IM presented in the explicit power port representation in Fig.4 is a passive system ${ }^{2}$ with the passive map $\left[\begin{array}{c}v_{r G} \\ \tau_{L G} \\ -\tau_{L M}\end{array}\right] \mapsto\left[\begin{array}{c}i_{r G} \\ -\omega_{G} \\ \omega_{M}\end{array}\right]$.

Proof. Consider the Fig. $4, \Sigma=\left[\begin{array}{c}\Sigma_{G} \\ \Sigma_{M}\end{array}\right]$ is passive $\Rightarrow\left[\begin{array}{c}v_{r G} \\ \tau_{L G} \\ -\tau_{L M}\end{array}\right] \mapsto\left[\begin{array}{c}i_{r G} \\ -\omega_{G} \\ \omega_{M}\end{array}\right]$ is passive ?

For this purpose, we have to prove that $\int\left(v_{r G}^{T} i_{r G}-\tau_{L G} \omega_{G}-\tau_{L M} \omega_{M}\right) \geq 0$. We know that each machine separately is passive (see [1])

$$
\begin{aligned}
\Sigma_{G} \text { is a passive system } & \Leftrightarrow \int\left(v_{r G}^{T} i_{r G}-\tau_{L G} \omega_{G}+i_{s M}^{T} v_{s M}\right) \geq 0 \\
\Sigma_{M} \text { is a passive system } & \Leftrightarrow \int\left(v_{s G}^{T} i_{s G}-\tau_{L M} \omega_{M}\right) \geq 0
\end{aligned}
$$

where equation (1) has been used in (20) and (21). Let's consider

$$
d \triangleq \int\left(v_{r G}^{T} i_{r G}-\tau_{L G} \omega_{G}+i_{s M}^{T} v_{s M}\right) \geq 0
$$

Using the energy conserving principle $\int i_{s M}^{T} v_{s M}=-\int i_{s G}^{T} v_{s G}$ yields

$$
d=\int\left(v_{r G}^{T} i_{r G}-\tau_{L G} \omega_{G}\right)-\int i_{s G}^{T} v_{s G}
$$

From (21) we have

$$
-\int i_{s G}^{T} v_{s G} \leq-\int \tau_{L M} \omega_{M}
$$

Finally (23) and (24) yields

$$
\int\left(v_{r G}^{T} i_{r G}-\tau_{L G} \omega_{G}-\tau_{L M} \omega_{M}\right) \geq d \geq 0
$$

Hence, the passivity of the DFIG interconnected to the IM is proven

\footnotetext{
${ }^{2}$ Passive systems are defined here with no causality relation assumed among the port variables (13). This, more natural, definition is more suitable for applications where power flow (and not signal behaviour) is the primary concern.
} 


\section{Zero dynamics}

For the IM speed control, we are interested in the internal behaviour of the system when the motor torque $\tau_{M}$ is constant. In addition, for practical considerations, we are interested in the control of the IM flux norm $\left|\lambda_{r M}\right|$, where $|\cdot|$ is the Euclidean norm.

For the study of the zero dynamics regarding these two outputs, we consider the DFIG-IM model $^{3}$ given by (18).

The control $\widehat{v}_{r G}$ is determined to obtain the desired equilibrium points of the DFIG-IM:

$$
\begin{gathered}
\ddot{\theta}_{G}=\ddot{\theta}_{M}=0, \quad \dot{\theta}_{G}=\dot{\theta}_{G}^{d}=\text { Constant }, \quad \dot{\theta}_{M}=\dot{\theta}_{M}^{d}=\text { Constant }, \\
\tau_{L G}=\tau_{L G 0}=\text { Constant }, \quad \tau_{L M}=\tau_{L M 0}=\text { Constant } \\
\widehat{\lambda}_{r M}^{T} \widehat{\lambda}_{r M}=\beta_{d}^{2}=\text { Constant }>0
\end{gathered}
$$

The IM mechanical dynamics show that the desired equilibrium points are obtained if:

$$
\tau_{M}=\tau_{M}^{d}=\tau_{L M 0}=\text { Constant }
$$

Hence:

$$
f \lambda_{s M G}^{T} J \widehat{\lambda}_{r M}=\tau_{M}=\tau_{M}^{d}=\text { Constant }
$$

The equation (27) can also be expressed by replacing $\lambda_{s M G}$ by its value given by the third line of the electrical subsystem (18):

$$
\frac{f}{d}\left(\dot{\widehat{\lambda}}_{r M}+c \widehat{\lambda}_{r M}-n_{M} \dot{\theta}_{M}^{d} J \widehat{\lambda}_{r M}\right)^{T} J \widehat{\lambda}_{r M}=\tau_{M}
$$

Hence

$$
\frac{f}{d}\left(\dot{\hat{\lambda}}_{r M}^{T} J \widehat{\lambda}_{r M}-n_{M} \dot{\theta}_{M}^{d} \widehat{\lambda}_{r M}^{T} \widehat{\lambda}_{r M}\right)=\frac{f}{d}\left(\dot{\hat{\lambda}}_{r M}^{T} J \widehat{\lambda}_{r M}-n_{M} \dot{\theta}_{M}^{d} \beta^{2}\right)=\tau_{M}=\tau_{M}^{d}=\text { cte }
$$

The relative degrees of the outputs $y_{1}=\beta^{2}=\widehat{\lambda}_{r M}^{T} \widehat{\lambda}_{r M}$ and $y_{2}=\tau_{M}=f \lambda_{s M G}^{T} J \widehat{\lambda}_{r M}$, regarding the input control $\widehat{v}_{r G}$, are 2 and 1 , respectively.

The zero dynamics of the mechanical subsystem (18) is stable, since the mechanical parameters are positive.

Following on we will analyze the zero dynamics of the electrical subsystem considering the equilibrium points such that (26), (27) and (28) are verified (we will omit the subscript $d$ ).

\section{- Consequence of (26):}

$$
\begin{aligned}
\frac{d \beta^{2}}{d t}=0 \Rightarrow & \dot{\hat{\lambda}}_{r M}^{T} \widehat{\lambda}_{r M}=0 \quad \text { et } \quad \hat{\lambda}_{r M}^{T} \dot{\hat{\lambda}}_{r M}=0 \\
0 & =\hat{\lambda}_{r M}^{T} \dot{\hat{\lambda}}_{r M}=d \widehat{\lambda}_{r M}^{T} \lambda_{s M G}-c \widehat{\lambda}_{r M}^{T} \widehat{\lambda}_{r M}+n_{M} \dot{\theta}_{M} \widehat{\lambda}_{r M}^{T} J \widehat{\lambda}_{r M}
\end{aligned}
$$

with the electrical

subsystem of (18), it comes: $0=d \widehat{\lambda}_{r M}^{T}\left(\lambda_{s M G}-\frac{c}{d} \widehat{\lambda}_{r M}\right)$

Hence, with (26), it comes as solution of $\lambda_{S M G}$ :

$$
\lambda_{s M G}=\frac{c}{d} \widehat{\lambda}_{r M}+\alpha J \widehat{\lambda}_{r M}, \quad \forall \alpha \in \mathbb{R}
$$

${ }^{3}$ The zero dynamics analysis is independent from the chosen frame. 
- Consequence of (27) and (28):

Replace (29) in (27):

$$
\tau_{M}=f \widehat{\lambda}_{r M}^{T}\left(\frac{c}{d} I_{2}-\alpha J\right) J \widehat{\lambda}_{r M}=f \alpha \widehat{\lambda}_{r M}^{T} \widehat{\lambda}_{r M}
$$

Since $\widehat{\lambda}_{r M}^{T} \widehat{\lambda}_{r M}=\beta^{2}=$ Constant:

$$
\tau_{M}=f \beta^{2} \alpha \Rightarrow \alpha=\frac{\tau_{M}}{f \beta^{2}}
$$

and with $\dot{\tau}_{M}=0$ :

$$
\dot{\tau}_{M}=f \beta^{2} \dot{\alpha} \Rightarrow \dot{\alpha}=0
$$

Recall of consequences of (26), (27) and (28):

At the equilibrium, the solutions of $\widehat{\lambda}_{r M}$ belong to the following set:

$$
\left\{\widehat{\lambda}_{r M} \in \mathbb{R}^{2} \mid \widehat{\lambda}_{r M}^{T} \widehat{\lambda}_{r M}=\beta^{2}>0, \widehat{\lambda}_{r M}^{T} \dot{\hat{\lambda}}_{r M}=0, \dot{\hat{\lambda}}_{r M}^{T} J \widehat{\lambda}_{r M}=\frac{d}{f} \tau_{M}+n_{M} \dot{\theta}_{M} \beta^{2}, \dot{\beta}=0\right\}
$$

At the equilibrium, the solutions of $\lambda_{s M G}$ belong to the following set:

$$
\left\{\lambda_{s M G} \in \mathbb{R}^{2} \mid \lambda_{s M G}=\frac{c}{d} \widehat{\lambda}_{r M}+\alpha J \widehat{\lambda}_{r M}, \alpha=\frac{\tau_{M}}{f \beta^{2}}, \dot{\alpha}=0\right\}
$$

Let take $\widehat{\lambda}_{r M}$ in the form :

$$
\widehat{\lambda}_{r M}=e^{J\left(\rho+n_{M} \theta_{M}\right)}\left[\begin{array}{l}
\beta \\
0
\end{array}\right]
$$

with the form (32) and the constrains of (30), it comes:

$$
\begin{gathered}
\dot{\beta}=0 \Rightarrow \dot{\hat{\lambda}}_{r M}=\left(\dot{\rho}+n_{M} \dot{\theta}_{M}\right) J \widehat{\lambda}_{r M} \text { and } \widehat{\lambda}_{r M}^{T} \dot{\hat{\lambda}}_{r M}=0 \\
\dot{\hat{\lambda}}_{r M}^{T} J \widehat{\lambda}_{r M}=\frac{d}{f} \tau_{M}+n_{M} \dot{\theta}_{M}^{d} \beta^{2} \Rightarrow\left(\dot{\rho}+n_{M} \dot{\theta}_{M}\right) \beta^{2}=\frac{d}{f} \tau_{M}+n_{M} \dot{\theta}_{M} \beta^{2} \Rightarrow \dot{\rho}=\frac{d}{f} \frac{\tau_{M}}{\beta^{2}}=d \alpha
\end{gathered}
$$

with $\alpha$ given by (31).

Hence the vectors $\lambda_{S M G}$ and $\widehat{\lambda}_{r M}$ are completely defined by the outputs $y_{1}$ and $y_{2}$.

Analyzing the behaviour of the dynamics of the state $\widehat{\lambda}_{r G}$ :

the substraction of the two upper lines of (18) give:

$$
\dot{\hat{\lambda}}_{r G}=(b+e) \lambda_{s M G}+\dot{\lambda}_{s M G}-c \widehat{\lambda}_{r M}+n_{M} \dot{\theta}_{M} J \widehat{\lambda}_{r M}
$$

By replacing $\lambda_{S M G}$ by its value given by (31), the state becomes:

$$
\dot{\hat{\lambda}}_{r G}=\left[\frac{c(b+e-d)}{d} I_{2}+\left(\alpha(b+e)+n_{M} \dot{\theta}_{M}\right) J\right] \widehat{\lambda}_{r M}+\left(\frac{c}{d} I_{2}+\alpha J\right) \dot{\hat{\lambda}}_{r M}
$$

Using the general form (32) and its derivative under the constrains (30), yields:

$$
\begin{aligned}
\dot{\hat{\lambda}}_{r G} & =\left[\left(\frac{c(b+e-d)}{d}-\alpha\left(\dot{\rho}+n_{M} \dot{\theta}_{M}\right)\right) I_{2}+\left(\alpha(b+e)+n_{M} \dot{\theta}_{M}+\left(\dot{\rho}+n_{M} \dot{\theta}_{M}\right) \frac{c}{d}\right) J\right] \widehat{\lambda}_{r M} \\
& =\left[c_{1} I_{2}+c_{2} J\right] \widehat{\lambda}_{r M}=M_{1} e^{J \gamma} \widehat{\lambda}_{r M}
\end{aligned}
$$


with $M_{1}=\sqrt{c_{1}^{2}+c_{2}^{2}}=$ Constant and $\gamma=\arctan \frac{c 2}{c 1}=$ Constant if $c_{1} \neq 0$, else $\gamma=\frac{\pi}{2}$. Then, with (32):

$$
\dot{\hat{\lambda}}_{r G}=M_{1} e^{J \gamma} e^{J\left(\rho+n_{M} \theta_{M}\right)}\left[\begin{array}{l}
\beta \\
0
\end{array}\right]=M_{1} \beta e^{J\left(\gamma+\rho+n_{M} \theta_{M}\right)}\left[\begin{array}{l}
1 \\
0
\end{array}\right]
$$

Consequently, $\widehat{\lambda}_{r G}$ is of the form:

$$
\widehat{\lambda}_{r G}=\frac{-M_{1} \beta}{\dot{\rho}+n_{M} \dot{\theta}_{M}} J e^{J\left(\gamma+\rho+n_{M} \theta_{M}\right)}\left[\begin{array}{l}
1 \\
0
\end{array}\right]+\text { Constant }
$$

because $\ddot{\rho}=\ddot{\theta}_{M}=0, \dot{\gamma}=0$ and $\dot{M}_{1}=0$.

We can then conclude that the dynamics of $\widehat{\lambda}_{r G}$ is stable if the desired operating point satisfies:

$$
\dot{\rho}+n_{M} \dot{\theta}_{M} \neq 0
$$

Consequently, the zero dynamics (the dynamics of $\widehat{\lambda}_{r G}$ ) is unstable when the desired operating point belongs to the slip line defined by:

$$
\dot{\rho}=-n_{M} \dot{\theta}_{M}
$$

or in terms of the controlled outputs $\beta^{2}$ and $\tau_{M}$ :

$$
\tau_{M}=-\frac{L_{r M}^{2}}{L_{m M}^{2} R_{r M}} n_{M} \dot{\theta}_{M} \beta^{2}
$$

With usual machine parameters, the operating point may belong to the slip line for very low speed which is not the case with the considered operating points

\section{Passivity-based controllers}

The PBC achieves the IM speed and rotor flux norm control with all internal signals remaining bounded under the condition $\dot{\rho}+n_{M} \dot{\theta}_{M} \neq 0$. From a practical point of view it is interesting to ensure the boundedness of the internal signals and in particular the stator current of the two machines. For this purpose, two classical controllers (Proportional and Proportional plus Integral) are applied or combined with the PBC on the stator current $i_{s G}$.

In this section we address the stability analysis of the following controllers:

1 PBC : Passivity Based control;

$2 \mathrm{PBC}+\mathrm{P}$ : Passivity Based control + Proportional action on the stator currents $i_{s G}$;

3 PBC + PI : Passivity Based control + Proportional plus Integral actions on the stator currents $i_{S G}$.

As defined in (3) a nested loop control configuration is adopted for the PBC control of the DFIG with the IM system. We propose to design first a torque tracking PBC for $\Sigma_{e}$, and then add a speed tracking loop around it. This leads to the nested-loop scheme depicted in Fig. 5 , where $C_{i l}$ is the inner-loop torque tracking $\mathrm{PBC}$ and $C_{o l}$ is an outer-loop speed controller, which generates the desired torque, and will be taken as a simple PI controller. The reader is referred to (1) for motivation and additional details on this control configuration. 


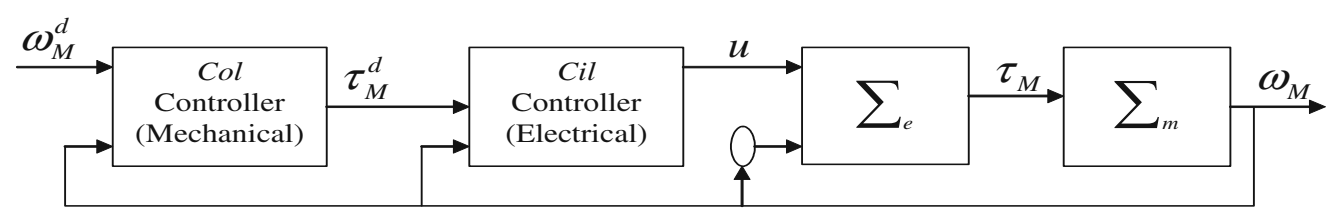

Fig. 5. Nested-loop control configuration.

\subsection{PBC}

To derive the torque tracking PBC we will shape the storage function $H_{\lambda}(\lambda)$, which has a minimum at zero, to take the form

$$
H_{\lambda}^{d}=\frac{1}{2} \tilde{\lambda}^{T} R^{-1} \tilde{\lambda} \geq 0
$$

where $\tilde{\lambda}=\lambda-\lambda^{d}$, with $\lambda^{d}$ a signal to be defined. As suggested in (1), we propose to establish the following relationship between $\lambda^{d}$ and $v_{r G}$ :

$$
B v_{r G}=\dot{\lambda}^{d}+R L^{-1}(\theta) \lambda^{d}
$$

Comparing with (12) we see that this, so-called implicit representation of the controller, is a "copy" of the electrical subsystem but evaluated along some desired trajectories. We will prove now that this control action indeed shapes the storage function as desired. Combining (34) with (12) yields the error equation for the fluxes

$$
\dot{\tilde{\lambda}}+R L^{-1}(\theta) \tilde{\lambda}=0 .
$$

The derivative of the desired energy function (33) along the trajectory of (35) is

$$
\dot{H}_{\lambda}^{d}=-\tilde{\lambda}^{T} L^{-1} \tilde{\lambda} \leq 0
$$

Hence, $\tilde{\lambda}(t) \rightarrow 0$ exponentially.

To complete our torque tracking design there are two remaining issues:

(i) find an explicit representation for the controller (34);

(ii) select $\lambda^{d}$ such that, for any given desired trajectory $\tau_{M}^{*}(t)$, we have

$$
\tilde{\lambda}(t) \rightarrow 0 \Rightarrow \tau_{M}(t) \rightarrow \tau_{M}^{*}(t)
$$

\subsection{PBC + P}

The $\mathrm{PBC}+\mathrm{P}$ controller is given by the equation below:

$$
B v_{r G}=\dot{\lambda}^{d}+R L^{-1}(\theta) \lambda^{d}+B K_{p}\left(i_{s G}-i_{s G}^{d}\right)
$$

where $K_{p}$ is a proportional positive gain. We have:

$$
\begin{aligned}
i_{s G}-i_{s G}^{d} & =\frac{1}{\Delta}\left(L_{21}^{\prime}\left(\lambda_{r G}-\lambda_{r G}^{d}\right)+L_{22}^{\prime}\left(\lambda_{s G M}-\lambda_{s G M}^{d}\right)+L_{23}^{\prime}\left(\lambda_{r M}-\lambda_{r M}^{d}\right)\right) \\
& =\frac{1}{\Delta}\left[\begin{array}{lll}
L_{21}^{\prime} & L_{22}^{\prime} I_{2} & L_{23}^{\prime}
\end{array}\right](\underbrace{\lambda-\lambda^{d}}_{\tilde{\lambda}})=\underbrace{\left[\begin{array}{lll}
0 & I_{2} & 0
\end{array}\right]}_{P} L^{-1}(\theta) \tilde{\lambda}
\end{aligned}
$$

with $L_{i j}^{\prime}\left(i=1^{-} 3, j=1^{-}, 3\right)$ and $\Delta$ are given by (10) and (11), respect. Then, 


$$
B v_{r G}=\dot{\lambda}^{d}+R L^{-1}(\theta) \lambda^{d}+K_{p} B P L^{-1}(\theta) \tilde{\lambda}
$$

The closed loop error dynamic can be written as the following:

$$
\dot{\tilde{\lambda}}=-R L^{-1}(\theta) \tilde{\lambda}-K_{p} B P L^{-1}(\theta) \tilde{\lambda}
$$

Consider the desired energy function given by (33), its derivative along the trajectories of (41) is:

$$
\begin{aligned}
\dot{H}_{\lambda}^{d} & =\tilde{\lambda}^{T} R^{-1} \dot{\tilde{\lambda}} \\
& =-\tilde{\lambda}^{T} \underbrace{L^{-1}(\theta)}_{>0} \tilde{\lambda}-\tilde{\lambda}^{T} \underbrace{R^{-1} B P L^{-1}(\theta)}_{\triangleq Q} \tilde{\lambda}
\end{aligned}
$$

To prove that the error dynamic (41) is stable, it's enough to prove that $Q$ is a positive semi-definite matrix:

$$
Q=R^{-1} B P L^{-1}(\theta)=\frac{1}{R_{1} \Delta}\left[\begin{array}{ccc}
L_{12}^{\prime T} & L_{22}^{\prime} & L_{23}^{\prime} \\
0 & 0 & 0 \\
0 & 0 & 0
\end{array}\right]
$$

$Q$ is positive semi-definite if:

$$
\begin{aligned}
X^{T} Q X \geq 0 \quad X \in \Re^{6 \times 1} \\
X^{T} Q X=\frac{1}{R_{1} \Delta} X^{T}\left[\begin{array}{ccc}
L_{12}^{\prime T} & L_{22}^{\prime} & L_{23}^{\prime} \\
0 & 0 & 0 \\
0 & 0 & 0
\end{array}\right] X \\
=\frac{1}{R_{1} \Delta} X^{T} \underbrace{\left[\begin{array}{ccc}
L_{12}^{\prime T} & \frac{1}{2} L_{22}^{\prime} & \frac{1}{2} L_{23}^{\prime} \\
\frac{1}{2} L_{22}^{\prime T} & 0 & 0 \\
\frac{1}{2} L_{23}^{\prime T} & 0 & 0
\end{array}\right]}_{\triangleq Q^{\prime}} X
\end{aligned}
$$

In order to prove that $Q$ is positive semi-definite (since $\Delta<0$ ), it's enough to prove that $Q^{\prime}$ is negative semi-definite.

$$
Q^{\prime}=\left[\begin{array}{ccc}
-L_{m G} L_{r M} e^{J n_{G} \theta_{G}} & \frac{1}{2} L_{r G} L_{r M} I_{2} & \frac{1}{2} L_{r G} L_{m M} e^{J n_{M} \theta_{M}} \\
\frac{1}{2} L_{r G} L_{r M} I_{2} & 0 & 0 \\
\frac{1}{2} L_{r G} L_{m M} e^{-J n_{M} \theta_{M}} & 0 & 0
\end{array}\right]
$$

We can see that all the sub-determinant of $Q^{\prime}$ are negative, hence the exponential stability of the $\mathrm{PBC}+\mathrm{P}$ controller is proven. 


\subsection{PBC + PI}

The PBC + PI controller is given by the equation below:

$$
B v_{r G}=\dot{\lambda}^{d}+R L^{-1}(\theta) \lambda^{d}+B\left(K_{p}\left(i_{s G}-i_{s G}^{d}\right)+K_{i} \int\left(i_{s G}-i_{s G}^{d}\right)\right)
$$

where $K_{p}$ and $K_{i}$ are the proportional and integral positive gains. We have:

$$
\begin{aligned}
& \int\left(i_{s G}-i_{s G}^{d}\right)=\frac{-1}{R_{2}}\left(\lambda_{s G M}-\lambda_{s G M}^{d}\right) \\
& =\frac{-1}{R_{2}} \underbrace{\left[\begin{array}{lll}
0 & I_{2} & 0
\end{array}\right]}_{P}(\underbrace{\lambda-\lambda^{d}}_{\tilde{\lambda}})
\end{aligned}
$$

Then,

$$
B v_{r G}=\dot{\lambda}^{d}+R L^{-1}(\theta) \lambda^{d}+K_{p} B P L^{-1}(\theta) \tilde{\lambda}-\frac{K_{i}}{R_{2}} B P \tilde{\lambda}
$$

The closed loop error dynamic is:

$$
\dot{\tilde{\lambda}}=-R L^{-1}(\theta) \tilde{\lambda}-K_{p} B P L^{-1}(\theta) \tilde{\lambda}+\frac{K_{i}}{R_{2}} B P \tilde{\lambda}
$$

Consider the desired energy function given by (33), it's derivative along the trajectories of (50) is:

$$
\begin{aligned}
\dot{V} & =\tilde{\lambda}^{T} R^{-1} \dot{\tilde{\lambda}} \\
& =-\tilde{\lambda}^{T} L^{-1}(\theta) \tilde{\lambda}-K_{p} \tilde{\lambda}^{T} R^{-1} B P L^{-1}(\theta) \tilde{\lambda}+\frac{K_{i}}{R_{2}} \tilde{\lambda}^{T} R^{-1} B P \tilde{\lambda} \\
& =-\tilde{\lambda}^{T} \underbrace{L^{-1}(\theta)}_{>0} \tilde{\lambda}+\tilde{\lambda}^{T} \underbrace{\left[-K_{p} R^{-1} B P L^{-1}(\theta)+\frac{K_{i}}{R_{2}} R^{-1} B P\right]}_{\triangleq M} \tilde{\lambda}
\end{aligned}
$$

To show that the error dynamic (50) is stable, it's enough to prove that $M$ is a negative semi-definite matrix:

$$
M=\frac{1}{R_{1}}\left[\begin{array}{ccc}
-\frac{K_{p}}{|\Delta|} L_{m G} L_{r M} e^{J n_{G} \theta_{G}} & {\left[\frac{K_{p}}{|\Delta|} L_{r G} L_{r M}+\frac{K_{i}}{R_{2}}\right] I_{2}} & \frac{K_{p}}{|\Delta|} L_{r G} L_{m M} e^{J n_{M} \theta_{M}} \\
0 & 0 & 0 \\
0 & 0 & 0
\end{array}\right]
$$

$M$ is a negative semi-definite matrix if: $X^{T} M X \leq 0 \quad X \in \Re^{6 \times 1}$

$$
X^{T} M X=\frac{1}{R_{1}} X^{T} \underbrace{\left[\begin{array}{ccc}
-\frac{K_{p}}{|\Delta|} L_{m G} L_{r M} e^{J n_{G} \theta_{G}} & {\left[\frac{K_{p}}{2|\Delta|} L_{r G} L_{r M}+\frac{K_{i}}{2 R_{2}}\right] I_{2}} & \frac{K_{p}}{2|\Delta|} L_{r G} L_{m M^{e}} e^{J n_{M} \theta_{M}} \\
{\left[\frac{K_{p}}{2 \Delta \mid} L_{r G} L_{r M}+\frac{K_{i}}{2 R_{2}}\right] I_{2}} & 0 & 0 \\
\frac{K_{p}}{2|\Delta|} L_{r G} L_{m M} e^{-J n_{M} \theta_{M}} & 0 & 0
\end{array}\right] X}_{\triangleq M^{\prime}}
$$

To prove that $M$ is a negative semi-definite matrix, it's enough to prove that $M^{\prime}$ is a negative semi-definite matrix, the calculus of the sub-determinants of this latter show that $M^{\prime}$ is a negative semi-definite matrix.

Hence the exponential stability of the PBC + PI controller is proven. 


\section{The construction for BDFTIG}

To establish the complete mathematical representation of the dynamic behaviour of the BDFTIG it is first necessary to clarify the kind of the electromechanical interconnection that exists between the cascaded machines. One of the simplest ways to connect these two machines is in the back-to-back method with no phase inversion on the rotor side, as shown by Figure 6.

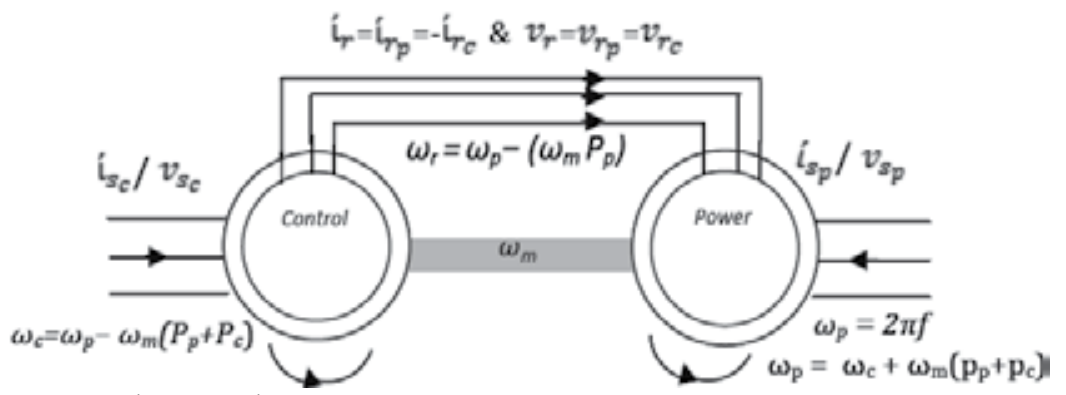

Fig. 6. BDFTIG Back-to-Back Connection

By this connection, the rotor currents produced by the two machines join in the subtractive style, and the rotor voltages have the same signs, i.e. $I_{r p}=-I_{r c}$ and $V_{r p}=V_{r c}$. The chosen connection really affects the distribution of the magnetic fields and flux inside the BDFTIG, producing the two counter-rotating torques as will be discussed in the following sections.

\subsection{Equivalent circuit analysis of the BDFTIG}

Figure 7- shows the equivalent circuit of the BDFTIG from which the electrical system equations can be derived.

Fig. 7. Equivalent Circuit of the BDFTIG

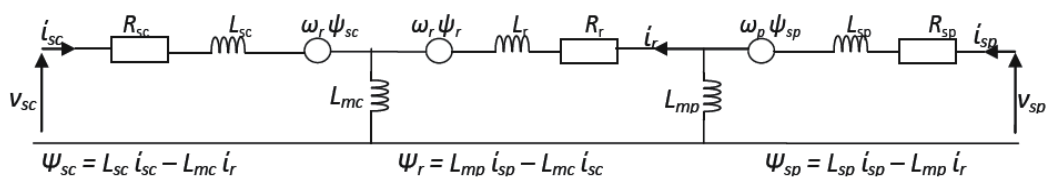

To simplify the controller algorithm, the machine quantities should be expressed in the d-q frame by employing Park's and Clark's transformation. The reason of this transformations is to remove as many time-varying quantities from the system as possible. By converting the three-phase machine to its two-phase equivalent and selecting the suitable reference frame, all the time-varying inductances in both the stator and the rotor are eliminated, allowing for a simple however complete dynamic model of the electric machine. From these equivalent circuits the electrical equations of BDFTIG can be determined as shown in the next section.

\subsection{Electrical system equations for BDFTIG}

Starting with the power machine, the general form of the vector equations of the BDFTIG can be written as:

$$
\begin{aligned}
v_{s p}^{q} & =R_{s p} i_{s p}^{q}+L_{s p} \frac{d i_{s p}^{q}}{d t}+\omega_{p} L_{s p} i_{s p}^{d}+L_{m p} \frac{d i_{r p}^{q}}{d t}+\omega_{p} L_{m p} i_{r p}^{d} \\
v_{s p}^{q} & =R_{s p} i_{s p}^{q}+\left(L_{s p} i_{s p}^{q}+L_{m p} i_{r p}^{q}\right) s+\left(L_{s p} i_{s p}^{d}+L_{m p} i_{r p}^{d}\right) \omega_{p}
\end{aligned}
$$




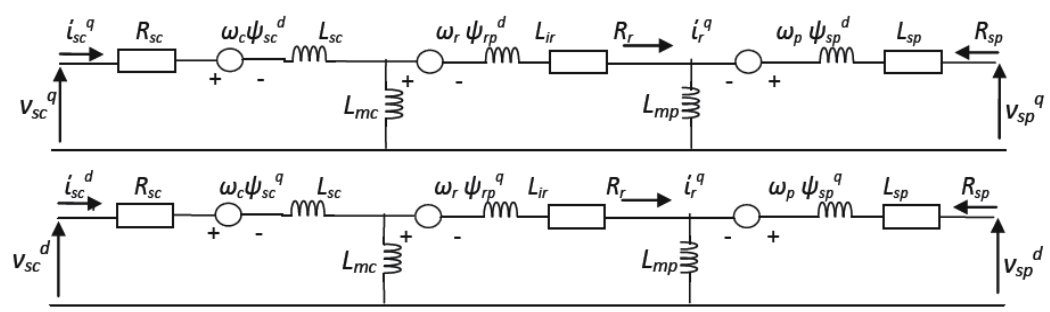

Fig. 8. Equivalent Circuits of d-q BDFTIG

The flux linkage current relations are:

$$
\begin{aligned}
\Psi_{s p}^{q} & =L_{s p} i_{s p}^{q}+L_{m p} i_{r p}^{q} \\
\Psi_{s p}^{d} & =L_{s p} i_{s p}^{d}+L_{m p} i_{r p}^{d} \\
v_{s p}^{q} & =R_{s p} i_{s p}^{q}+\frac{d \Psi_{s p}^{q}}{d t}+\omega_{p} \Psi_{s p}^{d} \\
v_{r p}^{q} & =R_{r p} i_{r p}^{q}+L_{r p} \frac{d i_{r p}^{q}}{d t}+\omega_{r} L_{r p} i_{r p}^{d}+L_{m p} \frac{d i_{s p}^{q}}{d t}+\omega_{r} L_{m p} i_{s p}^{d} \\
v_{r p}^{q} & =R_{r p} i_{r p}^{q}+\left(L_{r p} i_{r p}^{q}+L_{m p} i_{s p}^{q}\right) s+\left(L_{r p} i_{r p}^{d}+L_{m p} i_{s p}^{d}\right) \omega_{r}
\end{aligned}
$$

We have also:

$$
\begin{aligned}
\Psi_{r p}^{q} & =L_{r p} i_{r p}^{q}+L_{m p} i_{s p}^{q} \\
\Psi_{r p}^{d} & =L_{r p} i_{r p}^{d}+L_{m p} i_{s p}^{d} \\
v_{r p}^{q} & =R_{r p} i_{s p}^{q}+\frac{d \Psi_{r p}^{q}}{d t}+\omega_{p} \Psi_{r p}^{d} \\
v_{s p}^{d} & =R_{s p} i_{s p}^{d}+L_{s p} \frac{d i_{s p}^{d}}{d t}-\omega_{p} L_{s p} i_{s p}^{q}+L_{m p} \frac{d i_{r p}^{d}}{d t}-\omega_{p} L_{m p} i_{r p}^{q} \\
v_{s p}^{d} & =R_{s p} i_{s p}^{d}+\left(L_{s p} i_{s p}^{d}+L_{m p} i_{r p}^{d}\right) s-\left(L_{s p} i_{s p}^{q}+L_{m p} i_{r p}^{q}\right) \omega_{p} \\
v_{s p}^{d} & =R_{s p} i_{s p}^{d}+\frac{d \Psi_{s p}^{d}}{d t}-\omega_{p} \Psi_{s p}^{q} \\
v_{r p}^{d} & =R_{r p} i_{r p}^{d}+L_{r p} \frac{d i_{r p}^{d}}{d t}+\omega_{r} L_{r p} i_{r p}^{q}+L_{m p} \frac{d i_{s p}^{d}}{d t}+\omega_{r} L_{m p} i_{s p}^{q} \\
v_{r p}^{d} & =R_{r p} i_{r p}^{d}+\left(L_{r p} i_{r p}^{d}+L_{m p} i_{s p}^{d}\right) s+\left(L_{r p} i_{r p}^{q}+L_{m p} i_{s p}^{q}\right) \omega_{r} \\
v_{r p}^{d} & =R_{r p} i_{s p}^{d}+\frac{d \Psi_{r p}^{d}}{d t}+\omega_{p} \Psi_{r p}^{q}
\end{aligned}
$$

Electrical system equations for control machine:

$$
\begin{aligned}
v_{s c}^{q} & =R_{s c} i_{s c}^{q}+L_{s c} \frac{d i_{s c}^{q}}{d t}+\omega_{c} L_{s c} i_{s c}^{d}+L_{m c} \frac{d i_{r c}^{q}}{d t}+\omega_{c} L_{m c} i_{r c}^{d} \\
v_{s c}^{q} & =R_{s c} i_{s c}^{q}+\left(L_{s c} i_{s c}^{q}+L_{m c} i_{r c}^{q}\right) s+\left(L_{s c} i_{s p}^{d}+L_{m c} i_{r c}^{d}\right) \omega_{c}
\end{aligned}
$$


The flux linkage current relations are:

$$
\begin{aligned}
\Psi_{r c}^{q} & =L_{s c} i_{s c}^{q}+L_{m c} i_{r c}^{q} \\
\Psi_{s c}^{d} & =L_{s c} i_{s p}^{d}+L_{m c} i_{r c}^{d} \\
v_{s c}^{q} & =R_{s c} i_{s c}^{q}+\frac{d \Psi_{s c}^{q}}{d t}+\omega_{c} \Psi_{s c}^{d} \\
v_{r c}^{q} & =R_{r c} i_{r c}^{q}+L_{r c} \frac{d i_{r c}^{q}}{d t}+\omega_{r} L_{r c} i_{r c}^{d}+L_{m c} \frac{d i_{s c}^{q}}{d t}+\omega_{r} L_{m c} i_{s c}^{d} \\
v_{r c}^{q} & =R_{r c} i_{r c}^{q}+\left(L_{r c} i_{r c}^{q}+L_{m c} i_{s c}^{q}\right) s+\left(L_{r p} i_{r p}^{d}+L_{m c} i_{s c}^{d}\right) \omega_{r}
\end{aligned}
$$

and:

$$
\begin{aligned}
\Psi_{r c}^{q} & =L_{r c} i_{r c}^{q}+L_{m c} i_{s c}^{q} \\
\Psi_{r c}^{d} & =L_{r c} i_{r c}^{d}+L_{m c} i_{s c}^{d} \\
v_{r c}^{q} & =R_{r c} i_{r c}^{q}+\frac{d \Psi_{r c}^{q}}{d t}+\omega_{r} \Psi_{r c}^{d} \\
v_{s c}^{d} & =R_{r c} i_{s c}^{d}+L_{s c} \frac{d i_{s c}^{d}}{d t}-\omega_{c} L_{s c} i_{s c}^{q}+L_{m c} \frac{d i_{r c}^{d}}{d t}-\omega_{c} L_{m c} i_{r c}^{q} \\
v_{s c}^{d} & =R_{s c} i_{s c}^{d}+\left(L_{s c} i_{s c}^{d}+L_{m c} i_{r c}^{d}\right) s-\left(L_{s c} i_{s c}^{q}+L_{m c} i_{r c}^{q}\right) \omega_{c} \\
v_{s c}^{d} & =R_{s c} i_{s c}^{d}+\frac{d \Psi_{s c}^{d}}{d t}-\omega_{c} \Psi_{s c}^{q} \\
v_{r c}^{d} & =R_{r c} i_{r c}^{d}+L_{r c} \frac{d i_{r c}^{d}}{d t}+\omega_{c} L_{r c} i_{r c}^{q}+L_{m c} \frac{d i_{s c}^{d}}{d t}+\omega_{r} L_{m c} i_{s c}^{q} \\
v_{r c}^{d} & =R_{r c} i_{r c}^{d}+\left(L_{r c} i_{r c}^{d}+L_{m c} i_{s c}^{d}\right) s+\left(L_{r p} i_{r p}^{q}+L_{m c} i_{s c}^{q}\right) \omega_{r} \\
v_{r c}^{d} & =R_{r c} i_{r c}^{d}+\frac{d \Psi_{r c}^{d}}{d t}+\omega_{r} \Psi_{r c}^{q}
\end{aligned}
$$

As mentioned before, for the BDFTIG with the back-to-back configuration and with no phase inversion, the rotor currents of the individual machines have the opposite signs, the fluxes inside the rotor combine to produce the essential rotor flux, hence; $i_{r p}=-i_{r c}=i_{r}, \Psi_{r}=$ $\Psi_{r p}-\Psi_{r c}, v_{r p}=v_{r c}, 0=v_{r p}-v_{r c}$

$$
\begin{aligned}
0_{r}^{q} & =R_{r p} i_{r}^{q}+L_{r p} \frac{d i_{r}^{q}}{d t}+\omega_{r} L_{r p} i_{r}^{d}+L_{m p} \frac{d i_{s p}^{q}}{d t}+\omega_{r} L_{m p} i_{s p}^{d}+R_{r c} i_{r}^{q}+L_{r c} \frac{d i_{r}^{q}}{d t}+\omega_{r} L_{r c} i_{r}^{d} \\
& -L_{m c} \frac{d i_{s c}^{q}}{d t}-\omega_{r} L_{m c} i_{s c}^{d}
\end{aligned}
$$

But $L_{r}=L_{r p}+L_{r c}$ and $R_{r}=R_{r p}+R_{r c}$

$$
\begin{aligned}
& 0_{r}^{q}=R_{r} i_{r}^{q}+L_{r} \frac{d i_{r}^{q}}{d t}+\omega_{r} L_{r} i_{r}^{d}+L_{m p} \frac{d i_{s p}^{q}}{d t}+\omega_{r} L_{m p} i_{s p}^{d}-L_{m c} \frac{d i_{s c}^{q}}{d t}-\omega_{r} L_{m c} i_{s c}^{d} \\
& 0_{r}^{q}=R_{r} i_{r}^{q}+\left(L_{r} i_{r}^{q}+L_{m p} i_{s p}^{q}-L_{m c} i_{s c}^{q}\right) s+\left(L_{r} i_{r}^{d}+L_{m p} i_{s p}^{d}-L_{m c} i_{s c}^{d}\right) \omega_{r}
\end{aligned}
$$


The flux linkage current relations are:

$$
\begin{aligned}
\Psi_{r}^{q} & =L_{r} i_{r}^{q}+L_{m p} i_{s p}^{q}-L_{m c} i_{s c}^{q} \\
\Psi_{r}^{d} & =L_{r} i_{r}^{d}+L_{m p} i_{s p}^{d}-L_{m c} i_{s c}^{d} \\
0_{r}^{q} & =R_{r} i_{r}^{q}+\frac{d \Psi_{r}^{q}}{d t}+\omega_{r} \Psi_{r}^{d} \\
0_{r}^{q} & =R_{r} i_{r}^{d}+L_{r} \frac{d i_{r}^{d}}{d t}+\omega_{r} L_{r} i_{r}^{q}+L_{m p} \frac{d i_{s p}^{d}}{d t}+\omega_{r} L_{m p} i_{s p}^{q}-L_{m c} \frac{d i_{s c}^{d}}{d t}-\omega_{r} L_{m c} i_{s c}^{q} \\
0_{r}^{q} & =R_{r} i_{r}^{d}+\left(L_{r} i_{r}^{d}+L_{m p} i_{s p}^{d}-L_{m c} i_{s c}^{d}\right) s+\left(L_{r} i_{r}^{q}+L_{m p} i_{s p}^{q}-L_{m c} i_{s c}^{q}\right) \omega_{r} \\
0_{r}^{q} & =R_{r} i_{r}^{d}+\frac{d \Psi_{r}^{d}}{d t}+\omega_{r} \Psi_{r}^{q}
\end{aligned}
$$

The electrical torque creation in the power machine is governed by the same principles that apply to any induction machine. The general equation of the electrical torque in this case is simply:

$$
T_{e}=\frac{3}{2}\left(\frac{P}{2}\right) \Psi_{m} I_{r}
$$

In the d-q reference frame, however, the last equation is rearranged to show the torque as a function of certain control parameter. As the power machine is grid connected, it will have a constant voltage. The torque could be:

$$
T_{p}=\frac{3}{2}\left(\frac{P_{p}}{2}\right)\left(\Psi_{s p}^{q} i_{s p}^{d}-\Psi_{s p}^{d} i_{s p}^{q}\right)
$$

It is clear from the above equation that the only control variables are the $\mathrm{d}$-q components of the stator current, because the power machine stator fluxes are almost constant. Furthermore, when the controller reference frame is aligned with one of the flux components, the number of the control variables is reduced. To derive the electrical torque for the control machine, we can use the same general equation for the electrical torque. This case cannot be simplified because the stator fluxes of the control machine will be variable. The control machine torque must be expressed as a function of the excitation current and the purpose in this research is to provide a flexible power control of the BDFIG. So the next equation is the control machine torque, and it is given in terms of the future control quantities.

$$
T_{c}=-\frac{3}{2}\left(\frac{P_{c}}{2}\right) L_{m c}\left(i_{s c}^{d} i_{r}^{q}-i_{s c}^{q} i_{r}^{d}\right)
$$

The option of the rotor current as the second variable is clearly shown and that there exists an electric coupling between the two stators of the BDFTIG, which is achieved through the common rotor current. This reflects the behaviour of the inner workings of the BDFTIG. The total electric torque (Te) for the BDFTIG is the sum of the individual electrical torques of both machines:

$$
T_{e}=-\frac{3}{4}\left[P_{p}\left(\Psi_{s p}^{q} i_{s p}^{d}-\Psi_{s p}^{d} i_{s p}^{q}\right)+P_{c} L_{m c}\left(i_{s c}^{d} i_{r}^{q}-i_{s c}^{q} i_{r}^{d}\right)\right]
$$

The electric torque equation is defined by the friction and total inertia of the power and control machines:

$$
T_{e}=T_{L}+\left(B_{F}^{p}+B_{F}^{c}\right) \omega_{m}+\left(j_{s}^{p}+j_{s}^{c}\right) \frac{d \omega_{m}}{d t}
$$


Rearranging the last equation to derive the shaft speed:

$$
\frac{d \omega_{m}}{d t}=\frac{1}{j_{s}^{p}+j_{s}^{c}}\left[T_{e}-T_{L}-\left(B_{F}^{p}+B_{F}^{c}\right) \omega_{m}\right]
$$

Hence, the shaft speed is

$$
\omega_{m}=\frac{T_{e}-T_{L}}{\left(B_{F}^{p}+B_{F}^{c}\right)+\left(j_{s}^{p}+j_{s}^{c}\right) s}
$$

\subsection{Simulation of the BDFTIG Model}

The BDFTIG model was tested to determine if it was a true representation of the actual generator. Using Matlab/Simulink to test the BDFTIG, the main tests consisted of disabling one side of the BDFTIG and applying a constant AC voltage on the opposite side, at the same time as changing the load torque to allow both motoring and generation modes of operation. The short circuit test consisted of shorting the stator side of the control machine, and a natural speed of $900 \mathrm{rpm}$ was recorded, because both machines have four poles each, as shown in Figure 9. For the next test, the load torque was decreased at time 2.25 s to put the BDFTIG into the generation mode as shown in Figure 10. The system responded as expected by increasing its speed and moving into the super-synchronous mode of operation, the electrical torque changed at the same time as the load demand. In this section, the dynamic model of the generator was developed based on the selected d-q reference frame. The model was implemented and tested in MATLAB/Simulink. The simulation results verified that the model can correctly describe the dynamic behaviour of BDFTIG design.

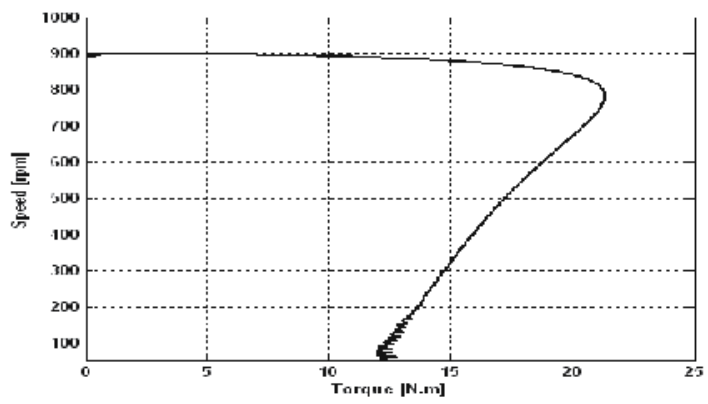

Fig. 9. Speed-Torque Curve of BDFTIG with short circuit test.

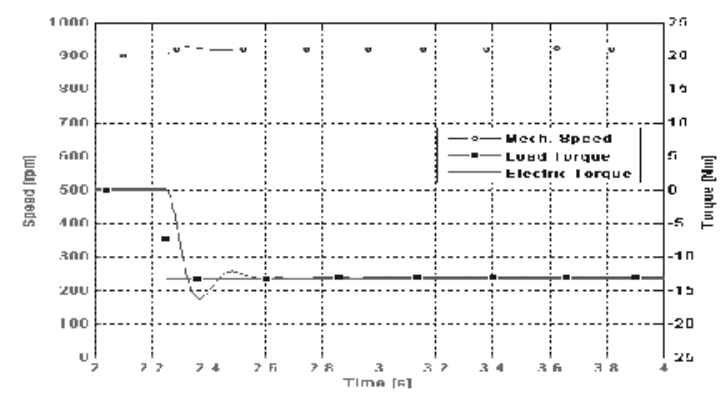

Fig. 10. Generation Mode of BDFTIG. 
In this section, the dynamic model of the generator was developed based on the selected $\mathrm{d}-\mathrm{q}$ reference frame. The model was implemented and tested in MATLAB/Simulink. The simulation results verified that the model can correctly describe the dynamic behaviour of BDFTIG design.

\section{Experimentation}

In order to validate the new controllers, experiments were conducted on a real system. The following controllers were implemented: PBC, PBC+Proportional action on stator currents, PI controller on stator currents, and a combination of PBC and PI control. The experiments were done in the IRII-UPC (Institute of Robotics and Industrial Informatics - University Polytechnic of Catalonia) where a $200 \mathrm{~W}$ DFIG interconnected with an IM prototype is available (see Fig. (11)). The setup was controlled using a computer working under RT-Linux operating system. With the PBC, only the position sensors of the Generator and the Induction machine were used for the control. For the Proportional and PI controllers of the electrical subsystem, measurement of the two stator currents were also needed. In order to show the behaviour of the system under different load conditions, a non-measured load torque was applied.

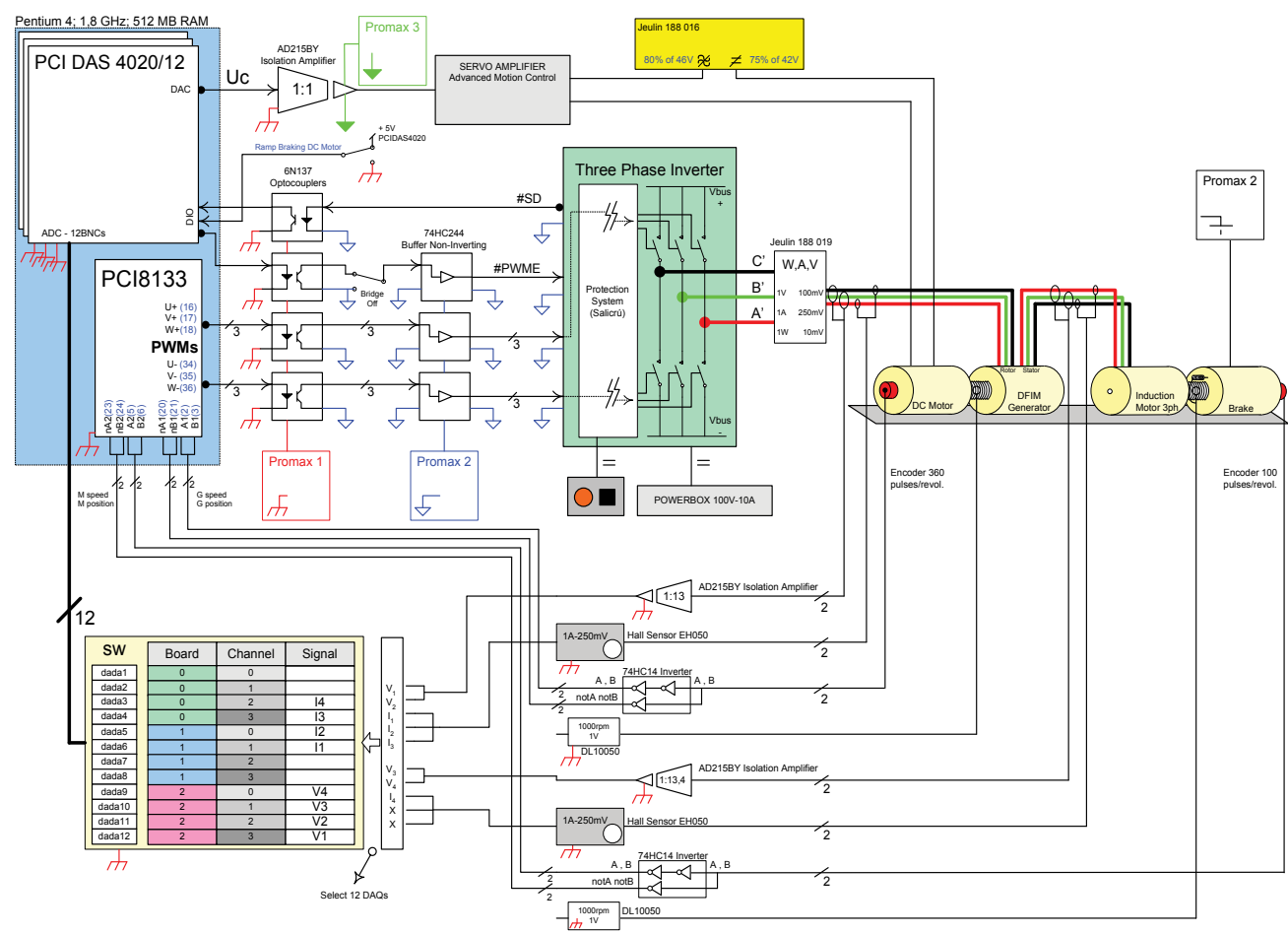

Fig. 11. Experimental setup

Since a load torque sensor was not available for the acquisition, we built an estimator of the resistive torque based on the measurement of the mechanical IM speed. 


\subsection{Estimation of the load torque}

The mechanical dynamics of the IM is given by:

$$
J_{M} \ddot{\theta}_{M}=\tau_{M}-\tau_{L M}-B_{M} \dot{\theta}_{M}
$$

Since the asymptotic stability of the electrical subsystem $\Sigma_{e}$ is proven we can consider that in the steady state $\tau_{M} \rightarrow \tau_{M}^{d}$ (exponentially). Then,we have in the steady state the following:

$$
J_{M} \ddot{\theta}_{M}=\tau_{M}^{d}-\underbrace{\tau_{L M}-B_{M} \dot{\theta}_{M}}_{\tau_{M L}}
$$

Hence, a linear load torque observer was designed (with $l_{1}, l_{2}$ are design parameters):

$$
\begin{aligned}
\dot{\hat{\omega}}_{m M} & =\left(\tau_{M}^{d}-\hat{\tau}_{M L}\right) / J_{M}+l_{1}\left(\hat{\omega}_{m M}-\omega_{m M}\right) \\
\dot{\hat{\tau}}_{M L} & =l_{2}\left(\hat{\omega}_{m M}-\omega_{m M}\right)
\end{aligned}
$$

\subsection{PBC}
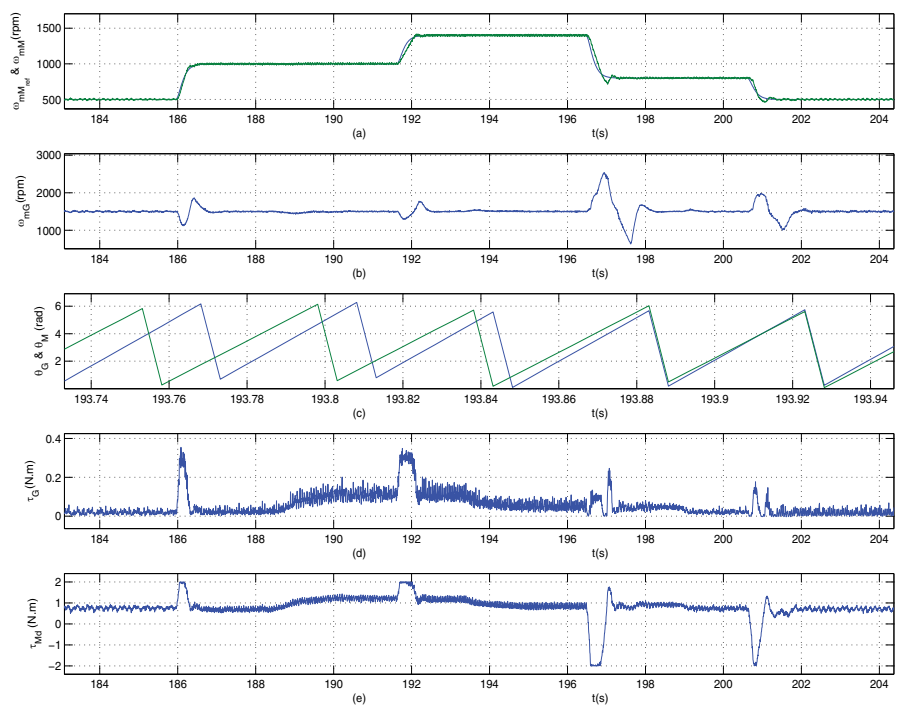

Fig. 12. PBC-(a) Regulated Motor speed and its reference. (b)Generator speed. (c) DFIG \& IM rotor position. (d) Generator torque (e) Motor desired torque.

Figure 12 presents the mechanical IM speed and its smooth reference, the mechanical DFIG speed, the DFIG and IM rotor positions, the DFIG torque $\tau_{G}$ and the IM desired torque $\tau_{M d}$. The real IM speed tracks the reference very well, i.e. low overshoot and no steady state error are observed. Figure 13 shows the stator currents $i_{s a}$ and $i_{s b}$, and their references over a suitable period of time. The stator currents do not track exactly their desired values but are bounded. This is because the goal of the PBC is to track the IM speed and to keep internal signals bounded.

Figure 14 shows the DFIG rotor currents $i_{r G a}$ and $i_{r G b}$, and their references over a period of time. Again, these currents are sinusoidal and bounded.

Figure 15 presents the DFIG rotor voltages $v_{r G a}$ and $v_{r G b}$, the IM rotor speed $\omega_{m M}$ and its estimation $\hat{\omega}_{m M}$, the estimated IM load torque $\hat{\tau}_{M L}$, and the estimated IM speed, given by 

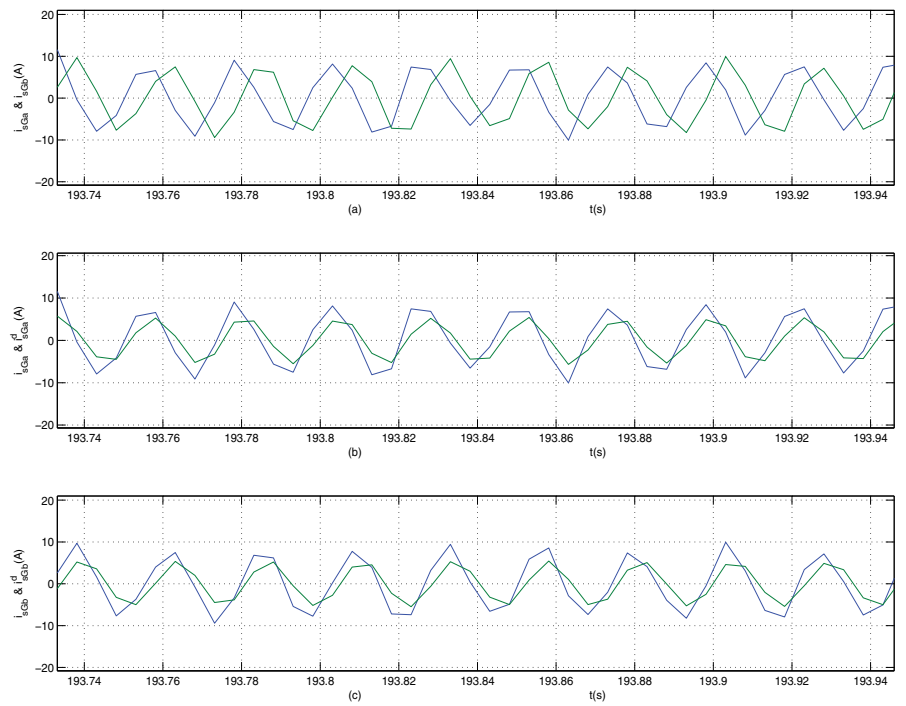

Fig. 13. PBC-(a) $i_{s a}, i_{s b}$ (b) $i_{s a}^{d}, i_{s a}$ (c) $i_{s b}^{d}, i_{s b}$
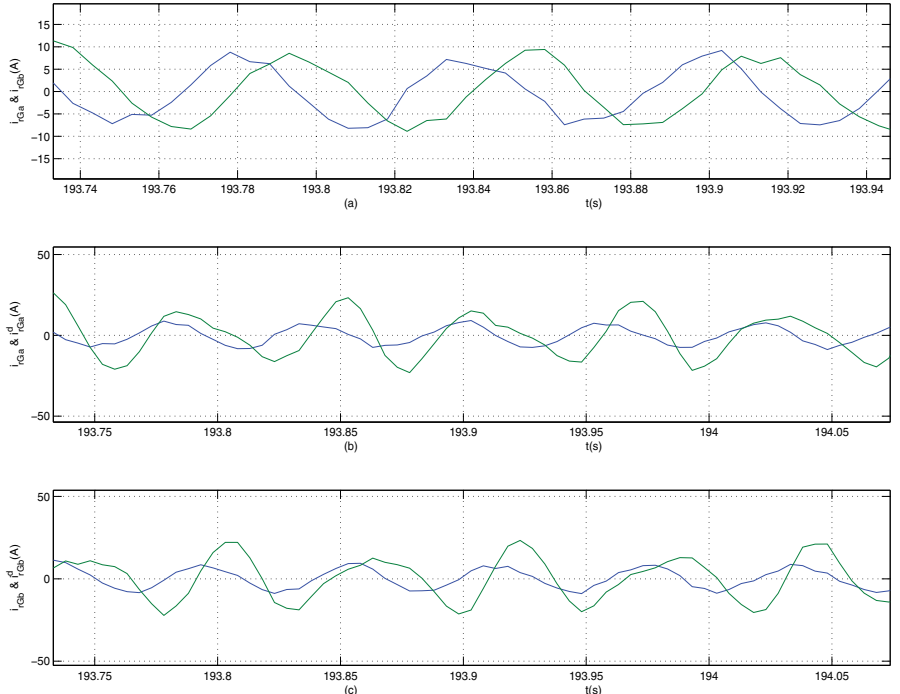

Fig. 14. PBC-(a) $i_{r G a}, i_{r G b}$ (b) $i_{r G a}^{d}, i_{r G a}$ (c) $i_{r G b}^{d}, i_{r G b}$. 

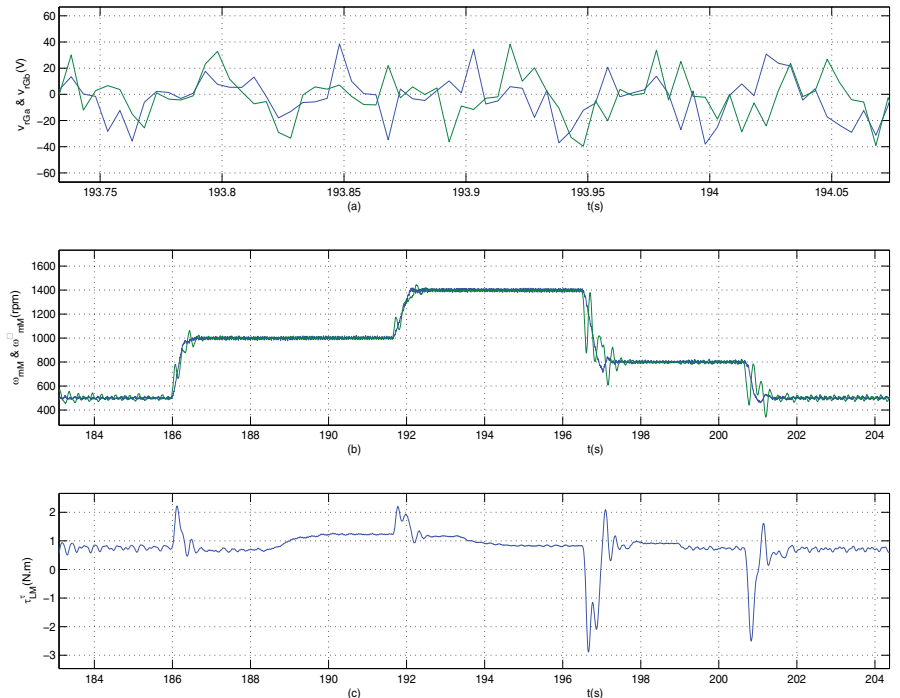

Fig. 15. PBC-(a) $v_{r G a}, v_{r G b}$ (b) $\omega_{m M}, \hat{\omega}_{m M}$ (c) $\hat{\tau}_{M L}$.

(76)-(77), is tracking the real speed. Hence, a good estimation of the real IM load torque is obtained. It has to be noticed that the IM rated torque is $0.7 \mathrm{Nm}$.

It can be concluded that the PBC provides good practical performance even when the applied load torque is twice the magnitude of the nominal load torque of the IM.

\subsection{PBC + P}
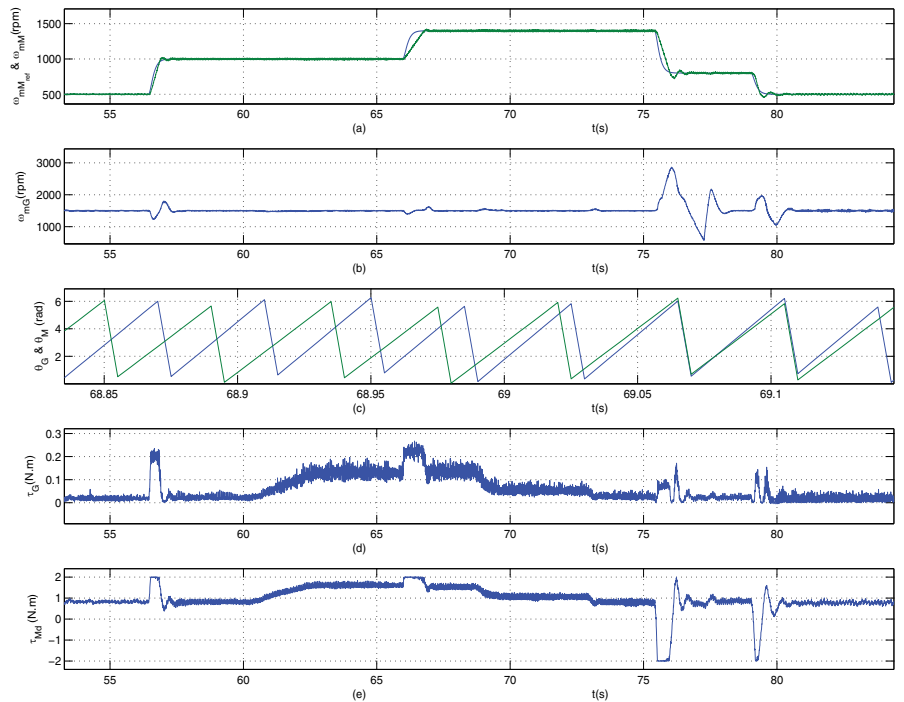

Fig. 16. PBC+P-(a) Regulated Motor speed and its reference. (b)Generator speed. (c) DFIG \& IM rotor position. (d)Generator torque (e) Motor desired torque.

As with the $\mathrm{PBC}$ alone, the results obtained with the $\mathrm{PBC}+\mathrm{P}$ are given in figures 16-19. On the whole, the system behaviour is the same as the PBC alone. One difference that is noticeable is 

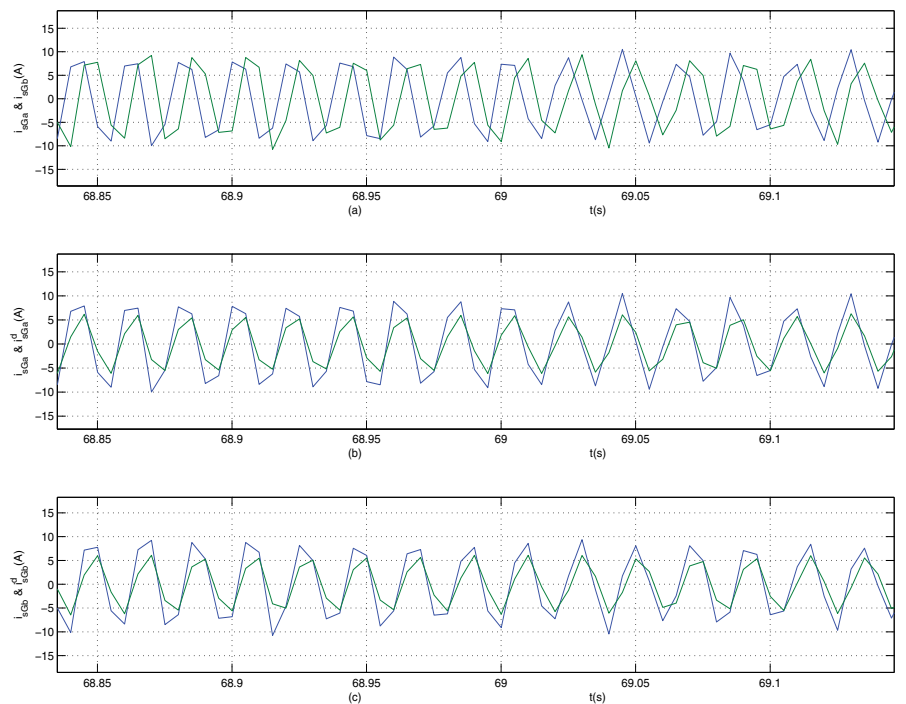

Fig. 17. PBC+P-(a) $i_{s G a}, i_{s G b}$ (b) $i_{s G a^{\prime}}^{d} i_{s G a}(\mathrm{c}) i_{s G b^{\prime}}^{d} i_{s G b}$.
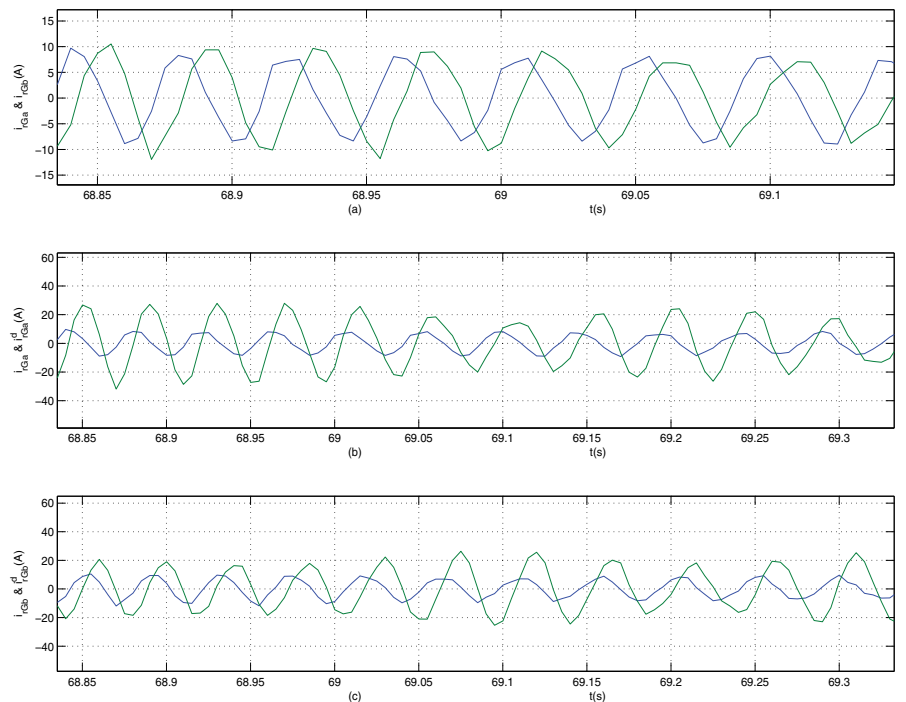

Fig. 18. PBC+P-(a) $i_{r G a}, i_{r G b}$ (b) $i_{r G a^{\prime}}^{d} i_{r G a}$ (c) $i_{r G b^{\prime}}^{d} i_{r G b}$.

the small error between the desired stator currents and the real ones thanks to the proportional controller.

The $\mathrm{PBC}+\mathrm{P}$ controller exhibits good practical performance but not significantly better than those obtained with the PBC alone.

\subsection{PBC + PI}

Again, as for the $\mathrm{PBC}$ and the $\mathrm{PBC}+\mathrm{P}$ controllers, figures 20-23 show the results. It can be seen in figure 21 that the integral actions on the stator currents do not decrease the error significantly between the real and desired values in comparison with the results for the $\mathrm{PBC}+\mathrm{P}$ 

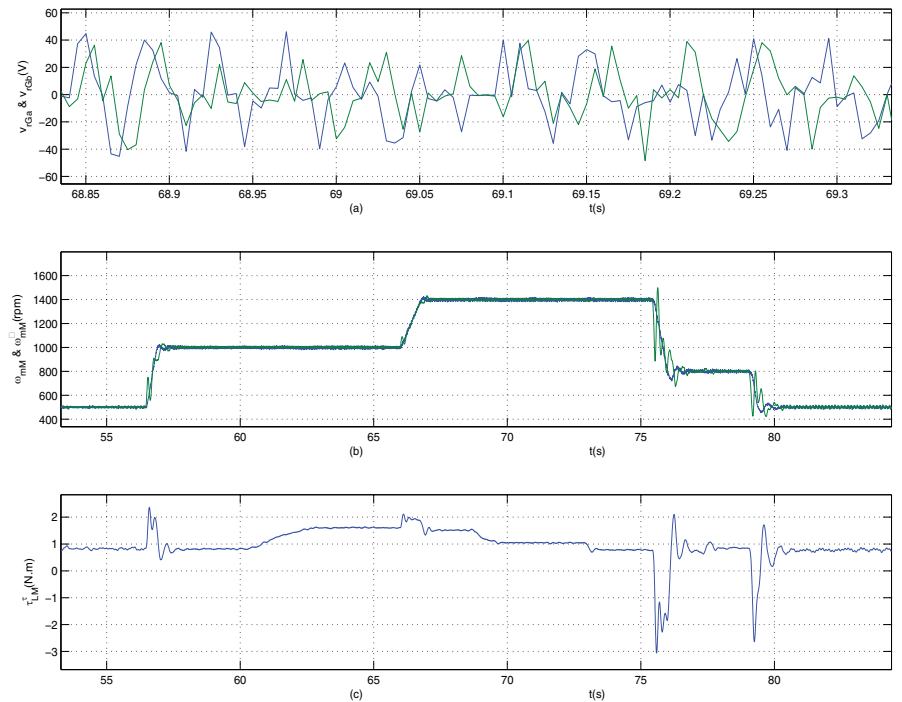

Fig. 19. PBC+P-(a) $v_{r G a}, v_{r G b}$ (b) $\omega_{m M}, \hat{\omega}_{m M}$ (c) $\hat{\tau}_{M L}$.
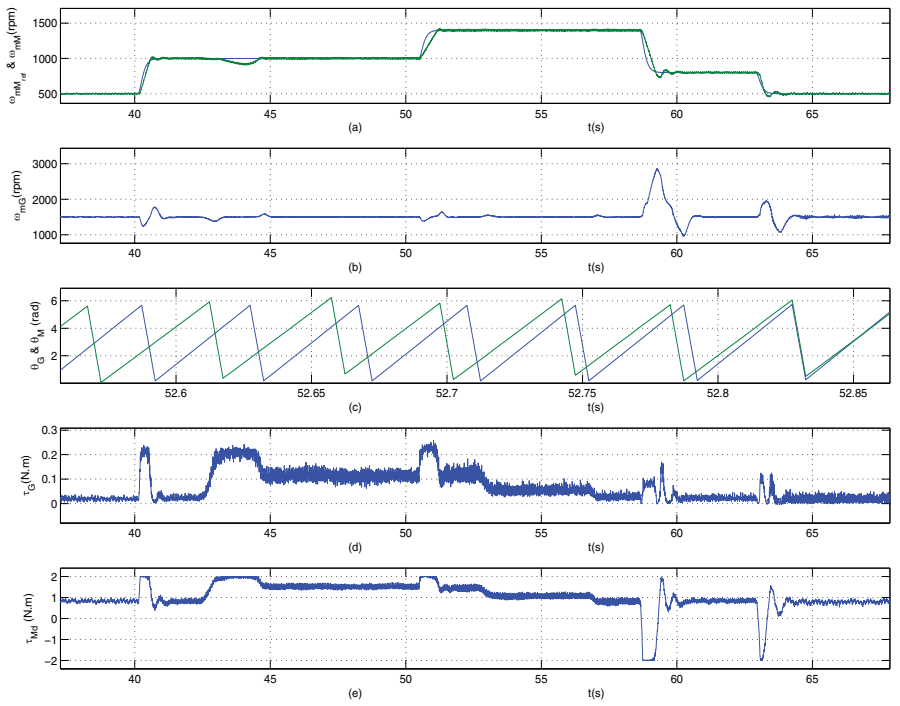

Fig. 20. PBC+PI-(a) Regulated Motor speed and its reference. (b)Generator speed. (c) DFIG \& IM rotor position. (d)Generator torque (e) Motor desired torque. 


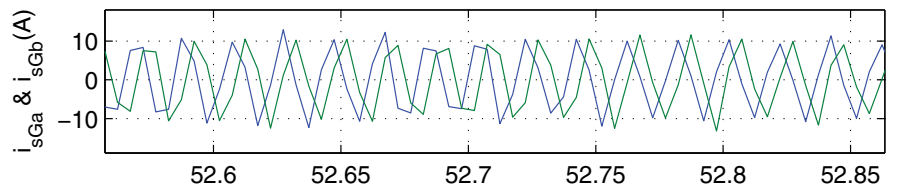

(a)

$t(s)$

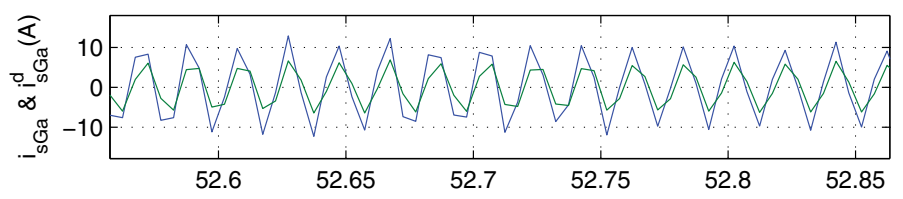

(b)

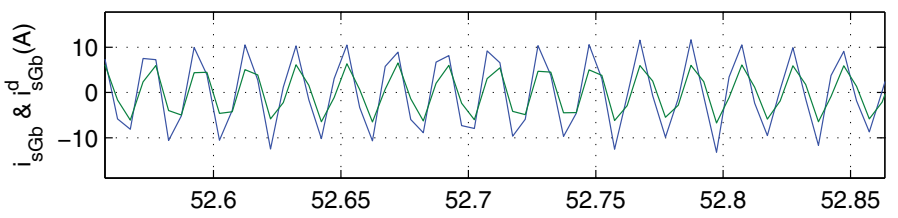

(c)

$t(s)$

Fig. 21. PBC+PI-(a) $i_{s G a}, i_{s G b}$ (b) $i_{s G a^{\prime}}^{d} i_{s G a}$ (c) $i_{s G b^{\prime}}^{d} i_{s G b}$.

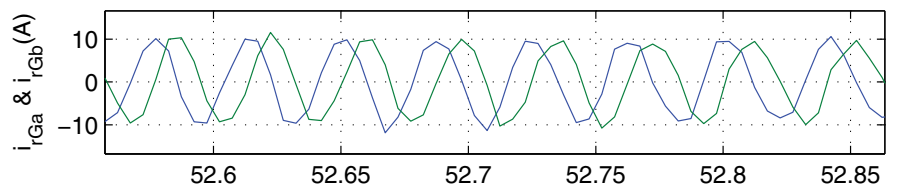

(a)

$\mathrm{t}(\mathrm{s})$

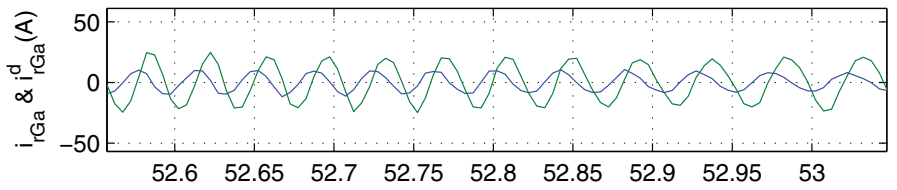

(b)

$t(s)$

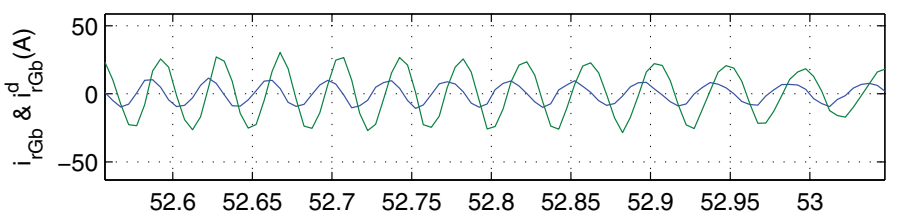

(c)

Fig. 22. PBC+PI-(a) $i_{r G a}, i_{r G b}$ (b) $i_{r G a^{\prime}}^{d} i_{r G a}$ (c) $i_{r G b^{\prime}}^{d} i_{r G b}$.

controller (see fig. 17). This is due to the fact that the reference values are sinusoidal and that the bandwidth of the PI controllers cannot be increased sufficiently experimentally.

It can be concluded that the PI action on the stator currents does not improve significantly the performance obtained with the $\mathrm{PBC}+\mathrm{P}$ controller.

\subsection{PI}

The PI control law (with $K_{p}$ and $K_{i}$ are proportional and integral gains) is given below:

$$
B v_{r G}=B\left(K_{p}\left(i_{s G}-i_{s G}^{d}\right)+K_{i}\left(i_{s G}-i_{s G}^{d}\right)\right)
$$




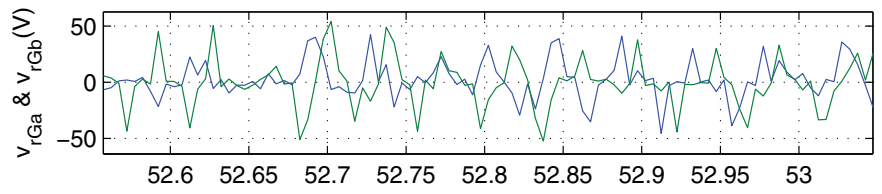

(a)

$\mathrm{t}(\mathrm{s})$

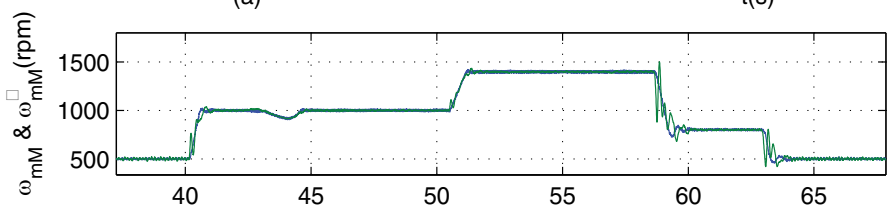

(b)

$t(s)$

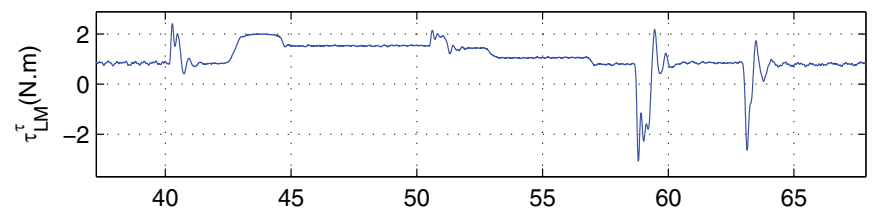

(c)

$t(s)$

Fig. 23. PBC+PI-(a) $v_{r G a}, v_{r G b}$ (b) $\omega_{m M}, \hat{\omega}_{m M}$ (c) $\hat{\tau}_{M L}$.
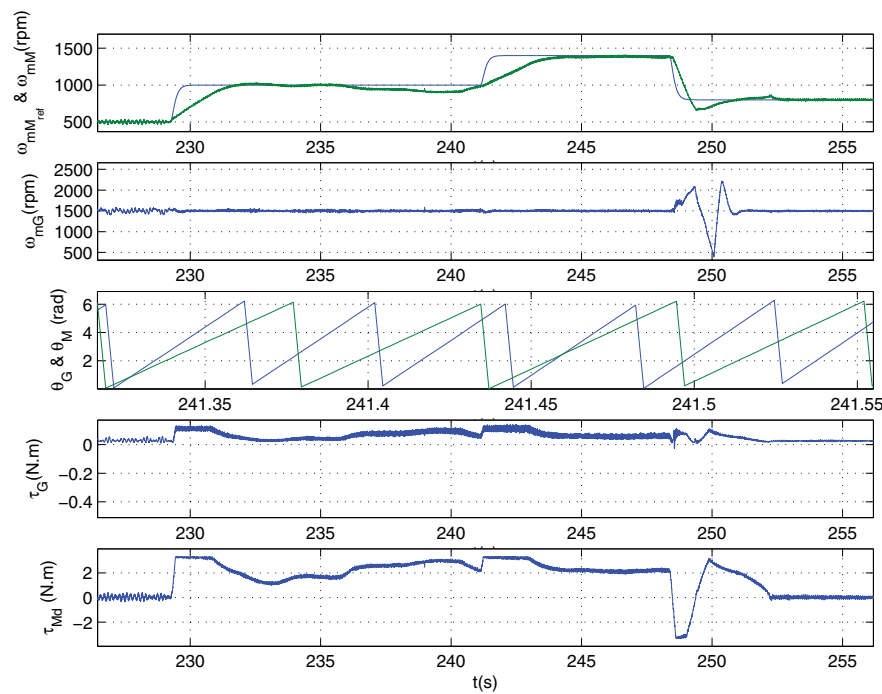

Fig. 24. PI-(a) Regulated Motor speed and its reference. (b)Generator speed. (c) DFIG \& IM rotor position. (d) Generator torque (e) Motor desired torque. 

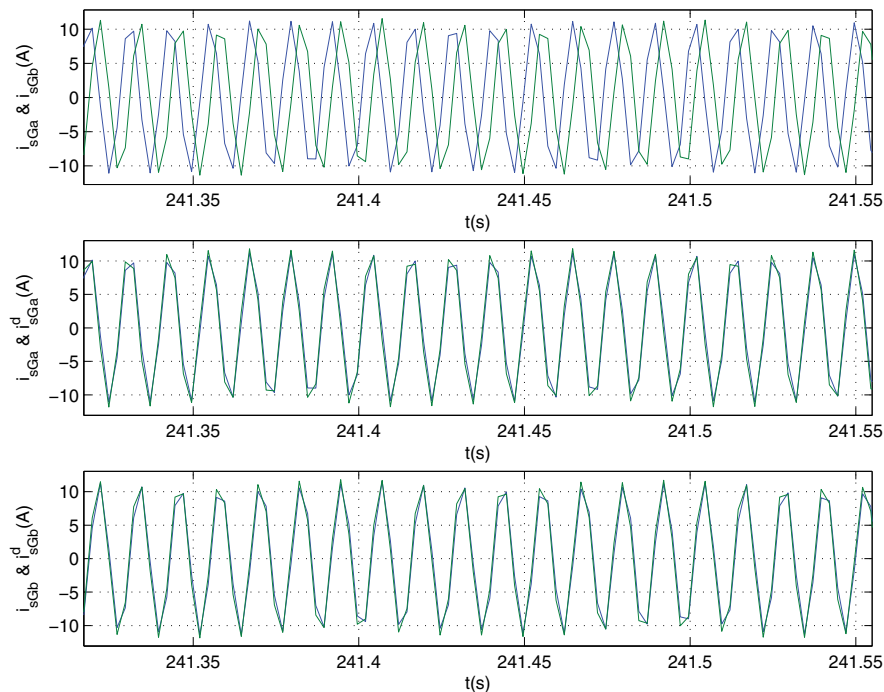

Fig. 25. PI-(a) $i_{s G a}, i_{s G b}$ (b) $i_{s G a^{\prime}}^{d} i_{s G a}$ (c) $i_{s G b}^{d}, i_{s G b}$.
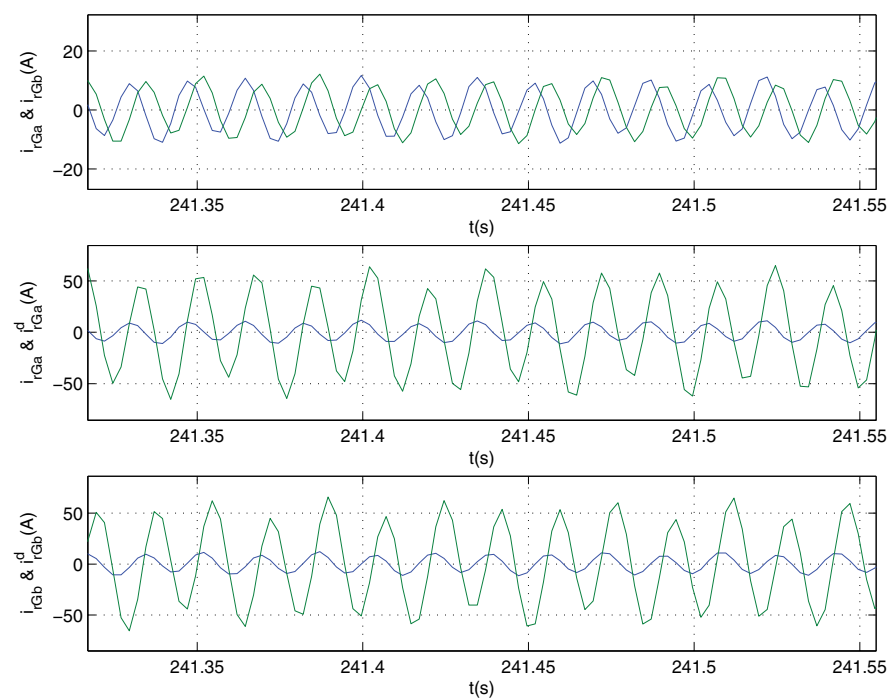

Fig. 26. PI-(a) $i_{r G a}, i_{r G b}$ (b) $i_{r G a^{\prime}}^{d} i_{r G a}$ (c) $i_{r G b}^{d}, i_{r G b}$.

Finally, in order to obtain a significant comparison between controllers, a PI-based control has been designed without a PBC, i.e. there is one PI controller for each stator current. Figures 24-27 show the results. These results show clearly that the system behaviour is much deteriorated in comparison with the results obtained with the previous controllers. Even if there is no IM speed error in the steady state, the speed does not track its reference during transients, and there is a speed error when a load torque is applied. This is mainly due to the saturation of the desired IM torque at a value four times its nominal value. Consequently, the stator currents are very large, i.e. their magnitude is about twice those currents with the PBC, and so significant stator losses can be expected. 

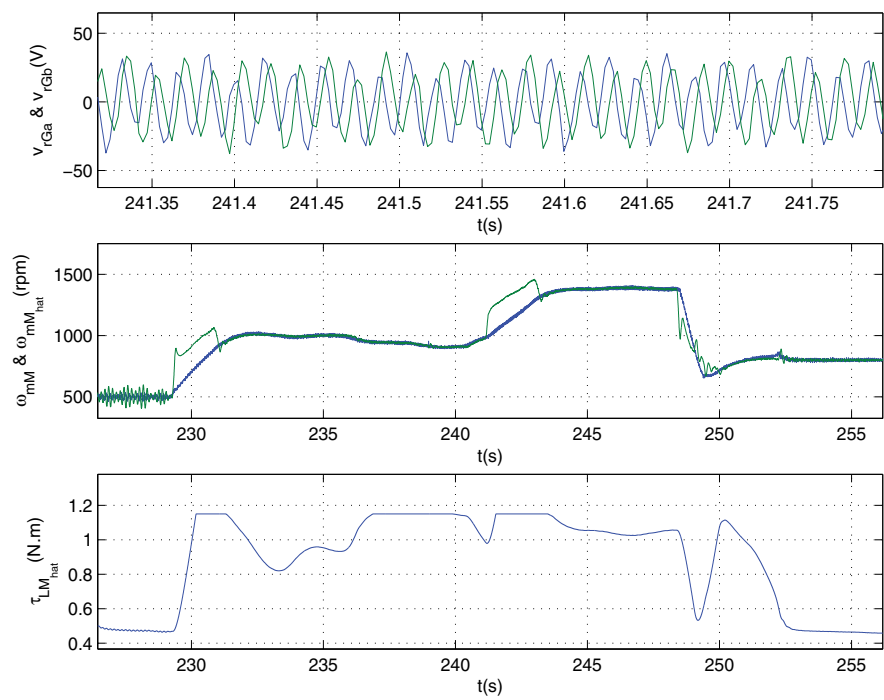

Fig. 27. PI-(a) $v_{r G a}, v_{r G b}$ (b) $\omega_{m M}, \hat{\omega}_{m M}$ (c) $\hat{\tau}_{M L}$.

These results show that the PI control alone of the stator currents is not efficient for the control of the DFIG+IM system. The PBC, with or without P or PI actions, shows much better performance.

\subsection{Robustness tests}

In order to highlight the performances of the controllers, and check their behaviour in the presence of machine parameter variations, a change in the DFIG and IM rotor and stator resistances is applied. In the real case, the resistances of a machine increase with temperature. In this case, all the resistances of the two machines used in the controllers are decreased by $40 \%$ when the "Switch on Parameters" signal value goes from 0 to 1 (see figure 31). This test has been carried out with the four controllers (i.e. PBC, PBC+P, PBC+PI and PI). The results show that all the controllers are robust to a large change in machine resistances. To be brief, only the results obtained with the PBC are reported here.

Figure 28 presents the mechanical IM speed and its smooth reference, the mechanical DFIG speed, the DFIG and IM rotor positions, the DFIG torque $\tau_{G}$ and the IM desired torque $\tau_{M d}$. The real IM speed tracks very well the reference, i.e. low overshoot and no steady state error are observed. Figure 29 shows the stator currents $i_{s a}$ and $i_{s b}$, and their references over a period of time. The stator currents do not track exactly the desired values but are bounded. This is because the goal of the PBC is to track the IM speed and to keep internal signals bounded.

Figure 30 shows the DFIG rotor currents $i_{r G a}$ and $i_{r G b}$, and their references over a period of time. Again, these currents are sinusoidal and bounded.

Figure 31 presents the control signals $v_{r G a}$ and $v_{r G b}$, the rotor IM speed $\omega_{m M}$ and its estimation $\hat{\omega}_{m M}$, and the "Switch on Parameters" signal. These results illustrate the robustness of the PBC when the parameters are varied.

\section{Conclusion}

Speed-torque tracking controllers for an IM powered by a DFIG have been presented. The joint system extracts energy from a primary mechanical source that is transformed by the 

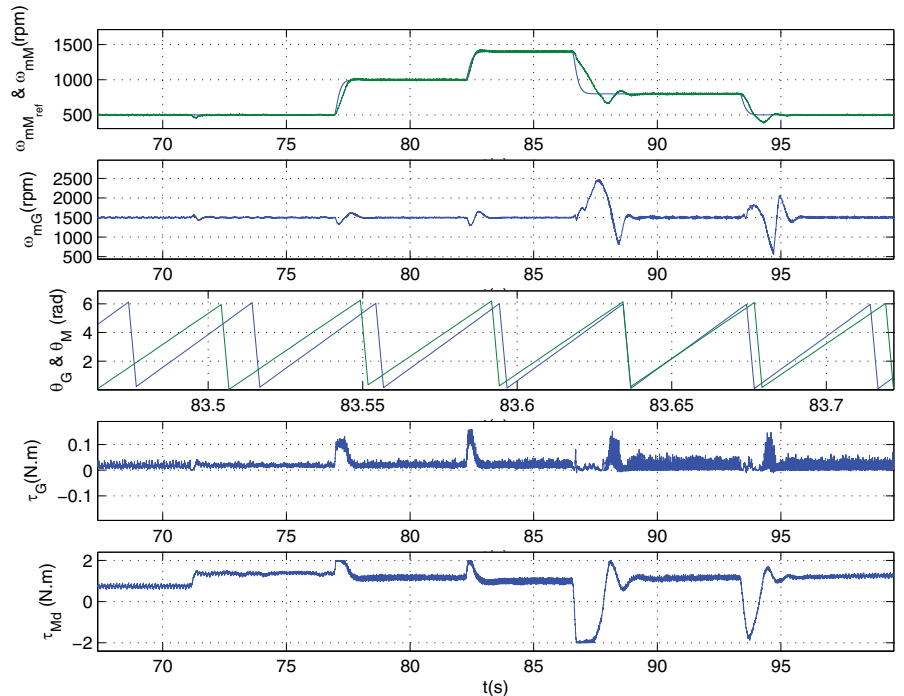

Fig. 28. PBC-robustness test-(a) Regulated Motor speed and its reference. (b)Generator speed. (c) DFIG \& IM rotor position. (d) Generator torque (e) Motor desired torque.
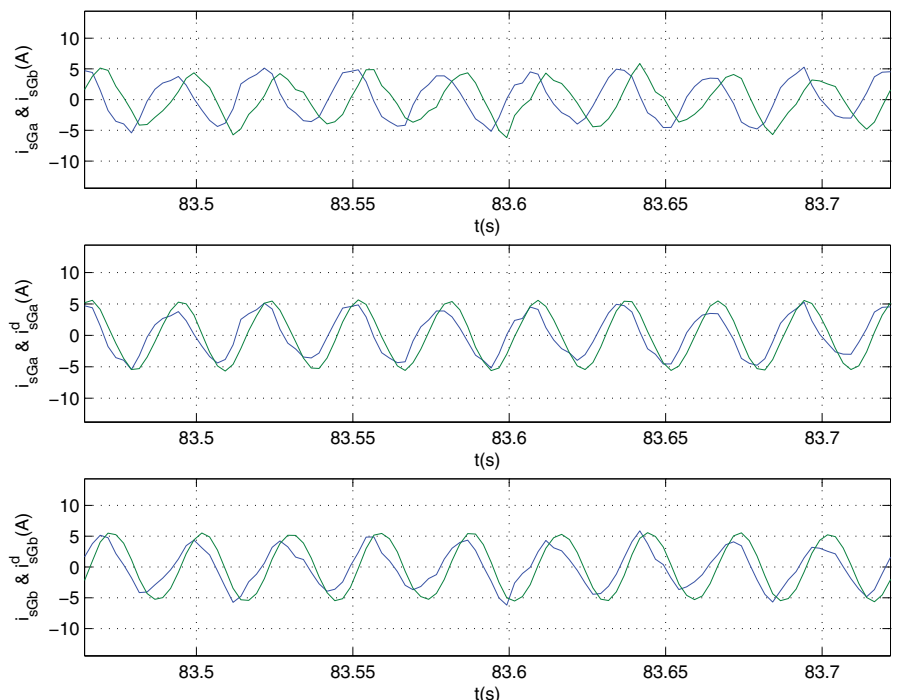

Fig. 29. PBC -robustness test-(a) $i_{s G a}, i_{s G b}$ (b) $i_{s G a^{\prime}}^{d} i_{s G a}$ (c) $i_{s G b^{\prime}}^{d} i_{s G b}$. 

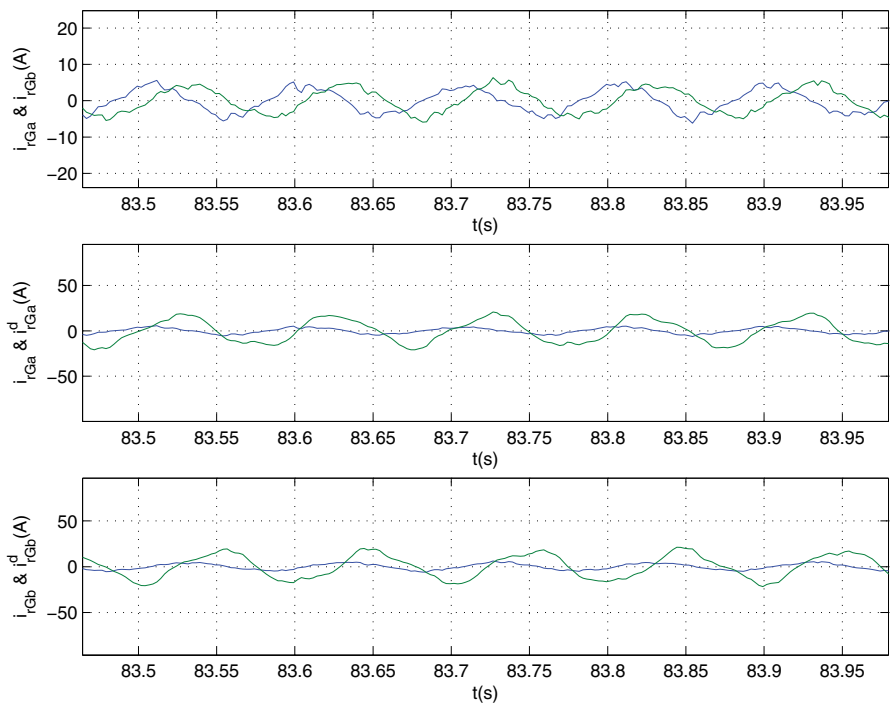

Fig. 30. PBC-robustness test-(a) $i_{r G a}, i_{r G b}$ (b) $i_{r G a^{\prime}}^{d} i_{r G a}$ (c) $i_{r G b^{\prime}}^{d} i_{r G b}$.
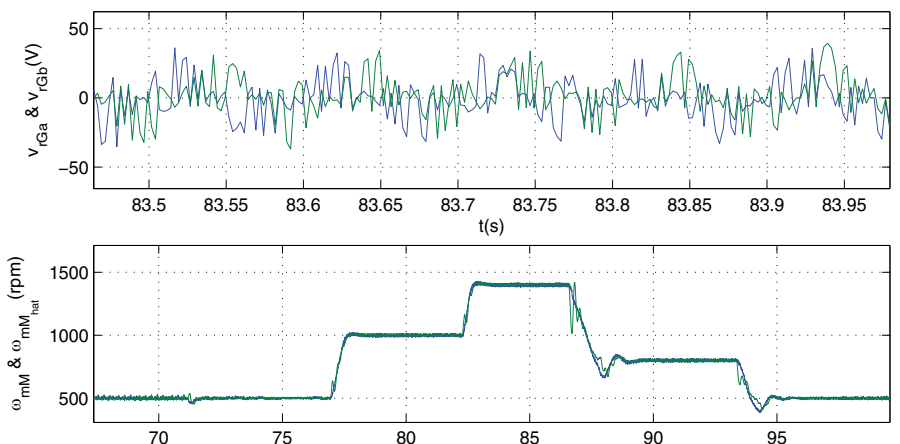

$t(s)$

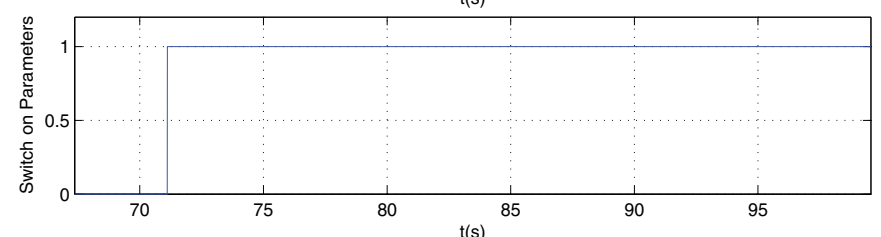

Fig. 31. PBC-robustness test-(a) $v_{r G a}, v_{r G b}$ (b) $\omega_{m M}, \hat{\omega}_{m M}$ (c) Switch.

DFIG, which at the same time controls the speed of the IM making use of the rotor voltage of the DFIG as a control variable. A complete stability proof for inner loop control is given. The proof of the overall scheme including the outer speed loop follows verbatim from (1) and is omitted here for brevity.

The main advantage of the $\mathrm{PBC}$ is that it requires the measurement of only two mechanical positions for the speed tracking. The PI controller applied to the inner loop provides good performance but saturation in the transient state can be observed. Robustness tests were performed to observe the behaviour of the controllers to machine parameter variations. All the proposed controllers were found to be robust towards variation in machine resistances. Also, a power flow analysis can be undertaken between the generator, the IM and the grid 
in order to optimize the efficiency of the overall system. A comparison of the experimental results of the proposed $\mathrm{PBC}, \mathrm{PI}, \mathrm{PBC}+\mathrm{P}$ and $\mathrm{PBC}+\mathrm{PI}$ algorithm is presented in Table 2 . It is based on the performance obtained practically with the different controllers. In addition to the comparison criteria of Table 2, it is proposed to check the following to see what their effects are on the controllers'performance:

- $e_{\omega_{M}}=\frac{1}{n T} \sum_{i=1}^{n}\left[\omega_{M}(i)-\omega_{R e f M}(i)\right]^{2}$ indication about the IM speed tracking error. Where $n$ is the length of the sampled data and $T$ is the sampling time;

- $e_{i_{s G a}}=\frac{1}{n T} \sum_{i=1}^{n}\left[i_{s G a}(i)-i_{s G a}^{d}(i)\right]^{2}$ indication about the stator current tracking error in the phase $a$;

- Observed magnitude of $i_{S G a}$;

- $P_{\mathrm{avg}_{G}}=\frac{1}{n} \sum_{i=1}^{n}\left[\tau_{G}(i) \omega_{G}(i)\right]$ indication about the rotor average value of the instantaneous absorbed power in the DIFG;

- $P_{\operatorname{avg}_{M}}=\frac{1}{n} \sum_{i=1}^{n}\left[\tau_{M}(i) \omega_{M}(i)\right]$ indication about the rotor average value of the instantaneous absorbed power in IM;

\begin{tabular}{|l||c|c|c|c|c|c|}
\hline & $R_{S}(\Omega)$ & $R_{r}(\Omega)$ & $L_{s}(m H)$ & $L_{r}(m H)$ & $L_{m}(m H)$ & $J\left(\mathrm{Nm}^{2} / \mathrm{rad}\right)$ \\
\hline \hline DFIG & 0.365 & 0.559 & 0.938 & 0.938 & 12.975 & $4.358 \times 10^{-3}$ \\
\hline IM & 0.5 & 0.2 & 1.2 & 1.2 & 9.00 & $1.1 \times 10^{-3}$ \\
\hline
\end{tabular}

Table 1. The parameters for DFIG and IM

\begin{tabular}{|c|c|c|c|c|}
\hline & PBC & $\overline{\mathrm{PBC}+\mathrm{P}}$ & $\overline{\mathrm{PBC}+\mathrm{PI}}$ & $\overline{\mathrm{PI}}$ \\
\hline $\begin{array}{l}\omega_{\text {RefM }} \\
{[\mathrm{rpm}]}\end{array}$ & $\begin{array}{c}500 \rightarrow 1000 \\
\rightarrow 1400 \rightarrow 800 \\
\left(1^{\text {st }} \text { order filter }\right) \\
\end{array}$ & $\begin{array}{c}500 \rightarrow 1000 \\
\rightarrow 1400 \rightarrow 800 \\
\left(1^{\text {st }} \text { order filter }\right) \\
\end{array}$ & $\begin{array}{c}500 \rightarrow 1000 \\
\rightarrow 1400 \rightarrow 800 \\
\left(1^{\text {st }} \text { order filter }\right) \\
\end{array}$ & $\begin{array}{c}500 \rightarrow 1000 \\
\rightarrow 1400 \rightarrow 800 \\
\left(1^{s t} \text { order filter }\right) \\
\end{array}$ \\
\hline$\tau_{L M}[\mathrm{~N} . \mathrm{m}]$ & $0.5 \rightarrow 1.45 \rightarrow 0.5$ & $0.5 \rightarrow 1.4 \rightarrow 0.5$ & $0.5 \rightarrow 1 \rightarrow 0.5$ & $0.5 \rightarrow 1.15 \rightarrow 0.5$ \\
\hline $\begin{array}{l}\text { settling time } \\
\text { of } \omega_{\text {Ref } M}\end{array}$ & $0.4 \mathrm{~s}$ & $0.4 s$ & $0.4 s$ & $0.4 s$ \\
\hline $\begin{array}{l}\text { settling time } \\
\text { of } \omega_{M}\end{array}$ & $0.1 \mathrm{~s}$ & $0.1 \mathrm{~s}$ & $0.1 s$ & $2 s$ \\
\hline$\omega_{G}[\mathrm{rpm}]$ & 1500 & 1500 & 1500 & 1500 \\
\hline$e_{\omega_{M}} \times 10^{5}$ & 2.9 & 4.8 & 5.7 & 38.6 \\
\hline$e_{i_{\mathrm{SG} Q}} \times 10^{3}$ & 3.7 & 2.65 & 2.68 & 0.25 \\
\hline $\begin{array}{c}\text { Observed magnitude } \\
\text { of } i_{s G a}[\mathrm{~A}]\end{array}$ & 5 & 7 & 8 & 10 \\
\hline$P_{\mathrm{avg}_{G}}[\mathrm{~W}]$ & 4.7 & 5.6 & 5 & 9 \\
\hline$P_{\text {avg }_{M}}[\mathrm{~W}]$ & 58.9 & 74.4 & 70.7 & 177.9 \\
\hline
\end{tabular}

Table 2. Comparison table of experimental results

If we take in account the problem of speed tracking of the IM interconnected to the DFIG and according to the robustness tests and the experimental results presented in Table 2 we can say that the PBC controller provided the best performance.

In addition, this paper has provided the detailed analysis of operational principles of the BDFTIG. 


\section{Acknowledgment}

The authors would like to express their gratitude to Jordi Riera, Enric Fossas and Miguel Allué from IRII-UPC, Barcelona, Spain for their help with the practical experiments.

\section{References}

[1] R. Ortega, A. Loria, P.J. Nicklasson, and H. Sira-Ramirez, "Passivity-based control of Euler-Lagrange systems," in Communications and Control Engineering. Berlin,Germany:Spring-Verlag, 1998.

[2] Liu, X., G. Verghese, J. Lang and M. Önder, Generalizing the Blondel-Park Transformation of Electrical Machines: Necessary and Sufficient Conditions, IEEE Trans. Circ. Syst., Vol. 36, No. 8, pp. 1085-1067, 1989.

[3] M. Becherif, R. Ortega, E. Mendes and S. Lee, "Passivity-based control of a doubly-fed induction generator interconnected with an induction motor," in CDC 2003.

[4] M. Becherif, "Contribution aux techniques de façonnement dénergie: Application à la commande des systèmes électromécaniques," PhD thesis, Université de Paris XI, France, 2004.

[5] W. Leonhard, "Control of electrical drives," Spring-Verlag, 1985.

[6] M.S. Vicatos and J.A. Tagopoulos, "Steady state analysis of a doubly-fed induction generator under sychronous operation," IEEE Trans. on Energy Conversion, vol.4, no.3, pp.495-501, 1989.

[7] F. Bogalecka, "Dynamics of the power control of a double fed induction generator connected to the soft power grid," ISIE International Symposium on Industrial Electronics, Budapest, pp.509-513, 1993.

[8] A. Mebarki and R.T. Lipczynsky, "Novel variable speed constant frequency generation system with voltage regualtion," EPE European Conference on Power Electronics and Applications, vol.2, pp.465-471, 1995.

[9] P. Caratozzolo, E. Fossas and J. Riera "Robust nonlinear control of an isolated motion system," CIEP International Power Electronics Congress, Mexico, 2002.

[10] R. Datta and V.T. Ranganathan, "Variable-speed wind power generation using doubly fed wound rotor induction machine-a comparision with alternative schemes," IEEE Trans. on energy conversion, vol.17, no.3, pp.414-421, 2002.

[11] S. Muller, M. Deicke, and Rik W. De Doncker, "Doubly fed induction generator systems for wind turbines," IEEE Industry Applications Magazine, vol., no., pp.26-33, May/June, 2002.

[12] P. Caratozzolo, E. Fossas, and J. Riera, "Nonlinear control of an isolated motion system with DFIG," IFAC International Federation of Automatic Control , 2002.

[13] A. J. van der Schaft, " $L_{2}-$ Gain and Passivity Techniques in Nonlinear Control," Springer-Verlag, Berlin, 1999. 


\title{
A RMRAC Parameter Identification Algorithm Applied to Induction Machines
}

\author{
Rodrigo Z. Azzolin ${ }^{1}$, Cristiane C. Gastaldini ${ }^{2}$, Rodrigo P. Vieira ${ }^{3}$ and \\ Hilton A. Gründling ${ }^{4}$ \\ 1,2,3,4 Federal University of Santa Maria \\ ${ }^{1}$ Federal University of Rio Grande \\ ${ }^{3}$ Federal University of Pampa \\ Brazil
}

\section{Introduction}

This chapter deals with the problem of parameter identification of electrical machines to achieve good performance of a control system. The development of an identification algorithm is presented, which in this case is applied to Single-Phase Induction Motors, but could easily be applied to other electrical machine. This scheme is based on a Robust Model Reference Adaptive Controller and measurements of stator currents of a machine with standstill rotor. From the obtained parameters, it is possible to design high performance controllers and sensorless control for induction motors.

Single-phase induction motors (SPIM) are widely used in fractional and sub-fractional horsepower applications, usually in locations where only single-phase energy supply is available. In most of these applications the machine operates at constant frequency and is fed directly from the AC grid with an ON/OFF starting procedure. In recent years, several researchers have shown that the variable speed operation can enhance the SPIM's efficiency (Blaabjerg et al., 2004; Donlon et al., 2002). In addition, other researchers have developed high performance drives for SPIM's, using Field-Oriented Control (FOC) and sensorless techniques (de Rossiter Correa et al., 2000; Vaez-Zadeh \& Reicy, 2005; Vieira et al., 2009b). However, the FOC associated with the sensorless technique demands accurate knowledge of electrical motor parameters to achieve good performance.

A good deal of research has been carried out in the last several years on parameter estimation of induction motors, mainly with regards to three-phase induction machines (Azzolin et al., 2007; Koubaa, 2004; Ribeiro et al., 1995; Velez-Reyes et al., 1989).

However, few methods have been proposed for automatic estimation in single-phase induction motors. One of them uses a classical method for electrical parameter identification (Ojo \& Omozusi, 2001), its implementation is onerous. In (Vieira et al., 2009a) a Recursive Least Squares (RLS) identification algorithm is used to obtain machine parameters. The identification results are good, although this method requires the design of filters to obtain the variable derivative, which can be deteriorated by noises.

In order to solve these problems, this chapter details a closed-loop algorithm to estimate the electrical parameters of a single-phase induction motor based on (Azzolin \& Gründling, 


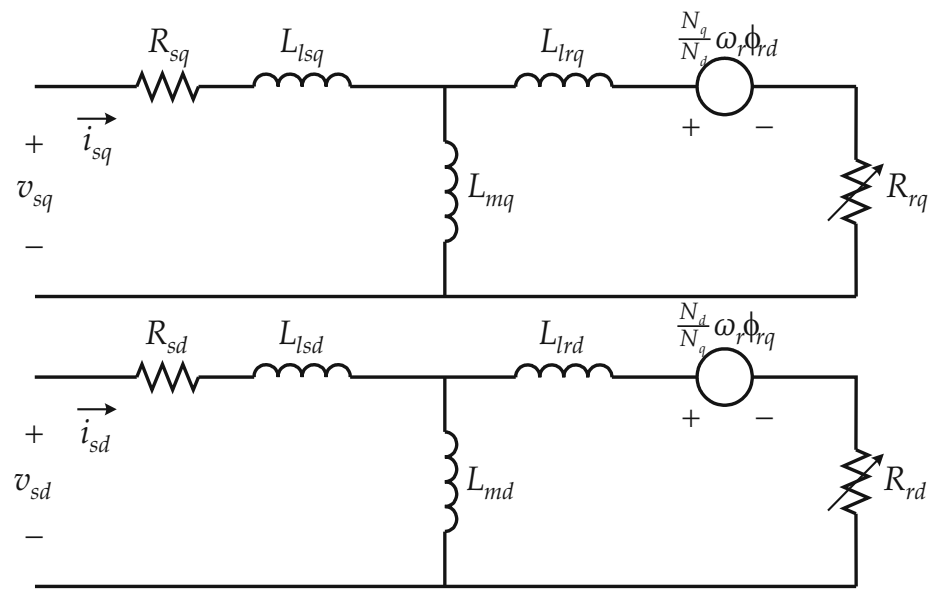

Fig. 1. Equivalent circuit of a SPIM.

2009). In (Azzolin \& Gründling, 2009) a Robust Model Reference Adaptive Controller (RMRAC) algorithm was used for parameter identification of three-phase induction motors. The RMRAC algorithm eliminates the use of filters to obtain derivatives of the signals. Thus, the objectives of this chapter are to apply the RMRAC algorithm in the electrical parameter identification of a SPIM and make use of the robustness of the system in dealing with of noise measurement.

As a result, the proposed parameter estimation procedure is divided into three steps:

(I) identification of the RMRAC controller gains;

(II) estimation of the transfer function coefficients of the induction motor at standstill;

(III) calculation of the stator resistance $R_{s i}$, rotor resistance $R_{r i}$, stator inductance $L_{s i}$, rotor inductance $L_{r i}$ and mutual inductance $L_{m i}$ using steps (I) and (II), where the index " $i$ " express the axes $\mathrm{q}$ or $\mathrm{d}$.

This chapter is organized as follows: Section 2 presents the induction machine model. A short review of the RMRAC algorithm applied to the identification system is presented in sections 3 and 4. Section 5 describes the assumptions and equations of Model Reference Control (MRC) while section 6 shows the proposed parameter identification algorithm. Sections 7 and 8 present the simulation and experimental results. Finally, chapter conclusions are presented in section 9 .

\section{Mathematical model of a single phase induction motor}

The SPIM equivalent circuit without the permanent split-capacitor can be represented by an asymmetrical two-phase induction motor as shown in Fig. 1 . In this figure $L_{l s i}$ and $L_{l r i}$ are the stator and rotor leakage inductances, $\omega_{r}$ is the speed rotor, $\phi_{r i}$ is the electromagnetic flux and $N_{i}$ is the number of turns for auxiliary winding or axis $\mathrm{d}$ and for main winding or axis $\mathrm{q}$. The stator and rotor inductances are relationship with the leakage and mutual inductance as a $L_{s i}=L_{l s i}+L_{m i}$ and $L_{r i}=L_{l r i}+L_{m i}$, respectively.

From Fig. 1 and from (Krause et al., 1986) it is possible to derive the dynamical model of a SPIM. The SPIM dynamical model in a stationary reference frame can be described by 1 . In this 
equation $\bar{\sigma}_{q}=L_{s q} L_{r q}-L_{m q}^{2}, \bar{\sigma}_{d}=L_{s d} L_{r d}-L_{m d^{\prime}}^{2} p$ is the poles pairs and $n$ is the relationship between the number of turns for auxiliary and for main winding $N_{d} / N_{q}$.

$$
\begin{gathered}
{\left[\begin{array}{c}
\dot{i}_{s q} \\
i_{s d} \\
i_{r q} \\
i_{r d}
\end{array}\right]=\left[\begin{array}{cccc}
-\frac{R_{s q} L_{r q}}{\bar{\sigma}_{q}} & -p \omega_{r} \frac{1}{n} \frac{L_{m q} L_{m d}}{\bar{\sigma}_{q}} & \frac{R_{r q} L_{m q}}{\bar{\sigma}_{q}} & -p \omega_{r} \frac{1}{n} \frac{L_{r d} L_{m q}}{\bar{\sigma}_{q}} \\
p \omega_{r} n \frac{L_{m q} L_{m d}}{\bar{\sigma}_{d}} & -\frac{L_{r d} R_{s d}}{\bar{\sigma}_{d}} & p \omega_{r} n \frac{L_{r q} L_{m d}}{\bar{\sigma}_{d}} & \frac{R_{r d} L_{m d}}{\bar{\sigma}_{d}} \\
\frac{L_{m q} R_{s q}}{\bar{\sigma}_{q}} & p \omega_{r} \frac{1}{n} \frac{L_{s q} L_{m d}}{\bar{\sigma}_{q}} & -\frac{L_{s q} R_{r q}}{\bar{\sigma}_{q}} & p \omega_{r} \frac{1}{n} \frac{L_{s q} L_{r d}}{\bar{\sigma}_{q}} \\
-p \omega_{r} n \frac{L_{s d} L_{m q}}{\bar{\sigma}_{d}} & \frac{L_{m d} R_{s d}}{\bar{\sigma}_{d}} & -p \omega_{r} n \frac{L_{s d} L_{r q}}{\bar{\sigma}_{d}} & -\frac{L_{s d} R_{r d}}{\bar{\sigma}_{d}}
\end{array}\right] .} \\
\cdot\left[\begin{array}{cc}
\frac{L_{s q}}{\bar{\sigma}_{q}} & 0 \\
i_{s d} \\
i_{r q} \\
i_{r d}
\end{array}\right]+\left[\begin{array}{cc}
0 & \frac{L_{r d}}{\bar{\sigma}_{d}} \\
-\frac{L_{m q}}{\bar{\sigma}_{q}} & 0 \\
0 & -\frac{L_{m d}}{\bar{\sigma}_{d}}
\end{array}\right]\left[\begin{array}{c}
v_{s q} \\
v_{s d}
\end{array}\right]
\end{gathered}
$$

From equation 1 it is possible to obtain the transfer functions in the axes $\mathrm{q}$ and $\mathrm{d}$ at standstill rotor $\left(\omega_{r}=0\right)$, where these equations are decoupled and presented in 2 and 3 .

$$
\begin{aligned}
& H_{q}(s)=\frac{i_{s q}(s)}{v_{s q}(s)}=\frac{s \frac{L_{r q}}{\bar{\sigma}_{q}}+\frac{R_{r q}}{\bar{\sigma}_{q}}}{s^{2}+s p_{q}+\frac{R_{r q} R_{s q}}{\bar{\sigma}_{q}}}, \\
& H_{d}(s)=\frac{i_{s d}(s)}{v_{s d}(s)}=\frac{s \frac{L_{r d}}{\bar{\sigma}_{d}}+\frac{R_{r d}}{\bar{\sigma}_{d}}}{s^{2}+s p_{d}+\frac{R_{r d} R_{s d}}{\bar{\sigma}_{d}}},
\end{aligned}
$$

where

$$
p_{q}=\frac{R_{s q} L_{r q}+R_{r q} L_{s q}}{\bar{\sigma}_{q}} \text { and } p_{d}=\frac{R_{s d} L_{r d}+R_{r d} L_{s d}}{\bar{\sigma}_{d}} .
$$

\section{Identification system}

The proposed electrical parameter identification system is shown in Fig. 2. This system is based on a RMRAC algorithm used to generate the control action $v_{s q}$ by the difference between the measured current $i_{s q}$ and the reference $i_{s q}^{*}$ at standstill rotor. In this test the auxiliary winding (or axis $\mathrm{d}$ ) is open while the main winding (or axis q) is identified.

The dotted box in Fig. 2 is detailed in Fig. 3 where the RMRAC control law applied to q axis is shown. In Fig. 3, the reference model and plant are given by

$$
W_{m}(s)=k_{m} \frac{Z_{m}(s)}{R_{m}(s)}
$$




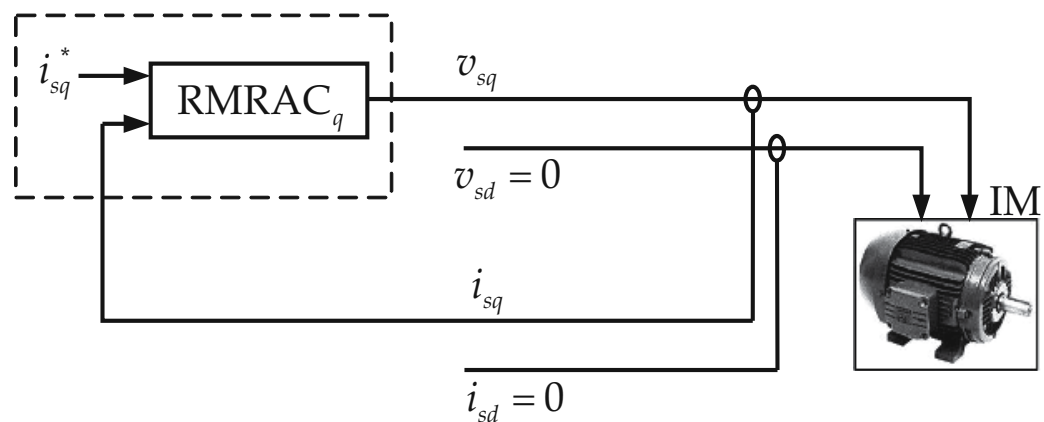

Fig. 2. Block diagram of the RMRAC identification system.

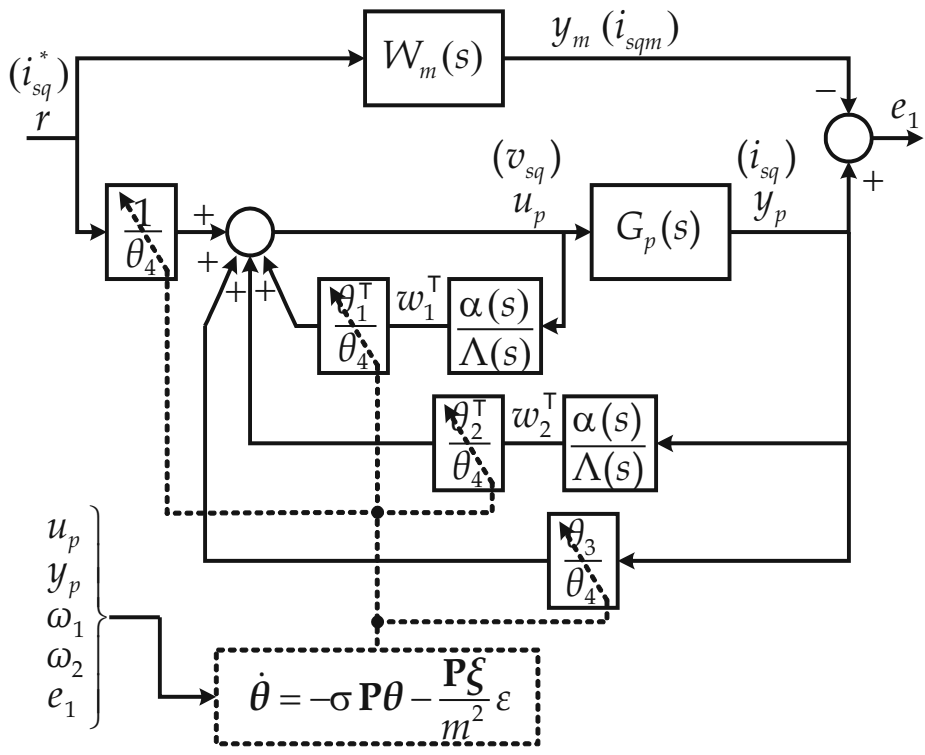

Fig. 3. RMRAC structure.

$$
G_{p}(s)=k_{p} \frac{Z_{p}(s)}{R_{p}(s)} .
$$

where $Z_{m}(s), R_{m}(s), Z_{p}(s)$ and $R_{p}(s)$ are monic polynomials and $k_{m}$ and $k_{p}$ are high frequency gains.

The general idea behind RMRAC is to create a closed-loop controller with gains that can be updated and change the response of the system. The output of the system $y_{p}$ is compared to a desired response from a reference model $y_{m}$. The controller gains vector $\boldsymbol{\theta}=\left[\begin{array}{llll}\theta_{1}^{\top} & \theta_{2}^{\top} & \theta_{3} & \theta_{4}\end{array}\right]^{\top}$ is updated by a gradient algorithm based on $e_{1}$ error. The goal is that the gains converge to ideal values that cause the plant response to match the response of the reference model. These gains can be obtained by a gradient algorithm presented in (Ioannou \& Sun, 1996; Ioannou \& Tsakalis, 1986) and described as follows. More details of RMRAC algorithms can be seen in (Câmara \& Gründling, 2004). 
The procedure described in Fig. 2 and Fig. 3 is applied to parameter identification of the $q$ axis or transfer function 2. However, the same procedure is used for parameter identification of the $\mathrm{d}$ axis or transfer function 3 .

\section{RMRAC gains adaptation algorithm}

The gradient algorithm used to obtain the control law gains is given by

$$
\dot{\boldsymbol{\theta}}=-\sigma \mathbf{P} \boldsymbol{\theta}-\frac{\mathbf{P} \xi}{m^{2}} \varepsilon
$$

with

$$
\dot{m}=\delta_{0} m+\delta_{1}\left(\left|u_{p}\right|+\left|y_{p}\right|+1\right), m(0)>\frac{\delta_{1}}{\delta_{0}}, \delta_{1} \geq 1
$$

and

$$
\begin{gathered}
\boldsymbol{\xi}=W_{m}(s) \mathbf{I} \boldsymbol{w}, \\
\boldsymbol{w}=\left[\begin{array}{llll}
w_{1}^{\top} & w_{2}^{\top} & y_{p} & u_{p}
\end{array}\right]^{\top},
\end{gathered}
$$

$w_{1}^{\top}, w_{2}^{\top}$ are auxiliary vectors, $\delta_{0}, \delta_{1}$ are positive constants and $\delta_{0}$ satisfies $\delta_{0}+\delta_{2} \leq \min \left(p_{0}, q_{0}\right)$, $q_{0} \in \Re^{+}$is such that the $W_{m}\left(s-q_{0}\right)$ poles and the $\left(\mathbf{F}-q_{0} \mathbf{I}\right)$ eigenvalues are stable and $\delta_{2}$ is a positive constant. The sigma modification $\sigma$ in 7 is given by

$$
\sigma=\left\{\begin{array}{clc}
0 & \text { if } \quad\|\boldsymbol{\theta}\|<M_{0} \\
\sigma_{0}\left(\frac{\|\boldsymbol{\theta}\|}{M_{0}}-1\right) & \text { if } M_{0} \leq\|\boldsymbol{\theta}\|<2 M_{0} \\
\sigma_{0} & \text { if } \quad\|\boldsymbol{\theta}\| \geq 2 M_{0}
\end{array}\right.
$$

where $M_{0}>\left\|\boldsymbol{\theta}^{*}\right\|$ and $\sigma_{0}>2 \mu^{-2} / R^{2}, R, \mu \in \Re^{+}$are design parameters. In this case, the parameters used in the implementation of the gradient algorithm are

$$
\left\{\begin{array}{l}
\delta_{0}=0.7 \\
\delta_{1}=1 \\
\delta_{2}=1 \\
\sigma_{0}=0.1 \\
M_{0}=10
\end{array}\right.
$$

More details of design of the gradient algorithm can be seen in (Ioannou \& Tsakalis, 1986). As defined in (Lozano-Leal et al., 1990), the modified error in 7 is given by

$$
\varepsilon=e_{1}+\boldsymbol{\theta}^{\top} \boldsymbol{\xi}-W_{m} \boldsymbol{\theta}^{\top} \boldsymbol{w}
$$

or

$$
\varepsilon=\phi^{\top} \xi+\mu \eta
$$

When the ideal values of gains are identified and the plant model is well known, the plant can be obtained by equation analysis of MRC algorithm described in the next section. 


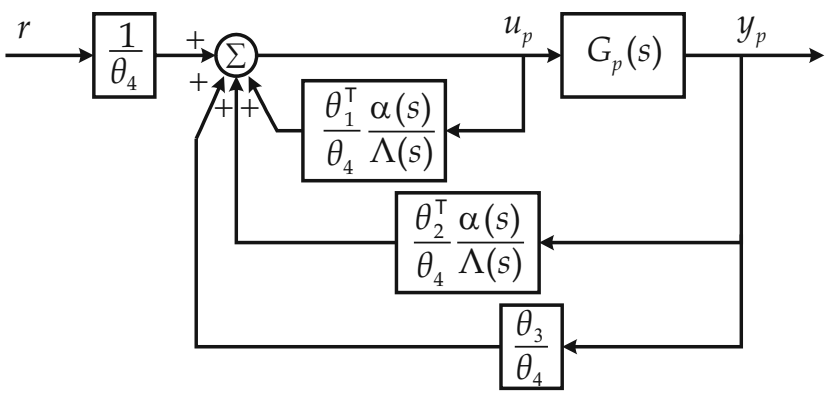

Fig. 4. MRC structure.

\section{MRC analysis}

The Model Reference Control (MRC) shown in Fig. 4 can be understood as a particular case of RMRAC structure, which is presented in Fig. 3. This occurs after the convergence of the controller gains when the gradient algorithm changes to the steady-state. It is important to note that this analysis is only valid when the plant model is well known and free of unmodeled dynamics and parametric variations.

To allow the analysis of the MRC structure, the plant and reference model must satisfy some assumptions as verified in (Ioannou \& Sun, 1996). These suppositions, which are also valid for RMRAC, are given as follow:

Plant Assumptions:

P1. $Z_{p}(s)$ is a monic Hurwitz polynomial of degree $m_{p}$;

P2. An upper bound $n$ of the degree $n_{p}$ of $R_{p}(s)$;

P3. The relative degree $n^{*}=n_{p}-m_{p}$ of $G_{p}(s)$;

P4. The signal of the high frequency gain $k_{p}$ is known.

Reference Model Assumptions:

M1. $Z_{m}(s), R_{m}(s)$ are monic Hurwitz polynomials of degree $q_{m}, p_{m}$, respectively, where $p_{m} \leq n ;$

M2. The relative degree $n_{m}^{*}=p_{m}-q_{m}$ of $W_{m}(s)$ is the same as that of $G_{p}(s)$, i.e., $n_{m}^{*}=n^{*}$.

In Fig. 4 the feedback control law is

$$
u_{p}=\frac{\theta_{1}^{\top}}{\theta_{4}} \frac{\alpha(s)}{\Lambda(s)} u_{p}+\frac{\theta_{2}^{\top}}{\theta_{4}} \frac{\alpha(s)}{\Lambda(s)} y_{p}+\frac{\theta_{3}}{\theta_{4}} y_{p}+\frac{1}{\theta_{4}} r,
$$

and

$$
\begin{array}{ll}
\alpha(s) \triangleq \alpha_{n-2}(s)=\left[s^{n-2}, s^{n-3}, \ldots, s, 1\right]^{\top} & \text { for } n \geq 2, \\
\alpha(s) \triangleq 0 & \text { for } n=1,
\end{array}
$$

$\theta_{3}, \theta_{4} \in \Re^{1} ; \theta_{1}^{\top}, \theta_{2}^{\top} \in \Re^{n-1}$ are constant parameters to be designed and $\Lambda(s)$ is an arbitrary monic Hurwitz polynomial of degree $n-1$ that contains $Z_{m}(s)$ as a factor, i.e.,

$$
\Lambda(s)=\Lambda_{0}(s) Z_{m}(s),
$$


which implies that $\Lambda_{0}(s)$ is monic, Hurwitz and of degree $n_{0}=n-1-q_{m}$. The controller parameter vector

$$
\boldsymbol{\theta}=\left[\begin{array}{llll}
\theta_{1}^{\top} & \theta_{2}^{\top} & \theta_{3} & \theta_{4}
\end{array}\right]^{\top} \in \Re^{2 n},
$$

is given so that the closed loop plant from $r$ to $y_{p}$ is equal to $W_{m}(s)$. The I/O properties of the closed-loop plant shown in Fig. 4 are described by the transfer function equation

$$
y_{p}=G_{c}(s) r
$$

where

$$
G_{c}(s)=\frac{k_{p} Z_{p} \Lambda^{2}}{\Lambda\left[\left(\theta_{4} \Lambda-\theta_{1}^{\top} \alpha\right) R_{p}-k_{p} Z_{p}\left(\theta_{2}^{\top} \alpha+\theta_{3} \Lambda\right)\right]},
$$

Now, the objective is to choose the controller gains so that the poles are stable and the closed-loop transfer function $G_{c}(s)=W_{m}(s)$, i.e.,

$$
\frac{k_{p} Z_{p} \Lambda^{2}}{\Lambda\left[\left(\theta_{4} \Lambda-\theta_{1}^{\top} \alpha\right) R_{p}-k_{p} Z_{p}\left(\theta_{2}^{\top} \alpha+\theta_{3} \Lambda\right)\right]}=k_{m} \frac{Z_{m}}{R_{m}} .
$$

Thus, considering a system free of unmodeled dynamics, the plant coefficients can be known by the MRC structure, i.e., $k_{p}, Z_{p}(s)$ and $R_{p}(s)$ are given by 21 when the controller gains $\theta_{1}^{\top}$, $\theta_{2}^{\top}, \theta_{3}$ and $\theta_{4}$ are known and $W_{m}(s)$ is previously defined.

\section{Parameter identification using RMRAC}

The proposed parameter estimation method is executed in three steps, described as follows:

\subsection{First step: Convergence of controller gains vector}

The proposed parameter identification method is shown in Fig. 2. In this figure the parameter identification of $\mathrm{q}$ axis is shown, but the same procedure is performed for parameter identification of $d$ axis, one procedure at a time.

A Persistent Excitant (PE) reference current $i_{s q}^{*}$ is applied at q axis of SPIM at standstill rotor. The current $i_{s q}$ is measured and controlled by the RMRAC structure while $i_{s d}$ stays at null value. The controller structure is detailed in Fig. 3 . When $e_{1}$ goes to zero, the controller gains go to an ideal value. Subsequently, the gradient algorithm is put in steady-state and the system looks like the MRC structure given by Fig. 4 . Therefore, the transfer function coefficients can be found using equation 21 .

\subsection{Second step: Estimation of $k_{p i}, h_{0 i}, a_{1 i}$ and $a_{0 i}$}

This step consists of the determination of the Linear-Time-Invariant (LTI) model of the induction motor. The machine is at standstill and the transfer functions given in 2 and 3 can be generalized as follows

where

$$
\frac{i_{s i}}{v_{s i}}=k_{p i} \frac{Z_{p i}(s)}{R_{p i}(s)}=k_{p i} \frac{s+h_{0 i}}{s^{2}+s a_{1 i}+a_{0 i}},
$$

$$
k_{p i}=\frac{L_{r i}}{\bar{\sigma}_{i}}, h_{0 i}=\frac{R_{r i}}{L_{r i}}, a_{1 i}=p_{i} \text { and } a_{0 i}=\frac{R_{s i} R_{r i}}{\bar{\sigma}_{i}},
$$


The reference model given by 5 is rewritten as

$$
W_{m}(s)=k_{m} \frac{Z_{m}}{R_{m}}=k_{m} \frac{s+z_{0}}{s^{2}+p_{1} s+p_{0}},
$$

and from the plant and reference model assumptions results

$$
\left\{\begin{array}{l}
m_{p}=1, n_{p}=2, n^{*}=1, \\
q_{m}=1, p_{m}=2, n_{m}^{*}=1,
\end{array}\right.
$$

The upper bound $n$ is chosen equal to $n_{p}$ because the plant model is considered well known and with $n=n_{p}$ only one solution is guaranteed for the controller gains. Thus, the filters are given by

$$
\left\{\begin{array}{l}
\Lambda(s)=Z_{m}(s)=s+z_{0} \\
\alpha(s)=z_{0}
\end{array}\right.
$$

Assuming the complete convergence of controller gains, the plant coefficients are obtained combining the equations 22, 24 and 26 in 21 and are given by

$$
\left\{\begin{array}{l}
k_{p i}=k_{m} \theta_{4 i}, \\
h_{0 i}=\frac{z_{0}}{\theta_{4 \mathrm{i}}}\left(\theta_{4 i}-\theta_{1 i}^{\top}\right), \\
a_{1 i}=p_{1}+k_{m} \theta_{3 i}, \\
a_{0 i}=p_{0}+k_{m} z_{0}\left(\theta_{2 i}^{\top}+\theta_{3 i}\right) .
\end{array}\right.
$$

6.3 Third step: $R_{s i}, R_{r i}, L_{s i}, L_{r i}$ and $L_{m i}$ calculation

Combining the equations 4,23 and using the values obtained in 27 after the convergence of the controller gains, we obtain the parameters of the induction motor:

$$
\left\{\begin{array}{l}
\hat{R}_{s i}=\frac{a_{0 i}}{k_{p i} h_{0 i}}, \\
\hat{R}_{r i}=\frac{a_{1 i}}{k_{p i}}-\hat{R}_{s i}, \\
\hat{L}_{s i}=\hat{L}_{r i}=\frac{\hat{R}_{r i}}{h_{0 i}}, \\
\hat{L}_{m i}=\sqrt{\hat{L}_{s i}^{2}-\frac{\hat{R}_{s i} \hat{R}_{r i}}{a_{0 i}} .}
\end{array}\right.
$$

In the numerical solution it-is considered that stator and rotor inductances have the same values in each winding.

\section{Simulation results}

Simulations have been performed to evaluate the proposed method. The machine model given by 1 was discretized by Euller technique under frequency of $f_{s}=5 \mathrm{kHz}$. The SPIM was performed with a square wave reference of current and standstill rotor. The SPIM used is a four-pole, $368 \mathrm{~W}, 1610 \mathrm{rpm}, 220 \mathrm{~V} / 3.4 \mathrm{~A}$. The parameters of this motor obtained from classical no-load and locked rotor tests are given in Table 1. 


\begin{tabular}{|c|c|c|c|}
\hline$R_{s q}$ & $R_{r q}$ & $L_{m q}$ & $L_{s q}$ \\
\hline $7.00 \Omega$ & $12.26 \Omega$ & $0.2145 \mathrm{H}$ & $0.2459 \mathrm{H}$ \\
\hline$R_{s d}$ & $R_{r d}$ & $L_{m d}$ & $L_{s d}$ \\
\hline $20.63 \Omega$ & $28.01 \Omega$ & $0.3370 \mathrm{H}$ & $0.4264 \mathrm{H}$ \\
\hline
\end{tabular}

Table 1. Motor parameter obtained from classical tests.

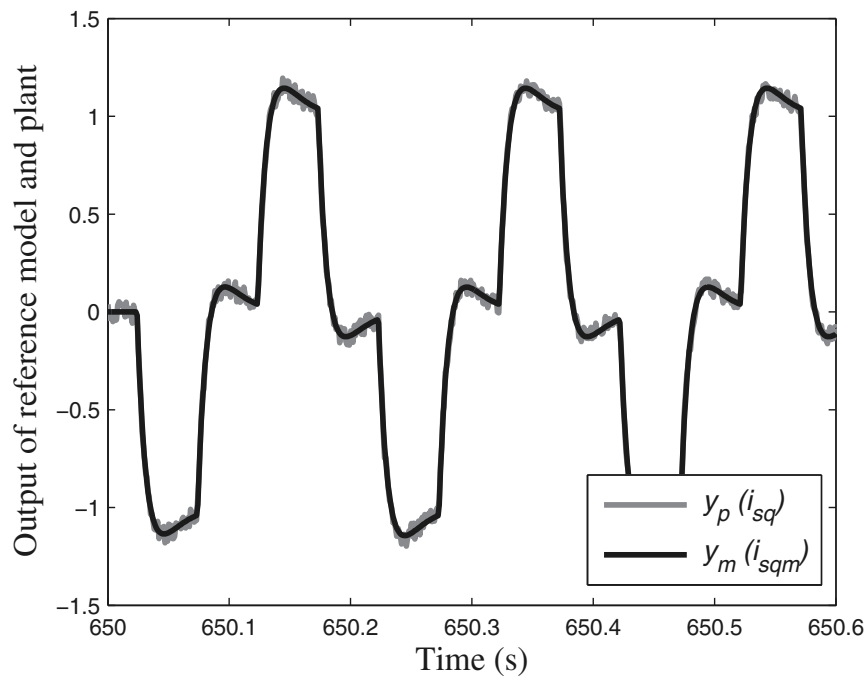

Fig. 5. Plant and reference model output.

The reference model $W_{m}(s)$ is chosen so that the dynamic will be faster than plant output $i_{s q}$. Thus, the reference model is given by

$$
W_{m}(s)=180 \frac{s+45}{s^{2}+180 s+8100},
$$

The induction motor is started in accordance with Fig. 2 with a Persistent Excitant reference current signal. A random noise was simulated to give nearly experimental conditions. Fig. 5 show the plant and reference model output after convergence of gains.

Fig. 6 shows the convergence of controller gains for parameter identification of $q$ axis. This figure shows that gains reach a final value after 600s, demonstrating that parameter identification is possible. The gain convergence of $d$ axis is shown in Fig. 7. Table 2 presents the final value of controller gains for the $\mathrm{q}$ and $\mathrm{d}$ axes, respectively.

\begin{tabular}{|c|c|c|c|}
\hline$\theta_{1 q}$ & $\theta_{2 q}$ & $\theta_{3 q}$ & $\theta_{4 q}$ \\
\hline-0.0096 & -1.0925 & 0.8332 & -0.0950 \\
\hline$\theta_{1 d}$ & $\theta_{2 d}$ & $\theta_{3 d}$ & $\theta_{4 d}$ \\
\hline-0.0164 & -0.6429 & 0.6885 & -0.0352 \\
\hline
\end{tabular}

Table 2. Final value of controller gains obtained in simulation.

The parameters of SPIM are obtained by combining the final value of controller gains from Table 2 with the equations 27, 28 and the reference model coefficients previously defined in 


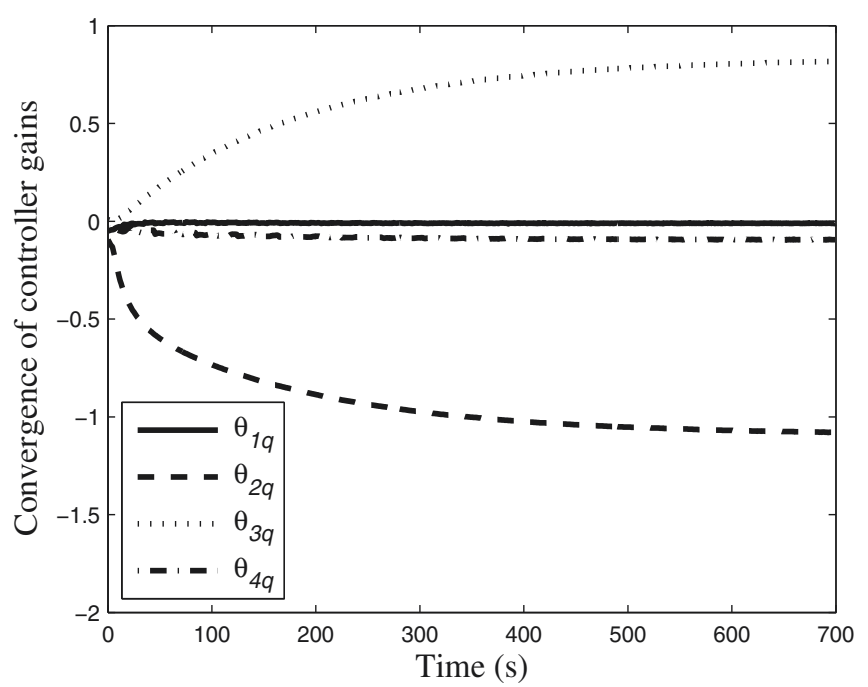

Fig. 6. Convergence of controller gains vector for $q$ axis.

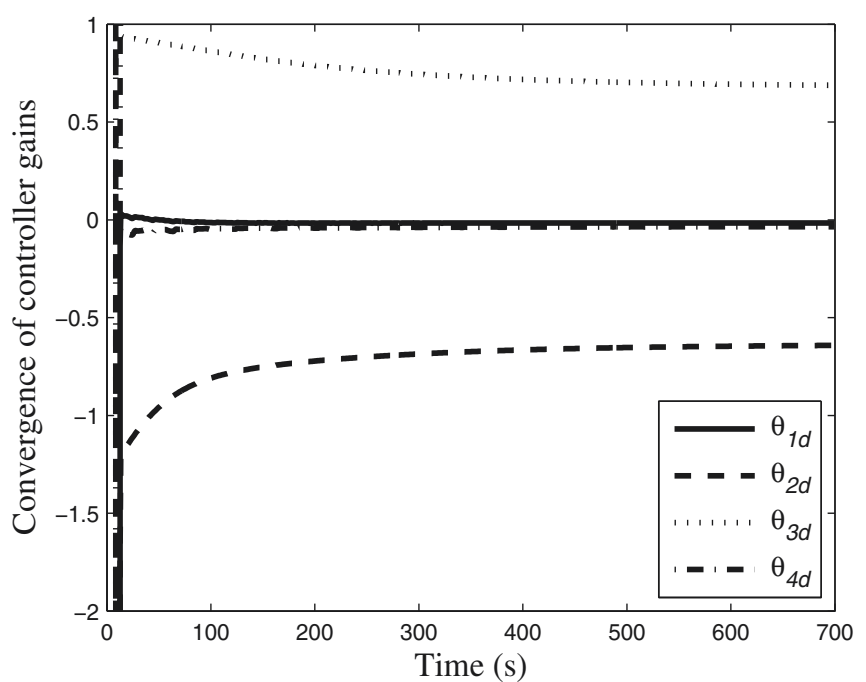

Fig. 7. Convergence of controller gains vector for $\mathrm{d}$ axis.

equation 29. The results are shown in Table 3. It is possible to observe in simulation that the electrical parameters converge to machine parameters, even with noise in the currents.

\begin{tabular}{|c|c|c|c|}
\hline$R_{s q}$ & $R_{r q}$ & $L_{m q}$ & $L_{s q}$ \\
\hline $7.07 \Omega$ & $12.21 \Omega$ & $0.2150 \mathrm{H}$ & $0.2462 \mathrm{H}$ \\
\hline$R_{s d}$ & $R_{r d}$ & $L_{m d}$ & $L_{s d}$ \\
\hline $20.22 \Omega$ & $27.67 \Omega$ & $0.3312 \mathrm{H}$ & $0.4292 \mathrm{H}$ \\
\hline
\end{tabular}

Table 3. Motor parameter identified in simulation. 


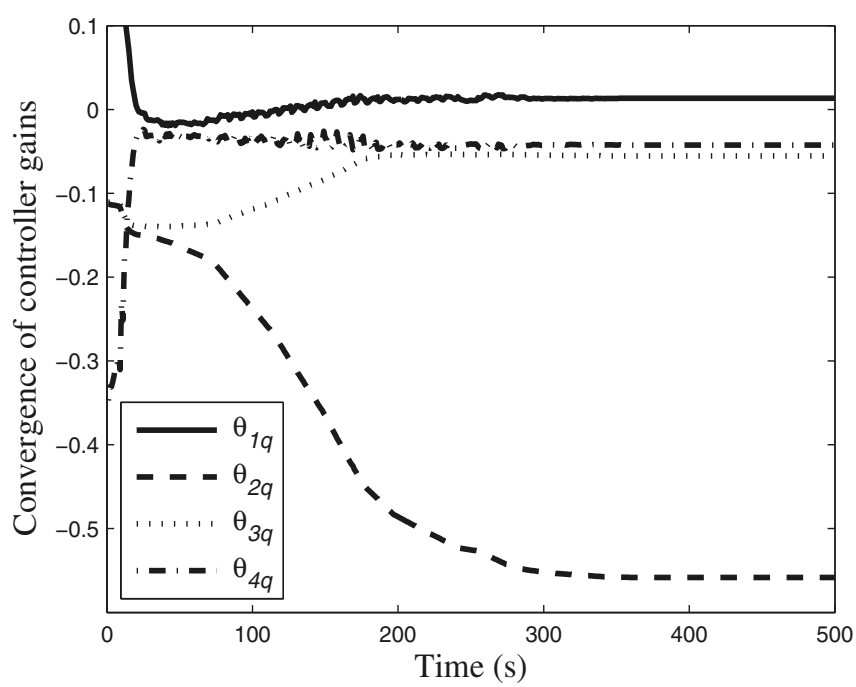

Fig. 8. Experimental convergence of controller gains for $q$ axis.

\section{Experimental results}

This section presents experimental results obtained from the induction motor described in simulation, whose electrical parameters obtained by classical no-load and locked rotor tests are shown in Table 1 . The drive system consists of a three-phase inverter controlled by a TMS320F2812 DSP controller. The sampling period is the same used in the preceding simulation.

Unlike the simulation, unmodeled dynamics by drive, sensors and filters, among others, are included in the implementation. This implies that the plant model is a little different from physical plant. As a result, there is a small error that is proportional to plant uncertainties and is defined here as a residual error. The tracking error $e_{1}$ can be minimized by increasing the gradient gain $\mathbf{P}$. However, increasing $\mathbf{P}$ in order to eliminate the residual error can cause divergence of controller gains and the system becomes unstable.

To overcome this problem a stopping condition was defined for the gain convergence. The identified stator resistance $\hat{R}_{s i}$ was compared to measured stator resistance $R_{s i}$ obtained from measurements. Thus, the gradient gain $\mathbf{P}$ must be adjusted until the identified stator resistance is equal to the stator resistance measurement.

Figure 8 presents the convergence of controller gains for $q$ axis. The gains reach a final value after 400s. Figure 9 presents the convergence of controller gains for $d$ axis. The value of the gain that resulted in $\hat{R}_{s i}=R_{s i}$ was $\mathbf{P}=20 \mathrm{I}$.

The plant output $i_{s q}$ and reference model output $i_{s q m}$ are shown in Fig. 10, after controller gain convergence, where it is possible to see the residual error between the two curves. The final values of controller gains, for axes $\mathrm{q}$ and $\mathrm{d}$, are shown in Table 4.

The parameters of SPIM are obtained by combining the final value of controller gains of Table 4 with the equations 27,28 and the reference model coefficients previously defined in equation 29. The results are shown in Table 5 . 


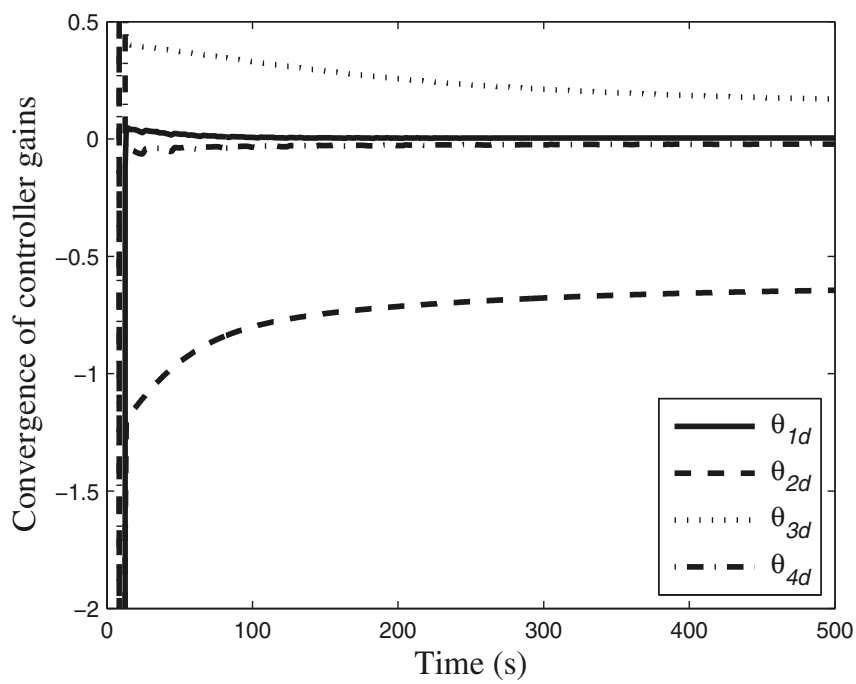

Fig. 9. Experimental convergence of controller gains for $\mathrm{d}$ axis.

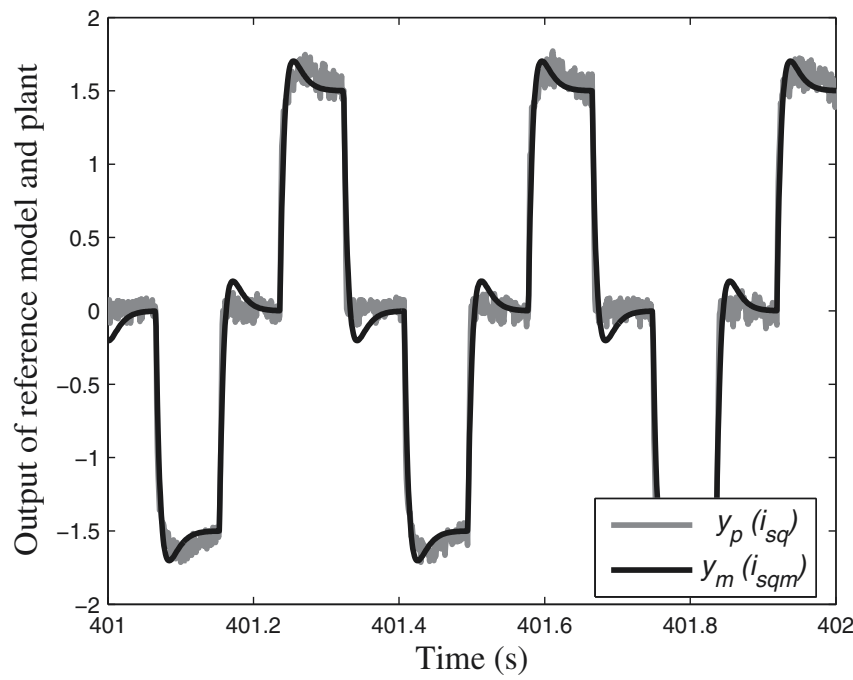

Fig. 10. Experimental plant and reference model output.

\begin{tabular}{|c|c|c|c|}
\hline$\theta_{1 q}$ & $\theta_{2 q}$ & $\theta_{3 q}$ & $\theta_{4 q}$ \\
\hline 0.0136 & -0.558 & -0.0555 & -0.0423 \\
\hline$\theta_{1 d}$ & $\theta_{2 d}$ & $\theta_{3 d}$ & $\theta_{4 d}$ \\
\hline 0.0042 & -0.6345 & 0.1560 & -0.0207 \\
\hline
\end{tabular}

Table 4. Final value of controller gains obtained in experimentation. 


\begin{tabular}{|c|c|c|c|}
\hline$R_{s q}$ & $R_{r q}$ & $L_{m q}$ & $L_{s q}$ \\
\hline $6.9105 \Omega$ & $15.4181 \Omega$ & $0.1821 \mathrm{H}$ & $0.2593 \mathrm{H}$ \\
\hline$R_{s d}$ & $R_{r d}$ & $L_{m d}$ & $L_{s d}$ \\
\hline $20.9438 \Omega$ & $34.9016 \Omega$ & $0.4926 \mathrm{H}$ & $0.6447 \mathrm{H}$ \\
\hline
\end{tabular}

Table 5. Motor parameter identified in experimentation.

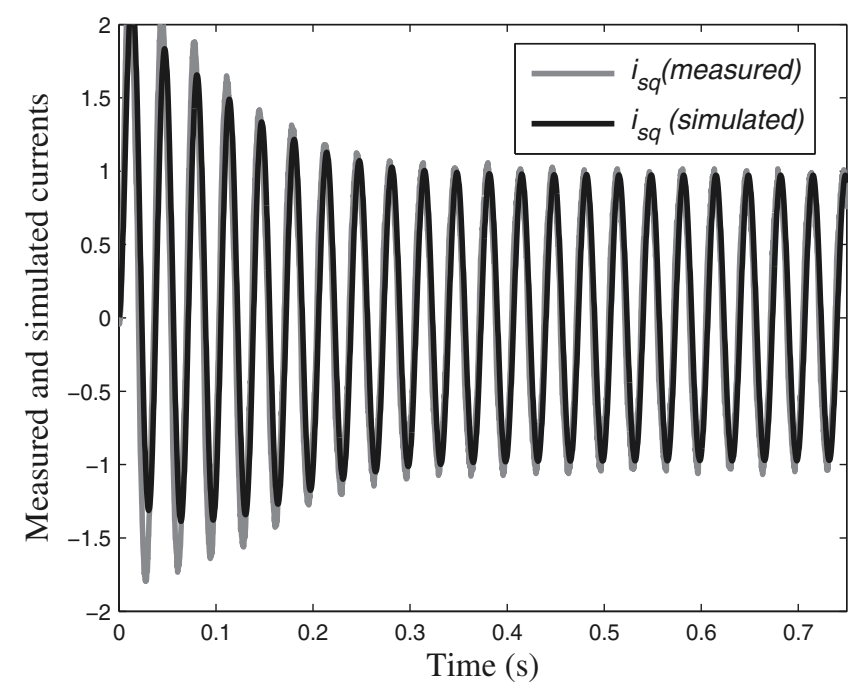

Fig. 11. Transient of comparison among measured and simulated currents from q axis.

\subsection{Comparison for parameter validation}

A comparative study was performed to validate the parameters obtained by the proposed technique. The physical SPIM was fed by steps of $50 \mathrm{~V}$ and $30 \mathrm{~Hz}$. The currents of main winding, auxiliary winding and speed rotor were measured.

Then, the dynamic model of SPIM given by 1 was simulated, using the parameters from Table 5 , under the same conditions experimentally, i.e., steps of $50 \mathrm{~V}$ and $30 \mathrm{~Hz}$. The measured rotor speed was used in the simulation model to make it independent of mechanical parameters. Thus, the simulated currents, from $q$ and $d$ windings, were compared with measured currents. Figures 11 and 12 shows the transient currents from axis $q$ and $d$, respectively, while Figures 13 and 14 shows the steady-state currents from axis $q$ and $d$, respectively.

From Figures 11-14, it is clear that the simulated machine with the proposed parameters presents similar behavior to the physical machine, both in transient and steady-state.

\section{Conclusions}

This chapter describes a method for the determination of electrical parameters of single phase induction machines based on a RMRAC algorithm, which initially was used in three-phase induction motor estimation in (Azzolin \& Gründling, 2009). Using this methodology, it is possible to obtain all electrical parameters of SPIM for the simulation and design of an high performance control and sensorless SPIM drives. The main contribution of this proposed work is the development of automated method to obtain all electric parameters of the induction machines without the requirement of any previous test and derivative filters. Simulation 


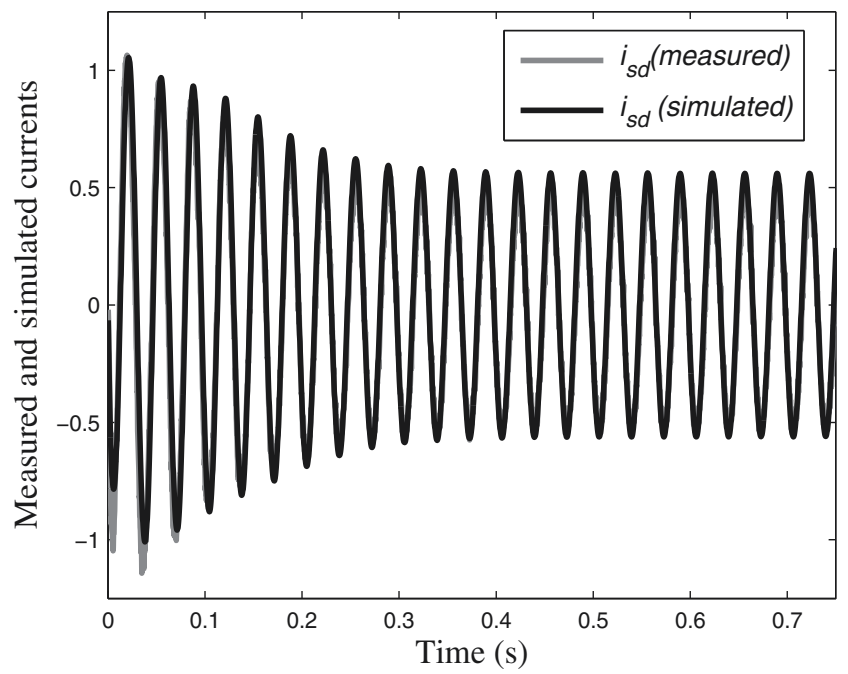

Fig. 12. Transient of comparison among measured and simulated currents from d axis.

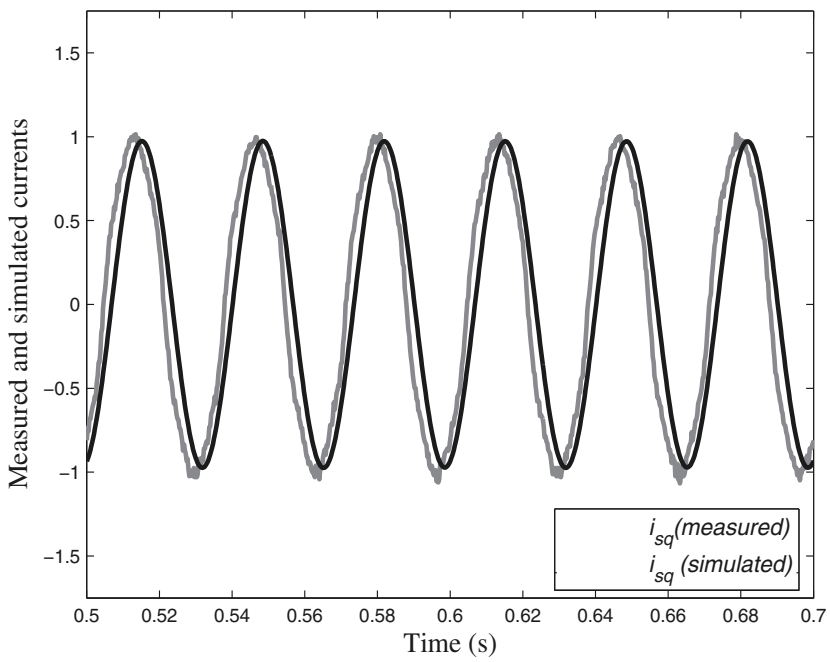

Fig. 13. Steady-state of comparison among measured and simulated currents from q axis.

results demonstrate the convergence of the parameters to ideal values, even in the presence of noise. Experimental results show that the parameters converge to different values in relation to the classical tests shown in Table 1. However, the results presented in Figures 11-14 show that the parameters obtained by proposed method present equivalent behavior to physical machine. 


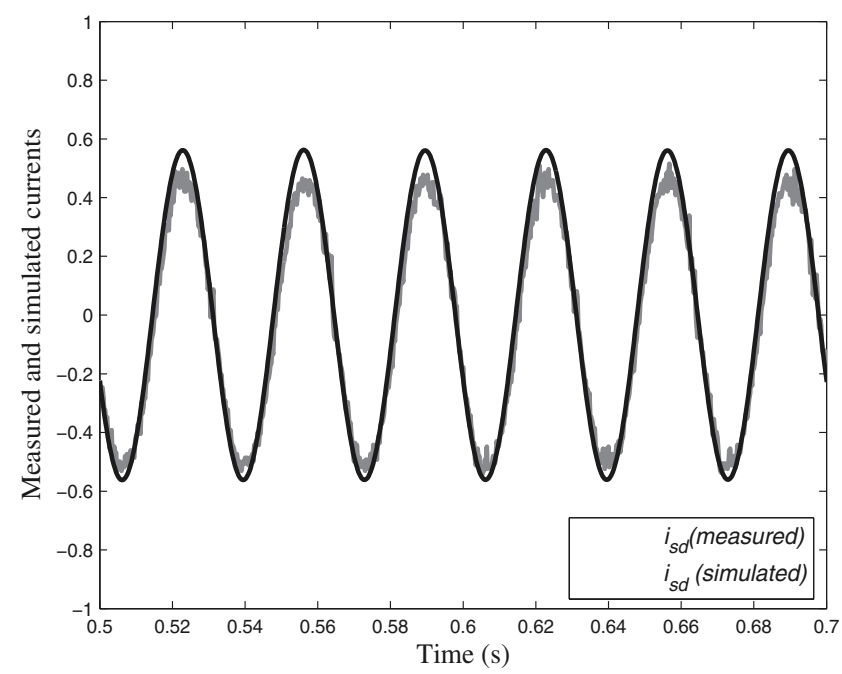

Fig. 14. Steady-state of comparison among measured and simulated currents from d axis.

\section{References}

Azzolin, R. \& Gründling, H. (2009). A mrac parameter identification algorithm for three-phase induction motors, Electric Machines and Drives Conference, 2009. IEMDC '09. IEEE International, pp. $273-278$.

Azzolin, R., Martins, M., Michels, L. \& Gründling, H. (2007). Parameter estimator of an induction motor at standstill, Industrial Electronics, 2009. IECON '09. 35th Annual Conference of IEEE, pp. $152-157$.

Blaabjerg, F., Lungeanu, F., Skaug, K. \& Tonnes, M. (2004). Two-phase induction motor drives, Industry Applications Magazine, IEEE 10(4): 24 - 32.

Câmara, H. \& Gründling, H. (2004). A rmrac applied to speed control of an induction motor without shaft encoder, Decision and Control, 2004. CDC. 43rd IEEE Conference on, Vol. 4, pp. $4429-4434$ Vol.4.

de Rossiter Correa, M., Jacobina, C., Lima, A. \& da Silva, E. (2000). Rotor-flux-oriented control of a single-phase induction motor drive, Industrial Electronics, IEEE Transactions on 47(4): $832-841$.

Donlon, J., Achhammer, J., Iwamoto, H. \& Iwasaki, M. (2002). Power modules for appliance motor control, Industry Applications Magazine, IEEE 8(4): 26 -34.

Ioannou, P. \& Sun, J. (n.d.). Robust Adaptive Control, Prentice Hall.

Ioannou, P. \& Tsakalis, K. (1986). A robust direct adaptive controller, Automatic Control, IEEE Transactions on 31(11): $1033-1043$.

Koubaa, Y. (2004). Recursive identification of induction motor parameters, Simulation Modelling Practice and Theory 12(5): 363 - 381.

URL: http://www.sciencedirect.com/science/article/B6X3C-4CJVR4N-1/2/bcc4bdf719a9c7 ad5b99750423f5ff23

Krause, P., Wasynczuk, O. \& Sudhoff, S. (n.d.). Analysis of Electric Machinery, NJ: IEEE Press. 
Lozano-Leal, R., Collado, J. \& Mondie, S. (1990). Model reference robust adaptive control without a priori knowledge of the high frequency gain, Automatic Control, IEEE Transactions on 35(1): $71-78$.

Ojo, O. \& Omozusi, O. (2001). Parameter estimation of single-phase induction machines, Industry Applications Conference, 2001. Thirty-Sixth IAS Annual Meeting. Conference Record of the 2001 IEEE.

Ribeiro, L., Jacobina, C. \& Lima, A. (1995). Dynamic estimation of the induction machine parameters and speed, Power Electronics Specialists Conference, 1995. PESC'95 Record., 26th Annual IEEE, Vol. 2, pp. $1281-1287$ vol.2.

Vaez-Zadeh, S. \& Reicy, S. (2005). Sensorless vector control of single-phase induction motor drives, Electrical Machines and Systems, 2005. ICEMS 2005. Proceedings of the Eighth International Conference on, Vol. 3, pp. 1838 -1842 Vol. 3.

Velez-Reyes, M., Minami, K. \& Verghese, G. (1989). Recursive speed and parameter estimation for induction machines, Industry Applications Society Annual Meeting, 1989., Conference Record of the 1989 IEEE, pp. 607 -611 vol.1.

Vieira, R., Azzolin, R. \& Gründling, H. (2009a). Parameter identification of a single-phase induction motor using rls algorithm, Power Electronics Conference, 2009. COBEP '09. Brazilian.

Vieira, R., Azzolin, R. \& Gründling, H. (2009b). A sensorless single-phase induction motor drive with a mrac controller, Industrial Electronics, 2009. IECON '09. 35th Annual Conference of IEEE, pp. $1003-1008$. 


\title{
Swarm Intelligence Based Controller for Electric Machines and Hybrid Electric Vehicles Applications
}

\author{
Omar Hegazy 1 , Amr Amin², and Joeri Van Mierlo ${ }^{1}$ \\ ${ }^{1}$ Faculty of Engineering Sciences Department of ETEC-Vrije Universiteit Brussel, \\ 2 Power and Electrical Machines Department, Faculty of Engineering - Helwan University, \\ ${ }^{1}$ Belgium \\ ${ }^{2}$ Egypt
}

\section{Introduction}

Swarm Intelligence in the form of Particle Swarm Optimization (PSO) has potential applications in electric drives. The excellent characteristics of PSO may be successfully used to optimize the performance of electric machines and electric drives in many aspects. It is estimated that, electric machines consume more than $50 \%$ of the world electric energy generated. Improving efficiency in electric drives is important, mainly, for two reasons: economic saving and reduction of environmental pollution. Induction motors have a high efficiency at rated speed and torque. However, at light loads, the iron losses increase dramatically, reducing considerably the efficiency. Swarm intelligence is used to optimize the performance of three applications; these applications are represented as follows:

- Losses Minimization of two asymmetrical windings induction motor

- Maximum efficiency and minimum operating cost of three-phase induction motor

- Optimal electric drive system for fuel cell hybrid electric vehicles.

In this chapter, a field-oriented controller that is based on Particle Swarm Optimization is presented. In this system, the speed control of two asymmetrical windings induction motor is achieved while maintaining maximum efficiency of the motor. PSO selects the optimal rotor flux level at each operating point. In addition, the electromagnetic torque is also improved while maintaining a fast dynamic response. A novel approach is used to evaluate the optimal rotor flux level by using Particle Swarm Optimization. PSO method is a member of the wide category of Swarm Intelligence methods (SI). The swarm intelligence is based on real life observations of social animals (usually insects), it is more flexibility and robust than any traditional optimization methods. PSO algorithm searches for global optimization for nonlinear problems with multi-objective. There are two speed control strategies explained in the next sections. These are field-oriented controller (FOC), and FOC based on PSO. The strategies are implemented mathematically and experimental. The simulation and experimental results have demonstrated that the FOC based on PSO method saves more energy than the conventional FOC method. 
In this chapter, another application of PSO for losses and operating cost minimization control is presented for the induction motor drives. Two strategies for induction motor speed control are proposed. These strategies are maximum efficiency strategy (MES), based PSO, and minimum operating cost Strategy. The proposed technique is based on the principle that the flux level in a machine can be adjusted to give the minimum amount of losses and minimum operating cost for a given value of speed and load torque.

In the demonstrated systems, the powertrain components sizing and the power control strategy are the only adjustable parameters to achieve optimal power sharing between sources and optimal design with minimum cost, minimum fuel consumption, and maximum efficiency for Electric Vehicles (EVs) and Hybrid Electric Vehicles (HEVs). Their selection greatly influences the performance of the drive system in Hybrid Electric Vehicles applications. In this section, the design and power management control are investigated and optimized by using Particle Swarm Optimization.

\section{Losses minimization of two asymmetrical windings induction motor}

In this section, a field orientation based on Particle Swarm Optimization (PSO) is applied to control the speed of two-asymmetrical windings induction motor. The maximum efficiency of the motor is obtained by the evaluation of optimal rotor flux at each operating point. In addition, the electro-magnetic torque is also improved while maintaining a fast dynamic response. In this section, a novel approach is used to evaluate the optimal rotor flux level. This approach is based on Particle Swarm Optimization (PSO). This section presents two speed control strategies. These are field-oriented controller (FOC) and FOC based on PSO. The strategies are implemented mathematically and experimental. The simulation and experimental results have demonstrated that the FOC based on PSO method saves more energy than the conventional FOC method [Hegazy, 2006; Amin et al., 2007; Amin et al., 2009]. The two asymmetrical windings induction motor is treated as a two-phase induction motor (TPIM). It is used in many low power applications, where three-phase supply is not readily available. This type of motor runs at an efficiency range of $50 \%$ to $65 \%$ at rated operating conditions.

The conventional field-oriented controller normally operates at rated flux at any values with its torque range. When the load is reduced considerably, the core losses become so high causing poor efficiency. If significant energy savings are required, it is necessary to optimize the efficiency of the motor. The optimum efficiency is obtained by the evaluation of the optimal rotor flux level. This flux level is varied according to the torque and the speed of the operating point.

PSO is applied to evaluate the optimal flux. It has the straightforward goal of minimizing the total losses for a given load and speed. It is shown that the efficiency is reasonably close to optimal.

\subsection{Mathematical model of the motor}

The d-q model of an unsymmetrical windings induction motor in a stationary reference frame can be used for a dynamic analysis. This model can take in account the core losses. The d-q model as applied to TPIM is described in [Hegazy, 2006; Amin et al., 2009]. The equivalent circuit is shown in Fig. 1. 

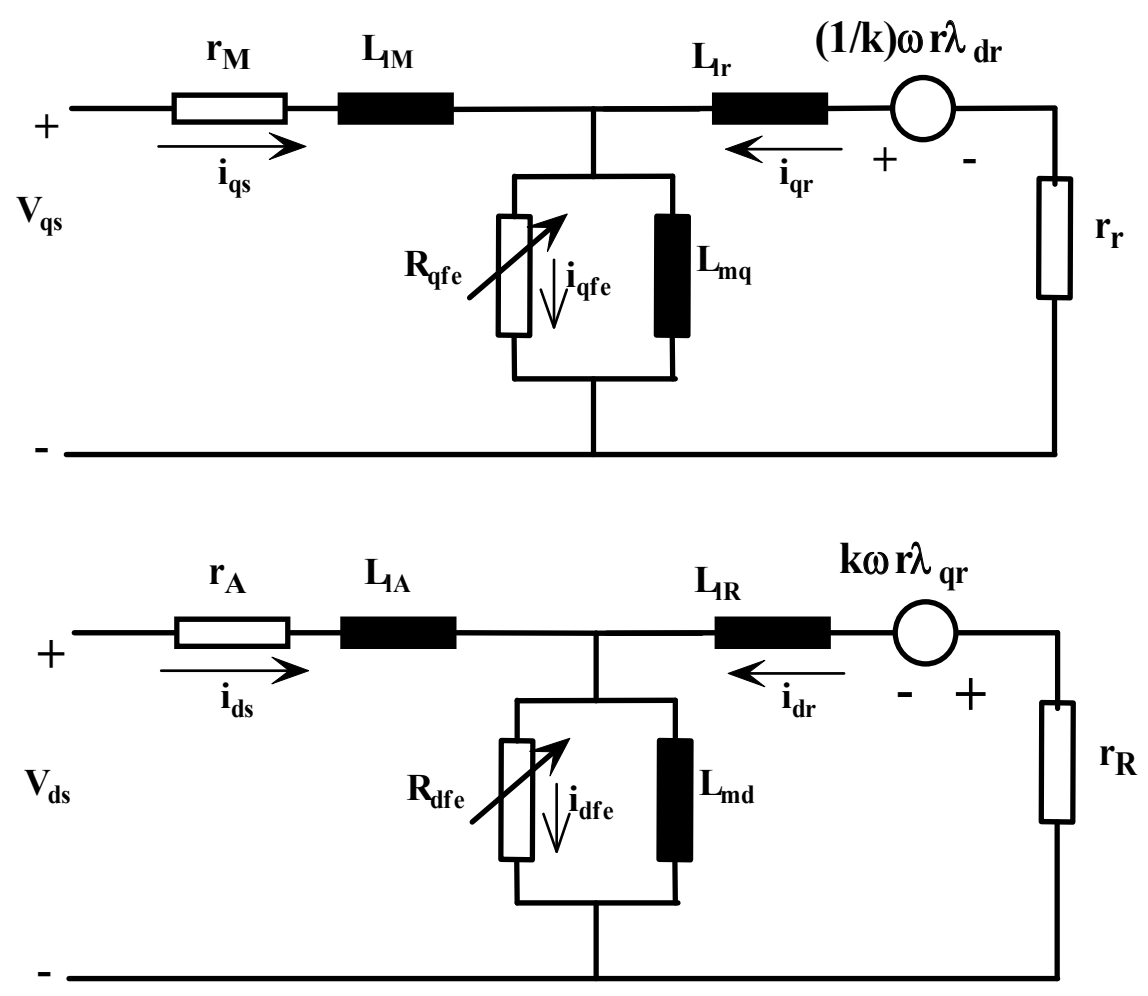

Fig. 1. The d-q axes two-phase induction motor Equivalent circuit with iron losses.

The machine model may be expressed by the following voltage and flux equations: Voltage Equations (1):

$$
\begin{gathered}
v_{q s}=r_{m} i_{q s}+p \lambda_{q s} \\
v_{d s}=r_{a} i_{d s}+p \lambda_{d s} \\
0=r_{r} i_{q r}-(1 / k)^{*} \omega_{r} \lambda_{d r}+p \lambda_{q r} \\
0=r_{R} i_{d s}+k^{*} \omega_{r} \lambda_{q r}+p \lambda_{d r} \\
0=-i_{q f e} R_{q f e}+L_{m q}\left(p i_{q s}+p i_{q r}-p i_{q f e}\right) \\
0=-i_{d f e} R_{d f e}+L_{m d}\left(p i_{d s}+p i_{d r}-p i_{d f e}\right)
\end{gathered}
$$

Flux Equations:

$$
\begin{aligned}
& \lambda_{q s}=L_{l m} i_{q s}+L_{m q}\left(i_{q s}+i_{q r}-i_{q f e}\right) \\
& \lambda_{d s}=L_{l a} i_{d s}+L_{m d}\left(i_{d s}+i_{d r}-i_{d f e}\right)
\end{aligned}
$$




$$
\begin{aligned}
& \lambda_{q r}=L_{l r} i_{q r}+L_{m q}\left(i_{q s}+i_{q r}-i_{q f e}\right) \\
& \lambda_{d r}=L_{l R} i_{d r}+L_{m d}\left(i_{d s}+i_{d r}-i_{d f e}\right)
\end{aligned}
$$

Electrical torque equation is expressed as:

$$
\begin{gathered}
T e=\frac{P}{2}\left(k L_{m q} i_{d r}\left(i_{q s}+i_{q r}-i_{q f e}\right)-\frac{1}{k} L_{m d} i_{q r}\left(i_{d s}+i_{d r}-i_{q f e}\right)\right. \\
T e-T_{l}=j_{m} p \omega_{r}+B_{m} \omega_{r}
\end{gathered}
$$

\subsection{Field-Oriented Controller [FOC]}

The stator windings of the motor are unbalanced. The machine parameters differ from the $d$ axis to the $q$ axis. The waveform of the electromagnetic torque demonstrates the unbalance of the system. The torque in equation (11) contains an AC term; it can be observed that two values are presented for the referred magnetizing inductance. It is possible to eliminate the AC term of electro-magnetic torque by an appropriate control of the stator currents. However, these relations are valid only in linear conditions. Furthermore, the model is implemented using a non-referred equivalent circuit, which presumes some complicated measurement of the magnetizing mutual inductance of the stator and the rotor circuits.

The indirect field-oriented control scheme is the most popular scheme for field-oriented controllers. It provides decoupling between the torque and the flux currents. The electric torque must be a function of the stator currents and rotor flux in synchronous reference frame [Popescu \& Navrapescu, 2000]. Assuming that the stator currents can be imposed as:

$$
\begin{gathered}
i_{d s}^{s}=i_{d s 1}^{s} \\
i_{q s}^{s}=k i_{q s 1}^{s}
\end{gathered}
$$

Where: $k=M_{s r d} / M_{s r q}$

$$
T_{e}=\frac{P}{2 L_{r}}\left[M_{s q r} i_{q s}^{s} \lambda_{d r}^{s}-M_{s d r} i_{d s}^{s} \lambda_{q r}^{s}\right]
$$

By substituting the variables $i_{d s}$, and $i_{q s}$ by auxiliary variables $i_{d s 1}$, and $i_{q s 1}$ into (15) the torque can be expressed by

$$
T_{e}=\frac{P M_{s d r}}{2 L_{r}}\left[i_{q s 1}^{s} \lambda_{d r}^{s}-i_{d s 1}^{s} \lambda_{q r}^{s}\right]
$$

In synchronous reference frame, the electromagnetic torque is expressed as:

$$
\begin{gathered}
T_{e}=\frac{P M_{s d r}}{2 L_{r}}\left[i_{q s 1}^{e} \lambda_{d r}^{e}-i_{d s 1}^{e} \lambda_{q r}^{e}\right] \\
T_{e}=\frac{P M_{s d r}}{2 L_{r}}\left[i_{q s 1}^{e} \lambda_{r}^{e}\right]
\end{gathered}
$$




$$
\begin{gathered}
i_{d s 1}^{e}=\frac{\lambda_{r}^{e}}{M_{s d r}} \\
\omega_{e}-\omega_{r}=\frac{M_{s d r}}{\tau_{r}{ }^{*} \lambda_{r}} i^{e}{ }_{q s 1}
\end{gathered}
$$

\subsection{Synchronous reference frame for losses model}

It is very complex to find the losses expression for the two asymmetrical windings induction motor with losses model. In this section, a simplified induction motor model with iron losses will be developed. For this purpose, it is necessary to transform all machine variables to the synchronous reference frame. The voltage equations are written in expanded form as follows [Hegazy, 2006; Amin et al., 2006; Amin et al., 2009]:

$$
\begin{gathered}
v_{q s}^{e}=r_{m} i_{q s}^{e}+L_{l m} \frac{d i_{q s}^{e}}{d t}+L_{m q} \frac{d i_{q m}^{e}}{d t}+\omega_{e}\left(L_{l a} i_{d s}^{e}+L_{m d} i_{d m}^{e}\right) \\
v_{d s}^{e}=r_{a} i_{d s}^{e}+L_{l a} \frac{d i_{d s}^{e}}{d t}+L_{m d} \frac{d i_{d m}^{e}}{d t}-\omega_{e}\left(L_{l m} i_{q s}^{e}+L_{m q} i_{q m}^{e}\right) \\
0=r_{r} i_{q r}^{e}+L_{l r} \frac{d i_{q r}^{e}}{d t}+L_{m q} \frac{d i_{q m}^{e}}{d t}+\frac{\omega_{s l}}{k}\left(L_{l R} i_{d r}^{e}+L_{m d} i_{d m}^{e}\right) \\
0=r_{R} i_{d r}^{e}+L_{l R} \frac{d i_{d r}^{e}}{d t}+L_{m d} \frac{d i_{d m}^{e}}{d t}-k^{*} \omega_{s l}\left(L_{l r} i_{q r}^{e}+L_{m q} i_{q m}^{e}\right) \\
i_{q s}^{e}+i_{q r}^{e}=i_{q f e}^{e}+i_{q m}^{e} \\
i_{d s}^{e}+i_{d r}^{e}=i_{d f e}^{e}+i_{d m}^{e} \\
v_{d m}^{e}=-\frac{\omega_{e} L_{l r} L_{m q s}}{L_{r}} i_{q s}^{e} \\
v_{q m}^{e}=\omega_{e} L_{m d s} i_{d s}^{e}
\end{gathered}
$$

Where:

$$
\mathrm{i}_{\mathrm{dfe}}^{\mathrm{e}}=\frac{\mathrm{v}_{\mathrm{qm}}^{\mathrm{e}}}{\mathrm{R}_{\mathrm{qfe}}} ; \mathrm{i}_{\mathrm{dfe}}^{\mathrm{e}}=\frac{\mathrm{v}_{\mathrm{dm}}^{\mathrm{e}}}{\mathrm{R}_{\mathrm{dfe}}}
$$

The losses in the motor are mainly:
a. Stator copper losses,
b. Rotor copper losses,
c. Core losses, and 
d. Friction losses

The total electrical losses can be expressed as follows

$$
\mathrm{P}_{\text {losses }}=\mathrm{P}_{\mathrm{cu} 1}+\mathrm{P}_{\mathrm{cu} 2}+\mathrm{P}_{\text {core }}
$$

Where:

$\mathrm{P}_{\text {cu1 }}$ : Stator copper losses

$\mathrm{P}_{\mathrm{cu} 2}$ : Rotor copper losses

$\mathrm{P}_{\text {core }}$ : Core losses

The stator copper losses of the two asymmetrical windings induction motor are caused by electric currents flowing through the stator windings. The core losses of the motor are produced from the hysteresis and eddy currents in the stator. The total electrical losses of motor can be rewritten as:

$$
P_{\text {losses }}=r_{m} i_{q s}^{2}+r_{a} i_{d s}^{2}+r_{r} i_{q r}^{2}+r_{R} i_{d r}^{e^{2}}+\frac{v_{q m}^{e^{2}}}{R_{q f e}}+\frac{v_{d m}^{e^{2}}}{R_{d f e}}
$$

The total electrical losses are obtained as follows:

$$
\begin{gathered}
P_{\text {losses }}=\left[r_{m}+\frac{r_{r} L_{m q s}^{2}}{L_{r}^{2}}+\frac{\omega_{e}^{2} L_{l r}^{2} L_{m q s}^{2}}{L_{r}^{2} R_{d f e}}\right]\left(\frac{T_{e}^{2} L_{r}^{2}}{P^{2}\left(\frac{L_{m d s}}{K}\right)^{2} \lambda_{r}^{2}}\right)+\left(r_{a}+\frac{\omega_{e}^{2} L_{m d s}^{2}}{R_{q f e}}\right) \frac{\lambda_{r}^{2}}{L_{m d s}^{2}} \\
\omega_{s l}=\frac{2 T_{e}{ }^{*} r_{r}}{P^{*} \lambda_{r}^{2}}
\end{gathered}
$$

Where:

$\omega_{\mathrm{e}}=\omega_{\mathrm{r}}+\omega_{\mathrm{sl}}$, and $\omega_{\mathrm{sl}}$ is the slip speed ( $\left.\mathrm{rad} / \mathrm{sec}\right)$.

Equation (31) is the electrical losses formula, which depends on rotor flux $\left(\lambda_{r}\right)$ according to operating point (speed and load torque). The total losses of the motor ( $\left.\mathrm{TP}_{\text {losses }}\right)$ are given as follows:

$$
\begin{gathered}
\mathrm{TP}_{\text {losses }}=\mathrm{P}_{\text {losses }}+\mathrm{P}_{\text {Fric }}=\mathrm{P}_{\text {in }}-\mathrm{P}_{\text {out }} \\
\text { Efficiency }(\eta)=\frac{P_{\text {out }}}{P_{\text {out }}+\mathrm{TP}_{\text {losses }}}
\end{gathered}
$$

Where:

Friction power losses $=\mathrm{F} * \omega_{\mathrm{r}}^{2}$, and

Output power $\left(P_{\text {out }}\right)=\mathrm{T}_{\mathrm{L}} * \omega_{\mathrm{r}}$.

\subsection{Losses minimization control scheme}

The equation (33) is the cost function, the total losses, which depends on rotor flux $(\lambda \mathrm{r})$ according to the operating point. Figure 2 presents the distribution of losses in motor and its variation with the flux. As the flux reduces from the rated value, the core losses decrease, 
but the motor copper losses increase. However, the total losses decrease to a minimum value and then increase again. It is desirable to set the rotor flux at the optimal value, so that the efficiency is optimum [Hegazy, 2006; Amin et al., 2006; Amin et al., 2009].

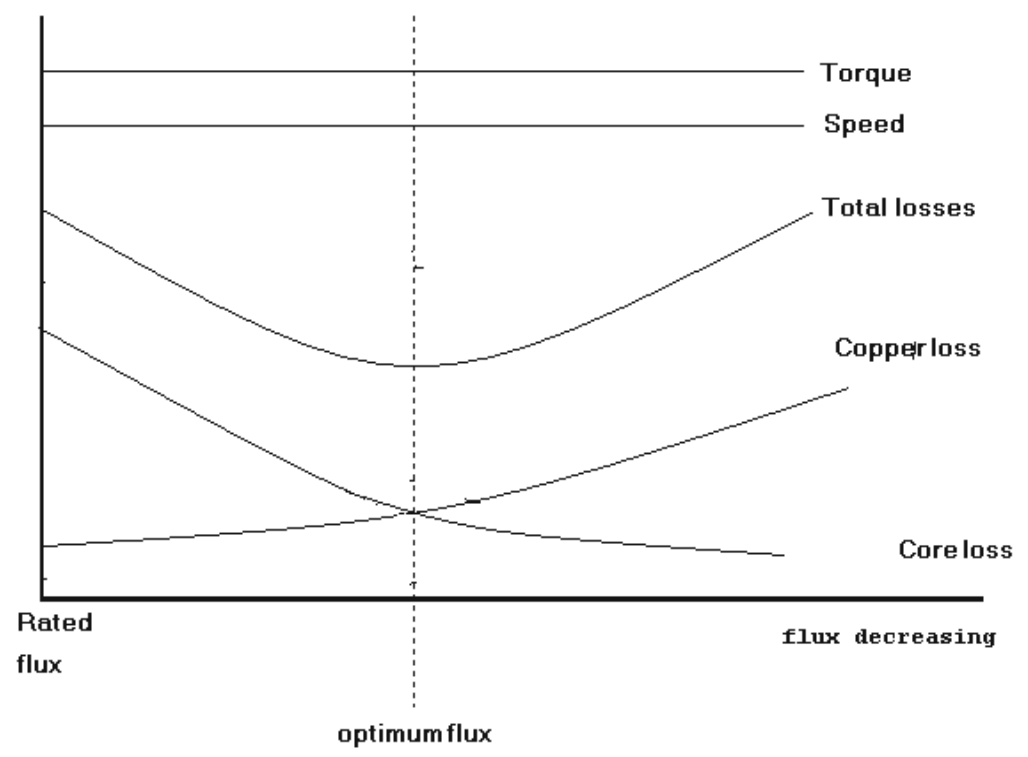

Fig. 2. Losses variation of the motor with varying flux

PSO is applied to evaluate the optimal flux that minimizes the motor losses. The problem can be formulated as follows:

$$
\text { minimize } \mathrm{TP}_{\text {losses }}=\Gamma\left(\lambda \mathrm{r}, \mathrm{T}_{\mathrm{L}}, \omega_{r}\right)
$$

\subsubsection{Particle Swarm Optimization (PSO)}

Particle swarm optimization (PSO) was originally designed and introduced by Eberhart and Kennedy [Ebarhart, Kennedy, 1995; Ebarhart, Kennedy, 2001]. Particle Swarm Optimization (PSO) is an evolutionary computation technique (a search method based on a nature system). It can be used to solve a wide range of optimization problems. Most of the problems that can be solved using Genetic Algorithms (GA) could be solved by PSO. For example, neural network training and nonlinear optimization problems with continuous variables can be easily achieved by PSO [Ebarhart, Kennedy, 2001]. It can be easily expanded to treat problems with discrete variables.

The system initially has a population of random solutions. Each potential solution, called a particle. Each particle is given a random velocity and is flown through the problem space. The particles have memory and each particle keeps track of its previous best position (call the pbest) and with its corresponding fitness. There exit a number of pbest for the respective particles in the swarm and the particle with greatest fitness is called the global best (gbest) of the swarm. PSO can be represented by the concept of velocity and position. The Velocity of each agent can be modified by the following equations: ( $36 \& 38$ ):

$$
\mathrm{v}^{\mathrm{k}+1}=w v_{i}^{k}+c_{1} r_{1}^{*}\left(\text { pbest }-s_{i}{ }^{k}\right)+c_{2} r_{2}{ }^{*}\left(\text { gbest }-s_{i}{ }^{k}\right)
$$




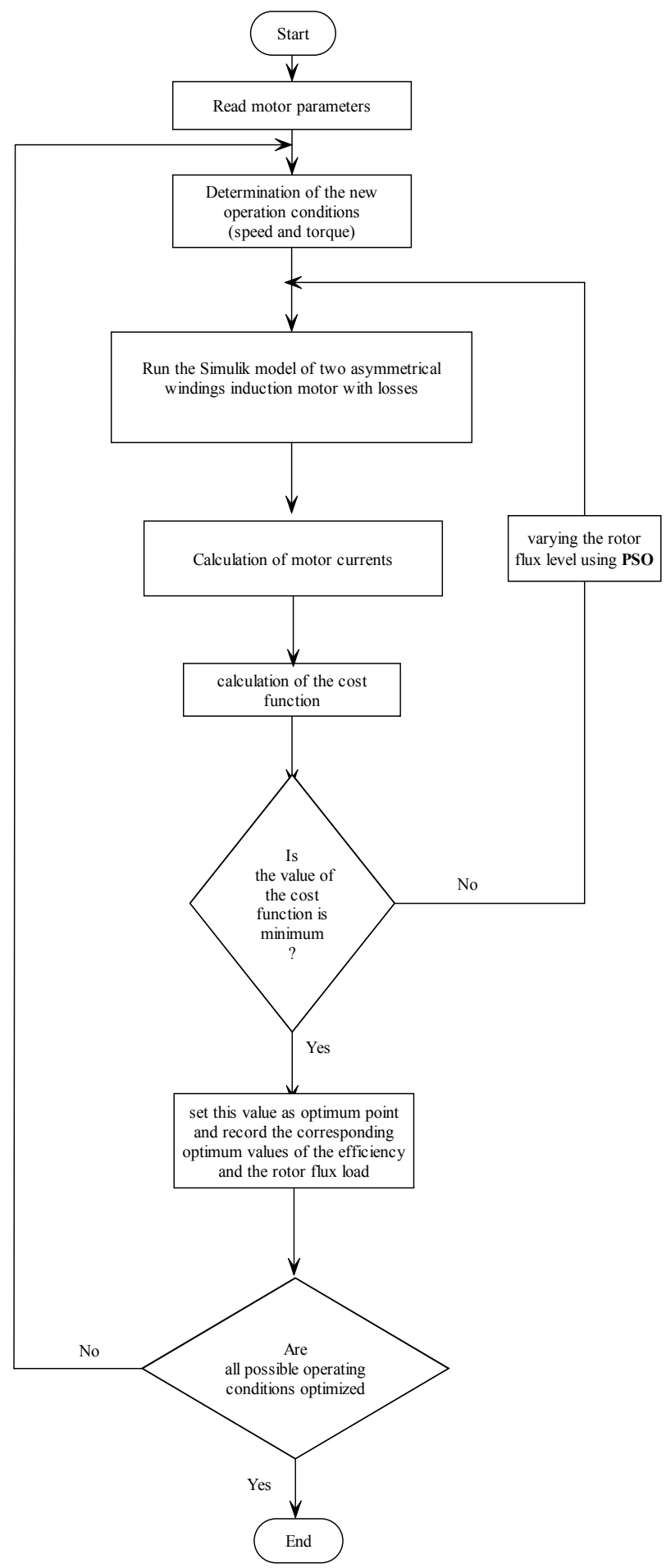

Fig. 3. The flowchart of the execution of the PSO [Hegazy, 2006) 


$$
\omega=\omega_{\max }-\frac{\omega_{\max }-\omega_{\min }}{i t e r_{\max }} * i t e r
$$

Using the above equations, a certain velocity can be calculated that gradually gets close to (pbest) and (gbest). The current position (searching point in the solution space) can be modified by the following equation:

$$
S_{i}^{k+1}=S_{i}^{k}+v_{i}^{k+1}
$$

Where:

$$
\begin{array}{ll}
\mathrm{v}^{\mathrm{k}} & : \text { Current velocity of agent } i \text { at iteration. } \\
\mathrm{v}_{\mathrm{i}} \mathrm{k}^{\mathrm{k}+1} & : \text { Modified velocity of agent } i \\
\mathrm{r}_{1}, \mathrm{r}_{2} & : \text { random number distributed [0,1], } \\
S_{\mathrm{k}} & : \text { current position of agent } i, \\
\omega & : \text { weight function for velocity of agent } i \\
\mathrm{c}_{1}, \mathrm{c}_{2} & : \text { positive constants; [c1 } \mathrm{c} 2<4] . \\
\omega_{\max } & : \text { Initial weight, } \\
\omega_{\min } & : \text { Final weight, } \\
\text { iter } & : \text { Maximum iteration number, } \\
\text { iter } & : \text { Current iteration number. }
\end{array}
$$

- In (35), the losses formula is the cost function of the PSO. The particle swarm optimization (PSO) technique is used for minimizing this cost function.

- The PSO is applied to evaluate the optimal rotor flux that minimizes the motor losses at any operating point. Figure 3 presents the flowchart of the execution of PSO, which evaluates the optimal flux by using MATLAB /SIMULINK.

The optimal flux is the input of the indirect rotor flux oriented controller. The indirect fieldoriented controller generates the required two reference currents to drive the motor corresponding to the optimal flux. These currents are fed to the hysteresis current controller of the two-level inverter. The switching pattern is generated according to the difference between the reference current and the load current through the hysteresis band. Figure 4 shows a whole control diagram of the proposed losses-minimization control system.

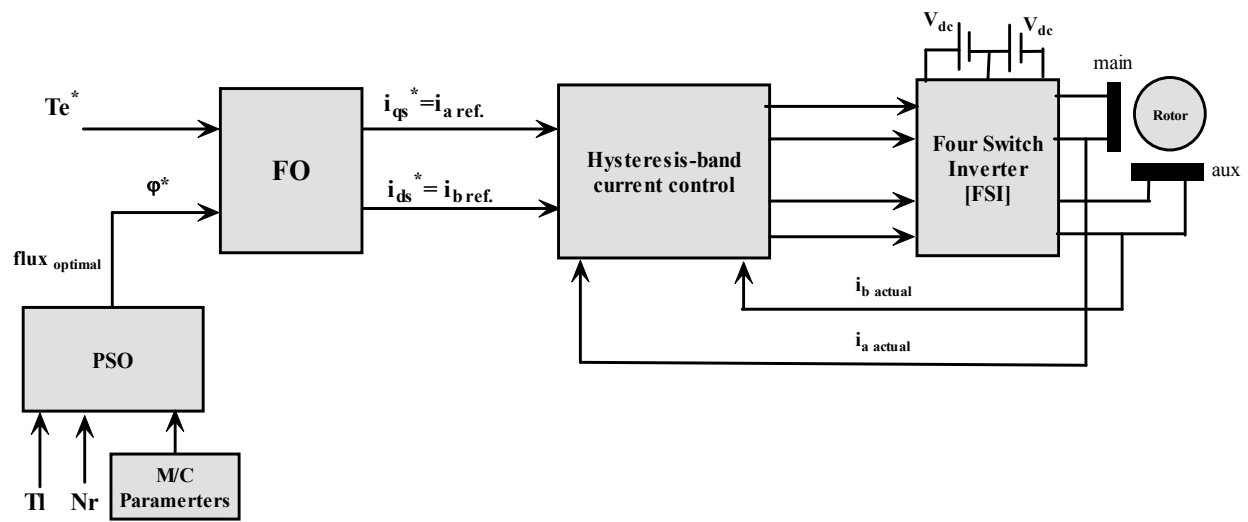

Fig. 4. The proposed losses minimization control system. 


\subsection{Simulation results}

In this section, the proposed application is implemented numerically using MATLABSIMULINK to validate the performance of the proposed control strategy. The motor used in this study has the following parameters, which were measured by using experimental tests. Table 1 shows motor parameters. The used parameters of the PSO are shown in Table 2.

\begin{tabular}{|c|c|}
\hline Rated power & $750 \mathrm{w}$ \\
\hline $\mathrm{V}$ & $220 \mathrm{v}$ \\
\hline $\mathrm{F}$ & $50 \mathrm{~Hz}$ \\
\hline $\mathrm{r}_{\mathrm{M}}$ & $4.6 \Omega$ \\
\hline $\mathrm{r}_{\mathrm{A}}$ & $10.6 \Omega$ \\
\hline $\mathrm{X}_{\mathrm{Lm}}$ & $4.31 \Omega$ \\
\hline $\mathrm{X}_{\mathrm{La}}$ & $7.1472 \Omega$ \\
\hline $\mathrm{rr}$ & $3.455 \Omega$ \\
\hline $\mathrm{X}_{\mathrm{Lr}}$ & $4.284 \Omega$ \\
\hline $\mathrm{X}_{\mathrm{mq}}$ & $89.65 \Omega$ \\
\hline $\mathrm{X}_{\mathrm{md}}$ & $169.43 \Omega$ \\
\hline $\mathrm{R}_{\mathrm{qfe}}$ & $1050 \Omega$ \\
\hline $\mathrm{R}_{\mathrm{dfe}}$ & $1450 \Omega$ \\
\hline $\mathrm{J}$ & $0.005776 \mathrm{~kg} . \mathrm{m} 2$ \\
\hline $\mathrm{B}$ & $0.00328 \mathrm{~N} . \mathrm{m} . \mathrm{sec} / \mathrm{r}$ \\
\hline Pole pair & 2 \\
\hline
\end{tabular}

Table 1. Motor Parameters

The optimal rotor flux provides the maximum efficiency at any operating point. There are six-cases of the motor operation are studied by using FOC based on PSO. PSO will evaluate the optimal rotor flux level. This flux is fed to the FOC module. Figure 5 shows the performance of the motor at case (1) $\left(\mathrm{T}_{\mathrm{L}}=0.25 \mathrm{PU}, \mathrm{N}_{\mathrm{r}}=0.5 \mathrm{~N}_{\text {rated }}\right)$, when PSO is applied sideby-side FOC as shown in Fig.4.

\begin{tabular}{|c|c|}
\hline Population size & 10 \\
\hline Max. iter & 50 \\
\hline c1 & 0.5 \\
\hline c2 & 0.5 \\
\hline Max. weight & 1.4 \\
\hline Min. weight & 0.1 \\
\hline r1 & {$[0,1]$} \\
\hline r2 & {$[0,1]$} \\
\hline Lower Bound & 0.2 \\
\hline Upper Bound & 2 \\
\hline
\end{tabular}

Table 2. PSO Algorithm parameter 


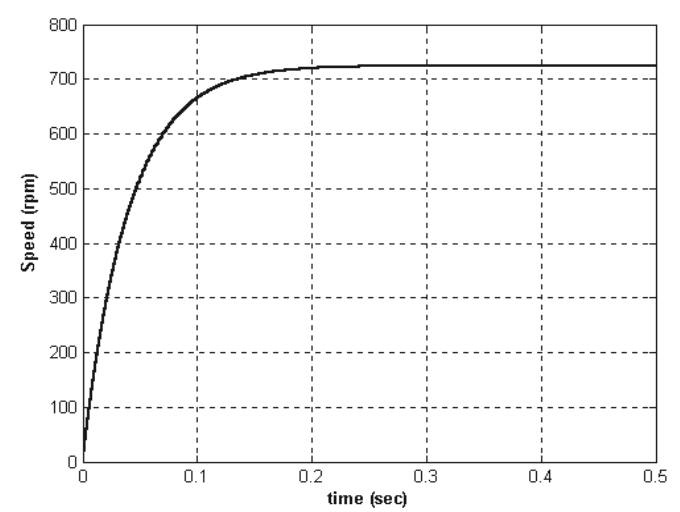

(a)

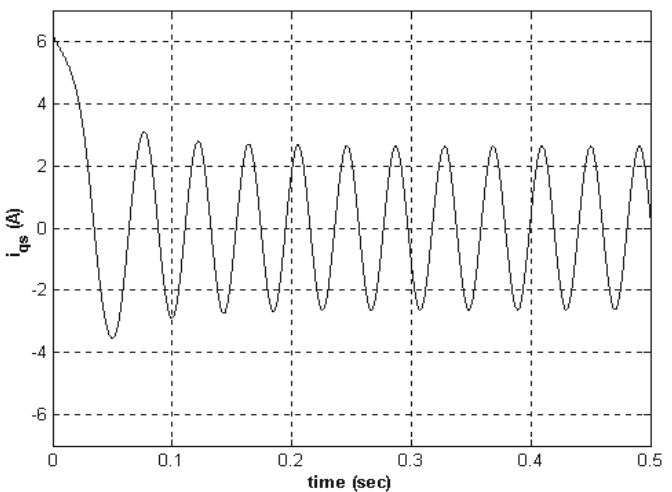

(c)

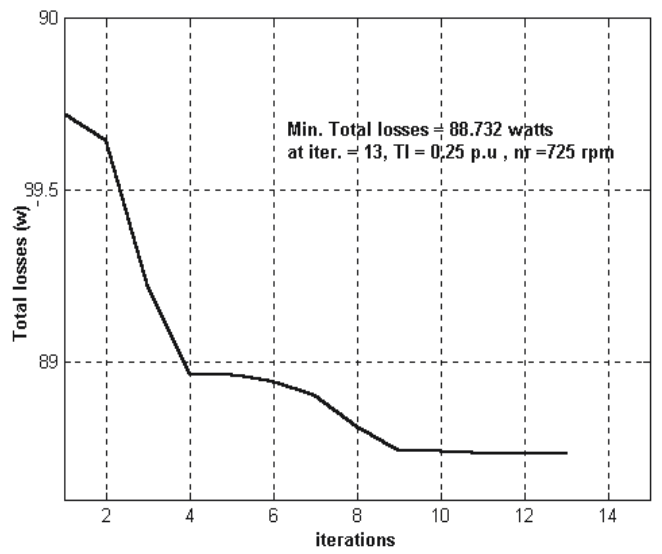

(e)

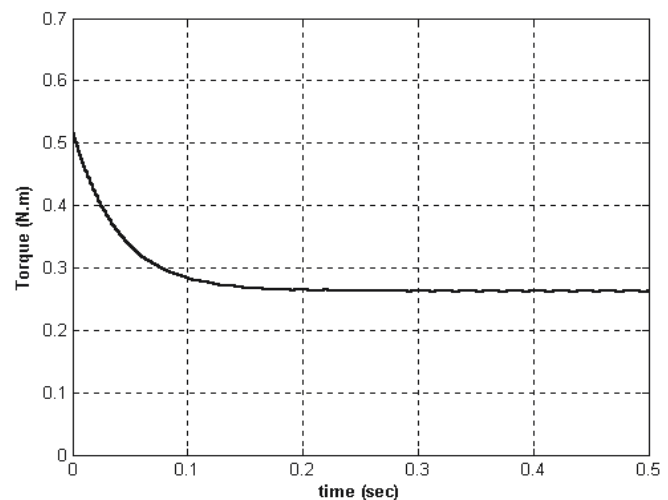

(b)

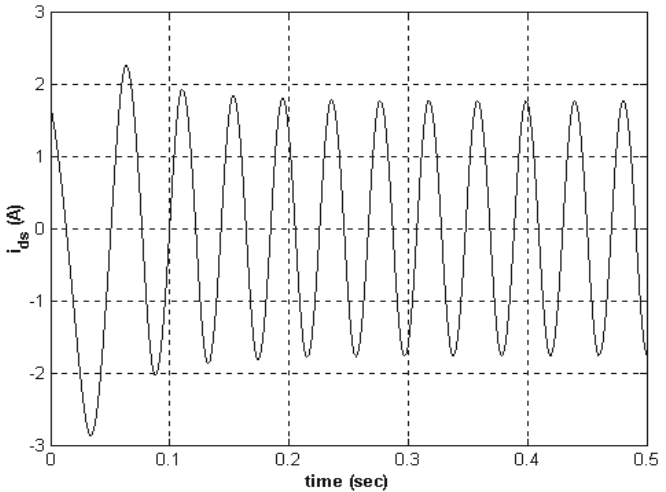

(d)

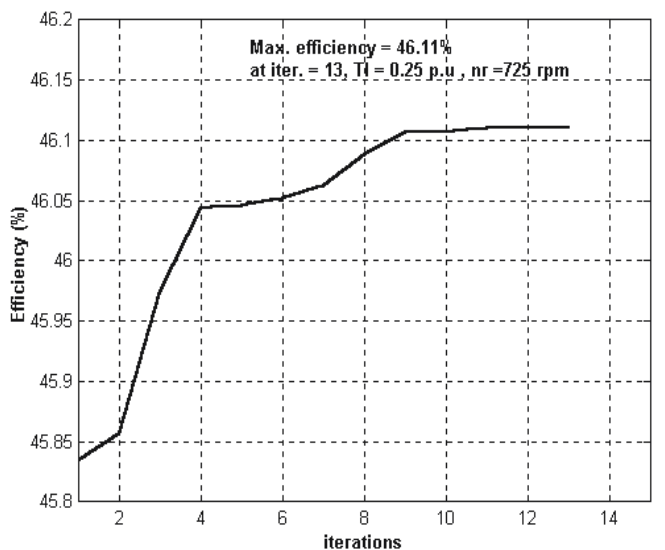

(f)

Fig. 5. Simulation results of the motor at case (1).Speed-time curve, (b) Torque-time curve, (c) The stator current in q-axis, (d) The stator current in d-axis, (e) Total Losses against iterations, (f) Efficiency against iterations 
Figure 6 illustrates the comparison between FOC and FOC based PSO control methods at different operating points. Figure 7 presents the optimal flux, which is obtained by applying PSO. Table 3 presents the summary of the results of FOC and FOC based PSO methods.

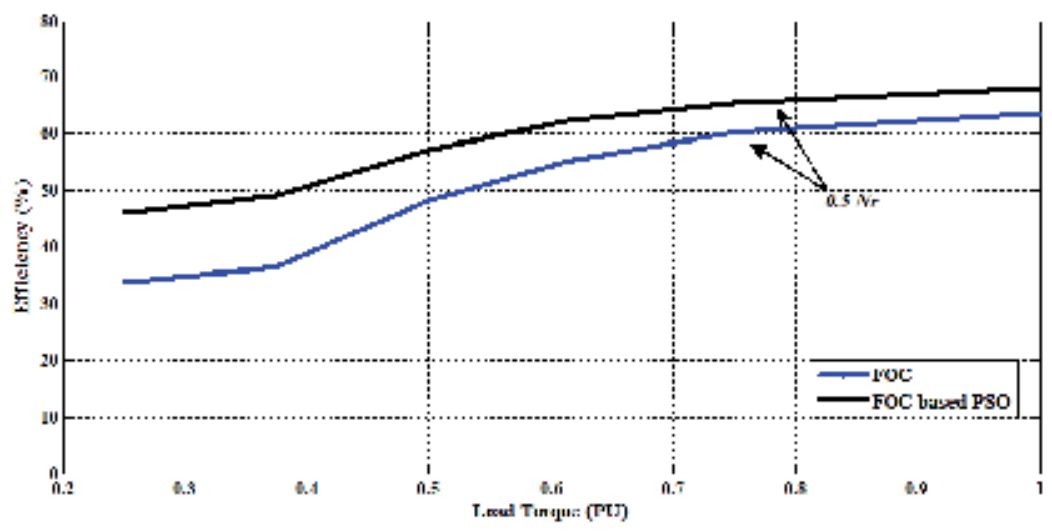

Fig. 6. the comparison between FOC and FOC based PSO

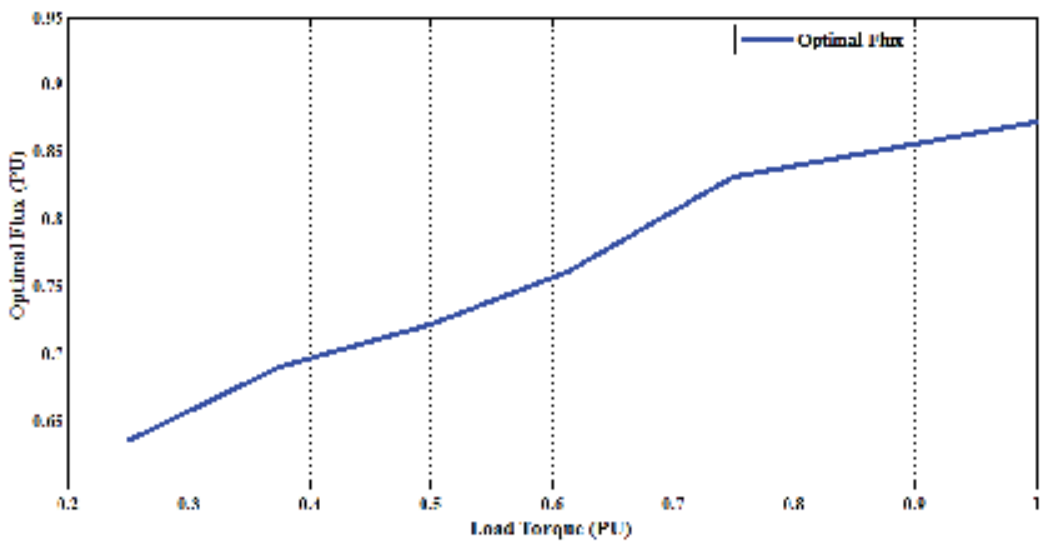

Fig. 7. The optimal flux at different load torque

\begin{tabular}{|c|c|c|c|c|c|c|c|}
\hline \multirow[b]{2}{*}{ Cases } & \multirow{2}{*}{$\begin{array}{c}T_{L} \\
(P U)\end{array}$} & \multirow{2}{*}{$\begin{array}{c}N_{r} \\
(r p m)\end{array}$} & \multicolumn{2}{|c|}{ FOC } & \multicolumn{2}{|c|}{ FOC based PSO } & \multirow[b]{2}{*}{$\begin{array}{c}\text { Improvement } \\
(\%)\end{array}$} \\
\hline & & & $\begin{array}{c}\lambda \\
(P U)\end{array}$ & $\eta(\%)$ & $\begin{array}{c}\lambda_{\text {Optimal }} \\
(\mathbf{P U})\end{array}$ & $\eta(\%)$ & \\
\hline (1) & 0.25 & $0.5 N_{\text {rated }}$ & 1 & 33.85 & 0.636 & 46.11 & 36.22 \\
\hline (2) & 0.375 & $0.5 N_{\text {rated }}$ & 1 & 36.51 & 0.6906 & 49.15 & 34.62 \\
\hline (3) & 0.5 & $0.5 N_{\text {rated }}$ & 1 & 48.21 & 0.722 & 57.11 & 18.46 \\
\hline (4) & 0.6125 & $0.5 N_{\text {rated }}$ & 1 & 55.15 & 0.761 & 62.34 & 13.04 \\
\hline (5) & 0.75 & $0.5 N_{\text {rated }}$ & 1 & 60.175 & 0.8312 & 65.31 & 8.53 \\
\hline (6) & 1 & $0.5 N_{\text {rated }}$ & 1 & 63.54 & 0.8722 & 68.15 & 7.26 \\
\hline
\end{tabular}

Table 3. Summary of the results of the two controllers 
In practical system, the flux level based on PSO at different operating points (torque and speed) is calculated and stored in a look up table (LUT). The use of look up table will enable the system to work in real time without any delay that might be needed to calculate the optimal point. The proposed controller would receive the operating point (torque and speed) and get the optimum flux ( $\left.\lambda_{\text {optimal }}\right)$ from the look up table. It will generate the required reference current. It is noticed that, the efficiency with the FOC based on PSO method is higher than the efficiency with the FOC method only.

\subsection{Experimental results}

To verify the validity of the proposed control scheme, a laboratory prototype is built and tested [Hegazy, 2006; Amin et al., 2006; Amin et al., 2009]. The basic elements of the proposed experimental scheme are shown in Fig. 8 and Fig. 9. The experimental results of the motor are achieved by coupling the motor to an eddy current dynamometer. The experimental results are achieved using two control methods:

- $\quad$ Field-Oriented Control [FOC], and

- $\quad$ Field-Oriented Control [FOC] based on PSO.

The reference and the actual motor currents are fed to the hysteresis current controller. The switching pattern of the two-level four-switch inverter [FSI] is generated according to the difference between the reference currents and the load currents. Figure 10 shows the experimental results of the motor with FOC at case (1), where the motor is loaded by $\mathrm{T}_{\mathrm{L}}=$ 0.25 PU. Figure 11 shows the experimental result of the motor with FOC based on PSO at case (1). The cases are summarized in Table 4.

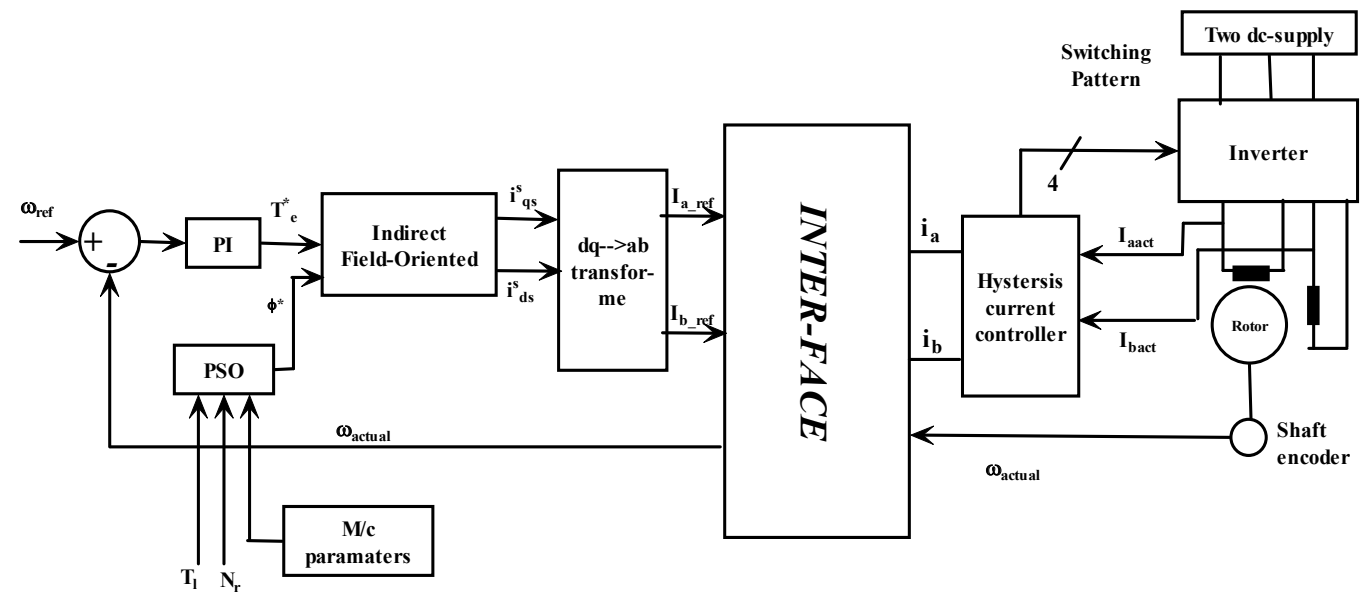

Fig. 8. Block diagram of the proposed experimental scheme [Hegazy, 2006; Amin et al., 2009]

\begin{tabular}{|c|c|c|c|c|c|c|c|}
\hline \multirow[b]{2}{*}{ Cases } & \multicolumn{3}{|c|}{ FOC } & \multicolumn{3}{|c|}{ FOC with PSO } & \multirow[b]{2}{*}{$\begin{array}{c}\text { Improvement } \\
(\%)\end{array}$} \\
\hline & $\begin{array}{c}\lambda \\
(P U\end{array}$ & $\begin{array}{c}\text { Power } \\
\text { Input (W) }\end{array}$ & $\begin{array}{c}\eta \\
(\%)\end{array}$ & $\begin{array}{c}\lambda \\
(P U\end{array}$ & $\begin{array}{c}\text { Power } \\
\text { Input }(W)\end{array}$ & $\begin{array}{c}\eta \\
(\%)\end{array}$ & \\
\hline (1) & 1 & 235 & 32.3 & 0.636 & 169 & 44.92 & 39.07 \\
\hline (2) & 1 & 323 & 35.2 & 0.690 & 243 & 47.06 & 33.69 \\
\hline
\end{tabular}

Table 4 . The summary of the two-cases 


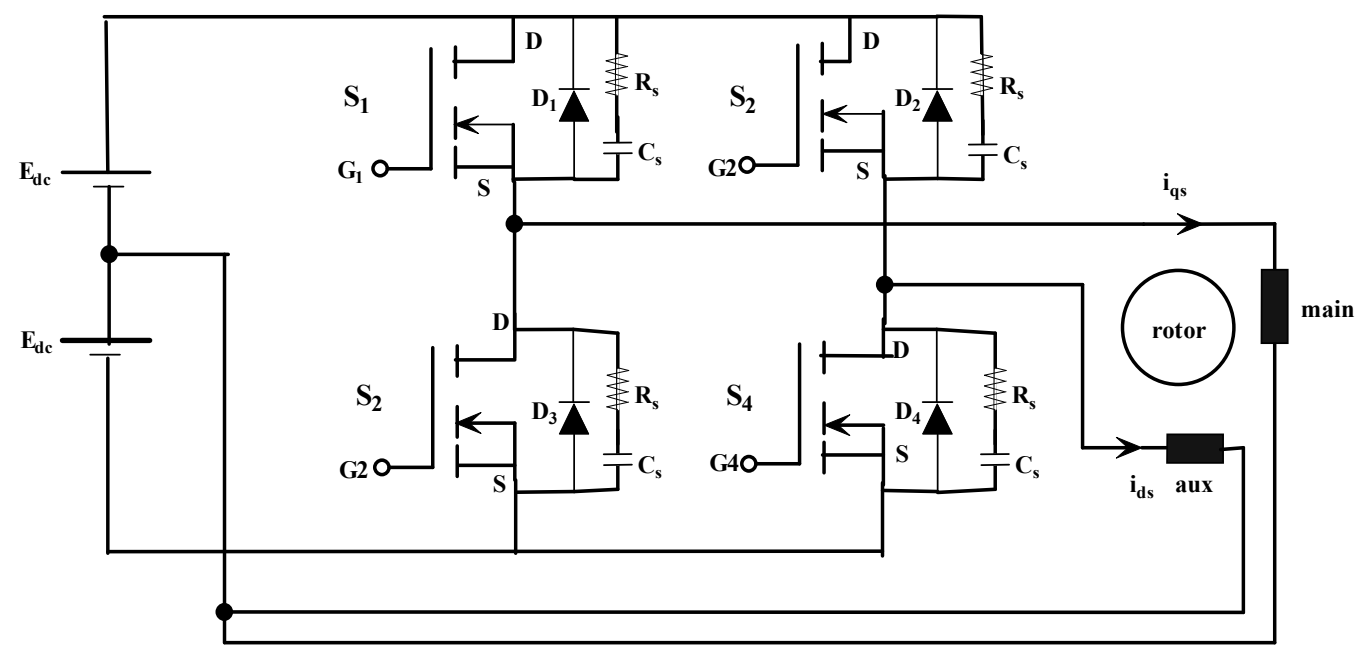

Fig. 9. The power circuit of Four Switch Inverter [FSI]

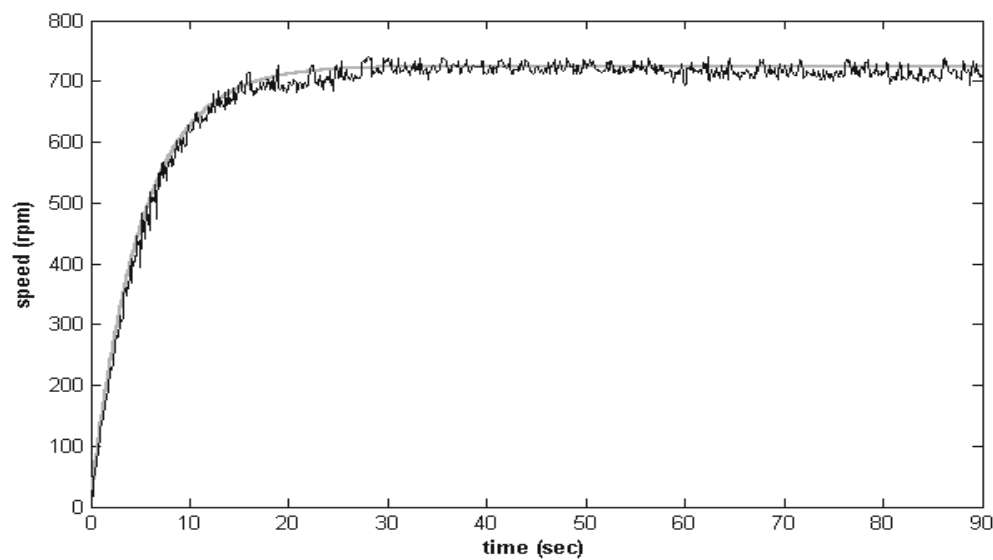

(a)

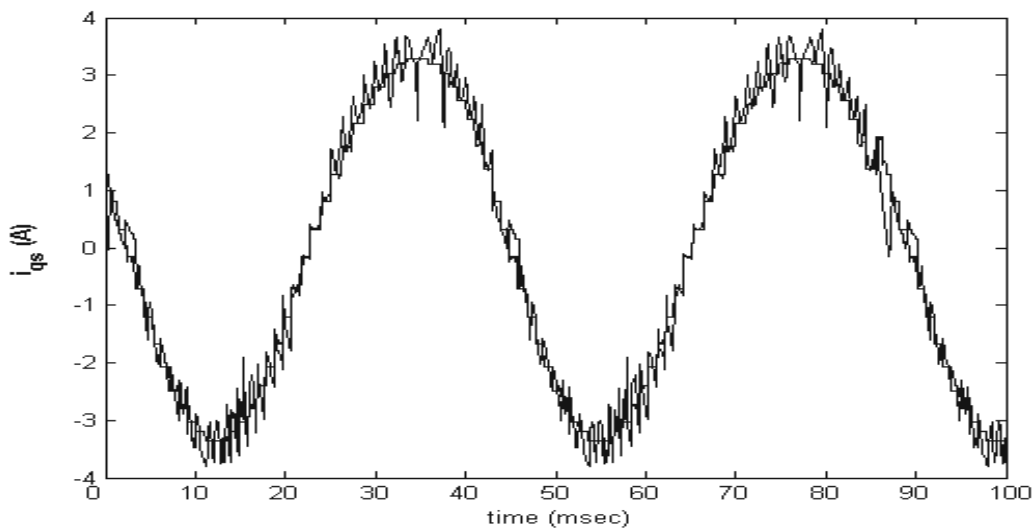

(b) 


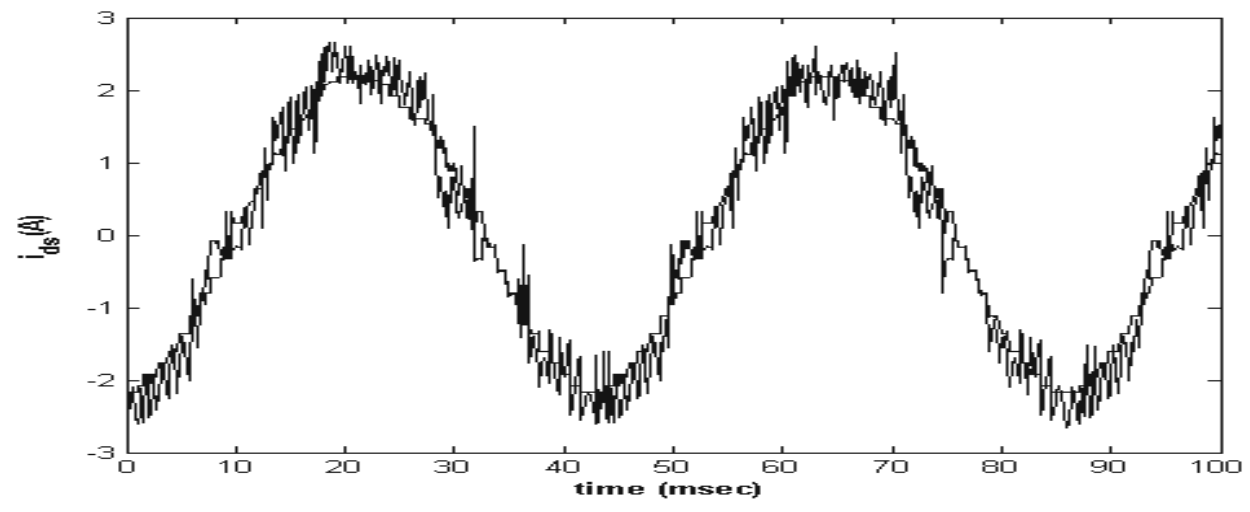

(c)

Fig. 10. Experimental results of FOC method; the reference and actual speed, (b) the reference and actual current in q-axis, (c) The reference and actual current in d-axis

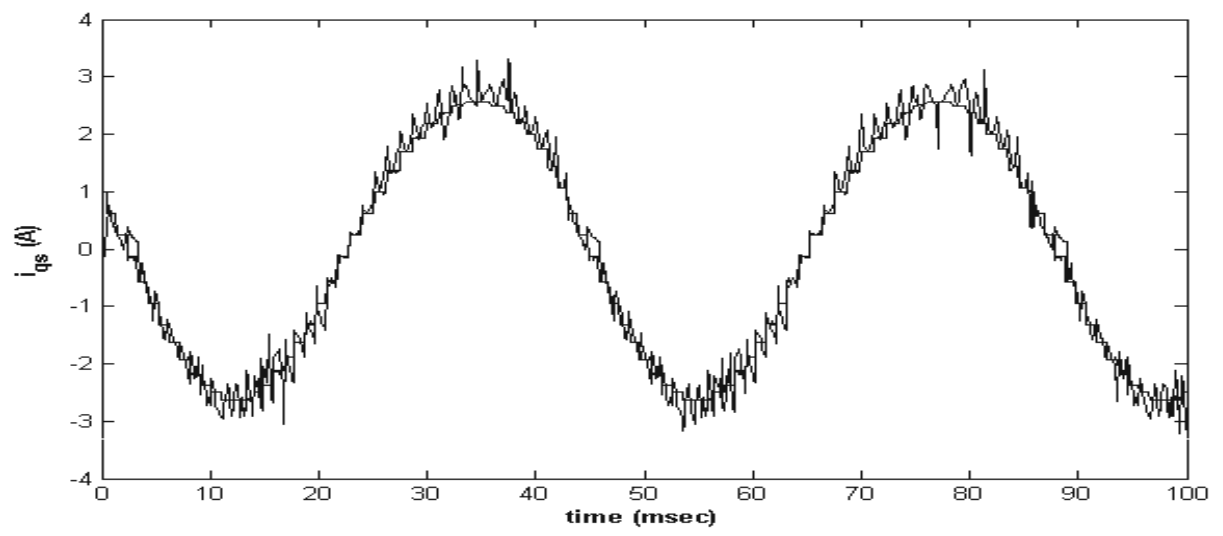

(a)

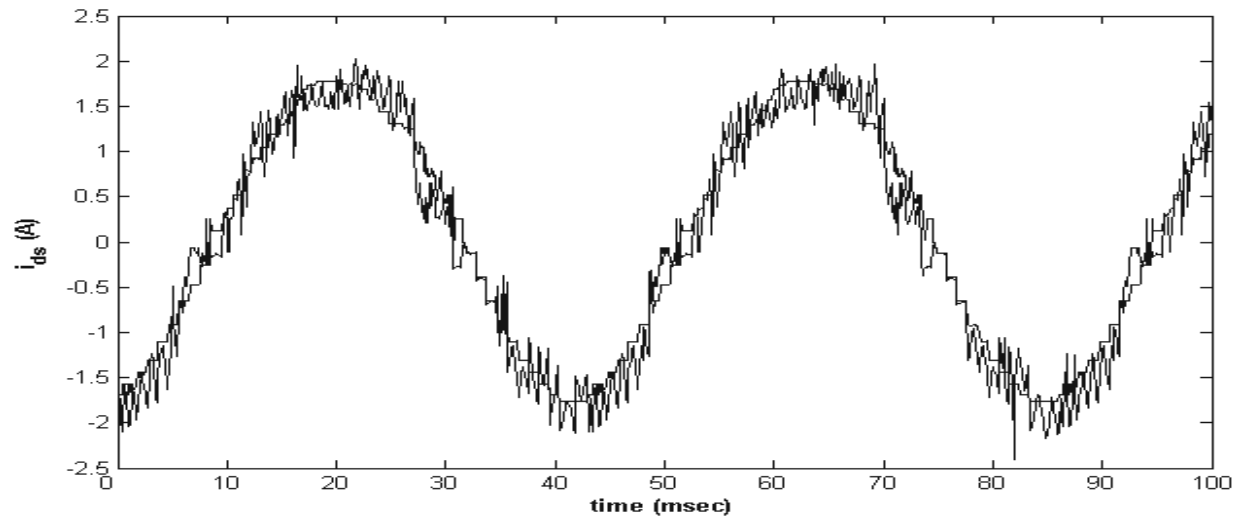

(b)

Fig. 11. Experimental results of FOC method based on PSO. (a) The reference and actual current in q-axis, the reference and actual current in d-axis 
Finally, these results demonstrate that, the FOC based on PSO method saves more energy than conventional FOC method. Thus, the efficiency with PSO is improved than it's at FOC.

\section{Maximum efficiency and minimum operating cost of induction motors}

This section presents another application of PSO for losses and operating cost minimization control in the induction motor drives. Two control strategies for induction motor speed control are proposed. Those two strategies are based on PSO and called Maximum Efficiency Strategy and Minimum Operating Cost Strategy [A. Hamid et al. 2006]. The proposed technique is based on the principle that the flux level in the machine can be adjusted to give the minimum amount of losses and minimum operating cost for a given value of speed and load torque. The main advantages of the proposed technique are; its simple structure. It is a straightforward maximization of induction motor efficiency and its operating cost for a given load torque. As was demonstrated, PSO is efficient in finding the optimum operating machine's flux level. The optimum flux level is a function of the machine operating point.

The main induction motor losses are usually split into five components: stator copper losses, rotor copper losses, iron losses, mechanical losses, and stray losses [Kioskeridis \& Margaris, 1996].

The efficiency that decreases with increasing losses can be improved by minimizing the losses. Copper losses reduce with decreasing the stator and the rotor currents, while the core losses essentially increase with increasing air-gap flux density. A study of the copper and core losses components reveals that their trends conflict. When the core losses increase, the copper losses tends to decrease. However, for a given load torque, there is an air-gap flux density at which the total losses is minimized. Hence, electrical losses minimization process ultimately comes down to the selection of the appropriate air-gap flux density of operation. Since the air-gap flux density must be variable when the load is changing, control schemes in which the (rotor, air-gap) flux linkage is constant will yield sub-optimal efficiency operation especially when the load is light. Then to improve the motor efficiency, the flux must be reduced when it operates under light load conditions by obtaining a balance between copper and iron losses.

The challenge to engineers, however, is to be able to predict the appropriate flux values at any operating points over the complete torque and speed range which will minimize the machines losses, hence maximizing the efficiency. In general, there are three different approaches to improve the induction motor efficiency especially under light-load.

\section{a. Losses Model Controller (LMC)}

This controller depends on a motor losses model to compute the optimum flux analytically. The main advantage of this approach is its simplicity and it does not require extra hardware. In addition, it provides smooth and fast adaptation of the flux, and may offer optimal performance during transient operation. However, the main problem of this approach is that it requires the exact values of machine parameters. These parameters include the core losses and the main inductance flux saturation, which are unknown to the users and change considerably with temperature, saturation, and skin effect. In addition, these parameters may vary due to changes in the operating conditions. However, with continuing improvement of evolutionary parameter determination algorithms, the disadvantages of motor parameters dependency are slowly disappearing.

b. Search Controller (SC)

This controller measures the input power of the machine drive regularly at fixed time intervals and searches for the flux value, which results in minimum power input for given 
values of speed and load torque. This particular method does not demand knowledge of the machine parameters and the search procedure is simple to implement.

However, some disadvantages appear in practice, such as continuous disturbances in the torque, slow adaptation (7sec.), difficulties in tuning the algorithm for a given application, and the need for precise load information. In addition, the precision of the measurements may be poor due to signal noise and disturbances. This in turn may cause the SC method to give undesirable control performance. Moreover, nominal flux is applied in transient state and is tuned after the system reaches steady state to an optimal value by numerous increments, thus lengthening the optimization process. Therefore, the SC technique may be slow in obtaining the optimal point. In addition, in real systems, it may not reach a steady state and so cause oscillations in the air gap flux that result in undesirable torque disturbances. For these reasons, this is not a good method in industrial drives.

\section{c. Look Up Table Scheme}

It gives the optimal flux level at different operating points. This table, however, requires costly and time-consuming prior measurements for each motor. In this section, a new control strategy uses the loss model controller based on PSO is proposed. This strategy is simple in structure and has the straightforward goal of maximizing the efficiency for a given load torque. The resulting induction motor efficiency is reasonably close to optimal. It is well known that the presence of uncertainties, the rotor resistance, for instance makes the result no more optimal. Digital computer simulation results are obtained to demonstrate the effectiveness of the proposed method.

\subsection{Definition of operating strategies}

The following definitions are useful in subsequent analyses. Referring to the analysis of the induction motor presented in [A. Hamid et al. 2006], the per-unit frequency is

$$
a=\frac{\omega_{e}}{\omega_{b}}=\frac{\omega_{s}+\omega_{r}}{\omega_{b}}
$$

The slip is defined by

$$
s=\frac{\omega_{s}}{\omega_{b}}=\frac{\omega_{s}}{\omega_{s}+\omega_{r}}
$$

The rotor current is given by

$$
I_{r}^{\prime}=\frac{\phi_{m}}{\sqrt{\left(\frac{r_{r}^{\prime}}{s a}\right)^{2}+X_{l r}^{\prime 2}}}
$$

The electromagnetic torque is given by

$$
T_{e}=\frac{\left(\frac{r_{r}^{\prime}}{s a}\right)}{\left(\frac{r_{r}^{\prime}}{s a}\right)^{2}+X_{l r}^{\prime 2}} \phi_{m}^{2}
$$


The stator current is related to the air gap flux and the electromagnetic torque as:

$$
I_{s}=\sqrt{\left(S_{1} \phi_{m}+S_{2} \phi_{m}^{3}+S_{2} \phi_{m}^{5}\right)^{2}+C_{L} \frac{T_{e}^{2}}{\phi_{m}^{2}}}
$$

Where

$$
C_{L}=1+2 \times \frac{x_{l r}}{x_{m}}
$$

The air gap flux is related to the electromagnetic torque as:

$$
\phi_{m}=\sqrt{\frac{s a}{r_{r}^{\prime}}} \sqrt{\left(\frac{r_{r}^{\prime}}{s a}\right)^{2}+X_{l r}^{\prime 2} \sqrt{T_{e}}}
$$

The efficiency is defined as the output power divided by the electric power supplied to the stator (inverter losses are included):

$$
\operatorname{Efficiency}(\eta)=\frac{P_{o u t}}{P_{i n}}
$$

\subsubsection{Maximum efficiency strategy}

In MES (Maximum Efficiency Strategy), the slip frequency is adjusted so that the efficiency of the induction motor drive system is maximized [A. Hamid et al. 2006].

The induction motor losses are the following:

1. Copper losses: these are due to flow of the electric current through the stator and rotor windings and are given by:

$$
P_{c u}=r_{s} I_{s}^{2}+r_{r}^{\prime} I_{r}^{\prime 2}
$$

2. Iron losses: these are the losses due to eddy current and hysteresis, given by

$$
P_{\text {core }}=K_{e}\left(1+S^{2}\right) a^{2} \varphi_{m}^{2}+K_{h}(1+S) a \varphi_{m}^{2}
$$

3. Stray losses: these arise on the copper and iron of the motor and are given by:

$$
P_{c u}=C_{s t r} \omega_{r}^{2} I_{r}^{\prime 2}
$$

4. Mechanical losses: these are due to the friction of the machine rotor with the bearings and are given by:

$$
P_{f w}=C_{f w}+\omega_{r}^{2}
$$

5. Inverter losses: The approximate inverter loss as a function of stator current is given by: 


$$
P_{i n v}=K_{1 i n v} i_{s}^{2}+K_{2 i n v} i_{s}
$$

Where: $K_{1 \text { inv }}, K_{2 \text { inv }}$ are coefficients determined by the electrical characteristics of a switching element where: K1inv= 3.1307e-005, K2inv=0.0250.

The total power losses are expressed by:

$$
\begin{aligned}
P_{\text {losses }} & =P_{c u}+P_{\text {core }}+P_{s}+P_{f w}+P_{i n v}=\left[r_{s} I_{s}^{2}+r_{r}^{\prime} I_{r}^{\prime 2}\right]+ \\
& {\left[K_{e}\left(1+S^{2}\right) a^{2} \varphi_{m}^{2}\right]+\left[K_{h}(1+S) a \varphi_{m}^{2}\right]+\left[C_{s t r} \omega_{r}^{2} I_{r}^{\prime 2}\right] } \\
& +\left[K_{1 i n v} i_{s}^{2}+K_{2 i n v} i_{s}\right]
\end{aligned}
$$

The output power is given by:

$$
P_{\text {out }}=T_{L} \times \omega_{r}
$$

The input power is given by:

$$
\begin{aligned}
P_{\text {in }}= & P_{\text {out }}+P_{\text {losses }}=P_{c u}+P_{\text {core }}+P_{s}+P_{f w}+P_{\text {inv }}=\left[r_{s} I_{s}^{2}+r_{r}^{\prime} I_{r}^{\prime 2}\right]+ \\
& {\left[K_{e}\left(1+S^{2}\right) a^{2} \varphi_{m}^{2}\right]+\left[K_{h}(1+S) a \varphi_{m}^{2}\right]+\left[C_{s t r} \omega_{r}^{2} I_{r}^{\prime 2}\right] } \\
& +\left[K_{1 i n v} i_{s}^{2}+K_{2 i n v} i_{s}\right]+T_{L} \times \omega_{r}
\end{aligned}
$$

The efficiency is expressed as:

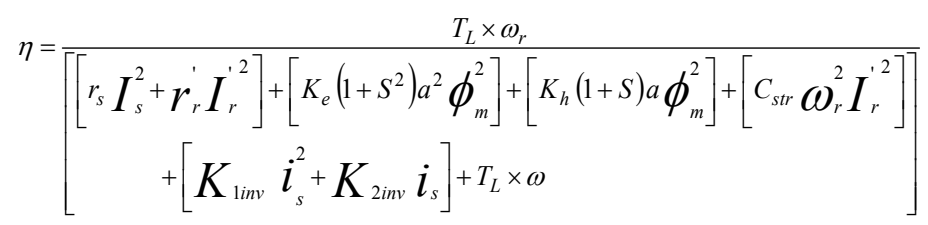

The efficiency maximization of the induction motor problem can be formulated as follows:

$$
\text { Maximize } \quad \eta\left(T_{L}, \omega_{s}, \omega_{r}\right)
$$

The maximization should observe the fact that the amplitude of the stator current and flux cannot exceed their specified maximum point.

\subsubsection{Minimum operating cost strategy}

In Minimum Operating cost Strategy (MOCS), the slip frequency is adjusted so that the operating cost of the induction motor is minimized. The operating cost of the induction machine should be calculated over the whole life cycle of the machine. That calculation can be made to evaluate the cost of the consumed electrical energy. The value of average energy cost considering the power factor penalties can be determined by the following stages [A. Hamid et al. 2006]:

1. If $0 \leq \mathrm{PF}<0.7$ 


$$
C=C_{0}\left[1+\left(\frac{0.9-P F}{0.01}\right) \times \frac{1}{100}\right]
$$

2. If $0.7 \leq \mathrm{PF} \leq 0.92$, If $\mathrm{PF} \geq 0.9, \mathrm{PF}=0.9$

$$
C=C_{0}\left[1+\left(\frac{0.9-P F}{0.01}\right) \times \frac{0.5}{100}\right]
$$

3. If $0.9 \leq \mathrm{PF} \leq 1$, If $0.95 \leq \mathrm{PF} \leq 1, \mathrm{PF}=0.95$

$$
C=C_{0}\left[1+\left(\frac{0.9-P F}{0.01}\right) \times \frac{0.7}{100}\right]
$$

If the average energy cost $C$ is calculated, it can be used to establish the present value of losses. The total cost of the machine is the sum of its initial cost plus the present worth value of losses and maintenance costs.

$$
P W_{L}=C \times T \times N \times P_{\text {out }} \times\left[\frac{1}{\eta}-1\right]
$$

Where:

$\mathrm{PW}_{\mathrm{L}}=$ present worth value of losses

$\mathrm{C} 0=$ energy cost per $\mathrm{kWh}$,

$\mathrm{C}=$ modified energy cost per $\mathrm{kWh}$

$\mathrm{T}$ = running time per year (Hrs / year)

$\mathrm{N}=$ evaluation life (years)

$\mathrm{P}_{\text {out }}=$ the output power $(\mathrm{kW})$

$\eta \quad=$ the efficiency

The operating cost minimization of the induction motor problem can be formulated as follows:

$$
\text { Minimize } P W_{L}\left(T_{L}, \omega_{s}, \omega_{r}\right)
$$

\subsection{Simulation results}

The simulation is carried out on a three-phase, $380 \mathrm{~V}, 1-\mathrm{HP}, 50 \mathrm{~Hz}$, and 4-pole, squirrel cage induction motor. The motor parameters are $R_{s}=0.0598, X_{l s}=0.0364, X_{m}=0.8564, X_{l r}=0.0546$, $\mathrm{R}_{\mathrm{r}}=0.0403, \mathrm{~K}_{\mathrm{e}}=0.0380, \mathrm{~K}_{\mathrm{h}}=0.0380, \mathrm{C}_{\mathrm{str}}=0.0150, \mathrm{C}_{\mathrm{fw}}=0.0093, \mathrm{~S}_{1}=1.07, \mathrm{~S}_{2}=-0.69, \mathrm{~S}_{3}=0.77$. For cost analysis, the following values were assumed: $\mathrm{C}_{0}=0.05, \mathrm{~N}=15, \mathrm{~T}=8000$. The task of PSO controller is to find that value of slip at which the maximum efficiency occurs. At certain load torque and rotor speed, the PSO controller determines the slip frequency $\omega_{\mathrm{s}}$ at which the maximum efficiency and minimum operating cost occur. The block diagram of the optimization process based on PSO is shown in Fig.12. To observe the improvements in efficiency using the suggested PSO controller, Fig. 13 shows the efficiency of the selected machine for all operating conditions using conventional methods (constant voltage to frequency ratio, field oriented control strategy) and using the proposed PSO controller at different rotor speed levels, $\mathrm{Wr}=0.2 \mathrm{PU}$, and $\mathrm{Wr}=1 \mathrm{PU}$ respectively [A. Hamid et al. 2006]. This figure shows that a considerable energy saving is achieved in comparison with the conventional method (field oriented control strategy and constant voltage to frequency 
ratio) especially at light loads and small rotor speed. Figure 14 compares the efficiency of the induction motor drive system under the maximum efficiency strategy with the minimum operating cost strategy at $\mathrm{Wr}=1 \mathrm{PU}$. It is obvious from the figure that the efficiency is almost the same for both strategies for all operating points.

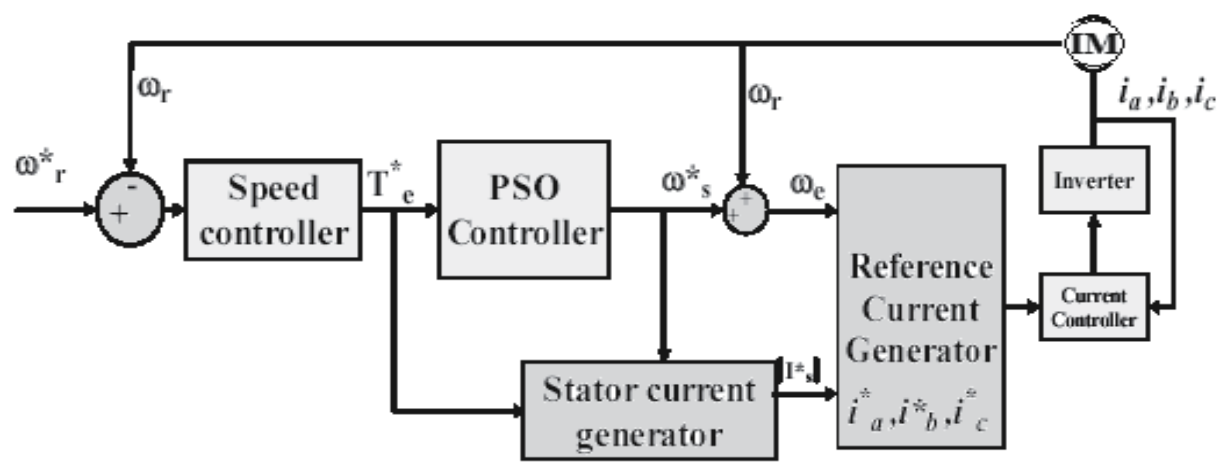

Fig. 12. The proposed drive system based on PSO controller

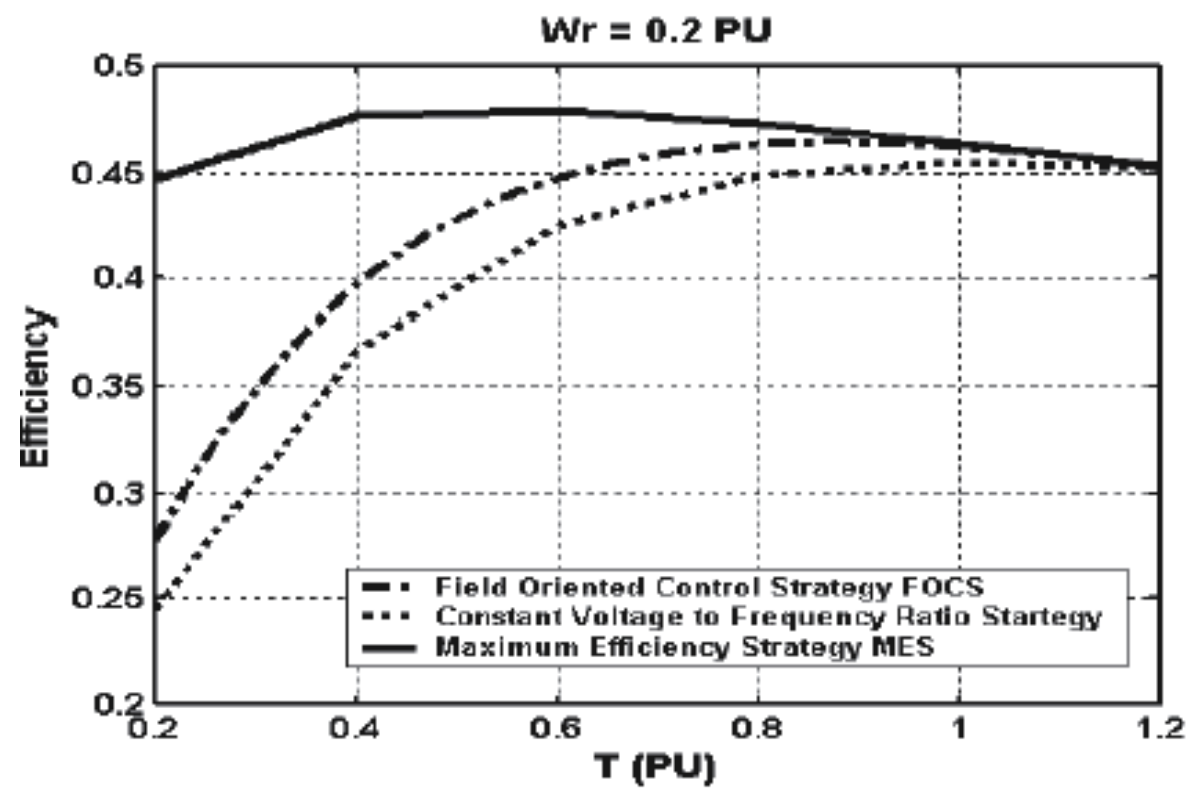

(a) 


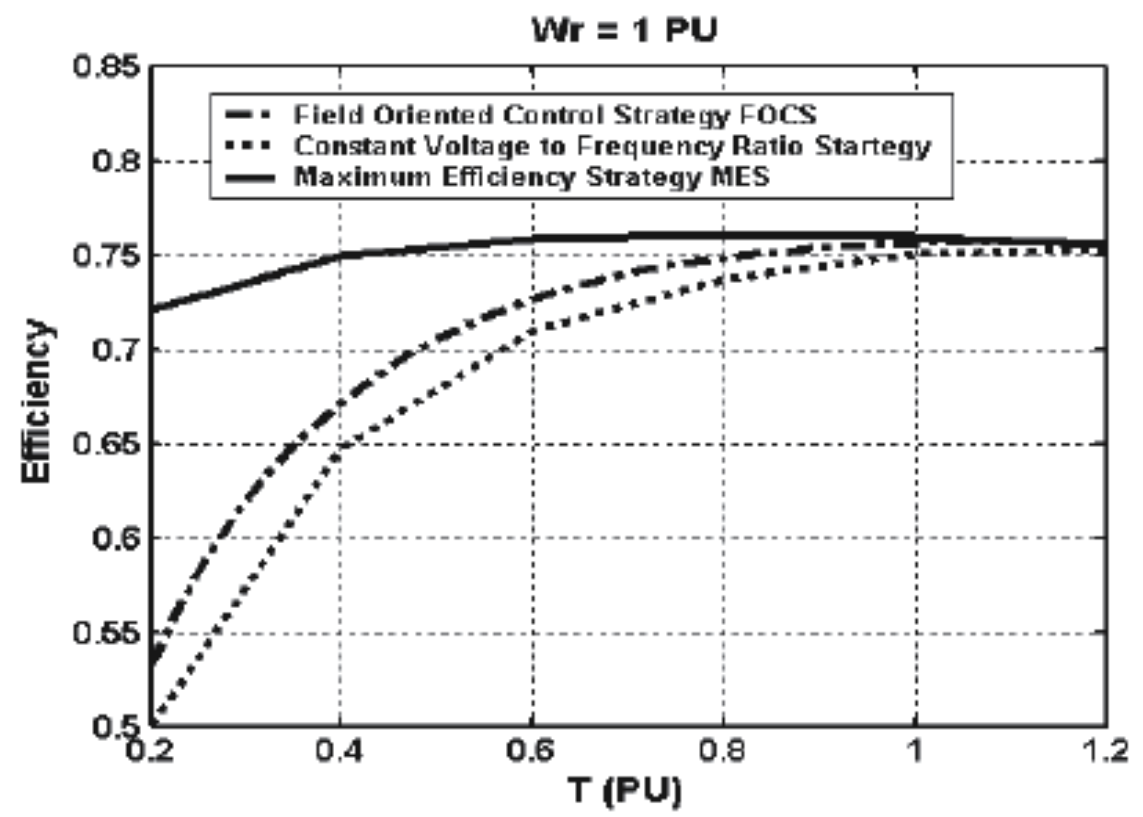

(b)

Fig. 13. The efficiency of the induction motor using the maximum efficiency strategy compared with the efficiency using the conventional methods at (a) $\mathrm{W}_{\mathrm{r}}=0.2 \mathrm{PU},(\mathrm{b}) \mathrm{W}_{\mathrm{r}}=1$ PU [A. Hamid et al. 2006].

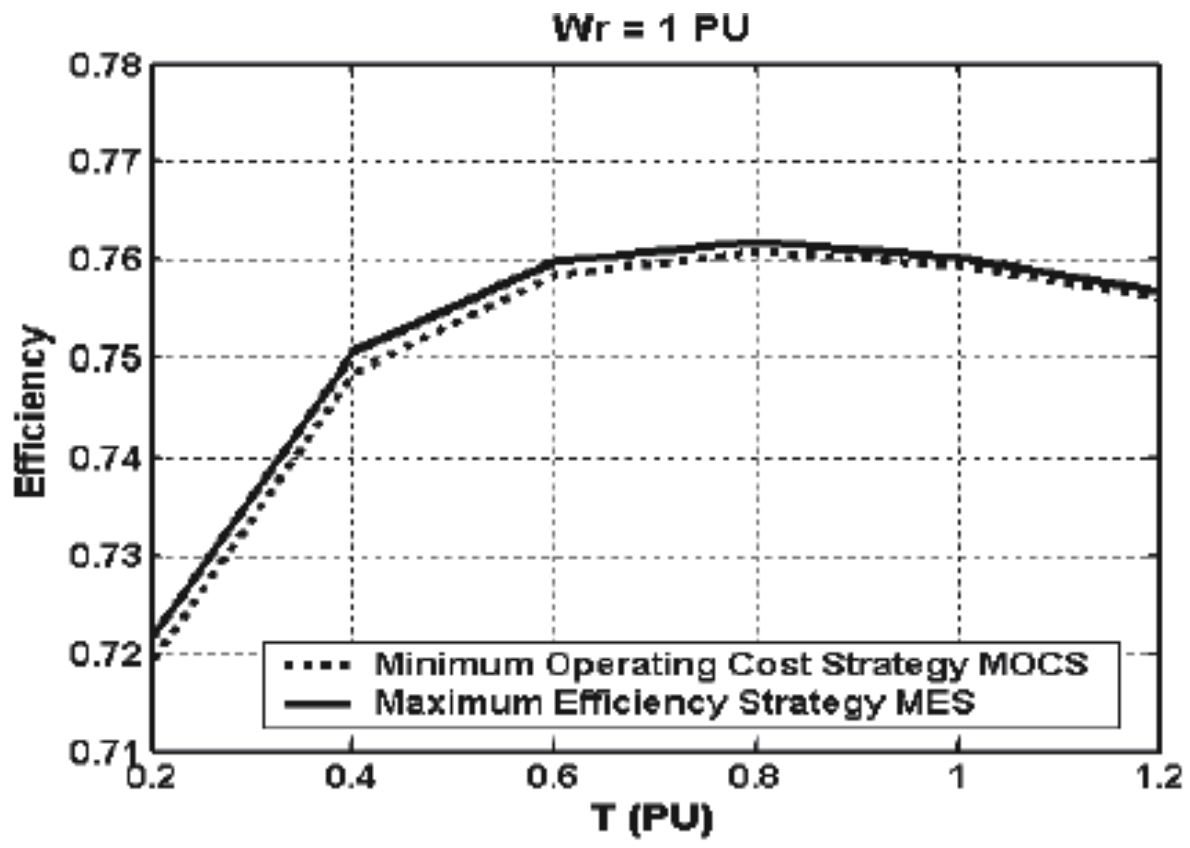

Fig. 14. The efficiency of the induction motor using the maximum efficiency strategy compared with the efficiency using minimum operating cost strategy at $\mathrm{Wr}=1 \mathrm{PU}$ 
Table 5 shows the efficiency comparison using few examples of operating points.

Figure 15 compares the power factor of the induction motor drive system under the maximum efficiency strategy with the minimum operating cost strategy at $\mathrm{Wr}=1 \mathrm{PU}$.

Finally, the proposed PSO-controller adaptively adjusts the slip frequency such that the drive system is operated at the minimum loss and minimum operating cost. It was found that the optimal system slip changes with variations in speed and load torque. When comparing the proposed strategy with the conventional methods field oriented control strategy and constant voltage to frequency ratio). It was found that a significant efficiency improvement especially at light loads for all speeds. On the other hand, small efficiency improvement is achieved at near rated loads (see Fig.13, and Fig.15).

\begin{tabular}{|c|c|c|c|c|}
\hline \multicolumn{5}{|c|}{ Efficiency comparison for $\omega_{\mathrm{r}}=1$ PU } \\
\hline $\mathrm{T}(\mathrm{PU})$ & $\begin{array}{c}\text { Constant } \\
\text { voltage to } \\
\text { frequency } \\
\text { ratio }\end{array}$ & $\begin{array}{c}\text { Field } \\
\text { oriented } \\
\text { control }\end{array}$ & $\begin{array}{c}\text { Maximum } \\
\text { efficiency } \\
\text { strategy }\end{array}$ & $\begin{array}{c}\text { Minimum } \\
\text { Operating } \\
\text { Cost } \\
\text { Strategy }\end{array}$ \\
\hline 0.2 & 0.5003 & 0.5330 & 0.7217 & 0.7193 \\
\hline 0.4 & 0.6482 & 0.6730 & 0.7506 & 0.7485 \\
\hline 0.6 & 0.7100 & 0.7271 & 0.7598 & 0.7584 \\
\hline 0.8 & 0.7384 & 0.7494 & 0.7618 & 0.7608 \\
\hline 1 & 0.7508 & 0.7569 & 0.7603 & 0.7595 \\
\hline 1.2 & 0.7544 & 0.7566 & 0.7568 & 0.7562 \\
\hline
\end{tabular}

Table 5. Some examples of efficiency comparison under different Load torque levels and $\mathrm{W}_{\mathrm{r}}$ $=1$ PU [A. Hamid et al. 2006].

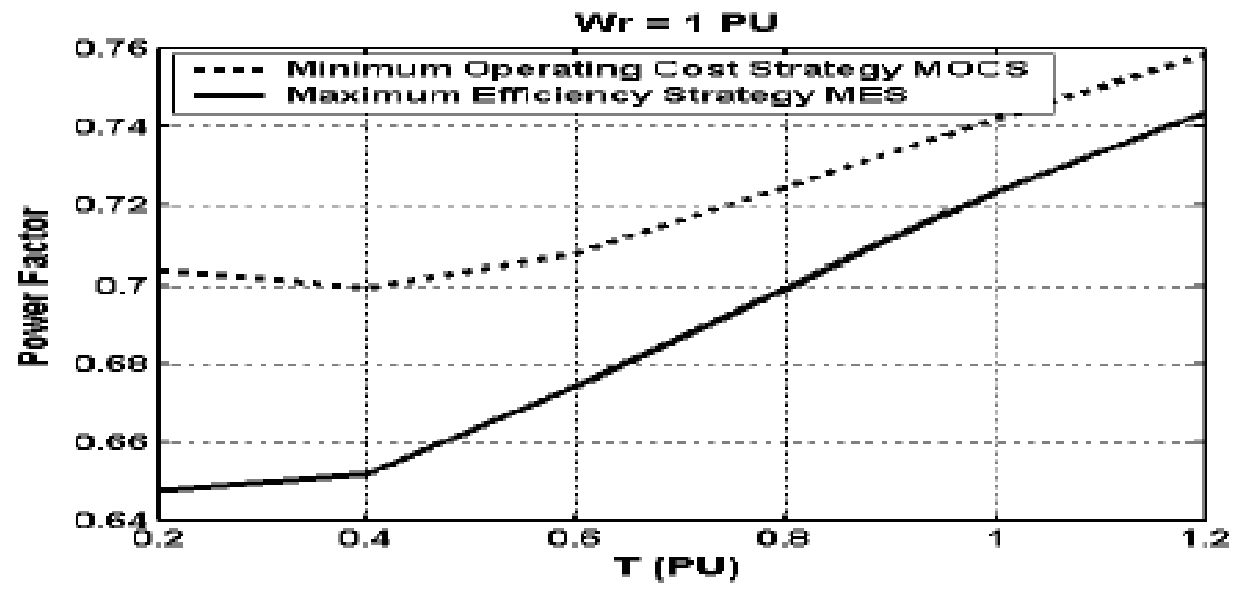

Fig. 15. The power factor of the induction motor using the maximum efficiency strategy compared with the efficiency using minimum operating cost strategy at $\mathrm{Wr}=1 \mathrm{PU}$ [A. Hamid et al. 2006]

\section{Optimal electric drive system for fuel cell hybrid electric vehicles}

Although there are various FC technologies available for use in vehicular systems, the proton exchange membrane FC (PEMFC) has been found to be a prime candidate, since 
PEMFC has higher power density and lower operating temperatures when compared to the other types of FC systems. A stand-alone FC system integrated into an automotive powertrain is not always sufficient to satisfy the load demands of a vehicle. Although FC systems exhibit good power capability during steady-state operation, the response of fuel cells during transient and instantaneous peak power demands is relatively poor. Thus, the FC system can be hybridized with supercapacitors (SC) or batteries to meet the total power demand of a hybrid electric vehicle (HEV) [Van Mierlo et. al, 2006; Paladini et. al, 2007].

In this section, a new control strategy based PSO algorithm is proposed for the Fuel Cell/Supercapacitor hybrid electric vehicles to optimize the electric drive system [Hegazy \& Van Mierlo, 2010]. Many factors influence on the performance of the electric drive system. These factors are mass, volume, size, efficiency, fuel consumption and control strategy. Therefore, the PSO is proposed to minimize the cost, the size and the mass of the powertrain sources (Fuel cell, and supercapacitor) as well as minimum fuel consumption and improves the efficiency of the system. PSO algorithm searches for global optimization for nonlinear problems with multi-objective. For a given driving cycle, the size and the cost of fuel cell and supercapacitor are minimized by identifying the best number of units of each, respectively. Three methods have been designed to achieve the optimal sizing. These are conventional method, trial and error, as was mentioned in [Wu \& Gao, 2006], GA, and PSO. In addition, the hydrogen consumption is minimized by the evaluation of the optimal power distribution between fuel cell (main source) and supercapacitor (auxiliary source). Three control strategies are implemented to minimize the hydrogen consumption and maintain the state of charge (SOC) of the supercapacitor ( SOCinitial =SOCfinal), which are control strategy based on Efficiency Map (CSEM), Control strategy based on PSO (CSPSO), and control strategy based on GA (CSGA).

\subsection{System description}

The power system configuration is illustrated in Fig.16. A hybrid fuel cell/supercapacitor vehicle utilizes a PEM fuel cell as the main power source and a supercapacitor as the auxiliary power source. A multiple-input power electronic converter (MIPEC) is proposed to interface the traction drive requirements. In the MIPEC, the FC is connected to DC Bus

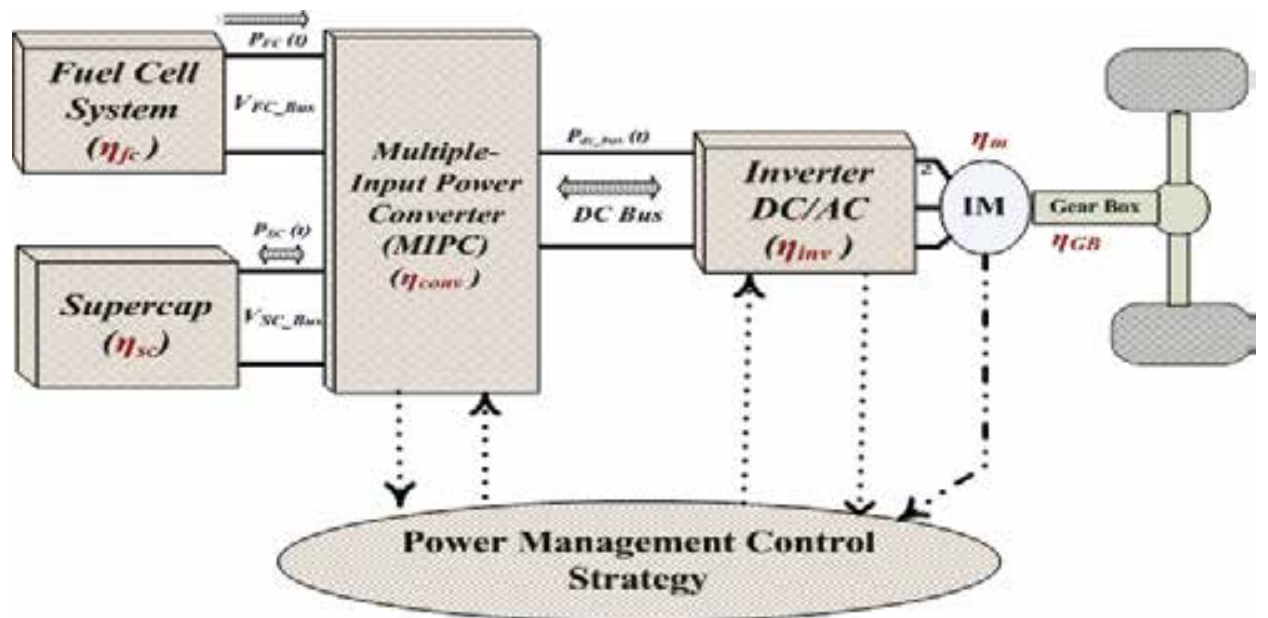

Fig. 16. The drive system of the Fuel Cell/Supercapacitor Hybrid Electric Vehicle 
via a Boost $D C / D C$ converter $\left(\eta_{B}=\eta_{\text {conv }}\right)$ and the supercapacitor is connected to DC Bus via a Buck/Boost converter $\left(\eta_{\mathrm{B} / \mathrm{B}}=\eta_{\text {conv }}\right)$. The desired value of the DC-Bus voltage is chosen to be $400 \mathrm{~V}$ with variations of $\pm 10 \%$ are permissible. The power supplied by the powertrain has to be obtaining from the power demand predicted by the dynamics of the vehicle. The efficiency of each component in the hybrid powertrain is taken into account. A detailed model of the powertrain is built in MATLAB /SIMULINK.

\subsubsection{Modeling of the vehicle power demand}

The load force of the vehicle consists of gravitational force, rolling resistance, aerodynamic drag force, and acceleration force. Hereby, the load power required for vehicle acceleration can be written as [Hegazy \& Van Mierlo, 2010; Hegazy et. al 2010]

$$
\begin{gathered}
P_{\text {load }}=\frac{\left(F_{g}+F_{\text {roll }}+F_{A D}+F_{a c c}\right) * V}{\eta_{G B}} \\
F_{g}=M \cdot g \cdot \sin (\alpha) \\
F_{\text {roll }}=M \cdot g \cdot f_{r} \cdot \cos (\alpha) \\
F_{A D}=0.5 \rho_{a} \cdot C_{D} \cdot A_{F} \cdot V^{2} \\
F_{a c c}=M \cdot \frac{d V}{d t} \\
V=\omega_{w} \cdot{ }_{w}
\end{gathered}
$$

The total electric power required from sources can be expressed as:

$$
P_{\text {req }}=\frac{P_{\text {load }}}{\eta_{m} \cdot \eta_{\text {Inv }} \cdot \eta_{\text {Conv }}}
$$

The parameters of the vehicle are given in Table 6. The analysis of FCHEV is performed with two standard driving cycles:

1. The Federal Test Procedure (FTP75) Urban;

2. The New European Driving Cycle (NEDC)

Suppose that the efficiencies of the motor $\left(\eta_{m}\right)$, inverter $\left(\eta_{\text {Inv }}\right)$, and MIPEC $\left(\eta_{\text {Conv }}=\eta_{B}=\eta \mathrm{B} / \mathrm{B}\right)$ are $0.90,0.94$ and 0.95 , respectively.

\begin{tabular}{|c|c|c|c|c|c|}
\hline $\mathrm{M}$ & Vehicle mass (kg) & 1450 & $\mathrm{~A}_{\mathrm{f}}$ & Front Area $(\mathrm{m} 2)$ & 2.13 \\
\hline $\mathrm{f}_{\mathrm{r}}$ & Rolling Resistance Coefficient & 0.013 & $\mathrm{r}_{\omega}$ & $\begin{array}{c}\text { Radius of the } \\
\text { wheel }(\mathrm{m})\end{array}$ & 0.28 \\
\hline $\mathrm{C}_{\mathrm{D}}$ & $\begin{array}{c}\text { Aerodynamic Drag } \\
\text { Coefficient (CD) }\end{array}$ & 0.29 & $\rho_{\mathrm{a}}$ & $\begin{array}{c}\text { Air density } \\
(\mathrm{kg} / \mathrm{m} 3)\end{array}$ & 1.202 \\
\hline
\end{tabular}

Table 6. Vehicle Parameters [Wu \& Gao, 2006] 


\subsection{Optimal powertrain design}

The first goal of optimization algorithm, PSO, is to minimize the cost, the mass, and the volume of the fuel cell (FC) and supercapacitor (SC). It is assumed that, the cost, the mass and the volume of the fuel cell and supercapacitor are a function of the number of the parallel units $N_{f c p}$ and $N_{s c p}$, respectively. The multi-objective criterion should be aggregated in a single objective function if the design objective is to embody a unique solution. The objective function can be formulated as follows:

$$
\begin{gathered}
F(x)=w_{1} \cos t+w_{2} \text { mass }+w_{3} \text { volume } \\
\cos t=C 1 . N f c s . N f c p+C 2 . N s c s . N s c p
\end{gathered}
$$

The coefficients of the terms in $\mathrm{F}(\mathrm{x})$ were chosen to reflect the importance of minimizing the cost, the mass and the volume. Suppose that $w 1, w 2$, and $w 3$ are $0.35,0.35$, and 0.3, respectively. Figure 17 presents the flowchart of the execution of PSO, which evaluates the optimal number of the FC units and the supercapacitor units by using MATLAB /SIMULINK. The layout of the fuel-cell stack and layout of the supercapacitor system are shown in Fig.18 (a) and (b), respectively. The constraints of the optimization problems are mentioned in [Hegazy \& Van Mierlo, 2010].

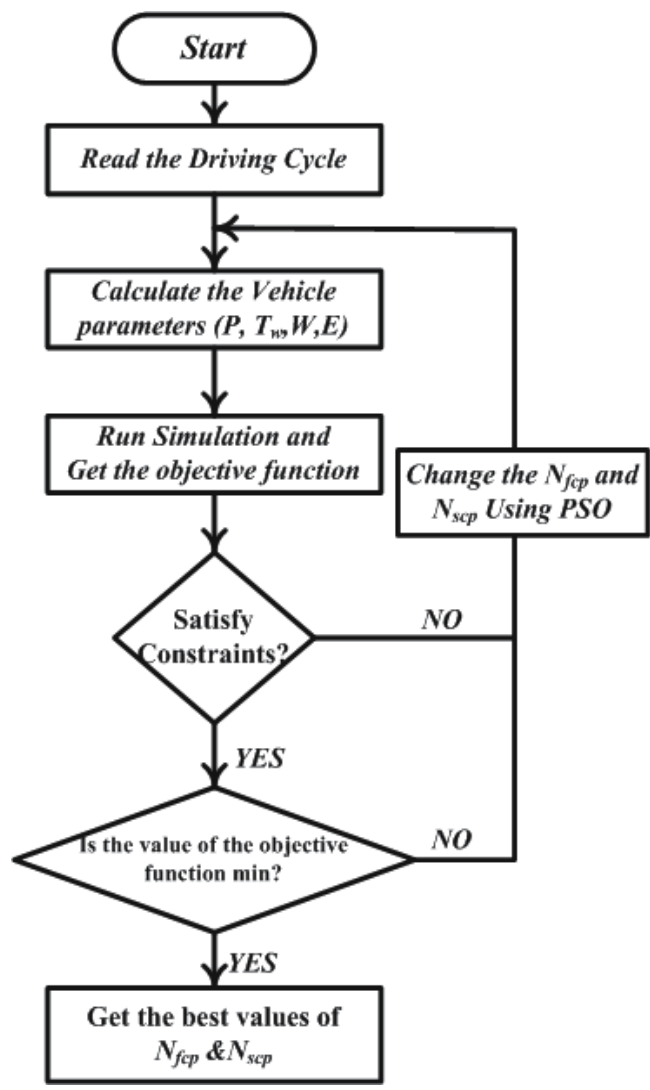

Fig. 17. The flowchart of the execution of PSO [Hegazy et. al 2010] 


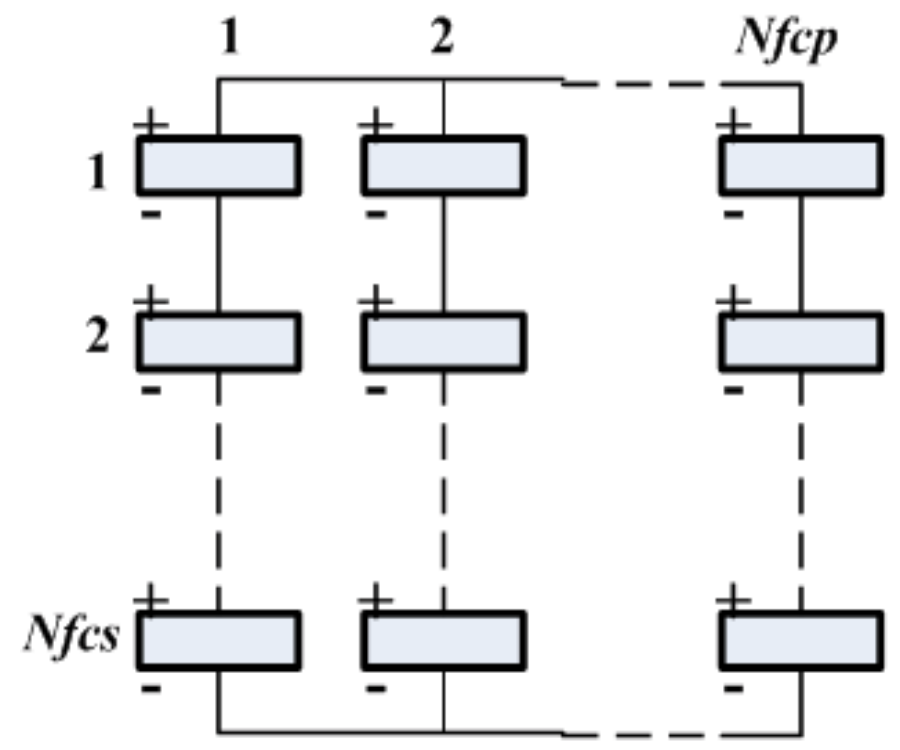

(a)

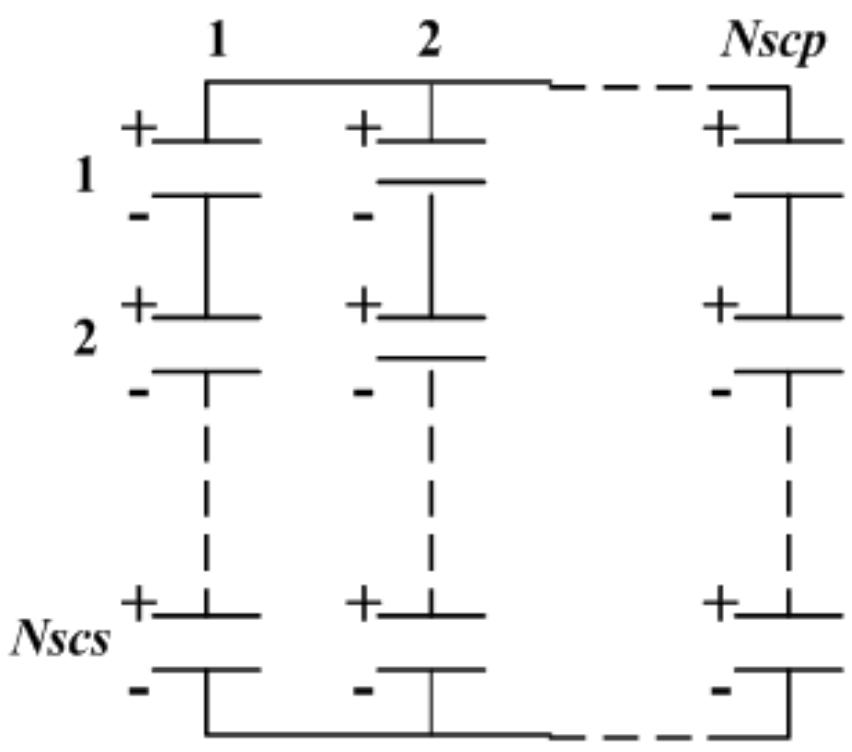

(b)

Fig. 18. (a) Layout of the FC; (b) Layout of the SC 
Based on minimizing the objective function $\mathrm{F}(\mathrm{x})$ in (68), the results of the optimal design and components sizing of the FC/SC powertrain are shown in Fig.19. The analyses and parameters of the FC and the SC are mentioned in [Hegazy \& Van Mierlo, 2010].

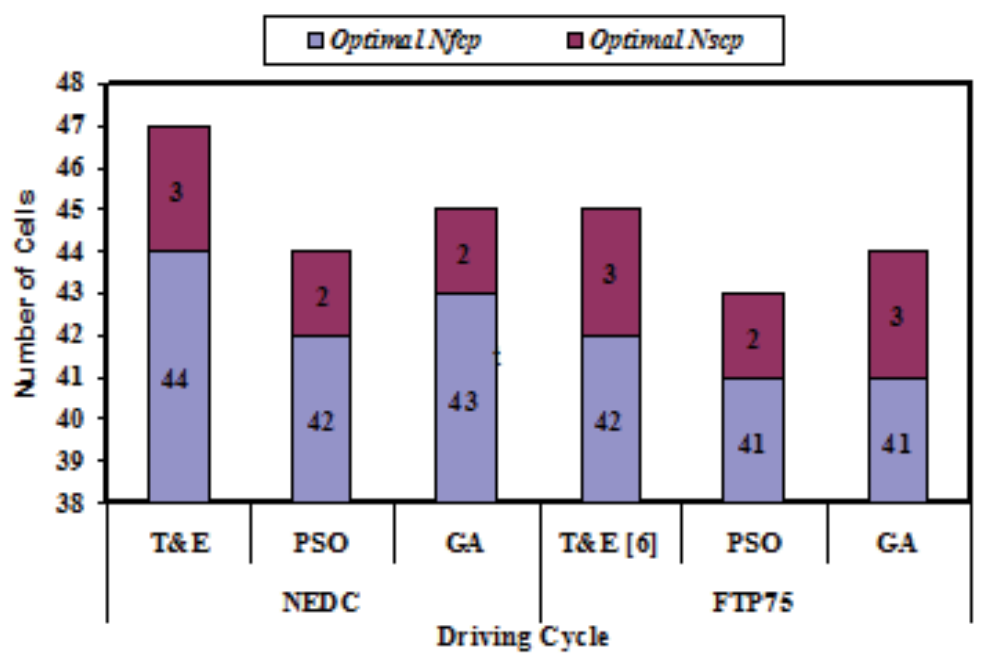

(a) The optimal numbers of cells of FC and SC

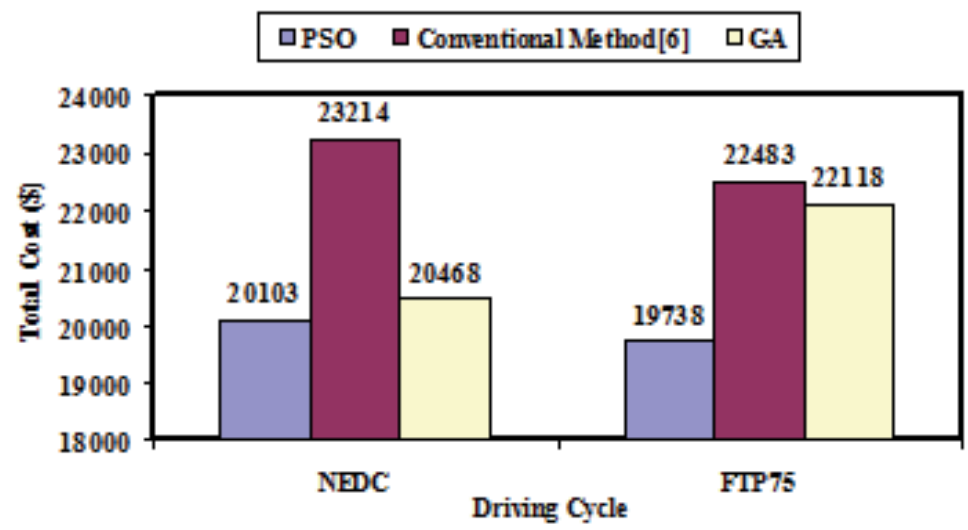

(b) The cost of the FC/SC components 


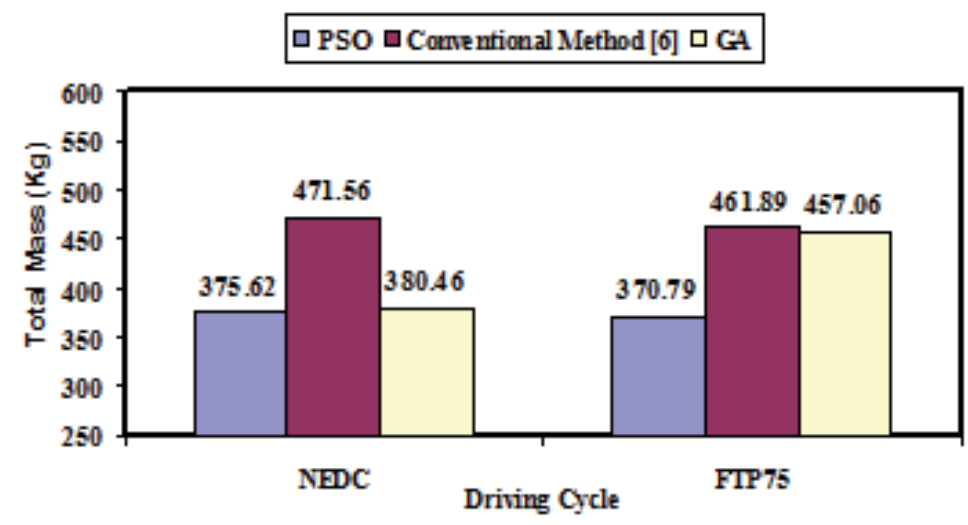

(c) The mass of the FC/SC components

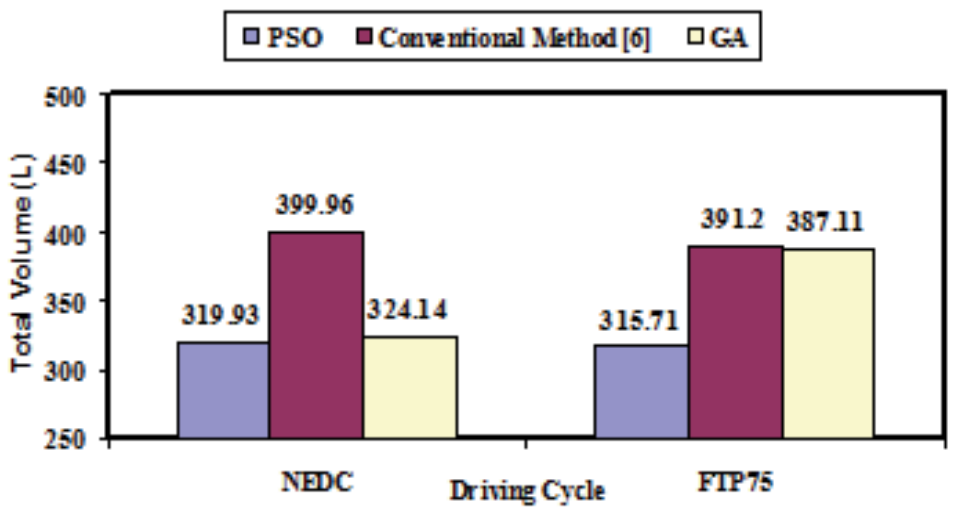

(d) The mass of the FC/SC components

Fig. 19. The Comparative of the optimal design between different methods for FC/SC HEV

\subsection{Optimal Power Control (OPC)}

The second goal of the PSO is to minimize the vehicle fuel, hydrogen, consumption while maintaining the supercapacitor state of charge. As a hybrid powertrain is under consideration, a power management strategy is required to define what both the FC and SC powers are. The global optimization algorithms, such as GA and dynamic programming (DP), achieve an optimal power control for FC/SC hybrid electric vehicle, which leads to the lowest hydrogen consumption and maintains the supercapacitor SOC [Sinoquet et. al 2009; Sundstrom \& Stefanopoulou 2006].

In this study, the optimal power control can be achieved by using PSO and GA for a given driving cycle. Suppose that the degree of hybridization of the fuel cell is $K_{f c}$ at time $t$ and $K_{s o c}$, Proportional controller gain, which used to adapt the SOC during charging from the FC. A balance equation can naturally be established, since the sum of power from both sources has to be equal to the required power at all times: 


$$
\begin{gathered}
P_{r e q}(t)=P f c(t)+P s c(t) \\
K f c(t)=\frac{P f c(t)}{P_{r e q}(t)}
\end{gathered}
$$

The net energy consumed from the FC at time $\mathrm{t}$ can be computed as follows:

$$
E f c(t)=\int_{0}^{t} \frac{P f c(t)}{\eta(P f c(t))} d t
$$

The cost function can be expressed as follows:

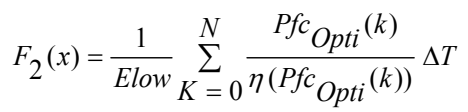

The Optimal fuel cell power output, $\mathrm{P}_{\mathrm{fcOpti}}$ is calculated based on the SOC of the supercapacitor and power demand, $P_{\text {req, }}$ as follows:

$$
P f c_{O p t i}(k)=K f_{c}(k) P_{r e q}(k)+K \operatorname{soc}(k)\left(P f c_{\max }-P f c_{\min }\right)\left[\frac{S O C_{r e f}-S O C(k)}{\left(S O C_{\max }-S O C_{\min }\right) / 2}\right]
$$

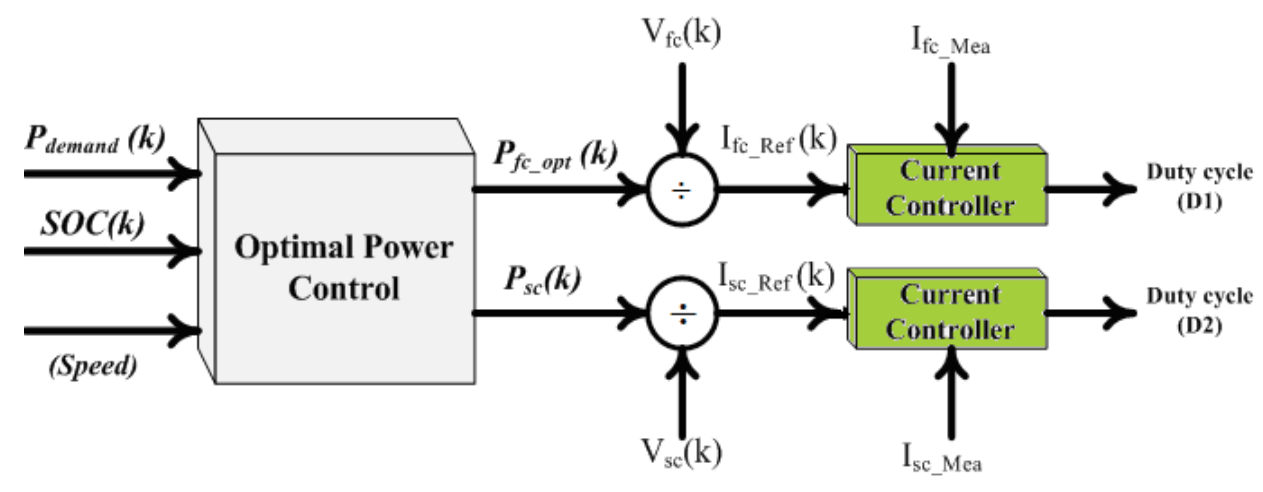

Fig. 20. The block diagram of the Optimal power Control

Where: $N=T / \Delta T$ is number of samples during the driving cycle, and $\Delta T=1$ s is the sampling time.

The block diagram of the optimal power control based on optimization algorithm is shown in Fig.20.

Based on minimizing the objective function $F_{2}(x)$ in (73), the results of the optimal power sharing based PSO and the comparative study for the FC/SC powertrain are summarized in Fig.21 [Hegazy et. al 2010]. 


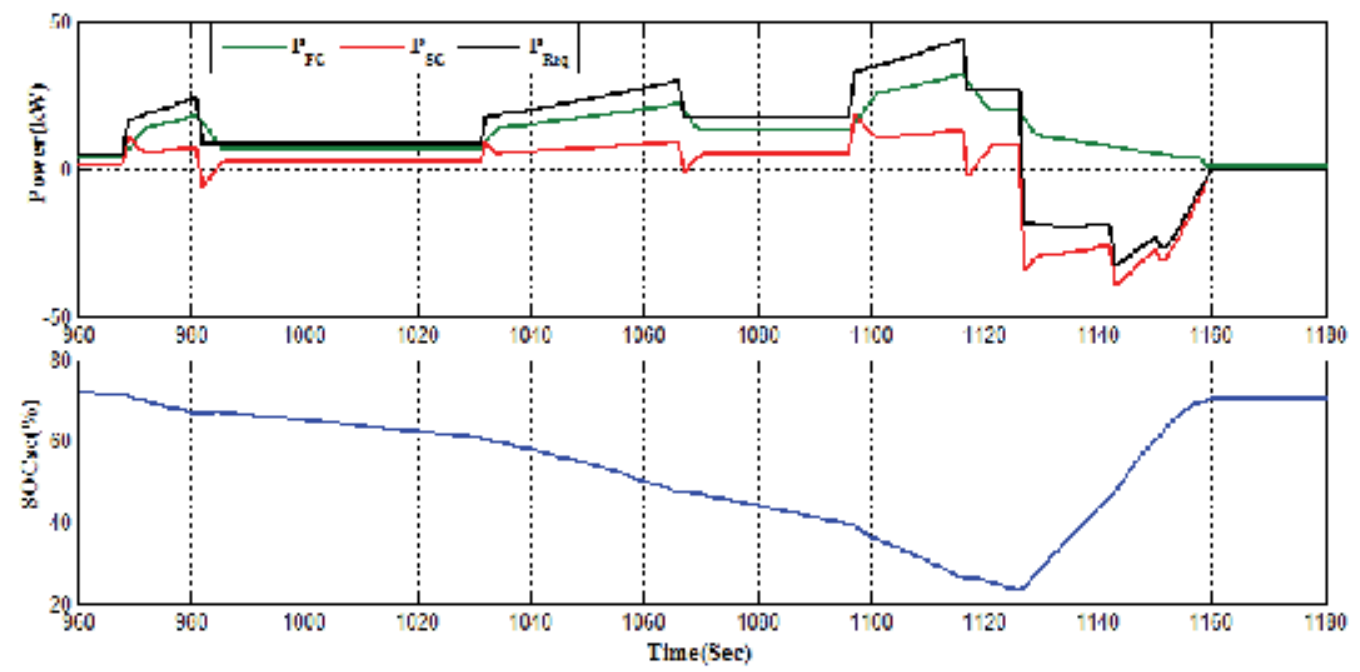

(a) The power sharing between FC and SC on NEDC driving cycle

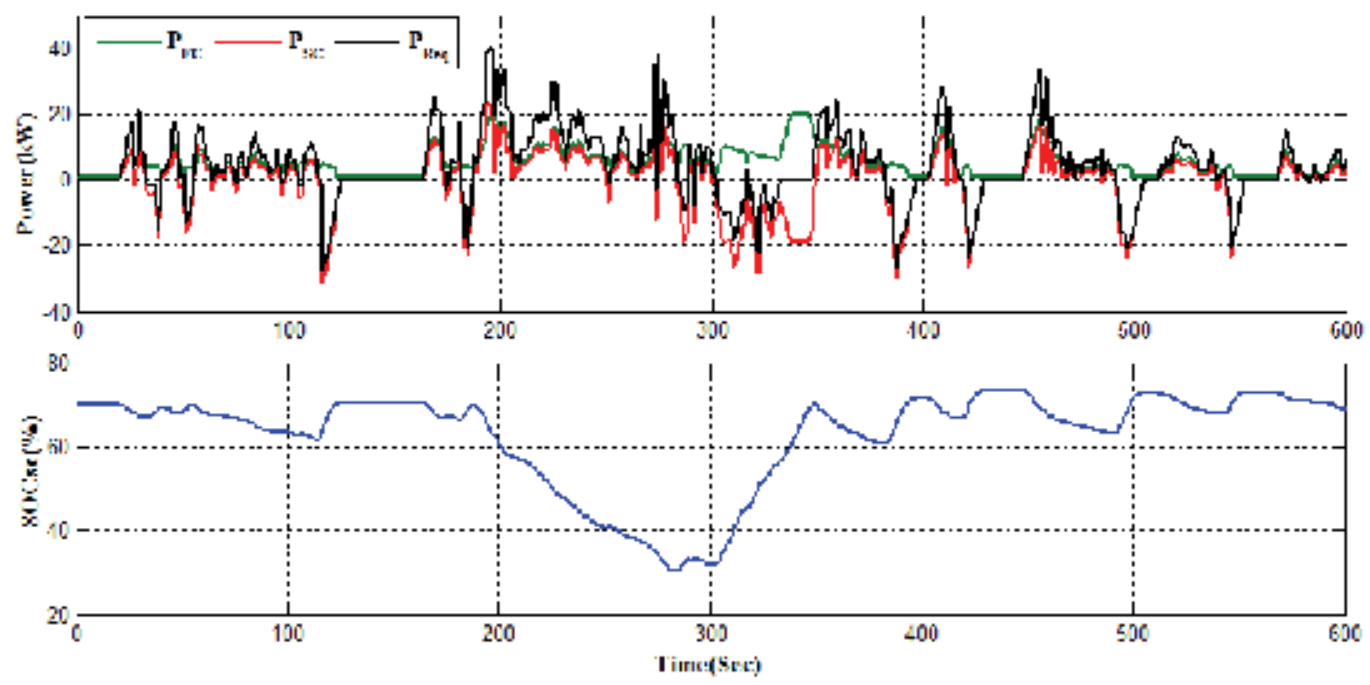

(b) The power sharing between FC and SC on FTP75 driving cycle 


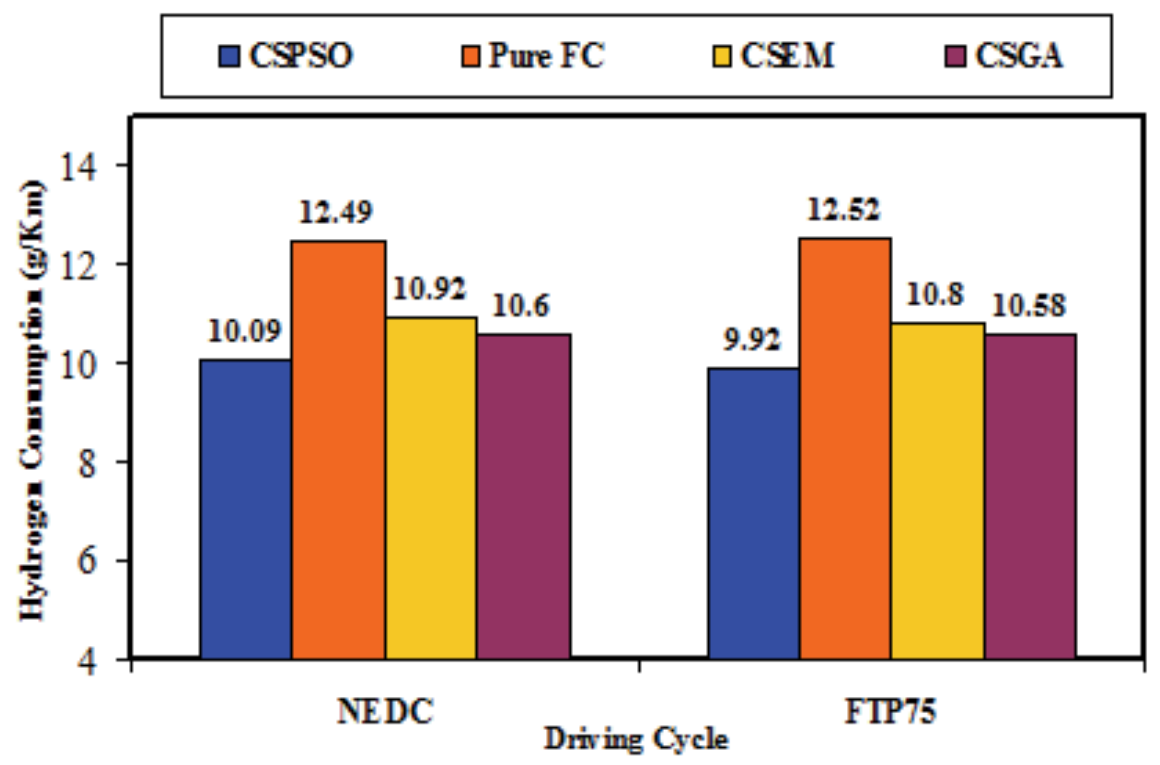

(c) The Comparative of the hydrogen consumption between control strategies

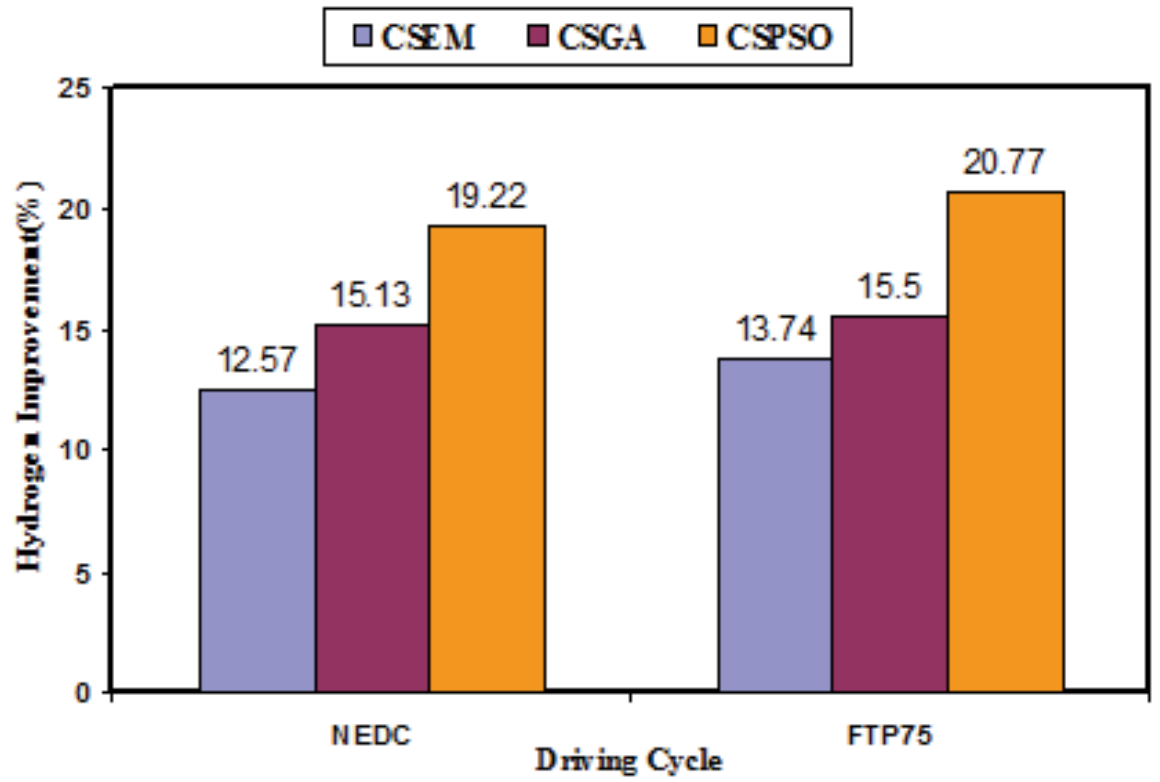

(d) The Hydrogen improvements with respect to pure fuel cell without SC

Fig. 21. The results of the optimal power Control for FC/SC 


\section{Conclusion}

This chapter deals with the applicability of swarm intelligence (SI) in the form of particles swarm optimization (PSO) used to achieve the best performance for the electric machines and electric drives. In addition, by analyzing and comparing the results, it is shown that control strategy based on PSO is more efficient than others control strategies to achieve the optimal performance for fuel cell/supercapacitor hybrid electric vehicles (FCHEV).

It is very important to note that, these applications were achieved without any additional hardware cost, because the PSO is a software scheme. Consequently, PSO has positive promises for a wide range of variable speed drive and hybrid electric vehicles applications.

\section{Index I}

List of principal symbols

$\omega_{\mathrm{e}} \quad$ : synchronous speed

$\omega_{\mathrm{r}} \quad$ : rotor speed

p : differential operator

$r_{m}, r_{a} \quad:$ main, auxiliary stator windings resistance

$r_{r} \quad$ : rotor winding resistance

$R_{\text {feq, } d} \quad$ : equivalent iron-loss resistance(d and q axis)

$L_{l m}, L_{l a} \quad$ : main, auxiliary stator leakage inductance

$L_{m d}, L_{m q} \quad$ : magnetizing inductance (d\& q axis)

$L_{l r} \quad$ : rotor leakage inductance

$K \quad$ : turns ratio auxiliary/main windings

$T_{e} \quad$ : electromagnetic torque

J : inertia of motor

$\lambda_{d s, q s} \quad:$ stator flux(d and q axis)

$\lambda_{d r, q r} \quad:$ rotor flux(d and q axis)

$V_{d s, q s} \quad:$ stator voltage ( $\mathrm{d}$ and $\mathrm{q}$ axis)

$i_{d s, q s} \quad:$ stator current (d and q axis)

M : mutual inductance

\section{References}

Amin. A. M. A., Korfally. M. I., Sayed. A. A. and Hegazy. O.T. M., (2009), Efficiency Optimization of Two Asymmetrical Windings Induction Motor Based on Swarm Intelligence, IEEE Transactions on Energy Conversion, Vol. 24, No. 1, March 2009

Amin. A. M. A., Korfally. M. I., Sayed. A. A. and Hegazy. O.T. M., (2006), Losses Minimization of Two Asymmetrical Windings Induction Motor Based on Swarm Intelligence, Proceedings of IEEE- IECON 06 , pp 1150 - 1155, Paris , France , Nov. 2006.

Amin. A. M. A., Korfally. M. I., Sayed. A. A. and Hegazy. O.T. M., (2007), Swarm Intelligence-Based Controller of Two-Asymmetrical Windings Induction Motor, accepted for IEEE. EMDC07, pp 953 -958, Turkey, May 2007.

Eberhart. R, Kennedy. J, (1995), A New Optimizer Using Particles Swarm Theory, Proc. 
Sixth International Symposium on Micro Machine and Human Science (Nagoya, Japan), IEEE Service Center, Piscataway, NJ, pp. 39-43,

A. Hamid Radwan H., Amin Amr. M. A., Ahmed Refaat S., and El-Gammal Adel A. A. (2006), New Technique For Maximum Efficiency And Minimum Operating Cost Of Induction Motors Based On Particle Swarm Optimization (PSO)", Proceedings of IEEE- IECON 06 , pp 1029 - 1034, Paris , France , Nov. 2006.

Hegazy Omar, (2006), Losses Minimization of Two Asymmetrical Windings Induction Motor Based on Swarm Intelligence, M.Sc., Helwan University, 2006.

Hegazy Omar, and Van Mierlo Joeri, (2010), Particle Swarm Optimization for Optimal Powertrain Component Sizing and Design of Fuel cell Hybrid Electric Vehicle, 12th International Conference on Optimization of Electrical and Electronic Equipment, IEEE OPTIM 2010

Hegazy Omar, Van Mierlo Joeri, Verbrugge Bavo and Ellabban Omar, (2010), Optimal Power Sharing and Design Optimization for Fuel Cell/Battery Hybrid Electric Vehicles Based on Swarm Intelligence, The 25th World Battery, Hybrid and Fuel Cell Electric Vehicle Symposium \& Exhibition (c) EVS-25 Shenzhen, China, Nov. 5-9, 2010.

Kennedy. J and Eberhart .R, (2001), Swarm Intelligence, Morgan Kaufmann Publishers, Inc., San Francisco, CA

Kioskeridis, I; Margaris, N., (1996), Losses minimization in scalar-controlled induction motor drives with search controllers" Power Electronics, IEEE Transactions, Volume: 11, Issue: 2, March 1996 Pages: 213 - 220

Popescu. M, Navrapescu. V, (2000) ,A method of Iron Loss and Magnetizing Flux Saturation Modeling in Stationary Frame Reference of Single and Two -Phase Induction Machines", IEE 2000, Conf. power Elec. \& Variable Speed Drives, 140146

Sundstrom Olle and Stefanopoulou Anna, (2006), Optimal Power Split in Fuel Cell Hybrid Electric Vehicle with different Battery Sizes, Drive Cycles, and Objectives, Proceedings of the 2006 IEEE International Conference on Control Applications Munich, Germany, October 4-6, 2006.

Van Mierlo Joeri, Cheng Yonghua, Timmermans Jean-Marc and Van den Bossche Peter, (2006), Comparison of Fuel Cell Hybrid Propulsion Topologies with SuperCapacitor, IEEE, EPE-PEMC 2006, Portorož, Slovenia

Wu Ying, Gao Hongwei, (2006) ,Optimization of Fuel Cell and Supercapacitor for Fuel-Cell Electric Vehicles, IEEE Transactions On Vehicular Technology, Vol. 55, No. 6, November 2006. 


\title{
Operation of Active Front-End Rectifier in Electric Drive under Unbalanced Voltage Supply
}

\author{
Miroslav Chomat \\ Institute of Thermomechanics AS CR, v.v.i. \\ Czech Republic
}

\section{Introduction}

Non-standard conditions in the power network such as voltage unbalance can negatively affect operation of electric drives. The unbalance can be caused by a failure in the network or by an unbalanced load in the electric vicinity of the affected drive. Unsymmetrical voltages at the input of a voltage source inverter cause pulsations in the DC link voltage when not properly taken care of. This may result in significantly reduced power capabilities and, therefore, limited controllability of the drive. This text deals with the effects of unbalanced voltage supply on the DC-link voltage pulsations, methods to address this problem and the additionally imposed constraints in operating regions of the rectifier.

\section{Control method}

A simplified scheme of the drive under investigation is shown in Fig. 1. The front-end controlled rectifier is connected to the mains through input filter inductors. The output current of the rectifier supplies the DC current to the output inverter and maintains the voltage across the DC-link capacitors constant at the same time. The value of this current can be controlled by suitable switching of solid-state elements in the front-end stage.

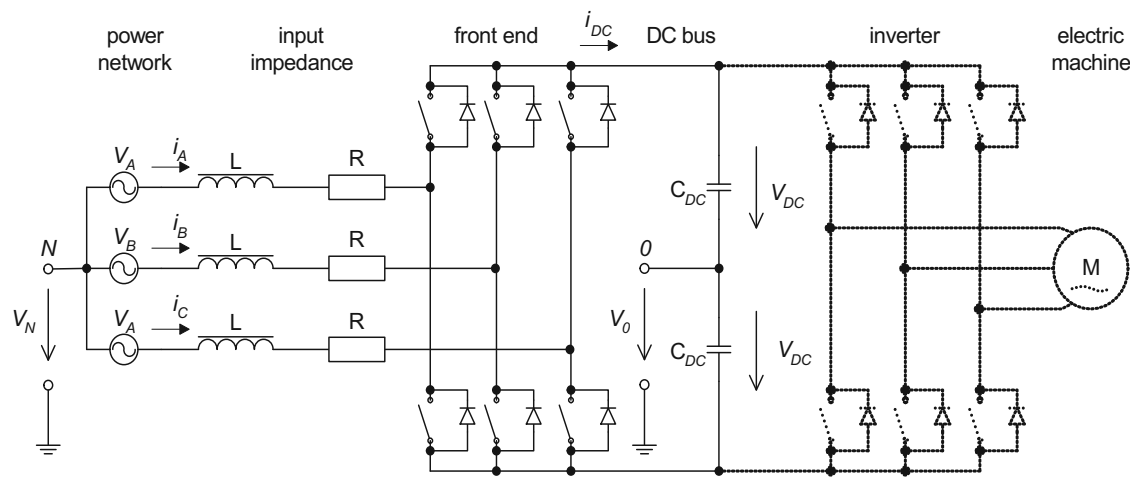

Fig. 1. Scheme of system under investigation.

Suitable control of the front-end AC/DC converter can be employed in order to draw constant input power from the power network even at unbalanced voltage supply 
(Stankovic \& Lipo, 2001; Lee et al., 2006; Cross et al., 1999; Song \& Nam, 1999). The switching functions for the front-end AC/DC converter are generated so that a constant voltage across the DC bus is maintained. Series combinations of inductance and resistance are considered at the input terminals of the inverter.

The system can be electrically described by the following set of ordinary differential equations (Chomat \& Schreier, 2005):

$$
\begin{aligned}
& v_{A}-L \frac{d i_{A}}{d t}-R i_{A}-v_{S A}+v_{N}-v_{0}=0, \\
& v_{B}-L \frac{d i_{B}}{d t}-R i_{B}-v_{S B}+v_{N}-v_{0}=0, \\
& v_{C}-L \frac{d i_{C}}{d t}-R i_{C}-v_{S C}+v_{N}-v_{0}=0,
\end{aligned}
$$

where

$$
\begin{aligned}
& v_{S A}=s_{A} \cdot V_{D C}, \\
& v_{S B}=s_{B} \cdot V_{D C}, \\
& v_{S C}=s_{C} \cdot V_{D C}
\end{aligned}
$$

are the voltages at the input of the inverter. The functions $s_{A}, s_{B}$, and $s_{C}$ are the corresponding unit switching functions of the particular phases of the front-end stage, which represent the fundamental harmonic components of the pulse width modulated output. Sinusoidal switching functions with the nominal frequency are considered throughout this paper, whereas the higher harmonics that would arise in a real power converter are neglected in the calculation for simplification. $V_{D C}$ represents one half of the overall DC-link voltage here. The voltage $v_{N}$ is the electric potential of the neutral of the mains and $v_{0}$ is the electric potential of the centre point of the capacitor bank in the DC bus. The DC-link current can be calculated from the phase currents and the switching functions according to

$$
i_{D C}=\frac{1}{2}\left(s_{A} i_{A}+s_{B} i_{B}+s_{C} i_{C}\right) .
$$

The coefficient $1 / 2$ takes into account the fact that currents in both positive and negative directions that flow through different current paths in the DC bus are produced by the rectifier.

An unbalanced system of phase quantities can advantageously be represented by phasors of positive and negative rotating sequences. It is not necessary to take zero-sequence quantities into account here as no neutral wire is considered in the system and, therefore, no zerosequence current can develop. The resulting rotating vector of such a quantity may then be written as

$$
\mathbf{x}=\mathbf{X}_{\mathbf{P}} e^{j \omega t}+\mathbf{X}_{\mathbf{N}} e^{-j \omega t}
$$


The subscripts $P$ and $N$ denote the positive and negative rotating sequences, respectively. Based on these assumptions, (1) - (3) and (4) - (6) may be rewritten in phasor form as

$$
\begin{gathered}
\mathbf{V}_{\mathbf{P}}-(R+j \omega L) \mathbf{I}_{\mathbf{P}}-\mathbf{S}_{\mathbf{P}} V_{D C}=0, \\
\mathbf{V}_{\mathbf{N}}-(R-j \omega L) \mathbf{I}_{\mathbf{N}}-\mathbf{S}_{\mathbf{N}} V_{D C}=0 .
\end{gathered}
$$

The solution of (9) and (10) for positive and negative sequence currents is

$$
\begin{aligned}
& \mathbf{I}_{\mathbf{P}}=\frac{\mathbf{V}_{\mathbf{P}}-\mathbf{S}_{\mathbf{P}} V_{D C}}{R+j \omega L}, \\
& \mathbf{I}_{\mathbf{N}}=\frac{\mathbf{V}_{\mathbf{N}}-\mathbf{S}_{\mathbf{N}} V_{D C}}{R-j \omega L}
\end{aligned}
$$

and the corresponding rotating vector of the input currents is therefore

$$
\mathbf{i}=\mathbf{I}_{\mathbf{P}} e^{j \omega t}+\mathbf{I}_{\mathbf{N}} e^{-j \omega t} .
$$

Similarly, we can formally introduce a rotating vector of the switching functions

$$
\mathbf{s}=\mathbf{S}_{\mathbf{P}} e^{j \omega t}+\mathbf{S}_{\mathbf{N}} e^{-j \omega t}
$$

Then the resulting instantaneous value of the current supplied into the DC link by the frontend converter from (7) can be written in the vector form as

$$
i_{D C}=\frac{1}{2} \frac{3}{2} \operatorname{Re}\{\mathbf{i} \cdot \overline{\mathbf{s}}\}
$$

where the bar over the symbol denotes the complex conjugate value. The term $3 / 2$ appears in (15) due to the transformation from rotating vector form to instantaneous quantities.

The resulting relation obtained after substituting (13) and (14) into (15) can be written as the sum of two separate current components and given as

$$
i_{D C}=i_{D C(\text { avg })}+i_{D C(2 \omega t)}
$$

where

$$
\begin{gathered}
i_{D C(a v g)}=\frac{3}{4} \operatorname{Re}\left\{\frac{\mathbf{V}_{\mathbf{P}}-\mathbf{S}_{\mathbf{P}} V_{D C}}{R+j \omega L} \overline{\mathbf{S}}_{\mathbf{P}}+\frac{\mathbf{V}_{\mathbf{N}}-\mathbf{S}_{\mathbf{N}} V_{D C}}{R-j \omega L} \overline{\mathbf{S}}_{\mathbf{N}}\right\}, \\
i_{D C(2 \omega t)}=\frac{3}{4} \operatorname{Re}\left\{\frac{\mathbf{V}_{\mathbf{P}}-\mathbf{S}_{\mathbf{P}} V_{D C}}{R+j \omega L} \overline{\mathbf{S}}_{N} e^{j 2 \omega t}+\frac{\mathbf{V}_{\mathbf{N}}-\mathbf{S}_{\mathbf{N}} V_{D C}}{R-j \omega L} \overline{\mathbf{S}}_{\mathbf{P}} e^{-j 2 \omega t}\right\} .
\end{gathered}
$$

The first component, (17), represents a DC component and the second, (18), represents a pulsating component with the frequency twice as high as that of the mains. The pulsating component is only produced when the negative sequence of either the input voltages or the switching functions is present. 
From (18), a condition for the elimination of the pulsating component in the DC link can be derived

$$
\operatorname{Re}\left\{\frac{\mathbf{V}_{\mathbf{P}}-\mathbf{S}_{\mathbf{P}} V_{D C}}{R+j \omega L} \overline{\mathbf{S}}_{N} e^{j 2 \omega t}\right\}=-\operatorname{Re}\left\{\frac{\mathbf{V}_{\mathbf{N}}-\mathbf{S}_{\mathbf{N}} V_{D C}}{R-j \omega L} \overline{\mathbf{S}}_{\mathbf{P}} e^{-j 2 \omega t}\right\} .
$$

As the real part of a complex number equals the real part of its conjugate value, (18) can also be written as

$$
\operatorname{Re}\left\{\frac{\mathbf{V}_{\mathbf{P}}-\mathbf{S}_{\mathbf{P}} V_{D C}}{R+j \omega L} \overline{\mathbf{S}}_{N} e^{j 2 \omega t}\right\}=-\operatorname{Re}\left\{\frac{\overline{\mathbf{V}}_{\mathbf{N}}-\overline{\mathbf{S}}_{\mathbf{N}} V_{D C}}{R+j \omega L} \mathbf{S}_{\mathbf{P}} e^{j 2 \omega t}\right\} .
$$

For the above equation to be satisfied at any time, the following must hold providing that there is non-zero input impedance

$$
\left(\mathbf{V}_{\mathbf{P}}-\mathbf{S}_{\mathbf{P}} V_{D C}\right) \overline{\mathbf{S}}_{\mathbf{N}}=-\left(\overline{\mathbf{V}}_{\mathbf{N}}-\overline{\mathbf{S}}_{\mathbf{N}} V_{D C}\right) \mathbf{S}_{\mathbf{P}}
$$

If the input voltages are known and the control is free to choose the positive sequence component of the switching functions, the negative sequence of the switching functions obtained from (21) is

$$
\mathrm{S}_{\mathbf{N}}=\frac{\overline{\mathrm{S}}_{\mathbf{P}} \mathrm{V}_{\mathbf{N}}}{2 \overline{\mathrm{S}}_{\mathbf{P}} V_{D C}-\overline{\mathbf{V}}_{\mathbf{P}}}
$$

It should be noted that the relation does not contain values of input resistance and inductance and is, therefore, the same for pure inductance as well as for pure resistance connected to the front end of the inverter.

As the amplitudes of the individual switching functions need to be less than or equal to one, the range of practical combinations of $\mathbf{S}_{\mathbf{P}}$ and $\mathbf{S}_{\mathbf{N}}$ is constrained. A simple, and rather conservative, condition to keep the switching functions in allowable limits can be written as

$$
\left|\mathbf{S}_{\mathbf{P}}\right|+\left|\mathbf{S}_{\mathbf{N}}\right| \leq 1
$$

For more precise evaluation of the constraints, we need to evaluate magnitudes of switching vectors in individual phases

$$
\begin{gathered}
\left|\mathbf{S}_{A}\right|=\left|\mathbf{S}_{\mathbf{P}}+\overline{\mathbf{S}}_{\mathbf{N}}\right|, \\
\left|\mathbf{S}_{B}\right|=\left|\mathbf{S}_{\mathbf{P}} a+\overline{\mathbf{S}}_{\mathbf{N}} a^{2}\right|, \\
\left|\mathbf{S}_{C}\right|=\left|\mathbf{S}_{\mathbf{P}} a^{2}+\overline{\mathbf{S}}_{\mathbf{N}} a\right|
\end{gathered}
$$

and limit the magnitude of each of them

$$
\left(\left|\mathbf{S}_{A}\right| \leq 1\right) \wedge\left(\left|\mathbf{S}_{B}\right| \leq 1\right) \wedge\left(\left|\mathbf{S}_{C}\right| \leq 1\right)
$$


For its operation, the above discussed control method requires to monitor the instantaneous values of the input phase voltages and of the DC-link voltage. Based on this information, a convenient combination of values of $\mathbf{S}_{\mathbf{P}}$ and $\mathbf{S}_{\mathbf{N}}$ can be chosen to produce the required value of the DC-link current and to satisfy the conditions in (22) and (23) at the same time. From $S_{P}$ and $S_{N}$, the switching functions for the individual legs of the rectifier are computed and switching pulses are generated for individual switching devices based on a particular pulse width modulation algorithm. The switching devices are considered to be transistors or thyristors with forced commutation.

\section{Operation of drive under unbalanced voltage supply}

\subsection{Numerical simulation of drive under unbalanced voltage supply}

Operation of the described system has been numerically simulated under various types of unbalanced voltage supply in order to investigate the effect of the unbalance on the system behavior and the influence of certain circuit parameters. The reference parameters of the input impedance were chosen to be $R=0.1 \Omega$ and $L=10 \mathrm{mH}$. The input phase voltages had nominal voltage amplitudes of $230 \mathrm{~V}$, nominal frequency of $50 \mathrm{~Hz}$, and mutual phase shifts of $120^{\circ}$ to form a three-phase voltage system in the case of the symmetrical system. The DC-link voltage was set to $560 \mathrm{~V}$ and the capacitor of $1000 \mu \mathrm{F}$ was used in the DC bus (Chomat et al., 2007).

First, operation of the investigated system under symmetrical voltage supply was simulated to obtain the reference case to compare with unbalanced operation. Figure 2 shows input phase voltages and currents and Figure 3 shows the DC-link current and voltage. It can be seen that both electrical quantities in the DC bus are smooth with no visible pulsations.
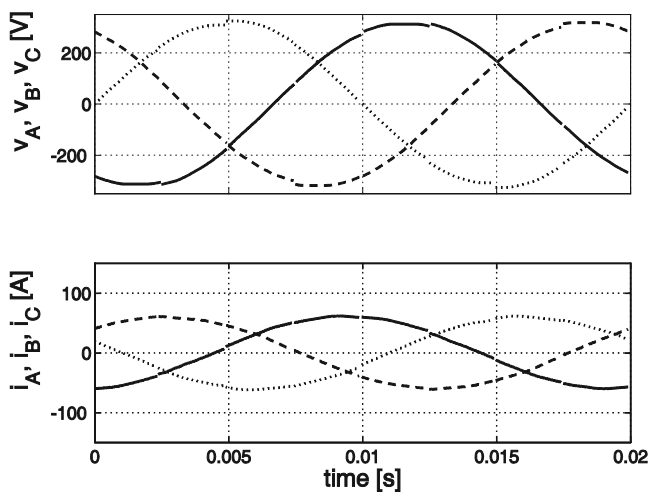

Fig. 2. Phase voltages and currents under symmetrical voltage supply.

Second, the unbalance caused by setting the magnitude of the voltage in phase $A$ to $200 V_{\text {RMS }}$ was investigated. Figures 4 and 5 show the corresponding quantities at the input of the rectifier and in the DC bus when no measures are taken to eliminate the pulsations by suitable modification of switching in the active front-end rectifier. The DC-link current and voltage contain significant pulsations that would make control of the drive more complicated. When the switching functions are modified in order to eliminate the effect of the supply voltage unbalance, the pulsations are nearly entirely eliminated, Figures 6 and 7 . This has also an effect on the input phase currents compared to the previous case. 

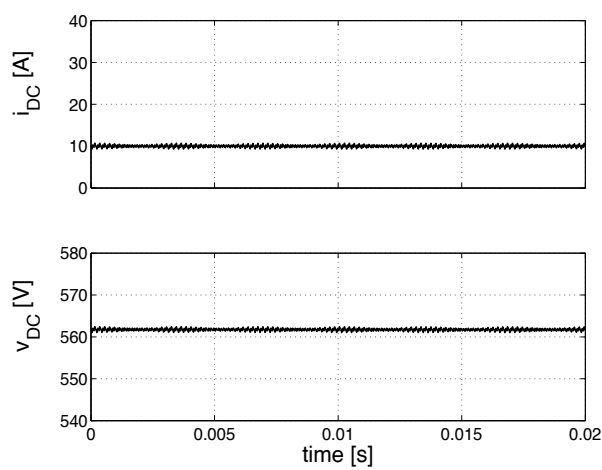

Fig 3. DC-link voltage and current under symmetrical voltage supply.
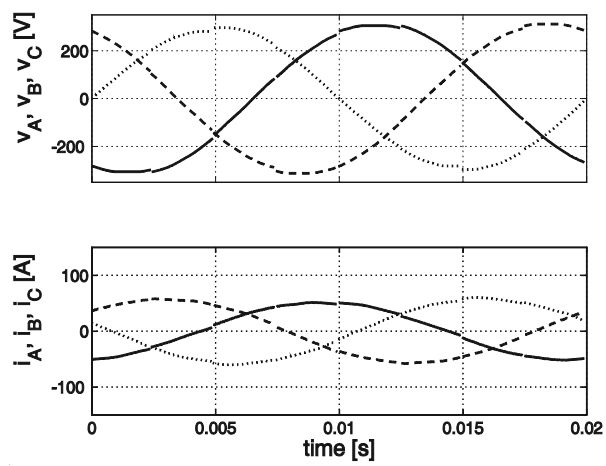

Fig 4. Phase voltages and currents under unbalanced voltage supply without compensation.
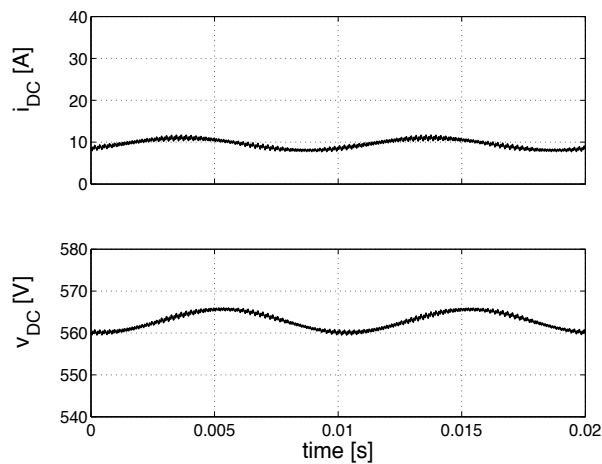

Fig. 5. DC-link voltage and current under unbalanced voltage supply without compensation. 

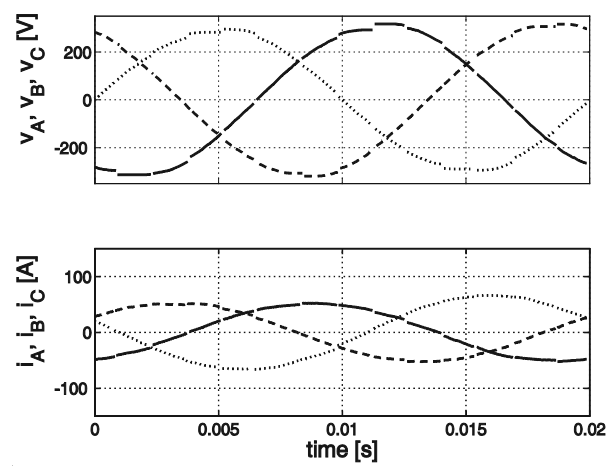

Fig. 6. Phase voltages and currents under unbalanced voltage supply with compensation.
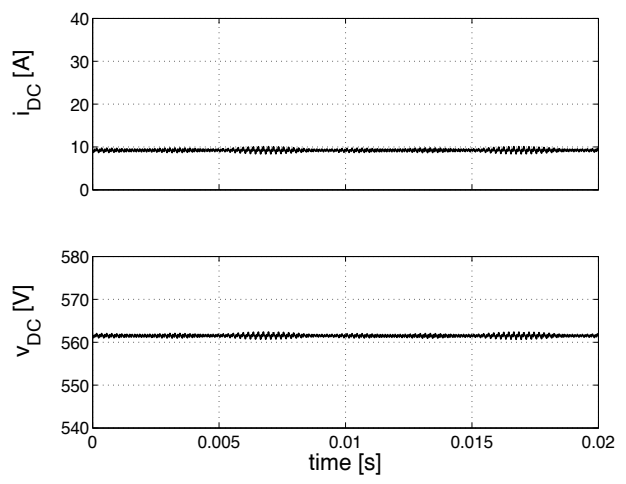

Fig. 7. DC-link voltage and current under unbalanced voltage supply with compensation.

When DC-link capacitor is reduced to have the capacity of only $500 \mu \mathrm{F}$ instead of $1000 \mu \mathrm{F}$, Figures 8 and 9, the DC-link voltage pulsations are increased twice as could be expected in case with no measures to eliminate the effect of the unbalance in the front-end rectifier. No change appears in the case when switching functions are modified to eliminate the effect of the unbalance, Figures 10 and 11.
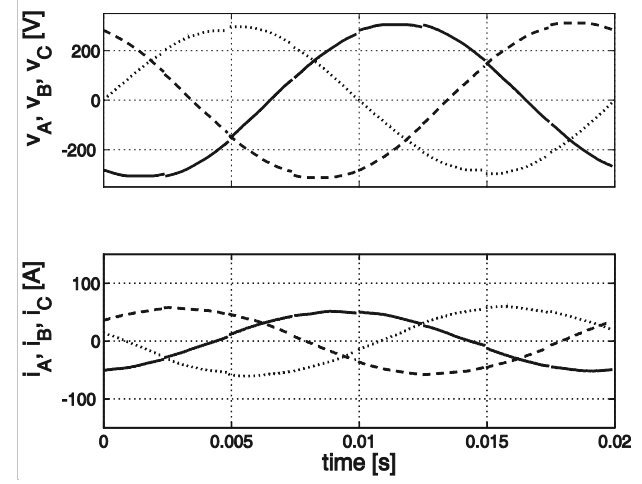

Fig. 8. Phase voltages and currents under unbalanced voltage supply with reduced DC-link capacitance without compensation. 

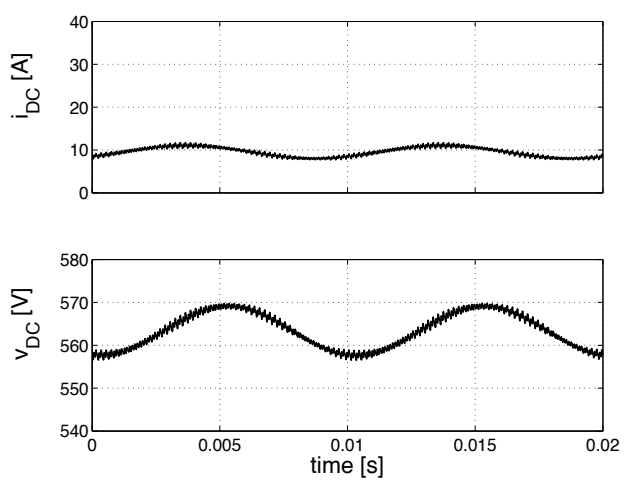

Fig. 9. DC-link voltage and current under unbalanced voltage supply with reduced DC-link capacitance without compensation.
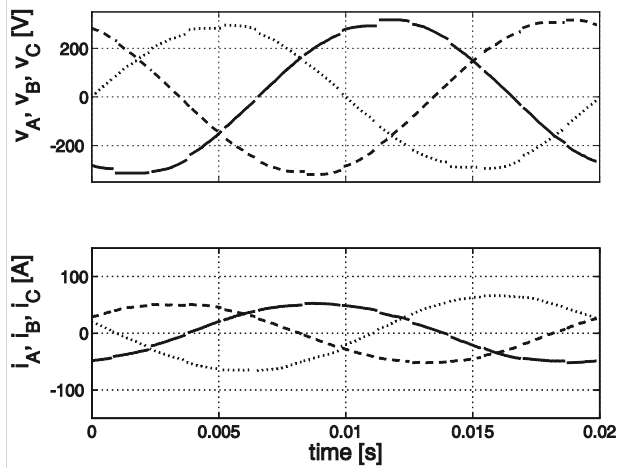

Fig. 10. Phase voltages and currents under unbalanced voltage supply with reduced DC-link capacitance with compensation.
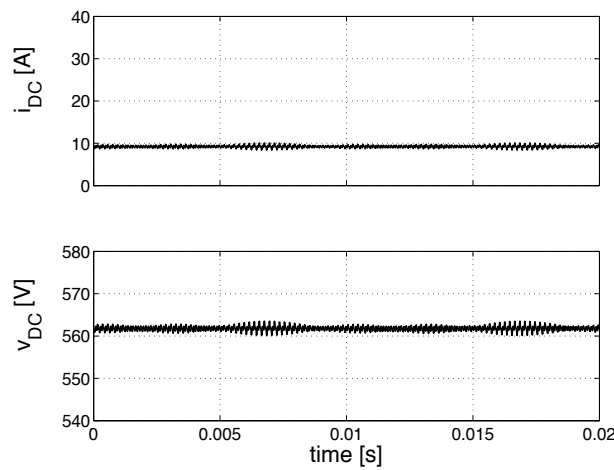

Fig. 11. DC-link voltage and current under unbalanced voltage supply with reduced DClink capacitance with compensation.

The change of the input inductance from $10 \mathrm{mH}$ to $5 \mathrm{mH}$ leads to an adequate increase in the input phase currents as well as in the DC-link current, Figures 12 to 15 . The relative amount of pulsations remain at about the same levels. 

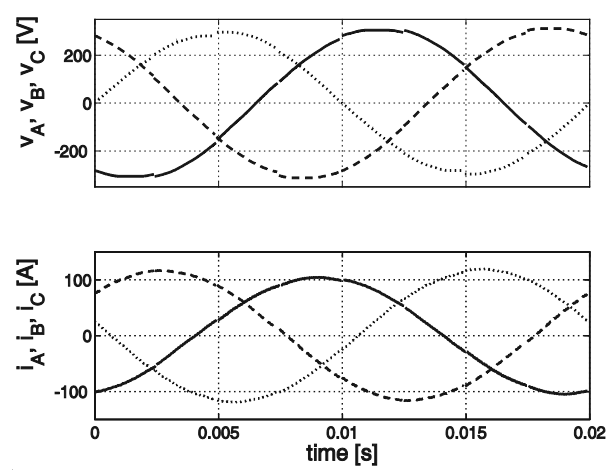

Fig. 12. Phase voltages and currents under unbalanced voltage supply with reduced input inductance without compensation.
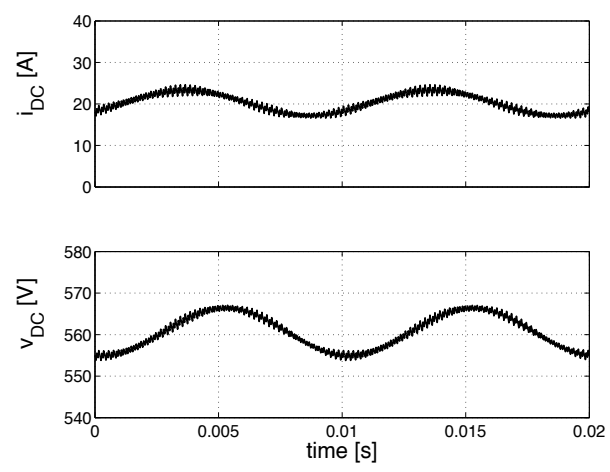

Fig. 13. DC-link voltage and current under unbalanced voltage supply with reduced input inductance without compensation.
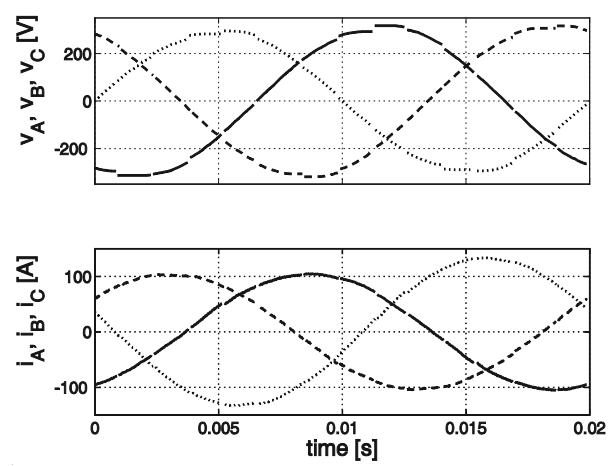

Fig. 14. Phase voltages and currents under unbalanced voltage supply with reduced input inductance with compensation. 

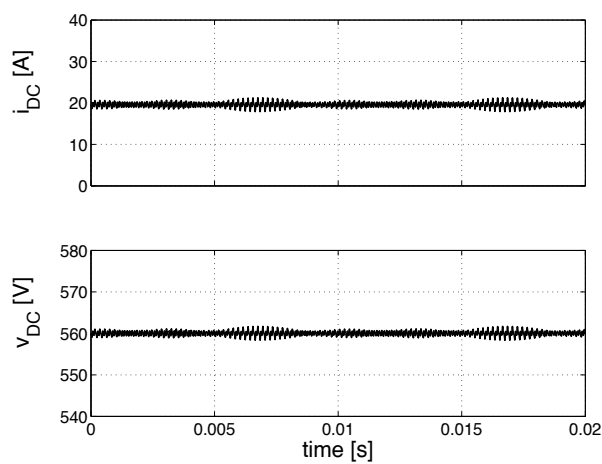

Fig. 15. DC-link voltage and current under unbalanced voltage supply with reduced input inductance with compensation.

Finally, the unbalance caused by shifting the voltage phasor of phase $A$ by $10^{\circ}$ was investigated. Corresponding results due to the changes in circuit parameters are illustrated in Figures 16 to 27. It can be noted that the effects are similar to the previous case of unbalance.
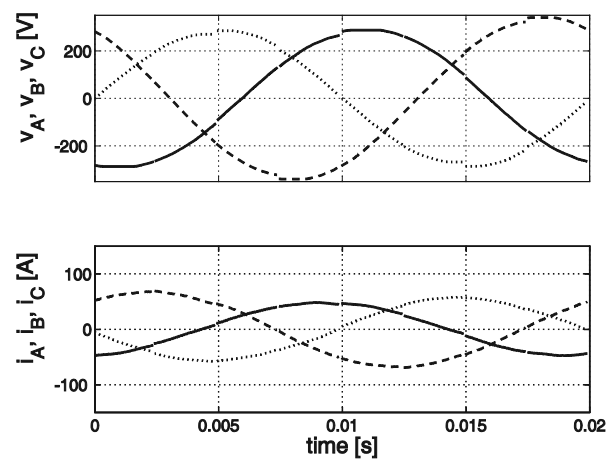

Fig. 16. Phase voltages and currents under unbalanced voltage supply without compensation.
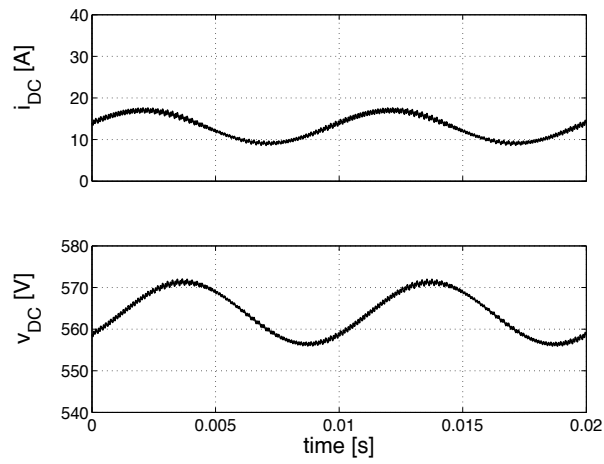

Fig. 17. DC-link voltage and current under unbalanced voltage supply without compensation. 

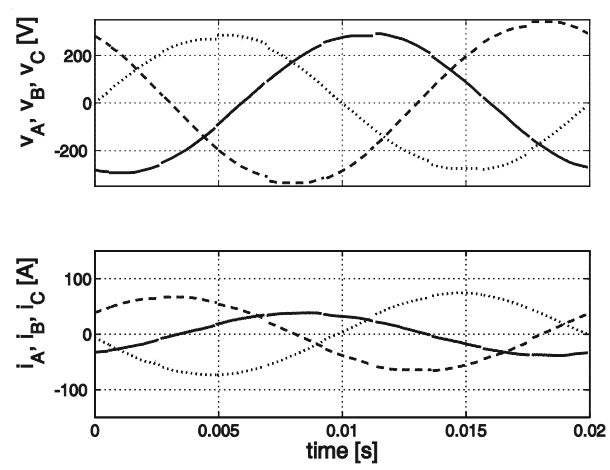

Fig. 18. Phase voltages and currents under unbalanced voltage supply with compensation.
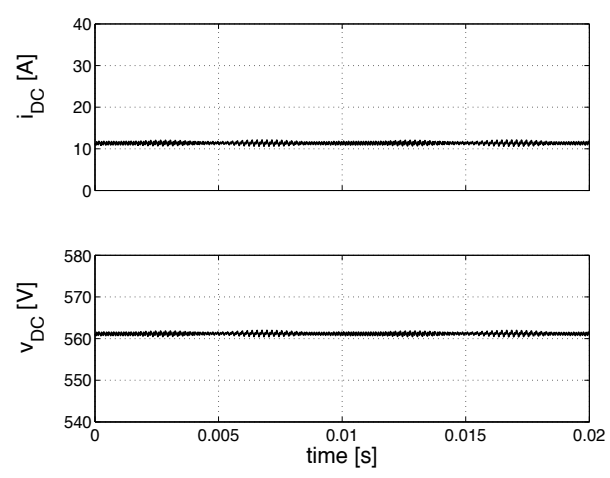

Fig. 19. DC-link voltage and current under unbalanced voltage supply with compensation.

The effect of reduction of the DC-link capacitor from $1000 \mu \mathrm{F}$ to $500 \mu \mathrm{F}$ is shown in Figures 20 through 23.
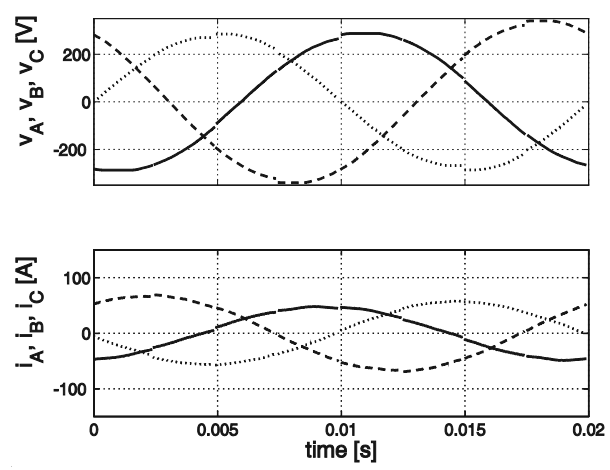

Fig. 20. Phase voltages and currents under unbalanced voltage supply with reduced DC-link capacitance without compensation. 

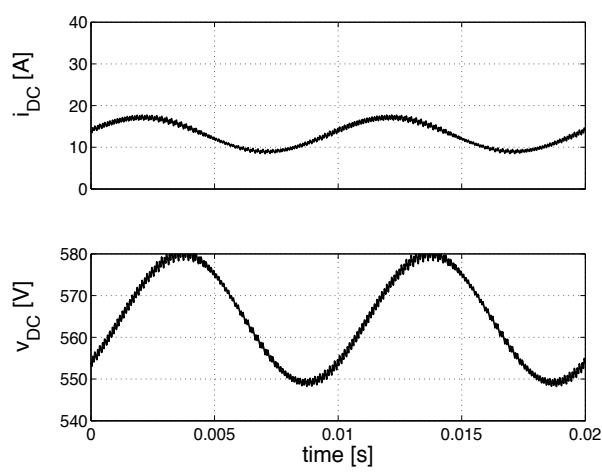

Fig. 21. DC-link voltage and current under unbalanced voltage supply with reduced DClink capacitance without compensation.
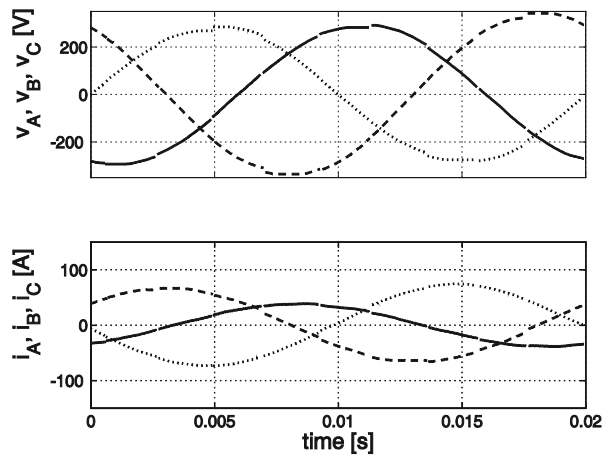

Fig. 22. Phase voltages and currents under unbalanced voltage supply with reduced DC-link capacitance with compensation.
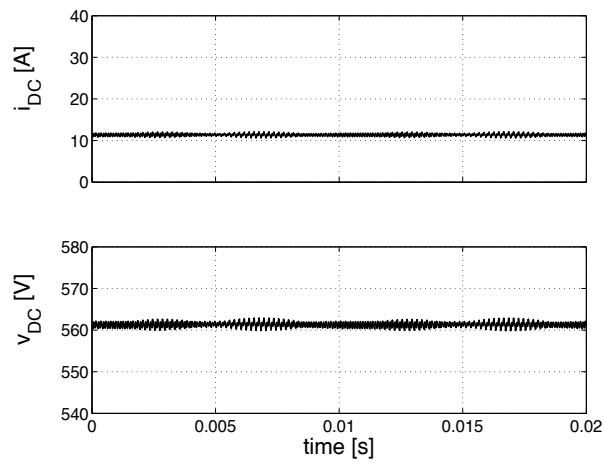

Fig. 23. DC-link voltage and current under unbalanced voltage supply with reduced DClink capacitance with compensation.

The corresponding situation for reduced input inductance from $10 \mathrm{mH}$ to $5 \mathrm{mH}$ is illustrated in Figures 24 to 27. 

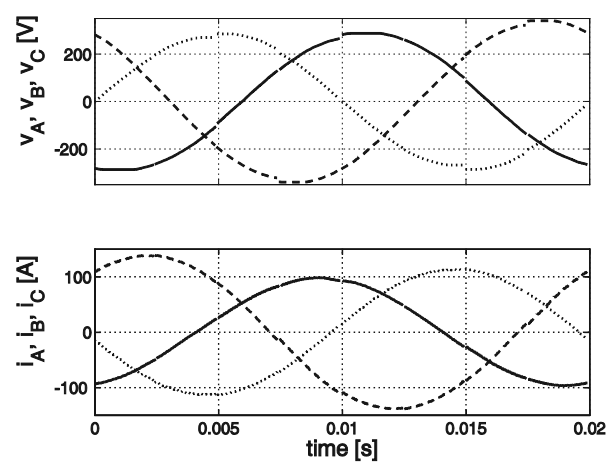

Fig. 24. Phase voltages and currents under unbalanced voltage supply with reduced input inductance without compensation.
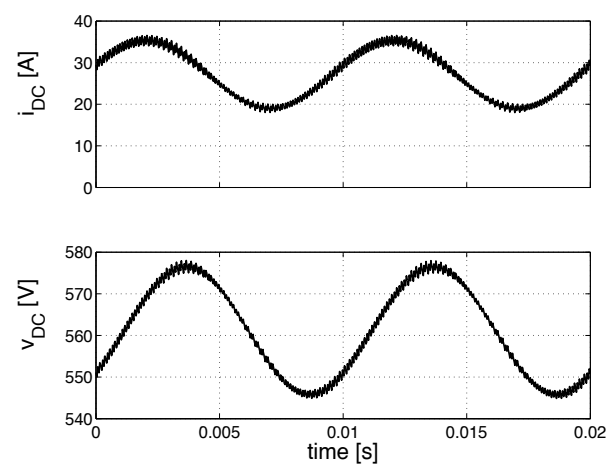

Fig. 25. DC-link voltage and current under unbalanced voltage supply with reduced input inductance without compensation.
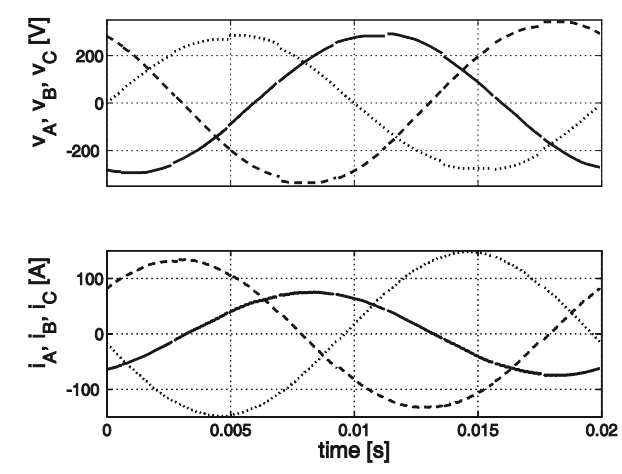

Fig. 26. Phase voltages and currents under unbalanced voltage supply with reduced input inductance with compensation. 

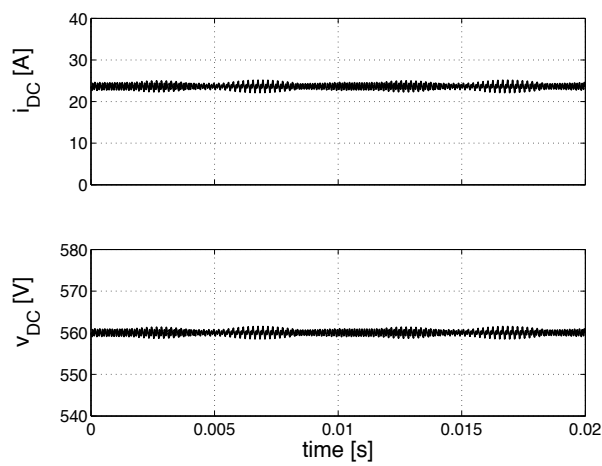

Fig. 27. DC-link voltage and current under unbalanced voltage supply with reduced input inductance with compensation.

\subsection{Limitation of control range due to unbalanced voltage supply}

The necessity to generate the negative sequence component of the switching functions in order to eliminate the effect of the supply-voltage unbalance on the DC-link voltage pulsations reduces the control range for the positive sequence component of the switching functions (Chomat et al., 2009). This is due to the fact that in individual phases the maximum of the switching function can only reach one at most at any given time. Another constraint results from the current rating of the converter. The resulting constraints depend on the value and type of the unbalance.

Analysis of the limitation corresponding to various types of unbalanced supply voltages has been carried out. The reference parameters of the input impedance were chosen to be $\mathrm{R}=0.1 \Omega$ and $\mathrm{L}=10 \mathrm{mH}$. The input phase voltages had nominal voltage amplitudes of $230 \mathrm{~V}$, nominal frequency of $50 \mathrm{~Hz}$, and mutual phase shifts of $120^{\circ}$ to form a three-phase voltage system in the case of the symmetrical system. The DC-link voltage was set to $400 \mathrm{~V}$. The choice of the positive sequence component of the switching functions from the available control range affects both the magnitude of the DC-link current and the currents in individual input phases. Figure 28 shows what magnitudes of the DC-link current correspond to the coordinates from the available control range. The unbalance was formed

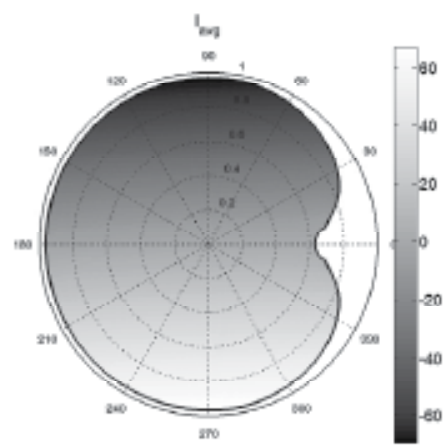

Fig. 28. DC-link current under unbalanced voltage supply $(L=10 \mathrm{mH}, R=0.1 \Omega$, $\left.V_{d c}=400 \mathrm{~V}\right)$. 
by setting the magnitude of the voltage in phase $A$ to 0.75 p.u. The corresponding maximal input phase current magnitude, calculated as the maximum of all the phase currents, is shown in Figure 29. It can be seen from Figure 28 that the resulting DC-link current decreases in the vertical direction of the operating region, whereas the maximal input current in Figure 29 decreases in the horizontal direction. The corresponding measure of the current unbalance is depicted in Figure 30 and the average power factor of all the three input phases is depicted in Figure 31.

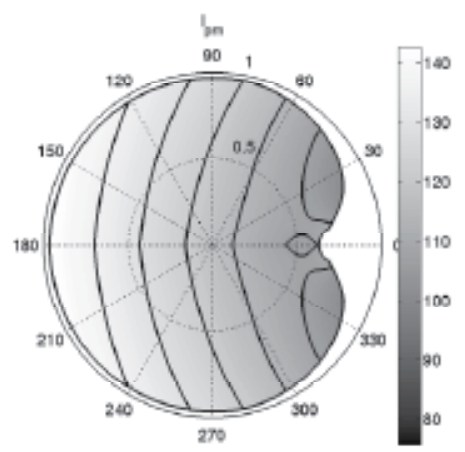

Fig. 29. Maximal input phase current under unbalanced voltage supply $(L=10 \mathrm{mH}$, $\left.R=0.1 \Omega, V_{d c}=400 \mathrm{~V}\right)$.

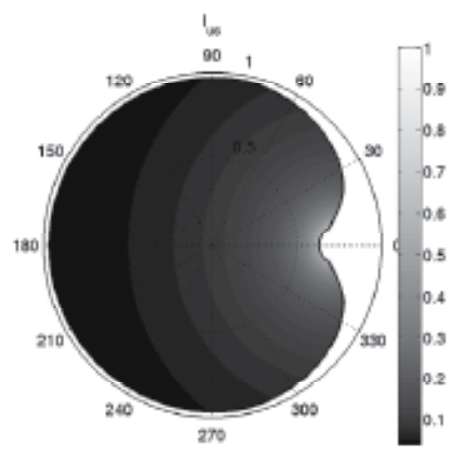

Fig. 30. Input current unbalance under unbalanced voltage supply $(L=10 \mathrm{mH}, R=0.1 \Omega$, $\left.V_{d c}=400 \mathrm{~V}\right)$.

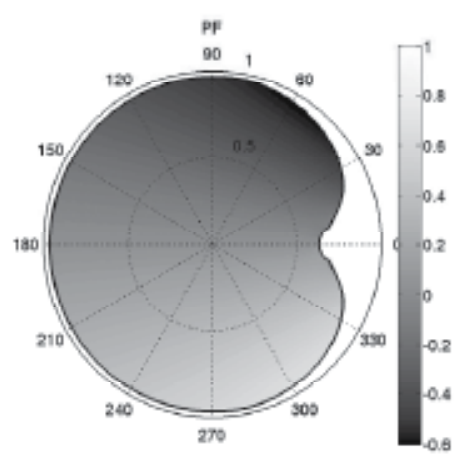

Fig. 31. Power factor under unbalanced voltage supply $\left(L=10 \mathrm{mH}, R=0.1 \Omega, V_{d c}=400 \mathrm{~V}\right)$. 
If we change the value of the input inductance from $10 \mathrm{mH}$ to $1 \mathrm{mH}$, the constraints caused by the switching functions remain the same as can be seen from Figures 32 through 35. However, both the DC-link current and the input current increased nearly ten times as the input reactance represents the main limiting factor for the currents entering the rectifier. The excessive values of the currents would, in a case of a real rectifier, impose additional restrictions to the operating regions resulting from current stress of electronic components in the bridge. This can also be considered in the shape of new borders of operating regions.

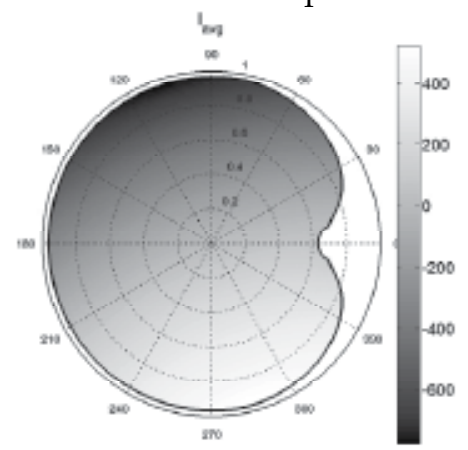

Fig. 32. DC-link current under unbalanced voltage supply $(L=1 \mathrm{mH}, R=0.1 \Omega$, $\left.V_{d c}=400 \mathrm{~V}\right)$.

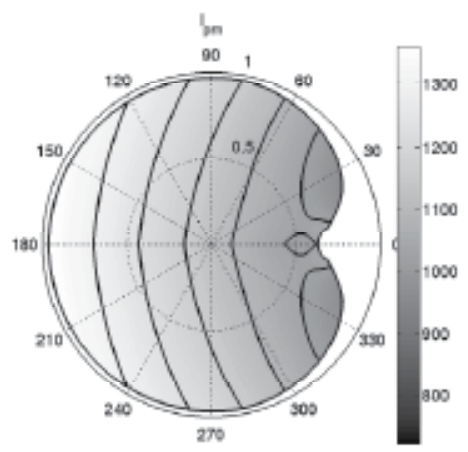

Fig. 33. Maximal input phase current under unbalanced voltage supply $(L=1 \mathrm{mH}, R=0.1 \Omega$, $\left.V_{d c}=400 \mathrm{~V}\right)$.

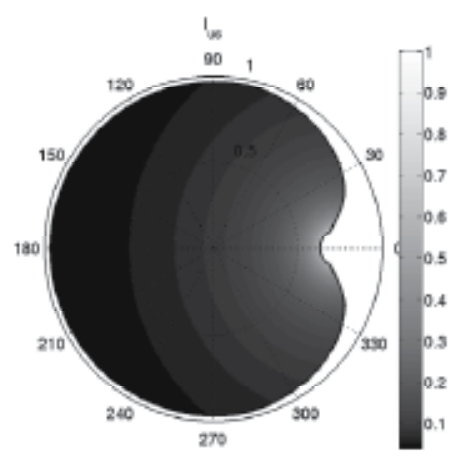

Fig. 34. Input current unbalance under unbalanced voltage supply $(L=1 \mathrm{mH}, R=0.1 \Omega$, $\left.V_{d c}=400 \mathrm{~V}\right)$. 


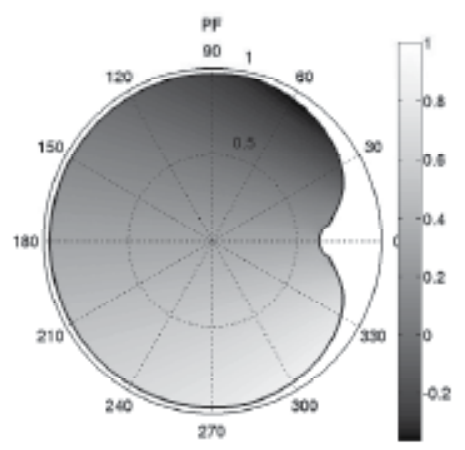

Fig. 35. Power factor under unbalanced voltage supply $\left(L=1 \mathrm{mH}, R=0.1 \Omega, V_{d c}=400 \mathrm{~V}\right)$.

A different situation arises when the input resistance is increased ten times to $1 \Omega$. The corresponding electrical quantities are shown in Figures 36 through 39. The increase in the DC-link and input phase currents is not as dramatic as the resistance plays less significant role in limiting the currents than the inductance. The values of the currents are similar to the ones in the first case.

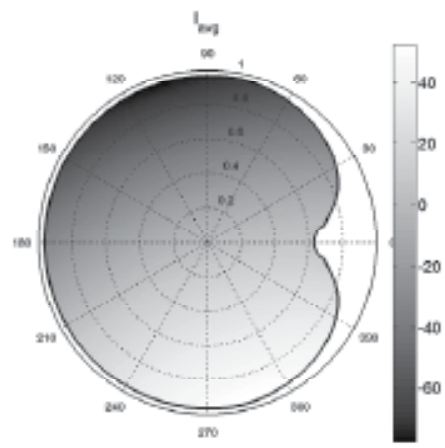

Fig. 36. DC-link current under unbalanced voltage supply $\left(L=1 \mathrm{mH}, R=1 \Omega, V_{d c}=400 \mathrm{~V}\right)$.

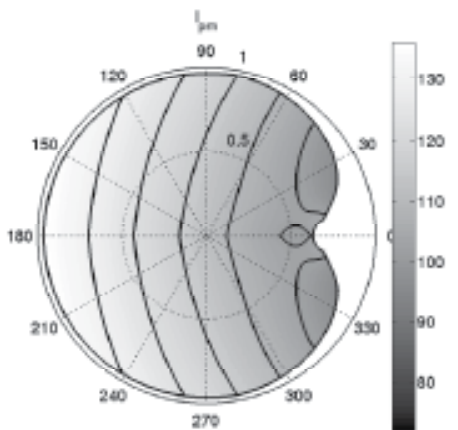

Fig. 37. Maximal input phase current under unbalanced voltage supply $(L=1 \mathrm{mH}, R=1 \Omega$, $\left.V_{d c}=400 \mathrm{~V}\right)$. 


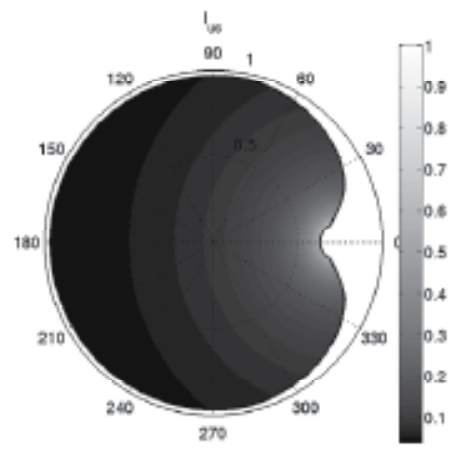

Fig. 38. Input current unbalance under unbalanced voltage supply $(L=1 \mathrm{mH}, R=1 \Omega$, $\left.V_{d c}=400 \mathrm{~V}\right)$.

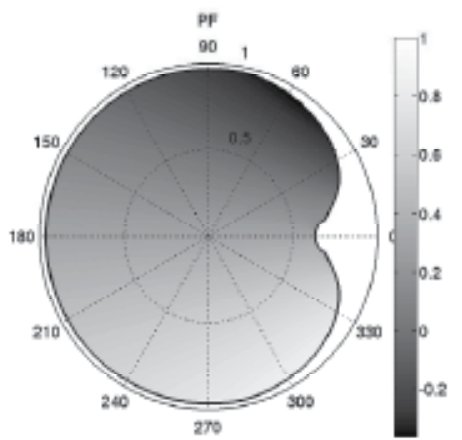

Fig. 39. Power factor under unbalanced voltage supply $\left(L=1 \mathrm{mH}, R=1 \Omega, V_{d c}=400 \mathrm{~V}\right)$.

A change in the DC-link voltage introduces, on the other hand, a noticeable change in the shape of constraints caused by the limitation of the switching functions. Figures 40 through 43 show the situation for the decrease in the DC-link voltage from $400 \mathrm{~V}$ to $200 \mathrm{~V}$ and Figures 45 through 47 show the situation for the increase to $600 \mathrm{~V}$. In the latter case, a rise of an isolated restricted area in the right hand side of the figure completely surrounded by available control space can be noticed.

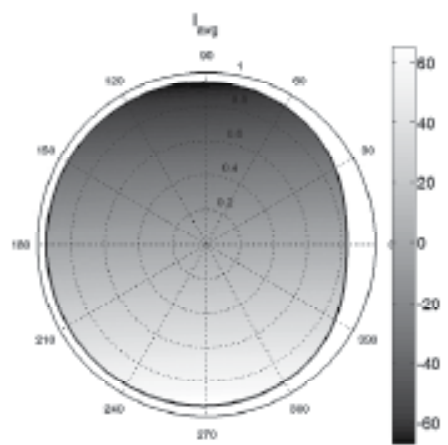

Fig. 40. DC-link current under unbalanced voltage supply $(L=10 \mathrm{mH}, R=0.1 \Omega$, $\left.V_{d c}=200 \mathrm{~V}\right)$. 


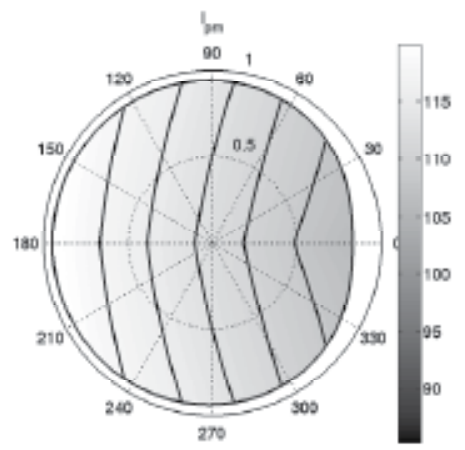

Fig. 41. Maximal input phase current under unbalanced voltage supply $(L=10 \mathrm{mH}$, $\left.R=0.1 \Omega, V_{d c}=200 \mathrm{~V}\right)$.

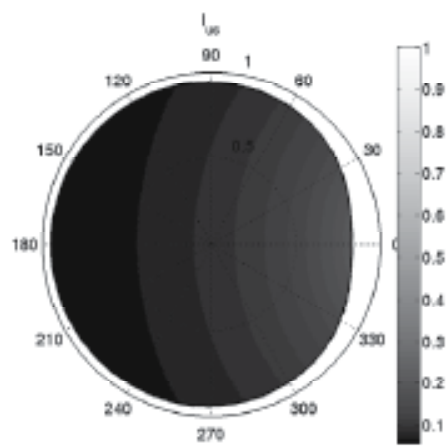

Fig. 42. Input current unbalance under unbalanced voltage supply $(L=10 \mathrm{mH}, R=0.1 \Omega$, $\left.V_{d c}=200 \mathrm{~V}\right)$.

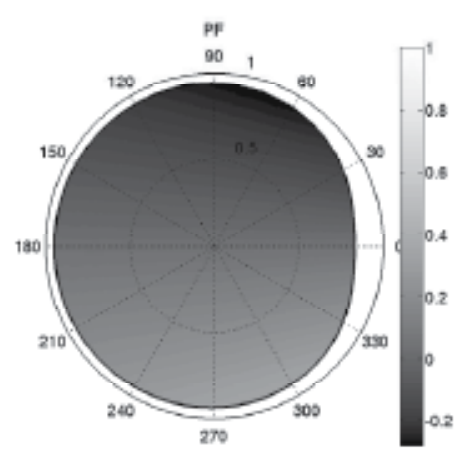

Fig. 43. Power factor under unbalanced voltage supply $(L=10 \mathrm{mH}, R=0.1 \Omega$, $V_{d c}=200 \mathrm{~V}$ ). 


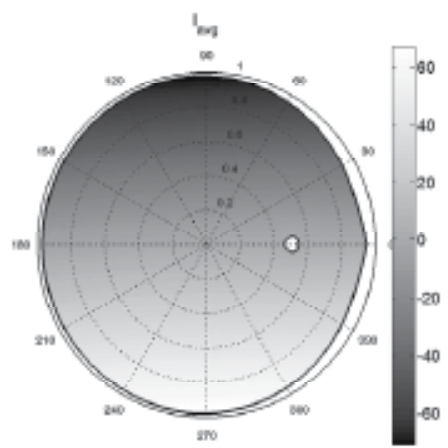

Fig. 44. DC-link current under unbalanced voltage supply $(L=10 \mathrm{mH}, R=0.1 \Omega$, $\left.V_{d c}=600 \mathrm{~V}\right)$.

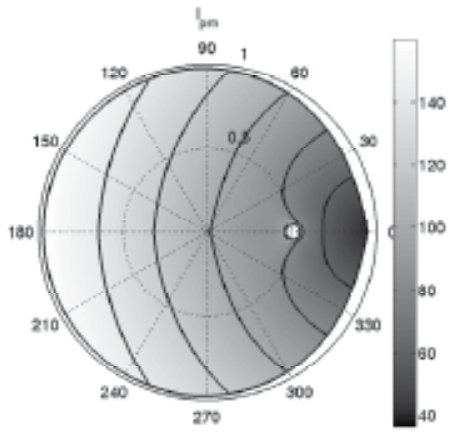

Fig. 45. Maximal input phase current under unbalanced voltage supply $(L=10 \mathrm{mH}$, $\left.R=0.1 \Omega, V_{d c}=600 \mathrm{~V}\right)$.

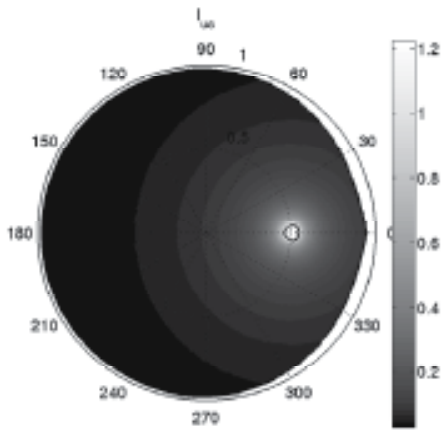

Fig. 46. Input current unbalance under unbalanced voltage supply $(L=10 \mathrm{mH}, R=0.1 \Omega$, $\left.V_{d c}=600 \mathrm{~V}\right)$.

Measurements on an experimental system identical to the simulated one have been carried out in order to verify the investigated method. The scope traces in Figure 48 show the measured current in phase $A$ and the DC link current when the negative-sequence in the supply voltage is not compensated for by the control method and the DC link current, therefore, contains significant component pulsating with a frequency of $100 \mathrm{~Hz}$, twice the 
fundamental network frequency. The case when unbalanced voltage system is compensated by the investigated control method is illustrated in Figure 49. It can be seen that the pulsating component of the DC link current has been effectively eliminated by the investigated method.

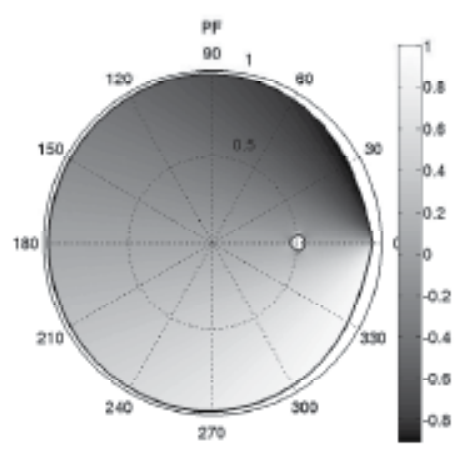

Fig. 47. Power factor under unbalanced voltage supply $\left(L=10 \mathrm{mH}, R=0.1 \Omega, V_{d c}=600 \mathrm{~V}\right)$.

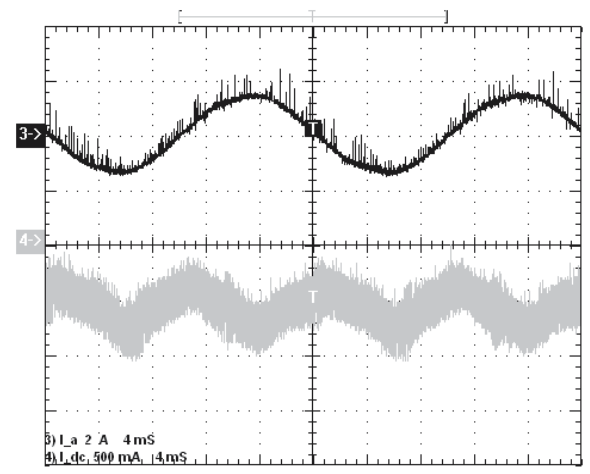

Fig. 48. Phase A current and DC-link current under unbalanced voltage supply without elimination of pulsating component.

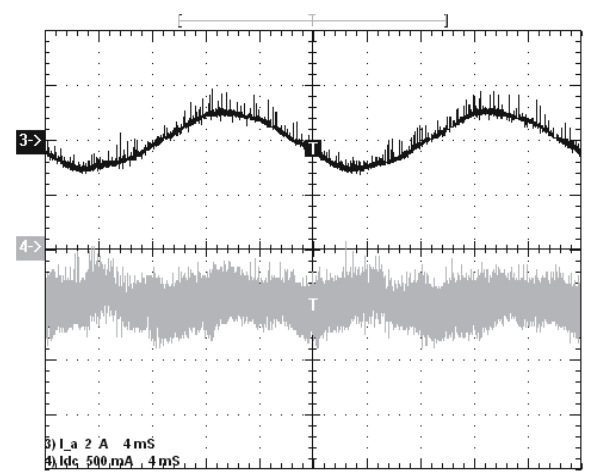

Fig. 49. Phase A current and DC-link current under unbalanced voltage supply with elimination of pulsating component. 


\section{Conclusion}

It has been shown in the article that it is possible to effectively compensate for the unbalanced voltage source at the input of a solid-state converter so that constant power flow into the DC bus is maintained. The results of simulations show that the choice of the operating point of front end converter may significantly affect the impact of the rectifier on the supplying power grid. It is possible to select the optimal operating point according to the chosen optimization criteria, which can be e.g. maximal power factor or current unbalance.

\section{Acknowledgment}

This work was supported by the Grant Agency of the Czech Republic under research grant No. 102/09/1273 and by the Institutional Research Plan AV0Z20570509.

\section{References}

Stankovic, A. V. \& Lipo, T. A. (2001). A Novel Control Method for Input Output Harmonic Elimination of the PWM Boost Type Rectifier Under Unbalanced Operating Conditions, IEEE Trans. on Power Electronics, 16, pp. 603-611, ISSN: 0885-8993.

Stankovic, A. V. \& Lipo, T. A. (2001). A Generalized Control Method for Input-Output Harmonic Elimination of the PWM Boost Type Rectifier Under Simultaneous Unbalanced Input Voltages and Input Impedances, Power Electronics Specialists Conference, pp. 1309-1314, ISBN: 0-7803-7067-8, Vancouver, Canada, June 2001.

Lee, K.; Jahns, T. M.; Berkopec, W. E. \& Lipo, T. A. (2006). Closed-form analysis of adjustable-speed drive performance under input-voltage unbalance and sag conditions, IEEE Trans. on Industry Applications, vol.42, no.3., pp.733-741, ISSN: 0093-9994.

Cross, A. M.; Evans, P. D. \& Forsyth, A. J. (1999). DC Link Current in PWM Inverters with Unbalanced and Non Linear Loads, IEE Proc.-Electr. Power Appl., vol. 146, no. 6, pp. 620-626, ISSN: 1350-2352.

Song, H. \& Nam, K. (1999). Dual Current Control Scheme for PWM Converter Under Unbalanced Input Voltage Conditions, IEEE Trans. on Industrial Electronics, 46, pp. 953-959, ISSN: 0278-0046.

Chomat, M. \& Schreier, L. (2005). Control Method for DC-Link Voltage Ripple Cancellation in Voltage Source Inverter under Unbalanced Three-Phase Voltage Supply, IEE Proceedings on Electric Power Applications, vol.152, no. 3, pp. 494-500, ISSN: 1350-2352.

Chomat, M.; Schreier, L. \& Bendl, J. (2007). Operation of Adjustable Speed Drives under Non Standard Supply Conditions, IEEE Industry Applications Conference/42th IAS Annual Meeting, pp. 262-267, ISBN: 978-1-4244-1259-4, New Orleans, USA, September 2007.

Chomat, M.; Schreier, L. \& Bendl, J. (2009). Influence of Circuit Parameters on Operating Regions of PWM Rectifier Under Unbalanced Voltage Supply, IEEE International Electric Machines and Drives Conference, pp.357-362, ISBN: 978-1-4244-4251-5, Miami, USA, May 2009.

Chomat, M.; Schreier, L. \& Bendl, J. (2009). Operating Regions of PWM Rectifier under Unbalanced Voltage Supply, International Conference on Industrial Technology, pp. 510 - 515, ISBN: 978-1-4244-3506-7, Gippsland, Australia, February 2009. 


\title{
Space Vector PWM-DTC Strategy for Single-Phase Induction Motor Control
}

\author{
Ademir Nied ${ }^{1}$, José de Oliveira ${ }^{1}$, Rafael de Farias Campos¹, \\ Seleme Isaac Seleme Jr. ${ }^{2}$ and Luiz Carlos de Souza Marques ${ }^{3}$ \\ ${ }^{1}$ State University of Santa Catarina \\ ${ }^{2}$ Federal University of Minas Gerais \\ ${ }^{3}$ Federal University of Santa Maria \\ Brazil
}

\section{Introduction}

Single-phase induction motors are widely used in fractional and sub-fractional horsepower applications, mostly in domestic and commercial applications such as fans, refrigerators, air conditioners, etc., operating at constant speed or controlled by an on/off strategy which can result in poor efficiency and low-power factor. In terms of construction, these types of motors usually have a main and an auxiliary stator winding, are asymmetrical and are placed 90 degrees apart from each other. The rotor is usually the squirrel-cage type. The asymmetry presented in the stator windings is due to the fact that these windings are designed to be electrically different so the difference between the stator windings currents can produce a starting torque (Krause et al., 1995). Since it has main and auxiliary stator windings, the singlephase induction motor is also known as a two-phase asymmetric induction motor.

In recent years, with the growing concern about low-cost operation and the efficient use of energy, the advance in motor drive control technology made it possible to apply these motors to residential applications with more efficiency. Different inverter topologies have been proposed to drive single-phase induction motors, providing ways to save energy. In dos Santos et al. (2010) different ac drive systems are conceived for multiple single-phase motor drives with a single dc-link voltage to guarantee installation cost reduction and some individual motor controls. In Wekhande et al. (1999) and Jabbar et al. (2004), Campos et al. (2007a) and Campos et al. (2007b), two topologies are considered. One is a Half-bridge inverter and the other is a three-leg inverter. The cost difference between the two topologies lays in the fact that the H-bridge inverter needs two large capacitors in the dc link rated for dc link voltage. Also, there is a need of two large resistors connected in parallel with the capacitors to balance the voltage of the capacitors.

Despite the fact that the three-leg inverter has more switches, the development of power modules and the need for just one capacitor in the dc link have decreased the topology cost. Along with the reduced cost, a more efficient use of the dc link voltage is achieved.

Besides the effort for developing more efficient driving topologies, many strategies to control single-phase motors have been proposed. In Jacobina et al. (1999), rotor-flux control, stator-flux control and direct torque control (DTC) (Takahashi and Noguchi, 1986) are analyzed. The main drawback of the two first strategies is that they use an encoder to obtain 
the speed signal. Since there is no need for speed and position signals, a DTC scheme appears to be a suitable solution. But it has some disadvantages such as current and torque distortions, variable switching frequency and low-speed operation problems (Buja and Kazmierkowski, 2004). In Neves et al. (2002), a DTC strategy is applied for a single-phase motor and the performance is improved with the use of pulse width modulation.

Along with control strategies and driver topologies, many researchers have investigated ways to optimize modulation techniques applied in single-phase induction motor drives. In Jabbar et al. (2004), space-vector modulation (SVPWM) is used to reduce the torque ripple and alleviate the harmonic content at the terminals of the single-phase induction motor being driving by a three-leg inverter. In Chaumit and Kinnares (2009) the proposed SVPWM method controls the two-phase voltage outputs of an unbalanced two-phase induction motor drive by varying the modulation index and voltage factors.

In this chapter, the authors are interested in studying the DTC strategy combined with the SVPWM applied to a three-leg inverter topology to drive a single-phase induction motor.

\section{Single-phase induction motor model}

A single-phase induction motor with main and auxiliary windings is designed to be electrically different. In order to make the motor self-starting, a capacitor is connected in series with the auxiliary winding.

When the windings of a single-phase induction motor are fed independently (i.e., using a voltage source inverter) one can consider a single-phase induction motor an example of an unsymmetrical two-phase induction motor.

In this section, the mathematical model of a single-phase induction motor will be derived. As is commonly done, the derivation of the motor model is based on classical assumptions:

- The stator and rotor windings are in space quadrature;

- The rotor windings are symmetrical;

- The magnetic circuit is linear and the air-gap length is constant;

- A sinusoidal magnetic field distribution produced by the motor windings appears in the air gap;

- The motor is a squirrel-cage type. Therefore the rotor voltages are zero.

Since the single-phase induction motor will be considered as acting as a two-phase system, to derive the dynamic motor model of the two-phase system, a common reference frame ( $a$ b) will be used, as shown in Fig. 1 .

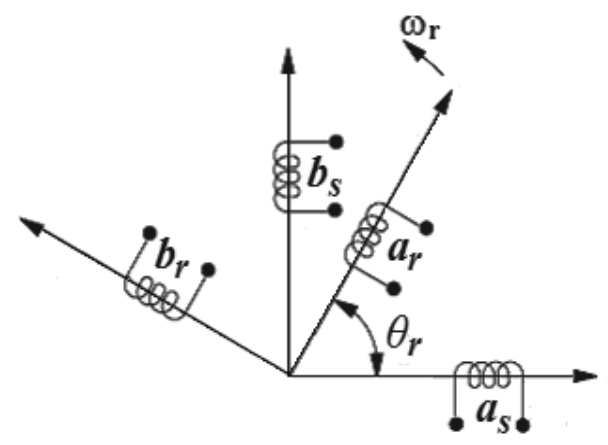

Fig. 1. Common reference frame $(a-b)$. 
Since the stator windings are in space quadrature, there is no magnetic coupling between them. The same consideration is applied to the rotor windings. According to Krause et al. (1995), the relations between the fluxes and currents can be established as:

$$
\left[\begin{array}{c}
\lambda_{a s} \\
\lambda_{b s} \\
\lambda_{a r} \\
\lambda_{b r}
\end{array}\right]=\left[\begin{array}{llll}
L_{a s a s} & L_{a s b s} & L_{a s a r} & L_{a s b r} \\
L_{b s a s} & L_{b s b s} & L_{b s a r} & L_{b s b r} \\
L_{a r a s} & L_{a r b s} & L_{a r a r} & L_{a r b r} \\
L_{b r a s} & L_{b r b s} & L_{b r a r} & L_{b r b r}
\end{array}\right]\left[\begin{array}{c}
i_{a s} \\
i_{b s} \\
i_{a r} \\
i_{b r}
\end{array}\right]
$$

In Equation (1), $L_{a s a s(b s b s)}$ is the stator windings self-inductance; $L_{\text {arar }(b r b r)}$ is the rotor windings self-inductance; $L_{a s b r(b r a s)}, L_{a r b s(b s a r)}$ and $L_{a s a r(a r a s)}, L_{b s b r(b r b s)}$ are the mutual inductance between the stator and rotor windings. Since the stator windings are in space quadrature and asymmetric, and the rotor windings are in space quadrature and symmetric, the following relations can be written:

$$
\begin{gathered}
L_{a s a s}=L_{a s} \\
L_{b s b s}=L_{b s} \\
L_{a s b s}=L_{b s a s}=0 \\
L_{a r b r}=L_{b r a r}=0 \\
L_{a r a r}=L_{b r b r}=L_{r}
\end{gathered}
$$

The self-inductances of stator and rotor are composed of a leakage inductance and a magnetizing inductance. That way, a new set of equations can be derived:

$$
\begin{gathered}
L_{a s}=L_{l a s}+L_{m a s} \\
L_{b s}=L_{l b s}+L_{m b s} \\
L_{r}=L_{l r}+L_{m r}
\end{gathered}
$$

where $\left(L_{l a s}, L_{l b s}\right)$ and $\left(L_{m a s}, L_{m b s}\right)$ indicate the stator leakage inductance and magnetizing inductance, respectively, and $L_{l r}$ and $L_{m r}$ indicate the rotor leakage inductance and magnetizing inductance, respectively. Since the rotor windings are assumed to be symmetric, Equation (9) expresses the rotor windings.

As shown in Fig. 1, there is an angular displacement between the stator and rotor windings establishing a magnetic coupling between them which results in a mutual inductance. The equation for the mutual inductances may be expressed in matrix form

$$
L_{s r}=\left[\begin{array}{cc}
L_{s r a} \cos \theta_{r} & -L_{s r a} \sin \theta_{r} \\
L_{s r b} \sin \theta_{r} & L_{s r b} \cos \theta_{r}
\end{array}\right]
$$

where $L_{s r a}$ and $L_{s r b}$ are the amplitude of the mutual inductances.

Thus, the Equation (1) can be rewritten as 


$$
\left[\begin{array}{c}
\lambda_{a s} \\
\lambda_{b s} \\
\lambda_{a r} \\
\lambda_{b r}
\end{array}\right]=\left[\begin{array}{cccc}
L_{a s} & 0 & L_{s r a} \cos \theta_{r} & -L_{s r a} \sin \theta_{r} \\
0 & L_{b s} & L_{s r b} \sin \theta_{r} & L_{s r b} \cos \theta_{r} \\
L_{s r a} \cos \theta_{r} & L_{s r b} \sin \theta_{r} & L_{r} & 0 \\
-L_{s r a} \sin \theta_{r} & L_{s r b} \cos \theta_{r} & 0 & L_{r}
\end{array}\right]\left[\begin{array}{l}
i_{a s} \\
i_{b s} \\
i_{a r} \\
i_{b r}
\end{array}\right]
$$

The voltage equations expressed in the common reference frame may be written as

$$
\begin{gathered}
{\left[\begin{array}{c}
v_{a s} \\
v_{b s}
\end{array}\right]=\left[\begin{array}{cc}
r_{a s} & 0 \\
0 & r_{b s}
\end{array}\right]\left[\begin{array}{l}
i_{a s} \\
i_{b s}
\end{array}\right]+\frac{d}{d t}\left[\begin{array}{l}
\lambda_{a s} \\
\lambda_{b s}
\end{array}\right]} \\
{\left[\begin{array}{l}
0 \\
0
\end{array}\right]=\left[\begin{array}{cc}
r_{r} & 0 \\
0 & r_{r}
\end{array}\right]\left[\begin{array}{l}
i_{a r} \\
i_{b r}
\end{array}\right]+\frac{d}{d t}\left[\begin{array}{l}
\lambda_{a r} \\
\lambda_{b r}
\end{array}\right]}
\end{gathered}
$$

Inserting Equation (11) in Equations (12) and (13), the single-phase induction motor model acting as a two-phase system can be mathematically expressed by

$$
\left[\begin{array}{c}
v_{a s} \\
v_{b s} \\
0 \\
0
\end{array}\right]=\left[\begin{array}{cccc}
r_{a s}+\frac{d}{d t} L_{a s} & 0 & \frac{d}{d t} L_{s r a} \cos \theta_{r} & -\frac{d}{d t} L_{s r a} \sin \theta_{r} \\
0 & r_{b s}+\frac{d}{d t} L_{b s} & \frac{d}{d t} L_{s r b} \sin \theta_{r} & \frac{d}{d t} L_{s r b} \cos \theta_{r} \\
\frac{d}{d t} L_{s r a} \cos \theta_{r} & \frac{d}{d t} L_{s r b} \sin \theta_{r} & r_{r}+\frac{d}{d t} L_{r} & 0 \\
-\frac{d}{d t} L_{s r a} \sin \theta_{r} & \frac{d}{d t} L_{s r b} \cos \theta_{r} & 0 & r_{r}+\frac{d}{d t} L_{r}
\end{array}\right]\left[\begin{array}{c}
i_{a s} \\
i_{b s} \\
i_{a r} \\
i_{b r}
\end{array}\right]
$$

The torque can be determined as

$$
T_{e}=\left[\begin{array}{c}
i_{a r} \\
i_{b r}
\end{array}\right]^{T} \frac{d\left(L_{s r}\right)^{T}}{d \theta_{r_{-} \text {mech }}}\left[\begin{array}{l}
i_{a s} \\
i_{b s}
\end{array}\right]
$$

Since $\theta_{r}=p \theta_{r_{-} \text {mech }}$, where $p$ is the number of pole pairs and $\theta_{r}$ is the electrical angle, and $\theta_{r_{-} \text {mech }}$ is the actual angular displacement of the rotor, the Equation (15) can be rewritten

$$
T_{e}=p \cdot\left[\begin{array}{l}
i_{a r} \\
i_{b r}
\end{array}\right]^{T} \frac{d\left(L_{s r}\right)^{T}}{d \theta_{r}}\left[\begin{array}{l}
i_{a s} \\
i_{b s}
\end{array}\right]
$$

In expanded form, (16) becomes

$$
T_{e}=p\left[-i_{a s} L_{s r a}\left(i_{a r} \sin \theta_{r}+i_{b r} \cos \theta_{r}\right)+i_{b s} L_{s r b}\left(i_{a r} \cos \theta_{r}-i_{b r} \sin \theta_{r}\right)\right]
$$

According to Krause et al. (1995), in order to obtain the motor mathematical model with constant parameters, it is necessary to transform all the variables to the stationary reference frame $(d-q)$ as shown in Fig. 2. That way $\theta_{r}=0$.

A transformation matrix is necessary in order to establish the new reference frame. Thus

$$
T=\left[\begin{array}{cc}
\cos \theta_{r} & \sin \theta_{r} \\
-\sin \theta_{r} & \cos \theta_{r}
\end{array}\right] ; T^{-1}=\left[\begin{array}{cc}
\cos \theta_{r} & -\sin \theta_{r} \\
\sin \theta_{r} & \cos \theta_{r}
\end{array}\right]
$$




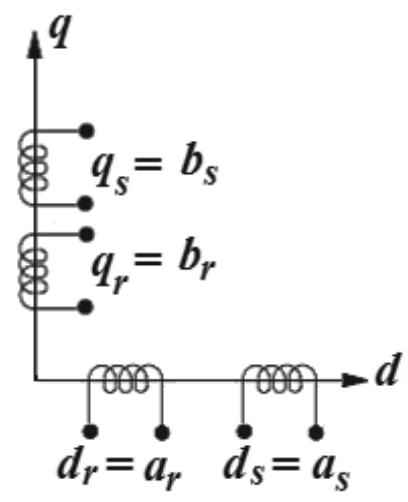

Fig. 2. Stationary reference frame $(d-q)$.

Applying the transformation matrix to Equations (14) and (17), then

$$
\left[\begin{array}{l}
v_{d s}^{s} \\
v_{q s}^{s} \\
0 \\
0
\end{array}\right]=\left[\begin{array}{cccc}
r_{d s}+\frac{d L_{d s}}{d t} & 0 & \frac{d L_{s r d}}{d t} & 0 \\
0 & r_{q s}+\frac{d L_{q s}}{d t} & 0 & \frac{d L_{s r q}}{d t} \\
\frac{d L_{s r d}}{d t} & p \omega_{r} L_{s r q} & r_{r}+\frac{d L_{r}}{d t} & p \omega_{r} L_{r} \\
-p \omega_{r} L_{s r d} & \frac{d L_{s r q}}{d t} & -p \omega_{r} L_{r} & r_{r}+\frac{d L_{r}}{d t}
\end{array}\right]\left[\begin{array}{c}
i_{d s}^{s} \\
i_{q s}^{s} \\
i_{d r}^{s} \\
i_{q r}^{s}
\end{array}\right]
$$

where the superscript $s$ denotes the stationary frame. The torque can also be expressed as

$$
p\left(T_{e}-T_{m}\right)=J \frac{d \omega_{r}}{d t}+B \omega_{r}
$$

This analytical approach represents the single-phase induction motor as an asymmetric twophase motor. The $d q$ voltages, currents, and fluxes for stator and rotor are, respectively: $v_{d s}^{s}, v_{q s}^{s}, i_{d s}^{s}, i_{q s}^{s}, i_{d r}^{s}, i_{q r}^{s}, \lambda_{d s}^{s}, \lambda_{q s}^{s}, \lambda_{d r}^{s}, \lambda_{q r}^{s}$. The terms $L_{d s}, L_{q s}, L_{r}, L_{s r d}, L_{s r q}$ denote the stator and rotor self-inductance and their respective mutual inductance. The stator and rotor resistance are denoted by $r_{d s}, r_{q s}, r_{r}$. The motor electromagnetic-torque and the load torque are indicated by $T_{e}$ and $T_{m}$, respectively. The moment of inertia, viscous friction coefficient and the motor speed are, respectively: $J, B$ and $\omega_{r}$.

One of the drawbacks of dealing with an asymmetric motor lies in the pulsating electromagnetic torque that occurs due to the unbalance between the stator variables, as can be seen in (19) and (20). To overcome this drawback and to create a symmetric model, a transformation of the stator variables employing the mutual inductances was proposed by Correa et al. (2004). The transformation matrix and its application can be written as 


$$
\begin{gathered}
S=\left[\begin{array}{ll}
1 & 0 \\
0 & n
\end{array}\right] \\
{\left[\begin{array}{l}
v_{d s}^{s} \\
v_{q s}^{s}
\end{array}\right]=S\left[\begin{array}{l}
v_{d s 1}^{s} \\
v_{q s 1}^{s}
\end{array}\right]} \\
{\left[\begin{array}{l}
i_{d s}^{s} \\
i_{q s}^{s}
\end{array}\right]=S^{-1}\left[\begin{array}{l}
i_{d s 1}^{s} \\
i_{q s 1}^{s}
\end{array}\right]} \\
{\left[\begin{array}{l}
\lambda_{d s}^{s} \\
\lambda_{q s}^{s}
\end{array}\right]=S^{-1}\left[\begin{array}{l}
\lambda_{d s 1}^{s} \\
\lambda_{q s 1}^{s}
\end{array}\right]}
\end{gathered}
$$

The transformation element can be written as $n=\frac{L_{s r d}}{L_{s r q}}$.

When these transformations are applied to the mathematical model of the motor, the unbalance between the main and auxiliary windings stator variables is eliminated. The new symmetrical model of the single-phase induction motor acting as a two-phase system can be given by the following equations:

$$
\begin{gathered}
{\left[\begin{array}{c}
v_{d s 1}^{s} \\
v_{q s 1}^{s}
\end{array}\right]=\left[\begin{array}{cc}
r_{d s} & 0 \\
0 & r_{q s}^{\prime}
\end{array}\right] \cdot\left[\begin{array}{l}
i_{d s 1}^{s} \\
i_{q s 1}^{s}
\end{array}\right]+\frac{d}{d t}\left[\begin{array}{c}
\lambda_{d s 1}^{s} \\
\lambda_{q s 1}^{s}
\end{array}\right]} \\
{\left[\begin{array}{c}
v_{d r}^{s} \\
v_{q r}^{s}
\end{array}\right]=\left[\begin{array}{cc}
r_{r} & 0 \\
0 & r_{r}
\end{array}\right] \cdot\left[\begin{array}{l}
i_{d r}^{s} \\
i_{q r}^{s}
\end{array}\right]+\frac{d}{d t}\left[\begin{array}{c}
\lambda_{d r}^{s} \\
\lambda_{q r}^{s}
\end{array}\right]+\omega_{r}\left[\begin{array}{cc}
0 & 1 \\
-1 & 0
\end{array}\right] \cdot\left[\begin{array}{l}
\lambda_{d r}^{s} \\
\lambda_{q r}^{s}
\end{array}\right]} \\
{\left[\begin{array}{l}
\lambda_{d s 1}^{s} \\
\lambda_{q s 1}^{s}
\end{array}\right]=\left[\begin{array}{cc}
L_{d s} & 0 \\
0 & L_{q s}^{\prime}
\end{array}\right] \cdot\left[\begin{array}{l}
i_{d s 1}^{s} \\
i_{q s 1}^{s}
\end{array}\right]+\left[\begin{array}{cc}
L_{s r d} & 0 \\
0 & L_{s r d}
\end{array}\right] \cdot\left[\begin{array}{l}
i_{d r}^{s} \\
i_{q r}^{s}
\end{array}\right]} \\
{\left[\begin{array}{l}
\lambda_{d r}^{s} \\
\lambda_{q r}^{s}
\end{array}\right]=\left[\begin{array}{cc}
L_{r} & 0 \\
0 & L_{r}
\end{array}\right] \cdot\left[\begin{array}{l}
i_{d r}^{s} \\
i_{q r}^{s}
\end{array}\right]+\left[\begin{array}{cc}
L_{s r d} & 0 \\
0 & L_{s r d}
\end{array}\right] \cdot\left[\begin{array}{l}
i_{d s 1}^{s} \\
i_{q s 1}^{s}
\end{array}\right]} \\
T_{e}=P L_{s r d}\left(i_{q s 1}^{s} i_{d r}^{s}-i_{d s 1}^{s} i_{q r}^{s}\right)
\end{gathered}
$$

where $L_{q s}^{\prime}=n^{2} L_{q s}$ and $r_{q s}^{\prime}=n^{2} r_{q s}$.

According to Correa et al. (2004), if the asymmetry that appears in the motor model depends only on the number of turns of each stator winding, then the ratio presented by $n$ will corresponds approximately to refer the auxiliary winding variables to the main winding. Also, if the inductances are slightly different, then $L_{q s}^{\prime} \approx L_{d s}$. 
Observing Equation (26) there is an asymmetry between the stator resistances. This term can be isolated

$$
\left[\begin{array}{c}
v_{d s 1}^{s} \\
v_{q s 1}^{s}
\end{array}\right]=\left[\begin{array}{cc}
r_{d s} & 0 \\
0 & r_{d s}
\end{array}\right] \cdot\left[\begin{array}{l}
i_{d s 1}^{s} \\
i_{q s 1}^{s}
\end{array}\right]+\frac{d}{d t}\left[\begin{array}{l}
\lambda_{d s 1}^{s} \\
\lambda_{q s 1}^{s}
\end{array}\right]+\Delta r
$$

The term $\Delta r$ is given by

$$
\Delta r=\left[\begin{array}{c}
0 \\
\left(n^{2} r_{q s}-r_{d s}\right) i_{q s 1}^{s}
\end{array}\right]
$$

When dealing with motor control, it is necessary to write the dynamic equations of the induction motor in an arbitrary reference frame. This transformation allows the $d-q$ variables to be treated as dc signals, which is commonly used in control theory.

If $\Delta r$ is considered negligible, a symmetric model of the single-phase induction motor in the arbitrary reference frame can be derived as shown in Fig. 3.

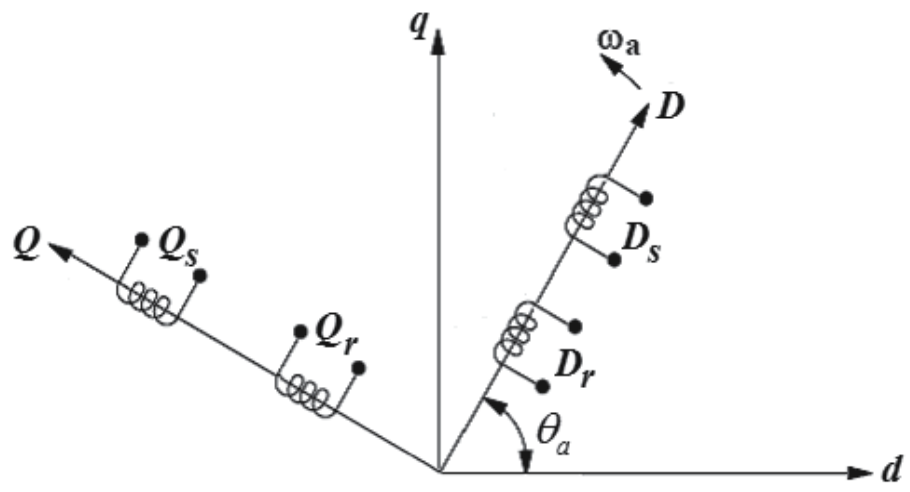

Fig. 3. Revolving reference frame.

The transformation matrix is given by

$$
T_{a}=\left[\begin{array}{cc}
\cos \theta_{a} & -\sin \theta_{a} \\
\sin \theta_{a} & \cos \theta_{a}
\end{array}\right] ; T_{a}^{-1}=\left[\begin{array}{cc}
\cos \theta_{a} & \sin \theta_{a} \\
-\sin \theta_{a} & \cos \theta_{a}
\end{array}\right]
$$

When applying the transformation to the motor model based on the stationary reference frame, given by (26) and (27), the resulting equations can be written in a different matrix form:

$$
\begin{aligned}
& {\left[\begin{array}{c}
v_{D s 1}^{a} \\
v_{\mathrm{Q} s 1}^{a}
\end{array}\right]=\left[\begin{array}{cc}
r_{s} & 0 \\
0 & r_{s}
\end{array}\right] \cdot\left[\begin{array}{l}
i_{D s 1}^{a} \\
i_{\mathrm{Q} s 1}^{a}
\end{array}\right]+\frac{d}{d t}\left[\begin{array}{l}
\lambda_{\mathrm{Ds} 1}^{a} \\
\lambda_{\mathrm{Q} s 1}^{a}
\end{array}\right]+\omega_{a}\left[\begin{array}{cc}
0 & -1 \\
1 & 0
\end{array}\right] \cdot\left[\begin{array}{l}
\lambda_{\mathrm{Ds} 1}^{a} \\
\lambda_{\mathrm{Q} s 1}^{a}
\end{array}\right]} \\
& {\left[\begin{array}{l}
0 \\
0
\end{array}\right]=\left[\begin{array}{cc}
r_{r} & 0 \\
0 & r_{r}
\end{array}\right] \cdot\left[\begin{array}{l}
i_{D r}^{a} \\
i_{\mathrm{Q} r}^{a}
\end{array}\right]+\frac{d}{d t}\left[\begin{array}{c}
\lambda_{D r}^{a} \\
\lambda_{\mathrm{Q} r}^{a}
\end{array}\right]+\left(\omega_{a}-p \omega_{r}\right)\left[\begin{array}{cc}
0 & -1 \\
1 & 0
\end{array}\right] \cdot\left[\begin{array}{l}
\lambda_{D r}^{a} \\
\lambda_{\mathrm{Q} r}^{a}
\end{array}\right]}
\end{aligned}
$$




$$
\begin{aligned}
& {\left[\begin{array}{l}
\lambda_{D s 1}^{a} \\
\lambda_{\mathrm{Q} s 1}^{a}
\end{array}\right]=\left[\begin{array}{cc}
L_{d s} & 0 \\
0 & L_{d s}
\end{array}\right] \cdot\left[\begin{array}{c}
i_{D s 1}^{a} \\
i_{\mathrm{Q} s 1}^{a}
\end{array}\right]+\left[\begin{array}{cc}
L_{s r d} & 0 \\
0 & L_{s r d}
\end{array}\right] \cdot\left[\begin{array}{c}
i_{D r}^{a} \\
i_{\mathrm{Q} r}^{a}
\end{array}\right]} \\
& {\left[\begin{array}{c}
\lambda_{\mathrm{D} r}^{a} \\
\lambda_{\mathrm{Q} r}^{a}
\end{array}\right]=\left[\begin{array}{cc}
L_{r} & 0 \\
0 & L_{r}
\end{array}\right] \cdot\left[\begin{array}{c}
i_{D r}^{a} \\
i_{\mathrm{Q} r}^{a}
\end{array}\right]+\left[\begin{array}{cc}
L_{s r d} & 0 \\
0 & L_{s r d}
\end{array}\right] \cdot\left[\begin{array}{c}
i_{D s 1}^{a} \\
i_{\mathrm{Q} s 1}^{a}
\end{array}\right]}
\end{aligned}
$$

The torque equation yields

$$
T_{e}=p L_{s r d}\left[i_{Q s 1}^{a} i_{D r}^{a}-i_{D s 1}^{a} i_{Q r}^{a}\right]
$$

\section{Conventional DTC scheme for single-phase induction motor}

The basic DTC scheme consists of a logical switching table, which calculates the right voltage vector to be applied by the inverter to obtain as fast a torque response as possible at every instant. The control is based on error between the references and estimated values of torque and flux magnitude and also uses the position of the estimated flux vector. The torque and flux magnitude error signals are the inputs to the torque and flux hysteresis controllers, respectively. That way, both the stator flux magnitude and the developed torque can be directly controlled by proper selection of stator voltage space vectors in order to reduce the torque and flux errors within the prefixed limits. The hysteresis determines the inverter switching frequency, which varies with the synchronous speed and load conditions. This technique achieves robust and fast torque response. Fig 4 shows the block diagram for the conventional DTC scheme applied to a single-phase induction motor.

To determine the proper voltage vectors to be applied, a logical switching table must be made. This can be achieved by dividing the $d q$ plane into six sectors. A power inverter will be employed in a three-leg configuration. That way the $d q$ plane has the form of an asymmetric hexagon, as shown in Fig 5. There are six active vectors and two zero vectors ( $V_{7}$ and $V o$ ). The zero vectors are used to reduce the torque. Since a single-phase induction motor can be viewed as a two-phase induction motor, there is no need for three-to-two phase transformation for the primary voltage and current. Another aspect concerning this method of control is the absence of field orientation which makes the control task a less complex issue.

So for the conventional DTC, the following equations are derived from (26) to estimate the stator flux

$$
\begin{aligned}
& \lambda_{s d}^{s}=\int\left(v_{s d}^{s}-r_{s d} i_{s d}^{s}\right) d t \\
& \lambda_{s q}^{s}=\int\left(v_{s q}^{s}-r_{s q} i_{s q}^{s}\right) d t
\end{aligned}
$$

The electromagnetic torque as a function of stator variables can be written as:

$$
T_{e}=p\left(i_{s q}^{s} \lambda_{s d}^{s}-i_{s d}^{s} \lambda_{s q}^{s}\right)
$$

Estimation of the stator flux by pure integration can present instability, especially at low speeds. This problem can be overcome by using a low-pass filter instead of a pure integrator or other filtering techniques presented in $\mathrm{Hu}$ (1998). 


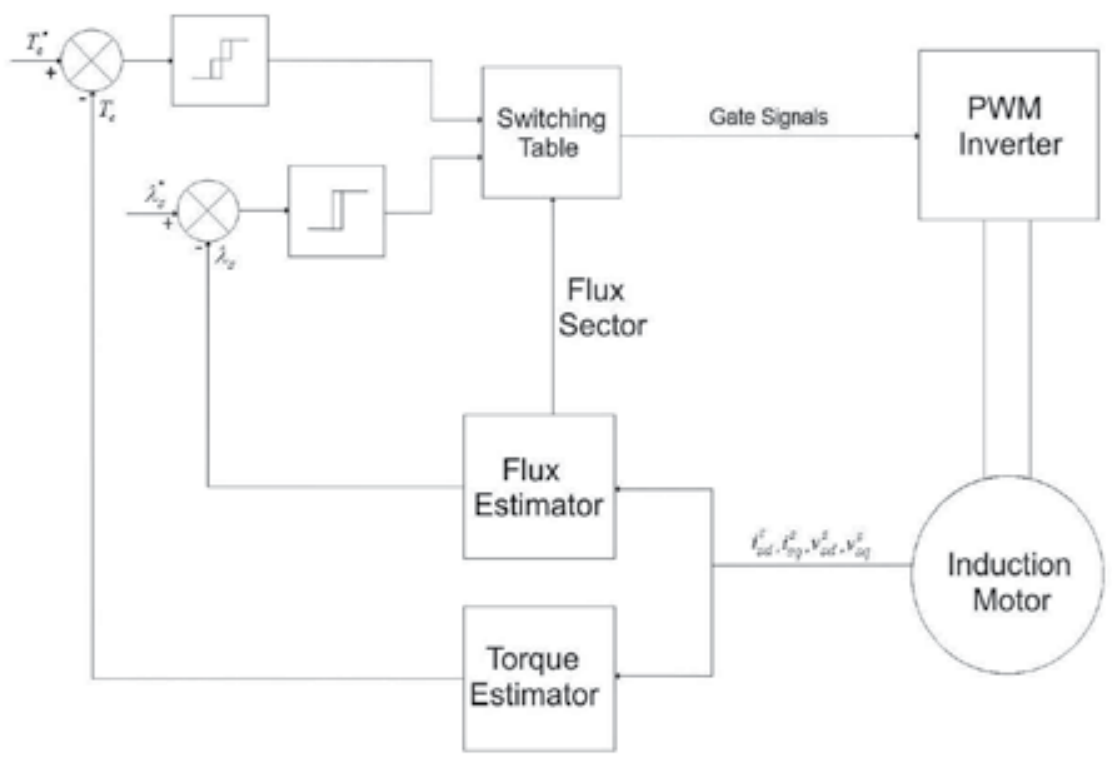

Fig. 4. Block diagram of the basic DTC scheme.

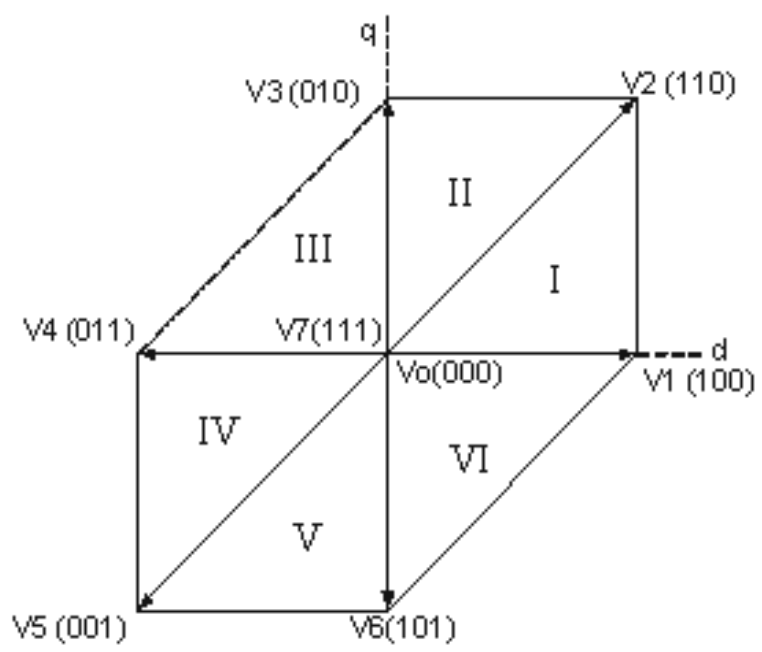

Fig. 5. Space-vectors hexagon.

Since DTC uses hysteresis control resulting in a so-called bang-bang control of the torque and flux, the result is a fast response of the control commands. However, the steady state performance is characterized by undesirable ripples in current, flux and torque. To avoid such effects, a high switching frequency should be delivered by the hysteresis loops. But the amplitude of the hysteresis band has a strong effect on those undesirable ripples mentioned above (Noguchi and Takahashi, 1997). To eliminate these issues, instead of using a switching table, a pulse width modulator can be used. Basically, the DTC scheme can be implemented by means of a closed-loop PI controller which will calculate the required stator 
voltage vector and then will be synthesized by a PWM technique (Jabbar et al., 2004). Therefore, the pulse width modulator is used to optimize the steady state drive performance.

\section{Space vector modulation technique}

To control the three-leg inverter driving a single-phase induction motor indirectly, space vector PWM can be employed. This approach is known to deliver less harmonic distortion in the output voltages applied to the phases of the induction motor. Consequently, the ripples in the stator current are diminished and a smooth flux and torque waveform is obtained.

In Correa et al. (2002), Tomaselli et al. (2004) and Jabbar et al. (2004), a space vector modulation applied to a three-leg inverter driving a single-phase motor is presented. As shown in Fig. 6, the spatial disposition of the space vectors differs from the one found when the same inverter system drives a three-phase induction motor. In the latter, the space vector displays a symmetric hexagon (Zhou, 2002). For the single-phase system, the space vectors form an asymmetric hexagon (Jabbar et al., 2004). Since the voltages supplied by the threeleg inverter to the motor phases are sinusoidal waves $90^{\circ}$ degrees apart from each other, the stator voltage vector can be decomposed into real and imaginary parts,

$$
v^{*}=v_{d s}^{s^{*}}+j v_{q s}^{s^{*}}
$$

The reference vector $v^{*}$ is used to determine the switching signals that will drive the threeleg inverter. The adjacent vectors $v_{d s}^{s^{*}}$ and $v_{q s}^{s^{*}}$ display the magnitude of the resulting switching vectors. Similar to the three-phase inverter feeding a three-phase induction motor, in a three-phase inverter feeding a two-phase induction motor there are eight voltage vectors to be applied (six active vectors and two zero vectors). Table I presents the eight voltage vectors that can be applied to the motor $\left(V_{n}\right.$ indicates the resulting space vectors with $n=0,1,2, \ldots, 7)$.

From the asymmetric hexagon, one can see that there are four vectors with amplitude $\mathrm{E}$ $\left(V_{1}, V_{3}, V_{4}, V_{6}\right)$, two vectors with amplitude $\sqrt{2} \mathrm{E}\left(V_{2}, V_{5}\right)$ and two null vectors $\left(V_{0}, V_{7}\right)$.

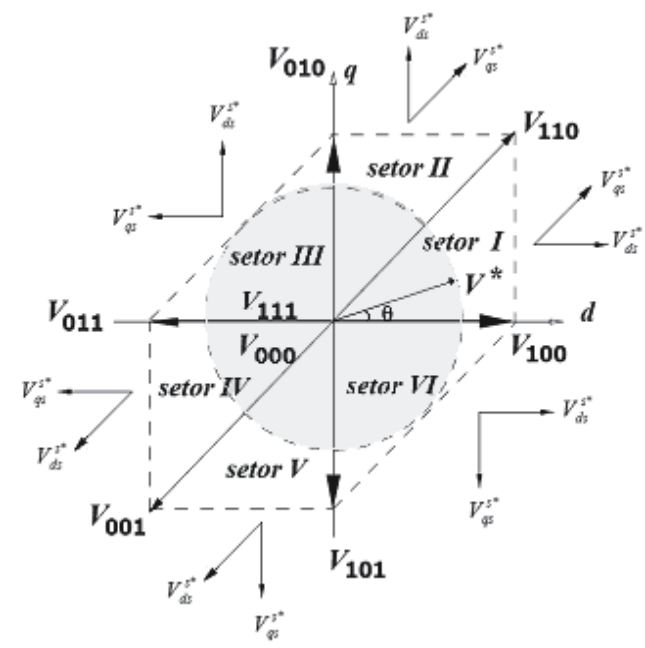

Fig. 6. Space Vectors spatial disposition. 


\begin{tabular}{|c||c|c|c|c|c|}
\hline$V_{n}$ & $\mathrm{~S}_{1}$ & $\mathrm{~S}_{2}$ & $\mathrm{~S}_{3}$ & $v_{d s}^{s}$ & $v_{q s}^{s}$ \\
\hline \hline$V_{0}$ & 0 & 0 & 0 & 0 & 0 \\
\hline$V_{1}$ & 1 & 0 & 0 & $\mathrm{E}$ & 0 \\
\hline$V_{2}$ & 1 & 1 & 0 & $\mathrm{E}$ & $\mathrm{E}$ \\
\hline$V_{3}$ & 0 & 1 & 0 & 0 & $\mathrm{E}$ \\
\hline$V_{4}$ & 0 & 1 & 1 & $-\mathrm{E}$ & 0 \\
\hline$V_{5}$ & 0 & 0 & 1 & $-\mathrm{E}$ & $-\mathrm{E}$ \\
\hline$V_{6}$ & 1 & 0 & 1 & 0 & $-\mathrm{E}$ \\
\hline$V_{7}$ & 1 & 1 & 1 & 0 & 0 \\
\hline
\end{tabular}

Table 1. Switching states and the adjacent vectors amplitude.

According to Jabbar et al. (2004), the reference vector and the adjacent vectors can be related by the following equation:

$$
T_{p w m} V^{*}=t_{d s} \cdot V_{d s}^{s^{*}}+t_{q s} \cdot V_{q s}^{s^{*}}
$$

In Equation (19), $t_{d s}$ and $t_{q s}$ are the durations in time in which the vectors $V_{d s}^{s^{*}}$ and $V_{q s}^{s^{*}}$ are within period $T_{p w m}$. The period of duration of the zero vectors can be defined by

$$
t_{0}=T_{p w m}-t_{d s}-t_{q s}
$$

\section{SVPWM-DTC Proposed Scheme}

As mentioned in Section 3, to optimize the steady state performance and to diminish the switching harmonics, a pulse width modulator can be applied. An improvement can be achieved when torque and stator flux magnitude are controlled by PI controllers using a closed-loop. The DTC strategy adapted to single-phase induction motors was discussed in Jacobina et al. (1999) and Neves et al. (2002). Fig. 7 shows the proposed scheme. The essence of DTC is kept, since its principle of accelerating the flux vector to increase torque is maintained, and there is no need for speed or position signals. The output signals of the PI controllers can be viewed as stator voltage components operating in Cartesian coordinates. After the reference frame transformation, the PWM is fed with the stator voltage components in the stationary frame. The control strategy relies on the stator flux orientation; therefore, the arbitrary reference frame should be aligned with the stator flux vector, $\theta_{a}=\theta_{s f}$.

The condition to achieve the field orientation can be expressed by

$$
\lambda_{D s 1}^{s f}=\lambda_{s} ; \lambda_{Q s 1}^{s f}=0
$$

To determine the dynamic equations for the proposed technique, some algebraic manipulations must be done. Taking the Equations (34), (35), (37), aligned with the stator reference frame, the designed control signals can be derived:

$$
v_{D s 1}^{s f}=\frac{\lambda_{s}}{\tau_{d s} \sigma_{s}}+\frac{d \lambda_{s}}{d t}-\frac{L_{s r d}}{\tau_{d s} \sigma_{s} L_{r}}\left[\frac{L_{r}}{L_{s r d}} \lambda_{s}-\frac{L_{r} L_{s d}}{L_{s r d}} i_{D s 1}^{s f}+L_{s r d} i_{D s 1}^{s f}\right]
$$




$$
v_{Q s 1}^{s f}=r_{q s} i_{Q s 1}^{s f}+L_{q s} \sigma_{s} \frac{d i_{Q s 1}^{s f}}{d t}+\frac{L_{s r d}}{L_{r}} \frac{d \lambda_{Q r}^{s f}}{d t}
$$

where,

$$
\sigma_{s}=1-L_{s r d}^{2} /\left(L_{r} L_{s d}\right)
$$

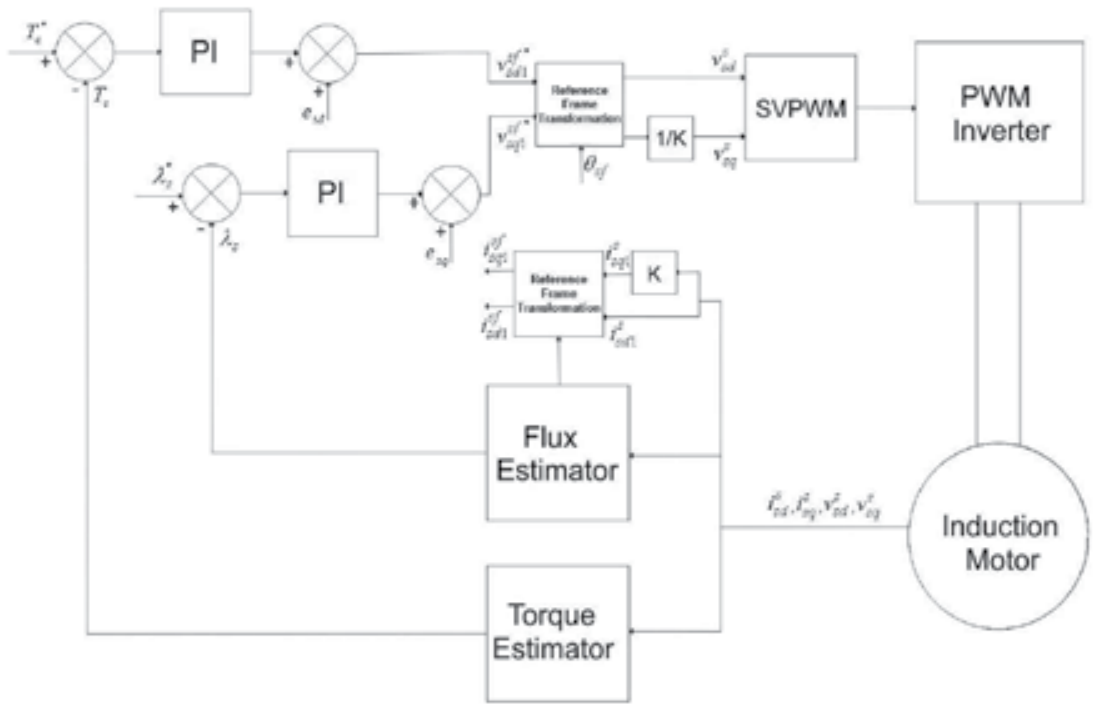

Fig. 7. SVPWM-DTC proposed scheme.

By manipulating (46) and (47) in the synchronous frame, the designed control signals can be obtained as:

$$
\begin{gathered}
v_{D s 1}^{s f}=\frac{\lambda_{s}}{\tau_{d s} \sigma_{s}}+\frac{d \lambda_{s}}{d t}+e_{d s} \\
v_{Q s 1}^{s f}=r_{q s} i_{Q s 1}^{s f}+L_{q s} \sigma_{s} \frac{d i_{Q s 1}^{s f}}{d t}+e_{q s}
\end{gathered}
$$

assuming the terms $e_{d s}$ and $e_{q s}$ as feed-forward elements and given by (51) and (52), and considering the terms that indicate the asymmetry and disturbance negligible:

$$
\begin{gathered}
e_{d s}=-\frac{L_{s r d}}{\tau_{d s} \sigma_{s} L_{r}}\left[\frac{L_{r}}{L_{s r d}} \lambda_{s}-\frac{L_{r} L_{d s}}{L_{s r d}} i_{s D 1}^{s f}+L_{s r d} i_{s D 1}^{s f}\right] \\
e_{q s}=\frac{L_{s r d}}{L_{r}}\left[\frac{r_{r} L_{s d}}{L_{s r d}} i_{s Q 1}^{s f}\right]
\end{gathered}
$$

The torque as function of stator flux and currents is given by: 


$$
T_{e}=p\left(i_{Q s 1}^{s f} \lambda_{D s 1}^{s f}-i_{D s 1}^{s f} \lambda_{Q s 1}^{s f}\right)
$$

A 4 poles, $1 / 4 \mathrm{HP}, 110 \mathrm{~V}, 60 \mathrm{~Hz}$, asymmetrical 2-phase induction machine was used with the following parameters expressed in ohms (Krause et al., 1995):

$r_{d s}=2.02 ;$

$X_{l d}=2.79 ; X_{m d}=66.8 ;$

$r_{q s}=7.14 ;$

$X_{l q}=3.22 ; X_{m q}=92.9 ;$

$r_{r}^{\prime}=4.12 ;$

$X^{\prime}{ }_{l r}=2.12$.

The total inertia is $J=1.46 \times 10^{-2} \mathrm{kgm}^{2}$ and $N_{s d} / N_{s q}=1.18$, where $N_{s d}$ is the number of turns of the main winding and $N_{s q}$ is the number of turns of the auxiliary winding. It was considered a squirrel cage motor type with only the $d$ rotor axis parameters.

In terms of stator-flux field-orientation

$$
T_{e}=p\left(i_{Q s 1}^{s f} \lambda_{s}\right)
$$

According to (49) and (50), the stator flux control can be accomplished by $v_{D s 1}^{s f}$ and torque control by $v_{\mathrm{Q} s 1}^{s f}$. The stator voltage reference values $v_{D s 1}^{s f^{*}}$ and $v_{\mathrm{Q} s 1}^{s f *}$ are produced by two PI controllers. The stator flux position is used in a reference frame transformation to orient the $d q$ stator currents. Although there is a current loop to decouple the flux and torque control, the DTC scheme is seen as a control scheme operating with closed torque and flux loops without current controllers (Jabbar et al., 2004).

\section{Simulation results}

Some simulations were carried out in order to evaluate the control strategy performance. The motor is fed by an ideal voltage source. The reference flux signal is kept constant at 0.4 $W b$. The reference torque signal is given by: $(0,1,-1,0.5) N m$ at $(0,0.2,0.4,0.6) s$, respectively. The SVPWM method used produced $d q$ axes voltages. The switching frequency was set to $5 \mathrm{kHz}$. Fig. 8 shows the actual value of the motor speed. In Fig. 9 and Fig. 10, the torque

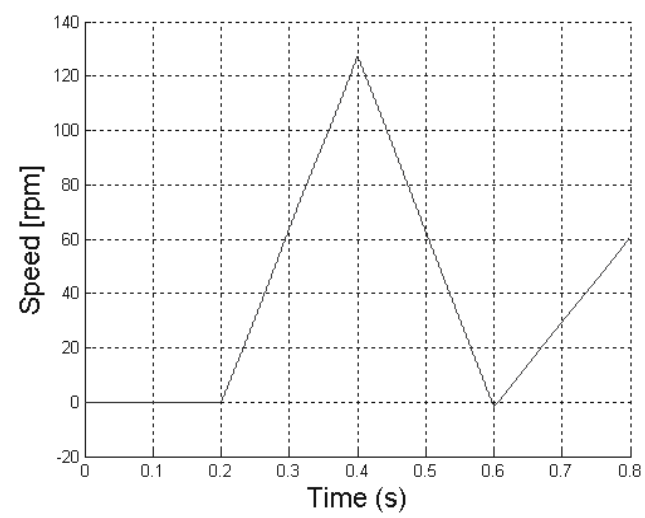

Fig. 8. Motor speed (rpm). 


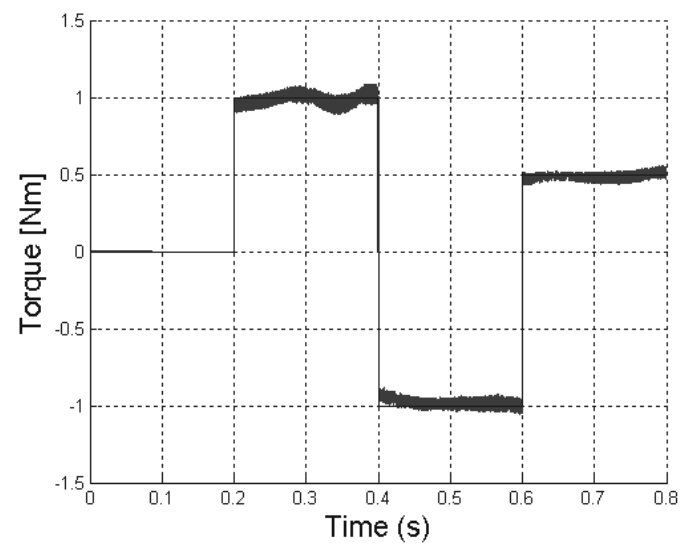

Fig. 9. Commanded and estimated torque (Nm).

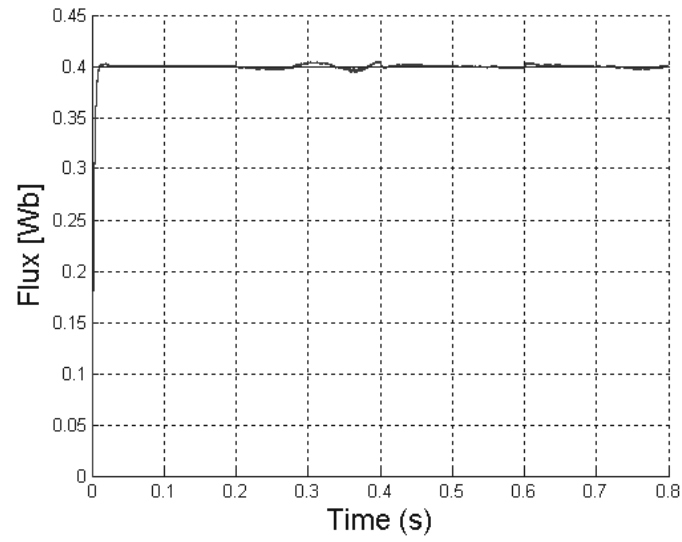

Fig. 10. Commanded and estimated flux

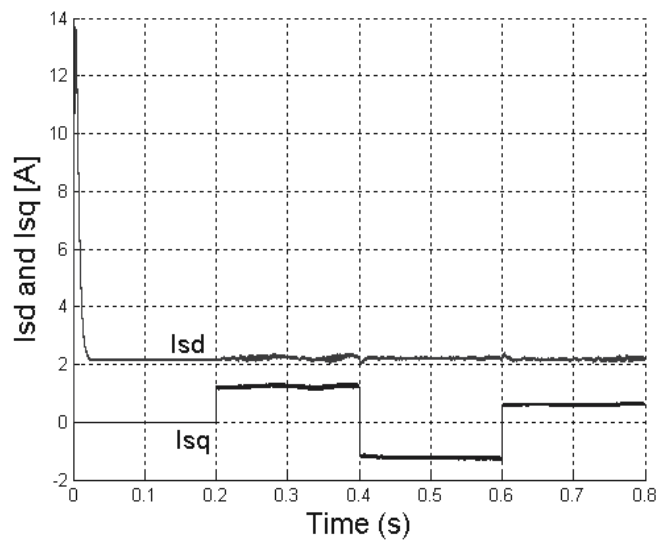

Fig. 11. Stator currents in stator flux reference frame. 
waveform and the flux waveform are presented. Although the torque presents some oscillations, the flux control is not affected. The good response in flux control can be seen. Fig. 11 shows the relation between the $d$ stator current component to the flux production and the $q$ stator current component to the torque production.

\section{Conclusion}

The investigation carried out in this paper showed that DTC strategy applied to a singlephase induction motor represents an alternative to the classic FOC control approach. Since the classic direct torque control consists of selection of consecutive states of the inverter in a direct manner, ripples in torque and flux appear as undesired disturbances. To minimize these disturbances, the proposed SVPWM-DTC scheme considerably improves the drive performance in terms of reduced torque and flux pulsations, especially at low-speed operation. The method is based on the DTC approach along with a space-vector modulation design to synthesize the necessary voltage vector.

Two PI controllers determine the $d q$ voltage components that are used to control flux and torque. Like a field orientation approach, the stators currents are decoupled but not controlled, keeping the essence of the DTC.

The transient waveforms show that torque control and flux control follow their commanded values. The proposed technique partially compensates the ripples that occur on torque in the classic DTC scheme. The proposed method results in a good performance without the requirement for speed feedback. This aspect decreases the final cost of the system. The results obtained by simulation show the feasibility of the proposed strategy.

\section{References}

Buja, G. S. and Kazmierkowski, M. P. (2004). Direct Torque Control of PWM Inverter-Fed AC Motors - A Survey, IEEE Transactions on Industrial Electronics, vol. 51, no. 4, pp. 744-757.

Campos, R. de F.; de Oliveira, J; Marques, L. C. de S.; Nied, A. and Seleme Jr., S. I. (2007a). SVPWM-DTC Strategy for Single-Phase Induction Motor Control, IEMDC2007, Antalya, Turkey, pp. 1120-1125.

Campos, R. de F.; Pinto, L. F. R.; de Oliveira, J.; Nied, A.; Marques, L. C. de S. and de Souza, A. H. (2007b). Single-Phase Induction Motor Control Based on DTC Strategies, ISIE2007, Vigo, Spain, pp. 1068-1073.

Corrêa, M. B. R.; Jacobina, C. B.; Lima, A. M. N. and da Silva, E. R. C. (2004).Vector Control Strategies for Single-Phase Induction Motor Drive Systems, IEEE Transactions on Industrial Electronics, vol. 51, no. 5, pp. 1073-1080.

Charumit, C. and Kinnares, V. (2009). Carrier-Based Unbalanced Phase Voltage Space Vector PWM Strategy for Asymmetrical Parameter Type Two-Phase Induction Motor Drives, Electric Power Systems Research, vol. 79, no. 7, pp. 1127-1135.

dos Santos, E.C.; Jacobina, C.B.; Correa, M. B. R. and Oliveira, A.C. (2010). Generalized Topologies of Multiple Single-Phase Motor Drives, IEEE Transactions on Energy Conversion, vol. 25, no. 1, pp. 90-99.

Jabbar, M. A.; Khambadkone, A. M. and Yanfeng, Z. (2004). Space-Vector Modulation in a Two-Phase Induction Motor Drive for Constant-Power Operation, IEEE Transactions on Industrial Electronics, vol. 51, no. 5, pp. 1081-1088. 
Jacobina, C. B.; Correa, M. B. R.; Lima, A. M. N. and da Silva, E. R. C. (1999). Single-phase Induction Motor Drives Systems, APEC'99, Dallas, Texas, vol. 1, pp. 403-409.

Krause, P. C.; O. Wasynczuk, O. and Sudhoff, S. D. (1995). Analysis of Electric Machinery. Piscataway, NJ: IEEE Press.

Neves, F. A. S.; Landin, R. P.; Filho, E. B. S.; Lins, Z. D.; Cruz, J. M. S. and Accioly, A. G. H. (2002). Single-Phase Induction Motor Drives with Direct Torque Control, IECON’02, vol.1, pp. 241-246.

Takahashi, I. and Noguchi, T. (1986). A New Quick-Response and High-Efficiency Control Strategy of an Induction Motor, IEEE Transactions on Industry Applications, vol. IA-22, no. 5, pp.820-827.

Noguchi, T. and Takahashi, I. (1997), High frequency switching operation of PWM inverter for direct torque control of induction motor, in Conf. Rec. IEEE-IAS Annual Meeting, pp. 775-780.

Wekhande, S. S.; Chaudhari, B. N.; Dhopte, S. V. and Sharma, R. K. (1999). A Low Cost Inverter Drive For 2-Phase Induction Motor, IEEE 1999 International Conference on Power Electronics and Drive Systems, PEDS'99, July 1999, Hong Kong.

$\mathrm{Hu}, \mathrm{J}$. and $\mathrm{Wu}, \mathrm{B}$. (1998). New Integration Algorithms for Estimating Motor Flux over a Wide Speed Range. IEEE Transactions on Power Electronics, vol. 13, no. 5, pp. 969977. 


\title{
The Space Vector Modulation PWM Control Methods Applied on Four Leg Inverters
}

\author{
Kouzou A, Mahmoudi M.O and Boucherit M.S \\ Djelfa University and ENP Algiers, \\ Algeria
}

\section{Introduction}

Up to now, in many industrial applications, there is a great interest in four-leg inverters for three-phase four-wire applications. Such as power generation, distributed energy systems [1-4], active power filtering [5-20], uninterruptible power supplies, special control motors configurations [21-25], military utilities, medical equipment[26-27] and rural electrification based on renewable energy sources[28-32]. This kind of inverter has a special topology because of the existence of the fourth leg; therefore it needs special control algorithm to fulfil the subject of the neutral current circulation which was designed for. It was found that the classical three-phase voltage-source inverters can ensure this topology by two ways in a way to provide the fourth leg which can handle the neutral current, where this neutral has to be connected to the neutral connection of three-phase four-wire systems:

1. Using split DC-link capacitors Fig. 1, where the mid-point of the DC-link capacitors is connected to the neutral of the four wire network [34-48].

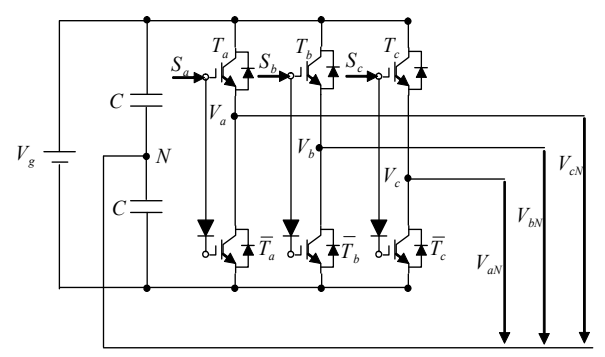

Fig. 1. Four legs inverter with split capacitor Topology.

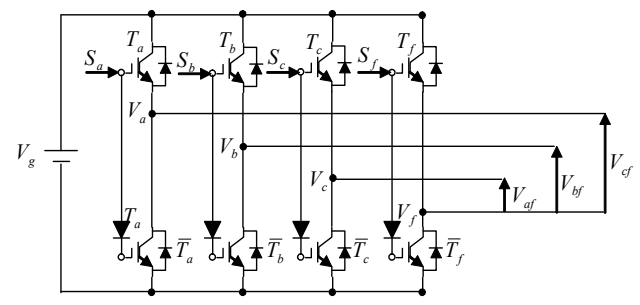

Fig. 2. Four legs inverter with and additional leg Topology. 
2. Using a four-leg inverter Fig. 2, where the mid-point of the fourth neutral leg is connected to the neutral of the four wire network,[22],[39],[45],[48-59].

It is clear that the two topologies allow the circulation of the neutral current caused by the non linear load or/and the unbalanced load into the additional leg (fourth leg). But the first solution has major drawbacks compared to the second solution. Indeed the needed DC side voltage required large and expensive DC-link capacitors, especially when the neutral current is important, and this is the case of the industrial plants. On the other side the required control algorithm is more complex and the unbalance between the two parts of the split capacitors presents a serious problem which may affect the performance of the inverter at any time, indeed it is a difficult problem to maintain the voltages equally even the voltage controllers are used. Therefore, the second solution is preferred to be used despite the complexity of the required control for the additional leg switches Fig.1. The control of the four leg inverter switches can be achieved by several algorithms [55],[[58],[60-64]. But the Space Vector Modulation SVM has been proved to be the most favourable pulse-width modulation schemes, thanks to its major advantages such as more efficient and high DC link voltage utilization, lower output voltage harmonic distortion, less switching and conduction losses, wide linear modulation range, more output voltage magnitude and its simple digital implementation. Several works were done on the SVM PWM firstly for three legs two level inverters, later on three legs multilevel inverters of many topologies [11],[43-46],[56-57],[6568]. For four legs inverters there were till now four families of algorithms, the first is based on the $\alpha \beta \gamma$ coordinates, the second is based on the $a b c$ coordinates, the third uses only the values and polarities of the natural voltages and the fourth is using a simplification of the two first families. In this chapter, the four families are presented with a simplified mathematical presentation; a short simulation is done for the fourth family to show its behaviours in some cases.

\section{Four leg two level inverter modelisation}

In the general case, when the three wire network has balanced three phase system voltages, there are only two independents variables representing the voltages in the three phase system and this is justified by the following relation :

$$
V_{a f}+V_{b f}+V_{c f}=0
$$

Whereas in the case of an unbalanced system voltage the last equation is not true:

$$
V_{a f}+V_{b f}+V_{c f} \neq 0
$$

And there are three independent variables; in this case three dimension space is needed to present the equivalent vector. For four wire network, three phase unbalanced load can be expected; hence there is a current circulating in the neutral:

$$
I_{L a}+I_{L b}+I_{L c}=I_{n} \neq 0
$$

$I_{n}$ is the current in the neutral. To built an inverter which can response to the requirement of the voltage unbalance and/or the current unbalance conditions a fourth leg is needed, this leg allows the circulation of the neutral current, on the other hand permits to achieve unbalanced phase-neutral voltages following to the required reference output voltages of 
the inverter. The four leg inverter used in this chapter is the one with a duplicated additional leg presented in Fig.1. The outer phase-neutral voltages of the inverter are given by:

$$
V_{i f}=\left[S_{a}-S_{f}\right] \cdot V_{g} \quad \text { where }: i=a, b, c
$$

$f$ designed the fourth leg and $S_{f}$ its corresponding switch state.

The whole possibilities of the switching position of the four-leg inverter are presented in Table 1. It resumes the output voltages of different phases versus the possible switching states

\begin{tabular}{|ccccc|}
\hline Vector & $S_{a} S_{b} S_{c} S_{f}$ & $V_{a f} / V_{g}$ & $V_{b f} / V_{g}$ & $V_{c f} / V_{g}$ \\
\hline$V^{1}$ & 1111 & 0 & 0 & 0 \\
\hline$V^{2}$ & 0010 & 0 & 0 & +1 \\
\hline$V^{3}$ & 0100 & 0 & +1 & 0 \\
\hline$V^{4}$ & 0110 & 0 & +1 & +1 \\
\hline$V^{5}$ & 1000 & +1 & 0 & 0 \\
\hline$V^{6}$ & 1010 & +1 & 0 & +1 \\
\hline$V^{7}$ & 1100 & +1 & +1 & 0 \\
\hline$V^{8}$ & 1110 & +1 & +1 & +1 \\
\hline$V^{9}$ & 0001 & -1 & -1 & -1 \\
\hline$V^{10}$ & 0011 & -1 & -1 & 0 \\
\hline$V^{11}$ & 0101 & -1 & 0 & -1 \\
\hline$V^{12}$ & 0111 & -1 & 0 & 0 \\
\hline$V^{13}$ & 1001 & 0 & -1 & -1 \\
\hline$V^{14}$ & 1011 & 0 & -1 & 0 \\
\hline$V^{15}$ & 1101 & 0 & 0 & -1 \\
\hline$V^{16}$ & 0000 & 0 & 0 & 0 \\
\hline
\end{tabular}

Table 1. Switching vectors of the four leg inverter

Equation (4) can be rewritten in details:

$$
\left[\begin{array}{c}
V_{a f} \\
V_{b f} \\
V_{c f}
\end{array}\right]=\left[\begin{array}{llll}
1 & 0 & 0 & -1 \\
0 & 1 & 0 & -1 \\
0 & 0 & 1 & -1
\end{array}\right] \cdot\left[\begin{array}{c}
S_{a} \\
S_{b} \\
S_{c} \\
S_{f}
\end{array}\right] \cdot V_{g}
$$

Where the variable $S_{i}$ is defined by:

$$
S_{i}=\left\{\begin{array}{l}
1 \text { if the upper switch of the leg } i \text { isclosed } \\
0 \quad \text { if the upper switch of the leg } i \text { is opened }
\end{array} \text { where: } i=a, b, c, f\right.
$$




\section{Three dimensional SVM in $a-b-c$ frame for four leg inverters}

The 3D SVM algorithm using the $a-b-c$ frame is based on the presentation of the switching vectors as they were presented in the previous table [34-35],[69-72]. The vectors were normalized dividing them by $V_{g}$. It is clear that the space which is containing all the space vectors is limited by a large cube with edges equal to two where all the diagonals pass by $(0,0,0)$ point inside this cube Fig. 3 , it is important to remark that all the switching vectors are located just in two partial cubes from the eight partial cubes with edges equal to one Fig. 4. The first one is containing vectors from $V^{1}$ to $V^{8}$ in this region all the components following the $a, b$ and $c$ axis are positive. The second cube is containing vectors from $V^{9}$ to $V^{16}$ with their components following the $a, b$ and $c$ axis are all negative. The common point $(0,0,0)$ is presenting the two nil switching vectors $V^{1}$ and $V^{16}$.

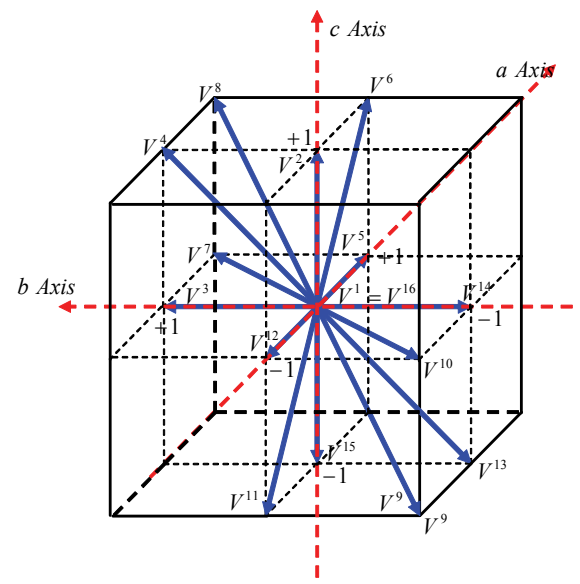

Fig. 3. The large space which is limiting the switching vectors

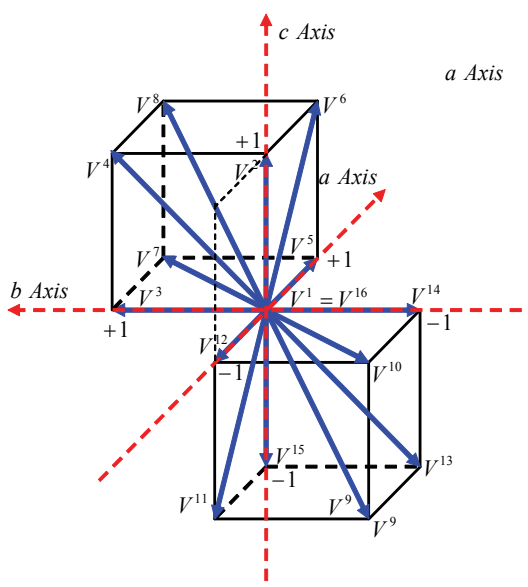

Fig. 4. The part of space which is limiting the space of switching vectors 


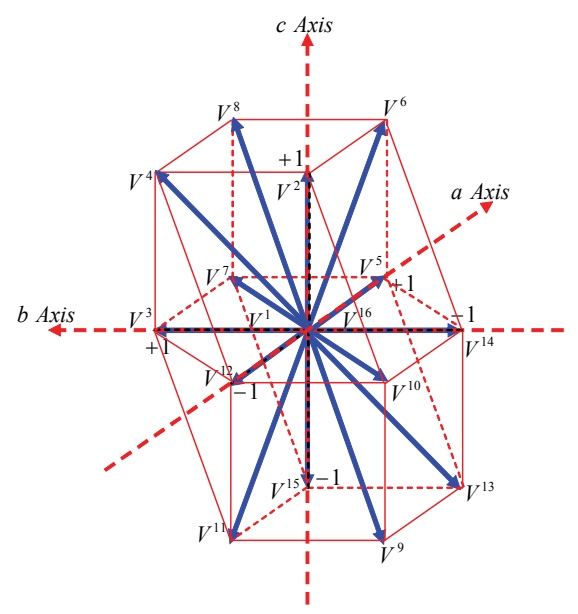

Fig. 5. The possible space including the voltage space vector (the dodecahedron) .

The instantaneous voltage space vector of the reference output voltage of the inverter travels following a trajectory inside the large cube space, this trajectory is depending on the degree of the reference voltage unbalance and harmonics, but it is found that however the trajectory, the reference voltage space vector is remained inside the large cube. The limit of this space is determined by joining the vertices of the two partial cubes. This space is presenting a dodecahedron as it is shown clearly in Fig. 5. This space is containing 24 tetrahedron, each small cube includes inside it six tetrahedrons and the space between the two small cubes includes 12 tetrahedrons, in Fig. 6 examples of the tetrahedrons given. In this algorithm a method is proposed for the determination of the tetrahedron in which the reference vector is located. This method is based on a region pointer which is defined as follows:

$$
R P=1+\sum_{i=1}^{6} C_{i} \cdot 2^{(i-1)}
$$

Where:

$$
C_{i}=\operatorname{Sign}(\operatorname{INT}(x(i)+1)) \quad i=1: 6
$$

The values of $x(i)$ are:

$$
x=\left[\begin{array}{c}
V_{\text {aref }} \\
V_{\text {bref }} \\
V_{c r e f} \\
V_{\text {aref }}-V_{\text {bref }} \\
V_{\text {bref }}-V_{c r e f} \\
V_{\text {aref }}-V_{c r e f}
\end{array}\right]
$$

Where the function Sign is: 


$$
\operatorname{Sign}(V)=\left\{\begin{array}{cc}
+1 & \text { if } V>1 \\
-1 & \text { if } V<1 \\
0 & \text { if } V=1
\end{array}\right.
$$
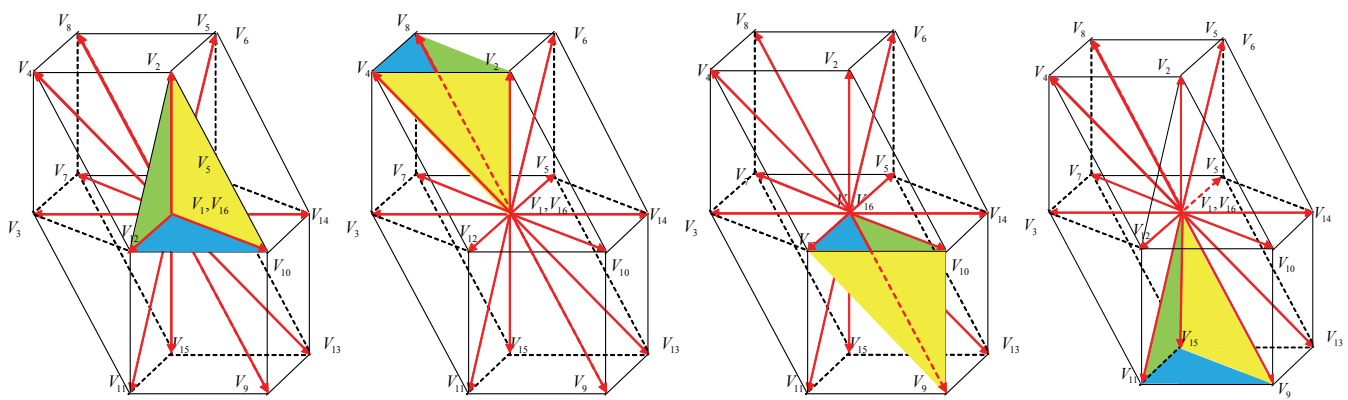

Fig. 6. The possible space including the voltage space vector (the dodecahedron).

\begin{tabular}{|cccccccc|}
\hline$R P$ & $V_{1}$ & $V_{2}$ & $V_{3}$ & $R P$ & $V_{1}$ & $V_{2}$ & $V_{3}$ \\
\hline 1 & $V^{9}$ & $V^{10}$ & $V^{12}$ & 41 & $V^{9}$ & $V^{13}$ & $V^{14}$ \\
\hline 5 & $V^{2}$ & $V^{10}$ & $V^{12}$ & 42 & $V^{5}$ & $V^{13}$ & $V^{14}$ \\
\hline 7 & $V^{2}$ & $V^{4}$ & $V^{12}$ & 46 & $V^{5}$ & $V^{6}$ & $V^{14}$ \\
\hline 8 & $V^{2}$ & $V^{4}$ & $V^{8}$ & 48 & $V^{5}$ & $V^{6}$ & $V^{8}$ \\
\hline 9 & $V^{9}$ & $V^{10}$ & $V^{14}$ & 49 & $V^{9}$ & $V^{11}$ & $V^{15}$ \\
\hline 13 & $V^{2}$ & $V^{10}$ & $V^{14}$ & 51 & $V^{3}$ & $V^{11}$ & $V^{15}$ \\
\hline 14 & $V^{2}$ & $V^{6}$ & $V^{14}$ & 52 & $V^{3}$ & $V^{7}$ & $V^{15}$ \\
\hline 16 & $V^{2}$ & $V^{6}$ & $V^{8}$ & 56 & $V^{3}$ & $V^{7}$ & $V^{8}$ \\
\hline 17 & $V^{9}$ & $V^{11}$ & $V^{12}$ & 57 & $V^{9}$ & $V^{13}$ & $V^{15}$ \\
\hline 19 & $V^{3}$ & $V^{11}$ & $V^{12}$ & 58 & $V^{5}$ & $V^{13}$ & $V^{15}$ \\
\hline 23 & $V^{3}$ & $V^{4}$ & $V^{12}$ & 60 & $V^{5}$ & $V^{7}$ & $V^{15}$ \\
\hline 24 & $V^{3}$ & $V^{4}$ & $V^{8}$ & 64 & $V^{5}$ & $V^{7}$ & $V^{8}$ \\
\hline
\end{tabular}

Table 2. The active vector of different tetrahedrons

Each tetrahedron is formed by three NZVs (non-zero vectors) confounded with the edges and two ZVs (zero vectors) $\left(V^{1}, V^{16}\right)$. The NZVs are presenting the active vectors nominated by $V_{1}, V_{2}$ and $V_{3}$ Tab. 2. The selection of the active vectors order depends on several parameters, such as the polarity change, the zero vectors ZVs used and on the sequencing scheme. $V_{1}, V_{2}$ and $V_{3}$ have to ensure during each sampling time the equality of the average value presented as follows:

$$
V_{\text {ref }} \cdot T_{z}=V_{1} \cdot T_{1}+V_{2} \cdot T_{2}+V_{3} \cdot T_{3}+V_{01} \cdot T_{01}+V_{016} \cdot T_{016} T_{z}=T_{1}+T_{2}+T_{3}+T_{01}+T_{016}
$$

The last thing in this algorithm is the calculation of the duty times. From the equation given in (9) the following equation can be deducted: 


$$
\left[\begin{array}{l}
V_{\text {aref }} \\
V_{\text {bref }} \\
V_{\text {cref }}
\end{array}\right]=\underbrace{\left[\begin{array}{lll}
V_{1 a} & V_{2 a} & V_{3 a} \\
V_{1 b} & V_{2 b} & V_{3 b} \\
V_{1 c} & V_{2 c} & V_{3 c}
\end{array}\right]}_{M} \cdot \frac{1}{T_{z}} \cdot\left[\begin{array}{c}
T_{1} \\
T_{2} \\
T_{3}
\end{array}\right]=M \cdot \frac{1}{T_{z}} \cdot\left[\begin{array}{c}
T_{1} \\
T_{2} \\
T_{3}
\end{array}\right]
$$

Then the duty times:

$$
\left[\begin{array}{c}
T_{1} \\
T_{2} \\
T_{3}
\end{array}\right]=T_{z} \cdot M^{-1} \cdot\left[\begin{array}{c}
V_{\text {aref }} \\
V_{\text {bref }} \\
V_{\text {cref }}
\end{array}\right]
$$

\section{3D-SVM in $\alpha-\beta-\gamma$ coordinates for four leg inverter}

This algorithm is based on the representation of the natural coordinates $a, b$ and $c$ in a new 3-D orthogonal frame, called $\alpha-\beta-\gamma$ frame [72-80], this can be achieved by the use of the Edit Clark transformation, where the voltage/current can be presented by a vector $V$ :

$$
V=\left[\begin{array}{c}
V_{\alpha} \\
V_{\beta} \\
V_{\gamma}
\end{array}\right]=C \cdot\left[\begin{array}{c}
V_{a} \\
V_{b} \\
V_{c}
\end{array}\right] \quad I=\left[\begin{array}{c}
I_{\alpha} \\
I_{\beta} \\
I_{\gamma}
\end{array}\right]=C \cdot\left[\begin{array}{c}
I_{a} \\
I_{b} \\
I_{c}
\end{array}\right]
$$

$C$ represents the matrix transformation:

$$
C=\frac{2}{3} \cdot\left[\begin{array}{ccc}
1 & -1 / 2 & -1 / 2 \\
0 & \sqrt{3} / 2 & -\sqrt{3} / 2 \\
1 / 2 & 1 / 2 & 1 / 2
\end{array}\right]
$$

When the reference voltages are balanced and without the same harmonics components in the three phases, the representations of the switching vectors have only eight possibilities which can be represented in the $\alpha-\beta$ plane. Otherwise in the general case of unbalance and different harmonics components the number of the switching vectors becomes sixteen, where each vector is defined by a set of four elements $\left[S_{a}, S_{b}, S_{c}, S_{f}\right]$ and their positions in the $\alpha-\beta-\gamma$ frame depend on the values contained in these sets Tab. 3 .

Each vector can be expressed by three components following the three orthogonal axes as follows:

$$
V^{i}=\left[\begin{array}{c}
V_{\alpha}^{i} \\
V_{\beta}^{i} \\
V_{\gamma}^{i}
\end{array}\right]
$$

Where:

$$
i=\overline{1,16}
$$


It is clear that the projection of these vectors onto the $\alpha \beta$ plane gives six NZVs and two $\mathrm{ZVs}$; these vectors present exactly the $2 \mathrm{D}$ presentation of the three leg inverters, it is explained by the nil value of the $\gamma$ component where there is no need to the fourth leg.

On the other side Fig. 7 represents the general case of the four legs inverter switching vectors. The different possibilities of the switching vectors in the $\alpha-\beta-\gamma$ frame are shown clearly, seven vectors are localised in the positive part of the $\gamma$ axis, while seven other vectors are found in the negative part, the two other vectors are just pointed in the $(0,0,0)$ coordinates, this two vectors are very important during the calculation of the switching times.

\begin{tabular}{|c|c|c|c|c|}
\hline Vector & $S_{a} S_{b} S_{c} S_{f}$ & $V_{\gamma}$ & $V_{\alpha}$ & $V_{\beta}$ \\
\hline$V^{9}$ & 0001 & -1 & 0 & 0 \\
\hline$V^{10}$ & 0011 & \multirow{3}{*}{$-\frac{2}{3}$} & $-1 / 3$ & $-1 / \sqrt{3}$ \\
\hline$V^{11}$ & 0101 & & $-1 / 3$ & $+1 / \sqrt{3}$ \\
\hline$V^{13}$ & 1001 & & $+2 / 3$ & 0 \\
\hline$V^{12}$ & 0111 & \multirow{3}{*}{$-\frac{1}{3}$} & $-2 / 3$ & 0 \\
\hline$V^{14}$ & 1011 & & $+1 / 3$ & $-1 / \sqrt{3}$ \\
\hline$V^{15}$ & 1101 & & $+1 / 3$ & $+1 / \sqrt{3}$ \\
\hline$V^{16}$ & 0000 & 0 & 0 & 0 \\
\hline
\end{tabular}

\begin{tabular}{|c|c|c|c|c|}
\hline Vector & $S_{a} S_{b} S_{c} S_{f}$ & $V_{\gamma}$ & $V_{\alpha}$ & $V_{\beta}$ \\
\hline$V^{1}$ & 1111 & 0 & 0 & 0 \\
\hline$V^{2}$ & 0010 & & $-1 / 3$ & $-1 / \sqrt{3}$ \\
\hline$V^{3}$ & 0100 & \multirow{2}{*}{$+\frac{1}{3}$} & $-1 / 3$ & $+1 / \sqrt{3}$ \\
\cline { 1 - 1 } \cline { 5 - 5 }$V^{5}$ & 1000 & & $2 / 3$ & 0 \\
\hline$V^{4}$ & 0110 & \multirow{2}{*}{$+\frac{2}{3}$} & $+\frac{1}{3} 3$ & 0 \\
\cline { 1 - 1 } \cline { 5 - 5 }$V^{6}$ & 1010 & & $-1 / \sqrt{3}$ \\
\hline$V^{7}$ & 1100 & & $+1 / 3$ & $+1 / \sqrt{3}$ \\
\hline$V^{9}$ & 1110 & +1 & 0 & 0 \\
\hline
\end{tabular}

Table 3. Switching vectors in the $\alpha \beta \gamma$ frame 


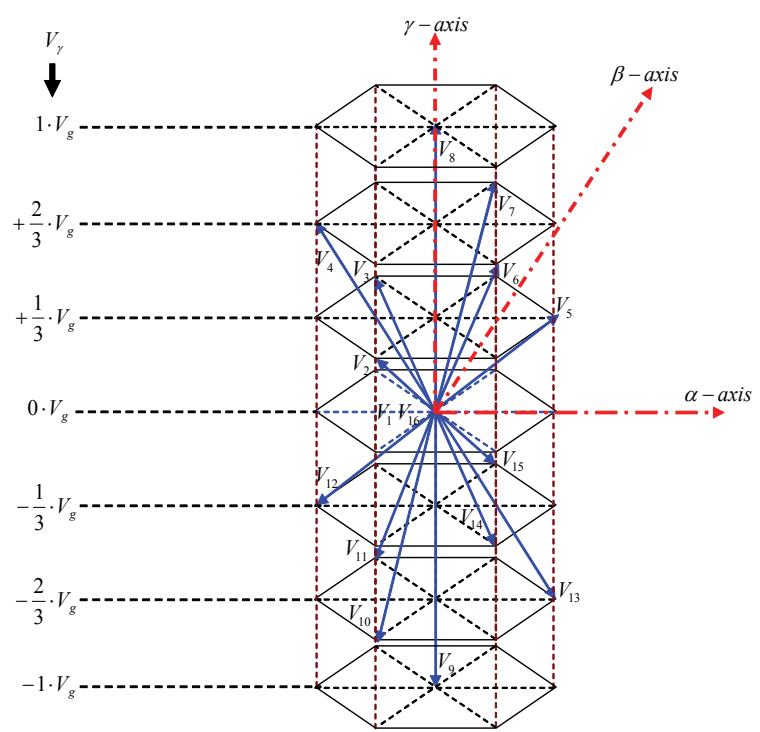

Fig. 7. Presentation of the switching vector in the $\alpha \beta \gamma$ frame

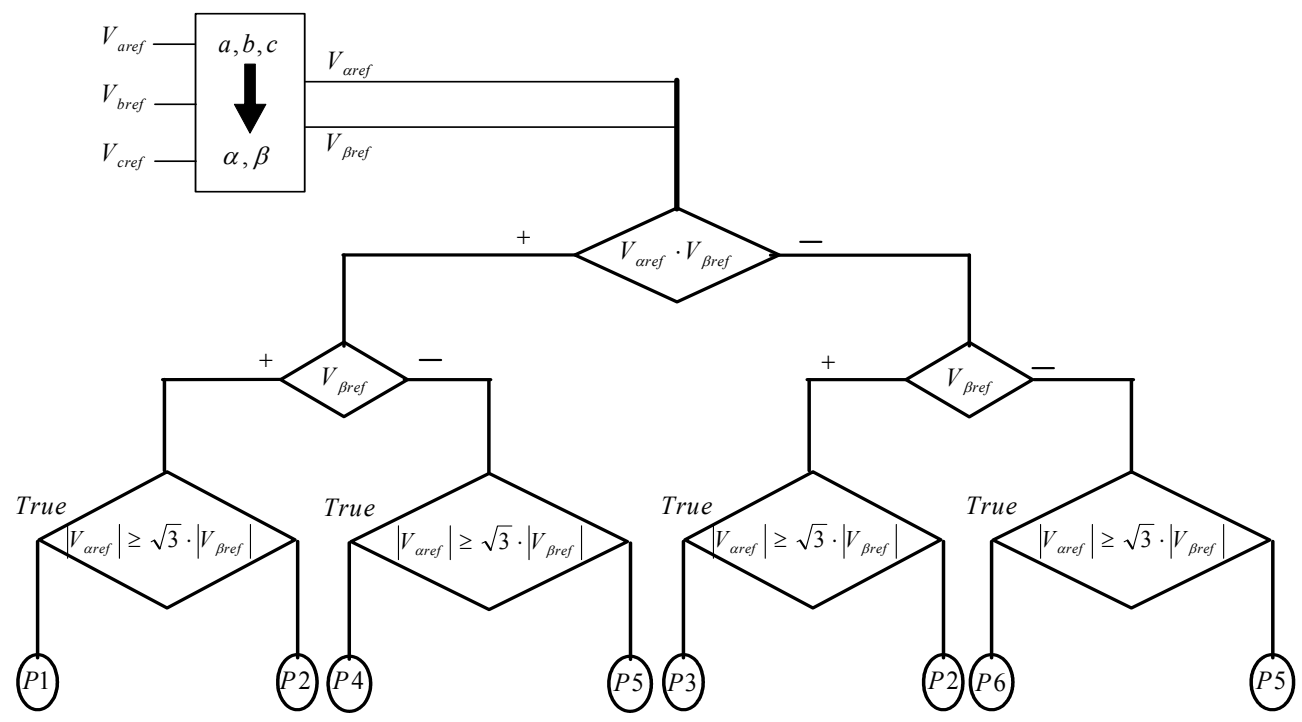

Fig. 8. Determination of the prisms

The position of the reference space vector can be determined in two steps.

1. Determination of the prism, in total there are six prisms. The flowchart in Fig.8 explains clearly, how the prism in which the reference space vector is found can be determined.

2. Determination of the tetrahedron in which the reference vector is located. Each prism contains four tetrahedrons Fig. 9, the determination of the tetrahedron in which the reference space vector is located is based on the polarity of the reference space vector components in $a-b-c$ frame as it is presented in Tab. 4. 

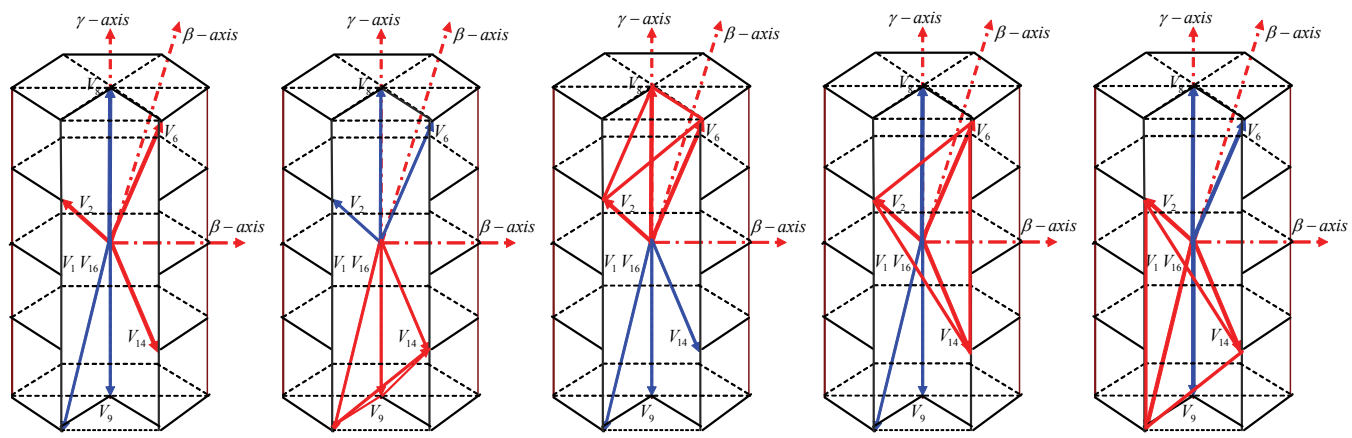

Fig. 9. Presentation of the switching vector in the $\alpha \beta \gamma$ frame

\begin{tabular}{|c|c|c|c|c|c|c|c|c|c|c|}
\hline \multirow{2}{*}{ Prism } & \multirow{2}{*}{ Tetrahedron } & \multicolumn{6}{|c|}{ Active vectors } & \multicolumn{3}{|c|}{$\begin{array}{c}\text { Reference vector } \\
\text { components }\end{array}$} \\
\hline & & $V_{1}$ & $V_{1}$ & $V_{2}$ & $V_{2}$ & $V_{3}$ & $V_{3}$ & $V_{a f}$ & $V_{b f}$ & $V_{c f}$ \\
\hline \multirow{4}{*}{$P_{1}$} & $T_{1}$ & $V^{15}$ & 1101 & $V^{13}$ & 1001 & $V^{5}$ & 1000 & $\geq$ & $\prec$ & $\prec$ \\
\hline & $T_{2}$ & $V^{5}$ & 1000 & $V^{7}$ & 1100 & $V^{15}$ & 1101 & $\geq$ & $\geq$ & $\prec$ \\
\hline & $T_{13}$ & $V^{9}$ & 0001 & $V^{13}$ & 1001 & $V^{15}$ & 1101 & $\prec$ & $\prec$ & $\prec$ \\
\hline & $T_{14}$ & $V^{8}$ & 1110 & $V^{7}$ & 1100 & $V^{5}$ & 1000 & $\geq$ & $\geq$ & $\geq$ \\
\hline \multirow{4}{*}{$P_{2}$} & $T_{3}$ & $V^{3}$ & 0100 & $V^{7}$ & 1100 & $V^{15}$ & 1101 & $\geq$ & $\geq$ & $\prec$ \\
\hline & $T_{4}$ & $V^{15}$ & 1101 & $V^{11}$ & 0101 & $V^{3}$ & 0100 & $\prec$ & $\geq$ & $\prec$ \\
\hline & $T_{15}$ & $V^{9}$ & 0001 & $V^{11}$ & 0101 & $V^{15}$ & 1101 & $\prec$ & $\prec$ & $\prec$ \\
\hline & $T_{16}$ & $V^{8}$ & 1110 & $V^{7}$ & 1100 & $V^{3}$ & 0100 & $\geq$ & $\geq$ & $\geq$ \\
\hline \multirow{4}{*}{$P_{3}$} & $T_{5}$ & $V^{12}$ & 0111 & $V^{11}$ & 0101 & $V^{3}$ & 0100 & $\prec$ & $\geq$ & $\prec$ \\
\hline & $T_{6}$ & $V^{3}$ & 0100 & $V^{4}$ & 0110 & $V^{12}$ & 0111 & $\prec$ & $\geq$ & $\geq$ \\
\hline & $T_{17}$ & $V^{9}$ & 0001 & $V^{11}$ & 0101 & $V^{12}$ & 0111 & $\prec$ & $\prec$ & $\prec$ \\
\hline & $T_{18}$ & $V^{8}$ & 1110 & $V^{4}$ & 0110 & $V^{3}$ & 0100 & $\geq$ & $\geq$ & $\geq$ \\
\hline \multirow{4}{*}{$P_{4}$} & $T_{7}$ & $V^{2}$ & 0010 & $V^{4}$ & 0110 & $V^{12}$ & 0111 & $\prec$ & $\geq$ & $\geq$ \\
\hline & $T_{8}$ & $V^{12}$ & 0111 & $V^{10}$ & 0011 & $V^{2}$ & 0010 & $\prec$ & $\prec$ & $\geq$ \\
\hline & $T_{19}$ & $V^{9}$ & 0001 & $V^{10}$ & 0011 & $V^{12}$ & 0111 & $\prec$ & $\prec$ & $\prec$ \\
\hline & $T_{20}$ & $V^{8}$ & 1110 & $V^{4}$ & 0110 & $V^{2}$ & 0010 & $\geq$ & $\geq$ & $\geq$ \\
\hline \multirow{4}{*}{$P_{5}$} & $T_{9}$ & $V^{14}$ & 1011 & $V^{10}$ & 0011 & $V^{2}$ & 0010 & $\prec$ & $\prec$ & $\geq$ \\
\hline & $T_{10}$ & $V^{2}$ & 0010 & $V^{6}$ & 1010 & $V^{14}$ & 1011 & $\geq$ & $\prec$ & $\geq$ \\
\hline & $T_{21}$ & $V^{9}$ & 0001 & $V^{10}$ & 0011 & $V^{14}$ & 1011 & $\prec$ & $\prec$ & $\prec$ \\
\hline & $T_{22}$ & $V^{8}$ & 1110 & $V^{6}$ & 1010 & $V^{2}$ & 0010 & $\geq$ & $\geq$ & $\geq$ \\
\hline \multirow{4}{*}{$P_{6}$} & $T_{11}$ & $V^{14}$ & 1011 & $V^{13}$ & 1001 & $V^{5}$ & 1000 & $\geq$ & $\prec$ & $\prec$ \\
\hline & $T_{12}$ & $V^{5}$ & 1000 & $V^{6}$ & 1010 & $V^{14}$ & 1011 & $\geq$ & $\prec$ & $\geq$ \\
\hline & $T_{23}$ & $V^{9}$ & 0001 & $V^{13}$ & 1001 & $V^{14}$ & 1011 & $\prec$ & $\prec$ & $\prec$ \\
\hline & $T_{24}$ & $V^{8}$ & 1110 & $V^{6}$ & 1010 & $V^{5}$ & 1000 & $\geq$ & $\geq$ & $\geq$ \\
\hline
\end{tabular}

Table 4. Tetrahedron determination.

By the same way, using (9) the duty times of the active vectors can be calculated using the following expression: 


$$
\left[\begin{array}{c}
V_{\text {aref }} \\
V_{\beta r e f} \\
V_{\gamma r e f}
\end{array}\right]=\underbrace{\left[\begin{array}{ccc}
V_{1 \alpha} & V_{2 \alpha} & V_{3 \alpha} \\
V_{1 \beta} & V_{2 \beta} & V_{3 \beta} \\
V_{1 \gamma} & V_{2 \gamma} & V_{3 \gamma}
\end{array}\right]}_{N} \cdot \frac{1}{T_{z}} \cdot\left[\begin{array}{c}
T_{1} \\
T_{1} \\
T_{1}
\end{array}\right]=N \cdot \frac{1}{T_{z}} \cdot\left[\begin{array}{c}
T_{1} \\
T_{1} \\
T_{1}
\end{array}\right]
$$

Finally:

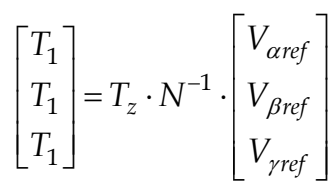

\section{3D-SVM new algorithm for four leg inverters}

A new method was recently proposed for the identification of tetrahedron and the three adjacent nonzero vectors [81]. It exposes the relationship between the reference voltages and the corresponding tetrahedron, on the other side the relationship between the three adjacent vectors and their duty times in each sampling period. This method is based on the idea that the three adjacent vectors are automatically in a tetrahedron, but it is not required to identify this tetrahedron. The authors of this method proposed two algorithms for the implementation of 3-D SVPWM where the phase angle is necessary to be determined. Each of the tetrahedrons is appointed by $T(x, y, z)$, it is composed of three non-zero vectors $V_{x}$, $V_{y}$ and $V_{z}$ as it is exposed in the other methods. On the other side the authors of this method have noticed that the shape of sliced prisms in two methods have the same shapes but with differences of scale and spatial position Fig. 10. On this basis the initial transformation used between the $a-b-c$ frame and $\alpha-\beta-\gamma$ frame is decomposed to three matrixes:

$$
\left[\begin{array}{l}
U_{\alpha} \\
U_{\beta} \\
U_{0}
\end{array}\right]=\frac{1}{3} \cdot\left[\begin{array}{ccc}
2 & 1 & 1 \\
0 & \sqrt{3} & -\sqrt{3} \\
1 & 1 & 1
\end{array}\right] \cdot\left[\begin{array}{l}
U_{a} \\
U_{b} \\
U_{c}
\end{array}\right]=T_{1} \cdot T_{2} \cdot T_{3} \cdot\left[\begin{array}{l}
U_{a} \\
U_{b} \\
U_{c}
\end{array}\right]
$$

Where:

$$
T_{1}=\left[\begin{array}{ccc}
\sqrt{2 / 3} & 0 & 0 \\
0 & \sqrt{2 / 3} & 0 \\
0 & 0 & \sqrt{1 / 3}
\end{array}\right], T_{2}=\left[\begin{array}{ccc}
\sqrt{2 / 3} & 0 & \sqrt{1 / 3} \\
0 & \sqrt{3} & 0 \\
-\sqrt{1 / 3} & 0 & \sqrt{2 / 3}
\end{array}\right], T_{3}=\left[\begin{array}{ccc}
1 & 0 & 0 \\
0 & \sqrt{1 / 2} & -\sqrt{1 / 2} \\
0 & \sqrt{1 / 2} & \sqrt{1 / 2}
\end{array}\right]
$$

The first matrix rotates the $a-b-c$ coordinates around the $a$ axis by an angle of $45^{\circ}$. Then the second matrix rotates the $a-b-c$ coordinates around the $b$ axis by an angle of $36.25^{\circ}$. Finally, the third matrix modifies its scale by multiplying the $a$ and $b$ axis by $\sqrt{2 / 3}$ and $\sqrt{1 / 3}$ respectively. After this transformation, it was noticed that the vector used in each tetrahedron are the same in either frames $a-b-c$ and $\alpha-\beta-\gamma$, of course with two 
different spatial positions, Hence, it is deducted that the duration of the adjacent vectors are independent of coordinates.

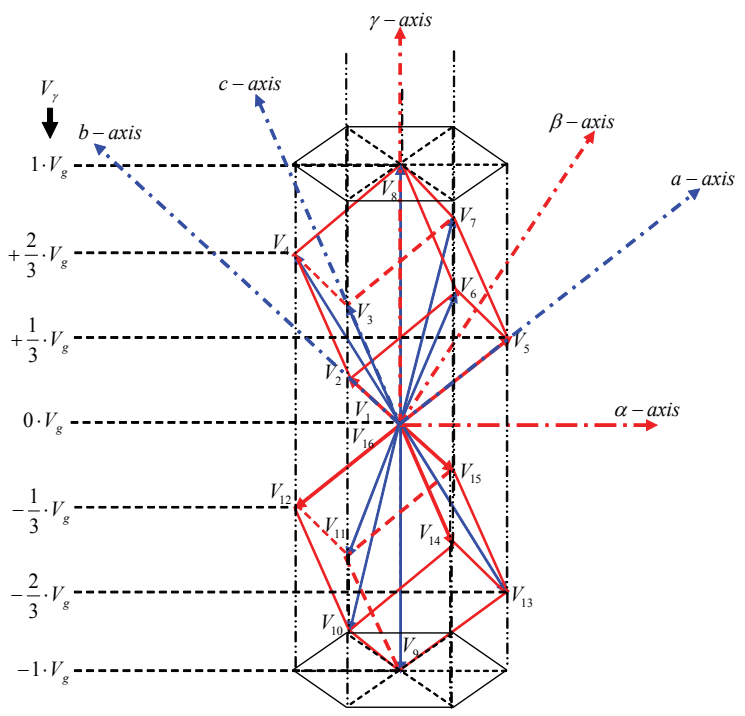

Fig. 10. Presentation of the switching vector in the $\alpha \beta \gamma$ frame

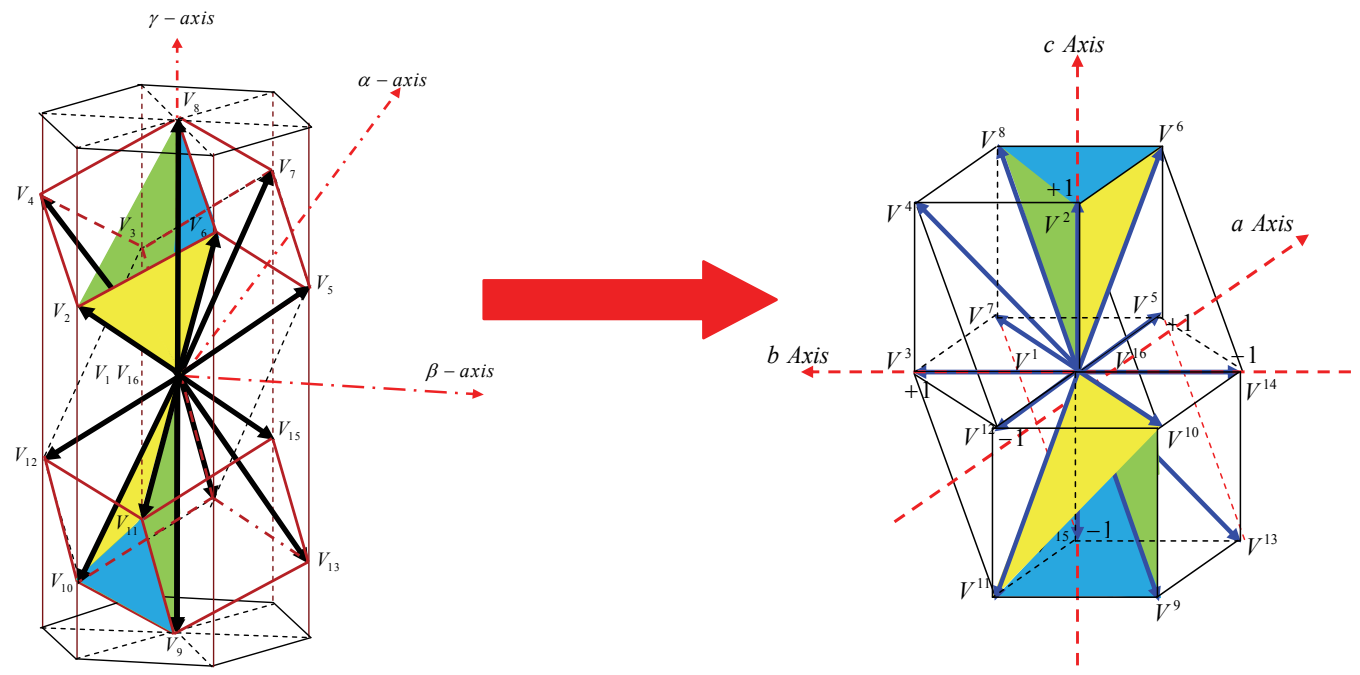

Fig. 11. Presentation of the switching vector in the $\alpha \beta \gamma$ frame

The determination of the tetrahedron can be extracted directly by comparing the relative values of $U_{a}, U_{b}, U_{c}$ and zero. The Zero value is used in the comparison for determining the polarity of the voltages in three phases. If the voltages $U_{a}, U_{b}, U_{c}$ and zero are ordered in descending order, the possible number of permutations is 24 which is equal to the number of Tetrahedron. Tab. 5 shows the relationship between Terahedrons and the 
order of $U_{a}, U_{b}, U_{c}$ and zero. Therefore the tetrahedron $T(x, y, z)$ can be determined without complex calculations. These elements are respectively denoted $U_{1}, U_{2}, U_{3}$ and $U_{4}$ in descending order.

$$
U_{1} \geq U_{2} \geq U_{3} \geq U_{4}
$$

For example for: $U_{a} \geq 0 \geq U_{c} \geq U_{b}$

It can be found that: $U_{1}=U_{a}, U_{2}=0, U_{3}=U_{c}, U_{4}=U_{b}$.

\begin{tabular}{|c|c|c|c|c|c|}
\hline Tetrahedron & Vecteurs & $U_{1} \geq U_{2} \geq U_{3} \geq U_{4}$ & Tetrahedron & Vecteurs & $U_{1} \geq U_{2} \geq U_{3} \geq U_{4}$ \\
\hline 1 & $T(1,3,7)$ & $0 \geq U_{c} \geq U_{b} \geq U_{a}$ & 13 & $T(4,5,7)$ & $U_{b} \geq 0 \geq U_{c} \geq U_{a}$ \\
\hline 2 & $T(1,3,11)$ & $0 \geq U_{c} \geq U_{a} \geq U_{b}$ & 14 & $T(4,5,13)$ & $U_{b} \geq 0 \geq U_{a} \geq U_{c}$ \\
\hline 3 & $T(1,5,7)$ & $0 \geq U_{b} \geq U_{c} \geq U_{a}$ & 15 & $T(4,6,7)$ & $U_{b} \geq U_{c} \geq 0 \geq U_{c}$ \\
\hline 4 & $T(1,5,13)$ & $0 \geq U_{b} \geq U_{a} \geq U_{c}$ & 16 & $T(4,6,14)$ & $U_{b} \geq U_{c} \geq U_{c} \geq 0$ \\
\hline 5 & $T(1,9,11)$ & $0 \geq U_{a} \geq U_{c} \geq U_{b}$ & 17 & $T(4,12,13)$ & $U_{b} \geq U_{a} \geq 0 \geq U_{c}$ \\
\hline 6 & $T(1,9,13)$ & $0 \geq U_{a} \geq U_{b} \geq U_{c}$ & 18 & $T(4,12,14)$ & $U_{b} \geq U_{a} \geq U_{c} \geq 0$ \\
\hline 7 & $T(2,3,7)$ & $U_{c} \geq 0 \geq U_{b} \geq U_{a}$ & 19 & $T(8,9,11)$ & $U_{a} \geq 0 \geq U_{c} \geq U_{b}$ \\
\hline 8 & $T(2,3,11)$ & $U_{c} \geq 0 \geq U_{a} \geq U_{b}$ & 20 & $T(8,9,13)$ & $U_{a} \geq 0 \geq U_{b} \geq U_{c}$ \\
\hline 9 & $T(2,6,7)$ & $U_{c} \geq U_{b} \geq 0 \geq U_{a}$ & 21 & $T(8,10,11)$ & $U_{a} \geq U_{c} \geq 0 \geq U_{b}$ \\
\hline 10 & $T(2,6,14)$ & $U_{c} \geq U_{b} \geq U_{a} \geq 0$ & 22 & $T(8,10,14)$ & $U_{a} \geq U_{c} \geq U_{b} \geq 0$ \\
\hline 11 & $T(2,10,11)$ & $U_{c} \geq U_{a} \geq 0 \geq U_{b}$ & 23 & $T(8,12,13)$ & $U_{a} \geq U_{b} \geq 0 \geq U_{c}$ \\
\hline 12 & $T(2,10,14)$ & $U_{c} \geq U_{a} \geq U_{b} \geq 0$ & 24 & $T(8,12,14)$ & $U_{a} \geq U_{b} \geq U_{c} \geq 0$ \\
\hline
\end{tabular}

Table 5. Determination of tetrahedron vectors

If an equality occurs between two elements, then the reference voltage is in the boundary between two neighboring tetrahedrons. If two neighboring equalities occur, then the reference voltage is within the boundary of six Tetrahedrons. If an equality is occurs between the first and the second element and at the same time an equality occurs between the third and fourth element, the reference voltage is within the boundary of four Tetrahedron.if three equalities occur, this means that the space vector is passing in the point $(0,0,0)$ connecting all the tetrahedrons. For example:

1. For $U_{c} \geq U_{a} \geq U_{b}=0$ the reference voltage is located in the interface of $T(2,10,11)$ and $T(2,10,14)$, which contains the two vectors $V_{2}$ and $V_{10}$.

2. For $U_{b} \geq U_{c}=0=U_{a}$ the reference voltage is parallel to $V_{4}$ and it is located in the interface among $T(4,5,7), T(4,5,13), T(4,6,7), T(4,6,14), T(4,12,13)$ and $T(4,12,14)$.

3. For $U_{b}=U_{c} \geq 0=U_{a}$ the reference voltage is parallel to $V_{6}$ and is located in the interface among $T(2,6,7), T(2,6,14), T(4,6,7)$ and $T(4,6,14)$.

4. For $U_{c}=U_{b}=U_{a}=0$ the reference voltage is nil. 
It is clear, that as the other methods the determination of the tetrahedron $T(x, y, z)$ allows the selection of the three vectors $V_{x}, V_{y}$ and $V_{z}$, and the calculation of the application duration of the switching states. These switching states have a binary format $x, y$ and $z$. Using the relationship between the tetrahedrons and the voltages $U_{a}, U_{b}, U_{c}$ and 0 Tab. 5 . The rule for the determination of switching states is derived as follows:

$$
x=2^{i}, y=x+2^{j}, z=y+2^{k}
$$

$i, j$ and $k$ are determined from the elements $U_{1}, U_{2}$ and $U_{3}$. Similarly the parameter $r$ can be deduced, this parameter is used subsequently for the calculation of the application durations of the three vectors.

$$
i=\left\{\begin{array}{ll}
0 & U_{1}=0 \\
1 & U_{1}=U_{c} \\
2 & U_{1}=U_{b} \\
3 & U_{1}=U_{a}
\end{array}, j=\left\{\begin{array}{ll}
0 & U_{1}=0 \\
1 & U_{1}=U_{c} \\
2 & U_{1}=U_{b} \\
3 & U_{1}=U_{a}
\end{array}, k=\left\{\begin{array}{ll}
0 & U_{1}=0 \\
1 & U_{1}=U_{c} \\
2 & U_{1}=U_{b} \\
3 & U_{1}=U_{a}
\end{array}, r= \begin{cases}0 & U_{1}=0 \\
1 & U_{1}=U_{c} \\
2 & U_{1}=U_{b} \\
3 & U_{1}=U_{a}\end{cases}\right.\right.\right.
$$

The determination of the duration of each vector is given by:

$$
\left[\begin{array}{c}
T_{1} \\
T_{2} \\
T_{3}
\end{array}\right]=\frac{T_{p}}{U_{d c}}\left[\begin{array}{lll}
a_{1} & a_{4} & a_{7} \\
a_{2} & a_{5} & a_{8} \\
a_{3} & a_{6} & a_{9}
\end{array}\right] \cdot\left[\begin{array}{l}
U_{a} \\
U_{b} \\
U_{c}
\end{array}\right]
$$

This method can be applied in both frames $a-b-c$ and $\alpha-\beta-\gamma$ in the same way. The switching states $x, y$ and $z$ or the voltage vectors $V_{x}, V_{y}$ and $V_{z}$ are independent of the coordinates and are determined only from the relative values of $U_{a}, U_{b}$ and $U_{c}$. All matrix elements $a_{i}$ take the values 0,1 or -1 . Therefore, the calculations need only the addition and subtraction of $U_{a}, U_{b}$ and $U_{c}$ except the coefficient $T_{p} / U_{d c}$. The $a_{i}$ values are determined from the following relations where they can be presented as a function of elementary relative voltages:

$$
\begin{aligned}
& a_{1}=\left\{\begin{array}{cc}
1 & U_{a}=U_{1} \\
-1 & U_{a}=U_{2} \\
0 & \text { otherwise }
\end{array}, a_{2}=\left\{\begin{array}{cc}
1 & U_{a}=U_{2} \\
-1 & U_{a}=U_{3} \\
0 & \text { otherwise }
\end{array}, a_{3}=\left\{\begin{array}{rr}
1 & U_{a}=U_{3} \\
-1 & U_{a}=U_{4} \\
0 & \text { otherwise }
\end{array}\right.\right.\right. \\
& a_{4}=\left\{\begin{array}{cc}
1 & U_{b}=U_{1} \\
-1 & U_{b}=U_{2} \\
0 & \text { otherwise }
\end{array}, a_{5}=\left\{\begin{array}{cc}
1 & U_{b}=U_{2} \\
-1 & U_{b}=U_{3} \\
0 & \text { otherwise }
\end{array}, a_{6}=\left\{\begin{array}{rr}
1 & U_{b}=U_{3} \\
-1 & U_{b}=U_{4} \\
0 & \text { otherwise }
\end{array}\right.\right.\right. \\
& a_{7}=\left\{\begin{array}{cc}
1 & U_{c}=U_{1} \\
-1 & U_{c}=U_{2} \\
0 & \text { otherwise }
\end{array}, a_{8}=\left\{\begin{array}{rr}
1 & U_{c}=U_{2} \\
-1 & U_{c}=U_{3} \\
0 & \text { otherwise }
\end{array}, a_{9}=\left\{\begin{array}{rr}
1 & U_{c}=U_{3} \\
-1 & U_{c}=U_{4} \\
0 & \text { otherwise }
\end{array}\right.\right.\right.
\end{aligned}
$$


If we substitute these values in (20) and according to the given definitions of $i, j, k$ and $r$, the application duration of adjacent vectors, can be expressed in (22), it shows that they are only depending on the relative voltage vectors $U_{1}, U_{2}, U_{3}$ and $U_{4}$.

$$
\left[\begin{array}{l}
T_{1} \\
T_{2} \\
T_{3}
\end{array}\right]=\frac{T_{p}}{U_{d c}} \cdot\left[\begin{array}{l}
U_{1}-U_{2} \\
U_{2}-U_{3} \\
U_{3}-U_{4}
\end{array}\right]
$$

\section{3D-SVM new algorithm for four leg inverters}

A new algorithm of tetrahedron determination applied to the SVPWM control of four leg inverters was presented by the authors in [82]. In this algorithm, a new method was proposed for the determination of the three phase system reference vector location in the space; even the three phase system presents unbalance, harmonics or both of them. As it was presented in the previous works the reference vector was replaced by three active vectors and two zero vectors following to their duty times [34-35], [69-80]. These active vectors are representing the vectors which are defining the special tetrahedron in which the reference vector is located.

In the actual algorithm the numeration of the tetrahedron is different from the last works, the number of the active tetrahedron is determined by new process which seems to be more simplifiers, faster and can be implemented easily. Form (5) and (12) the voltages in the $\alpha \beta \gamma$ frame can be presented by:

$$
\left[\begin{array}{c}
V_{\alpha} \\
V_{\beta} \\
V_{\gamma}
\end{array}\right]=C \cdot\left[\begin{array}{c}
S_{a}-S_{f} \\
S_{b}-S_{f} \\
S_{c}-S_{f}
\end{array}\right] \cdot V_{g}
$$

It is clear that there is no effect of the fourth leg behaviours on the values of the components in the $\alpha-\beta$ plane. The effect of the fourth leg switching is remarked in the $\gamma$ component. The representation of these vectors is shown in Fig. 12 -c-.

\subsection{Determination of the truncated triangular prisms}

As it is shown in the previous sections, the three algorithms are based on the values of the $a-b-c$ frame reference voltage components. In this algorithm there is no need for the calculation of the zero (homopolar) sequence component of the reference voltage. Only the values of the reference voltage in $a-b-c$ frame are needed. The determination of the truncated triangular prism (TP) in which the reference voltage space vector is located is based on four coefficients. These four coefficients are noted as $C_{0}, C_{2}, C_{3}$ and $C_{4}$. Their values can be calculated via two variables $x$ and $y$ which are defined as follows:

$$
x=\frac{V_{\alpha}}{\|V\|}
$$




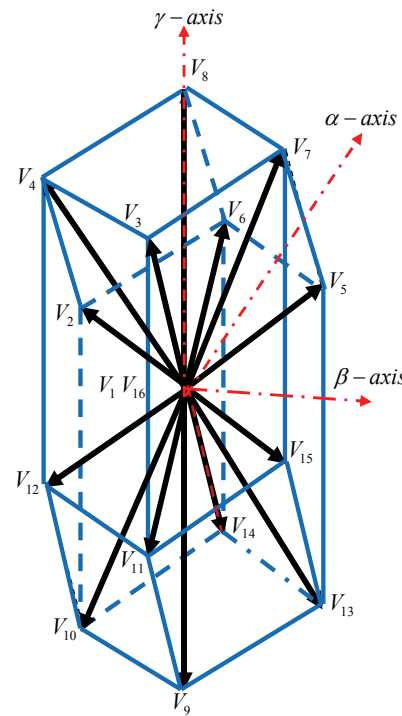

(a)

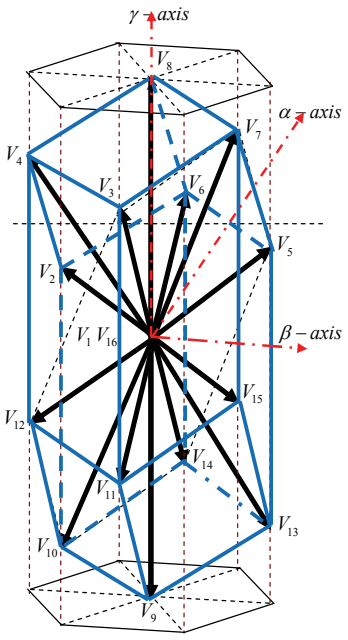

(b)

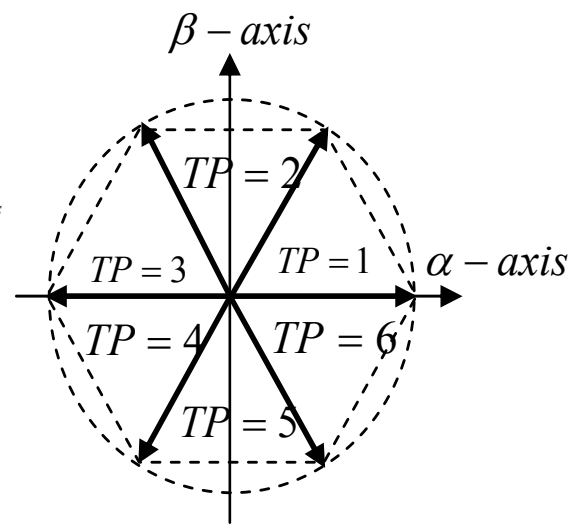

(c)

Fig. 12. Presentation of the possible switching vectors in $a-b-c$

$$
y=\frac{V_{\beta}}{\|V\|}
$$

Where:

$$
\|V\|=\sqrt{V_{\alpha}^{2}+V_{\beta}^{2}}
$$

The coefficients can be calculated as follows:

$$
\left[\begin{array}{l}
C_{0} \\
C_{1} \\
C_{2} \\
C_{3}
\end{array}\right]=\left[\begin{array}{c}
1 \\
\operatorname{INT}\left(\frac{5}{2}-x-\varepsilon\right) \\
\operatorname{INT}(1-y-\varepsilon) \\
\operatorname{INT}\left(\frac{5}{2}+x+\varepsilon\right)
\end{array}\right]
$$

$\varepsilon$ is used to avoid the confusion when the reference vector passes in the boundary between two adjacent triangles in the $\alpha \beta$ plane, the reference vector has to be included at each sampling time only in one triangle Fig. 12-c-. On the other hand, as it was mentioned in the first family works, the location of the reference vectors passes in six prism Fig. 12-b-, but effectively this is not true as the reference vector passes only in six pentahedron or six truncated triangular prism (TP) as the two bases are not presenting in parallel planes following to the geometrical definition of the prism Fig. 12-a-. The number of the truncated prism TP can be determined as follows:

$$
\mathrm{TP}=3 \mathrm{C}_{2}+\sum_{i=0}^{2}(-1)^{i} C_{i} C_{i+1}
$$




\subsection{Determination of the tetrahedrons}

In each TP there are six vectors, these vectors define four tetrahedrons. Each tetrahedron contains three active vectors from the six vectors found in the TP. The way of selecting the tetrahedron depends on the polarity changing of each switching components included in one vector. The following formula permits the determination of the tetrahedron in which the voltage space vector is located.

$$
T_{h}=4(T P-1)+1+\sum_{1}^{3} a_{i}
$$

Where:

$$
\begin{array}{cl}
a_{i}=1 \quad \text { if } & V_{i} \geq 0 \quad \text { else } a_{i}=0 \\
& i=a, b, c
\end{array}
$$

To clarify the process of determination of the $T P$ and $T_{h}$ for different three phase reference system voltages cases which may occurred. Figures 13 and 14 are presenting two general cases, where:

- $\quad$ Figures noted as ' $a$ ' present the reference three phase voltage system;

- Figures noted as ' $b$ ' present the space vector trajectory of the reference three phase voltage system ;

- $\quad$ Figures noted as ' $c$ ' present the concerned TP each sampling time, where the reference space vector is located;

- Figures noted as ' $\mathrm{d}$ ' present the concerned $T_{h}$ in which the reference space vector is located.

Case I: unbalanced reference system voltages

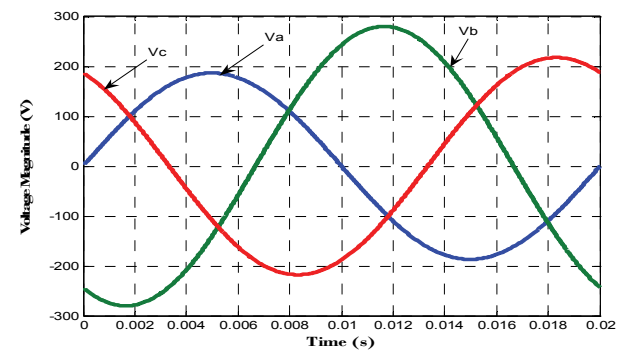

(a)

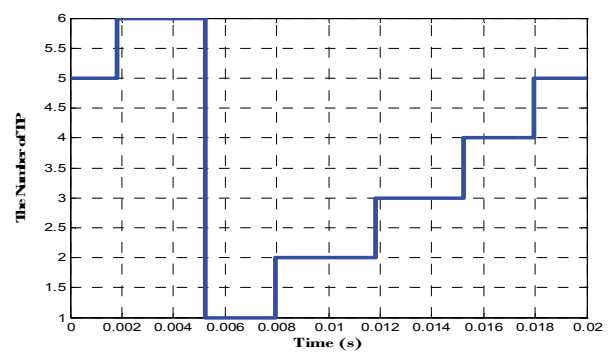

(c)

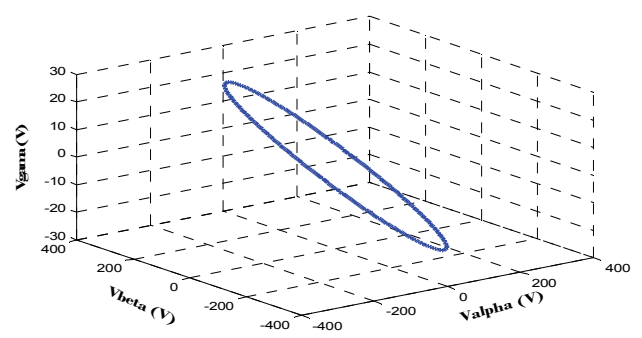

(b)

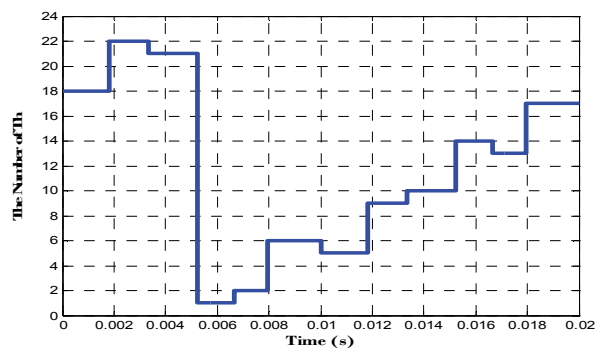

(d)

Fig. 13. Presentation of instantaneous three phase reference voltages, reference space vector, TP and $T_{h}$ 
Case II Unbalanced reference system voltages with the presence of unbalanced harmonics

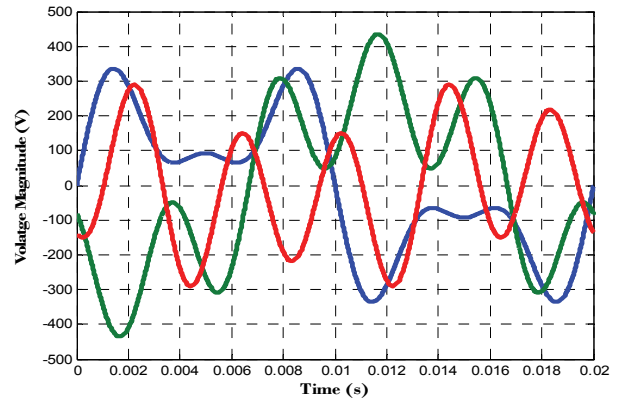

(a)

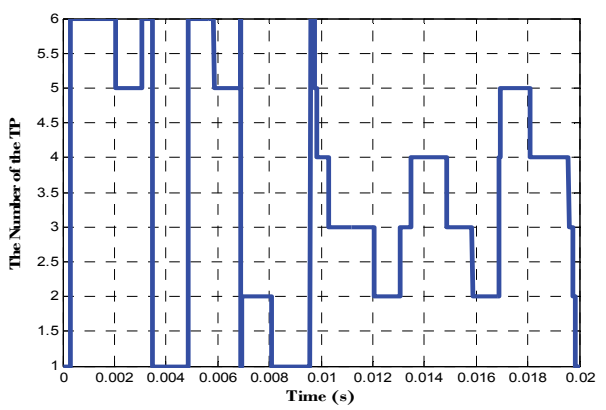

(c)

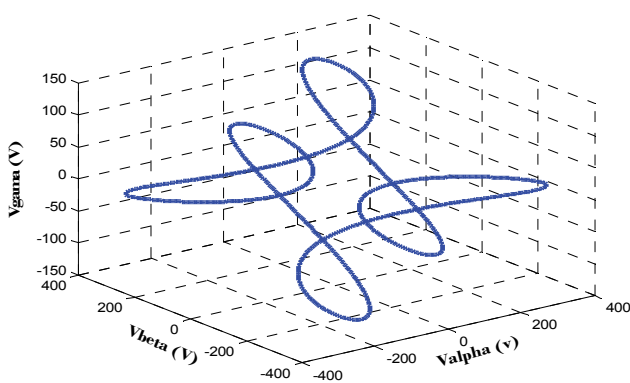

(b)

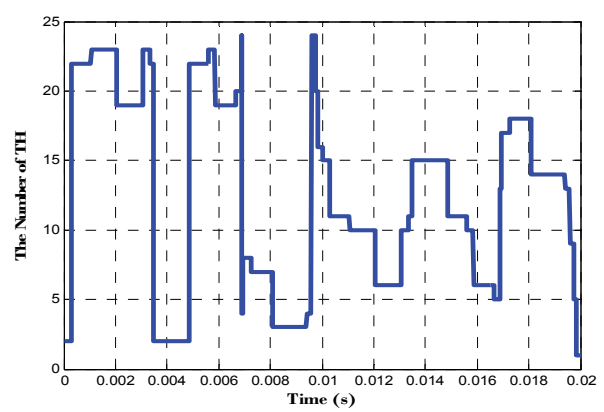

(d)

Fig. 14. Presentation of instantaneous three phase reference voltages, reference space vector, TP and $T_{h}$

\subsection{Calculation of duty times}

To fulfill the principle of the SVPWM as it is mentioned in (9) which can be rewritten as follows:

$$
V_{\text {ref }} \cdot T_{z}=\sum_{i=0}^{3} T_{i} \cdot V_{i}
$$

Where:

$$
T_{z}=\sum_{i=0}^{3} T_{i}
$$

In this equation the $a-b-c$ frame components can be used, either than the use of the $\alpha-\beta-\gamma$ frame components of the voltage vectors for the calculation of the duty times, of course the same results can be deduced from the use of the two frames. The vectors $V_{1}, V_{2}$ and $V_{3}$ present the edges of the tetrahedron in which the reference vector is lying. So each vector can take the sixteen possibilities available by the different switching possibilities. On the other hand these vectors have their components in the $\alpha-\beta-\gamma$ frame as follows: 


$$
V_{i}=\left[\begin{array}{c}
V_{\alpha i} \\
V_{\beta i} \\
V_{\gamma i}
\end{array}\right]=C \cdot\left[\begin{array}{c}
S_{a i}-S_{f i} \\
S_{b i}-S_{f i} \\
S_{c i}-S_{f i}
\end{array}\right] \cdot V_{g}
$$

From (30), (31) and (32) the following expression is deduced:

$$
\sum_{1}^{3} T_{i} \cdot\left[\begin{array}{l}
S_{a i}-S_{f i} \\
S_{b i}-S_{f i} \\
S_{c i}-S_{f i}
\end{array}\right]=\frac{1}{V_{g}} \cdot C^{-1} \cdot V_{r e f} \cdot T_{z}
$$

In the general case the following equation can be used to calculate the duty time for the three components used in the same tetrahedron:

$$
T_{i}=\sigma \cdot\left[\begin{array}{l}
\left(S_{a i}-S_{f i}\right) \cdot\left(\left(S_{b j}-S_{f j}\right) \cdot\left(S_{c k}-S_{f k}\right)-\left(S_{b k}-S_{f k}\right) \cdot\left(S_{c j}-S_{f j}\right)\right) \\
\left(S_{b i}-S_{f i}\right) \cdot\left(\left(S_{a k}-S_{f k}\right) \cdot\left(S_{c j}-S_{f j}\right)-\left(S_{a j}-S_{f j}\right) \cdot\left(S_{c k}-S_{f k}\right)\right) \\
\left(S_{c i}-S_{f i}\right) \cdot\left(\left(S_{a j}-S_{f j}\right) \cdot\left(S_{b k}-S_{f k}\right)-\left(S_{a k}-S_{f k}\right) \cdot\left(S_{b j}-S_{f j}\right)\right)
\end{array}\right]^{t} \cdot\left[\begin{array}{c}
V_{r e f a} \\
V_{r e f b} \\
V_{r e f c}
\end{array}\right]
$$

Where:

$$
\sigma=\frac{1}{\sum_{1}^{3}\left(S_{a i}-S_{f i}\right) \cdot\left[\left(S_{b j}-S_{f j}\right) \cdot\left(S_{c k}-S_{f k}\right)-\left(S_{b k}-S_{f k}\right) \cdot\left(S_{c j}-S_{f j}\right)\right]}
$$

Variable $j$ and $k$ are supposed to simplify the calculation where:

$$
j=i+1-3 \cdot \operatorname{INT}(i / 3) ; k=i+2-3 \cdot \operatorname{INT}((i+1) / 3) \quad i=1,2,3
$$

A question has to be asked. From one tetrahedron, how the corresponding edges of the existing switching vectors can be chosen for the three vectors used in the proposed SVPWM. Indeed the choice of the sequence of the vectors used for $V_{1}, V_{2}$ and $V_{3}$ in one tetrahedron depends on the SVPWM sequencing schematic used [108],[115], in one sampling time it is recommended to use four vectors, the fourth one is corresponding to zero vector, as it was shown only two switching combination can serve for this situation that is $V^{16}(0000)$ and $V^{1}$ (1111). On the other hand only one changing state of switches can be accepted when passing from the use of one vector to the following vector. For example in tetrahedron 1 the active vectors are: $V^{11}(1000), V^{3}(1001)$ and $V^{4}(1101)$, it is clear that if the symmetric sequence schematic is used and starts with vector $V^{1}$ then the sequence of the use of the other active vectors can be realized as follow:

$$
V^{1}, V^{11}, V^{3}, V^{4}, V^{10}, V^{4}, V^{3}, V^{11}, V^{1}
$$




\begin{tabular}{|c|c|c|c|c|c|c|c|c|c|}
\hline $\begin{array}{l}\text { Active } \\
\text { vector }\end{array}$ & $V^{1}$ & $V^{11}$ & $V^{3}$ & $V^{4}$ & $V^{10}$ & $V^{4}$ & $V^{3}$ & $V^{11}$ & $V^{1}$ \\
\hline $\begin{array}{l}S_{a} \\
S_{b} \\
S_{c} \\
S_{f}\end{array}$ & $\begin{array}{l}1 \\
1 \\
1 \\
1\end{array}$ & $\begin{array}{l}1 \\
1 \\
0 \\
1\end{array}$ & $\begin{array}{l}1 \\
0 \\
0 \\
1\end{array}$ & $\begin{array}{l}1 \\
0 \\
0 \\
0\end{array}$ & $\begin{array}{l}0 \\
0 \\
0 \\
0\end{array}$ & $\begin{array}{l}1 \\
0 \\
0 \\
0\end{array}$ & $\begin{array}{l}1 \\
0 \\
0 \\
1\end{array}$ & $\begin{array}{l}1 \\
1 \\
0 \\
1\end{array}$ & $\begin{array}{l}1 \\
1 \\
1 \\
1\end{array}$ \\
\hline$T_{a \cdot{ }_{0}}{ }^{1}$ & & & & & & & & & \\
\hline$T_{b} \cdot{ }_{0}^{1}$ & & & & & & & & & \\
\hline$T_{c} \cdot{ }_{0}^{1}$ & & & & & & & & & \\
\hline$T_{f \cdot \cdot}{ }^{1}$ & & & & & & & & & \\
\hline & $\frac{t_{0}}{4}$ & $\frac{t_{1}}{2}$ & $\frac{t_{2}}{2}$ & $\frac{t_{3}}{2}$ & $\frac{t_{0}}{2}$ & $\frac{t_{3}}{2}$ & $\frac{t_{2}}{2}$ & $\frac{t_{1}}{2}$ & $\frac{t_{0}}{4}$ \\
\hline
\end{tabular}

Otherwise, if it starts with vector $V^{16}$ then the sequence of the active vectors will be presented as follow Tab.9:

$$
V^{16}, V^{4}, V^{3}, V^{11}, V^{1}, V^{11}, V^{3}, V^{4}, V^{16}
$$

\begin{tabular}{|c|c|c|c|c|c|c|c|c|c|}
\hline $\begin{array}{l}\text { Active } \\
\text { vector }\end{array}$ & $V^{16}$ & $V^{4}$ & $V^{3}$ & $V^{11}$ & $V^{1}$ & $V^{11}$ & $V^{3}$ & $V^{4}$ & $V^{16}$ \\
\hline $\begin{array}{l}S_{a} \\
S_{b} \\
S_{c} \\
S_{f}\end{array}$ & $\begin{array}{l}0 \\
0 \\
0 \\
0\end{array}$ & $\begin{array}{l}1 \\
0 \\
0 \\
0\end{array}$ & $\begin{array}{l}1 \\
0 \\
0 \\
1\end{array}$ & $\begin{array}{l}1 \\
1 \\
0 \\
1\end{array}$ & $\begin{array}{l}1 \\
1\end{array}$ & $\begin{array}{l}1 \\
1 \\
0 \\
1\end{array}$ & $\begin{array}{l}1 \\
0 \\
0 \\
1\end{array}$ & $\begin{array}{l}1 \\
0 \\
0 \\
0\end{array}$ & $\begin{array}{l}0 \\
0 \\
0 \\
0\end{array}$ \\
\hline$T_{a \cdot{ }_{0}}{ }^{1}$ & & & & & & & & & \\
\hline$T_{b} \cdot{ }_{0}^{1}$ & & & & & & & & & \\
\hline$T_{c} \cdot{ }_{0}^{1}$ & & & & & & & & & \\
\hline$T_{f} \cdot{ }_{0}^{1}$ & & & & & & & & & \\
\hline & $\frac{t_{0}}{4}$ & $\frac{t_{1}}{2}$ & $\frac{t_{2}}{2}$ & $\frac{t_{3}}{2}$ & $\frac{t_{0}}{2}$ & $\frac{t_{3}}{2}$ & $\frac{t_{2}}{2}$ & $\frac{t_{1}}{2}$ & $\frac{t_{0}}{4}$ \\
\hline
\end{tabular}




\subsection{Applications}

To finalize this chapter two applications are presented here to show the effectiveness of the four-leg inverter. The first application is the use of the four-leg inverter to feed a balanced resistive linear load under unbalanced voltages. The second application is the use of the four-leg inverter as an active power filter, where the main aim is to ensure a sinusoidal balanced current circulation in the source side. In the two cases an output filter is needed between the point of connection and the inverter, in the first case an " $\mathrm{L}$ " filter is used, while for the second case an "LCL" filter is used as it is shown in Fig. 15 and Fig. 20.

\subsubsection{Applications1}

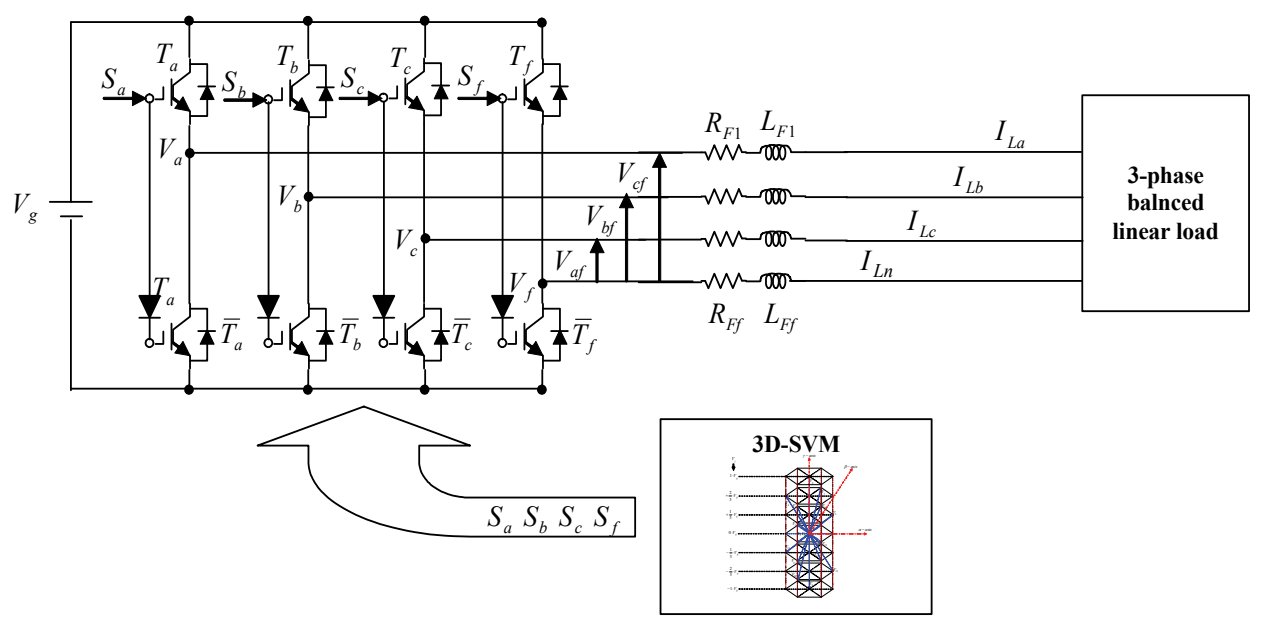

Fig. 15. Four-leg inverter is used as a Voltage Source Inverter 'VSI' for feeding balanced linear load under unbalanced voltages.

In this application, the reference unbalanced voltage and the output voltage produced by the four leg inverter in the three phases $a, b$ and $c$ are presented in Fig. 16. The currents in the four legs are presented in Fig. 17, it is clear that because of the voltage unbalance the fourth leg is handling a neutral current. To clarify the flexibility of the four leg inverter and the control algorithm used, Fig. 18 shows the truncated prisms and the tetrahedron in which the reference voltage space vector is located.

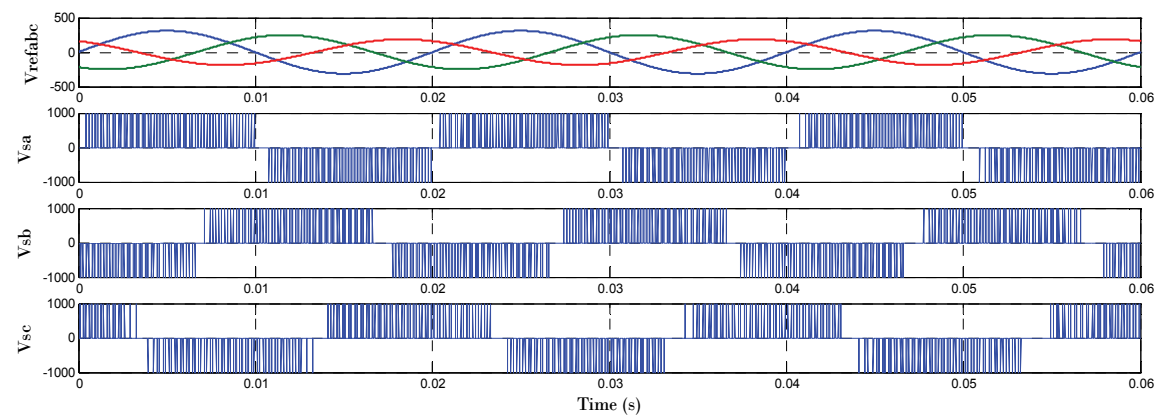

Fig. 16. Presentation of three phase reference voltages and the output voltage of the three legs. 


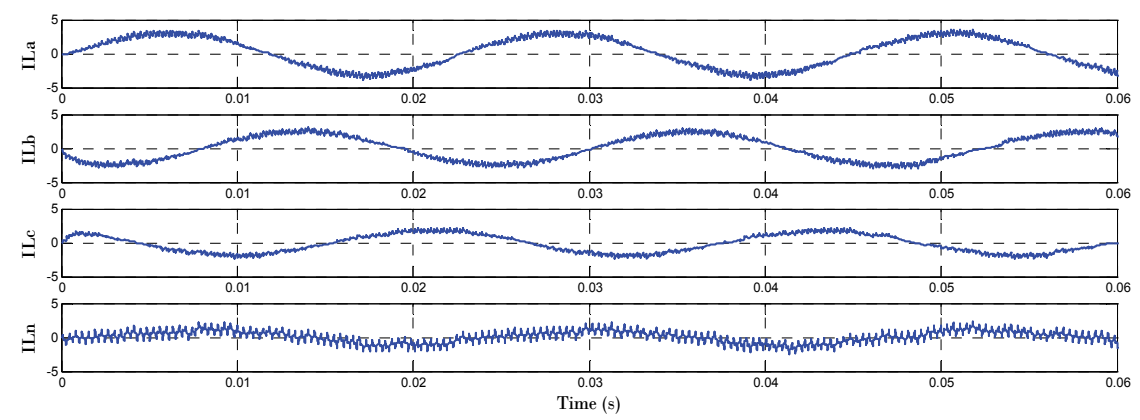

Fig. 17. Presentation of instantaneous load currents generated by the four legs
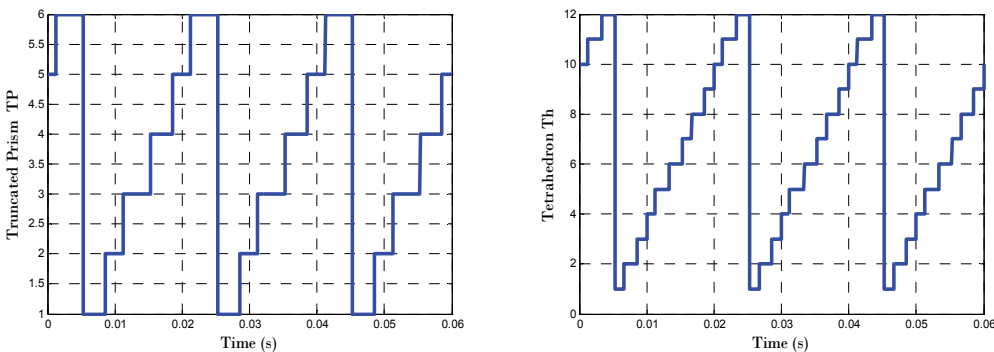

Fig. 18. Determination of the Truncated Prism TP and the tetrahedron $T_{h}$ in which the reference voltage space vector is located.

The presentation of the reference voltage space vector and the load current space vector are presented in the both frames $\alpha-\beta-\gamma$ and $a-b-c$, where the current is scaled to compare the form of the current and the voltage, just it is important to keep in mind that the load is purely resistive.
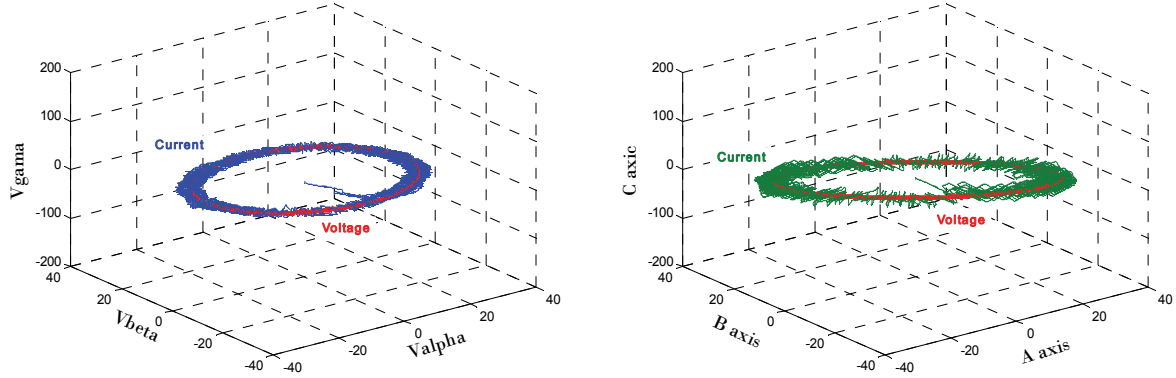

Fig. 19. Presentation of the instantaneous space vectors of the three phase reference system voltages and load current in $\alpha-\beta-\gamma$ and $a-b-c$ frames ( the current is multiplied by 10, to have the same scale with the voltage)

\subsubsection{Applications2}

The application of the fourth leg inverter in the parallel active power filtering has used in the last years, the main is to ensure a good compensation in networks with four wires, where the three phases currents absorbed from the network have to be balanced, sinusoidal 
and with a zero shift phase, on the other side the neutral wire has to have a nil current circulating toward the neutral of power system source. Figures 21, 22, 23 and 24 show the behavior of the four leg inverter to compensation the harmonics in the current. The neutral current of the source in nil as it is shown in Fig 24. Finally the current space vectors of the load, the active filter and the source in the both frames $\alpha-\beta-\gamma$ and $a-b-c$ are presented.

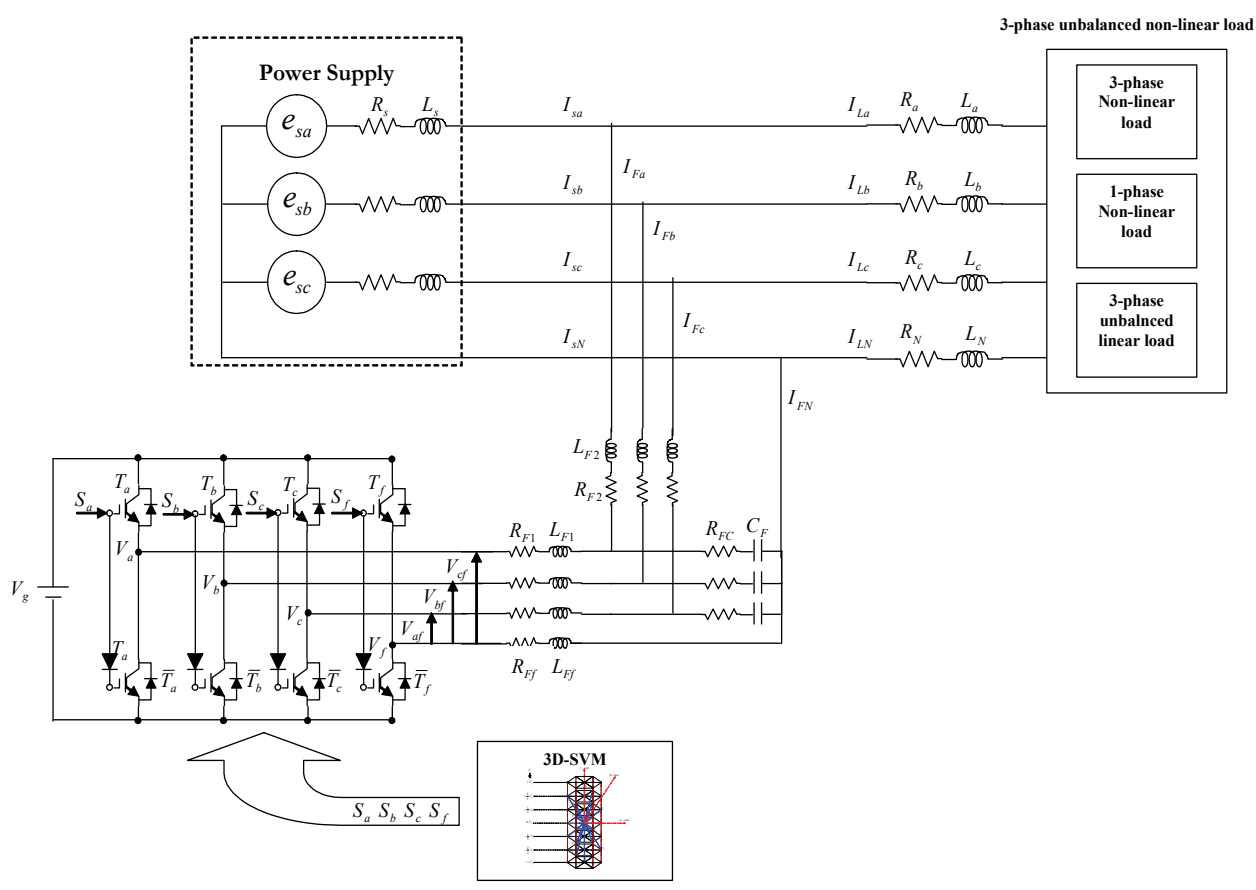

Fig. 20. Four-leg inverter is used as a Parallel Active Power Filter 'APF' for ensuring a sinusoidal source current.
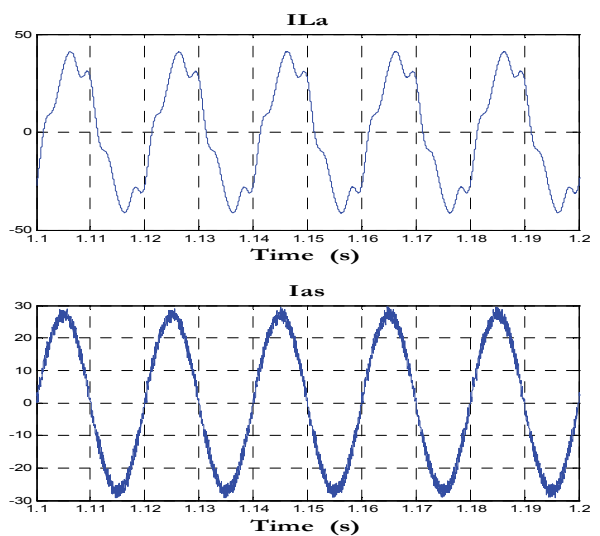
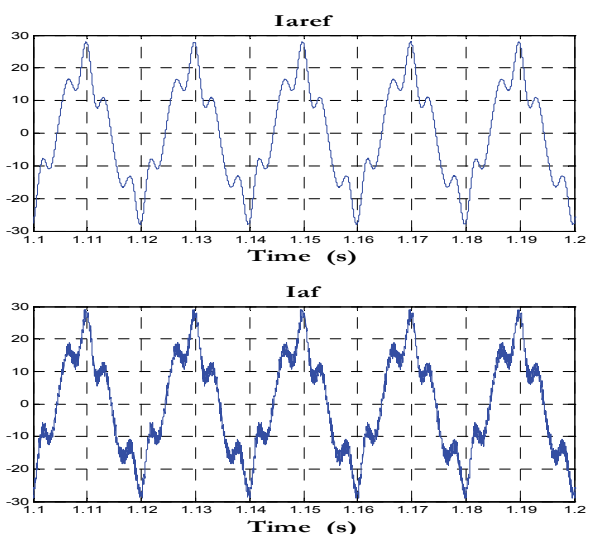

Fig. 21. Presentation of the instantaneous currents of Load, reference, active power filter and source of phase ' $a$ ' 


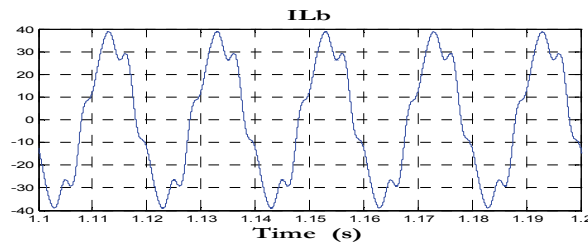

Ibs

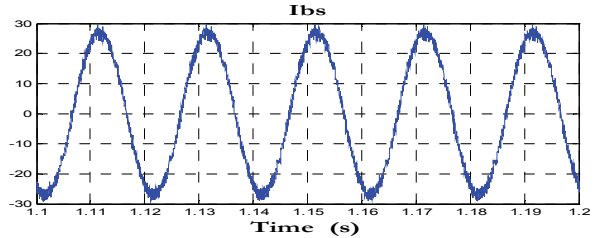

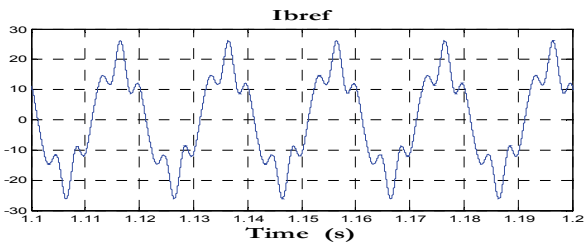

Ibf

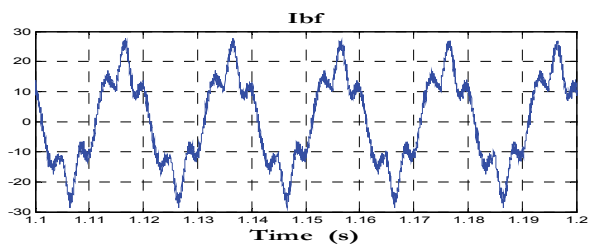

Fig. 22. Presentation of the instantaneous currents of Load, reference, active power filter and source of phase ' $b$ '

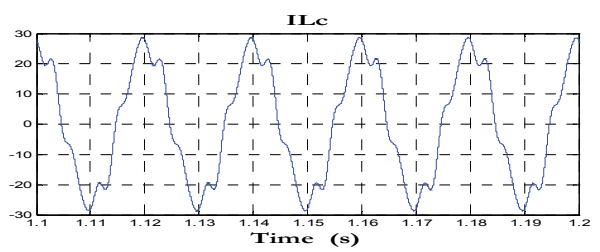

Ics

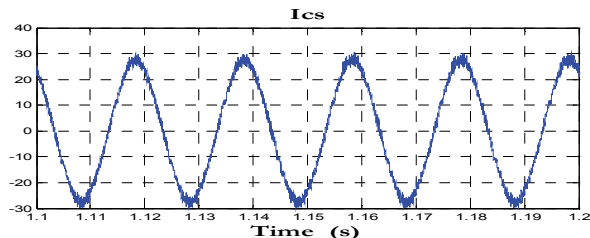

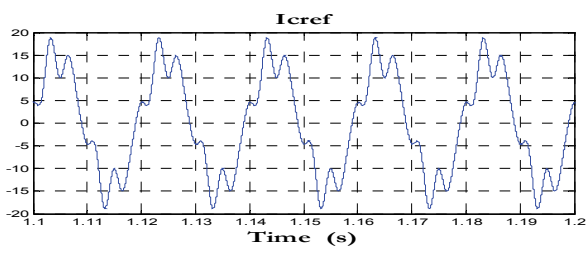

Icf

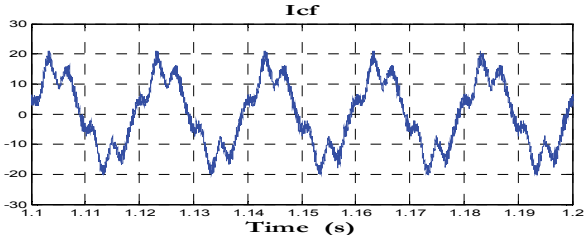

Fig. 23. Presentation of the instantaneous currents of Load, reference, active power filter and source of phase ' $c$ '
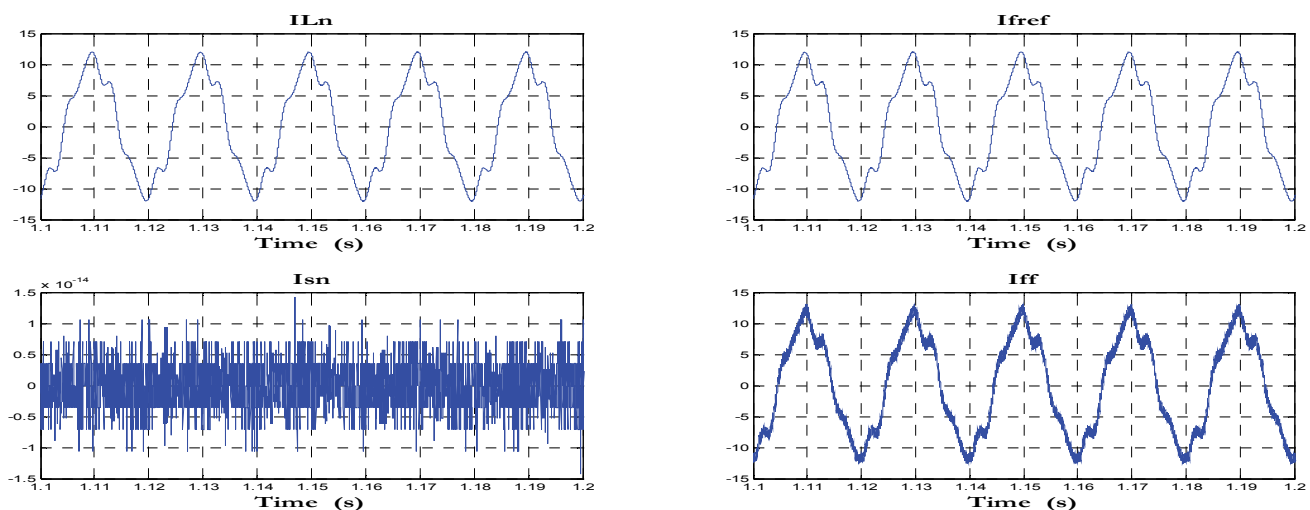

Fig. 24. Presentation of the instantaneous currents of Load, reference, active power filter and source of the fourth neutral leg ' $\mathrm{f}$ ' 

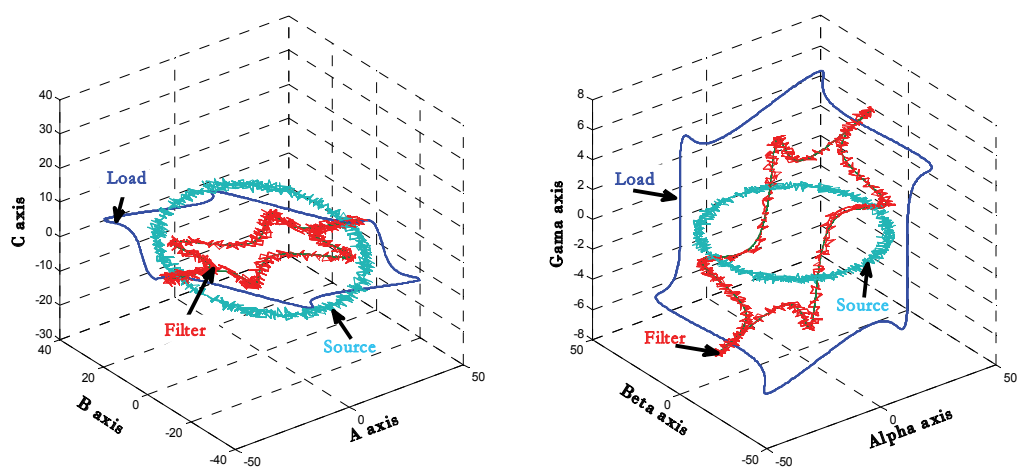

Fig. 25. Presentation of the instantaneous currents space vectors of the load, active power filter and the source in $\alpha-\beta-\gamma$ and $a-b-c$ frames

\section{Conclusion}

This chapter deals with the presentation of different control algorithm families of four leg inverter. Indeed four families were presented with short theoretical mathematical explanation, where the first one is based on $\alpha-\beta-\gamma$ frame presentation of the reference space vector, the second one is based on $a-b-c$ frame where there is no need for matrix transformation. The third one which was presented recently where the determination of the space vector is avoided and there is no need to know which tetrahedron is containing the space vector, it is based on the direct values of the three components following the three phases, the duty time can be evaluated without the passage through the special location of the space vector. The fourth method in benefiting from the first and second method, where the matrix used for the calculation of the duty time containing simple operation and the elements are just 0,1 and -1 . As a result the four methods can lead to the same results; the challenge now is how the method used can be implemented to ensure low cost time calculation, firstly on two level inverters and later for multilevel inverters. But it is important to mention that the SVMPWM gave a great flexibility and helps in improving the technical and economical aspect using the four leg inverter in several applications.

\section{References}

[1] Ionel Vechiu, Octavian Curea, Haritza Camblong, "Transient Operation of a Four-Leg Inverter for Autonomous Applications With Unbalanced Load," IEEE TRANSACTIONS ON POWER ELECTRONICS, VOL. 25, NO. 2, FEBRUARY 2010

[2] L. Yunwei, D. M. Vilathgamuwa, and L. P. Chiang, "Microgrid power quality enhancement using a three-phase four-wire grid-interfacing compensator," IEEE Trans. Power Electron., vol. 19, no. 1, pp. 1707-1719, Nov./Dec. 2005.

[3] T. Senjyu, T. Nakaji, K. Uezato, and T. Funabashi, "A hybrid power system using alternative energy facilities in isolated island," IEEE Trans. Energy Convers, vol. 20, no. 2, pp. 406-414, Jun. 2005.

[4] M. N. Marwali, D. Min, and A. Keyhani, "Robust stability analysis of voltage and current control for distributed generation systems," IEEE Trans. Energy Convers., vol. 21, no. 2, pp. 516-526, Jun. 2006. 
[5] C. A. Quinn and N. Mohan, "Active filtering of harmonic currents in three-phase, fourwire systems with three-phase and single-phase nonlinear loads," in Proc. IEEEAPEC'93 Conf., 1993, pp. 841-846.

[6] A. Campos, G.. Joos, P. D. Ziogas, and J. F. Lindsay, "Analysis and design of a series voltage unbalance compensator based on a three-phase VSI operating with unbalanced switching functions," IEEE Trans. Power Electron., vol. 10, pp. 269-274, May 1994.

[7] S.-J. Lee and S.-K. Sul, "A new series voltage compensator scheme for the unbalanced utility conditions," in Proc. EPE'01, 2001.

[8] D. Shen and P. W. Lehn, "Fixed-frequency space-vector-modulation control for threephase four-leg active power filters," in Proc. Inst.Elect. Eng., vol. 149, July 2002, pp. 268-274.

[9] Zhihong Ye; Boroyevich, D.; Kun Xing; Lee, F.C.; Changrong Liu “Active common-mode filter for inverter power supplies with unbalanced and nonlinear load" ThirtyFourth IAS Annual Meeting. Conference Record of the 1999 IEEE, Vol., pp. 18581863, 3-7 Oct. 1999.

[10] A. Julian, R. Cuzner, G. Oriti, and T. Lipo, "Active filtering for common mode conducted EMI reduction in voltage source inverters " Applied Power Electronics Conference APEC 98 , Anaheim, CA, 1998, pp. 934-939.

[11] Z. Lin, L.Mei, Z. Luowei, Z. Xiaojun, and Y. Yilin, “Application of a fourleg ASVG based on 3D SVPWM in compensating the harmful currents of unbalanced system," in Proc. IEEE Power Syst. Technol., 2002, vol. 2, pp. 1045-1050.

[12] P. Lohia, M. K. Mishra, K. Karthikeyan, and K. Vasudevan, “A minimally switched control algorithm for three-phase four-leg VSI topology to compensate unbalanced and nonlinear load," Trans. Power Electron., vol. 23, no. 4, pp. 1935-1944, Jul. 2008.

[13] C. A. Quinn, N. Mohan, "Active Filtering of harmonic Currents in Three-Phase, FourWire Systems with Three-Phase and Single-Phase Non-Linear Loads", IEEE Applied Power Electronics Conference (APEC), pp. 829-836, 1992.

[14] A. Nava-Segura, G. Mino-Aguilar, "Four-Branches-Inverter-Based-Active-Filter for Unbalanced 3-Phase 4-Wires Electrical Distribution Systems", IEEE Industry Applications Conference (IAS), pp. 2503-2508, 2000.

[15] P. Rodriguez, R. Pindado, J. Bergas, “Alternative Topology For Three-Phase Four-Wire PWM Converters Applied to a Shunt Active Power Filter", IEEE Proceedings of Industrial Electronics Society (IECON), pp. 2939-2944, 2002.

[16] N. Mendalek, "Modeling and Control of Three-Phase Four-Leg Split-Capacitor Shunt Active Power Filter," ACTEA '09. International Conference on Advances in Computational Tools for Engineering Applications, pp. 121-126. July 15-17, 2009 Zouk Mosbeh, Lebanon.

[17] Seyed Hossein Hosseini, Tohid Nouri2 and Mehran Sabahi, "A Novel Hybrid Active Filter for Power Quality Improvement and Neutral Current Cancellation," International Conference on Electrical and Electronics Engineering, ELECO 2009, pp. I-244 - I-248.2009.

[18] Valdez, A.A.; Escobar, G.; Torres-Olguin, R.E, “A novel model-based controller for a three-phase four-wire shunt active filter," 37th IEEE Power Electronics Specialists Conference, 2006. PESC '06.

[19] M. Aredes, K. Heumann, J. Hafner, "A Three-phase Four-Wire Shunt Active Filter Employing a Conventional Three-Leg Converter", European Power Electronics Journal, Vol. 6, no 3-4, pp 54-59, December, 1996. 
[20] Verdelho P., Marques G., “A Neutral Current Electronic Compensator", Industrial Electronics Conference, 1998.

[21] Fabien Meinguet, Johan Gyselinck, "Control Strategies and Reconfiguration of Four-Leg Inverter PMSM Drives in Case of Single-Phase Open-Circuit Faults," , pp. 299304,2009

[22] Fabien Meinguet*, Eric Semail§, lohan Gyselinck, "Enhanced Control of a PMSM Supplied by a Four-Leg Voltage Source Inverter Using the Homopolar Torque," Proceedings of the 2008 International Conference on Electrical Machines,2008

[23] R.L.A Ribeirol, C.B. Jacobina, A.M.N. Lima, E.R.C. da Silva, "A Strategy for Improving Reliability of Motor Drive Systems Using a Four-Leg Three-phase Converter," ,pp. 385-390,2001

[24] Drazen Dujic, Martin Jones, Member, "A General PWM Method for a $(2 n+1)$-Leg Inverter Supplying $\mathrm{n}$ Three-Phase Machines," IEEE Transaction on Industrial electronics, Vol. 56, No. 10,pp. 4107-4118, October 2009

[25] A. Bouscayrol, S. Siala*, M. Pietrzak-David, B. deFomel, "Four-Legged PWM inverters feeding two induction motors for a Vehicle drive applications,", Power Elecironi zsa nd Variable-Speed Drivers, pp. 700-705,26 - 28 October 1994,

[26] Tomáš Glasberger, Zdenek Peroutka, "Control of Power Supply Unit for Military Vehicles Based on Four-Leg Three-Phase VSI with Proportional-Resonant Controllers," 2008 13th International Power Electronics and Motion Control Conference (EPE-PEMC), pp. 1268-1273,2008

[27] Desheng Liu Miao Guan Lei Zhang Yong Wang, “ Research on the Three-Phase FourLeg Aeronautical Static Inverter Based on Three-dimensional Space Vector Modulation in abc Coordinates," The Ninth International Conference on Electronic Measurement \& Instruments, (ICEMI),pp. 729-732,2009

[28] Zdenĕk Peroutka, Tomáš Glasberger and Jan Molnár, “Design of New Diesel-Electric Power Supply Unit for Military Vehicles", 14th International Power Electronics and Motion Control Conference, EPE-PEMC 2010,pp. 101-108,2010

[29] Jing Li, Fang Zhuo, Jinjun Liu, Xianwei Wang, Bo Wen, Lin Wang, Song Ni, “Study on Unified Control of Grid-connected Generation and Harmonic Compensation in Dual-stage High-capacity PV system,",pp. 3336-3342,2009

[30] Said El-Barbari and W. Hofmann, "Control of a 4 Leg Inverter for Standalone Photovoltaic Systems,",pp.348-354,2001

[31] Yogesh K. Chauhan, Sanjay K. Jain, and Bhim Singh, "Transient Performance of ThreePhase Four-Wire Stand Alone Supply System with Static Converter Employed for Industrial Loads," 2008

[32] Salem Fouzey, Said El-Barbari4 W. Hofmann, Caner Unsalver, "A new space vector modulation scheme for three phase four wire inverter for standalone photovoltaic systems,"

[33] Egon Ortjohann, Arturo Arias, Danny Morton, Alaa Mohd, Nedzad Hamsic, Osama Omari, "Grid-Forming Three-Phase Inverters for unbalanced loads in hybrid power system,",pp. 2396-2399,2006

[34] Manuel A. Perales,M.M. Prats,Ramón Portillo,José L.Mora,José I.León, and Leopoldo G.Franquelo, "Three-Dimensional Space Vector Modulation in abc CoordinatesforFour-Leg Voltage Source Converters", IEEE Power Elect. Letters,Vol.1, No.4, pp 104-109, December 2003.

[35] M. M. Prats, L. G. Franquelo. J. I Leon. R. Portillo,E. Galvan and J. M. Carrasco, "A SVM3D generalized algorithm for multilevel converters".. Proc. IEEE IECON.'03, 2003. pp. 24-29. 
[36] M. Aredes, J. Hafner, and K. Heumann, "Three-phase four-wire shunt active filter control strategies," IEEE Trans. Power Electron., vol. 12, no. 2, pp. 311-318, Mar. 1997.

[37] C. J. Zhan, A. Arulampalam, and N. Jenkins, "Four-wire dynamic voltage restorer based on a three-dimensional voltage space vector PWM algorithm," IEEE. Trans. Power Electron., vol. 18, no. 4, pp.1093-1102, Jul. 2003.

[38] R. Faranda and I. Valade, "UPQC compensation strategy and design aimed at reducing losses," in Proc. IEEE Int. Symp. Ind. Electron., Jul. 2002, vol. 4, pp. 1264-1270.

[39] Jun Liang, Tim C. Green, Chunmei Feng, and George Weiss, "Increasing Voltage Utilization in Split-Link Four-Wire Inverters," IEEE TRANSACTIONS ON POWER ELECTRONICS, VOL. 24, NO. 6,pp. 1562-1569, JUNE 2009

[40] Changjiang Zhan, Atputharajah Arulampalam, Nicholas Jenkins, "Four-Wire Dynamic Voltage Restorer Based on a Three-Dimensional Voltage Space Vector PWM Algorithm," IEEE Trans. On Power electronics, Vol. 18, No. 4,pp.1093-1102, July 2003

[41] H. Pinheiro, F. Botterbn, C. Rech, L. Schuch, R. F. Camargo, H. L. Hey, H. A. Griindling, J. R. Pinheiro, "Space Vector Modulation for Voltage-Source Inverters: A Unified Approach,",pp. 23-29,2002

[42] Ning-Yi Dai, Chi-Seng Lam, Man-Chung Wong, Ying-Duo Han, “Application of 3D Direct PWM in Parallel Power Quality Compensators in Three-phase Four-wire Systems,", pp. 3220-3225,2008

[43] T.H. Nguyen, Paul K.W. Chan, Y. Shrivastava, S.Y.R. Hui, “A Three-Dimensional Space Vector Modulation Scheme for Three-Level Three-wired Neutral Point Clamped Converters,",pp. 2307-2314,2005

[44] Jing Tang, Man-Chung Wong, Yingduo Hun, "Novel Five-Level Inverter PWM Control in 3-Phase 4-Wire System for Power Quality," ,pp. 579-584,2002

[45] Ning-Yi Dai , Man-Chung Wong, Yin-Duo Han, "Controlling Tri-level Center-Split Power Quality Compensator by 3-Dimensional Space Vector Modulation," , pp. 1692-1697,2003

[46] Man-Chung Wong, Jing Tang, Ying-Duo Han, "Cylindrical Coordinate Control of Three-Dimensional PWM Technique in Three-Phase Four-Wired Trilevel Inverter," IEEE Transaction On Power Electronics, Vol. 18, No. 1,pp. 208-220, January 2003

[47] Ning-Yi Dai,Man-Chung Wong,Ying-Duo Han, "Three-Leg Center-Split Inverter Controlled by 3DSVM under dc Variation,",pp. 1362-1367

[48] Salem M. Ali, Marian P. Kazmierkowski, "PWM Voltage and Current Control of FourLeg VSI,",pp. 196-201,1998

[49] Ojo, O.; Kshirsagar, P.M. “Concise modulation strategies for four-leg voltage source inverters" IEEE 33rd Annual Power Electronics Specialists Conference, pesc 02. vol.1, pp. 238 - 243, 2002.

[50] Ojo, O.; Kshirsagar, P.M. “Concise modulation strategies for four-leg voltage source inverters" Power Electronics, IEEE Transactions on , Vol. 19, Issue: 1 , pp. 46 -53, Jan 2004.

[51] Liu, C., Peng, D., Lai, J., Lee, F.C., Boroyevich, D., Zhang, R. “Fourlegged converter 3-D SVM scheme over-modulation study" Applied Power Electronics Conference and Exposition, APEC Fifteenth Annual IEEE, Vol.1 , pp. 562 -568, 2000.

[52] Gan Dong, Olorunfemi Ojo, “Design Issues of Natural Reference Frame Current Regulators with Application to Four Leg onverters", Proc. Of IEEE-PESC '05, pp. 1370-1376. 
[53] Gan Dong, Olorunfemi Ojo, "Current Regulation in Four-Leg Voltage-Source Converters", IEEE Transactions on Industrial Electronics, vol.54, no 4, Aug. 2007, pp. 2095-2105.

[54] Fanghua Zhang, Yangguang Yan, "Selective Harmonic Elimination PWM Control Scheme on a Three-Phase Four-Leg Voltage Source Inverter," IEEE TRANSACTIONS ON POWER ELECTRONICS, VOL. 24, NO. 7, JULY 2009.

[55] M. J. Ryan, R. W. De Doncker, and R. D. Lorenz, “Decoupled control of a 4-leg inverter via a new 4$\urcorner 4$ transformation matrix," in Proc. IEEE Power Electron. Spec. Conf., 1999, pp. 187-192.

[56] Jang-Hwan Kim, Seung-Ki Sul,Prasad N. Enjeti, “A Carrier-Based PWM Method with Optimal Switching Sequence for a Multi-level Four-leg VSI," IAS,pp. 99-105, 2005

[57] Maxim A, Dybko, Sergey S. Tumaev, Sergey V. Brovanov, "A Power Losses Calculation in a Four-legged Three-level Voltage Source Inverter," 10th International Conference and seminar EDM, Sections VI, pp. 365-369, JULY 1-6, ERLAGOL,2009

[58] Worrajak Muangjai, Suttichai Premrudeepreechacharn, "Implementation of a Carrierbased Three-dimensional Space Vector PWM Technique for Three-phase Four-leg Voltage Source Converter with Microcontroller," ICIEA,pp. 837-841, 2009

[59] Praveen Kumar M, Mahesh Kumar Mishra, Sandeep Joseph, "Switching Minimization of Three-phase Four-leg Dynamic Voltage Restorer," 2009

[60] Salem M. Ali, Marian P. Kazmierkowski, "Current Regulation of Four-Leg PWMVSI,",pp. 1853-1858,1998

[61] Verdelho, P.; Marques, G.D, “A current control system based in a $\beta 0$ variables for a fourleg PWM voltage converter," Proceedings of the 24th Annual Conference of the IEEE Industrial Electronics Society, 1998. IECON '98. Vol. 3, pp. 1847 - 1852.

[62] Reza Nasiri, Student, Ahmad Radan, "Pole-Placement Control Strategy for 4-Leg Voltage-Source Inverters," 1st Power Electronic \& Drive Systems \& Technologies Conference,pp.74-79,2010

[63] V. Yaramasu, J. Rodriguez, B. Wu, M. Rivera, A. Wilson and C. Rojas, “A Simple and Effective Solution for Superior Performance in Two-Level Four-Leg Voltage Source Inverters: Predictive Voltage Control,"

[64] Nikhil Prabhakar, Mahesh K. Mishra, "Dynamic Hysteresis Current Control to Minimize Switching for Three-Phase Four-Leg VSI Topology to Compensate Nonlinear Load," IEEE Trans ON Power Electronics, Vol. 25, No. 8,pp. 1935-1942, August 2010

[65] N.V. Nho and M.J. Youn, "Carrier PWM algorithm with optimised switching loss for three-phase four-leg multilevel inverters," ELECTRONICS LETTERS Vol. 41 No. 1, 6th January 2005

[66] N.V. Nho and M.J. Youn, “Carrier PWM algorithm with optimised switching loss for three-phase four-leg multilevel inverters," ELECTRONICS LETTERS Vol. 41 No. 1, 6th January 2005

[67] Salvador Ceballos, Josep Pou, Jordi Zaragoza, José L. Martín, Eider Robles, Igor Gabiola, Pedro Ibáñez, "Efficient Modulation Technique for a Four-Leg Fault-Tolerant Neutral-Point-Clamped Inverter," IEEE Trans On Ind. Elect.Vol. 55, No. 3,pp. 10671074, March 2008

[68] O’ scar Lo'pez, Jacobo A' lvarez, Jesu's Doval-Gandoy, Francisco Freijedo, Alfonso Lago and Carlos M. Pen alver, "Four-Dimensional Space Vector PWM Algorithm for Multilevel Four-Leg Converters," 2008

[69] Fernando Botteron, Robinson F. de Camargo, Humberto Pinheiro Hilton A. Griindling, Jose R. Pinheiro and Helio L. Hey, "On the Space Vector Modulation and Limiting 
Algorithms for Three-phase Four-Leg Voltage Source Inverter in abc Coordinates," , pp. 12-17,2002

[70] Manuel A. Perales, M. M. Prats, Ramon Portillo, Jose L. Mora, Leopoldo G. Franquelo, "Three Dimensional Space Vector Modulation for four-leg inverters using natural coordinates,", pp. 1129-1134,2004

[71] Rui Wu, Donghua chen, shaojun xie, “ A Three-dimentsional Space Vector Modulation Algorithm in A-B-C Coordinate Implementation By FPGA",pp. 1017-1075,2005

[72] Man.Wong, J.Tang and Y.Han "Three-Dimensional Pulse-Width Modulation Technique in Three-level Power Inverters for Three-Phase Four-Wired System”, IEEE Trans. on Power Electronics, 16(3), pp.418 -427, May 2001.

[73] A.Kouzou, Mahmoudi M.O, Boucherit. M.S, "Comparative Study of the SVPWM 3D Algorithms Used for four-leg inverters", SSD09,6th International Multi-Conference on Systems, signals and Devices. Djerba Tunisia. 23-26 March 2009.

[74] Richard Zhang, V. Himamshu Prasad, Dushan Boroyevich and FredC.Lee, "ThreeDimensional Space Vector Modulation for Four-Leg Voltage-Source Converters", IEEE Transaction on Power Electronics, Vol.17, No.3, pp 314-326, May2002

[75] R. Zhang, D. Boroyevich, H. Prasad, H. Mao, F. C. Lee, and S Dubovsky, “A three-phase inverter with a neutral leg with space vector modulation," in Proc. IEEE-APEC'97 Conf., 1997, pp. 857-863.

[76] R. Zhang, H. Prasad, D. Boroyevich, and F. C. Lee, "Analysis and design of a threephase inverter with a neutral leg," in Proc. EPE'97 Conf., 1997, pp. 1.170-1.175.

[77] H. Prasad, D. Boroyevich, and R. Zhang, "Analysis and comparison of space vector modulation schemes for a four-leg inverter," in Proc. IEEEAPEC' 97 Conf., 1997, pp. 864-871.

[78] E. Ortjohann, A. Mohd, N. Hamsic, A. Al-Daib, M.Lingemann, " three-Dementional Space Vector Modulation Algorithm for Three-leg Four Wire voltage source Inverters," POWERENG,pp. 605-610, April 12-14,2007, Setubal, Portugal

[79] M. G. Villala, E. Ruppert F, « 3-D Space vector PWM for Three-leg four-wire Source Inverter," 35th annual IEEE Power electronics Specialists conference,pp. 3946-3951, Aachen, Germany,2004

[80] E. Ortjohann, A. Mohd, N. Hamsic, M. Lingemann, W. Sinsukthavorn, D. Morton, “A Novel Space Vector Modulation Control Strategy for Three-leg Four-Wire Voltage Source Inverters,"

[81] Xiangsheng Li, Zhiquan Deng, Zhida Chen, and Qingzhao Fei, "Analysis and Simplification of Three-dimensional Space Vector PWM for Three-phase Four-leg Inverters,"

[82] Kouzou A, Mahmoudi M.O, Boucherit M.S, "A new 3D-SVPWM algorithm for Four-leg Inverters," IEEE International Electric Machines and Drives Conference, IEMDC '09.pp. 1674 - 1681, May 2009. Miami, Florida, USA 



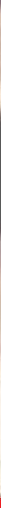

\section{Edited by Miroslav Chomat}

The subject of this book is an important and diverse field of electric machines and drives. The twelve chapters of the book written by renowned authors, both academics and practitioners, cover a large part of the field of electric machines and drives. Various types of electric machines, including three-phase and single-phase induction

machines or doubly fed machines, are addressed. Most of the chapters focus on modern control methods of induction-machine drives, such as vector and direct torque control. Among others, the book addresses sensorless control techniques, modulation strategies, parameter identification, artificial intelligence, operation under harsh or failure conditions, and modelling of electric or magnetic quantities in electric machines. Several chapters give an insight into the problem of minimizing losses in electric machines and increasing the overall energy efficiency of electric drives.

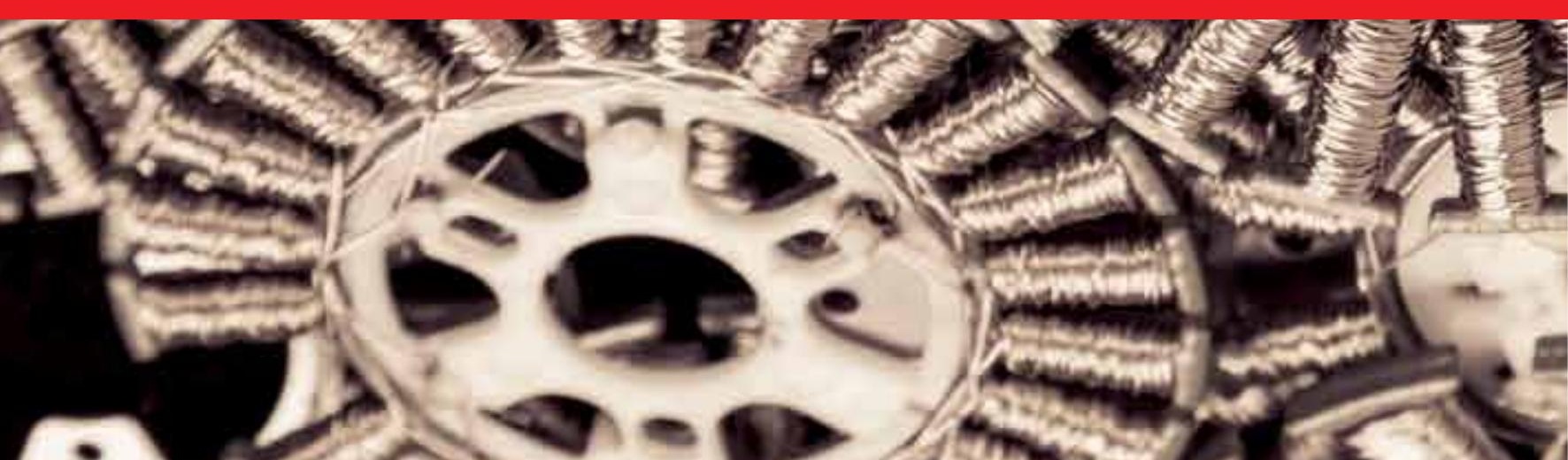

